Soil Hydrology and Biophysics 



\title{
Soil Hydrology and Biophysics
}

\author{
JOHN SELKER AND DANI OR
}

\author{
OREGON STATE UNIVERSITY \\ CORVALLIS, OR
}




\section{() (i) (2) (2)}

Soil Hydrology and Biophysics by John Selker E Dani Or is licensed under a Creative Commons Attribution-NonCommercial-ShareAlike 4.0 International License, except where otherwise noted.

Download for free at https://open.oregonstate.education/soilhydrologyandbiophysics

Publication and on-going maintenance of this textbook is possible due to grant support from Oregon State University Ecampus.

Suggest a correction 


\section{Contents}

$\underline{\text { Soil Hydrology and Biophysics }} \quad$ vii

1. Fundamentals of Soil Hydrology and Bio-Physics 1

2. Basic Relationships and the Soil Phases Under Equilibrium Conditions 45

3. Liquid Flow in Soils (Hydrodynamics) 124

4. Land Atmosphere Interactions 242

5. Heat Flow and Thermal Effects in Soils 243

6. Solute Transport in Soils and Vadose Materials 267

7. The Gas Phase in Soils 306

8. Bio-Physics of the Vadose Zone 307

9. Mechanics of Soil 392

10. Selected Applications Across Scales 393

Appendix I

References

Symbols used

Physical units and conversions $\quad 414$

Selected physical constants 422

Properties of liquid water 423

Creative Commons License $\quad 424$

Recommended Citations

Versioning 



\section{Soil Hydrology and Biophysics}

John Selker, Distinguished Professor, Oregon State University and

Dani Or, Professor, ETH-Zurich 



\section{Fundamentals of Soil Hydrology and Bio-Physics}

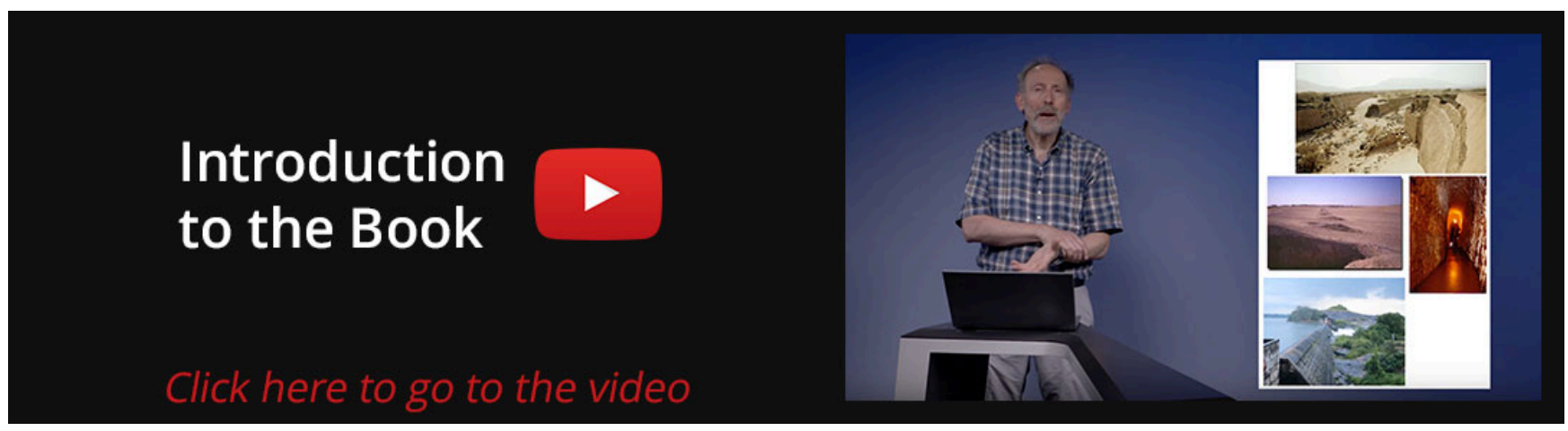

\section{Goals of the text}

The study of movement of water through soils and geologic materials is as old as irrigation itself (at least 4,000 years), so we let ourselves off the hook in terms of providing an exhaustive list of related texts or every application of this material. At the same time, it is important to be aware that there are a great number of excellent and up-to-date sources of related information. Where does this text fall between these alternatives, and why is it necessary at all? This text differs from the previous in several features. First, it is concerned with the presentation of this material as a discipline: a logically connected body of ideas that explains an aspect of nature. Specifically, we provide a framework for understanding the status and movement of energy, liquids, gases, and the solutes they might contain through natural porous media. We also lay out some of the most important boundary processes, including evaporation, infiltration, and vapor diffusion. We provide derivations and explanations for each concept and result so that these may form a solid foundation for the understanding of the systems which depend upon earth's subsurface. This means that fewer results can be presented in a given number of pages, but hopefully makes the ideas more accessible and generally applicable. In terms of scale, you will notice that we spend much more time working on the grain (say $0.1 \mathrm{~mm}$ ) and soil-sample (say $10 \mathrm{~cm}$ ) scale than the larger scales (from soil profile to global), but our goal is to provide the tools and frameworks that will describe large-scale processes mediated by the vadose zone. This means that we do take special efforts in the book to discuss the spatial structure of deep percolation, lateral flow in hillslopes and layered and soils, and limitation to Richards equation for large spatial scales. The topics and scope of the materials included in this book reflect on the disciplinary boundaries of modern soil hydrology and biophysics - we seek to address traditional soil physical properties and characterization, and basics of water flow, solute and heat transport, but also to inject advances in capillarity, in linking biological processes and agent with the physical environment and processes (biophysics).

Advances in measurement science, links with atmospheric sciences and remote sensing require a different approach to the scale of soil processes representation and special sections are devoted to linking energy balance and evaporation process with climate science and remote sensing. The historical links between soil physics and geotechnical engineering that were strong during the inception of these two sister disciplines in 1930's require strengthening, one cannot discuss root growth and earthworm activity in soil without understanding of soil mechanical properties. Similarly, linking pore spaces to transport properties requires understanding of how soils become compacted by farm implements or livestock grazing. Hence, we endeavor to reintroduce basic notions of soil mechanics into the mainstream soil physics.

Though we are not rigid in our approach to presentation, we have in mind four levels of understanding that guide our structure. We hope to serve students interested at each level, with the proviso that our responsibility for the fourth level is to provide a foundation which prepares the reader to achieve this expertise on their own. 
1. Conceptual. The reader achieving this level should have an accurate physically-based understanding of the basis of key results. This is achieved though text, diagrams and generic equations (i.e., simply algebraic balances of inputs and outputs). This is the level of understanding that we believe any well educated person interested in natural systems should attain. To get this level of understanding we provide more detailed introductions to topics and syntheses of material at the beginning and ends of sections and chapters. These elements provide a framework in which the more advanced readers will be able to organize the more detailed information which can otherwise get jumbled. The readers of this book are specifically encouraged to "skip ahead" to read the synthesis at the end of each chapter to help them build the conceptual framework which is helpful in learning and retaining higher level material.

2. Quantitative. The first step in predictive understanding is translation of concepts into quantified expressions. This generally involves application of conservation of mass, energy, and momentum to derive the relationships between fluxes, stores, and media. Many of the equations we obtain are very challenging to actually apply because they are based on complex characterizations of the constituents of an ecological system, and often have highly non-linear response behaviors. At this level of understanding the reader will have grasped the quantitative relationships between diverse interacting elements, but will is only expected to be able to solve a limited set of often simplified example problems.

3. Applied prediction. Here the reader will select the appropriate equations and parameters to a specific focused problem. Their solutions will be accurate for simple problems involving either homogeneous or simply structure settings. They will be able to correctly formulate and apply all required boundary, initial, and media conditions to obtain appropriate solutions to these problems.

4. Expert proficiency in arbitrary problems. Here the reader will be able to identify, as a function of scale, the appropriate approach to representing a problem that may span grain to landscape scales. Model selection, parameter estimation and upscaling, and uncertainty analysis are all required at this level of understanding.

From this framework it is clear that quantification is central to our view of the vadose zone. To give the chance for achievement of level 4 understanding, the reader must understand each of the underlying assumptions and limitations of each formulation. This is why we insist on deriving expressions from first principles. This does not mean that we go all the way back to quantum mechanics in each derivation. We state the assumptions employed for each scale clearly so that the reader can later discern the applicability of the expressions obtained. The mathematical treatment must be handled with vector analysis and partial differential equations in keeping with the systems we seek to describe. Though essential to accurate description of many vadose processes, tensors are used only sparingly, and with ample support so that readers without background in this notation can gain the key insights.

The anticipated audiences are those with interest in soil/vadose zone processes, and at least one year of college level calculus, physics, and chemistry (e.g., upper division undergraduates, graduates, and practicing hydrologists). The selection of topics is different than previous texts. We address many issues, such as hysteresis, scaling, geochemical and stochastic methods, in greater detail than has been typical. We believe that these topics are central to understanding the behavior of fluid and solutes in partially saturated media, and have been short-changed for too long, much to the detriment of the completeness of understanding this discipline. The style of writing is less formal than many texts; we hope this helps remove the long-standing barrier of formal jargon that has stifled understanding in this important area of geophysics. 


\section{An Introduction to the the "Critical Zone" and the Vadose Zone}

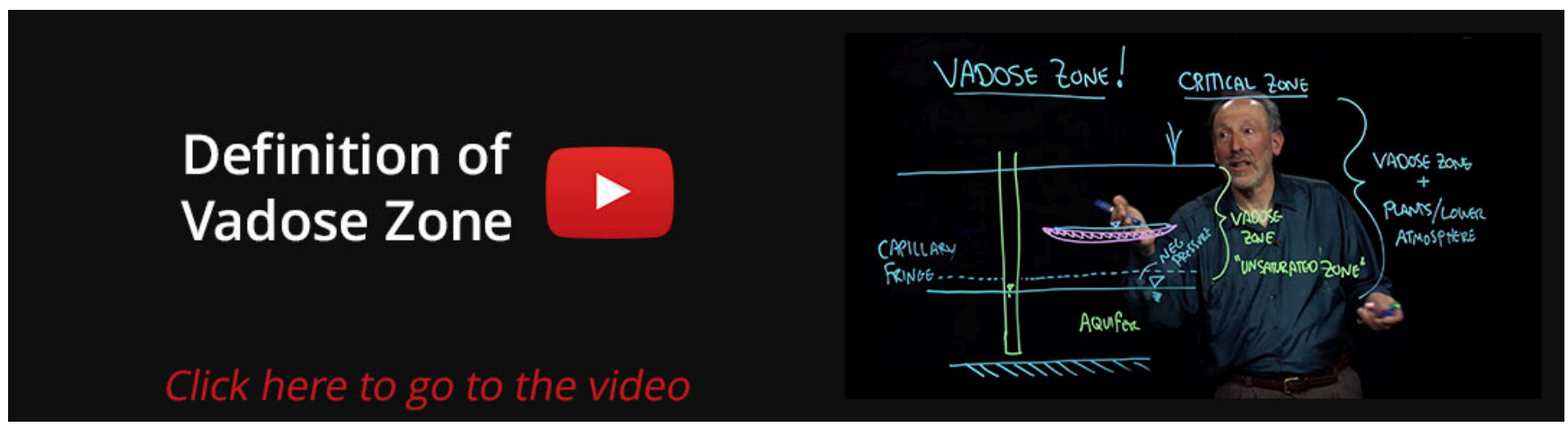

People have long sought to divide the earth into handy functional elements which could be considered independently. For instance there are atmospheric scientists who study the the gas that fills the space from where are feet touch the ground and above. There are the groundwater hydrologists who study aquifers. Of course, any such arbitrary delineation is not exact, for example, is the air in a cave or in the pore-space of the soil, the domain of an atmospheric scientist? Is the water perched on a clay layer far above the regional water table groundwater? So right from the getgo, we want you to realize that these constructs break down, and should be broken down, as soon as we consider processes at interfaces of the earth. For example, is the exchange of energy and mass at the ocean surface the domain of oceanography or atmospheric science? Clearly both, so we see these fields are not distinct, but overlapping.

This text focuses on the processes occurring in the geologic media which lies below the surface of the earth, but above the water table of the shallowest year-round aquifer which is often referred to as the vadose zone, or unsaturated zone (Figure 1.1). The water table is defined as the elevation where if a borehole were drilled into the very top of this shallowest aquifer, water would fill the hole to this height. This is equivalent to saying that the vadose zone is the region where the pressure of water is typically less than zero (it is under tension, or suction). The term vadose zone has not been strictly defined, so some people use it to refer to the "unsaturated zone" above an aquifer. This differs from our definition by excluding the capillary fringe, which is typically saturated, but above the water table, as well as regions which are only seasonally saturated. Frankly, there is little to be gained by splitting hairs in this definition, since the term is understood to be qualitative. 


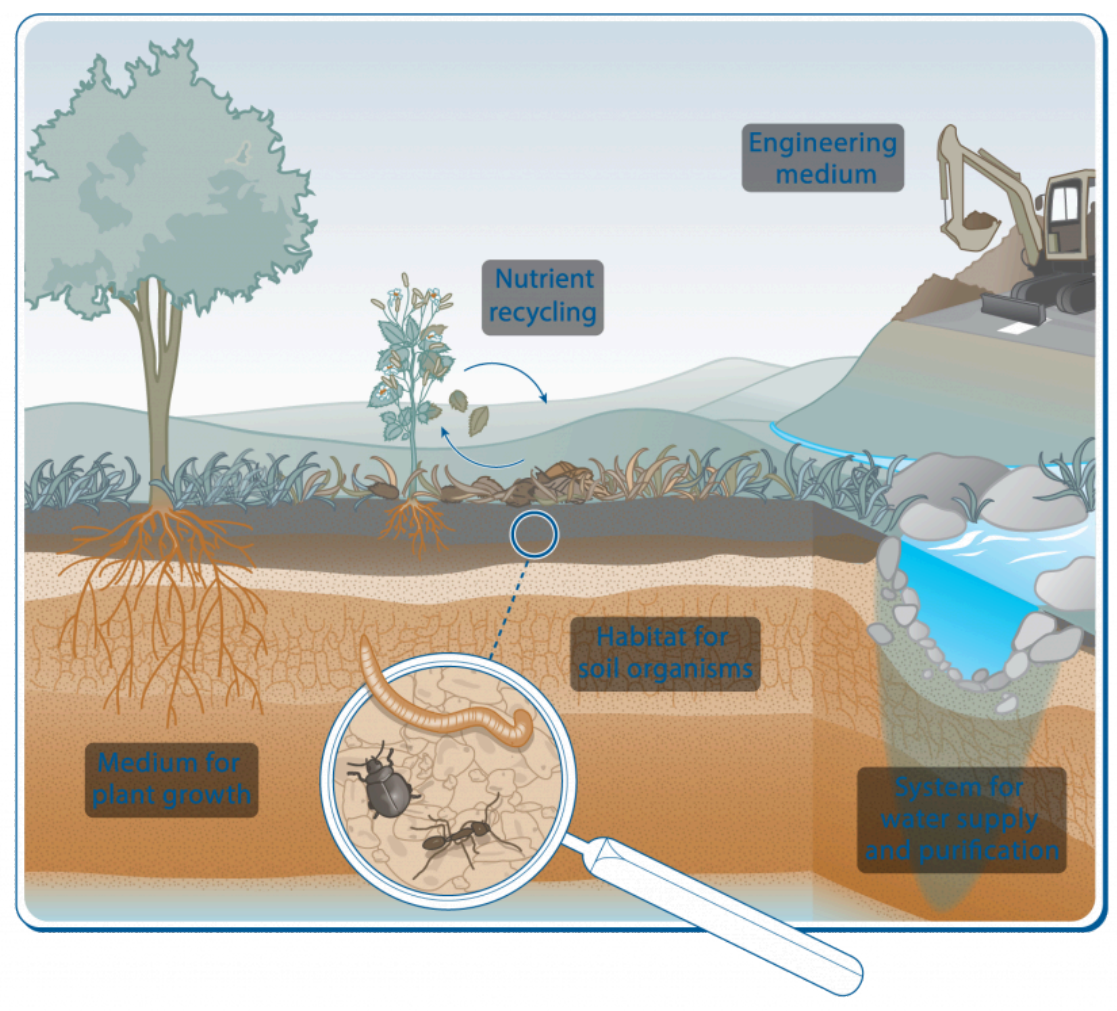

Figure 1.1. Illustration of a few of the diverse processes of interest in soil/vadose zone.

A few words about the extent and physical nature of the vadose zone are in order. Vadose zone depths go up to hundreds of meters (e.g., the US desert Southwest). For the most part, depths greater than 5 meters indicate that either there is a drainage in the area (e.g., gully, escarpment, valley, or canyon) or that people have been pumping ground water. As is shown later in Chapter 3, without the help of plant roots, water cannot evaporate from depths greater than a few meters below the soil surface, leaving vast supplies of groundwater under many areas which have been deserts for millennia (e.g., central Somalia, one of the hottest places on earth, where the water table can be reached easily with hand-dug wells).

The "soil" is typically defined as the upper portion of the earth's surface where the minerals have undergone significant weathering. The depth of a soil depends on the time, climate, position in the landscape, and "parent material" from which it was derived (Figure 1.2, and 1.3) 


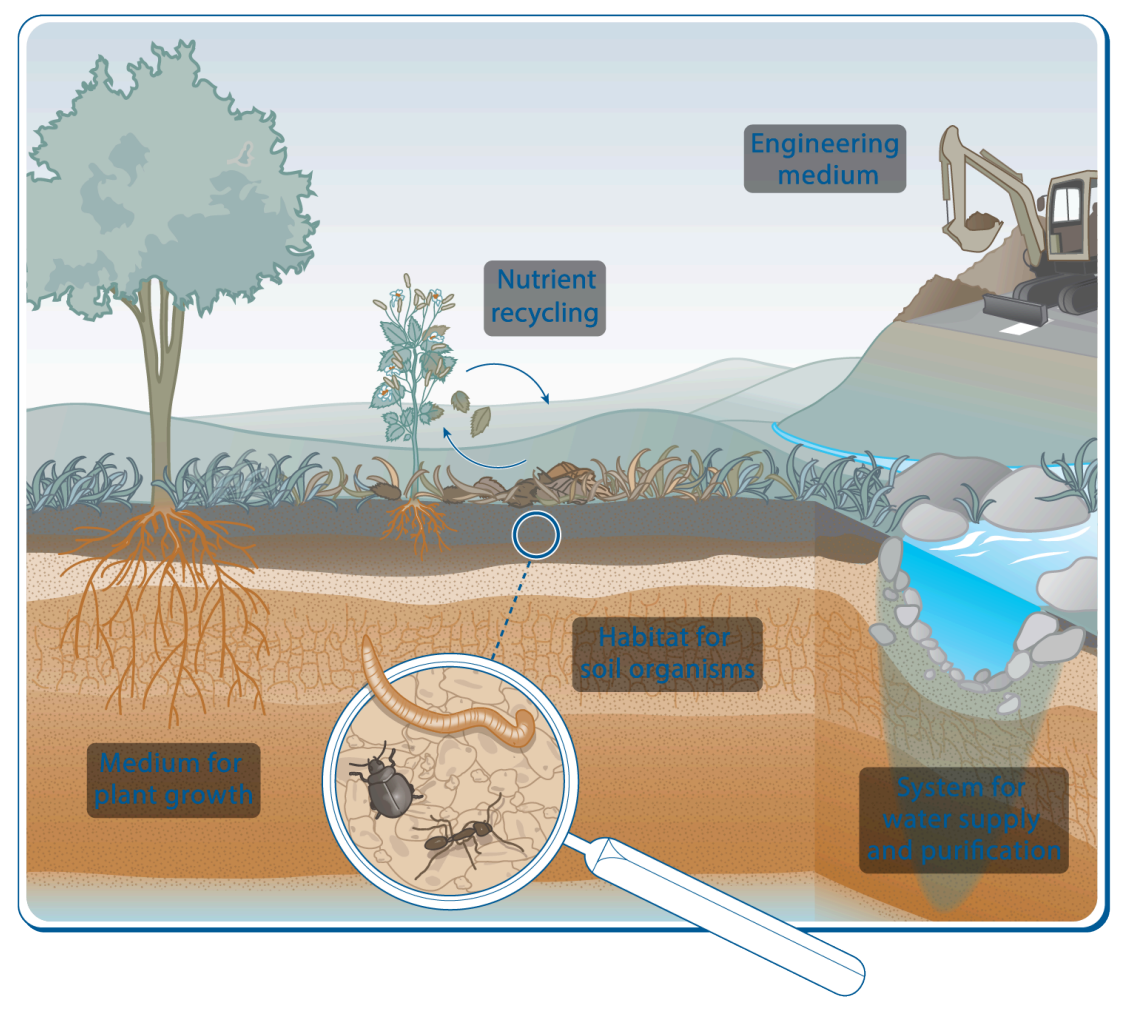

Figure 1.1. Illustration of a few of the diverse processes of interest in soil/vadose zone. 


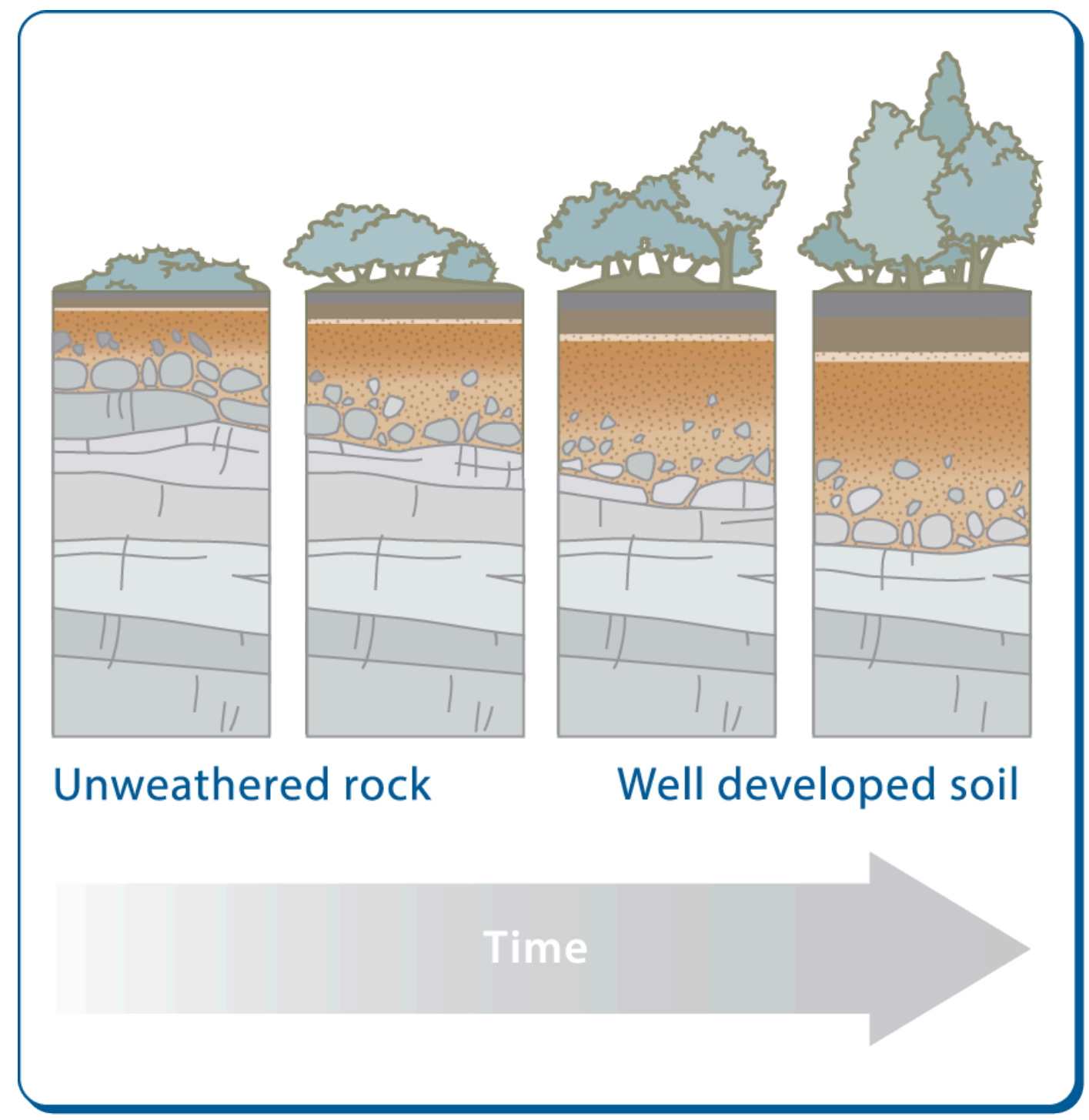

Figure 1.2 Illustration of the development of soil

from the "parent

material (rock or

deposited sediment) over

the course of time. 


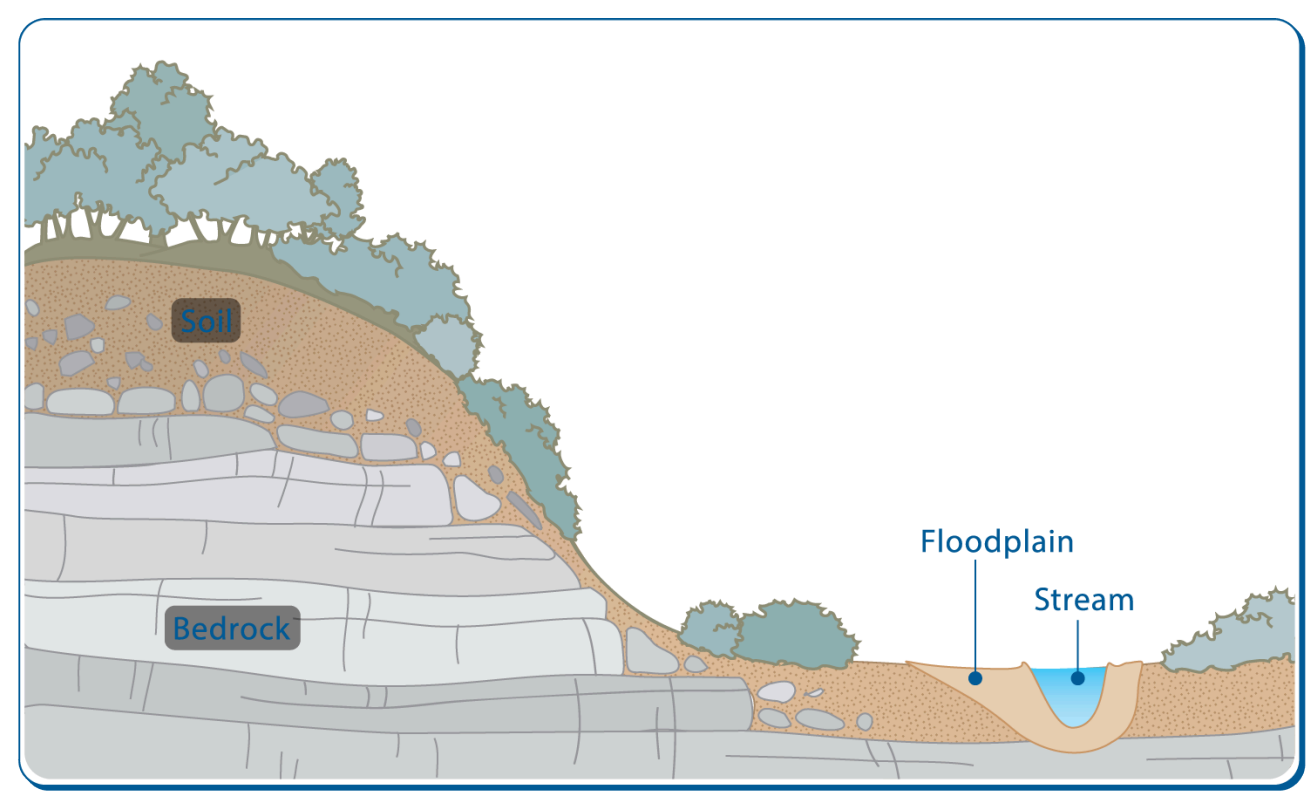

Figure 1.3 Illustration of the impact of landscape position on soil depth and character.

Although we admit to being biased in this account, the importance of the vadose zone to human existence cannot be overstated: The vast majority of plants depend on the nutrients and water stored in the vadose zone; almost all of the available groundwater (which accounts for more than half of all potable water) is generated by percolation through the vadose zone. The vadose zone is composed of highly reactive compounds (clays, organic acids, oxygen and water) which serve to purify water passing through, and provide nutrients for plants which root in it.

Study of processes in the vadose zone are included in many disciplines, most notably soil science and geology. It is also of significant interest to a wide range of professions, including civil, petroleum and geotechnical engineering, forest science and engineering, microbiology, and many others. It is, therefore, evident that no single text can adequately treat this topic in complete detail. In this text we focus on the fundamental physical and chemical properties of the vadose zone, and attempt to provide a quantitative framework for understanding the basic processes. Any of the topics covered here can be explored in greater depth by availing yourself of texts from an appropriate related discipline.

If one had to identify a single common feature of vadose environments it would be the heterogeneous nature of the materials. The vadose zone is typically composed of a structured weathered mineral surface soil overlying granular sedimentary materials. Soil properties typically vary laterally in a lognormal fashion (e.g., Figure 8.8 and the classic work of Biggar and Nielsen, 1976), with distinct vertically organized layers resulting from both sequential deposition and weathering, with varying mineralogy, structure, and chemistry. The complexity posed by such amalgams of materials presents great challenges to prediction of transport processes. Although this text deals primarily with deterministic approaches to quantifying transport processes, the nature of the vadose zone lends itself to stochastic methods, with the underlying message that it is often more useful and reasonable to predict the range of possible outcomes rather than the expected outcome, which may have a vanishingly small probability of occurring.

\section{Soil provision and regulatory functions in the biosphere}

Soil is a remarkably complex ecological systems, where the nature and dynamics of interactions among physical, chemical, and biological processes are intertwined according to the climatic region and mineral characteristics, and ecological inhabitants. Soil regulatory and life-supporting functions are derived from its position at the interface between the lithosphere, hydrosphere, and the atmosphere [Brady and Weil, 2003]. Soil provides an almost ideal framework for life, storing water, facilitating gas movement, providing diverse nutrients and elemental building blocks. For this reason soil is typically found to be the most biologically active compartment of the biosphere, supporting virtually all terrestrial life. This can be quantified, and the results are striking. Soil host the largest pool of biological diversity with an estimated $3 \times 10^{29}$ prokaryotic cells concentrated in approximately $10^{5} \mathrm{~km}^{3}$. In comparison, oceans 
contain approximately $10^{29}$ cells in a total volume of $10^{9} \mathrm{~km}^{3}$ [Whitman et al., 1998]. Thus, the mean soil prokaryotic density of soils exceeds that of the oceanic by a factor or 10,000. Beyond density of life, the biological diversity found in soils exceed values found in oceans by 3 to 4 orders of magnitude as well [Torsvik et al., 2002]. It has been stated that the diversity found in one ton of soil exceeds that found in all oceans combined [Curtis et al., 2002]. Of course this is not a competition, but does point to the extraordinarily rich environment for life provided by soils.

One element that explains the extent of bio-geochemical processes, biological activity and diversity found in soils may be attributed to the high degree of habitat heterogeneity within complex pore spaces [Crawford et al., 2005], the numerous interfacial functions and processes within soils, and to highly dynamic distribution of water and nutrients. Such variations affect connectivity and transport pathways of various fluxes resulting in a non-uniform and patchy resource distribution. Soil provides important ecosystem services such as:

- Serving as growth environment for higher plant roots and habitats for largest number of species in the biosphere

- Act as active water purification system and storage reservoir

- It is nature's recycling system

- Provide engineering foundations, materials and waste isolation

As an interface, soil (and other terrestrial surfaces) interact with the atmosphere at a wide range of time and spatial scales (Figure 1.2). Soil water storage and gradual provision for plant growth and evaporation extends the climatic memory of soil long after a rainfall event occurs. This point can be made concrete by noting that if a plant is to convert the sun's energy into fixed carbon, it requires about $5 \mathrm{~mm}$ of water per day. A typical soil profile can provide about $25 \%$ of its volume in water (a concept which we will later discuss known as field capacity), so a $1 \mathrm{~m}$ deep soil profile can store about $250 \mathrm{~mm}$ of plant available water, which will keep non-desert plants alive for about 50 days in absence of any added water. So we see that soil provides an essential bridge for sustained plant life between rainfall events.

While water is the most obvious requirement for plant life, soil organic carbon storage is both fundamental to terrestrial life and acts as a sort of capacitor that buffers atmospheric $\mathrm{CO}_{2}$ at time scales of seasons to decades (hence the importance of soil carbon sequestration potential in the discussion about climate change). Again, some numbers can be helpful to show the fundamental role in controlling atmospheric carbon. A typical soil organic matter mass fraction might be $1 \%$ for the upper $1 \mathrm{~m}$ (including all carbon constituents: roots, bugs, etc.), which works out to about $16 \mathrm{~kg} / \mathrm{m}^{2}$. If we average over the entire surface of the earth, assuming about $1 / 4$ has soil cover, we can attribute about $4 \mathrm{~kg} / \mathrm{m}^{2}$ to soil carbon (by the way, for a more rigorous estimate that comes to the same conclusion, see M. Köchy, R. Hiederer, A. Freibauer, Global distribution of soil organic carbon-Part 1: Masses and frequency distributions of SOC stocks for the tropics, permafrost regions, wetlands, and the world. Soil 1, 351-365 (2015). doi:10.5194/soil-1-351-2015). At 400 ppm, the atmosphere has about $1.3 \mathrm{~kg}$ carbon per square meter $\left(0.0004 \mathrm{kgCO}_{2}\right.$ air $\times 10$, 000air $\square \times 14 \mathrm{C}_{4} 4 \mathrm{CO}_{2}$ ). Wow: soils have about three times the carbon as does the atmosphere! This makes it clear why we need to be quite concerned about the management of this carbon if we are to limit atmospheric levels of carbon dioxide. Beyond these current climatic concerns, soil fulfill many interfacial functions at various scales ranging from molecular scale nutrient releases at a solid-liquid interface, to mediation of fluxes and exchanges with the atmosphere at the continental scale. These interfacial functions are facilitated by the position of soils at the Earth surface and their physico-chemical properties, namely a porous medium with significant interfacial area per unit volume.

\section{Soil as a natural body}




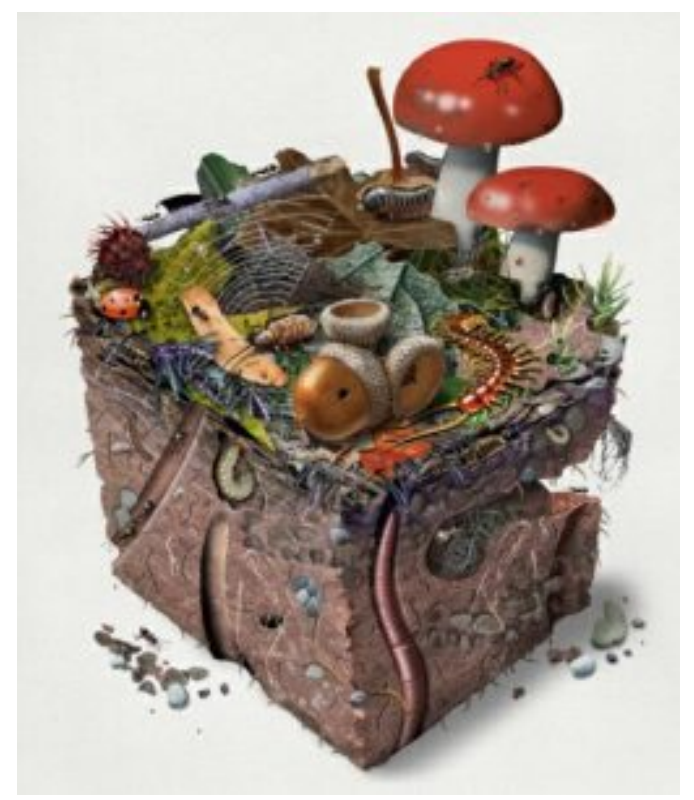

Figure 1.4 Illustration of the complex bio-physical environment found at the soil-atmosphere interface

[Dani - this seems like a good place for this image - do you have a citation and/or a higher-quality version?]

The diversity and scope of interconnected physical, chemical, and biological processes occurring in soil, reflect its centrality in many ecosystems, and highlight the functioning of soil as a natural body with far more complex emergent functions and processes than predicted from the collection of constituents. This is similar to oceans and lakes that host complex and interacting processes far beyond what would be predicted from consideration of the water in a lake [Brady and Weil, 2003]. Consequently, the study of physical environment of soils would not be complete without consideration of interactions with biological and chemical environments - as eloquently stated by Charles E. Kellogg (1938) "Essentially, all life depends upon the soil ... There can be no life without soil and no soil without life; they have evolved together." The consideration of these intrinsic links distinguishes the discipline of Soil Physics and sets it apart from other branches of science and engineering that study behavior of soils such as Geotechnical Engineering that focuses on the mechanical behavior of soils as engineering porous media, or Hydrology focusing exclusively on the role of water in soils. On the other hand, proper consideration of the impact of the soil physical environment on biological and chemical processes requires interdisciplinary perspective especially with engineering, ecology, biology, and atmospheric sciences to mention a few.

The study of soil physical environment, often referred to as Soil Physics is concerned with the application of physical principles to characterization of soil properties and behavior, to the quantitative understanding of dynamic exchange processes occurring in soils. For example, (i) the study of physical properties of soils such as particle and pore size distributions, water retention and hydraulic conductivity, gaseous diffusion, thermal properties, and soil mechanical behavior; (ii) the measurement, prediction, and control of physical processes taking place in and through the soil, such as water infiltration and redistribution, evaporation, solute and contaminant transport, and heat flow; and (iii) the study and control of physical conditions and processes affecting biological activity (most notably plant growth) such as atmospheric influences (i.e., radiation, rainfall, evapotranspiration) at the top boundary, and the groundwater at the lower boundary. We see that "physics" is a bit of a misnomer here, not adequately expressing the complex interaction of physical, biological, and chemical aspects of this field.

\section{The challenges of scale, heterogeneity and scarce information}

Traditionally, soil physics has been focused on processes occurring at the scales of intergranular pores (microns) to soil profiles (meters), however contemporary societal challenges require insights at much larger scales, those of 
catchments $(\mathrm{km})$ to continents and global. This essential shift in the scale of application of soil physics introduces challenges (hint: you can't just use the same equations), but also presents numerous opportunities. An obvious opportunity in the natural links with atmospheric and climate sciences and with global cycles of water, carbon, nitrogen and other nutrients. An additional aspects of large scale processes, is the emergence of phenomena that transcend the single soil profile scale, for example, local rainfall runoff in arid regions giving rise to vegetation patterns (Tigerbush in the Sahel and other places, Fig. 1a), or the striking differences in fluxes and vegetation patterns between north and south facing slopes (Fig. 1b).

The concern with process representation at multiple scales, introduces methodological and theoretical challenges that illustrate the limitation on application of theories developed for pore and sample scales to catchments and continents. It is not obvious whether governing equations and parameters for certain processes (water flow, evaporation, runoff) are applicable at all scales. For example, we will discuss principles of water flow in unsaturated soils where derivations and data are often obtained from core samples with dimensions of a few centimeters, yet, the same equations and parameters are applied in climate models over grids extending many kilometers. The governing equations describing water flow are based on representing the soil as an abstract continuum with certain effective (averaged) properties for which differential equations are applied. When considering flow across many tens of meters (as often required in the field) the transport parameters based on small-samples may not be applicable, and in some case, "scale-appropriate" properties and descriptions are needed. For example, pore-scale flow in soils is largely controlled by capillary forces, while landscape-scale flow is controlled largely by gravitational forces. The issue of flow processes across different scales within the soil continuum remains a challenge and is a topic of active research in many branches of hydrology and earth sciences (including soil physics). Nevertheless, to advance quantitative understanding, many derivations circumvent the issues of heterogeneity and scale dependency by making simplifying assumptions and by idealizing the real physical environment of the soil. The practical approaches and approximation made, and the tradeoffs necessary to provide solutions at different scales will be addressed throughout this book. Thoughout we hope you will ask yourself what are the limits on a particular set of ideas. The most fundamental lesson you should take away from this text is what processes have what impact at which scales. From this perspective you can expect to correctly construct a predictive framework for the processes of interest.

Soil covers most terrestrial surfaces spanning climatic conditions, topography, and underlying geologic formation and biomes. The diversity of these conditions, hence, factors that shape soil formation result in large degree of spatial heterogeneity in soil properties and physical environments. Questions to ponder: given soil is the ubiquitous substrate of the terrestrial world, how has biological evolution altered soil properties? If organisms thrive when water can both enter the soil, and drain to maintain aeration, how does the ecosystem effect the soil properties with respect to the climate in which the soil is found? The natural heterogeneity of soil present a challenge to the study of soil physical environment that must rely on incomplete information obtained from samples for making inferences on unsampled locations with unknown properties. The intimate relationship between soils and their environment, and the inherent element of uncertainty of our characterization of soils due to their complexity must be recognized in application of the principles outlined in this book to practical problems.

\section{History of investigation}

It is worthwhile to understand the historical context of the study of unsaturated flow, as it is a young field of study with some areas still early in their conceptual development. In the following section we will provide an overview of the development of this field to provide a framework for the entire text. The goal here is conceptual, while more comprehensive historical analyses may be found in documents dedicated to this subject (e.g. Gardner, 1972; Philip, 1972; Gardner, 1974).

Quantitative understanding of the movement of water below the soils surface was established by the work of Darcy, who in 1856 published his landmark study of the hydraulic properties of porous media. Darcy introduced the concept

10 | Fundamentals of Soil Hydrology and Bio-Physics 
of potential flow, whereby water in saturated media was observed to move in direct proportion to the gradient of potential energy, as well as the permeability of the media. Darcy obtained the governing equation for saturated water flow through porous media. What was needed next were some practical solutions to this equation.

But how could this equation be used in realistic contexts? In the mid 1870's Bousinesq extended Darcy's law to include the possibility that water might drain and fill the media, and developed an extension of Darcy's law to problems where there was a "free water surface" (i.e., the aquifer was not confined between impermeable boundaries). Bousinesq provided several very useful solutions to this equation of interest to dike designers, as well as farmers who wish to drain their fields. Unlike Darcy's equation which is linear in all parameters, the Bousinesq equation is strongly nonlinear. Solutions are far harder to come by, and it continues to provide fertile ground for research in mathematical hydrology to this day.

In 1899 Schlichter published "Theory of Flow Through Porous Media" which provided the first encyclopedic source of solutions to Darcy's governing equation. Schlichter made three-dimensional models of porous media, developing relationships between media geometry and permeability. He further provided a mathematical basis for the linearity of Darcy's Law based on creeping flow assumptions. Aside from providing a rigorous conceptual basis for Darcy's findings, Schlichter generated exact solutions for the flow and pressure fields around pumped wells, and other useful boundary conditions for problems of groundwater supply. [XXX - John: get scanned version of a few Schlichter figures]

Through the end of the 19th century the study of flow through porous media considered only the case of flow through saturated media. In 1907 Buckingham (the same person who later provided the fundamental basis for dimensional analysis which carries his name) extended Darcy's law to flow in unsaturated systems. In this regard, Buckingham proposed that the same governing equation could be applied, but that the conductivity term must be made a function of the degree of saturation of the media, and the term for hydraulic potential must be expanded to include capillary induced pressures which are less than zero. When applied to the unsaturated zone, the governing equation is often referred to as the Buckingham-Darcy equation to credit this contribution, although in this text we will maintain the shorter name for reasons of brevity.

The alert reader will have noticed by now that the folks who come up with governing equations are remembered for eternity, while the poor work-a-days who solve seem to be quickly forgotten. There are precious few exceptions to this rule, but there are two who did well in this regard. The Australian pair Green and Ampt (1911) decided to attack the key problem of how quickly water infiltrates into soil. They modeled the soil as a collection of capillary tubes which fill in parallel, considering only with infiltration which started from dry conditions and filled to saturation. Including both capillary and gravitational sources of potential, their approach is so powerful and accurate that it remains as the most widely used alternative to the full rigor of the governing equation for unsaturated flow, with new applications of this approach being published to this day.

Remarkably, although Buckingham set the stage at the turn of the century, the governing equation for flow through variably saturated porous media was to wait until after quantum mechanics was well established to be put on paper (some claim this is due to the fundamentally more complex nature of the issues in the vadose zone than in quantum mechanics). In the early 1920's, the members of Gardner's laboratory developed the basic tool required for the application of Buckingham's work: the tensiometer which allows direct measurement of the negative capillary pressures in soils via a porous ceramic cup. L.A. Richards extended this idea in the development of the tension plate, which allows laboratory measurement of the moisture content as a function of capillary pressure, as well as establishing controlled unsaturated flow experiments. In his stunningly prescient text, in 1926 Richardson combined the conservation of mass of water in unsaturated soils with the Buckingham-Darcy equation to develop the governing equation for unsteady water flow in unsaturated soils (page 108 of "Weather prediction by numerical process" 1922). In 1931, Richards independently derived the governing equation for unsaturated flow, which is most widely known as Richards equation, though for reasons of historical accuracy, we refer to this as the Richardson-Richards equation (RRE).

Around the same time, Haines (1930) pointed out that unsaturated porous media could have a variety of moisture contents at a given capillary pressure depending on the history of wetting. He pointed out that even in an idealized media the advancement (or retreat) of fluid into porous media was a highly non-linear process, proceeding in a sequence 
of "jumps" as the fluid proceeds (or recedes) from pore to pore. Still largely ignored, accounting for hysteretic behavior is often essential to understanding unsaturated flow processes.

Solutions to Richards equation have been slow in arriving for a variety of reasons. Firstly, Richards equation includes terms for pressure and moisture content, which are clearly interrelated. Although Buckingham (1907) had introduced a term, now called the soil-water diffusivity, which allowed the writing of Richards equation in terms of moisture content alone, only in 1956 was this method exploited by Bruce and Klute (1956). Using this re-parameterization, Richards equation takes the form of a diffusion equation, which is more conducive to solution. Since this time a number of additional analytical solutions have been presented, although the strong non-linearity of the equation has limited these to quite specialized boundary and initial conditions. The advent of the high-speed computer has at last allowed us to make wide use of Richards important result through implementation of numerical solutions.

Since the 1950's activity in the area of unsaturated flow processes has grown drastically, making a neat and comprehensive accounting for the later period more difficult. A few key contributions, however, do deserve note. A holy grail of vadose zone science has been a simple method to determine the permeability of material as a function of degree of saturation WITHOUT having to actually measure this directly (which is very difficult). This effort was kicked off by Schlichter (1899) who explored the effect of the packing of unconsolidated particles on pore geometry and permeability. This was followed on by the work of Kozeny (1927) and Carmen (1937) who provided theories for the permeability of saturated materials based on their particle size distribution. These results were then extended to unsaturated media in a series of significant contributions through the 1950's (Childs and Collis-George, 1950; Burdine, 1953; Marshall, 1958; and Millington and Quirk, 1959, 1961; and later, Mualem, 1976). These papers provide models which predict the permeability as a function of fluid content which can be fitted parametrically through the fluid content versus pressure relationship. This "trick" has been extended in recent years by inserting a parameterized form of the water retention function into the results of Burdine and Mualem to generate a very simply parameterized set of relationships for water retention and permeability (van Genuchten, 1980).

In 1956 Miller and Miller presented a generally applicable framework for understanding the relationship of the grain size of porous media to all of the capillary properties, now known as Miller Scaling Theory. This framework allows quantitative extrapolation of observed behavior in one soil with one liquid to soils of different grain size, and liquids with different properties, which fundamentally expanded our ability to generalize observations. In a second major advancement for the mathematical description of the soil/liquid interaction, in 1962 Poulovassilis introduced the concept of the independent domain model of hysteresis to describe fluid retention in natural porous media. This opened the possibility of quantitatively including the observations of Haines three decades earlier in flow modeling. Now another three decades hence, these ideas are at last being included in numerical models of vadose flow.

The 1970's began a period of coming to grips with the limitations of many of the assumptions that had been adopted in order to obtain solutions to the governing equations of flow. Nielson et al. (1973) and Biggar \& Nielsen (1976) were the first to quantitatively document the magnitude and importance of heterogeneity at the field scale in vadose materials. They showed that properties were not only variable, but varied in a lognormal fashion, such that the mean value of a property was of relatively little use in predicting the range of values which would be observed. Simultaneously, Hill \& Parlange (1972) showed that infiltration in homogeneous soils were far more complex than earlier thought. They experimentally demonstrated that advancing wetting fronts can be hydrodynamically unstable and breaking into vertical fingers of flow rather than the level orderly wetting process assumed for until this time. These developments were quickly followed by the observation of the importance of "macropores (the few very largest apertures in a media) in rapid movement of water and contaminants. Phenomena such as macropore and finger flow which give rise to rapid advance of solutes are referred to categorically as preferential flow. Each of these areas of research remain very active to this time, with literally hundreds of publications in the literature related to the effect of heterogeneity on transport, unstable wetting processes, and preferential flow.

TO ADD: Dani - history of biophysical process description; history of atmospheric interactions; history of pedotransfer functions 


\section{Relationship to saturated media}

Much of the theory and conceptual framework for understanding processes in the vadose zone have their origins in the study of flow in saturated systems. In saturated systems the geologic media are similar, stemming from essentially the same origins, and the primary liquid of interest, water, is dominant in both cases (Figure 1.5). One may refer to all water below the surface of the earth as "groundwater," while aquifers are made up of saturated media, while the pore space in the vadose zone is generally partially saturated with water, and partially filled with gas. While the great similarity has been very useful, it is also the source of many errors resulting from people naively applying concepts drawn from groundwater hydraulics to explain or predict processes in the vadose zone.

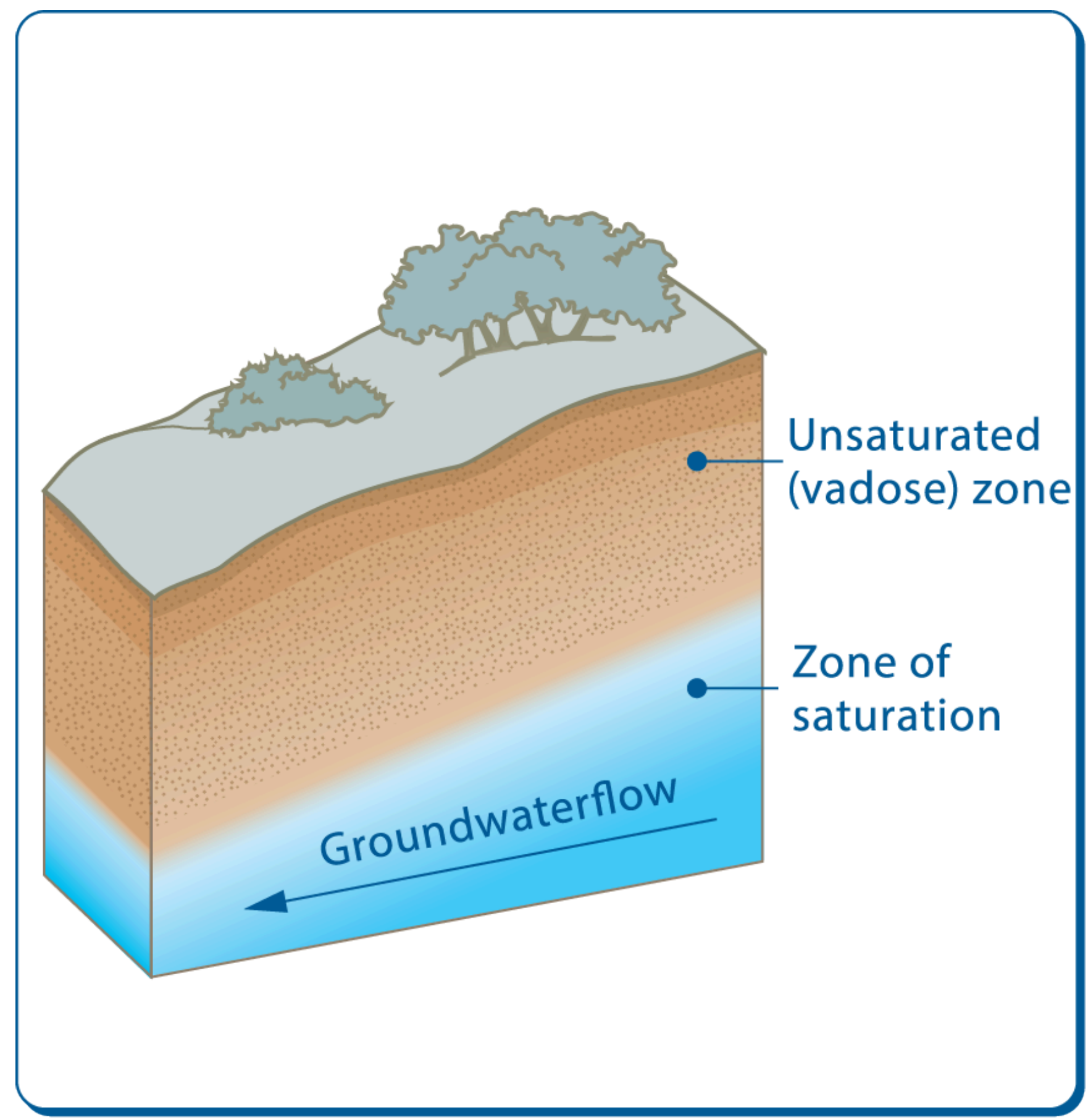

Figure 1.5 Illustration of the unsaturated, or "vadose" zone, and a saturated aquifer. 
The distinctions between processes in saturated and unsaturated media are most pronounced in three areas. First, capillarity, which gives rise to situations where flow occurs in isolated regions (e.g., fingered flow), and where flow will not cross between zones of differing texture (e.g., the capillary barrier effect). In a second related vein, partially saturated conditions extend the heterogeneity of hydraulic properties from the spatial domain, as observed in saturated media, into the temporal domain, with transport properties being direct functions of the occurrence of the transported fluid. Finally, there is the tremendous biochemical activity of a region which has continuous gas phase present. Transport via diffusion is two orders of magnitude faster in gas than liquid, and there is a generous supply of oxygen which supports an abundance of life. The take-home message is that one must be very careful in applying principles developed for application to saturated conditions to the vadose zone. The table show below expands upon these ideas.

Similarities:

- Both have governing equations for flow that are linear in the local potential gradient

- They share similar constitutive media (particles from clay to gravel)

Differences:

Table 1.1 Relationship of Vadose Zone to Saturated Zone

\begin{tabular}{|c|c|c|c|c|}
\hline Issue & \multicolumn{2}{|l|}{ Vadose Zone } & \multicolumn{2}{|c|}{ Saturated Zone } \\
\hline $\begin{array}{l}\text { Conductivity } \\
\text { at a point }\end{array}$ & \multicolumn{2}{|c|}{ A nonlinear function of moisture content. } & \multicolumn{2}{|l|}{ Constant. } \\
\hline $\begin{array}{l}\text { Density } \\
\text { Effects }\end{array}$ & \multicolumn{2}{|c|}{$\begin{array}{l}\text { Negligible influence for temperature and solute based } \\
\text { changes. }\end{array}$} & \multicolumn{2}{|c|}{$\begin{array}{l}\text { Temperature and solute based density differences can } \\
\text { dominate both static and dynamic disposition. }\end{array}$} \\
\hline $\begin{array}{l}\text { Spatial } \\
\text { variability }\end{array}$ & \multicolumn{2}{|c|}{$\begin{array}{l}\text { Lognormal distribution and a function of moisture } \\
\text { content and hence time. }\end{array}$} & \multicolumn{2}{|c|}{ Lognormal distribution fixed in time. } \\
\hline $\begin{array}{l}\text { Biological } \\
\text { and } \\
\text { chemical } \\
\text { activity }\end{array}$ & \multicolumn{2}{|c|}{$\begin{array}{l}\text { Often high in carbon and oxygen, leading to rapid } \\
\text { microbial metabolism. }\end{array}$} & \multicolumn{2}{|c|}{$\begin{array}{l}\text { Often anoxic with sparse carbon: comparatively slow } \\
\text { microbial activity. }\end{array}$} \\
\hline $\begin{array}{l}\text { Transport } \\
\text { mechanisms }\end{array}$ & $\begin{array}{l}\text { Advection } \\
\text { Dispersivity } \\
\text { Diffusion }\end{array}$ & $\begin{array}{l}0-10 \mathrm{~cm} / \text { day } \\
0.5-20 \mathrm{~cm}^{2} \\
0.1-.3 \mathrm{~cm}^{2} / \mathrm{s}\end{array}$ & $\begin{array}{l}\text { Advection } \\
\text { Dispersivity } \\
\text { Diffusion }\end{array}$ & $\begin{array}{l}0-100 \mathrm{~cm} / \text { day } \\
0.5-20 \mathrm{~cm} \\
0.00002 \mathrm{~cm}^{2} / \mathrm{s}\end{array}$ \\
\hline
\end{tabular}

\section{Contemporary Concerns with the Vadose Zone}

Almost all research related to vadose zone transport carried out prior to 1980 was directly related to agricultural applications. In the past two decades, however, a much broader range of disciplines have become interested in this region. Within the agricultural sector, the ever increasing population pressures on fresh water supplies, water conservation has driven more careful analysis of crop water requirements and salinity management. Both for agricultural and waste-disposal applications, the ability to model nutrient storage and transport is critical to maintaining aquifer quality. The increasing awareness of global warming has pointed to the critical role of soil-water in controlling the atmospheric energy budget, which dictates the thickness of the atmospheric boundary layer (e.g., Cuenca et al., 1996). A major area of activity in vadose zone research has been directed to the understanding of the migration and remediation of non-aqueous phase liquids (NAPLs) which have been introduced to the subsurface via leaking underground storage tanks and other un-monitored releases. The study of NAPLs has lead to greater awareness of the similarity of the problems addressed by individuals working with petroleum extraction and those working on problems related to the vadose zone as has been demonstrated historically by the work of Leverett $(1938,1941)$ and others. 
To illustrate a typical problem that might arise in the context of industrial contamination, consider this example. Suppose that 2,000 liters of some nasty NAPL spilled on a $10 \mathrm{~m} 2$ area above an aquifer that was at a depth of $10 \mathrm{~m}$. How much makes it to the aquifer? How long does it take? How much will stay in the material above the aquifer after a month? A year? How widely would you have to excavate to get al the material, and how might this depend upon the type of material spilled, the last time it rained, and the type of soil? A book could be written on this problem alone (with a catchy title like What to do When you Really Mess Up by Spilling a Bunch of Oil $10 \mathrm{~m}$ Above the Water Table). In fact, each of the questions we brought up about this problem could be the controlling factor in determining the fate of the oil. Here we figure that the only way to prepare for the wide range of possible vadose zone problems is to understand the basic processes of infiltration and flow through porous media, which is what we spend the next few hundred pages doing. With this basis you should be able to reason your way through the complexities of the real world.

\section{Basic and Derived Units and Dimensions}

All physical quantities are measured and expressed in units. The International System of Units, abbreviated SI, which is essentially the mks (meter-kilogram-second) system is accepted for scientific application and will be used in this text. The SI system is organized around seven basic units: length (meter, m), mass (kilogram, kg), time (second, s), temperature (Kelvin, K), electric current (Ampere, A), amount of substance (Mole, mol), and luminous intensity (candela, cd). Other physical quantities are expressed in derived units. Force, for example, is a derived unit Newton (kgm $\mathrm{x} \mathrm{m} / \mathrm{s} 2$ ). Fractions and multiples of the basic SI units are assigned conventional prefixes (Table 1.2).

Table 1.2 Base Units in the Systeme International (SI) and their Prefixes

\begin{tabular}{lll}
\hline Dimension/Property & SI Unit & Symbol \\
Length & Meter & $\mathrm{m}$ \\
Mass & Kilogram & $\mathrm{kg}$ \\
Time & Second & $\mathrm{s}$ \\
Electric current & Ampere & $\mathrm{A}$ \\
Temperature & Kelvin & $\mathrm{K}$ \\
Amount of substance & Mole & $\mathrm{mol}$ \\
Luminous intensity & candela & $\mathrm{cd}$ \\
\hline
\end{tabular}

Table 1.2 continued Base Units in the Systeme International (SI) and their Prefixes

\begin{tabular}{llllll}
\hline Fraction & Prefix & Symbol & Multiple & Prefix & Symbol \\
$10-1$ & deci & $\mathrm{d}$ & 10 & deca & $\mathrm{da}$ \\
$10-2$ & centi & $\mathrm{c}$ & 102 & hecto & $\mathrm{h}$ \\
$10-3$ & milli & $\mathrm{m}$ & 103 & kilo & $\mathrm{k}$ \\
$10-6$ & micro & $\mu$ & 106 & mega & $\mathrm{M}$ \\
$10-9$ & nano & $\mathrm{n}$ & 109 & giga & $\mathrm{G}$ \\
$10-12$ & pico & $\mathrm{p}$ & 1012 & tera & $\mathrm{T}$ \\
\hline
\end{tabular}

(adapted from: Sposito, 1989)

A dimension is a qualitative expression of a physical quantity or an attribute. It may be a basic dimension such as 
length $[\mathrm{L}]$, time $[\mathrm{t}]$, or mass $[\mathrm{M}]$, or a derived dimension such as volume $\left[\mathrm{L}^{3}\right]$, or density $\left[\mathrm{ML}^{-3}\right]$. Dimensional inspection is an important step in verifying the validity of an equation; the dimensions of all terms must be consistent. Writing the equation in dimensional form only, leaving out real values (numbers), enables algebraic manipulation of dimensions, i.e., dimensions may be divided, multiplied, and cancelled to simplify the dimensional equation in terms of basic dimensions.

\section{Example 1-1): Dimensions and Unit Conversion}

Dimensions: Find the dimensions of pressure in basic units

Solution: Pressure is force divided by the area of its action. The dimensions of force are $\left[\mathrm{MLt}^{-2}\right]$ and those of area are $\left[\mathrm{L}^{2}\right]$. Thus, the dimensions of pressure are 1.1

$$
\begin{aligned}
\mathrm{P} & =\frac{\mathrm{F}}{\mathrm{A}} \\
& =\frac{\left[\mathrm{MLt}^{-2}\right]}{\left[\mathrm{L}^{2}\right]} \\
(1.1) \quad= & =\left[\mathrm{MLt}^{2}\right]
\end{aligned}
$$

i)

Units: Convert a pressure of $2.7 \mathrm{~kg} / \mathrm{cm}^{2}$ into SI units $\left(\mathrm{Pa}=\mathrm{N} / \mathrm{m}^{2}\right)$

$$
2.70 \frac{\mathrm{kg}}{\mathrm{cm}^{2}}=2.70 \frac{\mathrm{kg}}{\mathrm{cm}^{2}} \frac{100^{2} \mathrm{~cm}^{2}}{\mathrm{~m}^{2}} \frac{9.806 \mathrm{~N}}{\mathrm{~kg}}=265000 \mathrm{~Pa}=265 \mathrm{kPa}
$$

ii)

Understanding of the transport of water and chemicals through natural porous media cannot be accomplished based purely on knowledge of the mathematical equations of flow and boundary conditions. The structure, setting, history and chemistry of the mineral system in the vadose zone all play central roles in the determination of flow and transport. In this section we will provide a very terse overview of the concepts and nomenclature used to describe soil mineralogy and classify soils in order to provide a context for the discussion of the properties of naturally occurring media.

\section{A Primer on Soils}

Soils are among the most complex systems found in nature. They represent a dynamic interaction of physical, chemical, 
and biological components and processes, acted upon by internal and external forces and influences, both biotic and abiotic. Soil physics is concerned with the application of physical principles to characterization of soil properties and to understanding the variety of dynamic processes occurring in soils. These include: (i) the study of physical properties of soils and other porous media, including but not limited to particle and pore size distributions, water retention and hydraulic conductivity, thermal capacity and conductivity, and soil strength; (ii) the measurement, prediction, and control of physical processes taking place in and through the soil, such as water infiltration and redistribution, solute and contaminant transport, and heat flow; and (iii) the study and control of physical conditions and processes affecting plant growth with respect to the zone comprising atmospheric influences (i.e., radiation, rainfall, evapotranspiration) at the top boundary, and the groundwater at the lower boundary.

\section{Components of Soils}

The particles making up the solid phase of the soil are products of weathering and other processes. The most important characteristics of these particles are their size distribution and shapes. These attributes affect pore geometry, total pore volume (porosity $n$ ), pore size distribution, and total solid surface area (an important attribute affecting many chemical and physical processes). The term soil texture refers to the size distribution and fractions of primary soil particles. The arrangement and organization of particles and voids is the soil structure. The particle size distribution is recorded by noting the fraction of the total soil mass which is made up of particles smaller than a given size (see Figure 1.1). The particles making up a soil are classified into size groupings based on their estimated diameters as obtained by sieving, sedimentation, or alternative methods (discussed below). As is discussed later in detail, the particle size distribution is very useful in estimating the soil's hydraulic properties such as the water retention characteristics and the hydraulic conductivity. In contemporary applications, particle size distribution data is often the basis of estimating the representative fractal dimension of the soil, which may then be used to deduce a number of feature of the soil as well as being the basis for hydraulic modeling of the soil (e.g., Arya and Paris, 1981; Rieu and Spositio, 1991a,b). There are several systems for classifying textural classes as can be seen in Fig.1.X. The textural designation for a soil is determined on the basis of mass ratios of the sand, silt and clay fractions. Wu et al. (1993) compared several techniques to characterize particle size distribution in soils. Their results indicated very similar results for the approaches they evaluated (Fig.1-7). The textural triangle in Fig.1-6 illustrates common textural classes for the USDA classification.

\section{I.5.I Sieve Analysis}

A known amount of soil is sieved through a stack of sieves of progressively smaller (from top to bottom) openings (Fig.1-4). The proportion of soil retained on each sieve is the proportion of the equivalent grain size. The grain size is actually bracketed between the retaining sieve opening-size and the opening-size immediately above it. Results are expressed as diameters; however, because particles are seldom spherical these diameters are to be regarded as effective diameters based on the sizes of the sieve openings. 


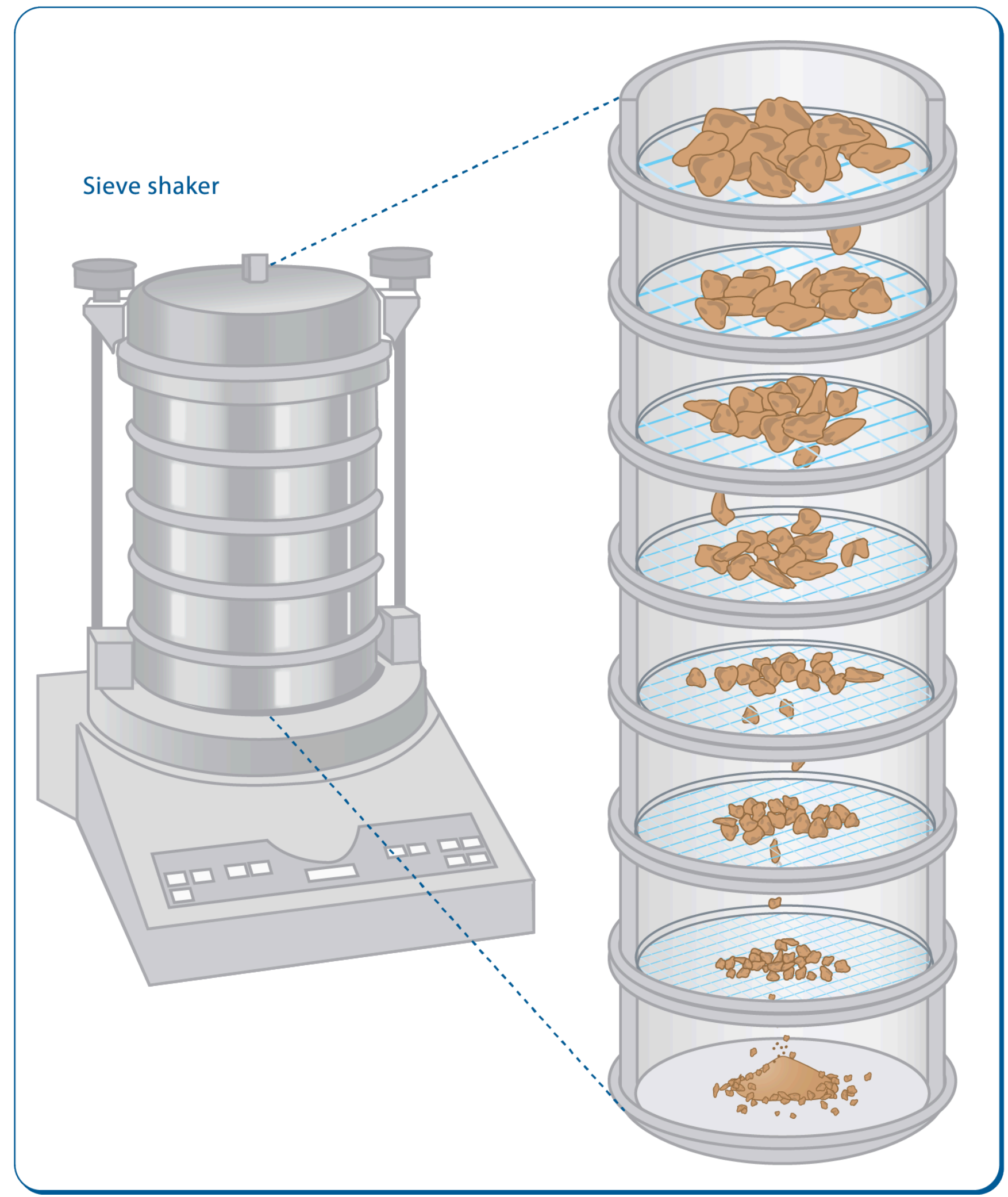

Figure 1.6 Stack of sieves and sieve shaker for particle size analysis with sieve openings decreasing in size from top to bottom of the stack. 


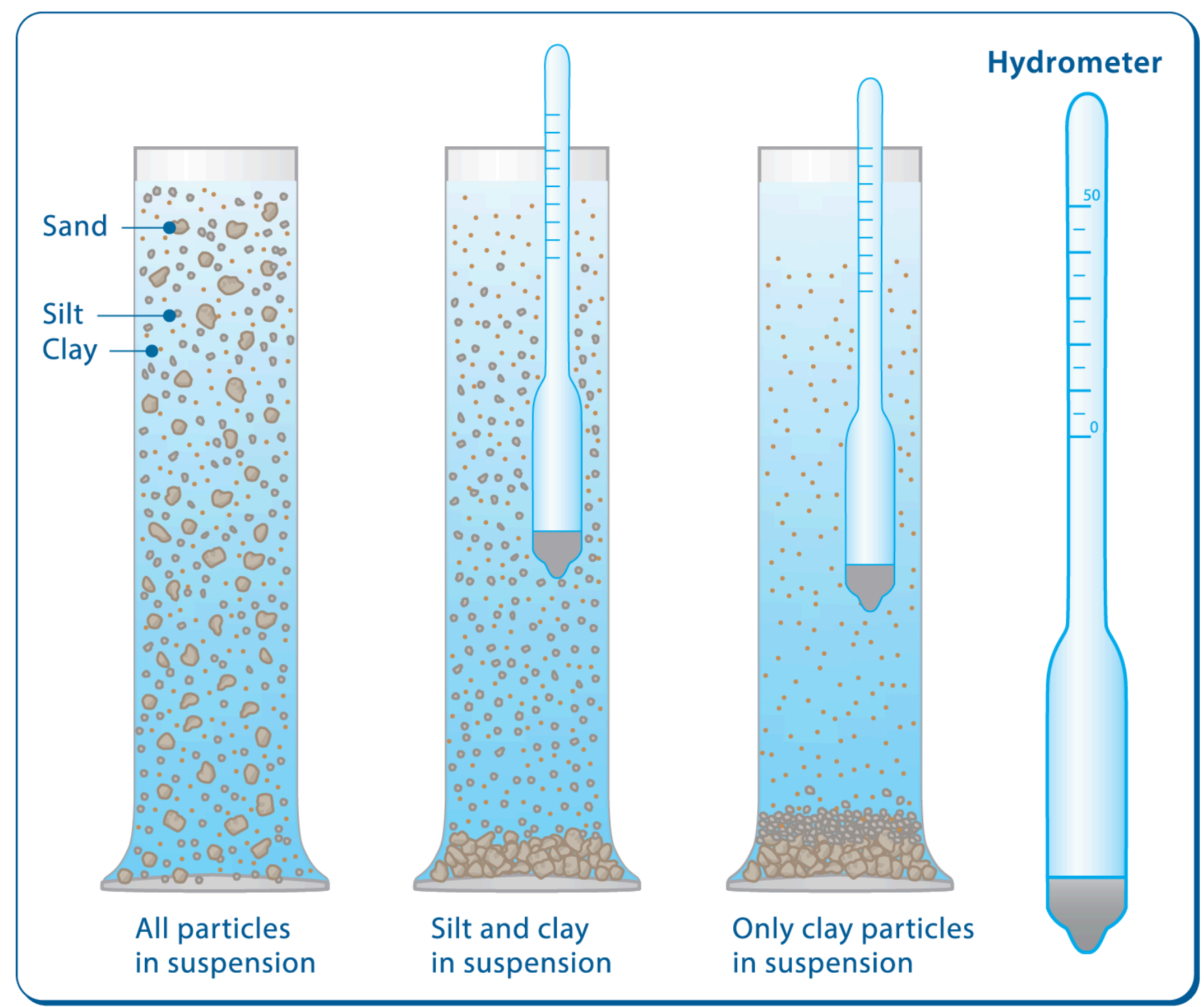

Figure 1.7 The hydrometer method employs a hydrometer which reports the mean density of the suspension surrounding the main bulb of the device. As particles settle our of the sample, the density of the solution decreases, and the hydrometer floats lower. The neck of the hydrometer is graduated so that the density of the solution is indicated by the position of the air-suspension interface. Using Stokes law for settling, the time at which a reading is made indicates the size of particles which have settled beyond the hydrometer, and so relating time and density one can compute the distribution of particle sizes in the suspension.

Laser light passing through suspensions of particles experiences diffraction which reveals the particle size distribution. This effect can be used to obtain the entire particle sizer distribution, from $\mathrm{nm}$ to $\mathrm{mm}$, in a single measurement (Figure 1.8). 


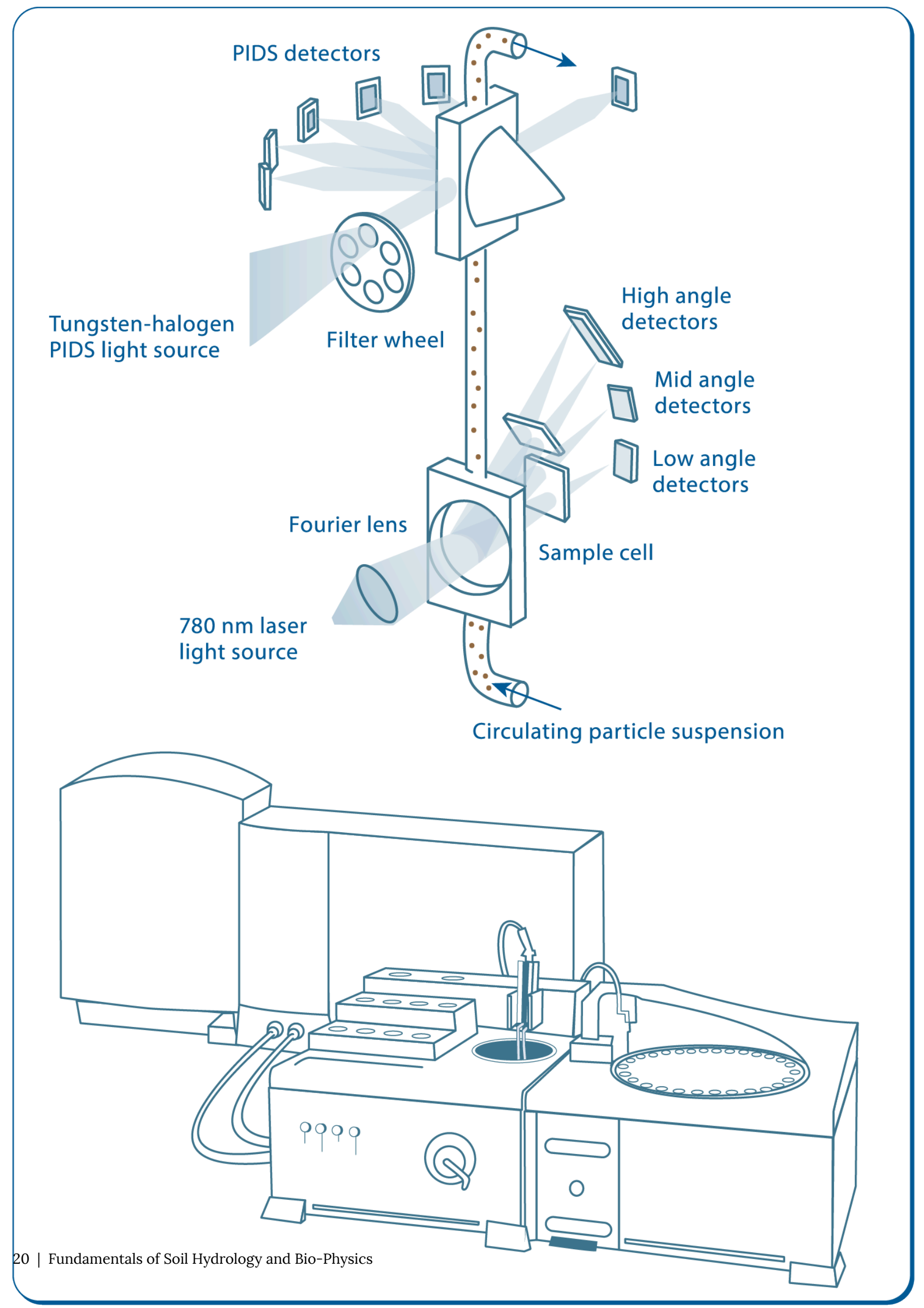




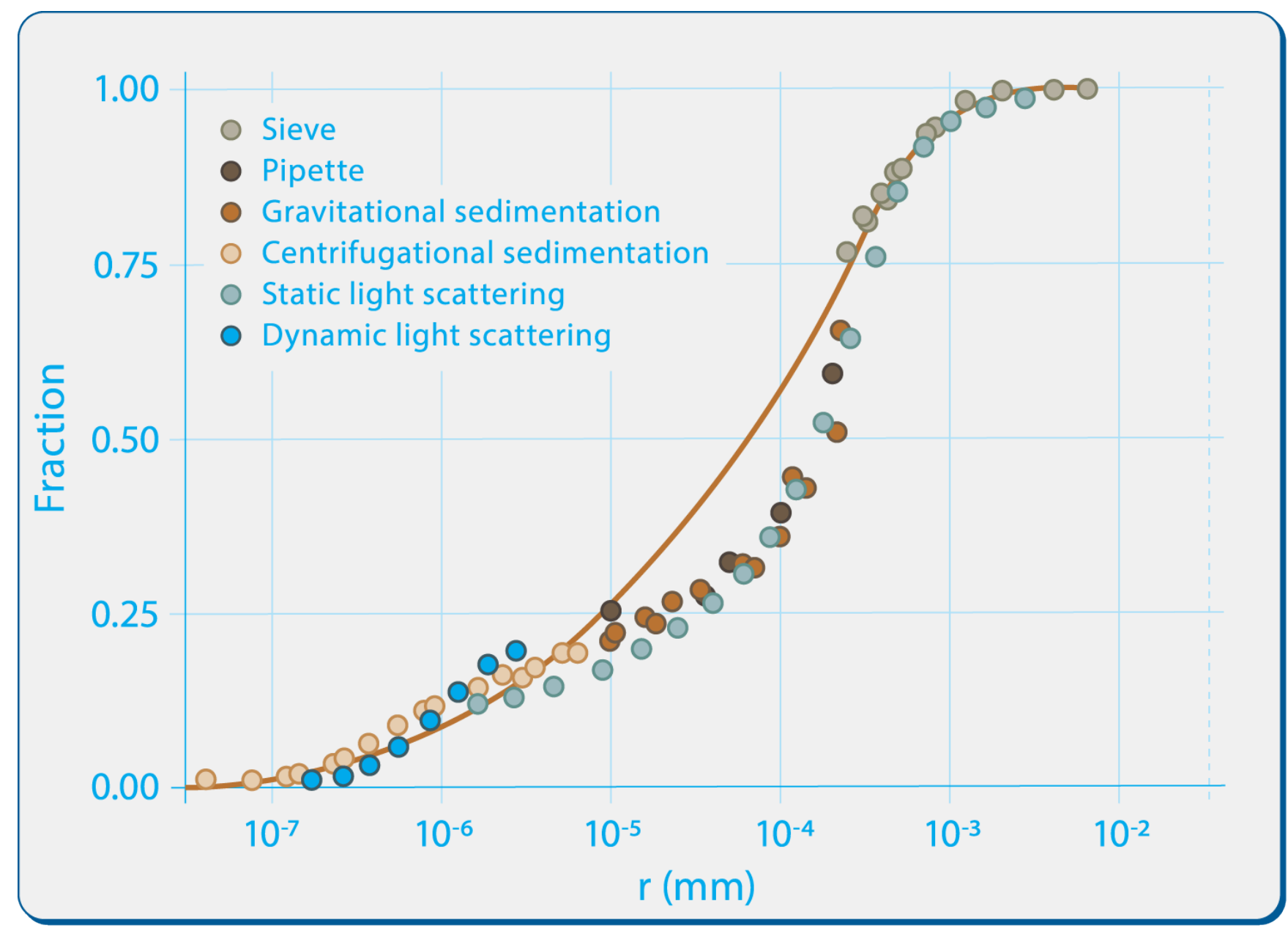

Figure 1.9 Typical plot of particle size distribution. Note that the particle size axis is often log-transformed to expand the size of the small particle size range. This semi-log plot of cumulative mass distribution as a function of soil particle radius illustrates the results obtained using various experimental methods. The solid line is best-fit of a power function (modified from Wu et al., 1993). 


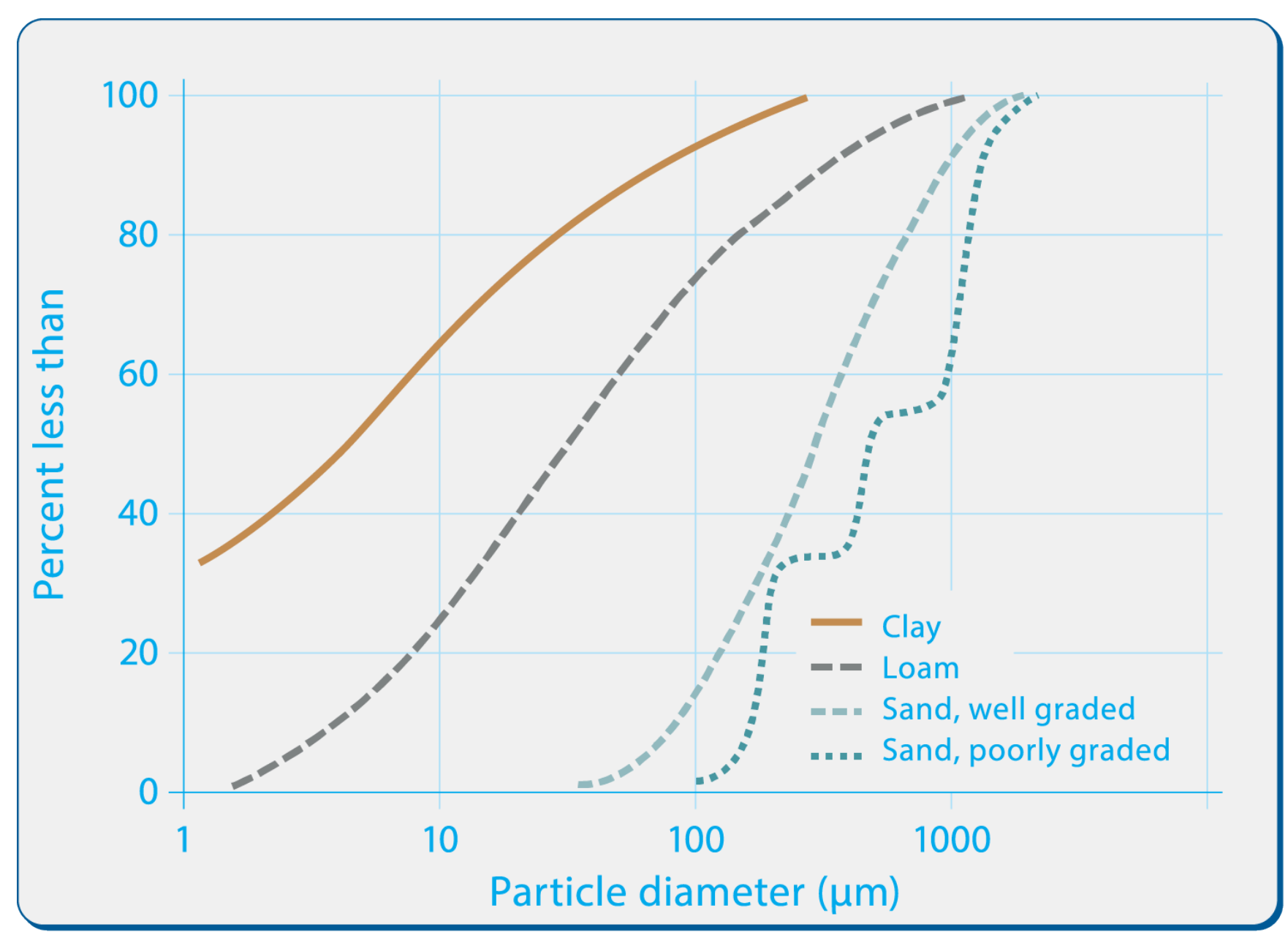

Figure 1.10 Example of particle size distribution versus cumulative mass of occurrence for several soils.

A plot of particle size distribution provides a great deal of information about a soil, yet the information is not in a readily applicable form. The particle size distribution of a soil may also be expressed by way of summary statistics of the distribution. These statistics are less complete in their information than the full distribution, but are often sufficiently revealing to be useful and more convenient. The most commonly used statistic is $d_{50}$, defined as the diameter of the particle for which half of the mass of the soil is made up of smaller particles (and half larger). Clearly $\mathrm{d}_{50}$ will have the units of length. The same notation is used to define other quantiles of the distribution, such as $\mathrm{d}_{10}, \mathrm{~d}_{60}$, for describing the diameter of particles for which $10 \%$ and $60 \%$ of the mass of particles are smaller. Having provided a number that tells the particle size of the "average" particle, it is also useful to provide an indication of how widely the particle size distribution is spread. To fulfill this need the uniformity coefficient, $\mathrm{U}$, has been defined

$$
\mathrm{U}=\frac{\mathrm{d}_{60}}{\mathrm{~d}_{10}}
$$

$\mathrm{U}$ is by definition greater than one, with typical natural porous media having $\mathrm{U}$ between 2 and 10 for well sorted and poorly sorted distributions, respectively.

The distribution of particles in media is also characterized by identifying the fraction of the particles which fall the soil into three size classes: clay, silt and sand. In the United States, clay size particles are those which are too small to settle freely in water $(<2 \mathrm{~m})$; silt size particles are those which freely settle, but are still to small to discriminate by eye $(<0.05 \mathrm{~mm})$; sands are those particles which are larger than silt, but smaller than pebbles $(<2.0 \mathrm{~mm})$. The sand category 
is subdivided into very coarse, coarse, medium fine and very fine sand categories, with class breaks at $1.0 \mathrm{~mm}, 0.5 \mathrm{~mm}$, $0.25 \mathrm{~mm}, 0.1 \mathrm{~mm}$ and $0.05 \mathrm{~mm}$ respectively. These size class divisions are essentially arbitrary and open to alternative definition, as shown in Figure 1.2. Clearly any particular soil will be made up of a combination of amounts of these three components. To systematize the combinations of these constituents, a textural triangle is used (Figure 1.11).

How do we determine the particle size distribution? Two complementary methods are typically employed. Sieve analysis allows identification of sand class particle distribution and settling tests are used for silt and clay classes. Procedurally, a sample is dispersed in water with the sand class particles separated from the silt and clay using a \#270 or \#320 sieve (the sand is retained on the sieve, while the silt and clay pass through the sieve openings). The sand is dried, and run through a stack of sieves of decreasing mesh openings to determine the distribution of particles within the sand class. The sizes of commonly used sieves are provided in Table 1.3. The silt and clay fractions are separated by suspending them in solution, and monitoring the density of the solution as a function of time at a particular elevation. By observing the rate at which the density of the solution decreases, it is straight forward to calculate the particle size distribution of the silt and clay (Klute, 1986).

The smallest screen for which sieving is practical is $0.05 \mathrm{~mm}$. This is also the smallest size defined as Sand; smaller particles are generally termed Silt and Clay. The size distribution of particles smaller than $0.05 \mathrm{~mm}$ (silt and clay) is determined by means of sedimentation. A soil sample is mixed in water with a dispersing agent (e.g., sodium hexametaphosphate) to separate (disperse) the particles. Physical dispersion to further improve particle-separation may be achieved using shakers or electrical mixers. Two techniques may be used to measure the density of the suspension at different times: the Hydrometer Method, or the Pipette Method of direct solution sampling. The amount of clay and silt in a water suspension and their average particle diameter may be found using Stokes' law. A spherical particle of density $r_{s}$ and radius $r$ falling through a liquid of density $r_{l}$ and viscosity $\eta$ is subject to three forces: (i) a downward gravitational

force $\mathrm{F}_{\mathrm{g}}=\rho_{\mathrm{s}}\left(4 \pi \mathrm{r}^{3} / 3\right) \mathrm{g}$ where $\mathrm{g}$ is acceleration of gravity; (ii) an upward buoyancy force $\mathrm{F}_{\mathrm{b}}=\rho_{\mathrm{l}}\left(4 \pi \mathrm{r}^{3} / 3\right) \mathrm{g}$ (the weight of fluid displaced by the particle); and (iii) an upward viscous drag force exerted by the surrounding liquid, found by Stokes to be $\mathrm{Fd}=6 \pi \mathrm{r} \eta \mathrm{V}$ where $\mathrm{V}$ is the settling velocity. The three forces rapidly equilibrate:

$$
\Sigma \mathrm{F}=0=\mathrm{F}_{\mathrm{g}}-\mathrm{F}_{\mathrm{b}}-\mathrm{F}_{\mathrm{d}}
$$

and the particle reaches a constant settling velocity $\mathrm{V}$ which may be obtained by inserting the expressions for the forces and rearranging

(1.5) $\mathrm{V}=\left(\rho_{\mathrm{s}}-\rho_{\mathrm{l}}\right) \mathrm{d}^{2} \mathrm{~g} / 18 \eta$

where $d=2 r$ (particle diameter). One can now find the time $t$ needed for a particle to settle through a distance $h$ :

$$
\mathrm{t}=18 \eta \mathrm{h} /\left(\rho_{\mathrm{s}}-\rho_{\mathrm{l}}\right) \mathrm{d}^{2} \mathrm{~g}
$$

In a solution containing a mixture of suspended particles, the rate of particle settlement will vary depending on their size. Given the height of the column and time after sedimentation commenced, we can calculate the largest particle diameter remaining in suspension. Successive liquid (suspension) density measurements at different times enable estimation of the particle size distribution for the soil sample. Examples of such particle size cumulative distributions are given in Fig.1-5 (top).

\section{Example 1-1): Sedimentation}

Use Stokes' law to calculate the time needed for: (a) sand particles (diameter $>50 \mu \mathrm{m}$ ) and (b) silt particles (>2 $\mu \mathrm{m})$ to settle out to a depth of $0.2 \mathrm{~m}$ in an aqueous suspension at $25^{\circ} \mathrm{C}$.

\section{Solution:}

(a) For the sand particles we take $\mathrm{h}=0.2 \mathrm{~m} ; \eta=0.89 \mathrm{mPa}$ or $\left(0.00089 \mathrm{~kg} \mathrm{~m}^{-1} \mathrm{~s}^{-2} \mathrm{~s}\right)$ at $25^{\circ} \mathrm{C} ; \mathrm{d}=50 \times 10^{-6} \mathrm{~m}$; $\mathrm{g}=9.81 \mathrm{~m} / \mathrm{s}^{2} ; \rho_{\mathrm{s}}=2650 \mathrm{~kg} / \mathrm{m}^{3} ;$ and $\rho_{\mathrm{l}}=1000 \mathrm{~kg} / \mathrm{m}^{3}$

$$
\mathrm{t}_{\text {sand }}=\frac{18 \cdot 0.2 \cdot 0.89 \cdot 10^{-3}}{\left(50 \cdot 10^{-6}\right)^{2} \cdot 9.81 \cdot(2650-1000)}=79 \mathrm{~s}
$$

(b) For the silt settlement time we need only change the diameter to $\mathrm{d}=2 \times 10^{-6} \mathrm{~m}$ : 
${ }_{(1.8)} t_{\text {silt }}=\frac{18 \cdot 0.2 \cdot 0.89 \cdot 10^{-3}}{\left(2 \cdot 10^{-6}\right)^{2} \cdot 9.81 \cdot(2650-1000)}=49485 \mathrm{~s}=13.74 \mathrm{~h}$

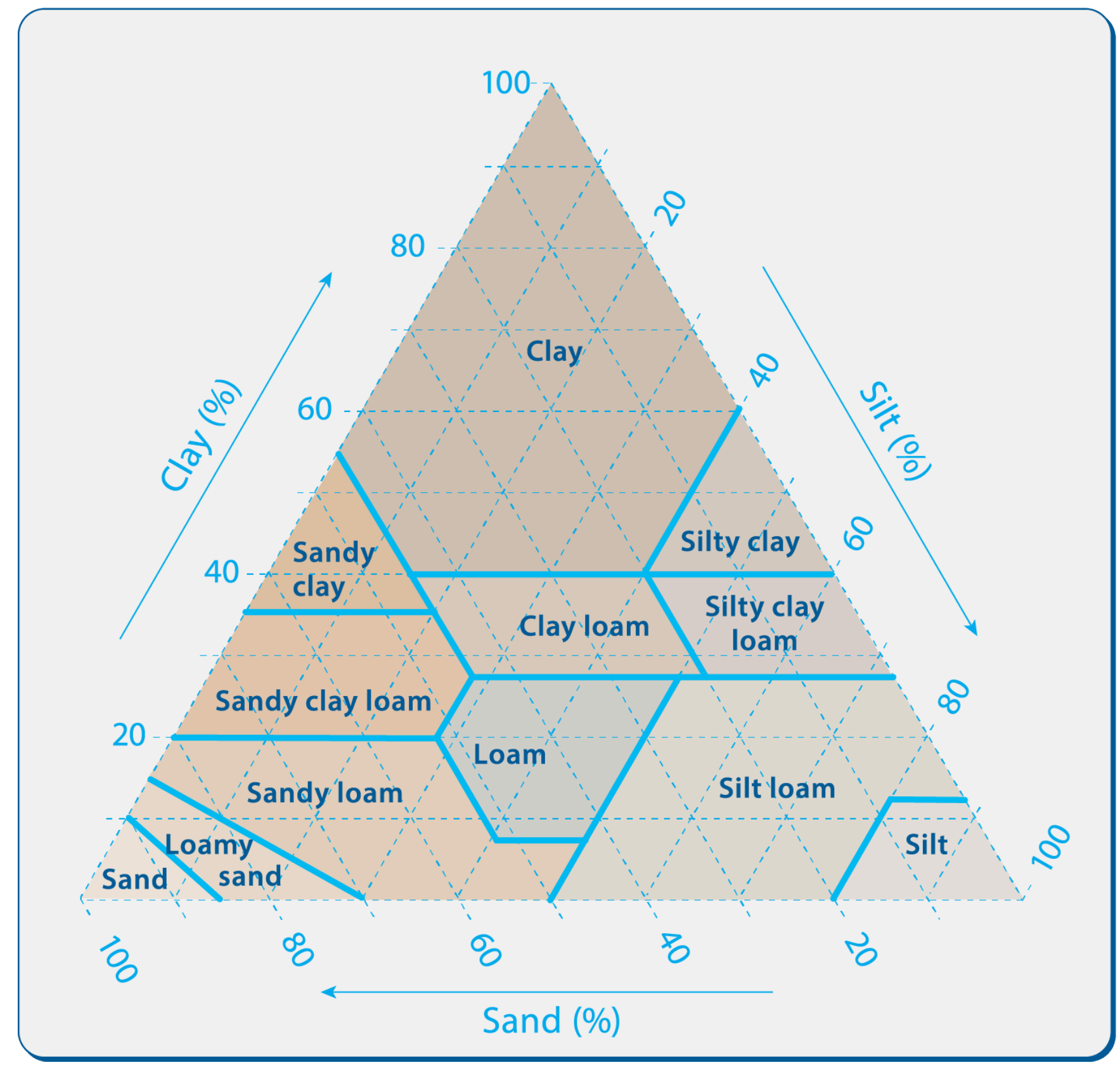

Figure 1.11 Soil textural triangle (from Soil Survey Staff, 1994.). 


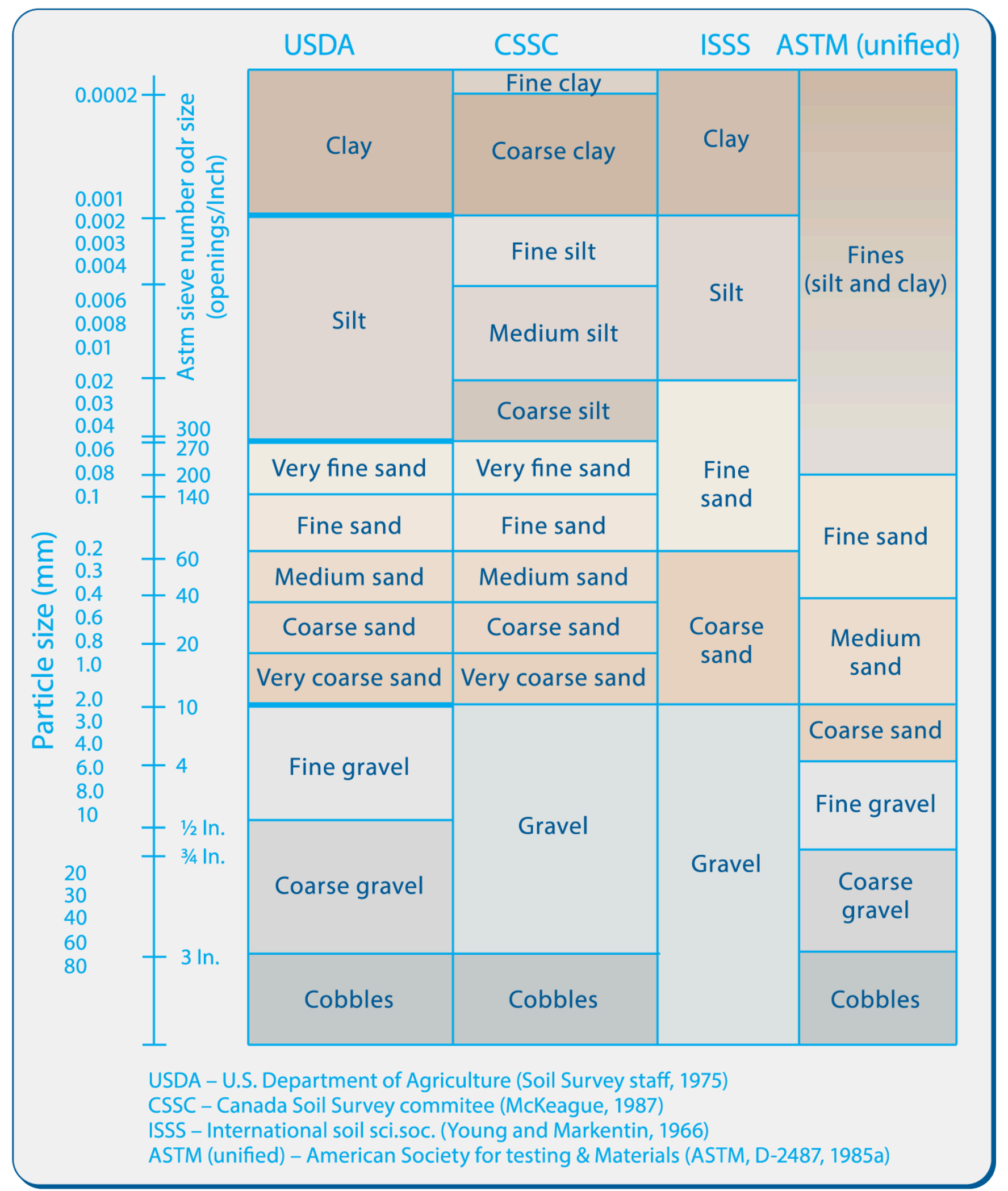

Figure 1.12 Major systems of classification of texture. 


\section{$F_{b}=\rho_{l}\left(4 \pi r^{2} / 3\right) g$ Buoyancy force}

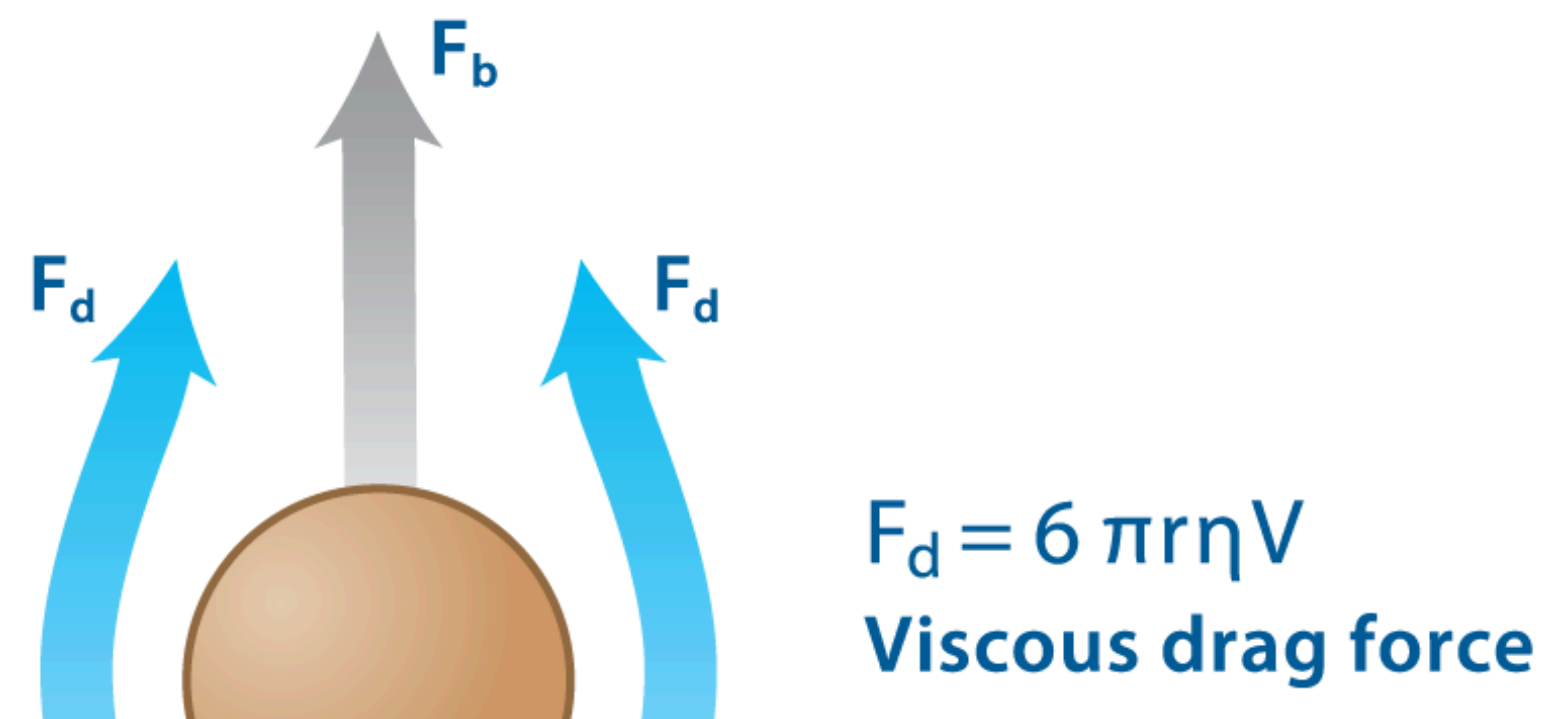

$F_{g}$

$F_{g}=\rho_{s}\left(4 \pi r^{3} / 3\right) g$ Gravitational force

Figure 1.13 Illustration of forces acting on a settling silt or clay particle as is employed in the pipette and hydrometer methods of particle size analysis.

Table 1.3 Standard Sieves and the size of their openings. 


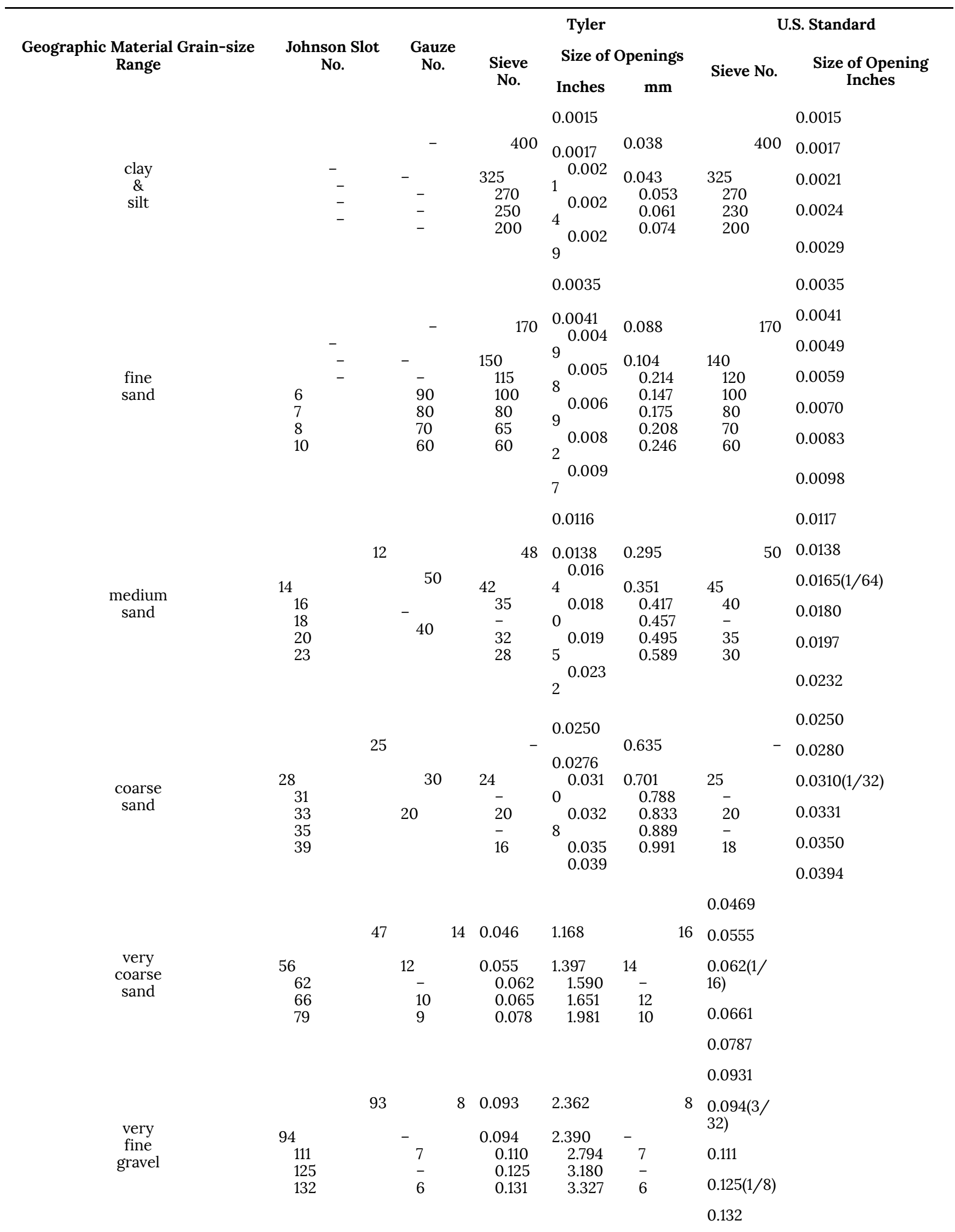




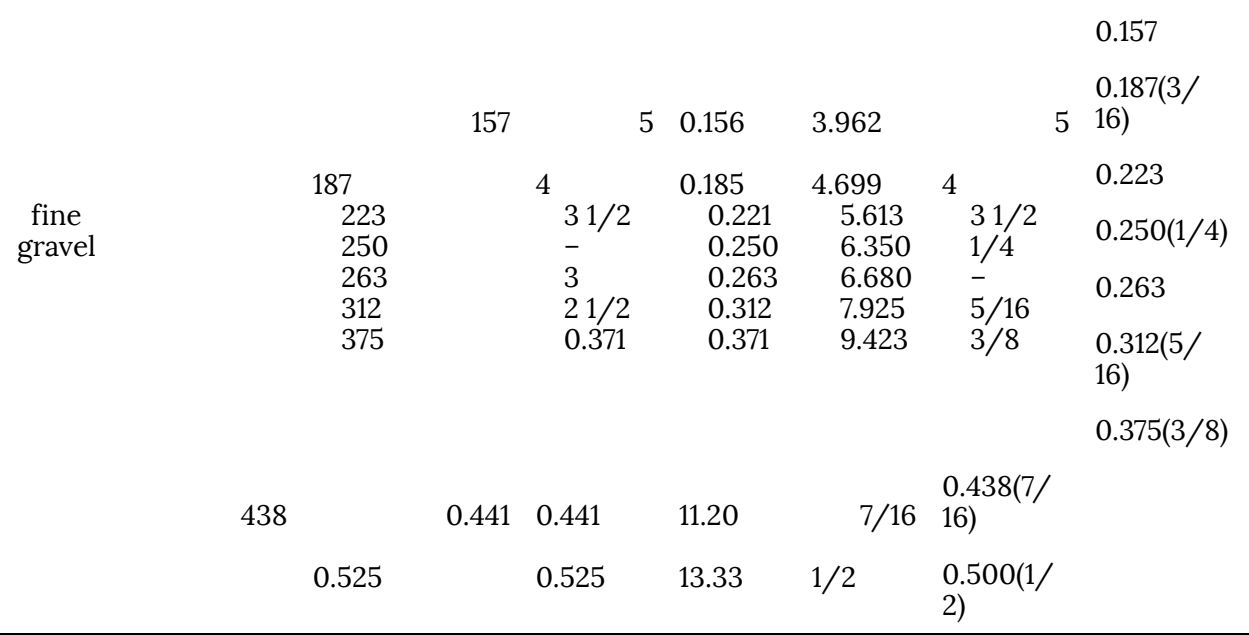

\section{Soil Classification}

Although the vadose zone often extends below the depth of soil, the classification system developed for soils provides the most useful and appropriate system for characterizing vadose zone materials, and is thus worth understanding. Classification of soil is based on the features of the soil as well as the formative processes that gave rise to what you see. Typically soil development extends to a depth of 1-2 meters, with the un-altered material residing below the depth of soil development being referred to the parent material. Landscapes are divided into regions of common soil characteristics (Figure 1.14). A designated soil is defined by identification of the structure of a characteristic profile, which is called a pedon. In the following sections we shall discuss the characteristics which make up a soil description. 


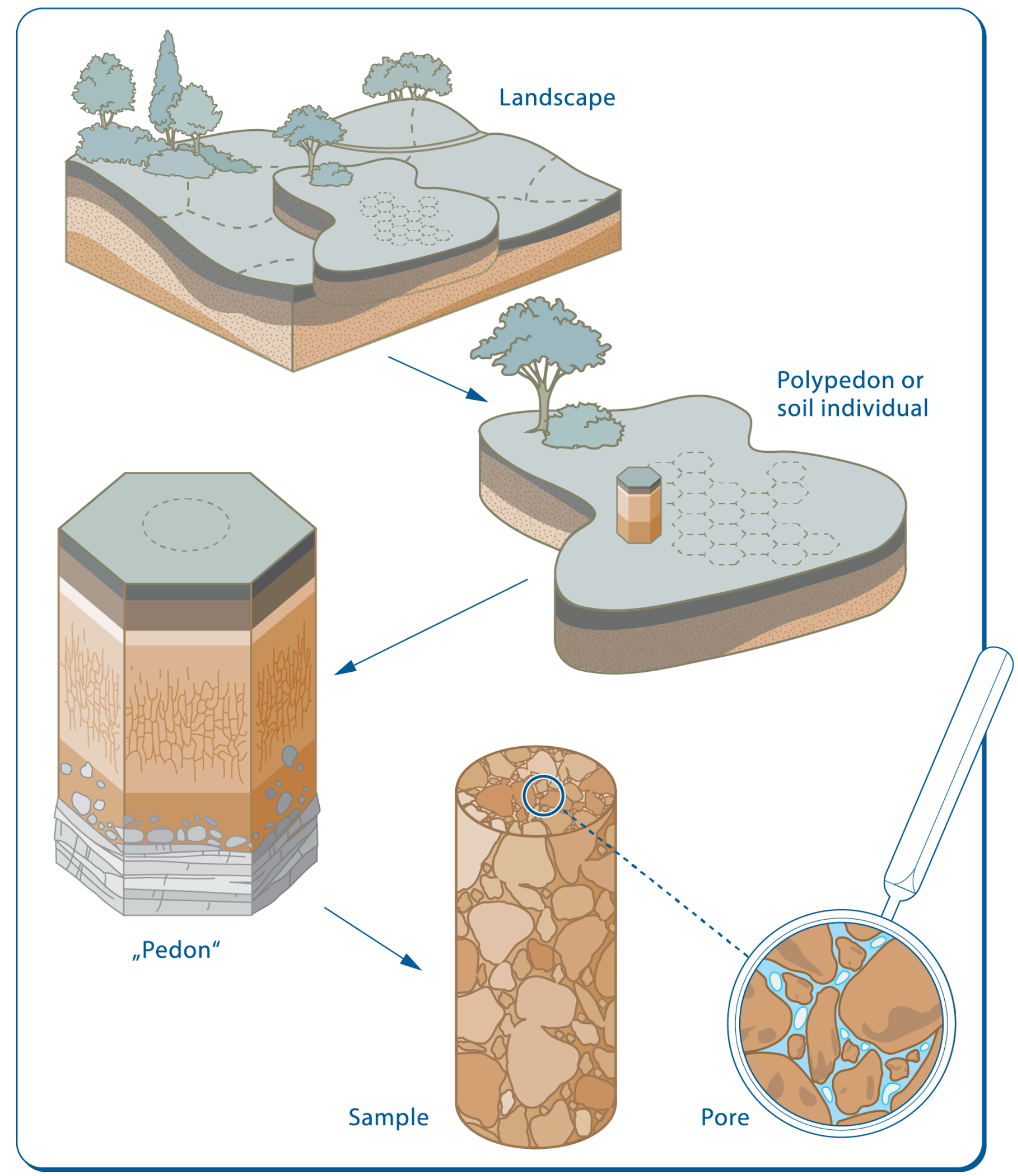

Figure 1.14 Illustration of delineation of a landscape into distinct and defined soil types ("polypedons"). Each portion of a pedon may be samples to define the local properites (e.g., porosity, color, water content, etc.). 


\section{Classification of Horizons and Layers}

Through the development of a soil it is usual for the soil profile to give rise to a sequence of bands, or horizons (Figure 1.15). Such a vertically stratified structure arises naturally from the aging process. For instance clay size particles may be physically carried with infiltrating water from the upper soil profile (in a process referred to as eluviation) and be deposited (a process called illuviation) in a tightly localized sheets (lamellae). This clay enriched region would be referred to as an argillic horizon. On the other hand minerals which have dissolved into solution may be carried upward through a soil profile driven by evaporation giving rise to concentrated bands of minerals at particular elevations. Mobility of ions is typically a function of the acidity of the soil solution, which also varies considerably with depth in a soil, thus leading to mobilization and precipitation of metals and other solutes in particular regions. Layering in soil may also arise from the depositional processes which established the parent material. In summary, the scale of variation in soils is typically much shorter in the vertical direction than in the horizontal. This being the case, soil types consist of particular arrangements of vertical layers (or horizons) of material. These layers may be very distinct, or almost indistinguishable. In the following sections we will introduce the terminology which is employed to describe the features of particular soil horizons. 


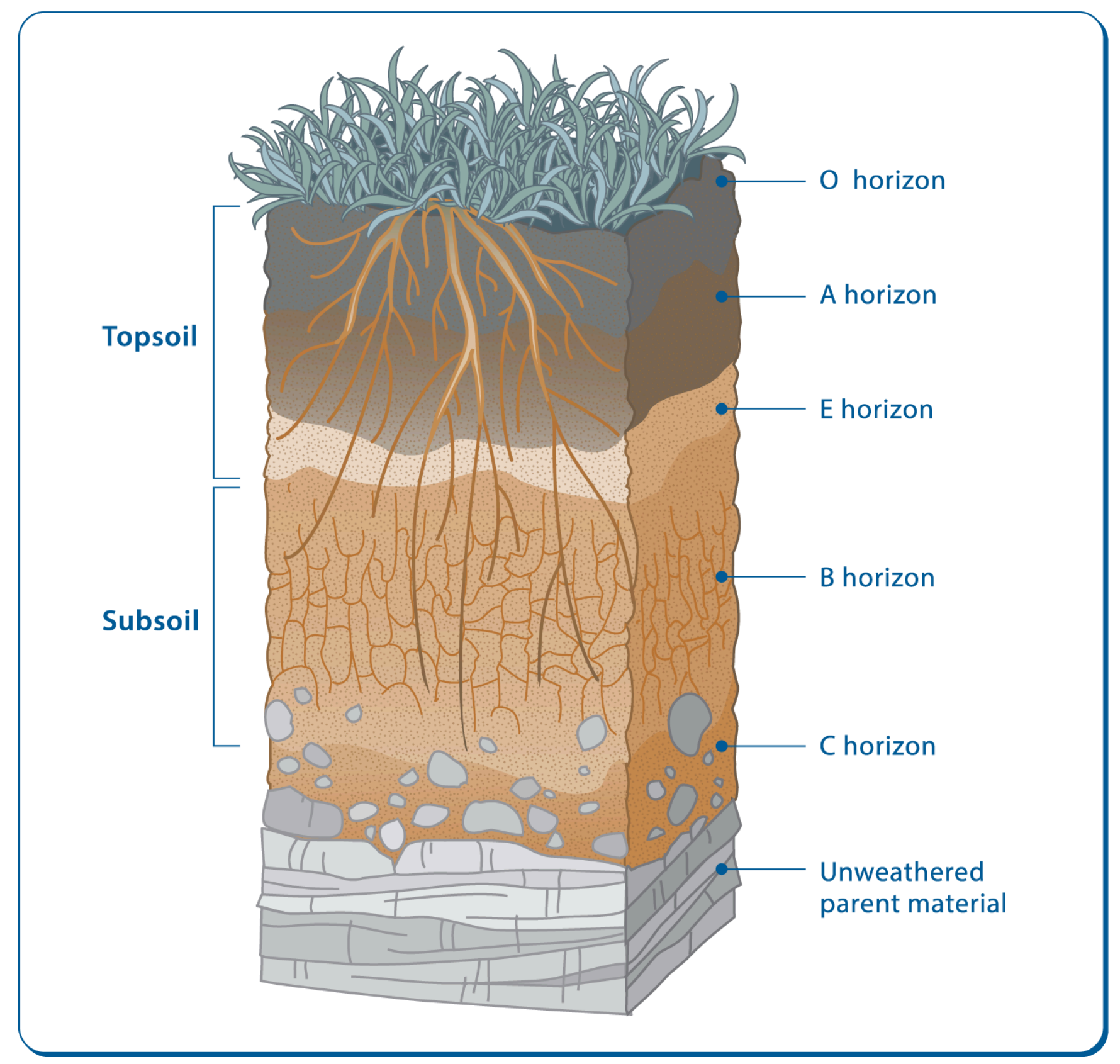

Figure 1.15 Illustration of typical soil horizons.

In the US system of classification, soil horizons are classified using a three symbol designation. We will describe the system of horizon classification by working through one particular designation: A'p1. Here "A" is what is referred to as the designation of master horizon. There are six master horizon designations; O, A, E, B, C, and R. In brief, O indicates that a horizon is dominated by organic matter, and may occur at any depth. An A horizon is the first mineral horizon in a soil, and may occur below only an O horizon. The A horizon is identified as either having enriched humic material or having properties altered by agricultural activities (e.g., plowing, grazing). An E horizon is a mineral horizon whose main feature is a loss of a combination of clay, iron and aluminum such that the remaining material is concentrated in sand, silt, and other resistant materials. Typically E horizons are lighter in color than the A horizon above it (due to a paucity of coatings of organic matter and iron oxides), or the B horizon below it, which will be enriched in iron and clays, which are highly colored. The B master horizon are simply soils which are bellow an A, E or O horizon and: (1) 
show significant alteration of structure from the soil's parent material, and (2) show evidence of movement of clays or other soil minerals or (3) show a block structure that results from changes in moisture content. The C horizon is simply soil material which is not bedrock, but shows little evidence of alteration from the parent material. The $\mathrm{R}$ designation indicates a hard bedrock layer too tough to penetrate with hand operated equipment. These descriptions are meant only to provide an idea of the meaning of these designations: for complete definitions, see the SCS Soil Taxonomy (Soil Survey Staff, 1994).

These major designations may be combined as either $\mathrm{AB}$ or $\mathrm{A} / \mathrm{B}$ if the horizon has some properties of the second designation (in this case $\mathrm{AB}$ would indicate an $\mathrm{A}$ horizon with some properties of a $\mathrm{B}$ ), or is composed of combinations of properties of the two designations respectively (A/B indicating a horizon with mixed A and B type properties). The lower case letter following the master designation indicates particular features of the master horizon. There are 22 such subordinate classifications. Examples include $\mathrm{k}$ to indicate accumulation of carbonates, $\mathrm{p}$ to indicate the influence of plowing, $\mathrm{n}$ to indicate the accumulation of sodium, etc. These subordinate designations may be used in multiple, simply depending upon the particular character of the horizon. Finally, Arabic numerals are employed to allow description of sequences of horizons with the same master designation, but with differing subordinate descriptions (e.g., Bk1 followed by Bn2). Whenever a horizon is designated, its vertical extent must also be reported.

\section{Classification of Color, Structure, and Texture}

Soil color provides critical information as to the genetic history of the soil, the biogeochemical environment of the soil, and how the observed soil strata differ in these regards. Typically dark colors are indicative of high organic content, while grayish coloration indicates reducing conditions, and reddish color indicates oxidizing conditions. Each of these conditions relates very closely to the typical hydraulic conditions of the site, often of greater use than a slew of lab analysis of soil cores.

Soil color is observed using a Munsell Color chart which provides a set of standard coupons encompassing the full range of soil colors (Figure 1.16). These standards are arranged by hue, value and chroma, which are summarized in an alpha-numerical coding shorthand. Thus, reading a complete soil description, one can reconstruct the sequence of horizon colors based on the field notes.

Figure 1.16 A typical page from a Munsell chart used to quantify soil color [NEED A PHOTO HERE, such as http://bebopandco.com/munsell-soil-color-book/page-from-the-munsell-colour-chart-book-soil-testtrench-157062/]

In addition to the average color of a soil, the pattern of coloration is informative. The condition referred to as mottling, whereby the color of the soil varies between grayish to reddish over a few $\mathrm{cm}$, is undoubtedly the most common and important pattern of coloration. Typically this indicates intermittent saturation, whence over periods of time the soil is either oxidizing or reducing the iron bearing compounds. The pattern of mottling (e.g., the placement, thickness and amount of each color) can be interpreted to reveal the history of the cycle of soil conditions. As in the case of average coloration, description of mottles has been refined to provide precise terminology of quantity, size, contrast and shape for use in field description (see the Soil Taxonomy, 1994, or; Vepraskas, M.J. 1992).

The structure of soil refers to the mechanical properties of the system. In describing structure one must identify the smallest repeated element which makes up the soil, which is referred to as a soil ped. The description of structure will include details of the size, strength, shape, and distinctness of the constituent peds.

This section is only intended to alert the reader to the basic features which are reported in a soil survey. For more detailed discussion of soil classification, refer to The Soil Taxonomy (Soil Survey Staff, 1994), or any of the many textbooks which focus on this subject.

Figure 1.17 The six climatic environments employed in soil classification, and their hydrologic character. 


\section{The High Points of Clay Mineralogy}

The clay constituents often dominate the hydraulic and chemical behavior of a geologic medium, and therefore warrant a more complete introduction than simply their size classification. In this section we will explore the basic structure of clay minerals to understand the nature of their reactive surfaces and differentiation between clay minerals.

There are two basic elementary building blocks for clay minerals: silica centered tetrahedra; and variously centered octahedra (Figure 1.18; Table 1.4). Each of these units can be built up into either chain structures (giving rise to minerals such as asbestos), amorphous structures (glasses), or sheet structures (phyllosilicates). The sheets are made up of either tetrahedra or octahedra in hexagonal unit elements.

Table 1.4 Ionic radius of various cations which could potentially replace $\mathrm{Si}^{4+}$ in tetrahedra or octahedra.

Ion

Ionic Radius (nm)

0.140

0.133

0.181

$\mathrm{F}^{-} \mathrm{Cl}^{-}$

$\mathrm{Si}^{4+}$

$\mathrm{Al}^{3+}$

$\mathrm{Fe}^{+3}$

$\mathrm{Mg}^{2}$

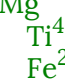

$\begin{array}{ll}\mathrm{Fe}^{2+} & 0.068 \\ \mathrm{Mn}^{2+} & 0.074\end{array}$

$\mathrm{Mn}^{2+} \quad 0.080$

$\mathrm{Na}^{+} \quad 0.097$

$\mathrm{Ca}^{2+} \quad 0.099$

$\mathrm{K}^{+}$

$\mathrm{Ba}^{2+}$

0.133

0.134

0.147
$\mathbf{R}_{\mathbf{X}}: \mathbf{R}_{\mathbf{O}}$

1.000

0.950

1.293

The cavity defined by the oxygen of these structures defines which ions could fit, which is 0.41 times that of oxygen for tetrahedra, and 0.732 times that of oxygen for octahedra. $\mathrm{R}_{\mathrm{x}}: \mathrm{R}_{\mathrm{O}}$ is the ratio of the radius of the cation to that of oxygen. Cations with less charge than silicon will result in a net charge on the structure, which will be negative, thus leading to a net negative charge on the phyllosilicate surface, attracting cations to bind to the surface.

Figure 1.18 The unit cell of the silica tetrahedra and the hexagonal ring structure of the silica sheet (a), and the octahedral unit cell and its ring and sheet structure referred to as the dioctahedral sheet. [we need a fresh version of this 
figure - Gabe: can you put us in touch with a person from the library who could make this figure? Seems like we could replicate som from Grim 1968 as seen at http://condor.wesleyan.edu/ethomas/ees123/sheet04.htm]

If we consider a cross-sectional view of the sheets which are made up by the octa- and tetrahedra, it is apparent that the sheets have the cations in the core of the sheet, with the exposed surfaces made up of oxygen and hydroxyls (so $\mathrm{OH}$ can replace $\mathrm{O}$ in the structure). Looking at these surfaces in plan view, we see that the surfaces are made up of hexagonal rings (Figure 1.18). The group of atoms in these rings act as a delocalized source of negative charge, and are referred to as a surface functional group. (a.k.a. SFG). Cations will be attracted to the center of these SFG's just above the surface of the sheet.

But now wait a minute. A bedrock tenet of the physical sciences is that all substances, including crystalline solids (clay minerals) and fluid water, too, are charge-balanced, i.e. their structures contain equal amounts of positive and negative charge such that their macroscopic, net charge is zero. How, then, can surface charge develop? It turns out that some of the charge (which sums to zero for a given crystal or particle) exists as adsorbed species, i.e. adsorbed species neutralize what would otherwise be a net charge within the solid structure.

Let's now look more closely at the development of negative charge within the clay structure. Charge development within layer-silicate crystal structure arises primarily from the distribution of cations in the sheets of which the layer silicates are comprised. Schematics of important sheet arrangements and charge distributions are shown in Figure 1.16. In the tetrahedral sheets, each silicon atom is surrounded or coordinated by four equidistant oxygen atoms; in the octahedral sheets, each aluminum (or magnesium) atom is coordinated by six equidistant hydroxide ions ( $\left.\mathrm{OH}^{-}\right)$and/ or oxygen atoms, which are essentially the same size. These coordinations within the sheets are dictated by the radius ratios of the respective cations and oxygen/hydroxide. The sheets are linked by the sharing of oxygen and hydroxide at the apices of the octahedra and tetrahedra comprising the sheets, in simple combinations called layers. Most of the layer silicates of interest to us are either 1:1 or 2:1 layer types (Figure 1.16). Most geochemistry, soil chemistry, and soil physics texts show diagrams of the atomic structures of sheets and their linkage in layers (e.g. Drever, 1988; Sposito, 1989; Jury et al., 1991; Bohn et al., 1979). 
a.

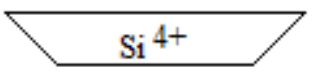

b.
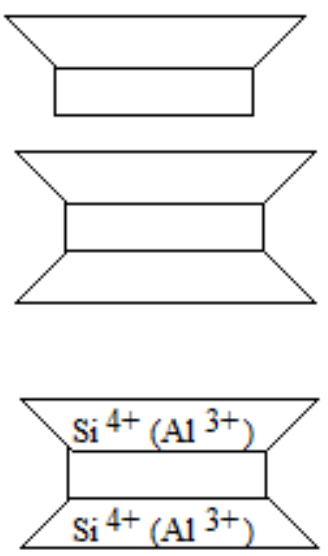

c.

d.
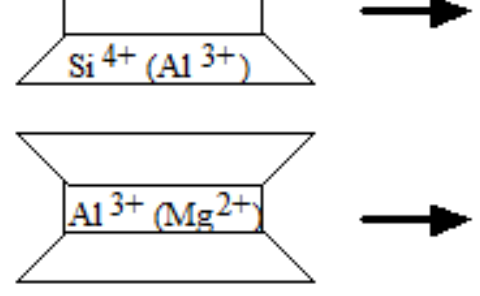

(K) $\left(\mathrm{K}^{+}\right)$

\section{1:1 layer}

2:1 layer
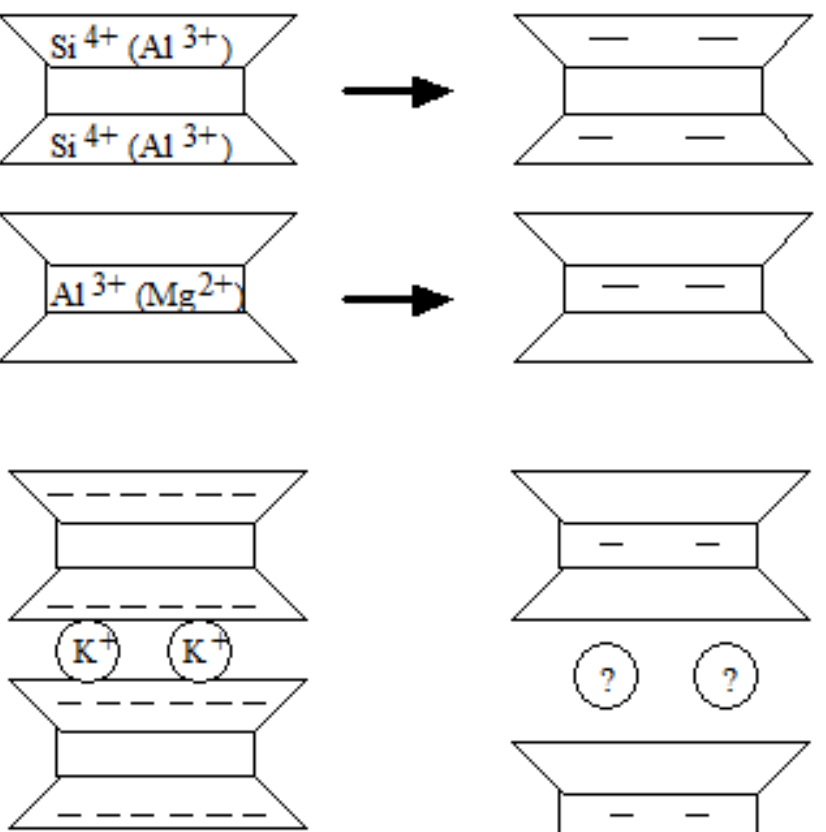

biotite

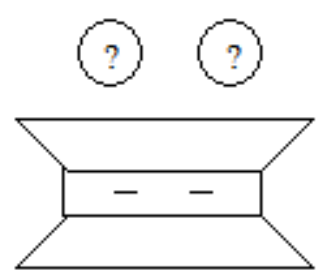

montmorillonite

Figure 1.16 Layer silicate crystal structures. (a) symbols for sheet types, showing predominant cation (which, in octahedral sheets, may be $\mathrm{Mg}^{+}$instead of $\mathrm{Al}^{+}$). (b) linkages of sheets in the most common layer types. (c) isomorphic substitution (of cation in parentheses) causes net negative layer charge in many 2:1 minerals. (d) locus and magnitude of layer charge determine interlayer bond properties and cation populations. $\mathrm{K}^{+}$is specifically adsorbed between biotite layers, whereas interlayer spacing and nonspecifically adsorbed cation population between montmorillonite layers varies depending on solution composition.

[this figure needs to be improved - can we look for a better version?]

In the chemically simplest of the layer types, the net layer charge is zero: cationic and anionic charges balance (e.g. kaolinite and pyrophyllite, Table 1.5). However, Nature is not always simple and the solutions from which these clays form 
usually contain concentrations of silicon, aluminum, and/or other cations which favor the selective incorporation of these cations into the tetrahedral and octahedral sheets as "substitutes" for $\mathrm{Si}_{4}^{+}$and $\mathrm{Al}^{+}$, respectively. This isomorphic substitution causes permanent (negative) layer charge.(Figure 1.16c). The locus and extent of this substitution determine the intensity of charge at the layer surface; this, in turn, determines how cations are bound between layers (Figure 1.16d). Nonspecifically adsorbed interlayer cations are exchangeable, a term we will elaborate on in section 4.5; such cations dominate the large cation exchange capacity (CEC) of 2:1 clays like montmorillonite and vermiculite (Table 1.5). Other 2:1 layer silicates like micas (biotite, muscovite) and chlorite have such large layer charge that interlayer cations are tightly, specifically bound, and are not exchangeable; these minerals do not develop large CECs (Table 1.5).

Table 1.5 Layer Silicate Properties (from Bohn, 1979, Table 3.6)

\begin{tabular}{|c|c|c|c|c|c|}
\hline Layer type & mineral & formula & layer charge* & $\begin{array}{l}\mathrm{CEC}^{* *} \\
(\mathrm{meq} \mathrm{g}-1)\end{array}$ & $\begin{array}{r}\text { Specif } \\
(\mathrm{m}\end{array}$ \\
\hline 1:1 & kaolinite & $\mathrm{Al}_{2} \mathrm{Si}_{2} \mathrm{O}_{5}(\mathrm{OH})_{4}$ & 0 & $0.03-0.2$ & \\
\hline 2:1 & montmorillonite & $\operatorname{Nax}\left[\left(\mathrm{Al}_{2}-\mathrm{xMGx}\right) \mathrm{Si}_{4} \mathrm{O}_{10}(\mathrm{OH}) 2\right]$ & $0.25-0.6$ & $0.8-1.5$ & 6 \\
\hline 2:1 & vermiculite & $\operatorname{Nax}\left[\mathrm{Mg}_{3}\left(\mathrm{Si}_{4}-\mathrm{xAlx}\right) \mathrm{O}_{10}(\mathrm{OH}) 2\right]$ & $0.6-0.9$ & $1.0-1.5$ & \\
\hline 2:1 & mica (illite) & $\mathrm{Kx}\left[\mathrm{Al}_{4}\left(\mathrm{Si}_{4}-\mathrm{xAlx}\right) \mathrm{O}(10 \mathrm{OH}) 2\right]$ & 1 & $0.1-0.4$ & \\
\hline 2:1:1 & chlorite & $\begin{array}{l}{\left[\mathrm{AlMg}_{2}(\mathrm{OH}) 6\right] \mathrm{x}} \\
\quad\left[\mathrm{mg}_{3}(\mathrm{Si} 4-\mathrm{xAlx}) \mathrm{O}_{10}(\mathrm{OH})_{2}\right]\end{array}$ & 1 & $0.1-0.4$ & \\
\hline \multicolumn{5}{|c|}{$\begin{array}{l}\text { * per formula unit } \\
\star * \text { includes values from Jury et al. (1991), Stumm and Morgan (1981), and Sposito (1989) }\end{array}$} & \\
\hline
\end{tabular}




\begin{tabular}{|c|c|c|c|c|c|}
\hline Mineral & Structure & $\begin{array}{l}\text { Surface } \\
\text { Area } \\
\left(\mathrm{m}^{2} / \mathrm{gr}\right)\end{array}$ & $\begin{array}{l}\text { Cation } \\
\text { Exchange } \\
\text { Capacity } \\
\text { (mol/kg) }\end{array}$ & Image & Citation \\
\hline Kaolinite & 1:1 & $7-30$ & 5 & 41 & $\begin{array}{l}\text { Image: } \\
\text { Shoval, S.; Yariv, S.; } \\
\text { Michaelian, K. H.; } \\
\text { Boudeulle, M.; } \\
\text { Panczer, G., Hydroxyl- } \\
\text { stretching Bands in } \\
\text { Polarized Micro- } \\
\text { raman Spectra of } \\
\text { Oriented Single- } \\
\text { Crystal Keokuk } \\
\text { Kaolinite, Clays and } \\
\text { Clay Minerals, Volume } \\
\text { 50, Number 1, 1 } \\
\text { February 2002, pp. } \\
\text { 56-62(7) } \\
\text { CEC:Chi M, C. and } \\
\text { R.A. Eggelton, Cation } \\
\text { Exchange Capacity of } \\
\text { Kaolinite, Clays and } \\
\text { Clay Minerals, vol. 47, } \\
\text { No. 2, 174-180, 1999. }\end{array}$ \\
\hline Smectite & $2: 1$ & $700-1000$ & 100 & & $\begin{array}{l}\text { Image: } \\
\text { Gitari, M.V., M. Tutu, H. } \\
\text { DeBeer, } \\
\text { Efficiency of ball } \\
\text { milled South African } \\
\text { bentonite clay for } \\
\text { remediation of acid } \\
\text { mine drainage, Journal } \\
\text { of Water Process } \\
\text { Engineering } \\
2015 \text { 8: } 227-240 . \\
\text { CEC: JAYNES, W.F. } \\
\text { and J.M. Bigham, } \\
\text { Multiple cation- } \\
\text { exchange capacity } \\
\text { measurements on } \\
\text { standard clays using a } \\
\text { commercial } \\
\text { mechanical extractor, } \\
\text { Clays and Clay } \\
\text { Minerals, Vol. } 34 \text {, } \\
\text { 1:93-98, 1986. }\end{array}$ \\
\hline
\end{tabular}

Nonetheless, the high-layer-charge 2:1 minerals and even the common 1:1 mineral kaolinite have cation exchange capacities which may be important in many settings. What accounts for these populations of nonspecifically adsorbed cations, if they do not populate interlayers? The explanation is considered to be the termination of crystal sheet structures at sheet edges.$$
\mathrm{Si}-\mathrm{OH}_{2}^{+} \leftrightarrow \mathrm{Si}-\mathrm{OH} \leftrightarrow \mathrm{Si}-\mathrm{O}^{-}
$$$$
\text { acid basic }
$$

Figure $1.17 \mathrm{pH}$-dependent charge at edge of kaolinite layer (modified from Bohn et al., 1979). 
Figure 1.17 shows this situation schematically at the edge of a kaolinite layer. Note that the charge on the hydroxyl functional groups (the reactive species on the solid surface) is $p \mathrm{H}$-dependent, that is, it depends on the activity of the $\mathrm{H}^{+}$ ion in solution, such that the net surface charge is positive at low $\mathrm{pH}$ (large $\mathrm{H}^{+}$activity) and negative at high $\mathrm{pH}\left(\mathrm{small} \mathrm{H}^{+}\right.$ activity). There must also exist some intermediate $\mathrm{pH}$ at which the net surface charge is zero, the isoelectric point. Thus at sufficiently high $\mathrm{pH}$ the negatively-charged kaolinite edge electrostatically adsorbs exchangeable cations, while at sufficiently low $\mathrm{pH}$ the edge would actually repel cations and adsorb exchangeable anions. Such $\mathrm{pH}$-dependent behavior is typical of any natural surface where structural oxygen atoms and hydroxyl ions are exposed at the solution interface, i.e. a variety of common solid surfaces including oxides, hydroxides, and organic matter (Table 1.6), such that it is an important component of the CEC of most soils.

Table 1.6 Surface properties of various solids

\begin{tabular}{|c|c|c|c|}
\hline Solid & type or formula & $\begin{array}{l}\text { pH dependence, } \\
\text { surface charge }\end{array}$ & isoelectric point ${ }^{(2)}$ \\
\hline kaolinite & 1:1 & extensive & $4-5$ \\
\hline smectites & $2: 1$ & slight & $* *$ \\
\hline mica (illite) & $2: 1$ & moderate & $* *$ \\
\hline vermiculite & $2: 1$ & slight & ** \\
\hline chlorite & $2: 2: 1$ & extensive & $* *$ \\
\hline quartz & $\mathrm{SiO}_{2}$ & extensive & $2-3$ \\
\hline feldspars & $\begin{array}{c}(\mathrm{K}, \mathrm{Na}) \mathrm{AlSi}_{3} \mathrm{O}_{3} \\
\mathrm{CaAl}_{2} \mathrm{Si}_{2} \mathrm{O}_{8}\end{array}$ & extensive & $2-3$ \\
\hline Fe-oxy-hydroxides & $\begin{array}{l}\mathrm{FeOOH} \\
\mathrm{Fe}(\mathrm{OH})_{3}\end{array}$ & extensive & $7-8$ \\
\hline Al oxy-hydroxides & $\begin{array}{l}\mathrm{AlOOH} \\
\mathrm{Al}(\mathrm{OH})_{3}\end{array}$ & extensive & $5-9$ \\
\hline humus & $\mathrm{C}, \mathrm{H}, \mathrm{O}$ & extensive & undefined \\
\hline \multicolumn{4}{|c|}{$\begin{array}{l}\text { (1) Bohn et al. (1979) } \\
\text { (2) Stumm and Morgan (1981) and Sposito (1989) } \\
\star * * \text { data not available }\end{array}$} \\
\hline
\end{tabular}

Specific adsorption can also occur at these surfaces. This changes the surface charge at a given $\mathrm{pH}$, but not the general $\mathrm{pH}$-dependence of surface charge (Sposito, 1989, section 7.4). The relative lack of organic matter in many subsoil vadose zones probably means that $\mathrm{pH}$-dependent charge is generally less important in subsoil vadose zones than in soils.

We have seen that clays are made up of layers of tetrahedral and octahedral sheets with cations bound in the surface functional groups holding the stacks together. Since these layers are only a few atomic radii thick and thousands of atomic radii in horizontal extent, clays have a pronounced thin plate structure. This means that clays have very high surface area to weight, with these surfaces holding both water and cations, making clays highly reactive. Most clays swell when they go from a dry to wet state due to the attraction of water between the plates of clay (Figure 1.18). As discussed further in section 4.4, clay layers and plates can dissociate from each other due to cation exchange under certain solution-chemistry conditions. 
The extensive platy structure also makes the water path though clays extremely tortuous, leading to very high resistance to flow of water. For this reason clay rich zones are often referred to as impermeable layers. Although this may be true in the saturated region, in the vadose zone clays often experience repeated wetting and drying which results in large shrinkage voids where water and other fluids can pass relatively directly.

$$
\mathrm{O}=\mathrm{H}_{2} \mathrm{O}
$$

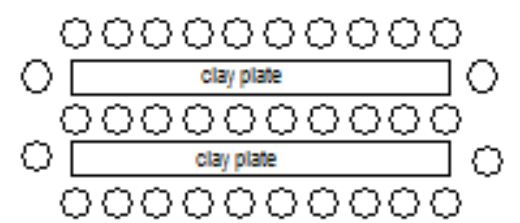

(a) Low moisture: clays bound

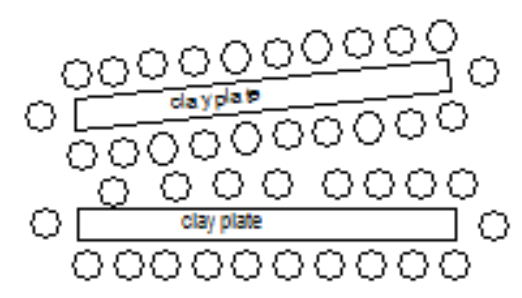

(b) High moisture: clays dissociated

Figure 1.18 Dissociation of clay with increasing moisture content gives rise to gross swelling.

To summarize: the range of properties found among clay minerals arises due to three main features: (1) the order of layering of tetrahedral and octahedral sheets; (2) the isomorphic substitutions in the sheets; and (3) the cations which are bound to the surface functional groups. A few examples, referring back to Table 1.5 and Figure 1.16, will make this clear. Kaolinite is a 1:1 clay mineral, indicating that its layers are made up of alternating octahedral and tetrahedral sheets. The unit cells of kaolinite have little isomorphic substitution. This being a very stable layering pattern, kaolinitic clays tend to be made up of thicker stacks of sheets, and therefore have relatively low specific surface and do not swell as much as other clay minerals. Montmorilonite, a member of the smectite family of clays, is a 2:1 mineral with octahedral sheets sandwiched between two tetrahedral sheets. There is also a great deal of isomorphic substitution of $\mathrm{Mg}^{+2}, \mathrm{Fe}^{+2}$, and $\mathrm{Fe}^{+3}$ for the $\mathrm{Al}^{+3}$ in the octahedral sheets. Since the octahedral sheet is sandwiched between tetrahedral sheets, the cations are in outer sphere complexes with the SFG's which may become hydrated. The net result of this is to generate large $\mathrm{CEC}$, much greater effective surface area, and very significant swelling with addition of water. Illite, on the other hand, is also a 2:1 mineral, but with the isomorphic substitution of $\mathrm{Al}^{+3}$ for the $\mathrm{Si}^{+4}$ in the tetrahedral sheet. This generates strong local charge in the area of the surface functional groups, binding potassium between the successive 2:1 units. As this bond is quite strong, there is little swelling with water, and the effective surface area is moderate, and the CEC is small.

\section{Review Questions}

1. Define the vadose zone.

2. Who first presented Darcy's law for unsaturated flow, and when.

3. List four major differences between flow processes in saturated and unsaturated conditions.

4. The size fractions for sand, silt and clay have particular values which depend upon the system of classification chosen (e.g., USDA vs FAO), yet there are physically based differences between the classes which are maintained between systems. For instance, sand includes grains that you can distinguish 
visually. What are the physical descriptions which define silt and clay?

5. If you were asked to provide one number to describe the particle size of a soil what would you choose. What if you were allowed two parameters? Define these parameters.

6. Name one processes by which surface soils become vertically stratified into layers over time. Name a process which leads to stratification of the parent material.

7. Name two factors which affect soil color.

8. Discuss how you might detect a region which experienced alternating periods of saturation and partial saturation in a soil by the color.

9. Define isomorphic substitution. What dictates what elements may be substituted into the crystal structure?

10. Why are cations attracted to clay surfaces? What is meant by anion exclusion?

11. What are typical surface areas for clays? How does this relate to the swelling of clay?

\section{Chapter 1 Problems}

1. Buckingham first presented Darcy's law for unsaturated flow in 1931.

2. Isomorphic substitution refers to the exchange of cations bound to surface function groups through either inner or outer sphere complexes.

3. Anions are excluded from the vicinity of clay surfaces due to osmotic forces due to gradients in ionic concentration.

4. A typical surface area for a clay would be $0.1 \mathrm{~km}^{2} / \mathrm{kg}$.

5. Find the textural class names for each of the tabulated soils using the textural triangle.

\begin{tabular}{llll}
\hline Soil Name & \% sand & \% silt & classification \\
Selker's special & 40 & 30 & \\
$\mathbf{5 4 2}$ goo & 15 & 70 & \\
finger-matic & 75 & 25 \\
slip-n-slide & 15 & 50 \\
typical dirt & 30 & 60 \\
\hline
\end{tabular}

6. Derive the basic dimensions of: (i) Energy; and (ii) Power.

Example: Derive the basic dimensions for Force:

$$
\mathrm{F}=\mathrm{ma}^{\prime}=\mathrm{m} \frac{\mathrm{v}}{\mathrm{t}}=\mathrm{m} \frac{\mathrm{s}}{\mathrm{t}^{2}} \quad\left[\mathrm{MLT}^{-2}\right]
$$

F.

Force

m........................... Mass

a'............................ Acceleration

v…......................... Velocity 


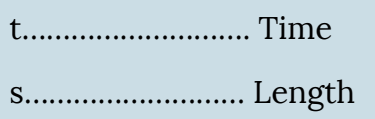

7. Show the basic dimensions of: (i) energy per unit of weight; and (ii) energy per unit of volume.

8. Given $\mathrm{P}=\rho \mathrm{gh}$, where $\rho$ is density (mass/volume), $\mathrm{g}$ is the acceleration of gravity, and $\mathrm{h}$ is height: (i) what are the basic dimensions of P? (ii) Check whether the dimensions of P match the definition of pressure as force divided by area.

9. Convert to SI units: (i) 5.21 dyne s $/ \mathrm{cm}^{2}$ known as poise - cgs units of viscosity; (ii) $8.5 \mathrm{~g} \mathrm{~cm} / \mathrm{s}^{2}$; (iii) a discharge of 1100 gpm (US gallons per minute); (iv) $25 \mathrm{cal} / \mathrm{cm}^{2}$.

10. What is the settling velocity in [m/sec] of: (i) sand (diameter $=0.1 \mathrm{~mm}$ ); (ii) silt ( $\mathrm{d}=0.01 \mathrm{~mm})$; and (iii) clay $(\mathrm{d}=0.001 \mathrm{~mm})$ particles, at a temperature of $10^{\circ} \mathrm{C}$ and at $20^{\circ} \mathrm{C}$ ? (Consult the tables in the Appendix for physical properties of water, and check your units and dimensions for consistency)

11. How long would it take for all sand particles to settle to the bottom of a $0.5 \mathrm{~m}$ long column at $30^{\circ} \mathrm{C}$ ? How long would it take for all silt particles to settle?

(Use the USDA classification for soil textural fractions to determine the particle diameters separating the sand, silt, and clay fractions)

12. Use the USDA textural triangle and determine the textural class for: (i) a soil having $25 \%$ sand, $60 \%$ silt, and $15 \%$ clay content; and (ii) a soil with $50 \%$ sand, $7 \%$ silt, and $43 \%$ clay content.

13. Estimate the specific surface area per unit of mass $\left(\mathrm{A}_{\mathrm{m}}\right)$ of a soil composed of $15 \%$ coarse sand (diameter $=1.0 \mathrm{~mm}), 35 \%$ fine sand $(\mathrm{d}=0.1 \mathrm{~mm}), 30 \%$ silt $(\mathrm{d}=0.02 \mathrm{~mm}), 10 \%$ kaolinite clay (average platelet thickness $=4 \times 10^{-5} \mathrm{~mm}$ ), and $10 \%$ montmorillonite clay (thickness $=1 \times 10^{-6} \mathrm{~mm}$ ). What percentage of the soil surface area is contributed by the montmorillonite? (Express specific surface area in $\mathrm{m}^{2} / \mathrm{g}$ )

14. A clod of soil displaces $70 \mathrm{ml}$ of water; the "wet" weight of the coated clod was $0.095 \mathrm{~kg}$ and the water content was $\theta_{\mathrm{m}}=0.1(\mathrm{~kg} / \mathrm{kg})$. What is the bulk density $\rho_{\mathrm{b}}$ of the clod, assuming that the volume of the paraffin coating was $5 \mathrm{ml}$ and its density $900 \mathrm{~kg} / \mathrm{m}^{3}$ ?

15. A porous medium is composed of spheres having equal radius $r_{1}$. (i) Find the porosity for cubic packing (use a cube edge of $4 \mathrm{r}_{1}$ ); (ii) find the ratio of porosities for a medium composed of different radii of spheres $r_{2}=r_{1} / m$ (m signifies an arbitrary number); (iii) find the ratio of surface areas for unit cubes composed of radii as in (i) and (ii).

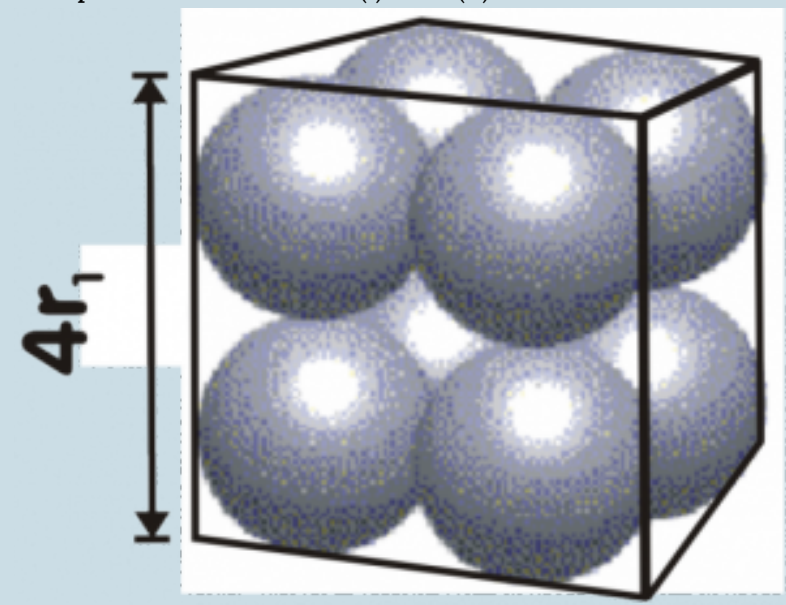

16. A cube is divided such that identical cubes are produced after each division (1->8->64 etc.). Given a cube edge of $\mathrm{L}$, find the number of cubes after $\mathrm{n}$ divisions and their total surface area. 


\section{Index}

A

$\begin{aligned} & \text { A horizon } \\ & \text { aquic } \\ & \text { argillic }\end{aligned}$
$\begin{aligned} & \text { asbestos } \\ & \text { B }\end{aligned}$
B horizon
biotite
Bousinesq
Buckingham
C
C horizon
capillarity
capillary fringe
cation
chlorite
chroma
clay
climate
coordination

D

d50

Darcy

Diffusion

diffusivity

dioctahedral

E

E horizon

eluviation

F

fingers

G

Green and Ampt

H

Haines jump

heterogeneity

horizons

hue

humic

hysteresis

I

illite

illuviation

independent domain model

interlayers

42 | Fundamentals of Soil Hydrology and Bio-Physics 
isoelectric point

K

kaolinite

L

lamellae

M

macropores

master horizon

Miller Scaling Theory

montmorillonite

montmorillontie

mottling

Munsell color chart

muscovite

N

NAPL

O

octahedra

P

parent material

particle size distribution

pebble

ped

pH-dependence

phyllosilicates

preferential flow

pyrophyllite

R

Richards

Richards equation

S

sand

Schlichter

settling analysis

SFG

sieve analysis

Sieves

silica

silt

smectite

soil classification

soil texture

soil triangle

subordinate classifications

suction

surface functional group

swelling

T

Fundamentals of Soil Hydrology and Bio-Physics | 43 
tensiometer

tension

tetrahedra

textural triangle

$\mathbf{U}$

uniformity coefficient

unsaturated zone

V

vadose zone

value

vermiculite

W

wetting front instability

$\mathbf{X}$

xeric 


\section{Basic Relationships and the Soil Phases Under Equilibrium Conditions}

Chapter 2: Basic Relationships and the Soil Phases Under Equilibrium Conditions

2.1 Where we are going

2.1.1 Soil as a Three-Phase System

2.1.2 Volume and Mass Relationships

2.1.3 Liquid Content

2.2 Characterization of the Solid Phase

2.2.1 Texture and systems of classification

2.2.2 Sieving

2.2.3 Sedimentation

2.2.4 Surface Area of Soil Particles

2.2.5 Soil Structure and Bulk Density

Aggregation, bio-pores, packing geometries

2.3 Characterization of the Liquid Phase

2.3.1 Soil Water Content Measurement Methods

Gravimetric, dielectric, neutron probe, cosmic ray backscatter, remote sensing via radar and gravitation (SMAP, SMOS, GRACE); direct, indirect, and surrogate methods, errors.

2.3.2 Applications of Soil Water Content Information - The Water Balance across scales of soil profile, field, catchment, continent, and globe and seconds to decades.

2.3.3 Field Capacity, Wilting Point and Plant-Available Soil Water

2.4 The Energy State of Soil Water

2.4.1 Total Soil Water Potential

2.4.2 Gravitational Potential

2.4.3 Matric Potential

2.4.3.1 Water Properties Affecting the Matric Potential

2.4.4 Pressure Potential

2.4.5 Solute Potential

2.4.6 Water Potentials Under Equilibrium Conditions (Hanks, 1992 - pp. 31-37)

2.4.6.1.1 Constructing Potential Diagrams under Equilibrium Conditions

2.4.6.1.2 Water Potentials Under Nonequilibrium Conditions

2.5 Bringing gas, solids and liquids together

2.5.1 Capillarity and Surface Tension

2.5.1.1 What is surface tension

2.5.1.2 Deriving the LaPlace Equation

2.5.1.3 Temperature dependence of surface tension

2.5.1.4 Surface absorption (Tuller and Or Equation) and the myth of dry soil

2.5.1.5 Contact angle

Idealized, impacts of surface texture and defects, hysteresis

2.5.2 Soil Water Content-Energy Relationships

2.5.2.1 Soil Water Characteristic (SWC)

2.5.2.2 Measurement of SWC Curves

2.5.2.3 Fitting Parametric SWC Expressions to Measured Data

Basic Relationships and the Soil Phases Under Equilibrium Conditions | 45 
2.5.2.4 Hysteresis in the Soil Water Characteristic Relation

2.5.2.4.1 The independent domain model for hysteresis

2.5.2.4.2 Analytical expressions for hysteresis

2.5.3 Measurement of Soil Water Potential Components

2.5.3.1 Tensiometer for Soil Matric Potential Measurement

2.5.3.2 Heat Dissipation in Rigid Porous Matrix for Measuring Matric Potential

2.5.3.3 The Psychrometric equation and Psychrometric methods for Measuring Water Potential

2.5.3.4 Piezometer for Measuring Hydrostatic Pressure Potential

2.5.3.5 Measurement of Soil Water Osmotic Potential

2.6 SUMMARY

2.7 Review Questions

2.8 REFERENCES

2.9 PROBLEMS

2.10 Appendices:

2.10.1 The thermodynamics of liquid and solid (Hamaker) interfaces

2.10.2 Advanced methods in soil moisture measurement (GPR, ERT, EMI, NMR, COSMOS

2.10.3 The independent domain model for hysteresis

\section{I Where we are going}

We are concerned with describing the retention and movement of fluids in natural porous media. This objective fits neatly into a single sentence, however the challenge of attaining this goal remains to be met to the degree required to solve many contemporary problems in environmental sciences and engineering. Nonetheless, there are many powerful tools at our disposal. In this chapter we will cover the basic physical properties upon which much of the remainder of the text rests. If all goes well you will get a "feel" for both the physical processes which control flow through porous media and the conceptual and mathematical approaches we employ to characterize the processes. Having a well balanced understanding from both perspectives is essential; you must be able to guide your application of mathematical solutions based on a clear and accurate intuitive concept of what you are trying to describe. How do you describe a chunk of moist porous medium? What are some basic features which would allow you to abstract that chunk of material in terms of a set of mathematical parameters which will assist in describing: the way in which it might dry; how rain falling on it would soak in (and how much would run off); and what would happen to it if you spilled some gasoline on it? We require a system of measurable quantities for a porous medium that may be used in addressing the broadest possible set of questions about how fluids will interact with it under a variety of conditions. Porous media is characterized by a set of parameters selected to provide as concisely as possible the greatest insight into the response of the media to a range of physical processes. In this section we identify the fundamental parameters employed in describing both the media and the fluid of interest, and introduce the fundamental equations of motion for fluids in porous media.

\section{I.I Soil as a Three-Phase System}

Before digging into the details of hydraulic properties and flow processes, we need to provide some background, definitions and quantitative measures. We divide natural porous media into three constituent phases: solid, liquid, and gaseous, with each of these phases consisting of an admixture of compounds.

The gaseous phase is generally dominated by the major constituents of the atmosphere; $\mathrm{N}_{2}, \mathrm{O}_{2}, \mathrm{CO}_{2}, \mathrm{H}_{2} \mathrm{O}$ vapor etc. Clearly, as microbes and plant roots respire, a soil's $\mathrm{CO}_{2}$ and $\mathrm{CH}_{4}$ content may become significantly elevated, while 
in industrially contaminated sites there may be significant levels of organic vapors. By and large we keep track of the state of the gaseous phase in the soil just as we do in the atmosphere; we consider the partial pressures of each of the constituents, and keep track of the partitioning of each compound. Primary parameters of interest, then, include the gas diffusivity, and the partial pressure versus temperature relationship, and the Henry's law coefficient (Henry's law states that at a given temperature, for low concentrations, there is a linear relationship between the partial pressure of the gas and the concentration of the material in the liquid phase). It is noteworthy that transport of gaseous phases are dominated by molecular diffusion which generates about $2 \mathrm{~cm} /$ day of spreading, while molecular diffusion in the liquid phase is 100 times less effective in contaminant mass movement. $\neq$ The movement of gaseous phase constituents is also a function of the changes in atmospheric pressure, which generates a pumping effect, as noted by Buckingham (1904) and Weeks (1979). Of course, gaseous phase movement can also be driven by liquid phase movement, as is the case of a saturated wetting front pushing gas down through a profile during flood irrigation. Gas phase transport has received renewed interest with the advent of soil vapor extraction (SVE) as a remediation technique for volatile organics. In this procedure, gas is pumped out of the vadose zone through a well, taking the volatile fraction of contamination with it.

Of the possible liquid phase constituents, water is by far of greatest interest. In the vadose zone it is valid to assume liquids are incompressible, since by definition liquids will only experience pressures which are negative, and generally with a magnitude of less than 10 atmospheres. In contrast, below the water table, groundwater commonly experiences extreme pressures (e.g. in cases of confined and very deep aquifers). Otherwise liquid phase properties are most often parameterized in terms of the interaction with solid and gaseous phases, such as contact angle, capillary pressure, volatility, etc., as is discussed later.

\section{Example}

What is the percent volumetric change in water in the vadose zone with a change in pressure from zero to -10 bars $\left(10\right.$ bars is $\left.10^{6} \mathrm{~N} / \mathrm{m}^{2}\right)$ ? Recall that the isothermal compressibility of water, $b_{\mathrm{w}}$, is about $4.8 \times 10^{-10} \mathrm{~m}^{2} / \mathrm{N}$. The percentage change in volume of water is $100 \times b_{\mathrm{w}} \mathrm{x}$ the change in pressure $=0.048 \%$. Since the material is mostly gas filled when the pressure is this low, this very small change in volume will be undetectable.

The solid phase may be as complicated or simple as you like. Typically composed of largely inert minerals (rock, gravel, sand, silt and clay), solid constituents may include roots, carbon, and other highly reactive substances. Note that in this discussion we have slighted the semi-solid or soft tissue "goo" such as microbes, plants and worms that are prevalent in the vadose zone. These components are very important to understanding the chemical behavior of the unsaturated zone, and are discussed in chapter 4 .

To get yourself in the proper frame of mind, pretend you are an ant. What humans consider to be uniform porous medium now looks lumpy and irregular. Although this may seem all too obvious, it is crucial to consider this problem in detail. To do this we will have to introduce the basic parameters used to conceptualize a porous media. First, let's identify the constituents which we will concern ourselves with: the mass and volume of each phase; gas, liquid and solid. As identified in Figure 2.1a, the volume and mass of phase i are identified as $\mathrm{V}_{i}$ and $\mathrm{M}_{\mathrm{i}}$ respectively. 


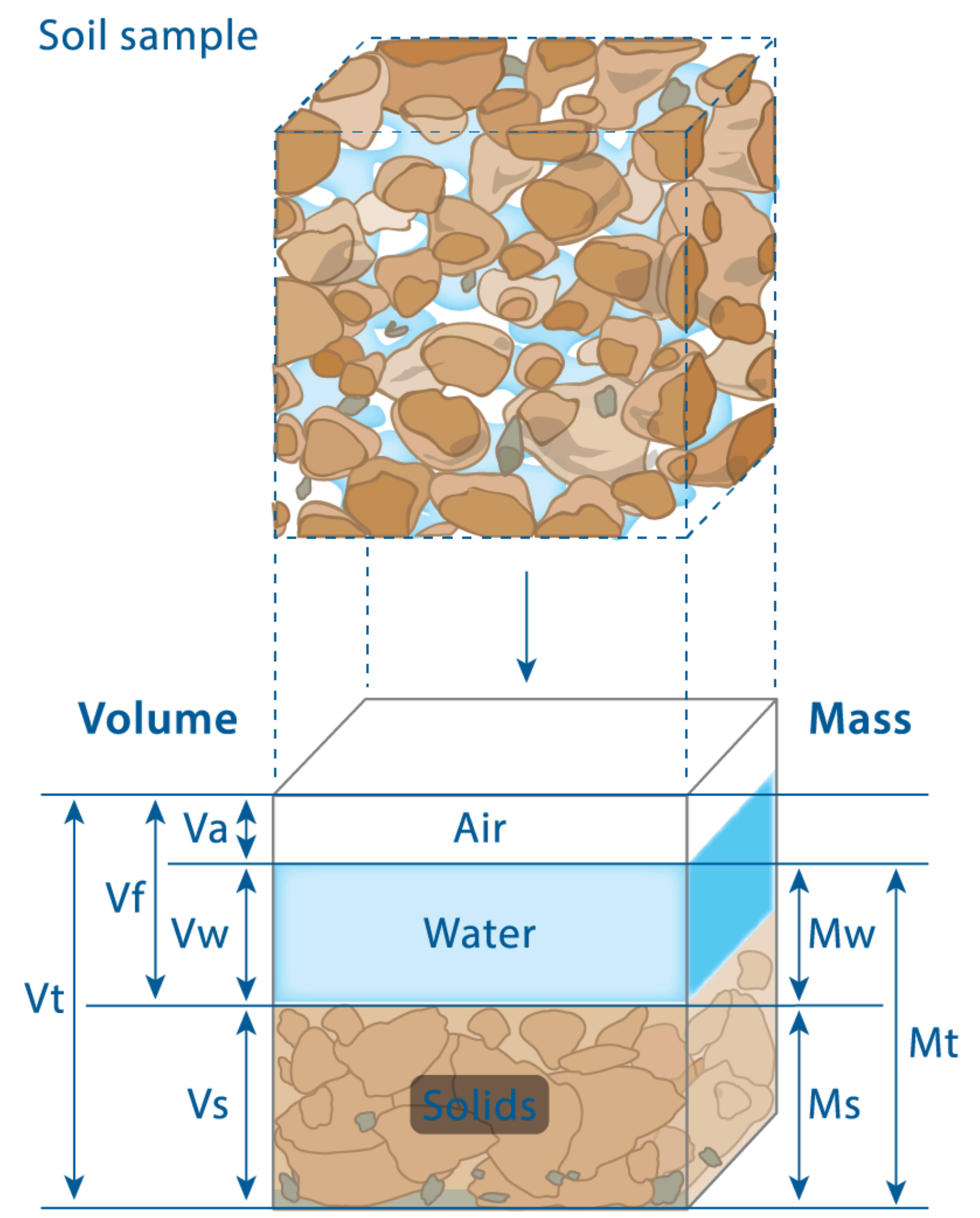

48 | Basic Relationshipfant the Soil Phases Under Equilibrium Conditions 
Figure 2.1a. Diagram of constituents of a sample of unsaturated porous media on volumetric and mass basis. Notice that the air and water phases are interchangeable on short time scales (down to seconds), while the other constituents are largely static over timescales of months and years.

\section{I.2 Volume and Mass Relationships}

We can now define the dry bulk density of the medium, which is the dry mass per unit volume of the medium, is denoted $\rho_{\mathrm{vb}}$.

$$
\begin{aligned}
\rho_{\mathrm{vb}} & =\frac{\text { mass in volume } \mathrm{V}}{\text { volume } \mathrm{V}} \\
\text { (1) } & =\frac{\mathrm{M}_{\mathrm{vs}}}{\mathrm{V}_{\mathrm{t}}}
\end{aligned}
$$

Here the subscripts " $\mathrm{V}_{\mathrm{t}}$ " denotes the total volume over which this density is calculated, "b" denotes bulk and "s" denotes the solid phase (without fluid). Typical units for density are grams per cubic centimeter, kilograms per cubic meter, or, heaven forbid, pounds per cubic foot. The upper limit on $\rho_{\mathrm{vb}}$ is the density of the pure solid mineral, often called the particle density, which we denote $\rho_{\mathrm{s}}$.

$$
\rho_{\mathrm{s}}=\frac{\text { mass of pure mineral }}{\text { volume of pure mineral }}
$$

$$
=\frac{\mathrm{M}_{\mathrm{s}}}{\mathrm{V}_{\mathrm{s}}}
$$

Notice that the value of $\rho_{\mathrm{S}}$ is independent of the value of $\mathrm{V}_{\mathrm{S}}$ (since the value of density is taken to be a material property independent of the volume selected), whereas as we shall soon see, $\rho_{\mathrm{vb}}$ is not quite as cooperative. The solid phase density is often denoted by the specific gravity of the material, denoted by s, which is simply the ratio of the solid density to that of water $\rho_{\mathrm{w}}$ :

(3) $\mathrm{s}=\rho_{\mathrm{s}} / \rho_{\mathrm{w}}$

Typical values of s are 2.65 for quartz (often used as the default value in lieu of further information), 2.54 for feldspar, 2.72 for calcite and 5.0 for pyrite.

Complementing these definitions, is that of porosity, or void fraction, which we denote by $\mathrm{n}_{\mathrm{v}}$. 


$$
\begin{aligned}
\mathrm{n}_{\mathrm{v}} & =\frac{\text { sample volume }- \text { volume of pure material }}{\text { sample volume }} \\
& =\frac{\mathrm{V}_{\mathrm{t}}-\mathrm{V}_{\mathrm{s}}}{\mathrm{V}_{\mathrm{t}}} \\
& =\frac{\text { void volume }}{\text { sample volume }} \\
& =1-\frac{\mathrm{V}_{\mathrm{s}}}{\mathrm{V}_{\mathrm{t}}} \\
& =1-\frac{\rho_{\mathrm{vb}}}{\rho_{\mathrm{s}}}
\end{aligned}
$$

$\mathrm{n}_{\mathrm{v}}$ indicates the fraction of the volume $\mathrm{V}$ which is not filled by solids. The bulk density of a medium, $\rho_{\mathrm{vb}}$, depends primarily on two characteristics of the medium: its particle size distribution and the medium's structure. Figure 2.1 illustrates that the size of particles alone does not dictate density: A set of media which are made up of single sizes of particles (and which are packed in the same arrangement) will have identical densities. Hence, the particle size distribution, and to a lesser degree the packing, strongly affect $\rho_{\mathrm{vb}}$. The material structure of the medium is also significant in determining $\rho_{\mathrm{vb}}$, particularly in the case of clays and very sharply angular aggregates.

So far so good; but what about this annoying V subscript? Can't we just forget about the sample volume? Not really, and it is worthwhile seeing why. Let's just run a quick "experiment" to make this clear. Imagine that you are in the middle of a large field with a tool which measures $\rho_{\mathrm{vb}}$ for any prescribed V. Start with the instrument set with V smaller than any individual grain in the soil (Figure 2.2). You will be sampling the density of either pure solid, pure void, or maybe some arbitrary mixture of the two. Now, holding the instrument steady, enlarge V continuously, all the while recording the values of $\rho_{\mathrm{vb}}$. Figure 2.2 shows two possible realizations of such an experiment. 
(a)
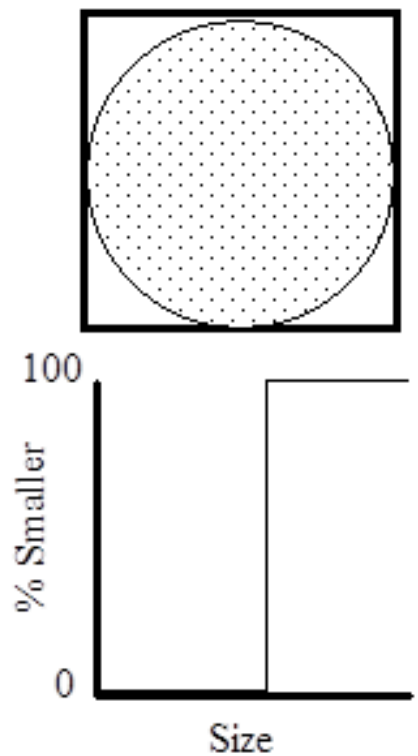

(b)
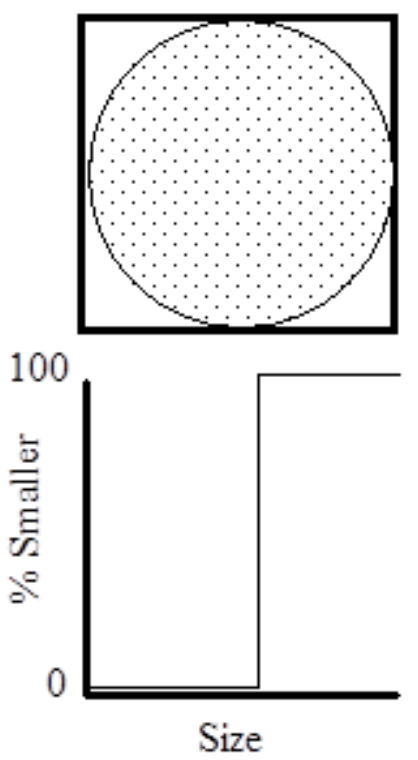
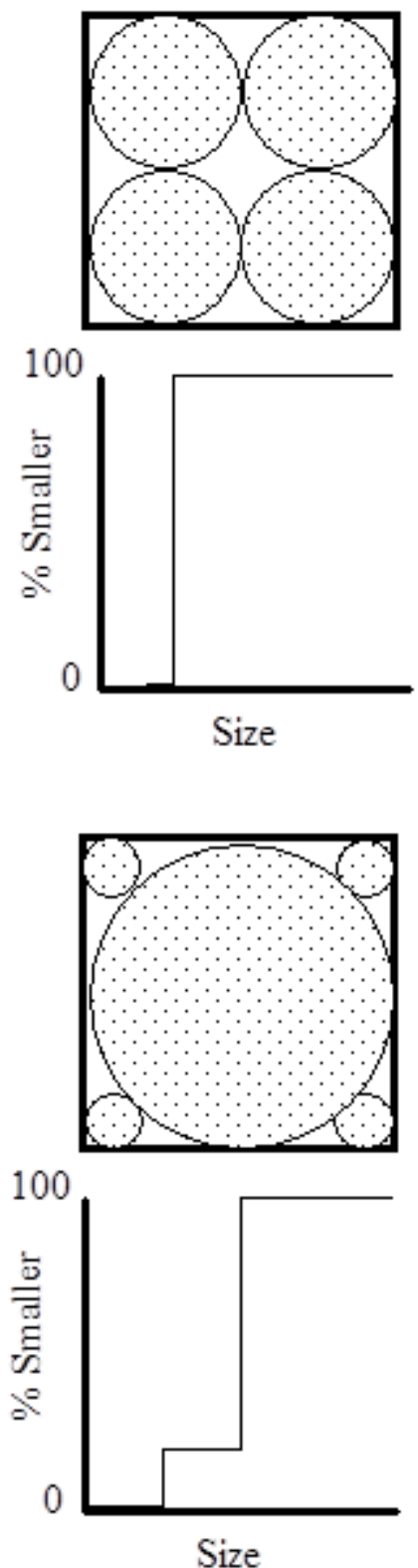
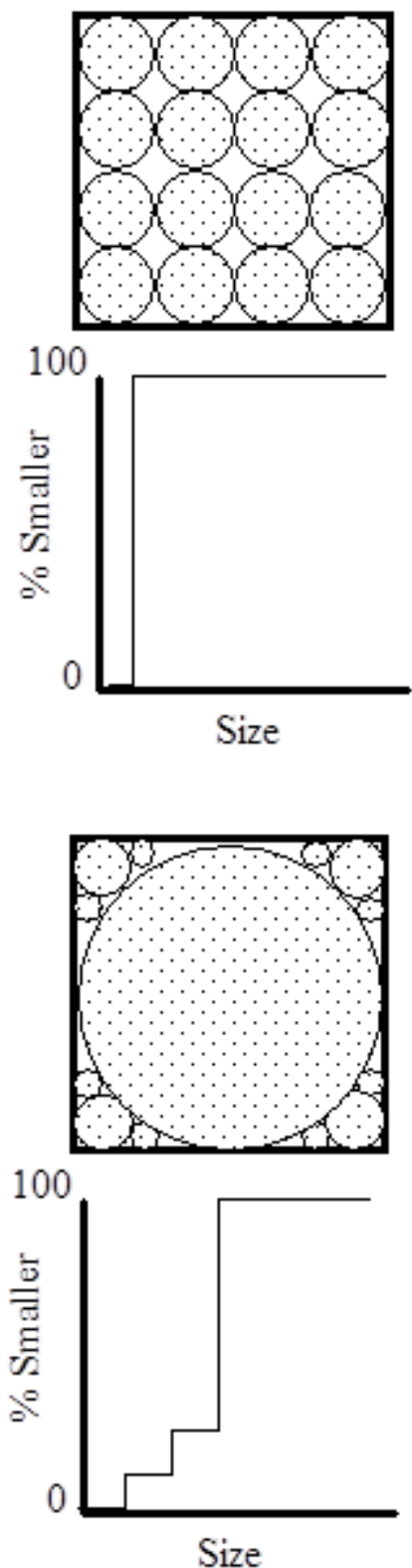

"Well Sorted"

"Poorly Sorted"

Figure 2.1 Dependence of bulk density on particle size distribution : (a) uniform particle size distribution gives uniform density for a given geometry of particle; (b) increasing the range of particle sizes gives rise to greater bulk density. Note that "well sorted" means that the particles fall within a narrow range of sizes, which is the opposite of "well graded," which refers to media containing a wide range of particle sizes (or "grades"). 


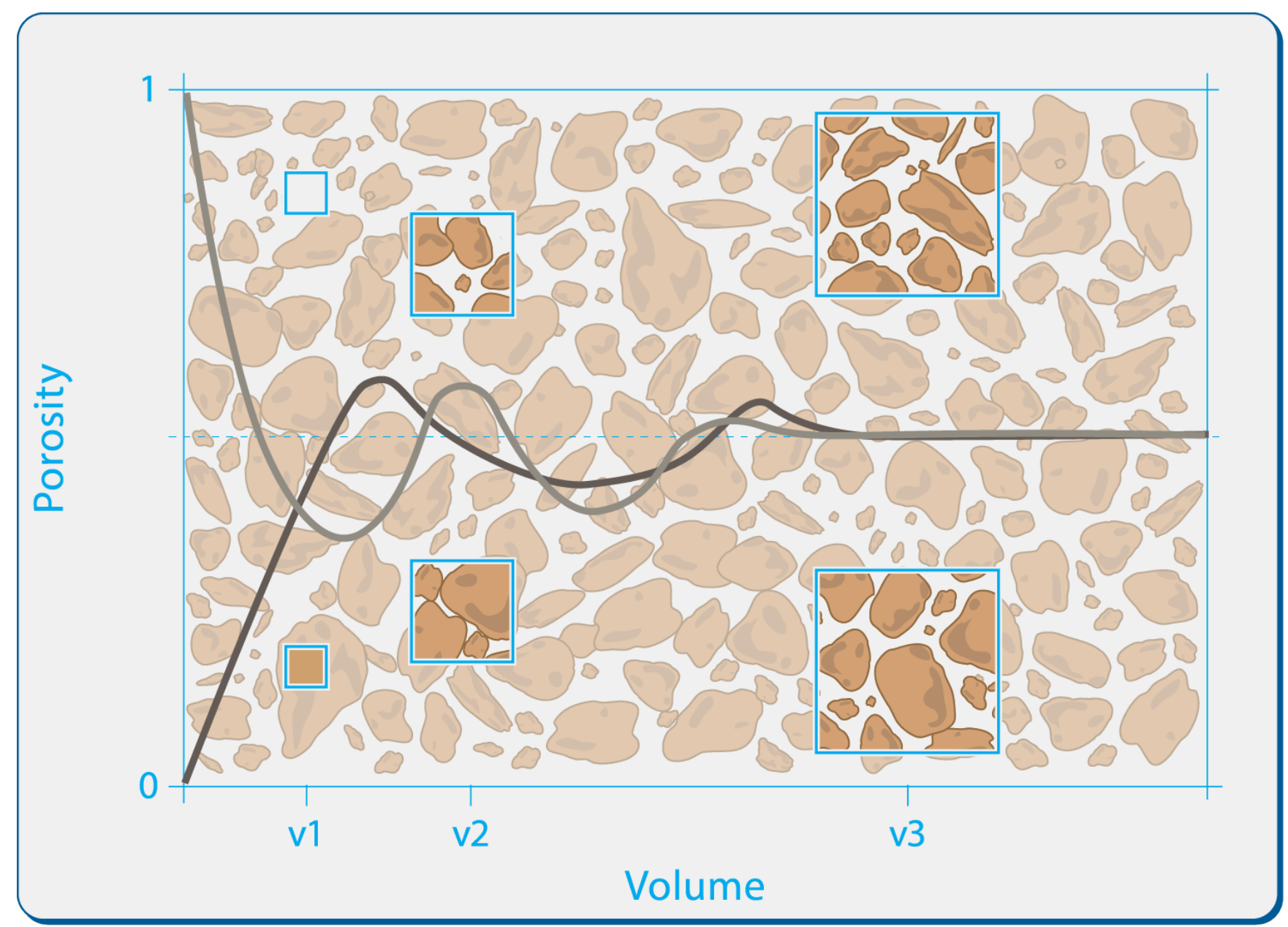

Figure 2.2 Bulk density as a function of control volume size. The Representative Elementary Volume (REV) for porosity is attained at the scale identified as v3. Note that the REV is not unique for any given media, but rather is a function of the parameter which is being characterized. Thus, since permeability depends on the largest connected apertures, which might be very widely spaced, the REV for permeability might be much larger than that found for porosity.

Although the measured density becomes "better behaved" as the control volume enlarges from the grain scale, there is no scale over which density is entirely constant. As the control volume increases, ever larger structural features of the setting are encompassed with differing properties, and thus the measured bulk density changes. The existence of such multi scale heterogeneity is widely accepted, and Dagan (1986) provides a very nice treatise on the topic. Keep this in mind: the value of all physical parameters in the vadose zone are a function of both position and sample volume.

We have two choices then: 1) throw up our arms, and figure that natural systems are hopelessly complex, or, 2) make some reasonable simplifying assumptions. For the sake of progress, we will opt for the latter option (however tempting the former). We will assume that there is some volume, much larger than the grains of the porous medium, yet smaller than the distance between dissimilar regions, which provides a representative sample of our porous medium. This volume is referred to as the Representative Elementary Volume (REV) for the system (Bear, 1972). Each extensive property of the medium is defined at a point by its value calculated taking $\mathrm{V}$ to be one REV about that point. We do get around to a more complete analysis of this problem in chapter 6 where we explicitly address scale dependent variability.

With that settled, we can proceed to complete our laundry list of definitions of physical properties of porous media (dropping the $\mathrm{v}$ subscripts since this is now implicitly understood):

Total Bulk Density : $\rho_{t}$ (solid and liquid phase) 
(5) $\rho_{\mathrm{t}}=\frac{\mathrm{M}_{\mathrm{t}}}{\mathrm{V}_{\mathrm{t}}}$

where $\mathrm{M}_{\mathrm{t}}$ is the total mass (solids and liquids).

Void Ratio: e (ratio of pore volume to solid volume)

(6)

$$
\mathrm{e}=\frac{\mathrm{V}_{\mathrm{a}}+\mathrm{V}_{\mathrm{w}}}{\mathrm{V}_{\mathrm{s}}}
$$

where $\mathrm{V}_{\mathrm{a}}$ is the air volume. Note that using our earlier definitions, we may relate the void ratio to the porosity:

$$
\mathrm{e}=\frac{\mathrm{V}_{\mathrm{a}}+\mathrm{V}_{\mathrm{w}}+\mathrm{V}_{\mathrm{s}}-\mathrm{V}_{\mathrm{s}}}{\mathrm{V}_{\mathrm{s}}}
$$

$$
=\frac{\mathrm{V}_{\mathrm{t}}-\mathrm{V}_{\mathrm{s}}}{\mathrm{V}_{\mathrm{s}}}
$$

$$
=\frac{1-\mathrm{V}_{\mathrm{s}} / \mathrm{V}_{\mathrm{t}}}{\mathrm{V}_{\mathrm{s}} / \mathrm{V}_{\mathrm{t}}}
$$

(7) $=\frac{\mathrm{n}}{1-\mathrm{n}}$

Table 2.1: Range of values found in bulk density and associated properties illustrated by particular cases. Particle density is taken as $2650 \mathrm{~kg} \mathrm{~m}^{-3}$.

\begin{tabular}{llll}
\hline \multirow{2}{*}{ Description } & Bulk Density, $\mathrm{r}_{\mathrm{b}}$ & Porosity, $\mathrm{n}$ & Void Ratio, $\mathrm{e}$ \\
& $\mathrm{kg} \mathrm{m}{ }^{-3}$ & & \\
Surface of wet clay soil & 1120 & 0.58 & 1.37 \\
Surface of loam soil & 1280 & 0.52 & 1.07 \\
Spheres (uniform size) open packing & 1390 & 0.48 & 0.91 \\
Subsoil of sandy texture & 1610 & 0.39 & 0.65 \\
Compacted sandy loam soil & 1900 & 0.28 & 0.39 \\
Spheres (uniform size) close packing & 1960 & 0.26 & 0.35 \\
Sandstone & 2120 & 0.20 & 0.25 \\
\multicolumn{2}{c}{ (Source: Marshall and Holmes, 1988; with permission of Cambridge University Press) } \\
\hline
\end{tabular}




\section{I.3 Liquid Content}

Central to most investigations of vadose zone processes is the quantification of liquid content (typically water, often referred to as the moisture content). This is expressed in either a mass or volumetric basis, as shown in $\underline{8}$ and $\underline{9}$, with the volumetric being more commonly employed, either as a water content or degree of saturation.

a. Mass basis (a.k.a., gravametric)

$$
\mathrm{w}=\frac{\mathrm{M}_{\mathrm{w}}}{\mathrm{M}_{\mathrm{s}}}=\frac{\mathrm{r}_{\mathrm{w}} \mathrm{V}_{\mathrm{w}}}{\mathrm{r}_{\mathrm{s}} \mathrm{V}_{\mathrm{s}}}=\frac{\mathrm{r}_{\mathrm{w}} \mathrm{V}_{\mathrm{w}}}{\mathrm{r}_{\mathrm{b}} \mathrm{V}_{\mathrm{b}}}
$$

b. Volume basis. $\theta$ is called the volumetric liquid content and $\mathrm{S}$ the degree of saturation:

$$
\begin{aligned}
& \theta=\frac{\mathrm{V}_{\mathrm{w}}}{\mathrm{V}_{\mathrm{t}}}=\mathrm{w} \frac{\rho_{\mathrm{b}}}{\rho_{\mathrm{w}}} \\
& \mathrm{S}=\frac{\theta-\theta_{0}}{\theta_{\mathrm{s}}-\theta_{0}}
\end{aligned}
$$

where:

$$
\begin{aligned}
& \theta_{\mathrm{O}}=\text { "residual" or "irreducible" liquid content. } \\
& \theta_{\mathrm{S}}=\text { saturated liquid content. }
\end{aligned}
$$

Equivalent Depth of Soil Water (Soil Water Storage): $D_{e}=\theta D$, where $D$ is the soil depth increment having water content $\theta$. $\mathrm{D}_{\mathrm{e}}$ provides a means for expressing water content in a given soil depth increment as soil water storage (Length), and for relating aboveground water dimensions (Length; as in rainfall, irrigation, or Et) to belowground dimensions (soil water content, $\mathrm{L}^{3}$ water $/ \mathrm{L}^{3}$ soil). This is very useful in water balance calculations, for example.

The typical units for moisture content are in mass per mass (for gravimetric water content, e.g., g/g) or volume per volume (for volumetric water content, e.g., $\mathrm{cm}^{3} / \mathrm{cm}^{3}$ ). Watch out though, because you will see water content in other units akin to the depth of storage; how about inches of water per foot of depth? Typically the units will indicate whether the values are on a volume or mass basis.

We need to take some time to iron out a few necessary fussy points regarding $\theta_{\mathrm{S}}$ and $\theta_{\mathrm{o}}$. Lets start with the easy one: what could be more straightforward than the concept of saturation? Just fill all the pores with water, right? Well, although this is simple in principle, it is rarely achieved in the vadose zone. Due to the complexity of the pore space, it is common for some pores to remain gas filled even if there is ponded water on the surface due. This is due to trapping in dead end pores and pores that become completely surrounded by water filled pores. For this reason, several terms have been proposed to describe the state of natural soil being as wet as it will get under normal conditions. This is referred to as a "satuated", "satiated", or "field saturated" condition. This can be used in the definition of degree of saturation if clearly specified. Typically, the field saturated conditions render $90 \%$ of the pore space filled with fluid (Bower, 1966).

Now, for this business of residual moisture content. Unless soil is heated to over $700{ }^{\circ} \mathrm{C}$, water held in hydrogen bonds will remain in place (Nagata et al., 1974, Figure 2.XX). This is clearly an extreme which will not be reached in typical natural settings, but the point is that the residual moisture content a particular medium will achieve is a function of the drying process. There are three drying processes which are widely considered in the definition of $\theta_{0}$ : gravity drainage, plant uptake, and atmospheric surface drying. The residual moisture content achieved under gravity drainage is referred to as "field capacity," $\theta_{\mathrm{fc}}$, which is defined as the condition which arises after a soil has been allowed to drain to a stable condition under gravity driven conditions (assuming an infinitely deep water table, for a detailed discussion, see Assouline, S., and D. Or, 2014, The concept of field capacity revisited: Defining intrinsic static and dynamic criteria for soil internal drainage dynamics, Water Resour. Res., 50, 4787-4802, doi:10.1002/2014WR015475). From an operational perspective, this is often assumed to be the conditions which occurs when the soil water is at a pressure of $-1 / 3$ bar, although clearly such a threshold is very much a function of the soil texture, ranging from $-1 / 30$ bar for coarse sands 
to -1 bar for clayey soils (Assouline et al., 2014). With more time, water eventually stops draining when the films of water break between soil particles leaving isolated pockets of water.

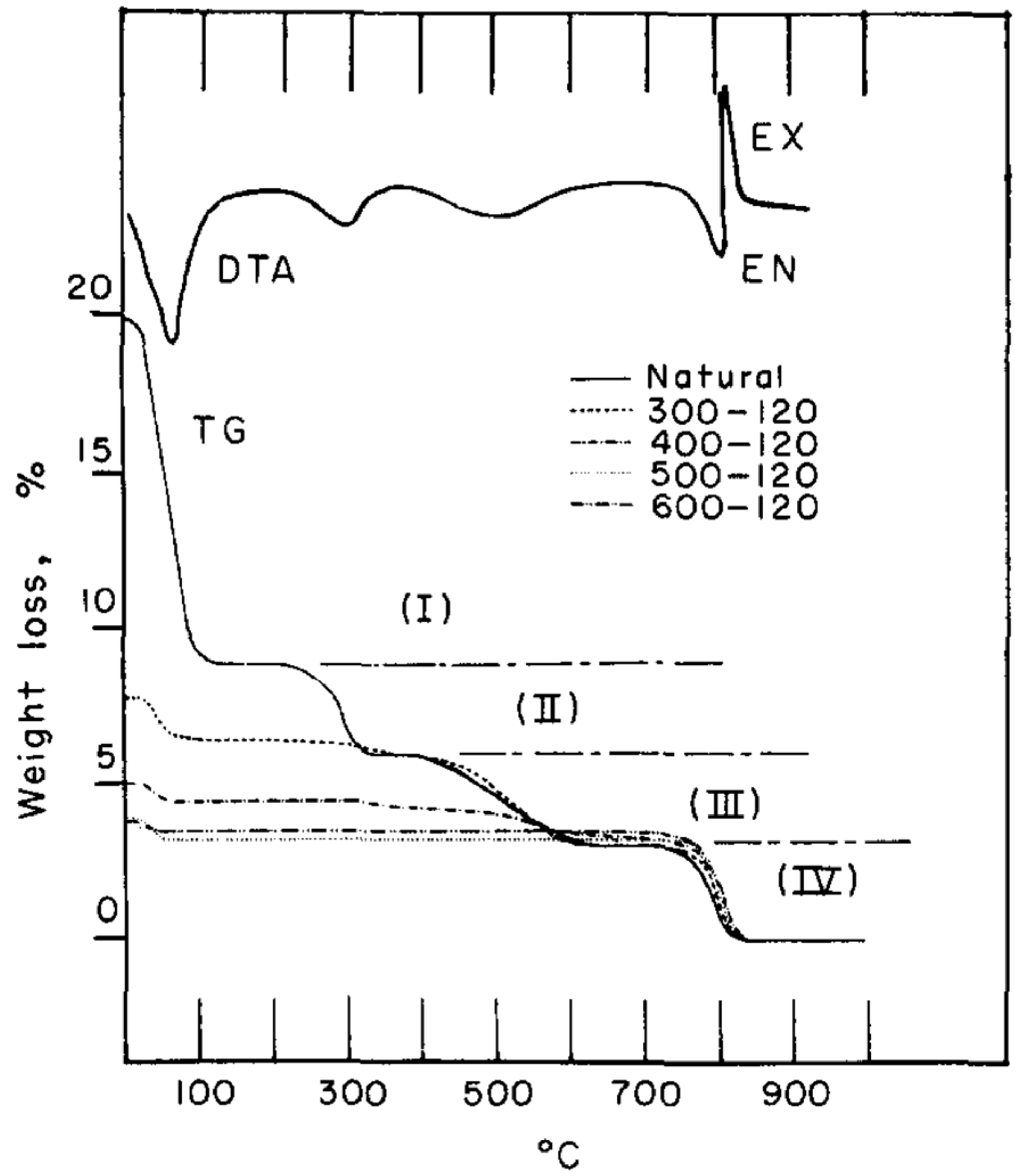

Figure 2.XX Experimental mass versus drying temperature for sepiolite clay, illustrating that 3\% of the mass is made up of bound water beyond $700{ }^{\circ} \mathrm{C}$ (with permission, Nagata et al., 1974). [Nagata, Hroshi, Susumu Shimoda, and Toshio Sudo. "On dehydration of bound water of sepiolite." Clays \& Clay Minerals. 1974.] 
The second commonly taken value for residual moisture content is referred to as the "permanent wilting point," $\theta_{\text {pwp. }}$ You might have guessed that this is the moisture content which is observed when plants can no longer retrieve water from soil (always lower than $\theta_{\mathrm{fc}}$ ). Since plants vary greatly in their ability to extract water, this is operationally defined as the moisture content when the water in the medium is a pressure of -15 bar. Since water no longer flows along continuous streams at moisture contents less than $\theta_{\mathrm{fc}}$ this measurement relies on vapor transport of water between pores, and so is necessarily slow in achieving equilibrium. The final method of drying is due to direct evaporation to the atmosphere. This process can bring the upper soil surface to pressures of less than -100 Bar. In summary, so long as you define the conditions over which a flow process will occur, there will be a real and measurable residual moisture content, but if you are unsure of the context where the parameter will be used, it is more reasonable to provide a range of possible values, or provide the extreme values just discussed. In fact, many soil scientists believe that $\theta_{\mathrm{o}}$ should be simply taken as 0 , regardless of the problem under study, as this is the only physically defensible value for arbitrary conditions. Table 2.1a and Figure 2.X provide some typical ranges for various parameters.

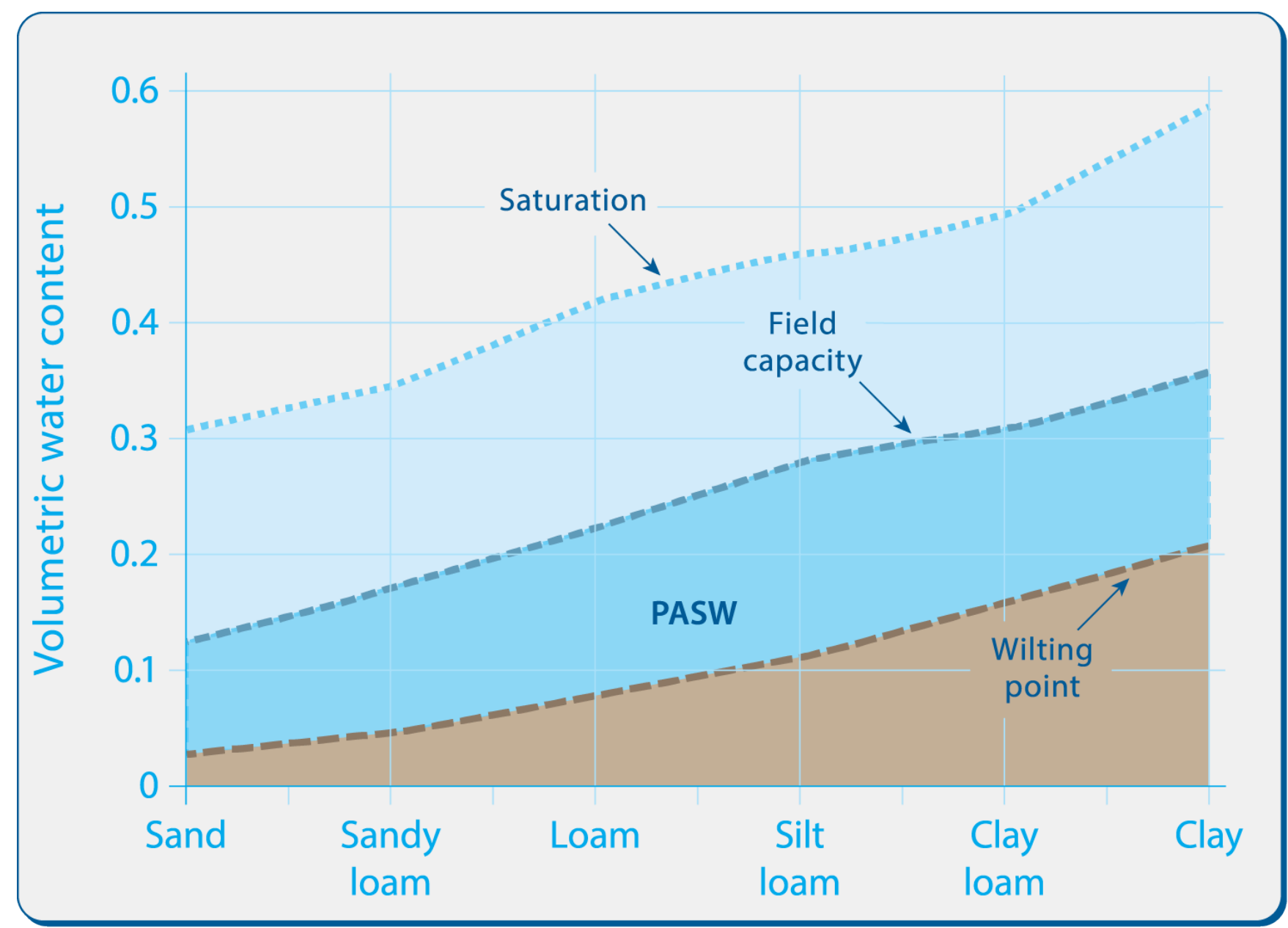

Figure 2.X Typical values of saturation, field capacity and permanent wilting point for a range of soil textures. The Plant Available Soil Water (PASW) indicates the volume fraction of the soil which might retain water following infiltration and drainage (i.e., field capacity), and be possible for the plant to extract (i.e., up to the PWP).

A primary practical importance of the field capacity and wilting point concepts is for determination of a range of plantavailable soil water (PASW; Fig.2.X). Soil water storage available for plant use is generally calculated as being between these two limits $\left(\theta_{\mathrm{FC}}-\theta_{\mathrm{WP}}\right)$, as water contents higher than $\theta_{\mathrm{FC}}$ are generally not sustained for long times. It takes only one to a few days for the attainment of field capacity after wetting if conditions permit. Plant available soil water storage is an important factor in the determination of irrigation amounts for a cropped field or other soil-plant system. For 
practical purposes irrigation amounts in excess of a soil's field capacity are lost to deep percolation and thus should be avoided.

A useful 'rule of thumb' in absence of other information is to estimate $\theta_{\mathrm{FC}}$ as $\theta_{\mathrm{S}} / 2$, and $\theta_{\mathrm{WP}}$ as $\theta_{\mathrm{FC}} / 2$; i.e., a soil exhibiting this property will have lost one-half it's saturated water content at field capacity, and another one-half the remaining water by wilting point.

Knowledge of soil water content is also important to geotechnical applications where the attainment of high bulk density by compaction minimizes future settlement and increases the soil strength. For each level of compactive effort there is an "optimal" water content at which maximum compaction is attained (this occurs at an approximate degree of saturation of 0.8). Hence, knowledge of the soil water content can assist a geotechnical engineer in determining how much water should be applied to a layer of earth fill to achieve optimal compaction. Such information is also useful for farmers who want to avoid excessive compaction of their fields by farm implements (see Hillel 1980, Chapter 14 for details).

Table 2.1a Typical values of physical properties for several soils.

\begin{tabular}{llllll}
\hline $\begin{array}{l}\text { Soil } \\
\text { Texture }\end{array}$ & $\begin{array}{l}\dagger \text { Porosity, } \\
\mathrm{n} \\
\left(\mathrm{cm}^{3} / \mathrm{cm}^{3}\right)\end{array}$ & $\begin{array}{l}\text { * Saturated Hydraulic Conductivity, } \\
\mathrm{K} \text { s } / \mathrm{sec})\end{array}$ & $\begin{array}{l}\dagger \text { Specific Density, } \\
\mathrm{s}\end{array}$ & $\begin{array}{l}\dagger \text { Field } \\
\text { Capacity } \\
\left(\mathrm{cm}^{3} / \mathrm{cm}^{3}\right)\end{array}$ & $\begin{array}{l}\begin{array}{l}\dagger \text { Permanent wilting } \\
\text { point } \\
\left(\mathrm{cm}^{3} / \mathrm{cm}^{3}\right)\end{array} \\
\text { Sandy }\end{array}$ \\
\hline 0.38 & $10^{-3}-10^{-1}$ & 1.65 & 0.09 & 0.04 \\
Sandy & 0.43 & $10^{-3}-10^{-2}$ & 1.50 & 0.14 & 0.06 \\
Loam & 0.47 & $10^{-3}-10^{-2}$ & 1.40 & 0.22 & 0.10 \\
Loam & 0.49 & $10^{-4}-10^{-3}$ & 1.35 & 0.27 & 0.13 \\
Clay Loam & 0.4 & 1.30 & 0.31 & 0.15 \\
Silty Clay & 0.51 & $10^{-7}-10^{-4}$ & 1.25 & 0.35 & 0.17
\end{tabular}

$\dagger$ †dapted from Israelson and Hanson, Irrigation Principles and Practices, Wiley and Sons, 1962.

*May vary beyond the stated range.

\section{Example 1-1): Volume-Mass Relationships}

\section{Problem Statement:}

The diameter of a cylindrical soil sample is $0.1 \mathrm{~m}$ and its height is $0.3 \mathrm{~m}$. (1) What was the gravimetric water content (W) if the wet mass was $4.12 \mathrm{~kg}$ and the dry mass was $3.18 \mathrm{~kg}$ ? (2) What was the soil bulk density, $\rho \mathrm{b}$ ? (3) What was the volumetric water content, $\theta_{\mathrm{V}}$ ?; and (4) What was the porosity (n), the void ratio (e), and the degree of saturation $(\mathrm{S})$ ?

\section{Solution:}

\section{(1) Gravimetric water content W}

$$
W=\frac{m_{w}}{m_{s}}=\frac{4.12-3.18}{3.18}=0.295\left[\frac{\mathrm{kg}}{\mathrm{kg}}\right]
$$

The gravimetric water content $\mathrm{W}$ is the mass of water relative to the mass of oven-dry soil. 
(2) Soil bulk density $\rho_{b}$

$$
\rho_{b}=\frac{m_{s}}{V_{t}}=\frac{3.18}{2.35 \cdot 10^{-3}}=1353\left[\frac{\mathrm{kg}}{\mathrm{m}^{3}}\right]
$$

The dry soil bulk density $\mathrm{rb}_{\mathrm{b}}$ is the ratio of the mass of oven dry soil to its bulk volume. The soil bulk volume is equal to the volume of the cylinder given as:

$$
V=A \cdot h=r^{2} \pi h=0.05^{2} \cdot \pi \cdot 0.3=2.35 \cdot 10^{-3}\left[\mathrm{~m}^{3}\right]
$$

\section{(3) Volumetric water content $\theta_{\mathbf{v}}$}

$$
\theta_{v}=\theta_{m} \frac{\rho_{b}}{\rho_{w}}=0.295 * \frac{1353}{1000}=0.399 \cong 0.4\left[\frac{\mathrm{m}^{3}}{\mathrm{~m}^{3}}\right]
$$

The volumetric water content $\theta_{\mathrm{v}}$ is the volume of water relative to the soil's total volume and may be expressed as gravimetric water content $\mathrm{W}$ times the ratio of bulk density $\rho_{\mathrm{b}}$ to water density $\rho_{\mathrm{W}}=1000 \mathrm{~kg} / \mathrm{m}^{3}$

\section{(4) Porosity $n$, void ratio e, and degree of saturation $S$}

$$
\begin{aligned}
& n=1-\frac{\rho_{b}}{\rho_{s}}=1-\frac{1353}{2650}=0.49 \\
& e=\frac{n}{1-n}=\frac{0.49}{1-0.49}=0.96 \\
& S=\frac{\theta_{v}}{n}=\frac{0.399}{0.489}=0.81=81 \%
\end{aligned}
$$

The porosity $\mathrm{n}$ is the ratio of pore volume to the total volume and may be expressed as 1 minus the ratio of bulk density to particle density. The particle density is assumed to be $2650 \mathrm{~kg} / \mathrm{m}^{3}$

\subsection{The Energy State of Soil Water}

Imagine a column of saturated soil that is allowed to drain: water will flow downward draining the upper soil, but why? What drives this motion? Water content alone is not sufficient to characterize the water status of soils. An additional critical attribute is the energy state of soil water. Soil water contains different forms and quantities of energy, as does any body of matter. Some familiar forms of energy are kinetic, potential, thermal, electrostatic, and chemical. The last aspect of characterizing the state of water in porous media requires the introduction of the energetic status of the liquid in the porous medium. While all of these quantities represent energy, we will discover that most liquid movement in porous media results from gradient in only the mechanical potentials (as opposed to electrostatic, chemical or thermal potential). In principle this is as simple as water flowing downhill in a stream: water is flowing from higher elevation (thus higher gravitational potential) to lower elevation under constant pressure (the gauge pressure of water at the airwater surface is zero), thus moving from areas of high gravitational potential to areas of lower potential with constant 
pressure potential. But given the differing roles of the different potentials, we must be deliberate in identifying these terms and their role in soil-water processes.

Let's start with the potential that you experience every time you move: Kinetic energy $\left(E_{k}\right)$ is acquired by virtue of motion and is proportional to the velocity squared $\left.\left.\left[\mathrm{E}<\mathrm{sub}>\mathrm{k}</ \mathrm{sub}>=1 / 2 \mathrm{mv}^{2}\right]_{\mathrm{k}=1 / 2} \operatorname{mv}^{\wedge}\{2\}\right]\right\}^{\prime \prime}$ title="Rendered by QuickLaTeX.com" height="20" width="256" style="vertical-align: -5px;">. Illustrating how different the primary processes are in natural porous media compared to daily life, because the movement of water in soils is generally quite slow (e.g., of the order of meters per year), its kinetic energy is almost always negligible. We call these slow-moving flows "creeping flow" to indicate that the forces of acceleration associated with changing kinetic energy are much smaller than the forces generated by viscosity and gradients in pressure. These flows are associated with Reynolds numbers less than 1. For every general rule there are special case exceptions, and in soils this can here occur during extreme events: flow along macropores can have velocities with kinetic energy that are appreciable. We will discuss this in detail in the context of dynamics of fluid in porous media, but for the moment we simply posit that potential energy is the primary driver of flow outside of these rather extreme cases.

With respect to potential energy we need to discriminate between terms which are mechanical from those which are electrostatic, chemical or thermal. The point here is that although hot water has more energy than cold water, this energetic difference does not cause the hot water to flow toward the cold water if they are in physical contact (think of a lake in summer with warm water on top and cool water below). Similarly, a pure water has higher osmotic potential than does a saline water, but when you have salty water below fresh water you will not see the salty water flow in bulk toward the fresh. So how can these chemical or thermal potentials give rise to movement of water? To translate chemical potential into mechanical potential requires there be an interface which selectively constrains the motion of selected components of the system (i.e., an charge barrier, permeable membrane, or gas-liquid interfaces). Some examples are helpful here. Consider a semi-permeable membranes that allows water to pass but excluding solutes. This can result in water delivery to the side with higher solute concentration, resulting in bulk water movement. At a clay surface with electrostatic bound cations population, when exposed to water, these cations will hydrate, pulling in bulk water into the clay. Systems with such selective regions can develop huge mechanical pressures (e.g., turgor in cells or the pressure drop across reverse-osmosis desalinization systems). Such interfaces occur in the natural world at roots, cell walls, and air-water interfaces (Figure 2.X123). While very important to life, these processes are somewhat esoteric in the case of the movement of bulk water in soils. 
COMPONENTS Of WATER POTENTIAL

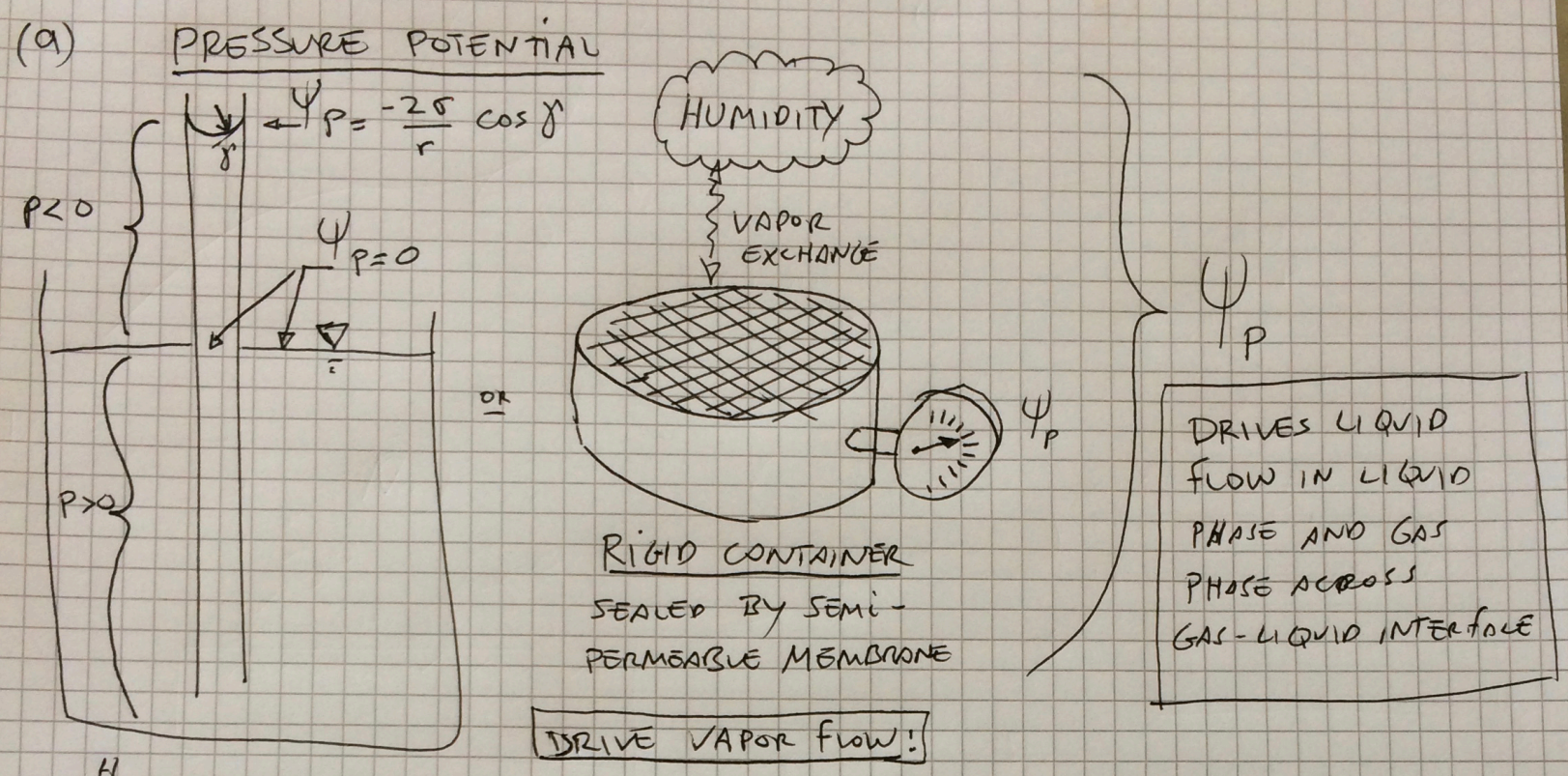

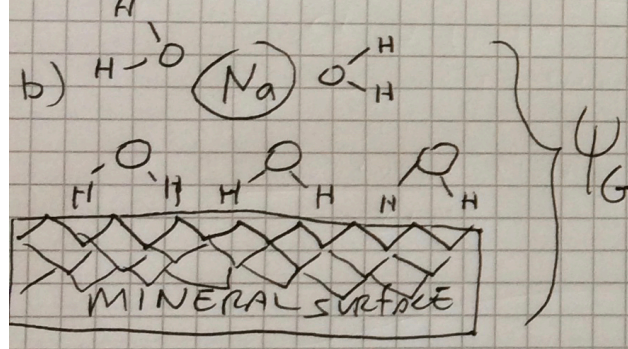

GOUY/STERN LAYER POTENTIAL
(C) OSMOTIC POTENTIAL

- Chemical potential M \& Solution concentrAtion C

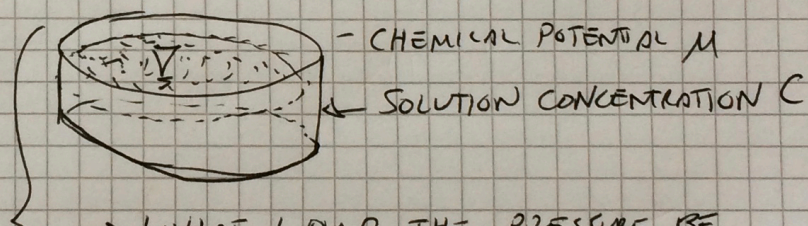

To $\{\rightarrow$ WHAT WOLD THE PRESSURE BE IN A RIGID CHAMBER WITH A BUSTERit from pure wares?

(CONVENION Of OSMOTIC POTENTIMC TO préssust potential)

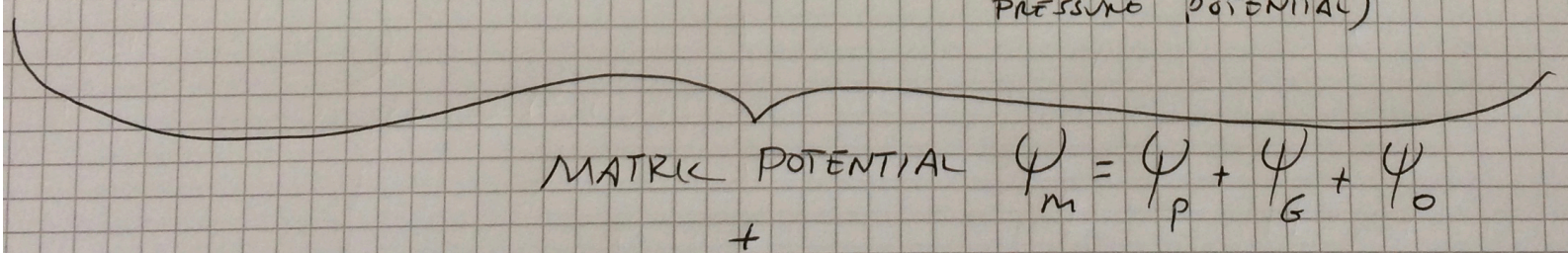

$+$

ELEcTION POTENTIAL $\Psi_{z}=z$

TOTAL POTENTIAL $\psi_{T}=\psi_{M}+\psi_{Z}$

BUT

TOtal mechanical potential $=\psi_{\text {mp }}=\psi_{p}+\psi_{z}$ DRIVING LUQUDDFOW

60 | Basic Relationships and the Soil Phases Under Equilibrium Conditions 
Figure 2.X123. The total water potential arises due to multiple mechanisms, including: (a) Pressure potential which can result from surface tension, where curved air-water interfaces give rise to pressure following the La Place equation, or if pure water is held in a rigid vessel with a semi-permeable membrane, the pressure can become very negative if the vessel is in a low-humidity environment; (b) Gouy-layer potential arising from water interactions at mineral surfaces, which generally includes contributions from dipole-dipole attraction of water to the mineral, electrostatic attraction of the positively charged portion of water to the negatively charged mineral surface, electrostatic attraction of the negatively charged portion of the water molecule to the cations bound to the mineral surface, and osmotic potential acting on the water as it dilutes the ions attracted to the minerals; and (c) the osmotic potential that can attract water vapor at saline water surfaces. (a)-(c) taken together are known as the matric potential (to the degree this term has a universally agreed upon definition), and may be expressed in units of pressure or head. When the gravitational potential is added this is known as the total water potential.

To predict fluid movement in the vadose zone we are generally concerned with keeping track of the total mechanical potential (TMP which we will indicate with the letter $\mathrm{H}$ ) of the fluid phase. The total mechanical potential is made up of the sum of the gravitational potential (due to elevation) and the pressure potential (the force per unit area exerted by the fluid on its boundary - Figure 2.X124). As we introduced above, fluid motion results from a gradient in the TMP. So we are concerned with gradients in the TMP. Still we need to define reference (or datum) in order to provide a frame of reference from which the TMP might vary. For pressure we typically define the atmospheric pressure as the datum (by assigning it a value of 0 ). This is the pressure of the water table. In selecting a datum for gravity, we choose an elevation which is convenient for each problem. In the lab this may be the elevation of the surface a pool of water, and in the field it is often taken to be the soil surface. We emphasize that the specific choice of datum is immaterial: fluid moves relative to local changes (gradients) in potential, so any constant added or subtracted from the total potential falls out when we take the derivative required to compute the flux.

So how are we going to add elevation to pressure to compute the TMP? We need to employ common units, and the most natural approach is to use the units of elevation: length. We typically denote the elevation with the variable $\mathrm{z}$ which becomes more positive in the upward direction. The pressure potential, however, most naturally has units of force per area(see Table 2.2), and can be calculated within a hydrostatic liquid by multiplying the distance to the water table (say, h) by the liquid density and the acceleration of gravity: $\psi_{\mathrm{g}}=\rho g h$. Here we have the trick which makes flow calculations work out: we can express the pressure in terms of the equivalent height of a column of water which would generate this pressure. This is the pressure "head," which we denote h, and has units exactly compatible with elevation. We denote the TMP as $\mathrm{H}$ and is equal to the sum of $\mathrm{h}$ and $\mathrm{z}$.

To get a feel for how this total mechanical potential works, let's consider the simplest possible case: a tank of water at rest (Figure 2.X124). Since there is no flow in the tank, the condition which is called "hydrostatic," we can be sure that the total mechanical potential is the same everywhere (remember water would flow towards lower potential, but we posited that the water was at rest). As mentioned, we may define our datum for pressure and elevation arbitrarily: here we chose the top of the water surface as the zero pressure (denoted with the letter $h$ when in units of head) datum and the bottom of the tank as the zero elevation datum. Clearly elevation increases with height in the tank, while pressure decreases such that the total mechanical, denoted with the letter $\mathrm{H}$ when measured in terms of head, potential is constant, consistent with the lack of flow in the tank. Clearly one may convert between units of pressure and equivalent water column height (Table 2.2). A word of caution is in order regarding the assumed density of the fluid which fills the pore space: what about cases of saline solutions, or non-aqueous phase liquids (NAPLs; hydrocarbons etc.)? We must be consistent in our units of pressure measurement, and in this case we will stick to height a column of pure water would be lifted (at $20 \mathrm{deg} \mathrm{C}$ ) regardless of the fluid under consideration.

Table 2.2 Conversions between common units of pressure. To convert the units given in the left column to that listed on the upper row, multiply by the conversion factor listed. 


\begin{tabular}{|c|c|c|c|c|c|c|}
\hline from: & kilo Pascal & bar & lb. $/ \mathrm{in}^{2}$ & cm water & ATM & mm Hg \\
\hline Kilo Pascal & 1 & 0.01 & 0.145038 & 10.1972 & 0.00986923 & 7.50062 \\
\hline bar & 100.000 & 1 & 14.5038 & $1,019.72$ & 0.986923 & 750.062 \\
\hline lb. $/ \mathrm{in}^{2}$ & 6.89476 & 0.0689747 & 1 & 70.3072 & 0.068046 & 51.7149 \\
\hline cm water & 0.0980665 & 0.000980665 & 0.0142233 & 1 & 0.000967841 & 0.735559 \\
\hline ATM & 101.325 & 1.01325 & 14.6960 & $1,033.23$ & 1 & 760 \\
\hline $\mathrm{mm} \mathrm{Hg}$ & 0.133322 & 0.0013332 & 0.0193368 & 1.35951 & 0.00131579 & 1 \\
\hline
\end{tabular}

People often wonder about pressures more negative than -1 atmosphere: isn't this somehow non-physical? We know from our kitchens that boiling occurs when the vapor pressure of the water reaches the atmospheric conditions, so why doesn't the pore water boil? Don't feel bad if you came up with these questions; in contemporary literature some authors still wondered if water pressures more negative than -1 ATM were physically possible (Gray and Hassanizadeh, 1991; Miller, 1994; Gray and Hassanizadeh, 1994). However, experiments have shown that liquid water can sustain negative pressures of up to 150 bars before vaporizing at room temperature since the vapor pressure of water is actually much less than one atmosphere at this temperature (e.g., Zheng et al., 1991) supporting Miller's position. This conclusion is confirmed in trees over $10 \mathrm{~m}$ in height, where the pore pressures more negative than - 1 bar are essential to the delivery of water to their leaves. Stable water at such negative pressures only occur in very small bodies of water (e.g., xylem of plants), but at such great negative pressures water will only be found in very small regions, as is discussed below. Thus a few points are clear: (a) negative pressures less than -1 bar arise commonly in porous media due to the forces listed in Table 2.1, and (b) it is not unreasonable to consider the fluid-dynamic behavior of water at pressures more negative than -1 bar.
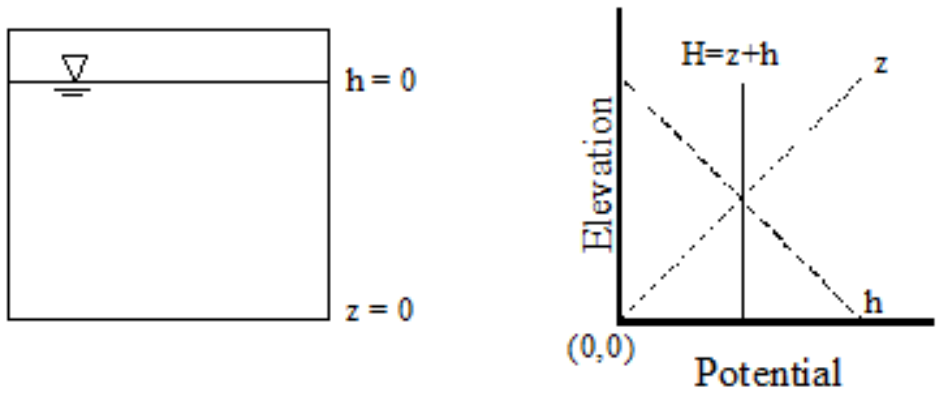

\begin{abstract}
Figure 2.X124. The terms of the total mechanical potential in a tank of water. The elevation potential in units of head (dotted line) always increases linearly with elevation. Since there is no movement in the tank, we know the total potential (solid line) is the same at all places, and therefore the pressure potential (dashed line) must increase with depth in a manner equal and opposite to the elevation, which agrees with our intuition that pressure goes up linearly with increasing depth in still water.
\end{abstract}




\section{Example 2-X: Gravitational Potential}

Figure XXX. Self-study of the elevation potential of a soil column to understand the role of selection of datum. All distance values shown are in $\mathbf{m m}$.

Case 1:

Point $\mathrm{A}$ is $100 \mathrm{~mm}$ above the datum and point $\mathrm{B}$ is $200 \mathrm{~mm}$ below the datum. Find the difference in gravitational potential: $\Psi_{\mathrm{z}}=\Psi_{\mathrm{zA}}-\Psi_{\mathrm{zB}}$ between the points.

\section{Solution:}

$\Psi_{\mathrm{zA}}=100 \mathrm{~mm}$ and $\Psi_{\mathrm{zB}}=-200 \mathrm{~mm}$, so $\Delta \Psi_{\mathrm{z}}=100-(-200)=300 \mathrm{~mm}$.

\section{Case 2:}

For the same two points A and B we now change the elevation of the reference to $100 \mathrm{~mm}$ above point A.

\section{Solution:}

$$
\Psi_{\mathrm{zA}}=-100 \mathrm{~mm} \text { and } \Psi_{\mathrm{zB}}=-400 \mathrm{~mm} \text {, so } \Delta \Psi_{\mathrm{z}}=-100-(-400)=300 \mathrm{~mm} \text {. }
$$

Notice we are not here dealing with the possible difference in pressure potential at points A and B, just their elevation potential. The key point here is that the choice of datum does not change the value of the difference in potential between two points.

\section{Total Soil Water Potential}

Definition of Total Soil Water Potential: While the Total Mechanical Potential (TMP, denoted H when measure in units of head) directly gives rise to flow, the energy state of water includes other factors, such as solute concentration and temperature. So the "Total Soil Water Potential" must include all of the quantities that would influence the amount of energy per unit quantity of soil water at equilibrium would be required to take water from some standard reference state to that of the water in the soil. Here we will take the standard reference state to be pure, free water, at the elevation of our datum. The total potential $\psi_{\mathrm{T}}$ may be expressed as the sum of the contributors to the total energy state:

(16) $\psi_{T}=\psi_{H}+\psi_{S}+\psi_{E}+\psi_{Z}+\psi_{P}$

where $\psi_{\mathrm{H}}$ is the thermal potential, $\psi_{\mathrm{S}}$ is solute or osmotic potential, $\psi_{\mathrm{E}}$ is the electrostatic potential, $\psi_{\mathrm{Z}}$ is gravitational potential, and $\psi_{\mathrm{p}}$ is pressure potential. Outside of engineered systems where electrical potentials are used to drive flow, and at the atomic scale near clay surfaces, the electrostatic potential rarely comes into 
play, so we will drop it from our accounting for the moment. For more information on this term we refer you to Revil and Jardani (2013). The difference in chemical and mechanical potentials between soil water and pure water at the same temperature is known as the soil water potential $\left(\psi_{\mathrm{w}}\right)$ :

(17) $\psi_{W}=\psi_{S}+\psi_{P}$

Note that the gravitational and thermal components $\left(\psi_{\mathrm{Z}}\right.$ and $\left.\psi_{\mathrm{H}}\right)$ are absent in this definition. Soil water potential is thus the result of inherent properties of soil water itself, and of its physical and chemical interactions with its surroundings, while total potential includes the effects of gravity (an "external" force), or its temperature. The idea is to define a soil state that can be measured, but where $\psi_{\mathrm{W}}$ for a sample of soil would not change if you walked from room to room in your laboratory, or put the sample on a high shelf.

Total soil water potential and its components may be expressed in several ways depending on what you choose for your "unit quantity of water." Potential may be expressed as (i) energy per unit of mass; (ii) energy per unit of volume; or (iii) energy per unit of weight. A summary of the resulting dimensions, common symbols and units are presented in Table 1-1. Note that energy has units of $\mathrm{ML}^{2} / \mathrm{t}^{2}$.

Table 1-1: Units, Dimensions and Common Symbols for Potential Energy of Soil Water

\begin{tabular}{|c|c|c|c|c|c|}
\hline Units & Symbol & Name & Dimensions & SI Units & cgs Units \\
\hline Energy/Mass & $\mu$ & Chemical Potential & $\mathrm{L}^{2} / \mathrm{t}^{2}$ & $\mathrm{~J} / \mathrm{kg}$ & $\mathrm{erg} / \mathrm{g}$ \\
\hline Energy/Volume & $\psi$ & Soil Water Potential, suction, or tension & $\mathrm{M} /\left(\mathrm{Lt}^{2}\right)$ & $\mathrm{N} / \mathrm{m}^{2}(\mathrm{~Pa})$ & $\mathrm{erg} / \mathrm{cm}^{3}$ \\
\hline Energy/Weight & $\mathrm{h}$ & Soil Water Head & $\mathrm{L}$ & $\mathrm{m}$ & $\mathrm{cm}$ \\
\hline
\end{tabular}

Note that only $\mu$ has actual units of potential; $\psi$ has units of pressure, and $h$ of head (L) of water. However, the above terminology is widely used in a generic sense in the soil and plant sciences (i.e., potential energy vs. units of potential). The various expressions of soil water energy status are equivalent. Specific relationships between the three potential energy expressions are derived from the relationship between volume and mass of water ( $\mathrm{M}_{\mathrm{w}}=\rho_{\mathrm{w}} \cdot \mathrm{V}_{\mathrm{w}}$ where $\rho_{\mathrm{w}}$ is the density of water), and between mass and weight $\left(\mathrm{W}_{\mathrm{w}}=\mathrm{g} \cdot \mathrm{M}_{\mathrm{w}}\right.$ where $\mathrm{g}$ is acceleration of gravity). The relationships may be summarized by:

$$
\mu=\frac{\Psi}{\rho_{w}}=\mathrm{g} \mathrm{h}
$$

\section{Example 2.X: Water Potential Conversions}

\section{Problem Statement:}

Convert a soil water head of $-0.01 \mathrm{~m}$ to $\mathrm{kPa}$ and to $\mathrm{J} / \mathrm{kg}$.

Solution:

(1) Conversion from $\mathrm{h}=-0.01 \mathrm{~m}$ to $\psi(\mathrm{kPa})$ : 
The conversion equation is $\Psi=\rho_{\mathrm{w}}$ gh where $\mathrm{g}=9.8 \mathrm{~m} / \mathrm{s}^{\mathrm{s}}$ and $\rho_{\mathrm{w}}=1000 \mathrm{~kg} / \mathrm{m}^{3}$.

Hence,

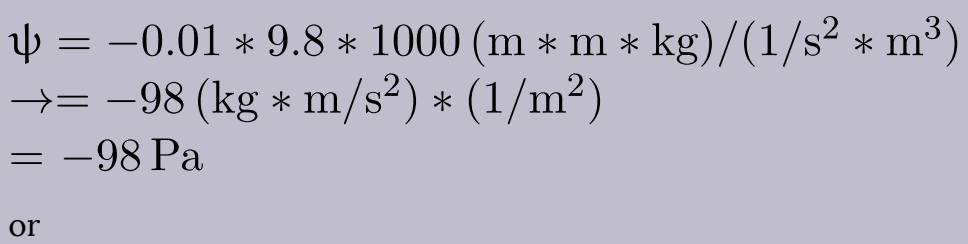

\section{$-0.098 \mathrm{kPa}$}

(note that $\mathrm{Pa}=\mathrm{N} / \mathrm{m}^{2}$, and $\mathrm{N}=\mathrm{kg} * \mathrm{~m} / \mathrm{s}^{2}$ ).

(2) Conversion from $\mathrm{h}=-0.01 \mathrm{~m}$ to $\mu(\mathrm{J} / \mathrm{kg})$ :

The conversion equation is $\mu=\mathrm{gh}$ where $\mathrm{g}=9.8 \mathrm{~m} / \mathrm{s}^{2}$, hence

$\mu=-0.01 * 9.8\left(\mathrm{~m} * \mathrm{~m} / \mathrm{s}^{2}\right) \rightarrow=-0.098\left(\mathrm{~m} * \mathrm{~m} / \mathrm{s}^{2}\right) ;$

we multiply and divide the expression by

$\mathrm{kg} \rightarrow=-0.098 \mathrm{~kg} * \mathrm{~m} * \mathrm{~m} /\left(\mathrm{kg} * \mathrm{~s}^{2}\right)$

which is

$-0.098 \mathrm{~J} / \mathrm{kg}$

(note that a Joule $\left.=\mathrm{N} * \mathrm{~m}=\left(\mathrm{kg} * \mathrm{~m} / \mathrm{s}^{2}\right) * \mathrm{~m}\right)$.

When do we employ total potential, versus mechanical potential, in the estimation of fluxes from high to low potential? As we discussed above, the osmotic potential can cause movement of water when there is an interface in the system which constrains the flux of selected constituents. For example, if there were two side-by-side cups of water solutions in a sealed box which could exchange water only in the vapor phase, then the air-water interfaces would act as constraints on the non-volatile solutes, while letting water be freely exchanged. If left long enough the water would move in the vapor phase until the water came to have the same total potential in the two cups. For instance, if one was pure water, and the other had a dissolved salt, then the pure water would tend to lose water in the vapor phase which would then condense into the saline solution, diluting the salt, and bringing the total potential of the two vessels closer to equal. Another important example is that of plant turgor: in contact with pure water, plants can obtain high cell pressures by having high dissolved concentrations in their tissue (low potential), so that the equilibrium total potential is obtained when the internal pressure in the tissue is high. Similarly, if the salt concentration in soil is too high, plants cannot create an energetic gradient to drive the saline water into the plant, and thus can wilt.

You will often hear of water described to be under "tension," "matric potential" or "suction." These terms all refer to the same quantity, the strength of attraction of water to a medium.This term is not rigorously tied to a particular physical mechanism, though some authors employ this term to identify the energy of interaction between water and solid surfaces (recall Figure 2.X illustrating the high energy of this interaction by way of the temperatures exceeding 700 C to remove all of this water - for a classic treatment of this see Brown and Montgomery, 1913, "Dehydration of Clays," Bureau of Standards Technologic Paper no. 21, Washington, D.C.: https://nvlpubs.nist.gov/nistpubs/nbstechnologic/ nbstechnologicpaperT21.pdf). While this is a perfectly fair energy term to quantify, it is important to recognize that, in general, solid surfaces will be at some intermediate state of water absorption, so any theoretical maximum value is rarely relevant. Generally speaking the energy per unit area of this interface is far in excess of the air-water surface tension, which theoretically should lead water to spread across all such surfaces. 


\section{Hydrostatics in Porous Media}

Having defined the parameterization of the porous media, and the units of the liquid phase potential, we can now discuss the interactions between soil and fluids. In principle there could be any number of fluids in these spaces, say $\mathrm{m}$, each of them filling some partial volume of the pore space; $\mathrm{V}_{1}, \mathrm{~V}_{2}, \ldots \mathrm{Vm}$. The only constraint on this system is that

$$
\sum_{i=1}^{m} \mathrm{~V}_{\mathrm{i}}=\mathrm{n}
$$

where we recall that $\mathrm{n}$ is the porosity. Fluids of primary interest are air and water; however other gases and nonaqueous phase liquids (e.g., gasoline and diesel in both liquid and gaseous forms) are also of significant interest. The focus of this text are on water and air, but the formulations are sufficiently general to allow direct extension to other fluids.

Why doesn't all the water just fall out of the porous media? There are at least four groups of forces contributing to the retention of water in soil, which are enumerated here in order of decreasing strength. Each of these forces has a connection to the structure of water, which has a polar nature, slightly negatively charged on the side where oxygen projects, and positively charged on the side where hydrogen is prominent. This means that water molecules have a highly dipolar electrostatic structure. Water molecules then develop a hydrogen bond between neighbors between their oxygen and hydrogen. Among other things, this leads to high bonding energy between water molecules, which leads to high surface tension, high latent heat of vaporization, and high solubility of ions. To summarize:

1. The hydrogen of water is attracted to the negative surface charge of mineral surfaces (Van der Waals attraction).

2. The periodic structure of the clay surfaces gives rise to an electrostatic dipole which results in an attractive force to the water dipole.

3. Osmotic force, caused by ionic concentration near charged surfaces, hold water.

4. Surface tension at water/air/solid interfaces maintains macroscopic units of water in pore spaces.

Table 2.1 Length scale ( $r$ ) of forces contributing to retention of water in soils.

\begin{tabular}{lll}
\hline Force & Attraction law & Range of influence \\
Van der Waals & $1 / \mathrm{r}^{7}$ & $1 \AA$ \\
& $1 / \mathrm{r}^{2}-$ ion & \\
Electrostatic - Dipole & $1 / \mathrm{r}^{6}-$ dipole & 5 \\
& & $100 \AA$ \\
Osmotic (double layer) & $1 / \mathrm{r}$ & $1 \mathrm{~mm}$ \\
\hline Surface Tension & $1 / \mathrm{r}$ & \\
\hline
\end{tabular}

These forces are discussed in detail in Sposito (1989) and Kirkham and Powers (1972) for readers who would like to learn more about these fascinating and fundamental relations. Attention in this text is focused on transport phenomena in the vadose zone. The first three forces listed in Table 2.1 act only at very short range, and essentially immobilize water. In contrast, surface tension produces effects which affect water in bulk, and is therefore more influential in transport.

\section{Surface Tension}

Surface tension is a measure of the energy required to generate an interface between two phases. Here we will be most 
concerned with the air-water interface, as shown in Figure 2.X2, but the concept applies to solid-gas interfaces, and liquid-solid interfaces. An intuitive understanding of surface tension can be obtained from a simple thought experiment. Imagine a block of water in a container that can be split in two (Figure 2.X3). For convenience think of each water molecule as being organized in a cubic lattice structure, with six bonds. Now we quickly split this block of water into two halves. The molecules on the new air/water surfaces are bound to only five of their neighbors. It took energy to break these bonds, which will be manifest as a free surface energy. Since the water surface has a constant number of molecules on its surface per unit area, the energy required to create these surfaces is directly related to the surface area created. This would suggest that surface tension should have units of energy per unit area, or force per unit length. 


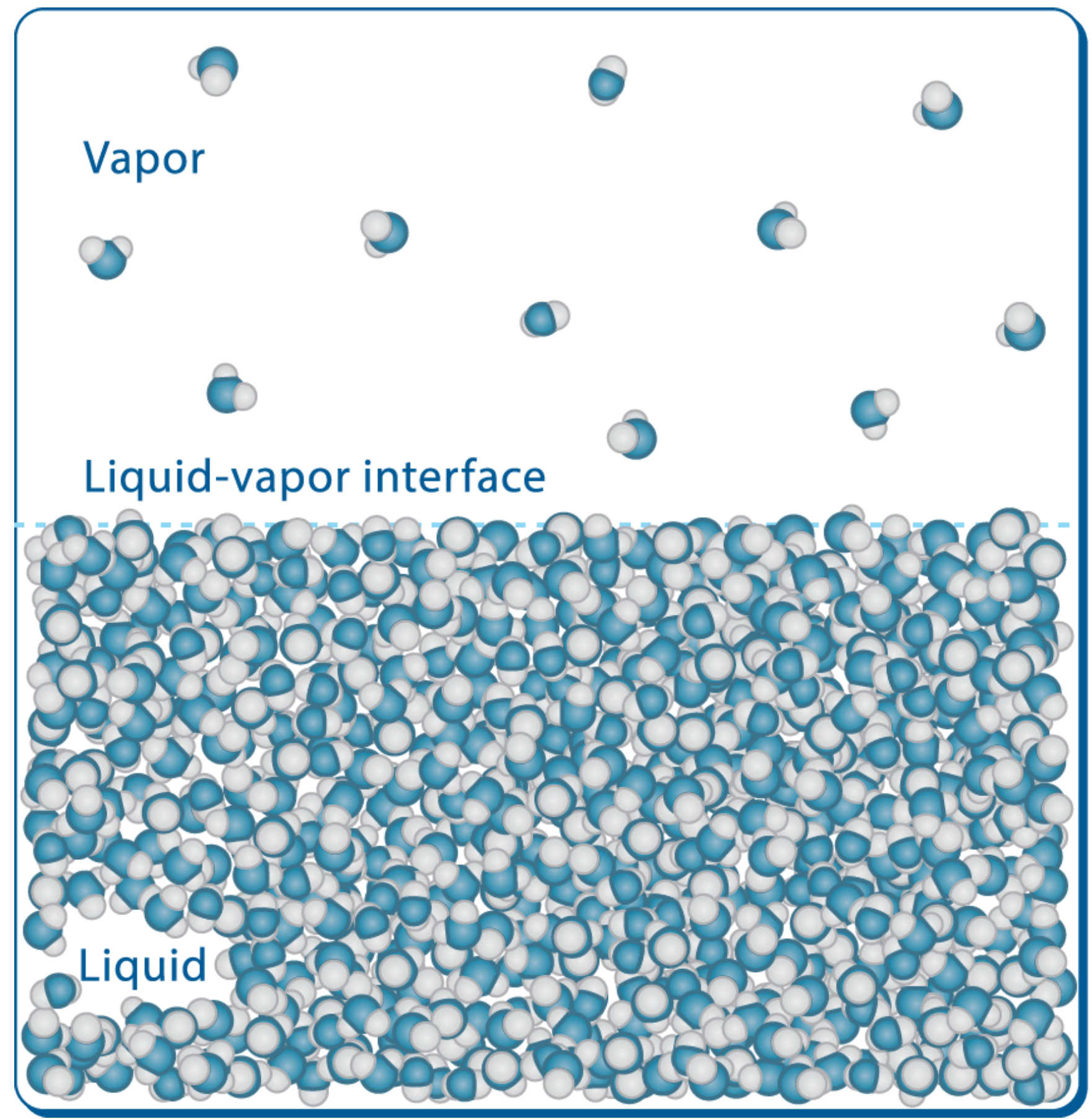

Figure 2.X2. Illustration of the tightly organized water molecules in the liquid state, and the constant adsorption of water vapor and desorption of liquid water from the air-water interface. The actual structure of water at the interface differs considerably from either the pure liquid or gaseous phases (e.g., Tong, Y., T. Kampfratha and R.K. Campen, Experimentally probing the libration of interfacial water: the rotational potential of water is stiffer at the air/water interface than in bulk liquid, Phys. Chem. Chem. Phys., 2016,18, 18424-18430, DOI 10.1039/C6CP01004K) in that there is a transition zone where the density of water moves between the liquid and vapor values. 


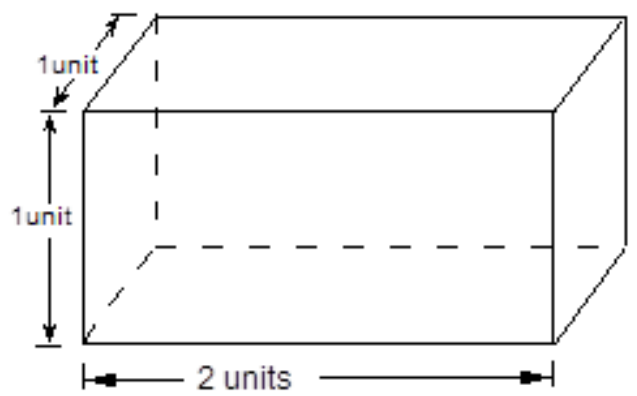

(a)
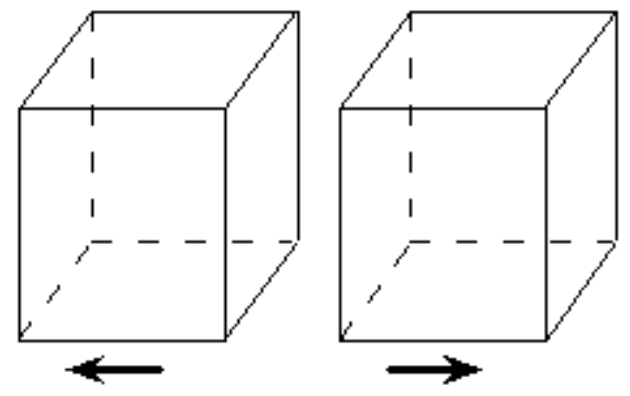

(b)

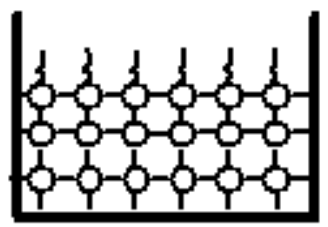

(c)

Figure 2.X3. Conceptual model of potential energy of broken bonds at liquid surface. The surface area of system (a) is 10 square units, while that of system (b) is 12 square units. System (b) has greater potential energy due to the broken surface bonds, as illustrated for an open vessel of water in figure (c).

It is easy to measure surface tension using the sliding wire set-up shown in Figure 2.X4. If the force measured on the scale is $\mathrm{F}$, and the width of the film is $\mathrm{L}$, then the surface tension, $\sigma$, is simply

$$
\sigma=\frac{F}{2 L}
$$

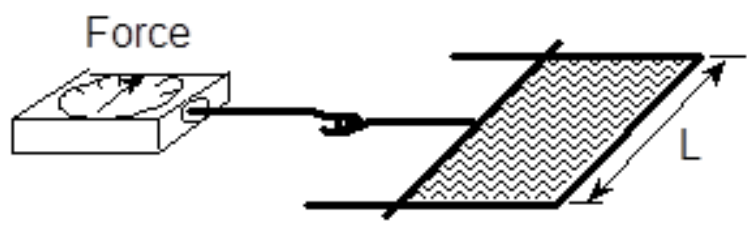

Figure 2.X4: Wire Frame set-up for measuring surface tension of air-liquid interfaces.

Wait a minute! How did that factor of 2 sneak into Equation $\underline{20}$ ? Simple: since the film is open on two sides, we are creating two air/water interfaces, and thus the effective length of a single-sided free surface is $2 \mathrm{~L}$. Note that you could also keep the entire problem in terms of energy per unit area in keeping with the thought experiment of the previous paragraph: carefully record the product of force required to stretch the film multiplied by the distance over which that force is exerted (to obtain the energy input) and divide by the area ( 1 x L). Table 2.3 lists some typical values of surface tension for fluid pairs.

Table 2.3 Some values of surface tensions for various interfaces (taken from the CRC Handbook of Chem. and Physics 68th ed., 1987). 


\begin{tabular}{|c|c|c|c|c|}
\hline Substance & Formula & Contacting & $\operatorname{Temp}\left({ }^{\circ} \mathrm{C}\right)$ & $\begin{array}{l}\sigma \\
\text { (dynes/cm) }\end{array}$ \\
\hline Water & $\mathrm{H}_{2} \mathrm{O}$ & air & 20 & 72.75 \\
\hline Acetone & $\mathrm{C}_{3} \mathrm{H}_{6} \mathrm{O}$ & air & 20 & 23.7 \\
\hline Benzene & $\mathrm{C}_{6} \mathrm{H}_{6}$ & $\begin{array}{l}\text { air } \\
\text { water }\end{array}$ & $\begin{array}{l}20 \\
20\end{array}$ & $\begin{array}{l}28.85 \\
35.00\end{array}$ \\
\hline Carbon Tetrachloride & $\mathrm{CCl}_{4}$ & $\begin{array}{l}\text { vapor } \\
\text { water }\end{array}$ & $\begin{array}{l}20 \\
20\end{array}$ & $\begin{array}{l}26.95 \\
45\end{array}$ \\
\hline Mercury & $\mathrm{Hg}$ & water & 20 & 375 \\
\hline
\end{tabular}

\section{Classical Derivation of Laplace's Equation}

We are concerned with how surface tension manifests itself in a porous medium. In particular, we would like to know the static fluid pressures which result from surface tension acting on fluid surfaces, which are curved within a porous medium. Consider the infinitesimal curved fluid surface which is shown in Figure 2.5. For the sake of generality, we allow for curvature along two axes of radius $r_{1}$ and $r_{2}$. To determine the pressure $p$ acting on the surface we simply add up the forces acting on the interface in the vertical direction. Since the system is at equilibrium, these forces must add to zero. The forces acting upward (which we will take as positive) result from $\mathrm{p}_{2}$ and $\mathrm{r}_{2}$

$$
\mathrm{F}_{\mathrm{up}}=\mathrm{p}_{2} \delta \mathrm{S}_{1} \delta \mathrm{S}_{2}+2 \sigma \delta \mathrm{S}_{1} \sin \left(\frac{\delta \theta_{2}}{2}\right)
$$

where $\sigma$ is the surface tension of the interface. Now since this is a very small patch, the angle $\delta \theta_{2}$ is very small, and we may use the small angle approximation for sine:

$$
\sin \left(\frac{\delta \theta_{2}}{2}\right) \approx \frac{\delta \theta_{2}}{2} \approx \frac{\delta S_{2}}{2 r_{2}}
$$

By symmetry, the expression for $\mathrm{F}_{\text {down }}$ is identical to Equation $\underline{21}$, substituting appropriate subscripts and signs. The net force on the surface is then given by

$$
\mathrm{F}_{\text {net }}=0=\text { upward forces }- \text { downward forces }
$$

$$
=\mathrm{p}_{2} \delta \mathrm{S}_{1} \delta \mathrm{S}_{2}+2 \sigma \delta \mathrm{S}_{1} \frac{\delta \mathrm{S}_{2}}{2 \mathrm{r}_{2}}-\mathrm{p}_{1} \delta \mathrm{S}_{2} \delta \mathrm{S}_{1}-2 \sigma \delta \mathrm{S}_{2} \frac{\delta \mathrm{S}_{1}}{2 \mathrm{r}_{1}}
$$

which, canceling $\delta \theta_{1} \delta \theta_{2}$ from all terms, simplifies to

$$
\mathrm{p}_{2}-\mathrm{p}_{1}-\frac{\sigma}{\mathrm{r}_{1}}+\frac{\sigma}{\mathrm{r}_{2}}=0
$$

or 
${ }_{(25)} \mathrm{p}_{2}-\mathrm{p}_{1}=\sigma\left(\frac{1}{\mathrm{r}_{1}}-\frac{1}{\mathrm{r}_{2}}\right)$

which is Laplace's Equation for pressure across an interface at a saddle point (an anticlastic surface). Notice that if both radii were of the same sign and magnitude (i.e., the surface was spherical) we have $r_{1}=-r_{2}=R$ in Figure 2.5, and Equation $\underline{25}$ would reduce to

$$
\text { (26) } \mathrm{p}=\frac{2 \sigma}{\mathrm{R}}
$$

which is the familiar form of Laplace's equation for pressure across a spherical fluid interface. Of course this is the same expression one obtains for the pressure behind the gas/liquid interface in a capillary tube of radius R. For the general case where $r_{1} \neq r_{2}$, we define the reduced radius of curvature, $R$, as 

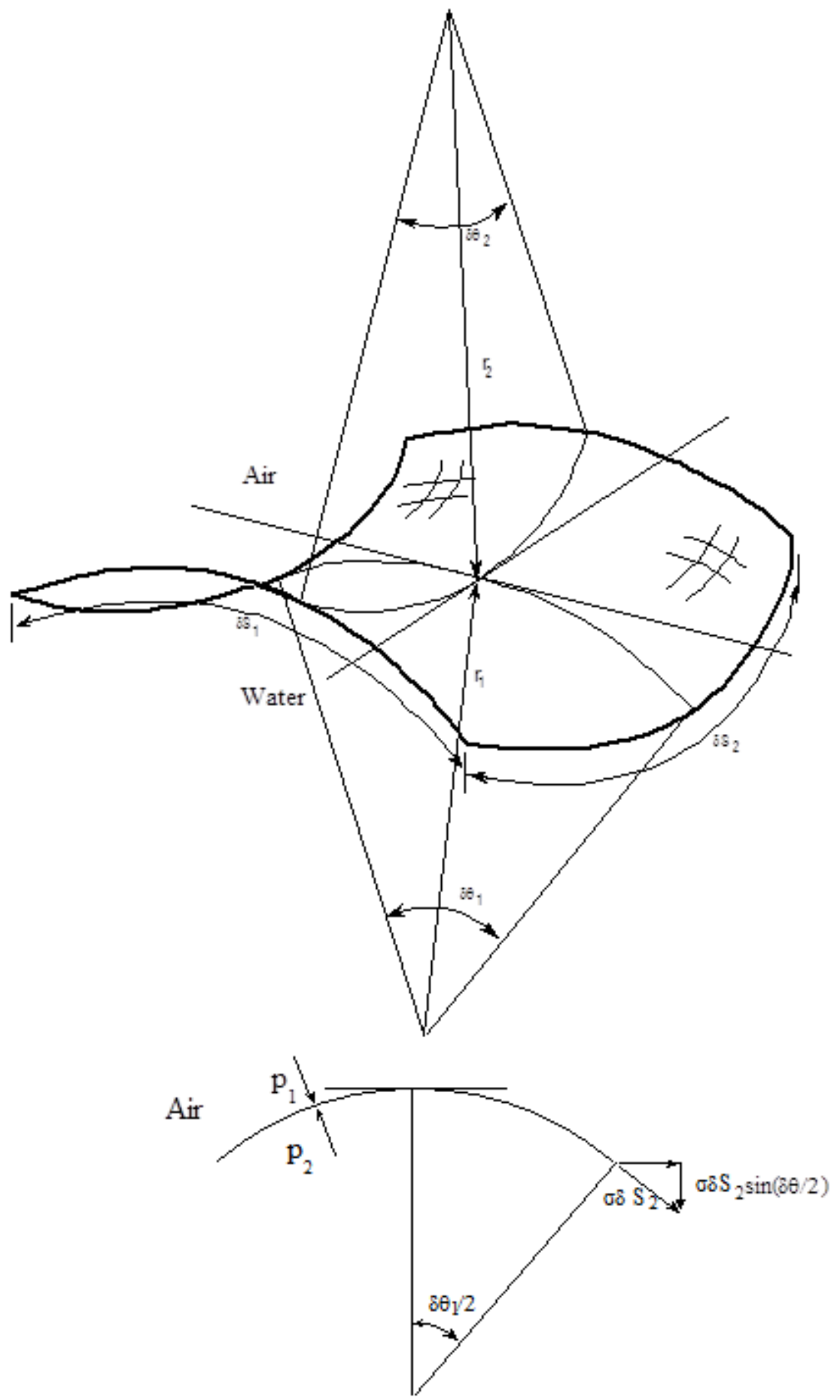

Figure 2.5. Definition sketches for a doubly curved (anticlastic) liquid surface.

(27) $\frac{2}{\mathrm{R}}=\left(\frac{1}{\mathrm{r}_{1}}+\frac{1}{\mathrm{r}_{2}}\right)$

or

72 | Basic Relationships and the Soil Phases Under Equilibrium Conditions 


$$
\text { (28) } \mathrm{R}=\frac{2 \mathrm{r}_{1} \mathrm{r}_{2}}{\mathrm{r}_{1}+\mathrm{r}_{2}}
$$

where we have used a sign convention for $r_{1}$ and $r_{2}$ such that they are taken to be positive if measured in the wetting fluid and negative if measured in the non-wetting fluid (typically air). Note that an element of water in a porous media often displays complex curved interfaces. Even the simplest case of two spheres in contact with water between has two characteristic radii, as shown in Figure 2.X

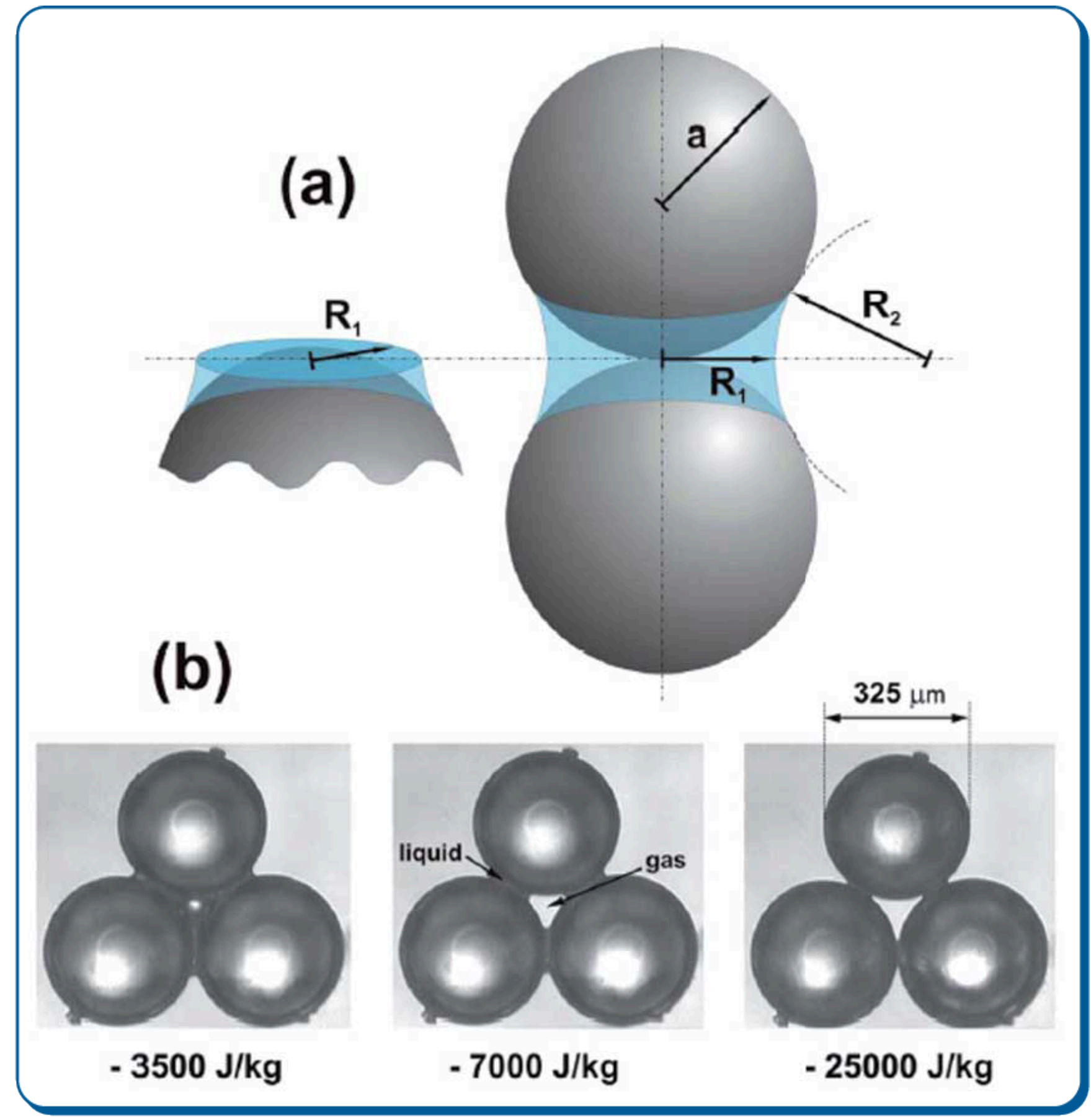

(a) Illustration of the combined convex and concave gas-liquid interface at the contact of two spherical particles as well as the progression of the water shape as a function of pressure (b) (from XXX with permission). 


\section{Introduction to the of Fluid Interfaces}

The derivation of Laplace's equation provided in section 2.3.2.1 is completely acceptable, but in this case there is good reason to look at this problem from another perspective, that of thermodynamics, to gain further insight into the workings of fluid interfaces. In the following sections we shall re-derive Laplace's equation, using a somewhat less intuitive and more formal approach. This will be a warm-up, for then we will push thermodynamics a bit further to obtain some very handy additional results.

The Thermodynamics of Flat Interfaces

Consider a small, partially filled container of water with a flat water-air interface. The system has water in three phases: liquid, gaseous and as a taut interfacial surface, denoted by subscripts ' $\mathrm{l}$ ', 'g', and 'a', respectively. We will denote the surface area as $\Sigma$ and the surface tension at the air/water interface as $\sigma$. Since there is no barrier to water moving between phases, the chemical potential of the water in each phase is the same, i.e.,

(29) $\mu_{\mathrm{g}}=\mu_{\mathrm{a}}=\mu_{\mathrm{l}}=\mu_{\infty}$

The subscript $\infty$ is used to indicate values of intensive parameters which are constant in all three phases (recall intensive parameters are those which do not depend upon the amount of material in question, in contrast to extensive parameters which do depend on the amount, or 'extent' of the material). We will consider a system at constant temperature, $\mathrm{T}$. We will use $\mathrm{N}_{\mathrm{i}}$ to denote the number of atoms, $\mathrm{S}_{\mathrm{i}}$ the entropy, $\mathrm{E}_{\mathrm{i}}$ the internal energy, $\mathrm{P}_{\mathrm{i}}$ the pressure and $\mathrm{V}_{\mathrm{i}}$ the volume in phase $\mathrm{i}$.

Considering the gas and liquid phases, we have three fundamental differential forms

$\mathrm{TdS}_{\mathrm{g}}=\mathrm{dE}_{\mathrm{g}}+\mathrm{P}_{\infty} \mathrm{dV} \mathrm{V}_{\mathrm{g}}-\mu_{\infty} \mathrm{dN}_{\mathrm{g}}$

$\mathrm{TdS}_{1}=\mathrm{dE}_{1}+\mathrm{P}_{\infty} \mathrm{dV} \mathrm{V}_{1}-\mu_{\infty} \mathrm{dN}_{1}-\sigma \mathrm{d} \Sigma$

(32) $\mathrm{TdS}_{\mathrm{a}}=\mathrm{dE}_{\mathrm{a}}-\sigma \mathrm{d} \Sigma$

(surface) (gas)

\section{(liquid)}

The total energy and entropy for the system are simply the sum of the components, i.e.

(33)

$$
\mathrm{S}=\mathrm{S}_{\mathrm{a}}+\mathrm{S}_{\mathrm{g}}+\mathrm{S}_{1}
$$

Since the surface is two-dimensional, we take the number of atoms in the surface to be zero in comparison to the atoms in the three-dimensional volumes of gas and liquid. So we may write

(35) $\mathrm{N}=\mathrm{N}_{1}+\mathrm{N}_{\mathrm{g}}$

Thus the fundamental differential form for the system is given by

(36) $\mathrm{TdS}=\mathrm{dE}+\mathrm{P}_{\infty} \mathrm{dV}-\mu_{\infty} \mathrm{dN}-\sigma \mathrm{d} \Sigma \quad$ (system)

Employing the Gibbs-Duhem relationship (i.e., for an exact differential, the differentiation may be shifted from the extensive to intensive variables maintaining equality) we find

(37) $\mathrm{S}_{\mathrm{a}} \mathrm{dT}=\Sigma \mathrm{d} \sigma$

or

(38) $\frac{\mathrm{S}_{\mathrm{a}}}{\Sigma}=\frac{\mathrm{d} \sigma}{\mathrm{dT}}$

which is the equation of state for the surface phase (analogous to the equation of state for an ideal gas $\mathrm{PV}=\mathrm{nRT}$ ), and relates the temperature dependence of surface tension to the magnitude of the entropy of the surface. 
Laplace's Equation from a sphere in Space

Now let's consider the effect of a curved air-water interface. Now $\mathrm{P}_{\mathrm{g}}$ and $\mathrm{P}_{1}$ are not equal, while $\mu_{\mathrm{g}}$ and $\mu_{1}$ remain equal. Considering a droplet floating in gas filled space of constant volume, the fundamental differential form for the system is given by

$$
T d S=d E+P_{g} d V g+P_{1} d V_{1}-\mu_{g} d N_{g}-\mu_{1} d_{1}-\sigma d \Sigma
$$

Considering a very small transfer of volume, $\delta \mathrm{V}$, between the two phases, we see the chemical potential terms would be equal and opposite, that the total change in energy in the system is unchanged (we are doing no work on the system), and the entropy would be constant to first order. Since the volume of the system is held constant from $\underline{39}$ we find

(40) $\left(\mathrm{P}_{1}-\mathrm{P}_{\mathrm{g}}\right) \delta \mathrm{V}-\sigma \delta \Sigma=0$

or defining $\mathrm{P}_{\mathrm{d}}$ as the pressure difference across the interface of the droplet, we have

$$
\mathrm{P}_{\mathrm{d}}=\sigma \frac{\delta \mathrm{S}}{\delta \mathrm{V}}
$$

We can calculate the differential on the right-hand side by noting that for a sphere $\mathrm{V}=\left(4 \pi \mathrm{r}^{3} / 3\right)$ and $\Sigma=4 \pi \mathrm{r}^{2}$, so $\delta \Sigma / \delta \mathrm{V}=2 / \mathrm{r}$, hence

$$
\mathrm{P}_{\mathrm{d}}=\frac{2 \sigma}{\mathrm{r}}
$$

which is Laplace's equation for the pressure across a curved interface where the two characteristic radii are equal (see 26).

Although you probably have had it with this equation by now, it is only fair to show the simple way to obtain this result. Consider the pressure balance across the middle of the droplet. We know the surface tension of the water about the center of the droplet must equal the pressure exerted across the area of the droplet by the liquid. The area of the droplet at its midpoint is $\pi r^{2}$ at pressure $P_{d}$, while the length of surface applying this pressure is $2 \pi r$ at tension $\sigma$. Equating these we obtain

$$
\mathrm{P}_{\mathrm{d}} \pi \mathrm{r}^{2}=2 \pi \mathrm{r} \sigma
$$

(43) $\mathrm{P}_{\mathrm{d}}=\frac{2 \sigma}{\mathrm{r}}$

as expected.

\section{Across Curved Interfaces}

The curved interface also affects the vapor pressure of the liquid species. Suppose we have our spherical water droplet in a fixed volume at equilibrium with the vapor phase. The chemical potential of the water in these two phases will be equal, and will remain equal through any reversible process, i.e.,

(44) $\mu_{1}=\mu_{\mathrm{g}}$

and

(45) $\delta \mu_{1}=\delta \mu_{\mathrm{g}}$

Writing the fundamental differential forms for the gas and liquid phases for a system with one mole of each species (following equations $\underline{30}$ and $\underline{31}$ ) we have

$$
\mathrm{TdS}_{\mathrm{g}}=\mathrm{dE} \mathrm{E}_{\mathrm{g}}+\mathrm{P}_{\mathrm{g}} \mathrm{dV} \mathrm{V}_{\mathrm{g}}-\mu_{\mathrm{g}} \mathrm{dN} \mathrm{N}_{\mathrm{g}}
$$


As before, the Gibbs-Duhem relation of the fundamental differential forms follows the principle that for an exact differential form, equality is maintained if differentiation for each term is exchanged between intensive and extensive variables, hence $\underline{46}$ and ?? may be written

(48) $\mathrm{S}_{\mathrm{g}} \mathrm{dT}=\mathrm{V}_{\mathrm{g}} \mathrm{dP} \mathrm{P}_{\mathrm{g}}-\mathrm{N}_{\mathrm{g}} \mathrm{d} \mu_{\mathrm{g}}$

(49) $\mathrm{S}_{1} \mathrm{dT}=\mathrm{V}_{1} \mathrm{dP}_{1}-\mathrm{N}_{1} \mathrm{~d} \mu_{1}$

Dividing $\underline{48}$ by $\mathrm{N}_{\mathrm{g}}$ and $\underline{49}$ by $\mathrm{N}_{\mathrm{l}}$ we get the equations in a per mole basis. By further assuming that temperature and surface tension remain constant, we obtain

(50) $\mathrm{v}_{\mathrm{g}} \mathrm{dP} \mathrm{P}_{\mathrm{g}}=\mathrm{d} \mu_{\mathrm{g}}$

(51) $\mathrm{v}_{1} \mathrm{dP}_{1}=\mathrm{d} \mu_{1}$

where $\mathrm{v}$ in lower case indicates the volume per mole. Now employing ?? we find

(52) $\mathrm{v}_{\mathrm{g}} \mathrm{dP}_{\mathrm{g}}=\mathrm{v}_{1} \mathrm{dP}_{1}$

which may be written

$$
\mathrm{d}\left(\mathrm{P}_{1}-\mathrm{P}_{\mathrm{g}}\right)=\frac{\mathrm{v}_{\mathrm{g}}-\mathrm{v}_{1}}{\mathrm{v}_{1}} \mathrm{dP} \mathrm{P}_{\mathrm{g}}
$$

Using Laplace's equation (e.g., 43 ) this may be re-written as

$$
\mathrm{d}\left(\frac{2 \sigma}{\mathrm{r}}\right)=\frac{\mathrm{v}_{\mathrm{g}}-\mathrm{v}_{1}}{\mathrm{v}_{1}} \mathrm{dP}_{\mathrm{g}}
$$

or

$$
\mathrm{dP}_{\mathrm{d}}=\frac{\mathrm{v}_{\mathrm{g}}-\mathrm{v}_{1}}{\mathrm{v}_{1}} \mathrm{dP} \mathrm{P}_{\mathrm{g}}
$$

We note that $\mathrm{v}_{\mathrm{l}}$ is approximately four orders of magnitude less than $\mathrm{v}_{\mathrm{g}}$, so we will suppose that $\left(\mathrm{v}_{\mathrm{g}}-\mathrm{v}_{\mathrm{l}}\right) / \mathrm{v}_{\mathrm{l}}$ can be accurately approximated by $\mathrm{v}_{\mathrm{g}} / \mathrm{v}_{\mathrm{l}}$, and we obtain

$$
\mathrm{d}\left(\frac{2 \sigma}{\mathrm{r}}\right)=\frac{\mathrm{v}_{\mathrm{g}}}{\mathrm{v}_{1}} \mathrm{dP}_{\mathrm{g}}
$$

Now assuming the vapor phase acts like an ideal gas, $\mathrm{P}_{\mathrm{g}} \mathrm{v}_{\mathrm{g}}=\mathrm{RT}$, whence $\underline{56}$ becomes

$$
\mathrm{d}\left(\frac{2 \sigma}{\mathrm{r}}\right)=\frac{\mathrm{RT}}{\mathrm{P}_{\mathrm{g} \mathrm{v}_{1}}} \mathrm{dP}_{\mathrm{g}}
$$

This may be integrated from a flat interface $(\mathrm{r}=\infty)$ to that with radius $\mathrm{r}$ to obtain

$$
\frac{2 \sigma}{\mathrm{r}}=\frac{\mathrm{RT}}{\mathrm{v}_{1}} \ln \left(\frac{\mathrm{P}_{\mathrm{g}}}{\mathrm{P}_{\infty}}\right)
$$

where $\mathrm{P}_{\infty}$ is the vapor pressure of water at temperature $\mathrm{T}\left(\mathrm{P}_{g} / \mathrm{P}_{\infty}\right.$ is the relative humidity of the soil air system). Taking $\mathrm{K}$ as the specific gas constant for water (i.e., $\mathrm{R}=\mathrm{R} / \mathrm{v}_{\mathrm{l}}$ ), and noting that the left-hand side of $\underline{58}$ is just $\mathrm{P}_{\mathrm{d}}$, this may also be written

$$
\mathrm{P}_{\mathrm{d}}=\overline{\mathrm{R}} \mathrm{T} \ln \left(\frac{\mathrm{P}_{\mathrm{g}}}{\mathrm{P}_{\infty}}\right)
$$

where $P_{d}$ is the pressure potential of the water. This very useful relation is known as the psychrometric equation, which provides the relationship between the humidity of the air in equilibrium with an air-water interface and the potential of the water. This allows the determination of very high negative potentials through measurement of the vapor pressure 
(or relative humidity) of water in porous media. For instance, at a pressure of -15 Bar (the permanent wilting point of many plants), $\mathrm{P}_{g} / \mathrm{P}_{\infty}$ is 0.99 . Measurement of $\mathrm{P}_{\mathrm{g}} / \mathrm{P}_{\infty}$ may be made in a number of ways. Most commonly a small thermocouple is cooled by application of a reverse current while its temperature is read with a second thermocouple. As the temperature of the first thermocouple attains the dew point of the vapor, its temperature decline is sharply reduced due to the energy of condensation of water on the device. Knowing the dew point, it is straightforward to obtain the relative humidity with good precision (Rawlins and Campbell, 1986).

\section{Temperature Dependence of Surface Tension}

It is often overlooked that all the measurements we take regarding water/media interactions are strongly temperature dependent. In many of the equations derived above we have treated surface tension as a constant property of a fluid. This is certainly not the case. The temperature dependence of air-water surface tension is illustrated below in Figure 2.6 where we see that for this pair, surface tension decreases at approximately one percent per $4^{\circ} \mathrm{C}$, which could give rise to discrepancies of greater than $5 \%$ between common laboratory and field conditions, or even through diurnal fluctuations.

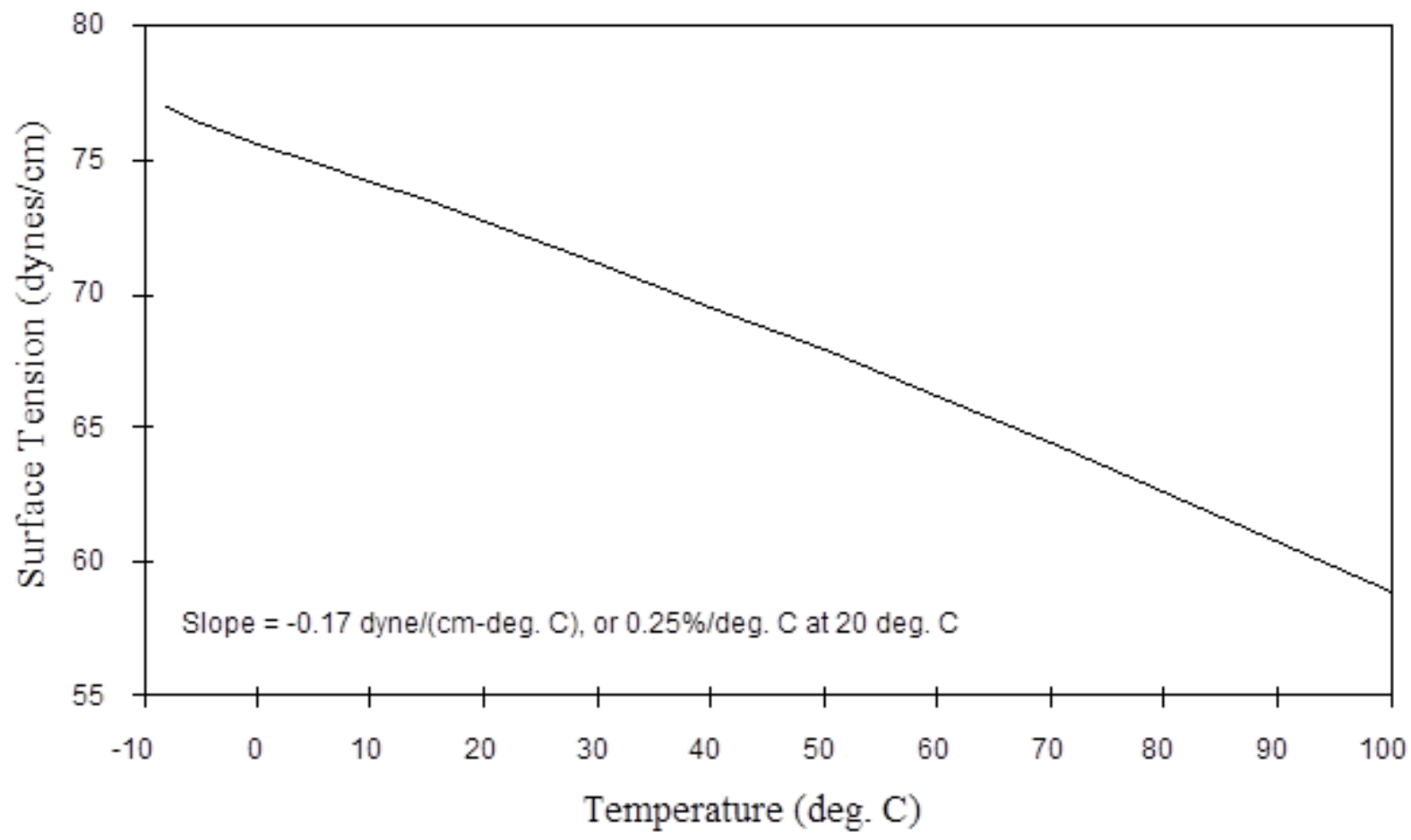

Figure 2.6. The temperature dependence of air-water surface tension (Data from the 68th ed. of the Handbook of Chemistry and Physics, 1987).

\section{The Characteristic Curves}

Now for the real excitement: putting the solid and fluid together! To do this, we will undertake yet another simple thought experiment. Take a funnel, and seal a flat porous plate in it to make a Buchner funnel, as shown in Figure 2.7. 
Select the porous plate so that its pores, of radius $r$, are much smaller than the pores in the soil sample. Now attach a tube to the bottom of the funnel, and fill the region below the porous plate to the end of the tube with water. This tube is fed from a beaker on a precision balance, which keeps track of the amount of water in the soil sample. By wetting the porous plate, we know from Equation $\underline{26}$ that air cannot enter the porous plate until a suction greater than Pentry

$$
\mathrm{P}_{\text {entry }}=\frac{2 \sigma}{\mathrm{r}_{\max }}
$$

is applied to the plate. Since the porous plate has pore radii smaller than the pore in the soil sample, we know that the plate will remain saturated as we draw the water from the sample. We now place a thin piece of dry soil on the porous funnel surface. The experiment will take place over three stages: (1) wetting, (2) drying, and (3) re-wetting. For stage (1), we incrementally bring the end of the tube up, finally attaining the elevation of the soil. At each lift we measure the amount of water drawn up by the soil, with pressures ranging from Pentry to 0 . Each measurement is taken allowing the system to come to a steady state. Measuring elevation, $\mathrm{H}$, as positive upward, the pressure applied to the water in this soil will be given by

(61) $\mathrm{P}_{\text {soil }}=\rho_{\mathrm{w}} \mathrm{gh}$

where $\rho_{\mathrm{W}}$ is the density of water and $\mathrm{h}$ is the pressure head of the water in the sample, which we will assume is much larger than the thickness of the sample 1. In stage (2) the end of the tube is incrementally lowered to apply a negative head to the water in the soil. Finally, in stage (3) we again lift the beaker to an elevation slightly below the height of the soil sample keeping track of the amount of water taken up by the sample. A typical plot illustrating how the soil sample's volumetric water content (equation \ref\{eq:volumetric_liquid\}) changes in response to pressure changes for this type of experiment is shown in Figure 2.8, with a few more lifts and drops thrown in for good measure. The vertical axis in Figure 2.8 represents the pressure in the sample, as reflected by the hanging height of water, $\mathrm{h}$. The horizontal axis shows the soil's volumetric moisture content.

The equilibrium relationship between pressure and moisture content is called the soil water characteristic (SWC). The SWC is an important soil property related to the distribution of pore space (sizes, interconnectedness), which is strongly affected by texture and structure, as well as related factors including organic matter content. The SWC is a primary hydraulic property required for modeling water flow, for irrigation management, and for many additional applications related to managing or predicting water behavior in the porous system. A SWC is a highly nonlinear function and is relatively difficult to obtain accurately. Because the pressure potential extends over several orders of magnitudes for the range of water contents encountered in the field, the potential is often plotted on a logarithmic scale (Figure 2.X). 


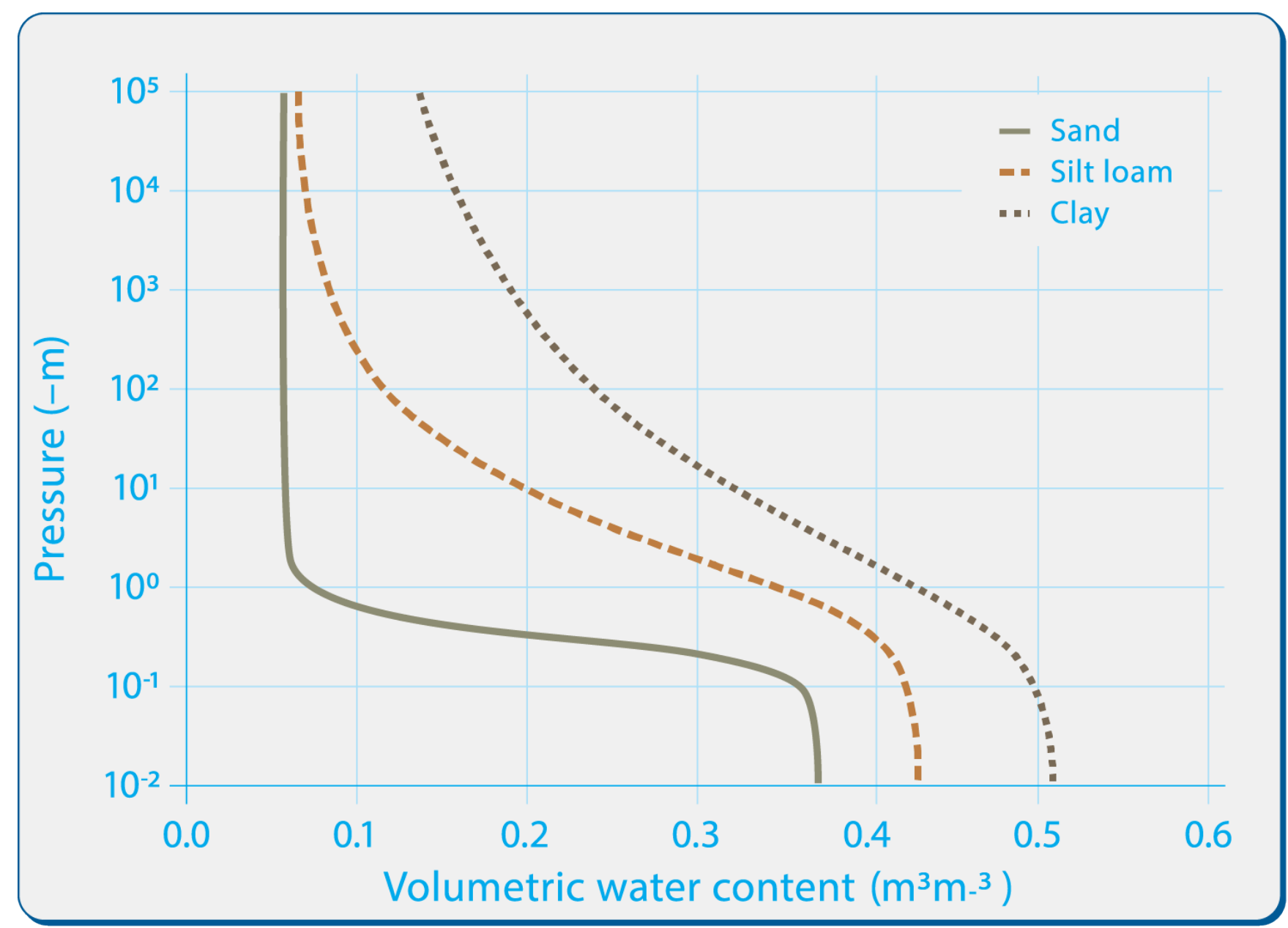

Figure 2.X SWC curves for soils of different textures demonstrating the reduced porosity (saturated water content) and the relatively large pores for coarse-textured media such as sand, as well as the extreme range of potentials required to drain soils of different texture.

This simple experiment illustrates most of the physics which control the retention and movement of fluids through porous media, so it is worth considering these results carefully. First consider the wetting curve alone, spanning the points labeled (1), (2) and (3) in Figure (2.8). We see that more water is taken up by the soil (i.e., the moisture content increases) as the beaker comes closer to the elevation of the soil (i.e., as the pressure of the feed water increases).

One can gain a basic understanding of the characteristic S-shape of this curve by relating it to the soil particle and pore size distributions. Consider a soil made up of particles with size distributed between $d_{\min }$ and $d_{\max }$ as shown in Figure 2.9. By passing the sample through successive sieves, from very fine to coarse, we may evaluate the percentage of the grains which have size greater than any given size, as plotted in Figure (2.9a) (see section 1.3.2). Without stretching your imagination too far, it is plausible that the pore size distribution in the original soil is quite similar to the particle size distribution. Now the ordinate goes from mass of particles, to volume of pores. The plot of pore size distribution which is obtained is quite similar to that of particle size, as shown in Figure (2.9b).

This is often conceptualized in terms of the soil being made of many differing pore sizes classes which could be represented as equivalent parallel capillary tubes of many radii (Figure 2.X). 
(a)

80 Basic Relationships and the Soil Phases Under Equilibrium Conditions 

Using Equation 26, we can equate pore size to the capillary filling pressure of each pore, and re-plot the pore size distribution as a filling pressure plot; Figure (2.9c). Notice the volume of pores which are filled in Figure (2.9c) is simply the degree of saturation. At last, in Figure (2.9d), we have obtained the wetting curve from the particle size distribution. The idea here is to point out the connection between the wetting characteristic curve and the physical properties of the medium. A similar argument is often used when trying to determine how a medium will drain using the particle size distribution (e.g., Arya and Paris, 1981).

A)

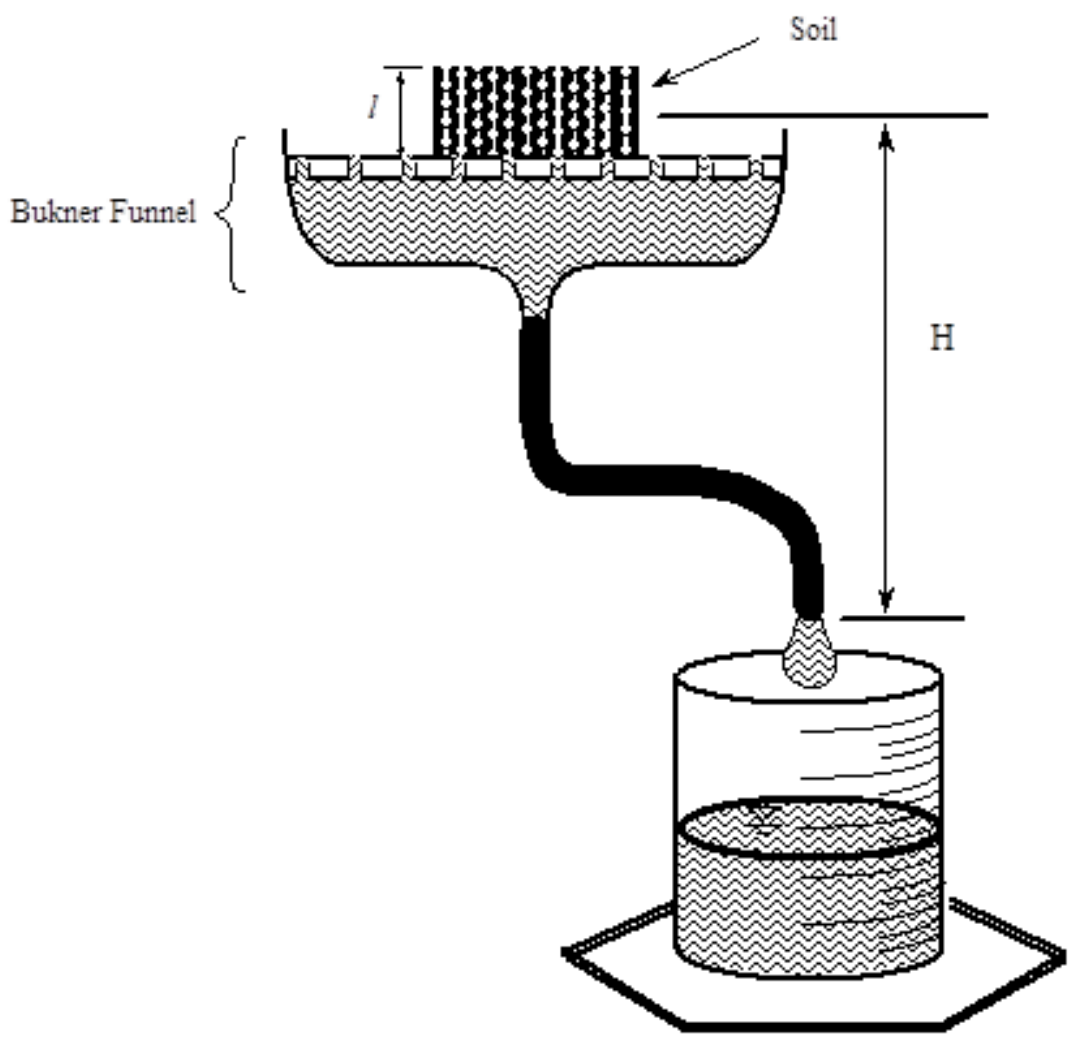

B)

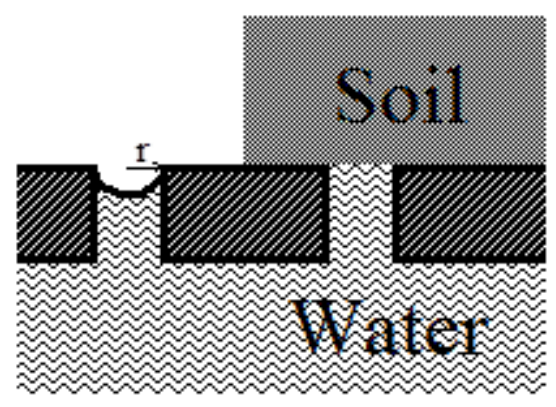

Figure 2.7. (a) Conceptual illustration of Bukner funnel experiment to determine characteristic draining curve; (b) close-up view of idealized porous plate. 


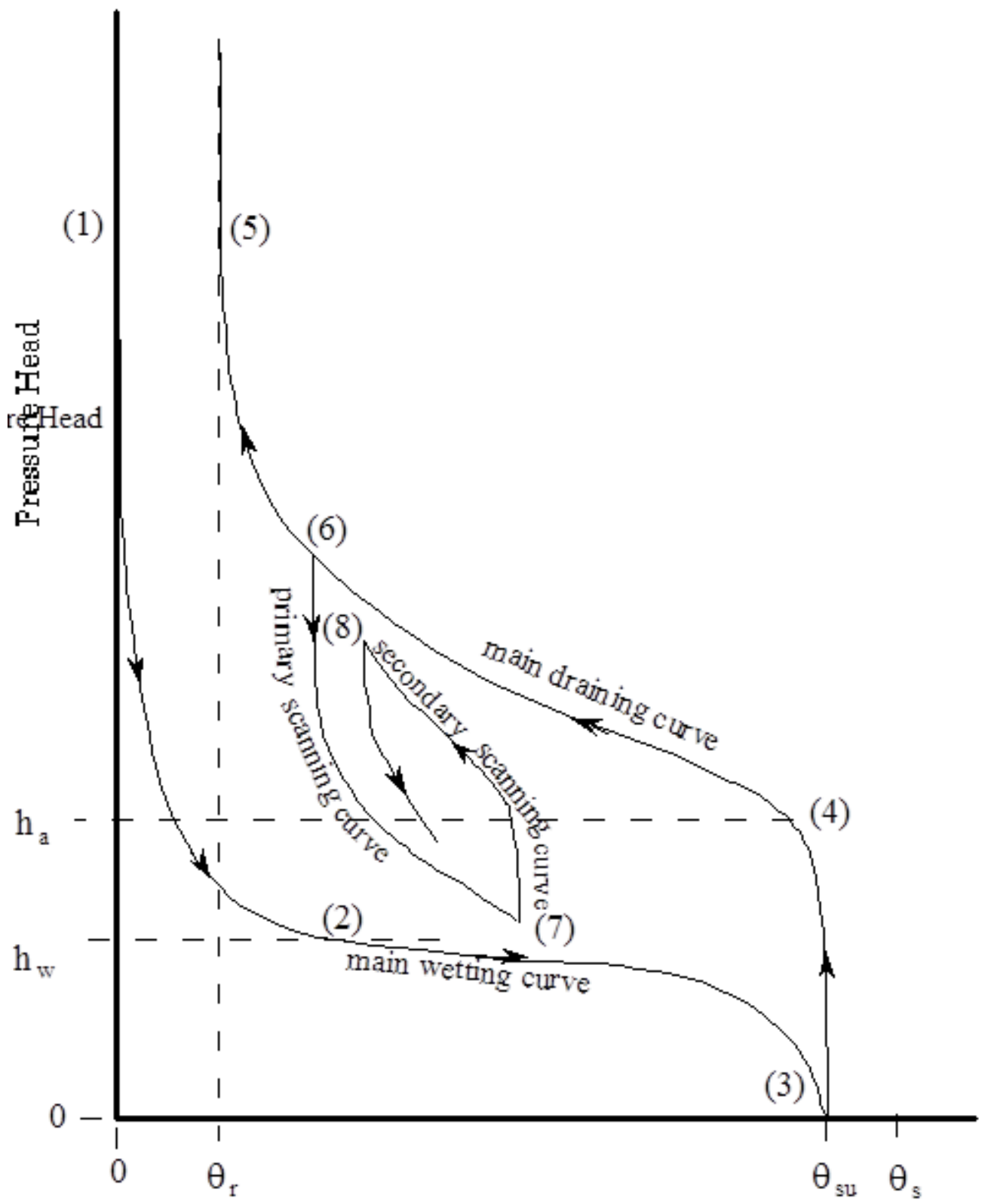

Figure 2.8. The main characteristic curves for wetting, draining, and one set of scanning curves. 


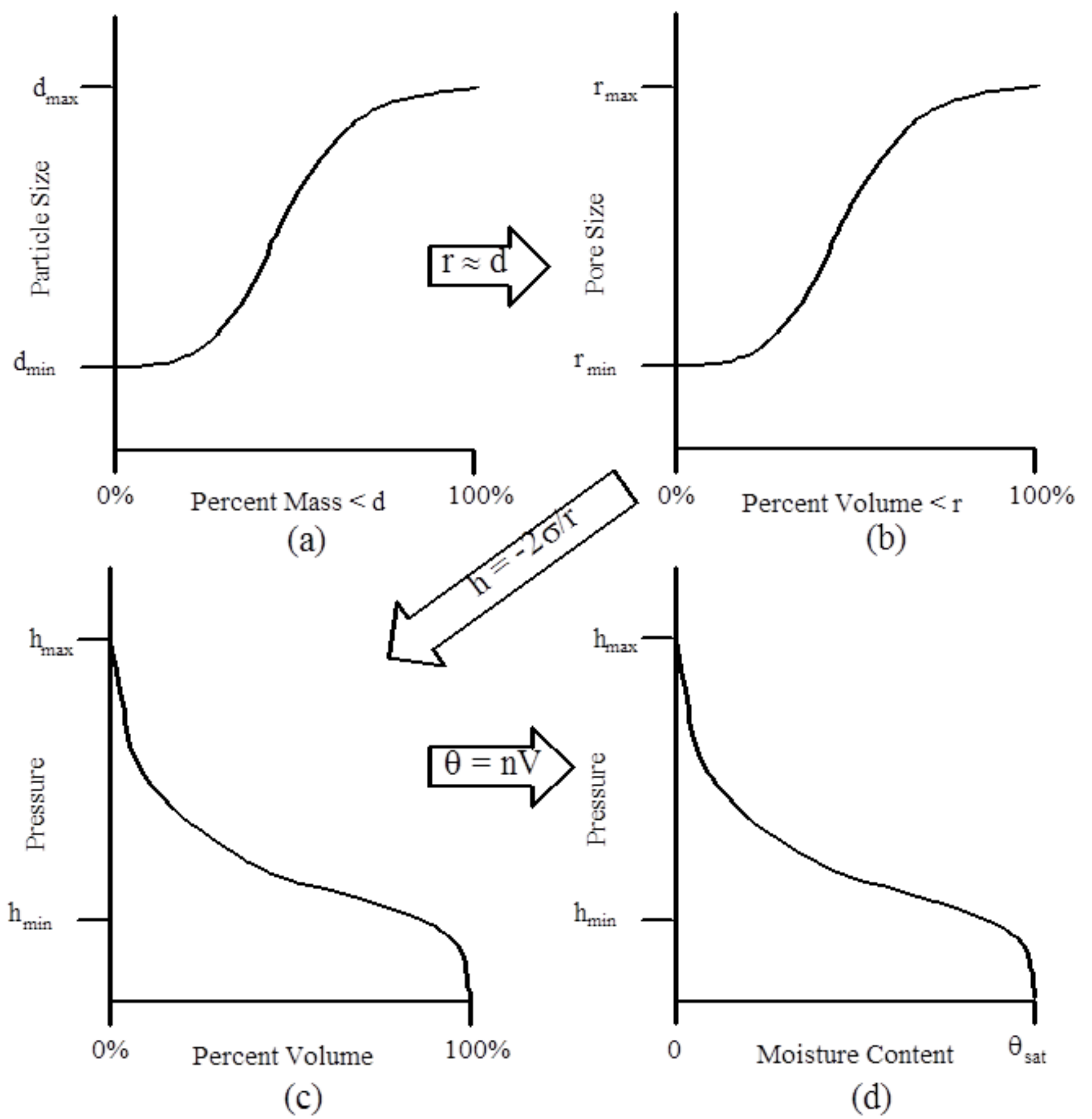

Figure 2.9. Conceptual relationship between (a) particle size distribution; (b) pore size distribution; (c) pore volume occupied vs pore filling pressure; and (d) the characteristic wetting curve.

Now let's follow the process of draining, going from points 3 to 4 and 5 in Figure (2.8). Why isn't this curve following right along the wetting curve? To understand this we shall consider the filling and emptying of a single pore, as shown in Figure (2.10). The idea represented in this figure again follows directly from Equation $\underline{26}$, and were introduced by Haines in 1930.

The wetting process for this pore is illustrated in stages 1 through 3 of Figure 2.8 and (b) of Figure 2.10, while the draining process is illustrated in stages 4 through 7 of Figure 2.8 and (c) and (d) of Figure 2.10. For any given pressure, there are two states that this pore may be in, depending on whether it is in the process of filling or draining. In this sense 
the saturation of the pore is a function of both the pressure and the history of the pore. For this reason, the wetting and drying curves for soil are referred to as "hysteretic."

Figure 2.10 shows that the filling process is held up until the pressure at the soil is sufficient to fill the largest pores in the medium. On the other hand, once the medium is filled, it cannot drain significantly until the pressure becomes sufficiently negative to pull the water out of the pore necks that lead into the medium. Clearly between the pressures of $-2 \sigma / \mathrm{r}_{2}$ and $-2 \sigma / \mathrm{r}_{1}$ the moisture content is a function of not only the pore pressure, but what the history of wetting has been.

Based on the ideas contained in Figure (2.9), $\mathrm{h}_{\mathrm{W}}$ can be identified as the pressure at which the largest group of pore bodies fill. From Figure (2.9) and Equation 26, this indicates that the largest pores in the medium are of radius $r_{\max }=2 \sigma /$ $h_{w}$. Similarly, the pressure $h_{a}$ is dictated by the size of the typical pore throats, which have a radius of approximately $2 \sigma /$ $h_{\mathrm{a}}$.

Why doesn't the soil drain completely, and have point \#5 in Figure (2.8) return to the dry state? There are two phenomena which account for this behavior, with relative magnitude depending on the chemical and physical properties of the medium. First, any water which entered into chemical bonds with charged surfaces in the medium cannot be removed through simple fluid tension: the bonds holding them are as strong as surface tension, and so they cannot be ripped away by the bulk fluid. Furthermore, even in an inert porous medium, fluid can be held in the very small radius regions at particle contacts. These isolated donut shaped pockets of water, referred to as "pendular," remain in place, no longer hydraulically connected to the fluid reservoir (as shown in Figure 2.10 (d)). 

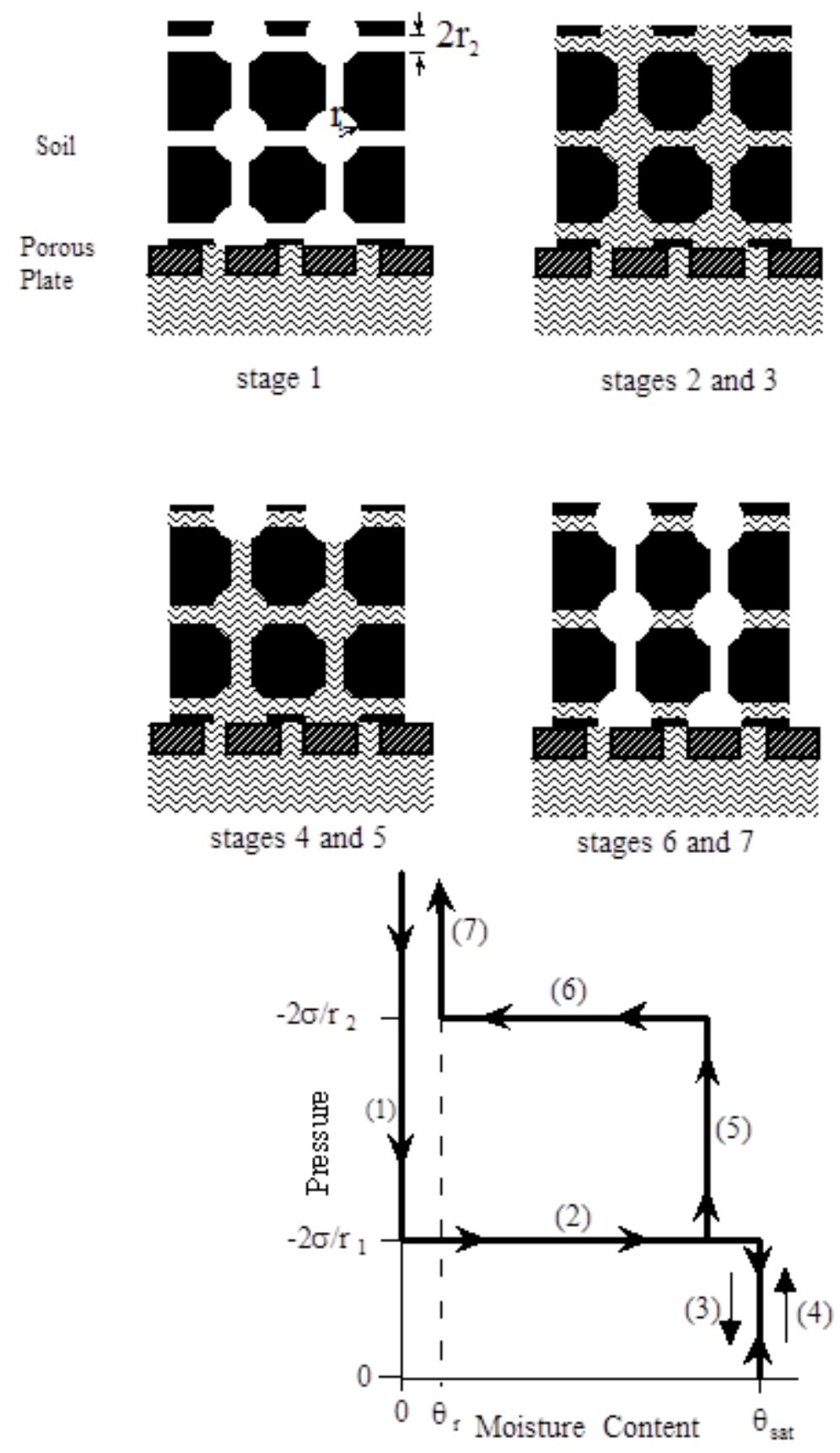

Figure 2.10. Illustration of a "Haines Jump" cycle for a set of equal size pores with equal size necks connecting them. In (1) we see that no water enters until the head becomes greater than $-2 \sigma / r_{1}$. When this pressure is exceeded, the pores will suddenly fill (2). In the draining process ((4), (5), and (6)) first the outer pores will drain, and finally, when the head becomes less than $-2 \sigma / r_{2}$ all but the isolated pores (shown as the horizontal ones here) will suddenly drain as air can finally enter the necks.

\section{Contact Angle; Sources and Effects}

The main hysteretic mechanism illustrated in Figure 2.10 is referred to as the "ink bottle effect," due to the similarity of the behavior of bottles of ink with very narrow spouts. Another source of hysteretic behavior is hysteresis in the contact 
angle. Although contact angle was mentioned before in passing, it is now time to take a closer look at this interesting corner of fluid behavior.

Figure 2.11 illustrates a cross-section of a straight-line contact between a fluid and a solid. What should the angle of contact between the solid and fluid be, and why? Well, since the system is at equilibrium, we should be able to balance the forces at the point of contact. Considering only the component of forces acting in the horizontal direction, we find

(a)

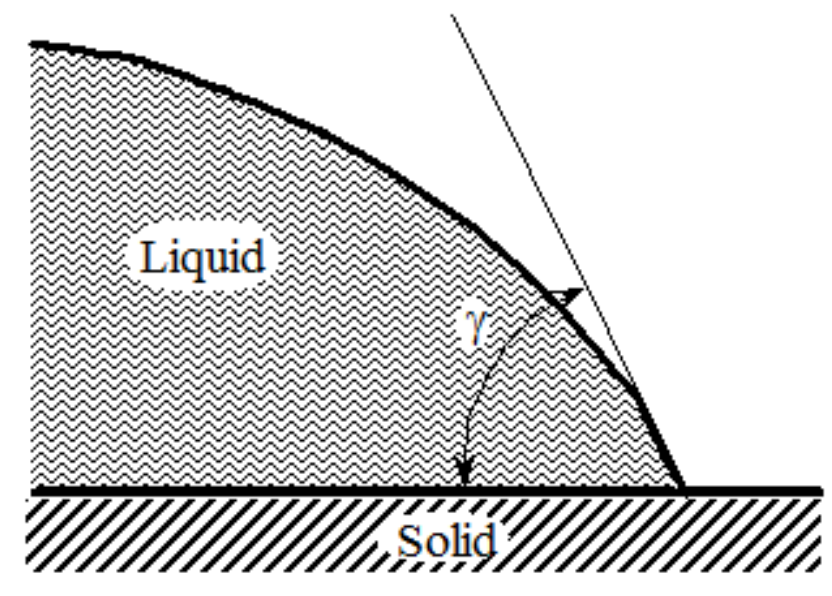

(b)

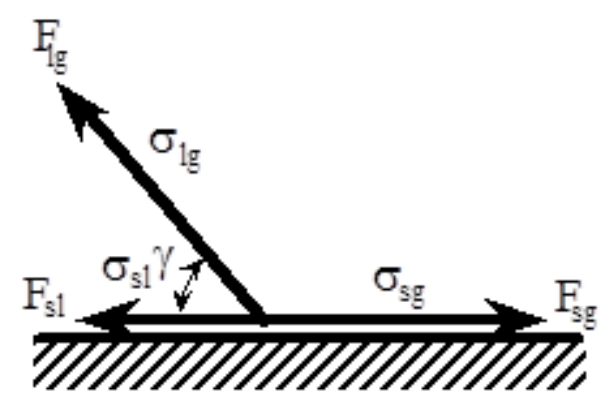

Figure 2.11. (a) A Cross-section of a line contact between a solid plane with a line of fluid/air contact at equilibrium. (b) The force balance at the point of contact.

(62) $\Sigma \mathrm{F}=0=\mathrm{F}_{\mathrm{sl}}-\mathrm{F}_{\mathrm{sg}}+\mathrm{F}_{\mathrm{lg}} \cos \gamma$

where $\mathrm{F}_{\mathrm{sg}}$ is the solid-gas surface force per unit length, $\mathrm{F}_{\mathrm{sl}}$ is the solid-liquid force, $\mathrm{F}_{\mathrm{lg}}$ is the liquid-gas surface force and $\gamma$ is the contact angle. Per unit length, $\mathrm{F}_{\mathrm{ij}}=\sigma_{\mathrm{ij}}$, so this may be put in terms of the relative surface tensions

(63) $\sigma_{\mathrm{sg}}=\sigma_{\mathrm{sl}}+\sigma_{\mathrm{lg}} \cos \gamma$

which is the Youngs-Laplace equation; the relationship between the contact angle and the three surface tensions. Solving [2.56] for the contact angle we find

$$
\gamma=\cos ^{-1}\left(\frac{\sigma_{\mathrm{sg}}-\sigma_{\mathrm{sl}}}{\sigma_{\mathrm{lg}}}\right)
$$

Care needs to be taken when applying these results, as there are some physical limits on possible values of $\gamma$ : the contact angle is bounded by $0 \leq \gamma \leq 180$. So if the operand of $\cos ^{-1}$ is greater than 1 , then $g$ will be $0^{\circ}$, while if the value is less than -1 , the value will be $180^{\circ}$. Since it is often true that $\left(\sigma_{\mathrm{sl}}-\sigma_{\mathrm{sg}}\right)>\sigma_{\mathrm{lg}} \backslash$ upsigma_\{lg\}\}" title="Rendered by QuickLaTeX.com" height="20" width="125" style="vertical-align: -6px;"> for water, the contact angle for water going into geologic material is often taken to be $0^{\circ}$. 

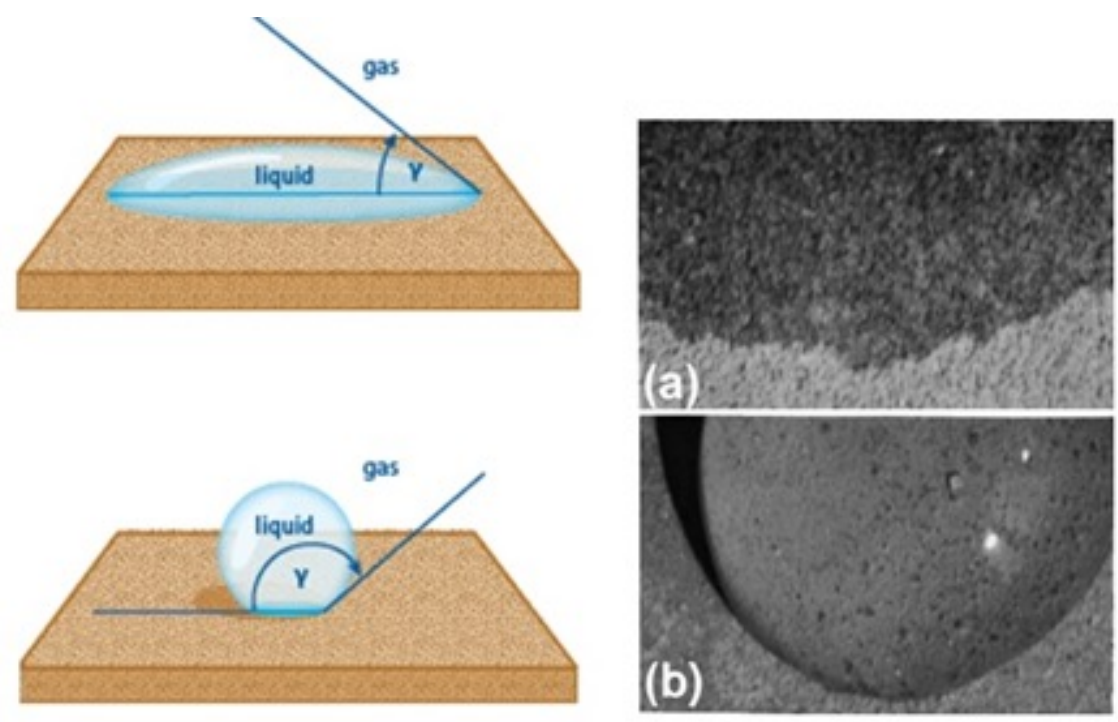

Figure 2.XX: Illustration of wetting and non-wetting surfaces. (a) Wettable silt soil surface $\left(\gamma \sim 0^{\circ}\right)$ (b) Treated waterrepellant silt soil $\left(\gamma \sim 70^{\circ}\right)$ (Bachmann et al., 2000) ]

Now how does this contact angle relate to hysteresis? As shown in Figure 2.12, the contact angle often exhibits a different value in the advancing and receding cases. This is the reason a drop of water on a flat plate will not start to move as soon as you tilt the plate: more energy is required to remove the water from the trailing edge of the plate than is given up by the sum of the gravitational potential plus the energy released wetting the plate. This is sometimes referred to as the rain-drop effect, perhaps referring to how a raindrop moves down a window pane. The relevance of the raindrop effect to capillary hysteresis is simply an extension of the observation regarding the plate and drop: a medium will retain water more vigorously than it will absorb water (Bartell and Wooley, 1933).

That this rain-drop effect exists is well known to any child who has bemoaned a rainy day at the window (without it all the droplets would run off the pane), but what is the physical basis? Consider the effect which might result from a scratch on a solid surface as shown in Figure 2.13. At the microscopic level the Youngs-Laplace equation is adhered to, but from a macroscopic point of view, the drip cannot advance until the apparent contact angle is quite large. Similarly, upon retreat the macroscopic contact angle will be much smaller than the true microscopic magnitude (Figure 2.13). The same pair of effects would result from a smudge of oil or other contamination which caused a local reduction in the microscopic contact angle. 

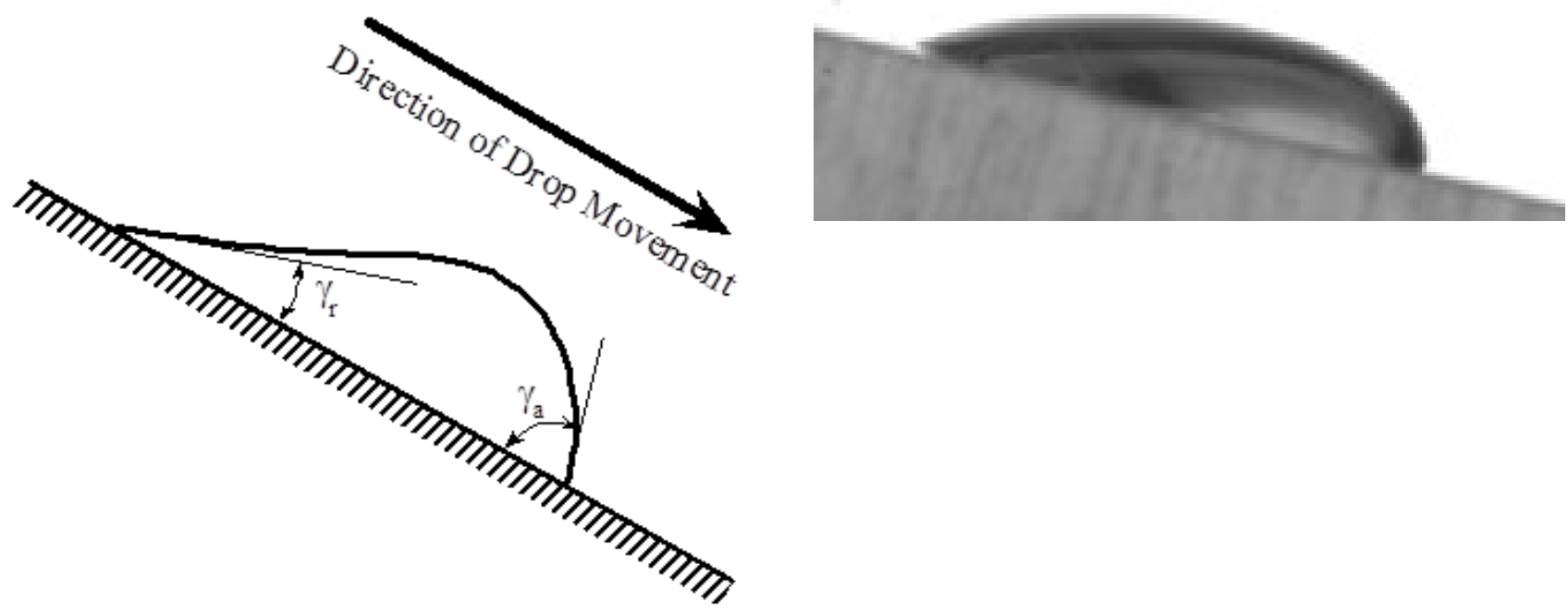

Figure 2.12. The rain-drop effect of contact angle hysteresis between advancing and receding contact angle, geometrically (left) and as seen (right). 


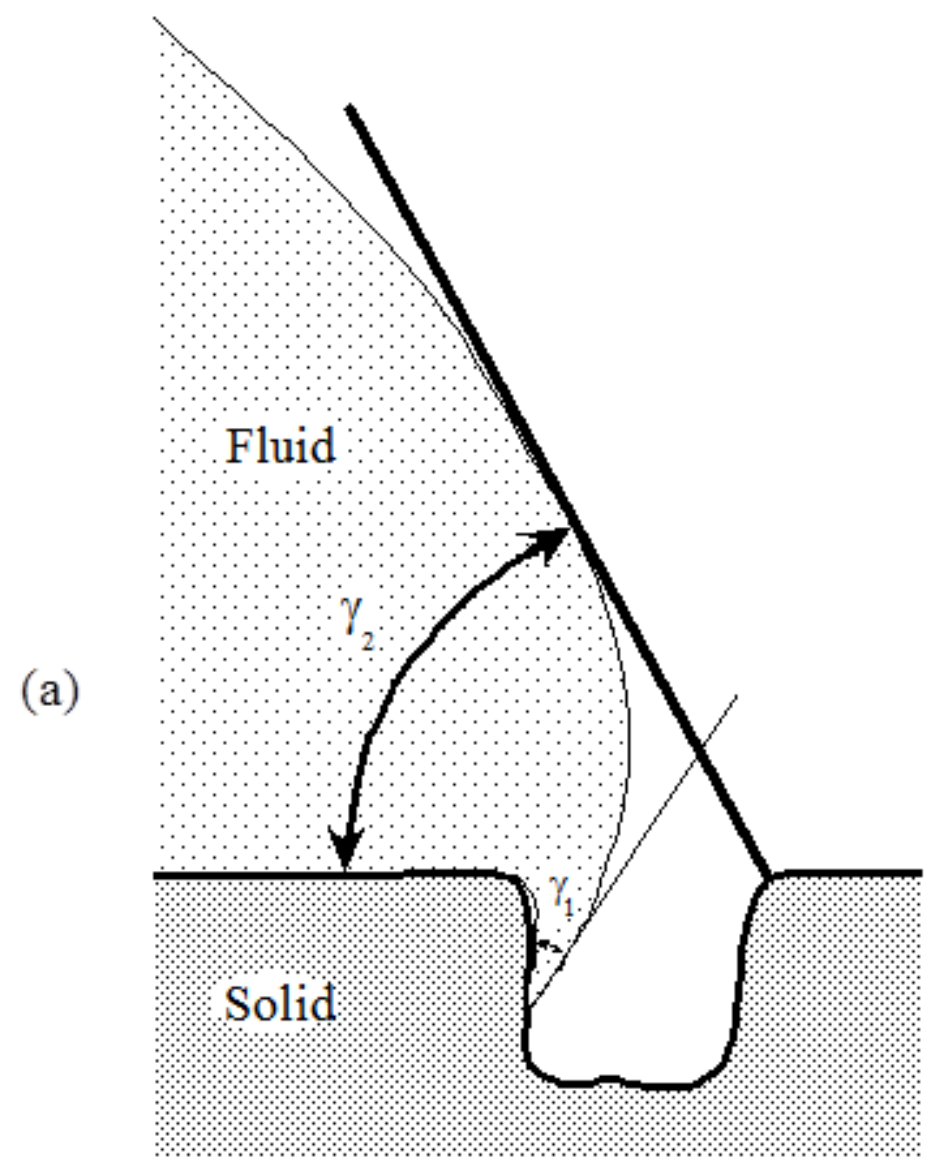

(b)

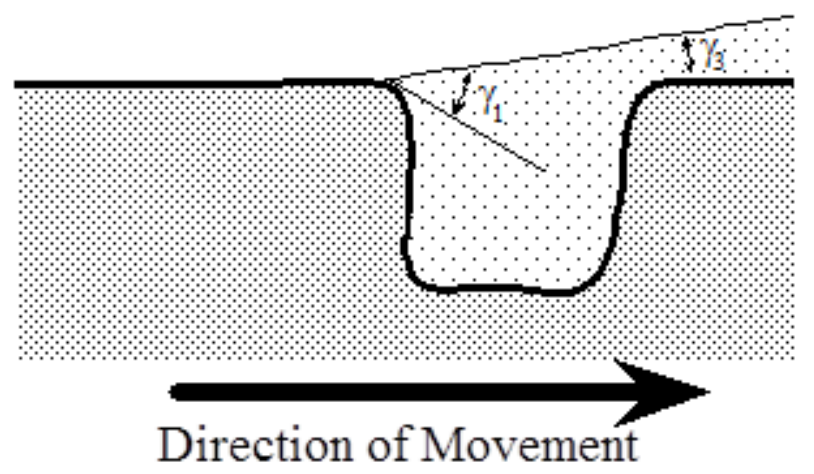

Figure 2.13. Illustration of the effect of a microscopic defect on the apparent contact angle for advancing and receding fluid. Although the microscopic contact angle is constant $\left(\gamma_{1}\right)$, the advancing macroscopic contact angle is $\gamma_{2}$ and the receding wetting front with macroscopic contact angle $\gamma_{3}$ as a result of the surface defect.

A fine cylindrical tube (capillary tube) is often taken as the simplest model for capillary attraction. Given its prominence in describing capillary processes, and because it is a useful conceptual model, we now discuss this system. Figure 2.14 shows two possible capillary tubes. The tube on the left has a zero-contact angle between the tube and the liquid, while the tube on the right has a non-zero contact angle. 


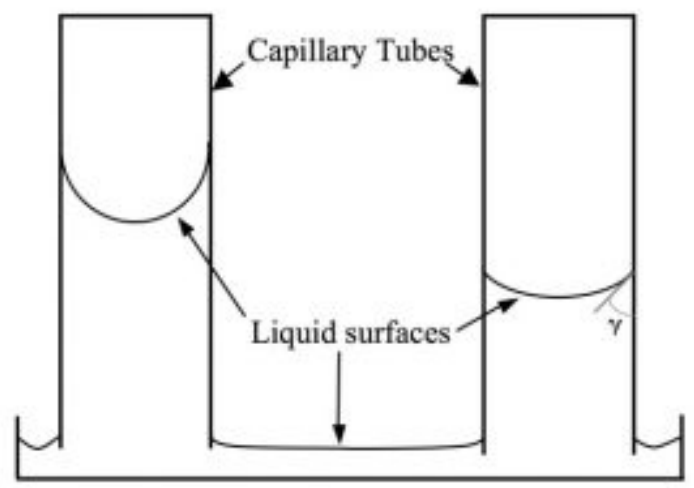

Figure 2.14. Examples of capillary tubes or radius $r$ drawing up liquid from a reservoir. (a) With the same liquid, but capillaries with different contact angle (the tube on the left has a zero contact angle between the liquid and tube and the right tube is non-zero) we see the capillary rise is reduced; (b) The maximum capillary rise is dictated by the relative surface energy of the air-solid and liquid-solid interfaces. If the gas-liquid interface had infinite energy, the interface in the tube would be a straight line with a 90-degree contact angle and the interface at the height dictated by the solid interfacial energies. If it happened that the solid-gas energy was very high, the liquid would coat the entire inside of the tube, and the visible gas-liquid interface would have a zero contact angle to the solid. Intermediate values of the gas-liquid interfacial energy give rise to contact angles between 0 and 90 degrees. The lowest interface shown in (b) illustrates a reduction in solid-gas interfacial energy (which reduced the capillary rise), but yet with a high gas-liquid interfacial energy, meaning the contact angle is between 0 and 90 degrees.

So why does the water go up into the tubes? Because energy is liberated when this liquid coats solid surfaces. The ultimate height of the capillary surface is that which balances the energy required to pull the liquid up against gravity and the energy liberated when the liquid coats the solid surfaces. Thus, the derivative of the gravitational energy and surface tension terms are equal. The gravitational $\left(E_{G}\right)$ and surface $\left(E_{S}\right)$ energies of the system are

$$
\mathrm{E}_{\mathrm{G}}=\int_{0}^{\mathrm{h}} \rho \mathrm{g} \pi \mathrm{r}^{2} \mathrm{~h}^{\prime} \mathrm{dh}^{\prime}=\frac{\rho g \pi \mathrm{r}^{2} \mathrm{~h}^{2}}{2}
$$

and

$$
\mathrm{E}_{\mathrm{S}}=\int_{0}^{\mathrm{h}} 2 \pi \mathrm{r}\left(\sigma_{\mathrm{sl}}-\sigma_{\mathrm{sg}}\right) \mathrm{dh}^{\prime}=2 \pi \mathrm{r}\left(\sigma_{\mathrm{sl}}-\sigma_{\mathrm{sg}}\right) \mathrm{h}
$$

taking the reservoir surface as a datum, for liquid of density $\rho$. A stable elevation demands that the rate of change in these equal and opposite with change in height, so we find

$$
\rho g \pi r^{2} h=-2 \pi r\left(\sigma_{\mathrm{sl}}-\sigma_{\mathrm{sg}}\right)
$$

or

$$
\mathrm{h}=-\frac{2\left(\sigma_{\mathrm{sl}}-\sigma_{\mathrm{sg}}\right)}{\rho g r}
$$


Which is quite akin to our good friend the Laplace equation, but only the same if . The tricky part here, which is often unappreciated, is that the gas-liquid interfacial energy does not show up in this result, nor does the contact angle. Many people write 67 in terms of the gas-liquid interfacial energy and the contact angle using ?? but this really obscures the important physical nature of the process, as pointed out by Selker and Schroth (1998).

We should look more closely at the case of the zero contact angle, which contains two special cases: (1) $\sigma-\sigma_{\mathrm{sg}}=\sigma_{\mathrm{lg}}$ and (2) $\sigma-\sigma_{\mathrm{sg}}=\sigma_{\mathrm{lg}}$. In case (1), the result of $\underline{67}$ is applicable. But in the case (2), when the surface energy of the gas-liquid interface is relatively small, the liquid will spontaneously spread in a thin film over the entire inner surface of the capillary tube. Now the capillary rise of the liquid in the tube is simply a matter of the pressure which is generated by a curved film of water that is tangential to the walls of the tube. In this case the radius of the curved surface is the radius of the tube wall and the pressure is given by Laplace's equation for a spherical surface $\underline{42}$ ).

\section{The Independent Domain Model of Hysteresis}

If you confine yourself to the conceptual model presented in Figure (2.10), you will always be on either the main wetting or main draining curve, but Figure (2.8) shows that if one stops draining at some point short of $\theta_{\mathrm{r}}, \theta\{\Psi\}$ will depart from both the main wetting and main draining characteristic curves. This can be understood and accounted for through a simple extension of Figure (2.10). We noted that the key features of filling and draining of a single pore had to do with that pore's neck and body radii, which we will refer to as $r_{n}$ and $r_{b}$ respectively. With this in mind, consider categorizing a medium by neck and body pore radius. As before, we will immediately translate these radii into equivalent capillary pressures via equation 26. So the idea is that we can characterize any medium as the aggregation of independent pores, each of which has a characteristic filling pressure $\left(\mathrm{h}_{\mathrm{f}}\right)$ dictated by the body radius and emptying pressure $\left(\mathrm{h}_{\mathrm{e}}\right)$ controlled by the neck radius. [Although we appeal to equation $\underline{26}$ as a motivation, we can simply measure the filling and emptying pressures, so this is really just a crutch for visualizing the system.] Finally, we assume each pore is hydraulically connected to the bulk medium so that if a pressure is established at one of the medium's boundaries, all the pores will experience that pressure. Each pore responds to changes in pressure independently (without regard to the state of the surrounding pores). This perspective was brought to vadose hydrology by A. Poulovassilis (1962).

The essence of this assumption is that the filling and draining of each pore is determined strictly by that pore's geometry, regardless of the connection of that pore to surrounding pores (thus the term "independent"). This assumption is certainly fine for the wet end of the characteristic curve, when water fills most pores, but when the medium is quite dry it is possible the pores will become isolated hydraulically by being surrounded by empty pores. In this case the independent domain assumption breaks down for the isolated pore since it cannot drain regardless of the level of pressure exerted on the boundaries of the sample. We may expect then that the independent domain assumption will not lead to good predictions of residual moisture content, which must be carefully defined based on the process of interest, as discussed in section 2.2 . 


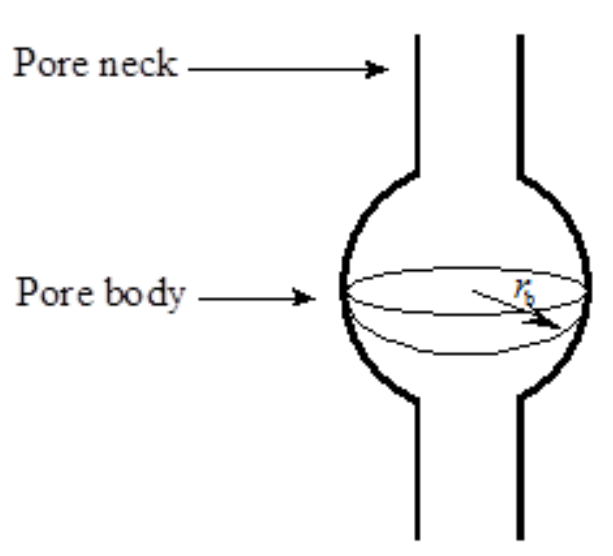

Filling

$$
\mathrm{h}_{\mathrm{f}}=\frac{2 \sigma}{\mathrm{r}_{\mathrm{b}}}
$$

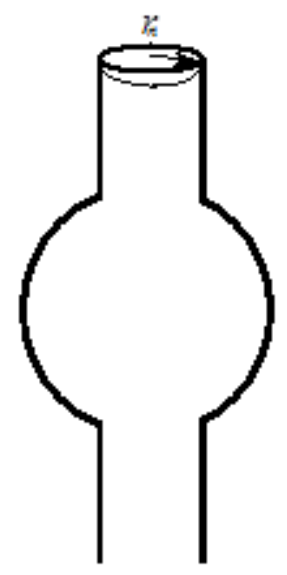

\section{Emptying}

$$
\mathrm{h}_{\mathrm{e}}=\frac{2 \sigma}{\mathrm{r}_{\mathrm{n}}}
$$

Figure 2.15. Cartoon of the characteristic radii for a pore and that pore's characteristic filling and emptying pressures.

Pores will have a range of magnitudes for he and hf which can be described by a joint probability density function, as plotted in Figure 2.16. Note that the distribution functions for $h_{e}$ and $h_{f}$ are not independent, since pores with very small necks are more likely to have similarly small bodies. The total volume under the curve in Figure 2.16 is 1 , corresponding to the fact that all pores will have some combination of the two characteristic radii, which is stated mathematically in equation $\underline{68}$.

$$
\int_{0}^{\infty} \int_{0}^{\infty} f\left(h_{e}, h_{f}\right) d h_{e} d h_{f}=1
$$

Pore necks are, by definition, smaller than pore bodies, so the absolute value of he is necessarily larger than $h_{f}$ for a given pore. So we may draw a line at $45^{\circ}$ on the $h_{e}-h_{f}$ plane and know that all pores will lay below that line, as indicated in Figures 2.16 and 2.17. 


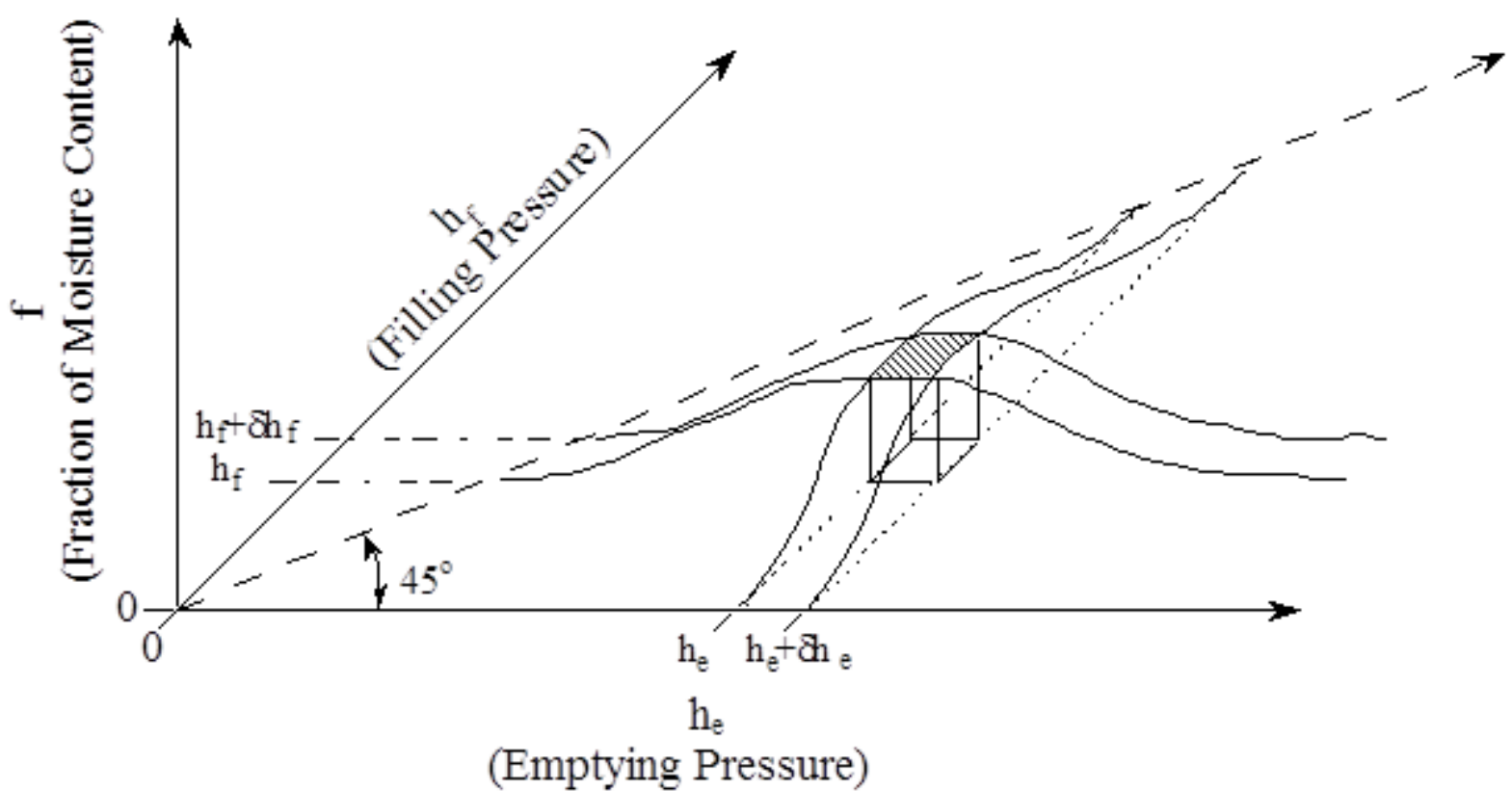

Figure 2.16. Illustration of the probability density function moisture content as a function of filling and emptying pressures.

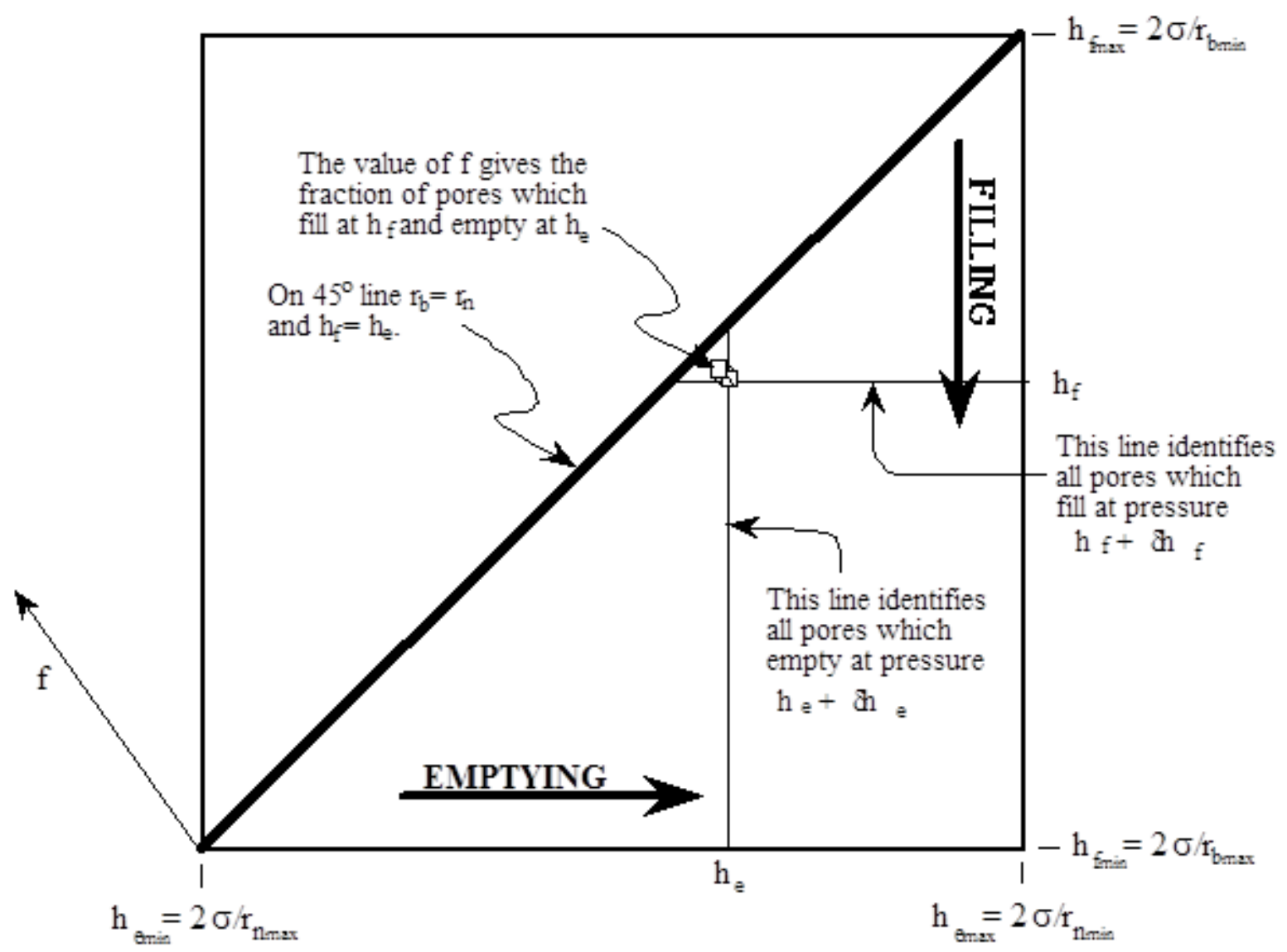

Figure 2.17. Identification of key features of the joint density function employed in the independent domain model of hysterisis given in Figure 2.16. The radius of the pore body is $r_{\mathrm{b}}$ and that of the neck radius is $r_{\mathrm{n}}$. 
To understand the density function plot more intuitively, it is useful to consider the physical interpretation of several of the points indicated in Figure 2.17. You see that the end-points of the pressure scales are defined by the largest and smallest bodies and necks. The appeal to Laplace's equation here is primarily heuristic: the exact radii identified are not what you would find under a microscope, but provide a useful conceptual model. If we consider some horizontal line with a value $\mathrm{h} 1$ going from the right boundary $\left(\mathrm{h}_{\mathrm{e}_{\max }}\right)$ to the $45^{\circ}$ line, this delineates all of the pores which fill at pressure h1. Since the filling pressure is determined by the body radius, we can consider these to be the pores with some body radius $r_{b}$ such that $h_{f}=2 \sigma / r_{1}$. Similarly, the vertical line shown in Figure 2.17 at pressure he defines all the pores which empty at pressure he. Realizing that the pore neck diameter determined the draining pressure, we can again associate all of these pore as having neck diameters of rn such that $h_{e}=2 \sigma / r_{n}$. The intersection of these two lines corresponds to the pores which fill at $h_{f}$ and empty at he, and the height of $f$ at this point indicates the fraction of pores which have this particular set of characteristic radii.

With this understanding of how to organize filling and emptying of pores, it is straightforward to obtain the various characteristic curves (once you have determined the joint density function). Applying this method boils down to figuring out the range over which to integrate the density function. As an example, consider the series of characteristic curves shown in Figure 2.8 within the context of the independent domain model. Starting from point 1 where the medium is dry, all the pores are empty. So now we will go from $h=-\infty$ to $h=0$. Through this sequence of pressures we want to add up all the pores which fill regardless of their emptying pressure. Thus we sweep downward through hf adding up all the pores with emptying pressures between $\mathrm{h}$ to $-\infty$, integrating the density function as we go. This first wetting process is illustrated in Figures 2.18a and 2.18b.

Having saturated the sample, to obtain the main draining curve we follow the same procedure. This time the pressure starts at 0 and becomes more negative. Since all the pores are initially filled, we need to integrate along vertical lines all the pores which empty at a given pressure regardless of the pressure at which they filled (Figure 2.18c). 
Filling from 1 to 2

(a)

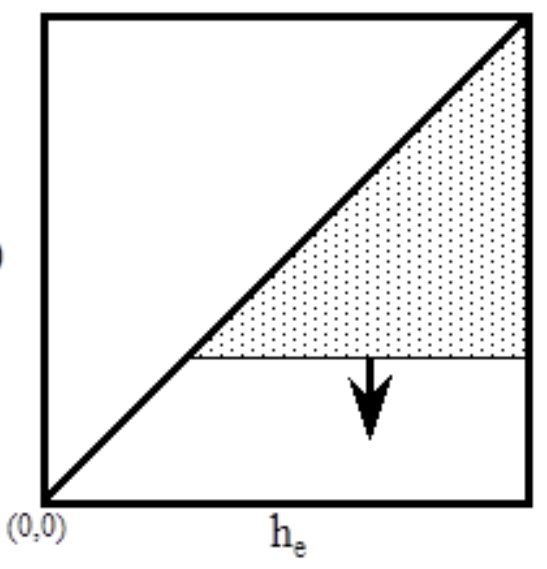

$h_{f}$

\section{Emptying from 3 to 6}

(c)

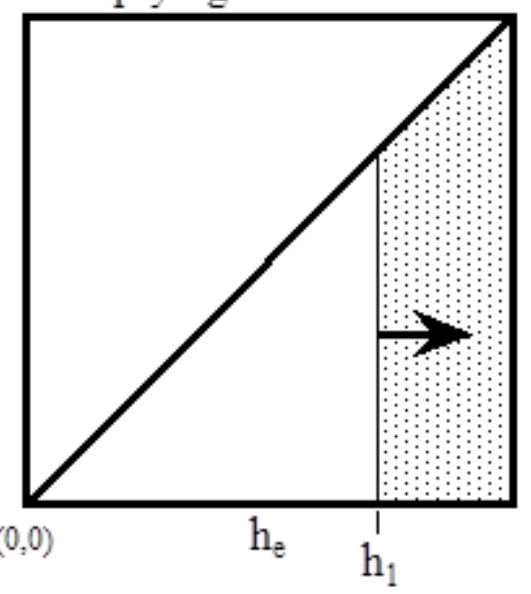

(b)

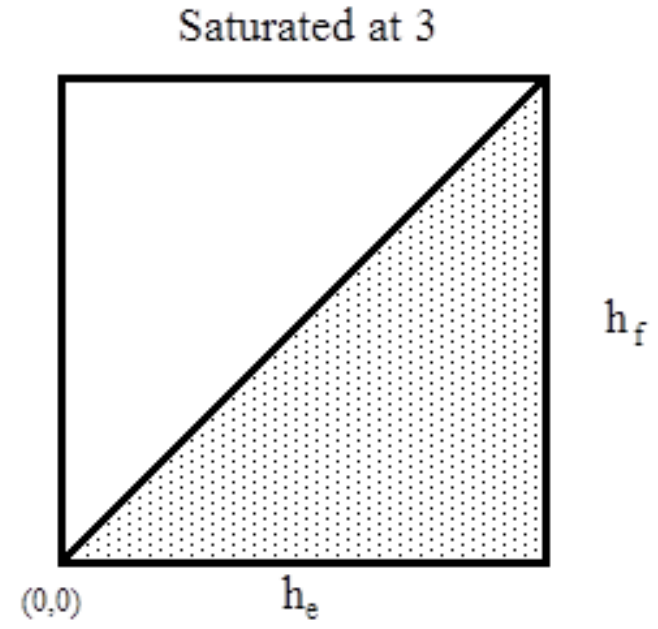

Filling from 6 to 7

(d)

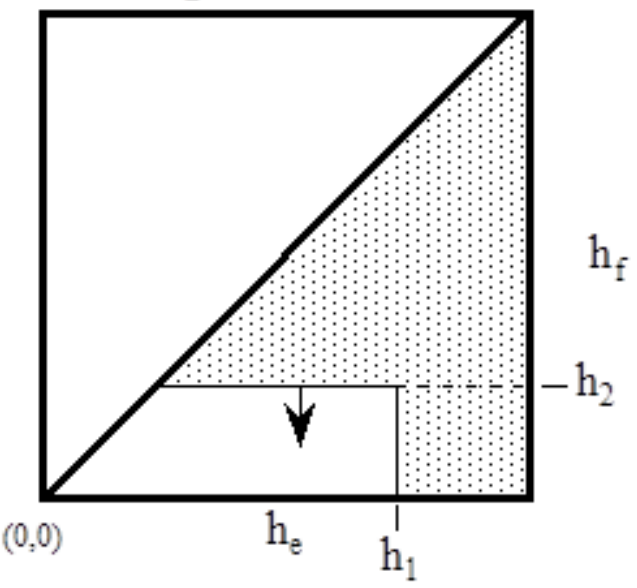

(e)

\section{Emptying from 7 to 8}

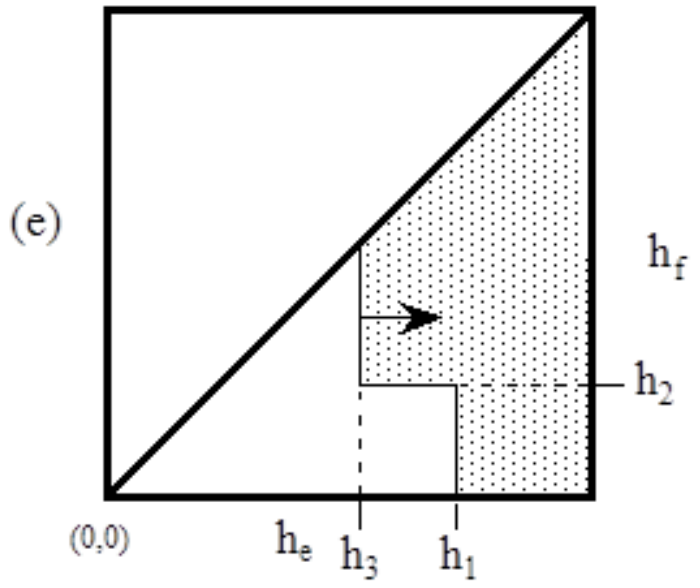

Figure 2.18. Mapping of the water filled pores through the sequence of wetting and draining characteristic curves presented in Figure 2.8.

Things get interesting when we stop prior to emptying all the pores, and turn around and start to re-fill the medium. Pores which are already filled with water cannot be refilled, which is the only feature which has changed from the 
main filling procedure. We still sweep down with a horizontal line, but now we only add the pores which fill between pressures $h_{1}$ and $h_{f}$ (Figure 2.18d). Figure 2.18e continues this sequence, now following the draining process. So once the joint density function is known, it is simply a matter of determining which area of the domain to integrate over to determine the moisture content for any sequence of pressure changes. This procedure is quite amenable to numerical implementation.

This procedure can be stated mathematically as integrating over the domain of filled pores. We set up a few of the integrals which would be evaluated in Figure 2.8 as examples. In the main wetting process (Figure 2.18a), we simply carry out the integral

$$
\theta(\mathrm{h})=\int_{-\infty}^{h} \int_{-\infty}^{h_{f}}\left\{\left(h_{e}, h_{f}\right) d h_{e} d h_{f}\right.
$$

Supposing we take the system to saturation, and now start draining the medium to obtain the main draining curve. Having filled all the pores to start with, we must calculate how much has been drained, and subtract that from 1 :

$$
\theta(\mathrm{h})=1-\int_{0}^{\mathrm{h}} \int_{0}^{\mathrm{h}_{\mathrm{e}}} \mathrm{f}\left(\left(\mathrm{h}_{\mathrm{e}}, \mathrm{h}_{\mathrm{f}}\right) \mathrm{dh}_{\mathrm{f}} \mathrm{dh}_{\mathrm{e}}\right.
$$

Suppose now that we stop draining at a pressure $\mathrm{h}_{1}$ and start refilling (Figure 2.18d). For convenience, let's define the moisture content at the completion of the draining as

$$
\theta\left(h_{1}\right)=\int_{-\infty}^{h_{1}} \int_{-\infty}^{h_{\mathrm{f}}}\left\{\left(h_{\mathrm{e}}, \mathrm{h}_{\mathrm{f}}\right) \mathrm{dh}_{\mathrm{e}} \mathrm{dh} \mathrm{h}_{\mathrm{f}}\right.
$$

In refilling, only the pores which were empty should be counted. So the integral will be restricted to the range of emptying pressures from $\mathrm{h}_{\mathrm{f}}$ to $\mathrm{h}_{6}$ :

$$
\theta(h)=\theta\left(h_{1}\right)+\int_{h_{1}}^{h} \int_{h_{f}}^{h_{1}} f\left(h_{e} h_{f}\right) d h_{e} d h_{f}
$$

The only trick here is to keep careful track of the range of integration, with some bookkeeping: you have to keep track of the turning points of the soil's history. By turning points, we mean the occasions when the soil either stopped wetting and began to dry, or vise-versa (i.e., there was a change in sign in the time derivative of the moisture content). A notation for recording the turning points was presented by Enderby (1955), and adopted by Everett (1955) and Mualem (1974)

$$
\theta\{h\}=\theta\left\{\begin{array}{ll}
h_{1} & h h_{0} \\
h_{2} &
\end{array}\right\}
$$

Figure 2.19. Experimental primary drying scanning curves (solid lines) and theoretical points obtained using the independent domain model (small circles). Also included is the joint density function for this medium. Reproduced with permission from Poulovassilis (1962).

In this notation, subscripts denote the order of pressures, and relative position indicates whether the transition was wetting or drying. In the case shown, the medium wetted from $h_{0}$ to $h_{1}$, dried to $h_{2}$, and then re-wetted to the present pressure h.

In $\underline{73}$ we have written the relationship between pressure and moisture content using curly brackets \{\} . Later in this text, and in general in the literature, this relationship is written as a function with regular parentheses (), but this really is inappropriate, as $\theta$ is not a function of $\mathrm{h}$ but is a functional of $\mathrm{h}$. That is to say, there is not a one-to-one mapping between $\theta$ and $\mathrm{h}$ without consideration of the antecedent conditions. Of course we can relate $\theta$ and $\mathrm{h}$ from a known initial state and through a known sequence of either $\theta$ or $h$ as stated in $\underline{73}$, without requiring any knowledge of the other independent variables (most notably time). The ability of this model to reproduce hysteretic scanning curves is demonstrated in Figure 2.19 which is reproduced from Poulovassilis (1962). 
So we have set up the conceptual framework for this method of modeling hysteresis, but how do we obtain the joint density function practically? You can just carry out a terrific number of experiments where you map out the entire domain of possible filling and draining pressures to obtain $f\left(h_{e}, h_{f}\right)$ by brute force. While this would appear to be impractical, with numerically controlled valves and scales, this is not infeasible using an automated pressure cell. However, in 1973 Mualem introduced a simplification of this model which greatly eased the task of determining the density function.

Mualem (1973) noted that the joint density function $\mathrm{f}\left(\mathrm{h}_{\mathrm{e}}, \mathrm{h}_{\mathrm{f}}\right)$ could be well approximated by the product of two univariate density functions

(74) $\mathrm{f}\left(\mathrm{h}_{\mathrm{e}} \mathrm{h}_{\mathrm{f}}\right) \approx \mathrm{g}\left(\mathrm{h}_{\mathrm{e}}\right) \mathrm{l}\left(\mathrm{h}_{\mathrm{f}}\right)$

where $\mathrm{g}()$ and $\mathrm{l}()$ are probability density functions that depend only on $\mathrm{h}_{\mathrm{e}}$ and $\mathrm{h}_{\mathrm{f}}$, respectively. This constrains, for instance, the filling pressure distributions to be the same, up to a constant multiplier, along any draining pressure line, as illustrated in Figure 2.20. Using Mualem's formulation, we only need the main filling and emptying curves to obtain g() and 1(), and from these results all the hysteretic results can be derived. Mualem showed that this formulation maintained its ability to reproduce scanning curves (Figure 2.21).

Parlange (1976) went on to show that an even more constraining similarity model based on data from the main draining curve alone is sufficient to reproduce the full family of scanning curves. This was demonstrated to be capable of fitting experimental data by Hogarth et al. in 1988, as shown in Figure 2.22, reproduced from their work.

We have gone into a fair bit of detail in how to handle hysterisis, but have not mentioned when and why it must be included when describing vadose zone processes. First off, to understand the general character of many processes, dealing specifically with hysterisis is not critical. For instance in irrigated agriculture, as long as you know the moisture content at field capacity and the permanent wilting point, you may not worry about the exact pressure the soil achieves at intermediate water contents. On the other hand, hysterisis has been shown to be central to the nature of fingered flow processes (see Chapter 3), as well as the movement of NAPLs (e.g., Lenhard and Parker, 1987; Lenhard et al., 1991). Beyond these very specific settings the literature does not provide general guidance on when and where hysterisis is most important. Beyond the examples given, we really don't know when hysterisis will play a central role.

If hysterisis has been shown to be important to several important processes, and in general we can't be sure hysterisis will not be influential, why haven't more people included consideration of hysterisis in the description of vadose zone processes? We think this is simply a matter of practical constraints. None of the analytical solutions to flow, and precious few of the numerical codes for modeling flow in the vadose zone consider the possibility of hysterisis. Until we have convenient numerical codes which include hysteretic effects, the possible effects of hysterisis will continue to be ignored in quantitative analyses. Of course nature doesn't care if hysterisis is hard to model or easy to model: it happens! Failing access to a useful model which includes hysterisis, a responsible approach is to qualitatively assess the potential impact of hysterisis on a problem. Given the rapid increase in availability and sophistication of numerical models, we believe that hysterisis will be included in many popular models in the coming decade, and is in fact available in research models at this time (White et al., 1995). 


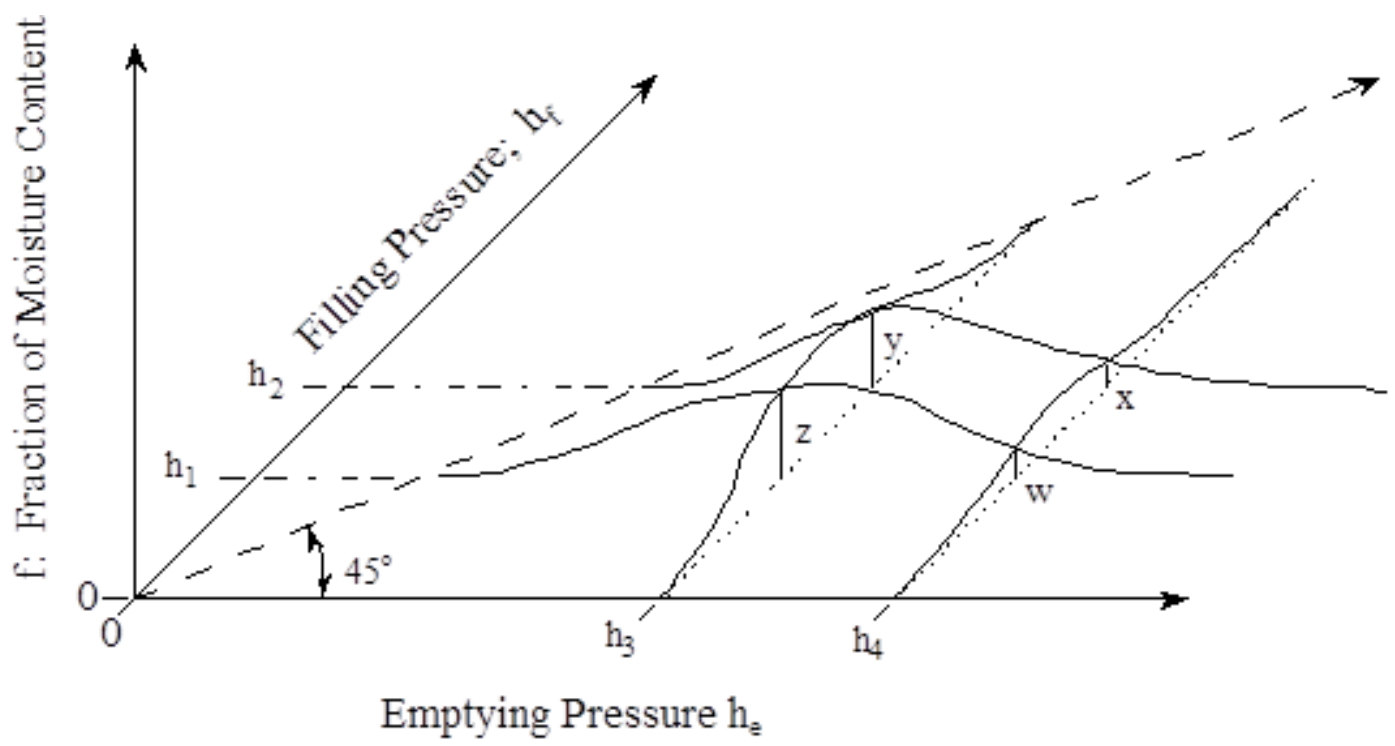

Figure 2.20. Illustration of the implication of the similarity assumption introduced by Mualem (1973) for the independent domain emptying and filling density function.

Geometric Relations of Mualem Similarity:

$$
\begin{aligned}
& \text { Emptying pressure: } \frac{\mathrm{x}}{\mathrm{y}}=\frac{\mathrm{g}\left(\mathrm{h}_{4}\right)}{\mathrm{g}\left(\mathrm{h}_{3}\right)}=\alpha \text { and } \mathrm{f}\left(\mathrm{h}_{4}, \mathrm{~h}\right)=\alpha \mathrm{f}\left(\mathrm{h}_{3}, \mathrm{~h}\right) \text { for any } \mathrm{h} \\
& \text { Filling Pressure: } \frac{\mathrm{z}}{\mathrm{y}}=\frac{\mathrm{l}\left(\mathrm{h}_{1}\right)}{\mathrm{l}\left(\mathrm{h}_{2}\right)}=\beta \text { and } \mathrm{f}\left(\mathrm{h}, \mathrm{h}_{1}\right)=\beta \mathrm{f}\left(\mathrm{h}, \mathrm{h}_{2}\right) \text { for any } \mathrm{h}
\end{aligned}
$$

Figure 2.21. Mualem's demonstration of the ability of the independent domain model with the similarity assumption to reproduce experimental scanning curves found by Poulovassilis (1970) (Reproduced from Mualem, 1974, with permission).

Figure 2.22. Primary draining curves as found experimentally and reproduced using the Parlange similarity model form of the independent domain model (Reproduced from Hogarth et al., 1988).

\section{[Insert "Measurement of water potential" here]}

\section{Measurement of Water Content}

The ability to accurately measure water content in time and space is central to the study of vadose zone hydrology. Remarkably, the ability to measure this critical parameter continues to present many challenges, with significant developments continuing to come forward.

The benchmark for water content measurements is the direct determination by oven drying (Gardner, 1986). In this method a sample of soil is gathered from the field and weighed. It is then placed in a $100-110^{\circ} \mathrm{C}$ oven until it's weight has stabilized. The mass basis water content may then be computed using $\underline{8}$. Though simple to describe, even this method is subject to significant error and interpretation. For instance, if stones are avoided, either as an unplanned feature of the method of collection or by design, the value of water content obtained will be systematically higher than the true field value. Furthermore, much of the water bound to clay remains in place at these temperatures. Thus you are not obtaining the total water content, but that which can be liberated under these specific conditions. Further discussion of these 
issues is presented by Gardner (1986). Perhaps the most problematic aspects of this method are that it is destructive and cannot be completed in the field in a timely manner which renders the method inappropriate for many applications.

There are a variety of field methods, all of which use indirect measurements of bulk soil physical properties to infer a moisture content. We will discuss three methods that illustrate the key physical properties that can be related to water content. These are the neutron thermalization method (neutron probe), the gamma ray attenuation method (gamma probe) and the dielectric method (time domain reflectometry).

Neutron Probe

The neutron probe has long been the most accurate method of measuring water content in the field. The method relies on the thermalization, or slowing, of neutrons. Relatively high energy neutrons are directed into the medium from a radioactive source, with a detector provided to observe the resulting number of slow neutrons that are back-scattered. These neutrons then collide with the various atoms in the soil. The loss of energy which occurs upon each collision is far greater when the collision is with an object of mass similar to the neutron. For instance, in a single collision the loss of energy from a neutron to hydrogen is about 4,000 time that of a collision with oxygen (Weinberg and Wigner, 1958). So the presence of hydrogen is closely correlated with the production of thermalized neutrons.

Since water is the largely hydrogen, the rate of thermalized neutron production can be calibrated to the surrounding volumetric water content. Site calibration is required to account for the local effects of the mineral and organic components and structurally bound water in the soil which will also contribute to neutron thermalization. The calibration equation is typically written in the form

(75) $\theta=a+b\left(I / I_{0}\right)$

where $\theta$ is the volumetric water content, $\mathrm{I}_{\mathrm{o}}$ is the incident neutron flux, I is the thermalized neutron count, and $\mathrm{a}$ and $\mathrm{b}$ are calibration parameters that depend upon the neutron source strength, soil properties, and detector characteristics. Since the soil characteristics will vary significantly in the features which will effect this calibration (e.g., organic matter), proper calibration must be carried out for each measurement location. This presents some logistical difficulties. First, the measurement must be taken at least two moisture contents to be able to obtain the two parameters. Therefore one must either come to the field twice, under differing moisture regimes, or one must carry out the calibration in the lab. Since the calibration requires a volume of soil at least as large as the instrument's sphere of influence (about $15 \mathrm{~cm}$ in wet soil and $75 \mathrm{~cm}$ in dry soil), and in light of the sensitivity to bulk density, it is impractical to obtain sufficient samples to calibrate the probe using disturbed laboratory samples. This leaves only in situ calibration as being a viable option. This calibration is typically carried using the samples obtained from the original probe installation, then returning to the field when a second measurement can be made when the soil has quite different moisture content. In fact, while two points are sufficient in theory, a reliable calibration generally requires many more measurements, typically more like ten samples.

A great deal of work has been carried out to obtain estimates of the error associate with neutron probe measurements, calibration of measurements made close to the soil surface, and other logistical issues. A good starting place to learn about these practical concerns is Gardner (1986). Use of the neutron probe method has diminished in the past decade due to the logistical issues involved with handling and transporting radioactive sources. Yet due to the precision which may be obtained, the large sphere of influence of measurements, and low cost of operation, this method remains a mainstay of vadose moisture measurement.

Gamma Attenuation

The gamma attenuation method of liquid content measurement relies on the adsorption of gamma rays. The measurement is made by locating the sample under study between a gamma source and detector. The attenuation of the beam follows Beer's law

$$
\mathrm{I}_{\lambda} / \mathrm{I}_{0 \lambda}=\exp \left(\sum \alpha_{\mathrm{i} \lambda} \mathrm{x}_{\mathrm{i}}\right)=\mathrm{C} \exp \left(\alpha_{\mathrm{w} \lambda} \mathrm{x}_{\mathrm{w}}\right)
$$

where the subscript $\lambda$ denotes the gamma frequency of interest, $\mathrm{I}_{\mathrm{o} \lambda}$ is the detected gamma intensity without sample, $\mathrm{I}_{\lambda}$ is the detected gamma intensity after passing through the sample, $\alpha_{i \lambda}$ is the attenuation coefficient for material $\mathrm{i}$, and $\mathrm{x}_{\mathrm{i}}$ is the path length of gamma travel in material $i$. If all of the features of the sample are constant except for liquid content, 
the attenuation of non liquid components can be identified with a single multiplicative constant $\mathrm{C}$. For the fluid content, $\mathrm{x}_{\mathrm{i}}=\mathrm{T} \theta_{\mathrm{i}}$ where $\mathrm{T}$ is the beam path length in the sample.

Each gamma energy will be attenuated at differing rates for a given liquid. By using multiple frequencies of gamma radiation it is possible to track the content of multiple variable constituents. For this reason, the dual frequency gamma method is the most common method of documenting the joint movement of water/oil systems (e.g., Schiegg, 1990), and may be used to document swelling effects (Gardner et al., 1972).

Due to the need to place the sample between source and detector, the gamma method is not often convenient to apply in the field, but is widely used in laboratory environments. Furthermore, given the use of intense radioactive sources, the gamma method is limited to highly controlled settings. For an excellent overview of this method, see Gardner (1986).

Time Domain Reflectometry

The measurement of water content using time domain reflectometry (TDR), although long standing (e.g., FellnerFeldegg, 1969), has rapidly grown in popularity with the advent of affordable TDR systems. The TDR method can be configured with multiplexing to allow nearly continuous automated measurement at multiple locations with a precision sufficient for most applications. Furthermore, TDR requires less calibration than the neutron probe method, and eliminates the use of radioactive sources. Finally, TDR can be used to measure bulk electrical conductivity in parallel with water content, which can be very useful in tracer and salinity studies. Disadvantages of the method are a relatively small sphere of influence ( $<10 \mathrm{~cm}$ radius), and the high cost of probes (on the order of $\$ 100$ per measurement location). Due to the significant benefits of TDR and developmental nature of this technology, we will provide a slightly more indepth discussion of this method.

TDR depends upon the measurement of travel time of an electromagnetic wave down a wave guide which is either inserted or laid on the soil surface (Selker et al., 1993; Maheshwarla et al., 1995). Travel time increases with the square root of increase in the real component of the dielectric surrounding the probe. Since the mineral portion of porous media typically have constant dielectric (between 2-5) at frequencies > $50 \mathrm{MHz}$ (Hoekstra and Delaney, 1974; Campbell, 1990), and liquid water in such media has a dielectric of 70-80 (temperature dependent), variation in signal travel time along the probes is attributed to variations in water content. Typical accuracy of TDR measurements better than $+/-2 \%$ volumetric (Roth et al., 1990).

With the travel times of signals along TDR probes being in the order of nanoseconds, the precise measurement of this travel time has only been economically feasible in the last 15 years with the advent of inexpensive dedicated TDR oscilloscopes. The specific implementation of the TDR method has been intimately tied to the characteristics of the measurement tools. In particular, the Tektronix 1502 series TDR meters which were designed for field evaluation of signal line integrity have been the primary instrument employed in TDR moisture measurement. A key determinant of spatial resolution in TDR is the rise time of the device; approximately $2 \times 10^{-10} \mathrm{~s}$ for the Tektronix 1502 series. This dictates the minimum probe length that can be used. The change in travel time along a probe, $\mathrm{D}_{t}$, for a change in travel velocity from $v_{1}$ to $v_{2}$ is given by

(77) $\mathrm{D}_{\mathrm{t}}=2\left(\mathrm{~L} / \mathrm{v}_{1}-\mathrm{L} / \mathrm{v}_{2}\right)$

where $\mathrm{L}$ is the probe length, with the factor 2 coming from the fact that two-way travel time is observed. Solving for $\mathrm{L}$, we may calculate the minimum probe length for a given ability to measure $\mathrm{D}_{\mathrm{t}}$, and a given tolerance in velocity change. For example, when considering a dry soil-water system with a dielectric of 10, one can ask what minimum probe length allows determination of a change 2 percent in volumetric moisture content. This would create a change in dielectric of about 1.5 , with $\mathrm{v}=3 \times 10^{8} \mathrm{~m} / \mathrm{s} \mathrm{x} \mathrm{K}^{1 / 2}$, the minimum desirable probe length is approximately $12 \mathrm{~cm}$. Measurement accuracy can be increased, or the probe length decreased, when using a faster TDR system. Kelly et al. (1995) determined that probes as short as $0.02 \mathrm{~m}$ could be used with a TDR system which had a $25 \mathrm{ps}$ rise time. The physical limitations of the frequency dependence of the dielectric of water become significant at these time scales. The period of molecular resonance of water is of the order of $20 \mathrm{ps}$. The real dielectric of water drops dramatically for signals with periods in this range, while the complex component (which is an energy loss term) climbs (Merabet and Bose, 1988). The combined loss of propagation delay and energy for single components with periods $<40$ ps obviates any advantage of operating at frequencies in excess of about $10 \mathrm{Ghz}$. In addition to considerations of length, issues of spacing, conductor diameter, 
and number of wave guides are all significant in determining the sampling volume and sensitivity of the device (Knight, 1992; Whalley, 1993).

An additional limitation experienced in the application of TDR has been signal loss due to bulk conductivity of the porous media, which essentially shorts out the signal sent down the TDR probe (Arcone, 1986). The loss of signal strength due to the electrical conductivity of the media can be exploited to provide estimates of soil solution salinity, as demonstrated by Dalton et al. (1984) and many others. Signal loss difficulties are also encountered using Ground Penetrating Radar (GPR) methods in fine-textured media, as will be described later in this chapter. The signal loss problem can in part be mitigated through the use of minimum length insulated probes (e.g., Kelly et al., 1995), but it requires individual calibration curves for each probe geometry. In addition, high conductivity conditions reduces signal energy due to eddy currents established within the media, thereby limiting the range of applicability of the insulated probes to solutions with concentration of smaller than one molar total salinity, about 25 times greater than can be accommodated by un-insulated probes.

TDR methods are well established in vadose zone monitoring, yet there are various areas where significant enhancements can be achieved. One recent advancement is the development of multilevel shorting-diode TDR probe (Cook et al., 1992), which allows the monitoring of a linear moisture profile with the installation of a single probe. Another opportunity for enhancement of TDR methods lies in the interpretation of wave forms. At present, most TDR signals are interpreted with an empirical relationship between moisture content and travel time as developed by Topp et al. (1980). More recently, authors have derived physically-based methods to interpret the signals (Herkelrath et al., 1991; Heimovaara, 1993; Whalley, 1993), thereby allowing a more clear distinction between dielectric effects on the signal by the solid and liquid phase of the soil matrix. At this time, explicit electromagnetic models for signal propagation are being developed which predict the exact shape of the reflected wave form. These models can be used in a forward modeling mode, but the ultimate objective is to estimate phase contributions by inverse modeling using the full TDR trace as the basis of estimation of moisture content and soil conductivity, which will make more complete use of the wave form obtained from a TDR system.

Measurement of moisture content using TDR will continue to grow in the research community, but rapid adoption in the commercial sector has been hampered by the relatively high cost of TDR systems and the requirement of careful signal interpretation. New products have reduced these concerns, with increasingly robust signal interpretation software and more affordable meters. The TDR method has yet to be packaged into a system which is broadly accessible to individuals in the commercial sector. Nevertheless, TDR is continuing to grow in popularity in the measurement of moisture content within the vadose zone. Competing methods of moisture content measurement through sensing of the soil dielectric are under development or marketed (e.g., Campbell Scientific). As shown by Hoekstra and Delaney (1974) and Campbell (1990), these measurements must be made at frequencies of greater than $50 \mathrm{MHz}$ to obtain a calibration which is independent of soil type.

\section{Measurement of Hydraulic Conductivity}

Hydraulic conductivity is one of the most important parameters in characterizing the transport of fluids and solutes through the vadose zone, but presents significant practical difficulty in measurement. In vadose applications, knowledge of the saturated conductivity is often of limited use, as these conditions are rare in most locations. Yet reliable methods of measuring unsaturated conductivity are very few. One of the most common approaches to obtaining the unsaturated conductivity relationship is to measure both the saturated conductivity and the water retention curve, and estimate the unsaturated conductivity based on a conceptual model relating permeability to water content (van Genuchten, 1980). This process is facilitated through application of computer programs written expressly for this purpose, such as RETC (van Genuchten et al., 1991).

The only direct method of measuring unsaturated conductivity in the field is through a pressure/moisture content profile method. The most widely used approach is the "instantaneous profile method" (IPM). The IPM requires that an 
area of land be subjected to ponded infiltration until the entire profile of interest is saturated. The site is instrumented with devices to measure both the moisture content and pressure with depth and time. At $t=0$ the ponding is ceased, and the site is covered with an impermeable barrier to avoid evaporative losses. Pressure and water content measurements are made in time, with measurements extending to periods of weeks and months if low permeability values are required. Using the water content measurements one can calculate the flux at various depths by way of the total time rate of change in water content in the profile above the height of interest. By observing the local pressure gradients, as measured with tensiometers, one then has values for $\mathrm{q}$ and $\mathrm{dh} / \mathrm{dz}$ in Darcy's law, and it is straightforward to solve for $\mathrm{K}$. A very nice added benefit of this method is that the joint pressure/water content measurements provide an in situ determination of the water retention relationship. Although the IPM is a direct measurement, due to the large number of measurements that need to be made over long periods of time, it is difficult to carry out, and often provide results which are less than satisfactory (e.g., Selker, 1994).

The remaining methods of measurement rely on an analytical solution for the flow from some simple geometric boundary condition. The solution must predict the time rate of infiltration in terms of the desired parameters. The nonlinearity in Richards equation generally leads to the adoption of a solution with very restrictive assumptions in order to allow development of a general solution. The two most widely used assumptions are (1) that the infiltration process may be approximated by a sharp wetting front followed by a region of nearly saturated media (Green and Ampt infiltration: see Chapter 3) and (2) that the hydraulic conductivity function may be approximated by the Gardner (1958) relationship

(78) $\mathrm{K}=\mathrm{K}_{\mathrm{s}} \exp (\alpha \mathrm{h})$

where $\alpha$ is a parameter approximately equal to $-1 / h_{\mathrm{we}}$, and thus is large for coarse textured soils (on the order of 10 $\mathrm{m}^{-1}$ for sands) and small for fine textured soils (on the order of $0.1 \mathrm{~m}^{-1}$ for clays).

The best control over the boundary at which infiltration occurs is obtained by carrying out surface measurements. Ring infiltration methods cover a broad set of these tests. In general these method involve establishing a prescribed pressure over a circular area and observing the resulting infiltration. The scale of these measurements ranges from $100 \mathrm{~cm}^{2}$ (Ankeny et al., 1988) to $4 \mathrm{~m}^{2}$ (ASTM, 1990). An approach that make use of the Green and Ampt concepts was introduced by Bouwer, and is discussed in section 3.1.1.3. Here we will introduce the ponded double ring method, and some newer tension infiltrometer approaches.

By far and a way the most common hydraulic test carried out for to estimate soil hydraulic properties is the double ring infiltrometer (Figure 2.40). The rings are inserted deep enough to preclude leakage from the outer ring, and to have the tops of the rings level with each other. A constant water level is quickly established in both rings to the same level, and the infiltration of water may be measured by watching the drop in water level on a floating ruler, or by using a Marriott bottle constant head water source.

The data may be analyzed using any of several infiltration models. Below we list the Horton Equation, the two-term Philip model, the Green and Ampt model and the Brutsaert model.

Horton Equation (1940): In this empirical infiltration model the rate of infiltration, i, is given by

(79) $\mathrm{i}=\mathrm{i}_{\mathrm{f}}+\left(\mathrm{i}_{0}-\mathrm{i}_{\mathrm{f}}\right) \exp (-\beta \mathrm{t})$

where if is the infiltration rate after long time, $i_{o}$ is the initial infiltration rate and $\beta$ is and empirical soil parameter. Integrating this with time yields the cumulative infiltration

$$
\mathrm{I}=\mathrm{i}_{\mathrm{f}} \mathrm{t}+\frac{\mathrm{i}_{0}-\mathrm{i}_{\mathrm{f}}}{\beta}(1-\exp (-\beta \mathrm{t}))
$$

The model is widely used, but since the parameters obtained are not directly associated with physically measurable soil properties (e.g., conductivity), it is of limited use except for predicting infiltration.

The two term Philip model (1957) is a simplification of the infinite series Philip introduced for infiltration, and is widely used. The result given by

(81) $\mathrm{i}=0.5 \mathrm{St}^{1 / 2}+\mathrm{A}$

or for cumulative infiltration

(82) $\mathrm{I}=\mathrm{St}^{1 / 2}+\mathrm{At}$ 
has the advantage of being closely related to measurable soil physical parameters (i.e., $\mathrm{S}$ is the soil sorptivity and $\mathrm{A}$ is typically taken to be an estimate of field saturated conductivity).

The Green and Ampt Model (1911) for constant head infiltration is derived in Chapter 3, and is also useful in interpreting infiltration results. The Green and Ampt result for constant head infiltration is given by

$$
\mathrm{t}=\frac{\mathrm{n}}{\mathrm{K}_{\mathrm{sat}}}\left\{\mathrm{L}-\left(\mathrm{h}_{\mathrm{f}}+\mathrm{d}\right) \ln \left[\frac{\mathrm{h}_{\mathrm{f}}+\mathrm{d}+\mathrm{L}}{\mathrm{h}_{\mathrm{f}}+\mathrm{d}}\right]\right\}
$$

where $\mathrm{L}$ is the depth of wetting front penetration, $\mathrm{n}$ is the porosity of the soil, $\mathrm{d}$ is the depth of ponding, and $\mathrm{h}_{\mathrm{f}}$ is the water entry pressure for the soil. The cumulative infiltration is simply $\mathrm{I}=\mathrm{nL}$. To use this equation you must find the values of $\mathrm{K}_{\mathrm{sat}}$ and $\mathrm{h}_{\mathrm{f}}$ which give the best fit to the data.

The Brutsaert Model. Brutsaert (1977) revisited the infinite series solution of Philip (1957) and developed a very convenient result that provides an excellent approximation to the full series solution for infiltration, and is written explicitly in the physical variables of interest. For the rate of infiltration Brutsaert (1977) found

$$
\mathrm{i}=\mathrm{K}_{\mathrm{sat}}+0.5 \mathrm{St}^{-1 / 2}\left[1+\beta\left(\frac{\mathrm{K}_{\mathrm{sat}} \mathrm{t}^{1 / 2}}{\mathrm{~S}}\right)\right]^{-2}
$$

where $S$ is the sorptivity and $1 / 3<\beta<1$ is a soil parameter related to the distribution of pore sizes. For soils with very wide pore size distributions you should take $\beta$ close to 1 , for most other soils $\beta=2 / 3$ is quite good, while for uniform pore size soils, $\beta=1 / 3$ is suggested. The cumulative infiltration using the Brutsaert model is simply

$$
\mathrm{I}=\mathrm{K}_{\mathrm{sat}} \mathrm{t}+\frac{\mathrm{S}^{2}}{\beta \mathrm{K}_{\mathrm{sat}}}\left\{1-\left[1+\beta\left(\frac{\mathrm{K}_{\mathrm{sat}} \mathrm{t}^{1 / 2}}{\mathrm{~S}}\right)\right]^{-1}\right\}
$$

Fitting this equation to infiltration data allows ready determination of both $\mathrm{K}_{\text {sat }}$ and $\mathrm{S}$. 


\section{Typical Double Ring Infilrometer Set-up}

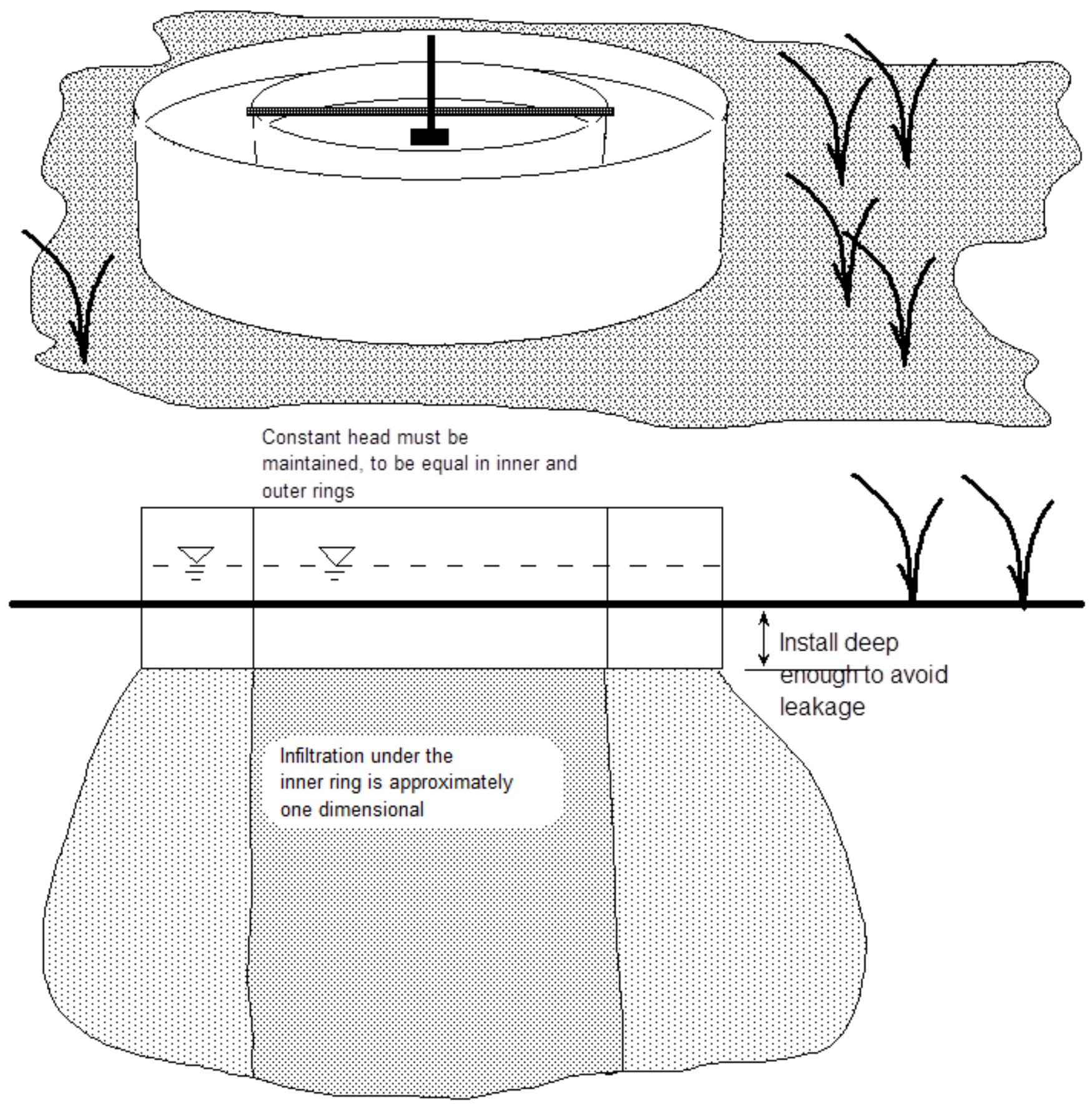

Figure 2.40. Typical double ring infiltrometer installation. Ring diameters employed vary widely.

At negative pressures, features of textural interfaces and fissures cause drastic reduction in unsaturated permeability, while at near-zero pressure macroporosity can greatly increase permeability. Thus, the ability to measure hydraulic properties of vadose materials in-situ at realistic negative pressures has been recognized as an area in need of attention.

Controlled infiltration under tension to determine unsaturated water retention and transport properties has been considered for decades, however, only in the past few years have experimental and analytical tools been improved to make the technique practical in the field. Clothier and White (1981) introduced the "sorptivity tube" which allowed for 
fixed pressure $(<0)$ application of water to a soil using a Marriott bottle supply with a sintered glass porous interface. This design was tested and improved, culminating in the fabric interface device presented by Perroux and White (1988). This device provided ready adjustment of interface pressure, could be made with a wide range of contact areas, and was robust and simple enough to be used conveniently in the field. Developed in parallel, Ankeny et al. (1988) fitted the tension infiltrometer with two pressure transducers which allowed automated acquisition of both the pressure at the soil interface and the flux from the device. Further, this device was fitted with three adjustable bubble tubes that allowed for rapid evaluation of flux from a series of interface pressures. A key development in interpretation of the results of these devices was identification of the utility of the result of Wooding (1968) to disk infiltrometer (Ankeny et al., 1991). Wooding found that using a Gardner exponential conductivity function $\underline{78}$ the flux into a circular wetted area of radius $\mathrm{r}$, at steady state, was

$$
\mathrm{Q}=\pi \mathrm{r}^{2} \mathrm{~K}_{\mathrm{s}} \exp (\alpha \mathrm{h})\left(1+\frac{4}{\pi \alpha \mathrm{r}}\right)
$$

where $\mathrm{Q}$ (measured) is the flux into the soil over a device of radius $\mathrm{r}$ (measured), $\mathrm{h}$ (measured) is the pressure at which the infiltration boundary, $\mathrm{K}_{\mathrm{S}}$ (unknown) is the saturated hydraulic conductivity, and $\alpha$ (unknown) is as defined in ??. By carrying out infiltration tests at two or more pressures, or with two or more radius disks, one may solve for both of the unknowns in $\underline{86}$.

One significant shortcoming of Wooding's approach is the notion of a well-defined steady-state condition, and the time required to achieve this condition. As shown by Smettem et al. (1995), these effects may take hours to become small, and the outflow might appear to be steady far before the true steady state has been achieved. Haverkamp et al. (1994) present an approximate solution for the time-dependent rate of infiltration for one and three dimensional cases as

$$
\begin{aligned}
& I_{1 d}=S_{0} t^{1 / 2}+\left(K_{n}+\frac{2-\beta}{3}\left(K_{0}-K_{n}\right)\right) t \\
& I_{3 d}=S_{0} t^{1 / 2}+\left(K_{n}+\frac{\gamma S_{0}}{\left(\theta_{0}-\theta_{n}\right) r}+\frac{2-\beta}{3}\left(K_{0}-K_{n}\right)\right) T
\end{aligned}
$$

where $S_{o}$ is the sorptivity, $\beta$ is a shape parameter $(0<\beta<1), \gamma$ is a parameter with value between 0.6 and 0.8 which Smettem et al. (1994) found to be well approximated by $0.75, K_{o} \& K_{n}$, and $\theta_{o} \& \theta_{n}$ are the conductivity and moisture content at the antecedent and instrument pressures respectively. As shown by Smettem et al. (1995), a double ring tension infiltrometer may be made in which the outflow of the inner ring may be assumed to be one-dimensional, and that of the combined inner and outer rings three-dimensional.

Smettem et al. (1995) show that a good estimate of sorptivity may be obtained using $\underline{88}$ by plotting $\mathrm{I}_{3 \mathrm{~d}} / \mathrm{t}^{1 / 2}$ against time. We see that $\underline{88}$ then reduces to

(89) $\mathrm{I}_{3 \mathrm{~d}} / \mathrm{t}^{1 / 2}=\mathrm{S}_{0}+\mathrm{At}$

for some constant A. By fitting a straight line to $\underline{89}$, the $\mathrm{t}=0$ value of this relationship provides an estimate of $\mathrm{S}_{\mathrm{o}}$. Smettem et al. found this value to be consistent with values obtained from one-dimensional infiltration tests. This result may be used in conjunction with $\underline{86}$ or $\underline{88}$ for $\mathrm{K}_{\mathrm{s}}$.

These measurements are typically made at a series of tensions and often with two sizes of circular sources. The measurements require access to a horizontal soil surface, thereby limiting application of this technique to the first few meters of the vadose zone, although a down-hole tension infiltrometer designs has also been developed (Sisson and Honeycutt, 1994).

An alternative method of interpreting tension infiltrometer results is to carry out the estimation of sorptivity and hydraulic conductivity functions by inverting a numerical simulation of the process (Simunek et al., 1995 and 1996). Since this method makes full use of the early time data, it holds the promise of significantly increasing the utilization of site-specific hydraulic properties by reducing the experimental time required to obtain reliable values. The accuracy and practicality of these methods has also been explored recently, comparing a number of methods both for field and 
laboratory application (e.g., Logsdon and Jaynes, 1993; Gupta et al., 1993; Stolte et al., 1994; Cook and Broeren, 1994). It is clear that each of the present implementations of the tension infiltrometer method have difficulty under some situations, but the outlook for improved methods of measurement appears quite bright in the coming years using this technique.

To determine the hydraulic properties of vadose materials at depth, down-hole methods are essential. This is an area of active development, with some very useful techniques available. The method most widely employed is to auger a hole of constant radius to the depth at which the measurement is required. A constant head of water is then established in the hole, while the rate of infiltration is observed. Several tools have been developed for this test, and generally consist of a Marriott bottle source of water with an extension tube to reach the required depth. Such tools can easily be made without access to specialized equipment, as shown in Figure 2.41.

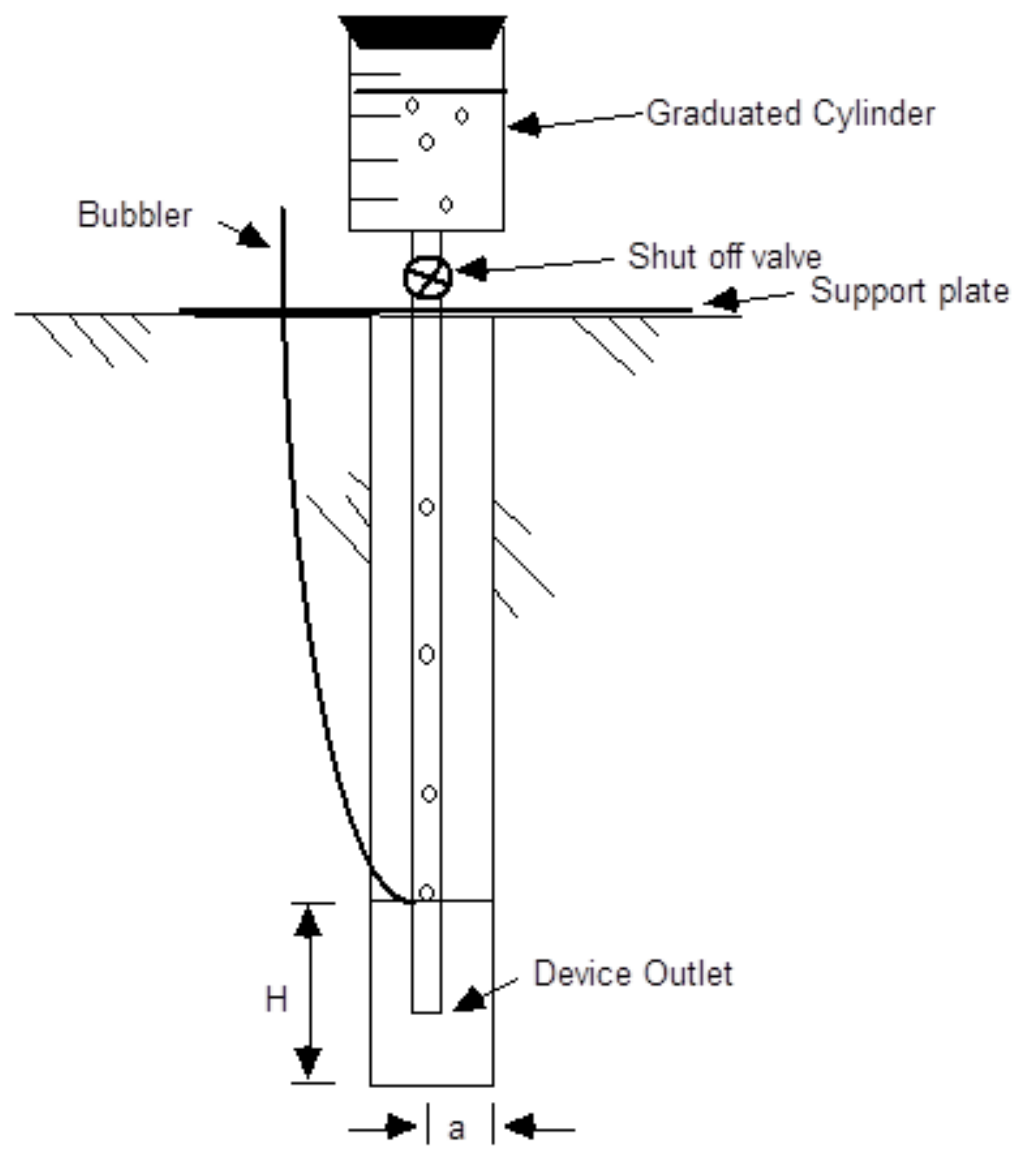

Figure 2.41Figure 2.41. Simple down-hole constant head permeameter. The graduated cylinder is filled prior to the test, then the device is lowered into the soil until the support plate sits on the soil surface. The test is commenced by opening the shut off valve, at which time the bubble tube establishes a zero pressure level at its point of entry to the drop pipe.

The analysis of constant head well permeameter can be carried out using a number of formulations. The original work directed to analysis of such data was presented by Glover (Zangar, 1953). As presented by Elrick and Reynolds (1993), Glover found that at steady state the rate of infiltration from such a permeameter could be approximated by

$$
\mathrm{Q}=2 \pi \mathrm{H}^{2} \mathrm{~K}_{\mathrm{fs}} / \mathrm{C}
$$

where $\mathrm{K}_{\mathrm{fs}}$ is the "field saturated" hydraulic conductivity and $\mathrm{C}$ is a dimensionless shape coefficient reflecting the geometry of the wetted area. This was estimated by Glover to be

$$
\mathrm{C}=\sinh ^{-1}(\mathrm{H} / \mathrm{a})-\left[(\mathrm{a} / \mathrm{H})^{2}+1\right]^{1 / 2}+\mathrm{a} / \mathrm{H}
$$


This solution assumes complete saturation in the wetted region, and does not include gravity driven flow (Elrick and Reynolds, 1993). For ratios of $\mathrm{H} / \mathrm{a}<2$, this solution has been shown to be reasonably accurate, while for $\mathrm{H} / \mathrm{a}>10$, it under estimates the correct value of $\mathrm{C}$ by about $50 \%$, leading to an error in $\mathrm{K}_{\mathrm{fs}}$ of about $40 \%$. To account for the effects of gravity and unsaturated flow effects, Elrick et al. (1989) provided the solution

$$
\mathrm{K}_{\mathrm{fs}}=\frac{\mathrm{CQ}}{2 \pi \mathrm{H}^{2}+\pi \mathrm{a}^{2} \mathrm{C}+2 \pi \mathrm{H} / \alpha^{\text {ast }}}
$$

Elrick and Reynolds (1992a and 1992b) suggest that $\alpha^{*}$ may be estimated based on field conditions using the values given in Table 2.5. Alternately, tests using multiple depths of ponding may be carried out, providing for multiple equations from which the values of $\mathrm{K}_{\mathrm{fs}}$ and $\alpha^{*}$ may be estimated using regression analysis. Difficulties with this approach have been reported (e.g. Elrick and Reynolds, 1992b) due to heterogeneity in soils which will yield inconsistent values of the parameters depending upon the depth of ponding. This can lead to non-physical values of estimated parameters (e.g., negative conductivity), and thus should only be employed in soils without significant vertical variability in properties.

Table 2.5 Suggested values for $\alpha^{\star}$ as provided by Elrick and Reynolds (1992).

$\alpha^{*}$ Comments

$\left[\mathrm{m}^{-1}\right]$

$\infty$ Essentially the Glover Solution. Probably applicable to gravels.

36 Coarse sands and highly structured soils.

12 Most structured soils and medium and fine sands.

4 Unstructured fine-textured soils.

1 Compacted clays (e.g., clay liners).

0 The Gardner solution (Reynolds and Elrick, 1985). Pressure and gravity contributions negligible.

\section{Review Questions}

1. What are the units of surface tension and why?

2. What is the definition of the reduced radius of curvature and what is the pressure drop across a curved interface?

3. If the local atmosphere in a soil is, at equilibrium, below the saturated vapor pressure for water, is there any liquid water for plants?

4. What is a Haines Jump?

5. What is the raindrop effect?

6. What is hysteresis?

7. What are the two basic assumptions of the independent domain model for hysteresis?

8. Is Darcy's law linear? in which terms?

9. When does Darcy's Law break down? What are the Reynolds number criteria?

10. Is the unsaturated version of Darcy's law hysteretic in moisture content? Pressure? Why?

11. What are the two relationships that go into the derivation of Richards equation?

12. Is Richards equation linear in any terms? (Consider $\mathrm{K}, \theta, \mathrm{D}, \mathrm{h}, \mathrm{x}, \mathrm{z}$ )

13. What is the point of using the $\mathrm{D}$ parameterization instead of $\mathrm{K}$ in Richards equation? 
14. Why have so many people developed functional relationships for $\mathrm{D}$ and $\mathrm{K}$ ?

15. What are the basic assumptions of Miller Similarity?

16. List the dependence of the following terms on the characteristic grain size of the media (don't worry about the effects of viscosity and surface tension): $\mathrm{K}, \mathrm{S}, \mathrm{h}$.

17. What is a NAPL, an LNAPL, and a DNAPL?

18. Explain why the observation well depth of a NAPL is a poor indicator of true "free NAPL" content at a site.

19. What is a tensiometer, and how does it work?

\subsection{Problems}

1. The raindrop effect illustrates hysteresis in fluid/solid contact angle.

2. A Haines jump is an Olympic sporting event developed by Sir Walter Haines in the late 1920's.

3. For a soil at equilibrium, if the soil gas has a relative humidity of 0.5 , the moisture content is approximately $0.5 \theta \mathrm{s}$.

4. Darcy's law breaks down for high flow when the flow regime becomes non-laminar at a Reynolds number of about 100. Below this value flux and gradient in potential are linearly related.

5. Darcy's law breaks down for Reynolds number greater than 1 due to turbulence at the pore scale.

6. Since conductivity is hysteretic in moisture content, even if you know the local gradient in pressure, it is not possible to be sure of the local flux at a given moisture content without knowledge of the history of the wetting at this point.

7. Under steady state conditions, Richards equation reduces to Darcy's law for unsaturated flow since conservation of mass is satisfied.

8. The units of surface tension are energy per area.

9. Poiseuille's law tells us that conductivity follows a linear relationship with average pore size.

10. The Green and Ampt infiltration model would be applicable to rapid infiltration processes for a falling head situation.

11. A soils sample taken from the field with a volume of $42 \mathrm{~cm}^{3}$ weighed 29 grams, when saturated weighed 32 grams, and when dried weighed 21 grams. Calculate the soil's moisture content (both volumetrically and on a mass basis), saturated moisture content, porosity, bulk density, particle density, and void ratio.

12. For a fluid with surface tension of $2 \mathrm{~N} / \mathrm{m}$, and with curvature defined by the two radii $-0.02 \mathrm{~m}$ and $-0.01 \mathrm{~m}$, what is the pressure in the liquid if the gas pressure is $2 \mathrm{~N} / \mathrm{m}^{3}$ ? Express your result in units of $\mathrm{N} /$ $\mathrm{m}^{2}$.

13. Assume that we have a medium made up of pores identical to the one shown below. Assume that the total porosity of the medium is $28 \%$. Plot the wetting and draining curve for the medium in the axis shown below. Assume a surface tension $\sigma$ and a contact angle of $\gamma$. (You may assume that $L>>R$ ). 


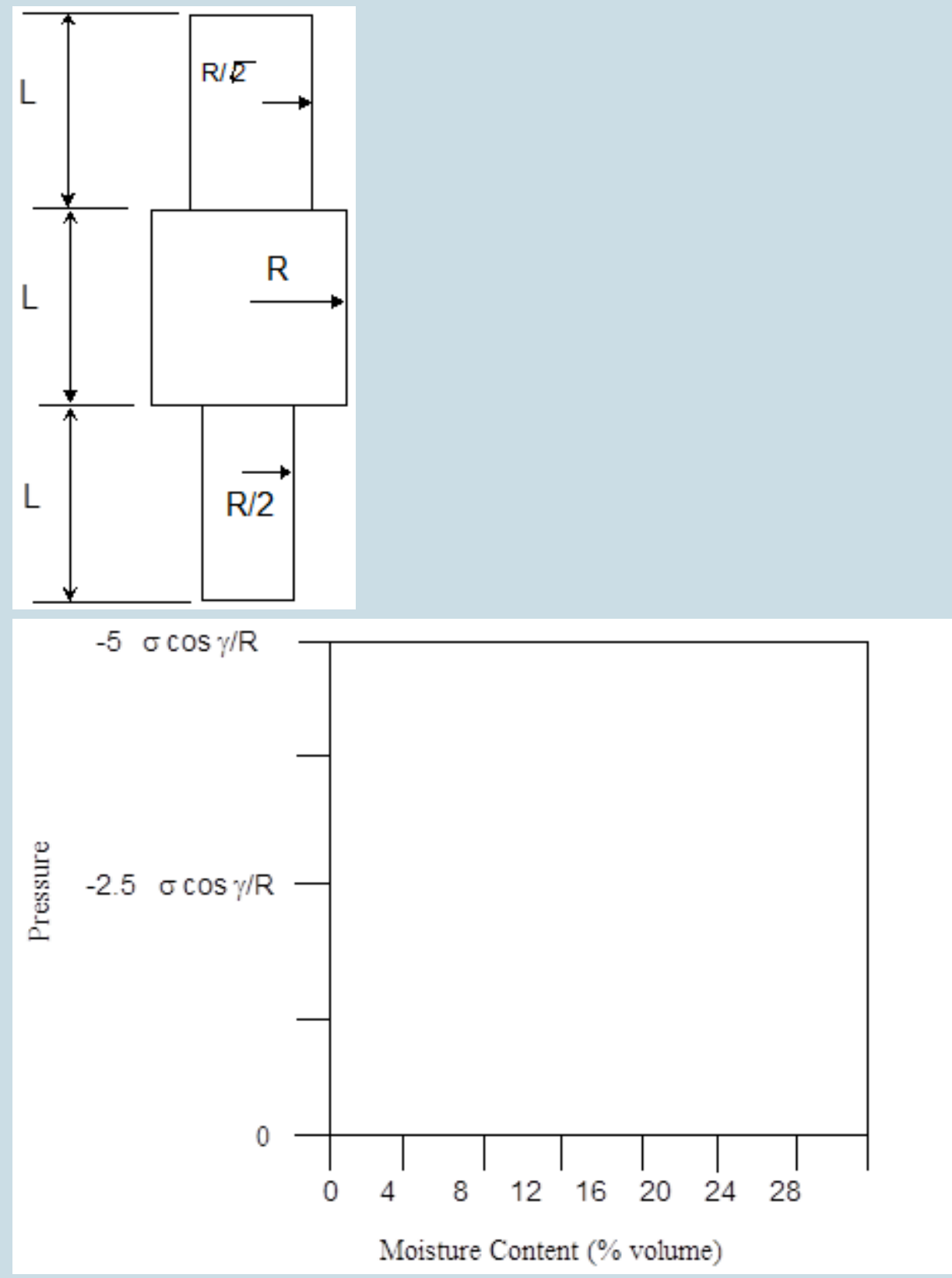

14. A soil is found to respond as if it were made up of a capillary tube bundle as shown on the right. For each capillary of radius $\mathrm{R}$ there are two tubes of radius $\mathrm{R} / 2$, and four tubes of radius $\mathrm{R} / 4$. If the saturated conductivity of the medium is $\mathrm{K}_{\mathrm{s}}$, calculate the unsaturated conductivity and pressure potential of the medium for the three possible values of $\theta$ (assume that there is no hysteresis and write the range of $\Psi$ in units of $2 \sigma \cos \gamma / \mathrm{R}, \theta$ in units of $\theta / \theta_{\mathrm{S}}$ and $\mathrm{K}(\theta)$ in terms of $\left.\mathrm{K} / \mathrm{K}_{\mathrm{S}}\right)$. Put the results in the table given.

\begin{tabular}{lll}
\hline $\begin{array}{l}\text { Moisture Content } \\
\left.\text { (units of } \theta / \theta_{\mathrm{S}}\right)\end{array}$ & Conductivity & Range of $\mathrm{h}$ \\
(units of $\left.\mathrm{K} / \mathrm{K}_{\mathrm{S}}\right)$ & (units of $2 \sigma \cos \gamma / \mathrm{R})$ \\
\hline
\end{tabular}

15. Solve for and plot the values of $\mathrm{k}(\mathrm{h})$ for $\mathrm{h}=-9.2,-26.7,-49.4,-69.1$, and estimate $\mathrm{k}(-90 \mathrm{~cm}) \mathrm{using}$ the data 
obtained in a steady-state, homogeneous, vertical column experiment shown below.

\begin{tabular}{ll}
\hline Pressure $(\mathrm{cm})$ & Elevation $(\mathrm{cm})$ \\
0.0 & 0 \\
-9.2 & 10 \\
-18.2 & 20 \\
-26.7 & 30 \\
-34.9 & 40 \\
-42.4 & 50 \\
-49.4 & 60 \\
-55.5 & 70 \\
-60.9 & 80 \\
-65.4 & 90 \\
-69.1 & 100 \\
\hline
\end{tabular}

\section{$\mathrm{Q}=2 \mathrm{ml} / \mathrm{min}$, area $=100 \mathrm{~cm}^{\wedge} 2$}

$\mathrm{z}=100 \mathrm{~cm}$

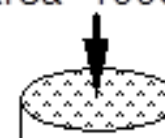

$\mathrm{z}=0 \mathrm{~cm}$

$\mathrm{K}(-9.2)=$

$\mathrm{K}(-26.7)=$

$\mathrm{K}(-49.4)=$

$\mathrm{K}(-69.1)=$ 
$\mathrm{K}(-90)=$

16. Plot the characteristic scanning curves for $\theta(\mathrm{h})$ for the normalized $\mathrm{f}\left(\mathrm{h}_{\mathrm{e}}, \mathrm{h}_{\mathrm{f}}\right)$ data and the following wetting and drying history.

$$
\theta(h)=\theta\left\{\begin{array}{lr}
-40 & -30 \\
0 & -10
\end{array}\right\}
$$

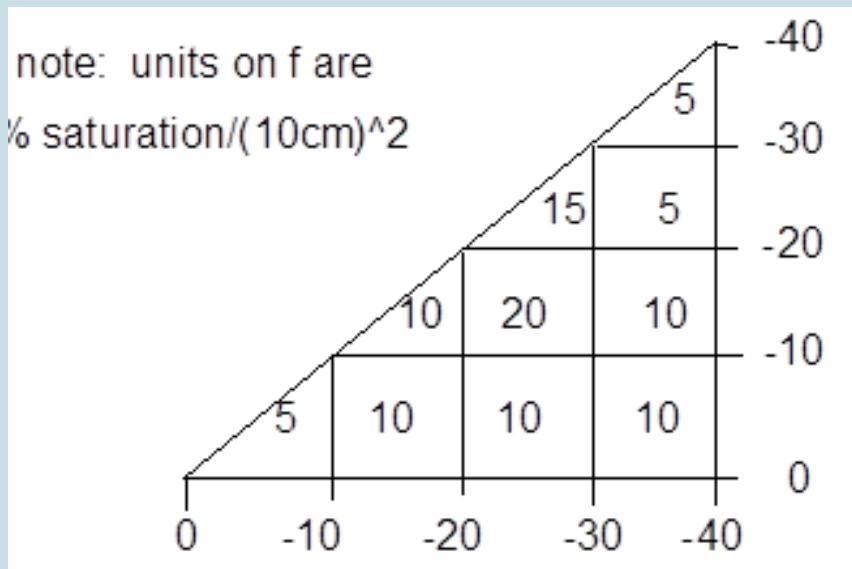

17. Relative Humidity in the soil is useful for calculating pressure head at high suctions using the psychrometric equation. In semi-arid climates, soil water pressures may exceed -50 bars (about $-750 \mathrm{psi}$, or $-500 \mathrm{~m}$ of head!). Make a graph of relative humidity vs. pressure head over the range of 0 to -50 bars.

18. Porous filters can be obtained with average pore size of 0.2 microns in radius. [Of course, the largest pores are those that dictate the air entry pressure of the plates.] Such porous plates can be used to dewater soil at high pressures to obtain the pressure-moisture content relationship. Assuming that the largest continuously connected pores through the plate are 2.5 times this size, and that the plate is initially completely saturated with water, at what pressure will the plate let air pass through? Express your answer in units of $\mathrm{N} / \mathrm{m}^{2}$, psi, Bars, atmospheres, and $\mathrm{cm}$ of water.

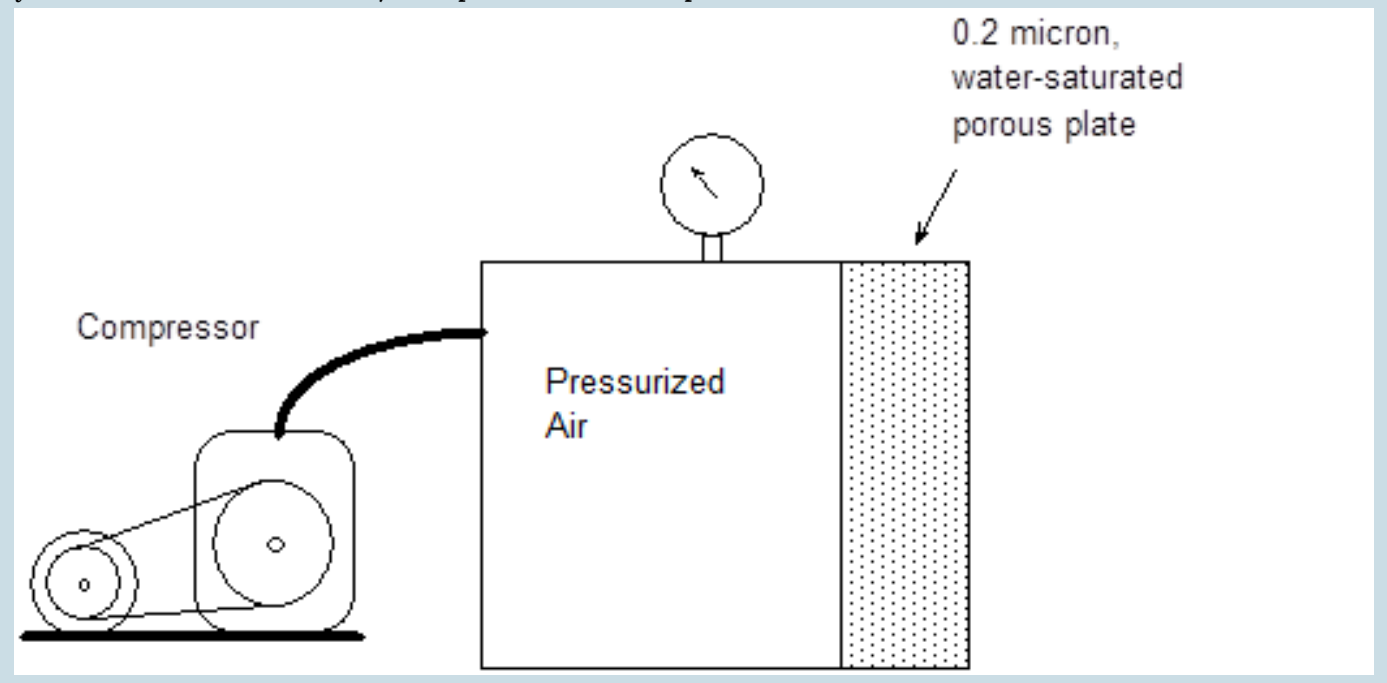

1. Calculate the capillary pressure in $\mathrm{kPa}$ for the water shown between the two spheres (State your 
assumptions).

2. What is the force between the spheres? [Think about balancing the forces of the surface tension, the contact force between the spheres and the pressure of the water. It is easiest to calculate about the plane of symmetry.]

3. Why does wet sand stick to your hands? What about making sand castles with dry sand vs. wet sand?

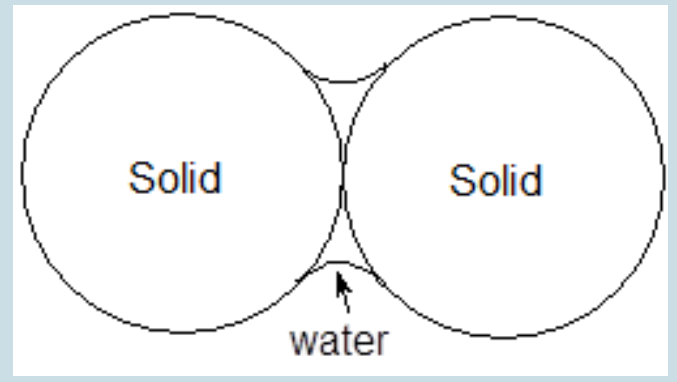

The scale of the drawing is $1 \mathrm{~cm}=0.1 \mathrm{~mm}$.

19. Another way to obtain the Laplace equation is to balance the forces between surface tension and internal pressure for a sphere floating in space. Carry out this calculation for the force balance across the middle of a sphere of water in air. Calculate the pressure in a $0.1 \mathrm{~mm}$ sphere of water.

20. Characteristic draining curves(adapted from similar problem by D.B. Stephens).

Yechezkel Mualem has done a great service to folks studying the flow through the vadose zone with the publication of the text A Catalogue of the Hydraulic Properties of Unsaturated Soils in 1976. This catalogue collects the characteristic draining and wetting curves, bulk density, particle size distribution, and conductivity. You will find two typical entries from this catalogue attached; one for "Gilat Loam", and the other for "Sinai Sand." Consider a soil profile as shown below, which might have resulted from a glacial deposition (In solving this problem, ignore the effects of hysteresis and express pressure in the units cm of water).

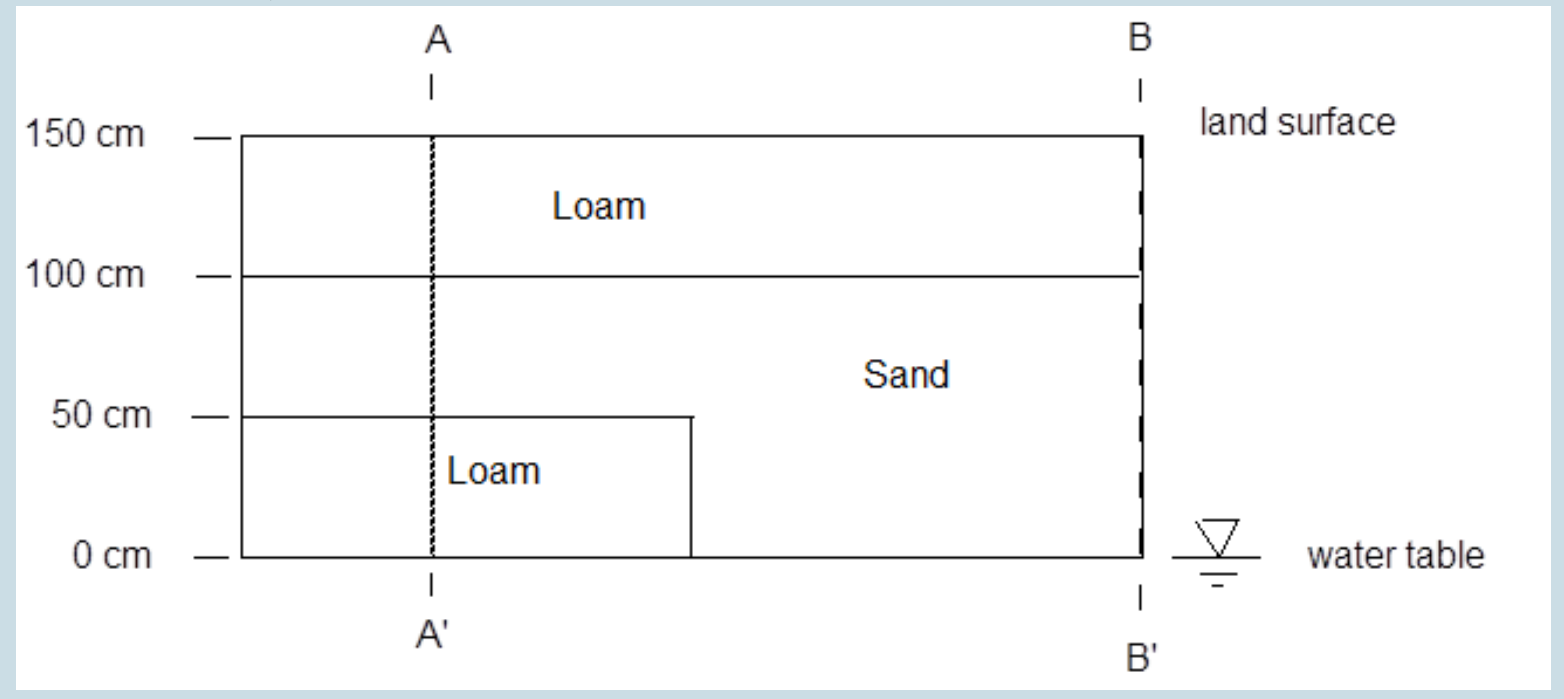

1. Plot the characteristic draining curves for the two soils using Mualem's data. 
2. Plot the pressure versus elevation for the profile for the transects labeled A-A' and B-B' assuming that the profile is at steady state with the water table at $150 \mathrm{~cm}$ below the soil surface, as shown.

3. Plot the moisture content versus elevation for the transects labeled A-A' and B-B'.

4. Suppose that the soil started by being saturated to the soil surface, and then was drained to have the water table at a depth of $150 \mathrm{~cm}$, as shown. How much water (in $\mathrm{cm}$ depth) drained from the profile?

21. Hysteresis Curves using Independent Domain Model. (after problem by Dr. W. Brutsaert, Cornell University).

Using the data for the pore space density for Adeline Dune Sand (Talsma, 1970) given below, draw the pressure vs. moisture content for the following pressure series:

(93)

$$
q(y)=q\left\{\begin{array}{rrr}
0 & -30 & \\
-100 & -70 & -50
\end{array}\right\}
$$

\begin{tabular}{|l|l|l|l|l|l|l|l|l||}
\hline \\
\hline
\end{tabular}

water content units: $10^{-4}\left(\mathrm{~cm}^{3}\right.$ water $/ \mathrm{cm}^{3}$ soil $) /(10 \mathrm{~cm} \text { water })^{2}$

22. The diameter of a soil cylinder is $0.12 \mathrm{~m}$ and its height is $0.28 \mathrm{~m}$.

(i) What was the gravimetric water content $\left(\theta_{\mathrm{m}}\right)$ if the wet weight was $5.5 \mathrm{~kg}$ and the dry weight was 
$4.25 \mathrm{~kg}$ ? (ii) What was the soil bulk density $\rho$ b (assume particle density of $2650 \mathrm{~kg} / \mathrm{m}^{3}$ ); and (iii) what was the volumetric water content $\theta_{\mathrm{v}}$ ?

23. What were the porosity (n), the void ratio (e), and the degree of saturation (S) of the soil sample in problem 5 ?

24. How many 200-liter barrels of water would be stored in the top $1.2 \mathrm{~m}$ of 1 hectare of land if the average $\theta_{\mathrm{V}}$ was 0.25 ? What would be the volume of air-filled pores if the porosity (n) was 0.45 ? How deep would 80 $\mathrm{mm}$ of water wet a soil with $\rho_{\mathrm{b}}=1250 \mathrm{~kg} / \mathrm{m}^{-3}$ and initial $\theta_{\mathrm{v}}=0.14 \mathrm{~m}^{3} / \mathrm{m}^{3}$ ? Assume complete saturation immediately after wetting, and $\rho_{\mathrm{s}}=2650 \mathrm{~kg} / \mathrm{m}^{3}$. What would the final depth of wetting be if the soil then drained internally to a field capacity water content of $\theta_{\mathrm{v}}=0.26$ ?

25. Derive the following relations:

(i) $\mathrm{e}=\mathrm{n} /(1-\mathrm{n})$; (ii) $\theta_{\mathrm{v}}=\mathrm{Sn}$; (iii) $\mathrm{n}_{\mathrm{a}}=\mathrm{n}(1-\mathrm{S})$ where $\mathrm{n}_{\mathrm{a}}=\mathrm{V}_{\mathrm{a}} / \mathrm{V}_{\mathrm{t}}$ is the air-filled porosity.

Example: derive $\mathrm{n}=1-\rho_{\mathrm{b}} / \rho_{\mathrm{s}}$

$\mathrm{n}=\frac{\rho_{\mathrm{s}}-\rho_{\mathrm{b}}}{\rho_{\mathrm{s}}}=\frac{\frac{\mathrm{M}_{\mathrm{s}}}{\mathrm{V}_{\mathrm{s}}}-\frac{\mathrm{M}_{\mathrm{s}}}{\mathrm{V}_{\mathrm{t}}}}{\frac{\mathrm{M}_{\mathrm{s}}}{\mathrm{V}_{\mathrm{s}}}}=1-\frac{\mathrm{V}_{\mathrm{s}}}{\mathrm{V}_{\mathrm{t}}}=\frac{\mathrm{V}_{\mathrm{t}}-\mathrm{V}_{\mathrm{s}}}{\mathrm{V}_{\mathrm{t}}}$

$\Rightarrow \quad$ since $V_{\mathrm{t}}-\mathrm{V}_{\mathrm{s}}=\mathrm{V}_{\mathrm{f}}$ we obtain the definition of porosity as $\mathrm{n}=\frac{\mathrm{V}_{\mathrm{f}}}{\mathrm{V}_{\mathrm{t}}}$

26. The following measurements were collected for the calibration of a neutron probe:

\begin{tabular}{cccccccc}
\hline Count Ratio - CR & 1.7 & 1.5 & 1.2 & 1.0 & 0.8 & 0.6 & 0.4 \\
Water Content qm $[\mathrm{kg} / \mathrm{kg}]$ & 0.27 & 0.23 & 0.17 & 0.15 & 0.11 & 0.09 & 0.06 \\
\hline
\end{tabular}

The Bulk density was $1300 \mathrm{~kg} / \mathrm{m}^{3}$. Determine the calibration equation for the neutron probe and plot the data with the calibration line.

27. The distance to a TDR trace initial reflection $\left(\mathrm{x}_{1}\right)$ was $5.01 \mathrm{~m}$ and to the final reflection $\left(\mathrm{x}_{2}\right) 5.63 \mathrm{~m}$. Given a probe length of $0.2 \mathrm{~m}$ and $\mathrm{Vp}=0.99$; (i) What is the bulk dielectric constant $\varepsilon_{\mathrm{b}}=\left[\left(\mathrm{x}_{2}-\mathrm{x}_{1}\right) /(\mathrm{L} \mathrm{Vp})\right]^{2}$, and (ii) Using Topp's equation, determine the volumetric water content of the soil.

28. For a soil with $\rho_{b}=1300 \mathrm{~kg} / \mathrm{m}^{3}$ what would be the dielectric constant ( $\left.\varepsilon_{\mathrm{b}}\right)$ at $\theta_{\mathrm{v}}=0.22$ ? (hint: use Eq.18 ).

29. What would be the volume of drainage flowing into $1.5 \mathrm{~m}$ deep drains from a 2.15 hectare field with $\theta_{\mathrm{vFC}}=0.22 \mathrm{~m}^{3} / \mathrm{m}^{3}$ and initial $\theta_{\mathrm{v}}=0.18 \mathrm{~m}^{3} / \mathrm{m}^{3}$ following $150 \mathrm{~mm}$ of rain?

30. Given a depth-dependent field capacity as: $\theta_{\mathrm{vFC}}=0.26-0.03 \mathrm{z}$, where $\mathrm{z}$ is the depth in $\mathrm{m}$, and assuming a constant wilting point at $\theta_{\mathrm{vWP}}=0.04 \mathrm{~m}^{3} / \mathrm{m}^{3}$, compute the volume $\left(\mathrm{m}^{3}\right)$ of plant-available soil water in the top $1.5 \mathrm{~m}$ of a 25 hectare field.

31. What is the gauge reading of a tensiometer if the matric potential $\psi \mathrm{m}=-2500 \mathrm{~mm}$ and the gauge is 300 
$\mathrm{mm}$ above the porous cup?

32. What is hydraulic potential $\psi_{\mathrm{h}}$ for a situation where $\psi_{\mathrm{m}}=-280 \mathrm{~mm}$ and the reference elevation is 520 $\mathrm{mm}$ below the point of interest?

33. The cups of tensiometers 1 and 2 are at a depth of 0.6 and $0.8 \mathrm{~m}$, respectively. The gauges are $0.2 \mathrm{~m}$ above the soil surface. The gauge in tensiometer 1 indicates $\psi$ gauge $=-0.9 \mathrm{~m}$. (i) Draw the potential diagram (use weight basis or head) assuming static equilibrium conditions (use the soil surface as reference level). (ii) What is the gauge reading in tensiometer 2 ?

34. If the $\mathrm{EC}$ of a soil solution was $21 \mathrm{dS} / \mathrm{m}$, and $\psi_{\mathrm{m}}=-280 \mathrm{kPa}$, what was the relative humidity of the soil air at $20^{\circ} \mathrm{C}$ ?

35. What should be the effective diameter of the largest pore in a tensiometer cup used to measure pressure heads of $-8.0 \mathrm{~m}$ ?

36. What is the height of rise in clean glass capillaries having diameters of $0.01,0.05,0.3$, and $1.5 \mathrm{~mm}$, when dipped vertically into a pool of pure water?

37. What would be the effect on the height of capillary rise of problem 27 if the capillaries were tipped vertically into a pool of mercury, with the contact angle mercury-glass being $148^{\circ}$. Explain the differences.

38. Use the SWC and van Genuchten parameters $\alpha=0.356 \mathrm{~m}^{-1}, \mathrm{n}=1.501, \theta_{\mathrm{r}}=0.039 \mathrm{~m}^{3} / \mathrm{m}^{3}$, and $\theta_{\mathrm{s}}=0.418$ $\mathrm{m}^{3} / \mathrm{m}^{3}$ to estimate plant available soil water between field capacity $\left(\psi_{\mathrm{m}}=-3 \mathrm{~m}\right)$ and wilting point $\left(\psi_{\mathrm{m}}=-150\right.$ $\mathrm{m})$. What are the values of $\theta_{\mathrm{vFC}}$ and $\theta_{\mathrm{vWP}}$ ?

39. Fit the van Genuchten and Brooks and Corey parametric models for Soil Water Characteristics to the following experimental data.

\begin{tabular}{cccccccccc}
\hline Matric Potential $[-\mathrm{m}]$ & 0.01 & 1 & 2 & 3 & 5 & 8 & 61 & 110 & 323 \\
Water Content $\left[\mathrm{m}^{3} / \mathrm{m}^{3}\right]$ & 0.433 & 0.347 & 0.300 & 0.263 & 0.222 & 0.183 & 0.116 & 0.093 & 0.066 \\
\hline
\end{tabular}

40. What is the volume $\left(\mathrm{m}^{3}\right)$ of plant-available soil water stored in the top $1 \mathrm{~m}$ of a $2 \mathrm{~m}^{2}$ area of Sarpy loam soil that is characterized by the following SWC VG model parameters: $\alpha=2.79 \mathrm{~m}^{-1} ; \mathrm{n}=1.6 ; \theta_{\mathrm{s}}=0.4 \mathrm{~m}^{3} / \mathrm{m}^{3}$; and $\theta_{\mathrm{r}}=0.032 \mathrm{~m}^{3} / \mathrm{m}^{3}$

41. The relative humidity measured with a psychrometer at $25^{\circ} \mathrm{C}$ is $0.45(45 \%)$. What is the water potential yw of a soil sample in equilibrium with ambient air?

42. A uniformly packed, U-shaped soil column has one end suspended in a container of water as shown in the figure at the right. The water level in the container has been kept constant and evaporation has been prevented from the open end for a period sufficient that the soil column has reached equilibrium. Thus there is no net flow in the column.

Find: The hydraulic potential $\psi_{\mathrm{h}}$ and its components $\psi_{\mathrm{p}}, \psi_{\mathrm{m}}$, and $>_{\mathrm{z}}$ for points A through $\mathrm{F}$ in the soil column. List your results. 


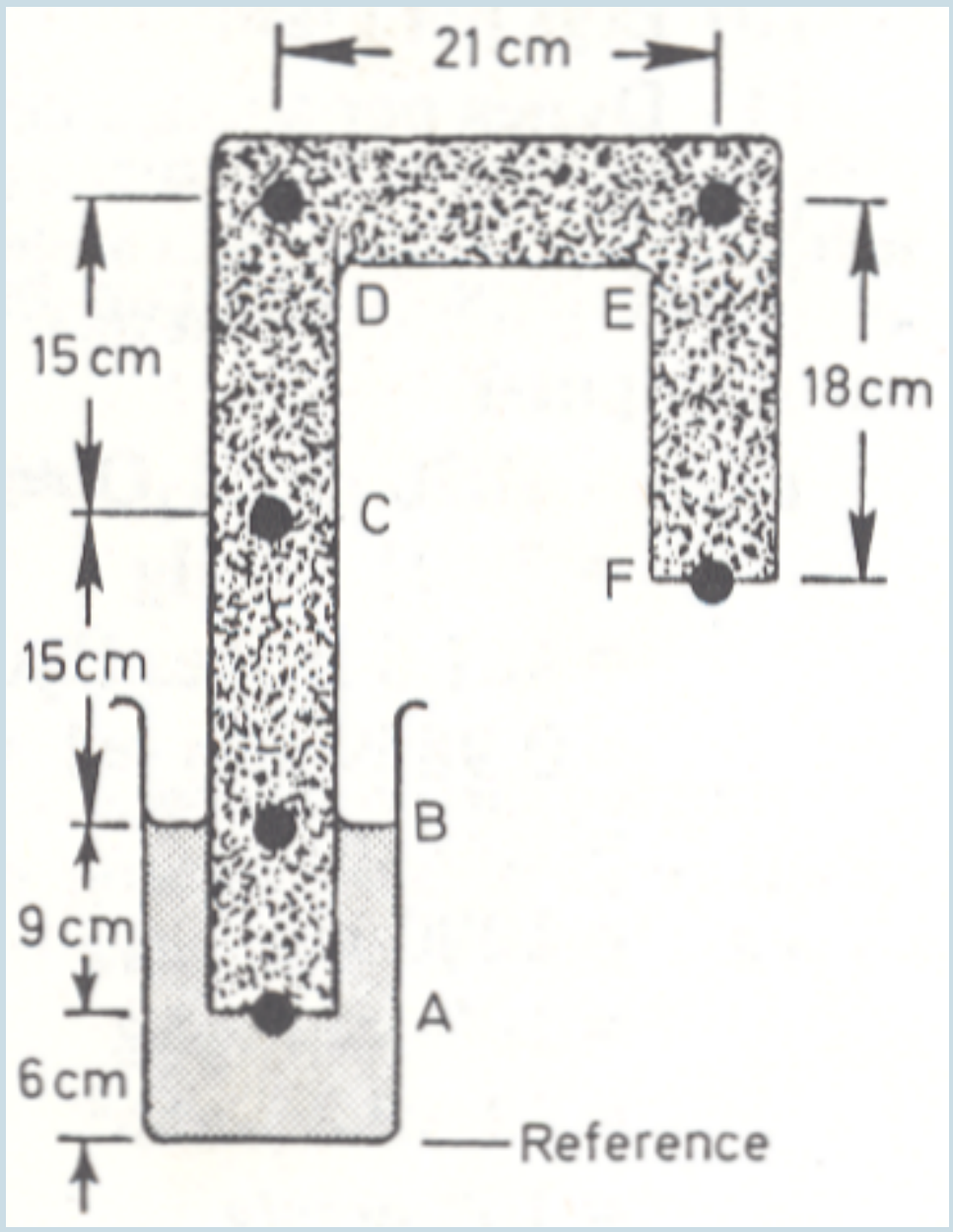

\section{Index}

A

admixture

anticlastic

atmospheric pumping

B

Beer's law

Brutsaert Infiltration model

Buchner funnel

Buckingham

Bukner funnel

bulk density

C

calcite

capillary tube 
characteristic curves

\section{Characteristic Curves}

chemical potential

conductivity, down hole measurement

constant head permeameter

constituents

contact angle

conversions

creeping flow

curved fluid surface

D

Darcian Length

Darcian velocity

Darcy's law

datum

dead end pore

density

dew point

dielectric

diffusion

diffusivity

dipole

DNAPL

double ring infiltrometer

down-hole tension infiltrometer

Droplet

dynamic viscosity

E

entropy

F

feldspar

field capacity

field saturated

Fokker-Planck equation

free product

functional

fundamental differential form

G

gamma attenuation

gamma method, dual frequency

Gardner conductivity

gas movement

gas permeability

gaseous

geometric similarity

Gibbs-Duhem relationship

GPR

gravitational potential

118 | Basic Relationships and the Soil Phases Under Equilibrium Conditions 
Green and Ampt

ground penetrating radar

gypsum block

H

Hagen Poiseuille

Hagen-Poiseuille Equation

Haines jump

Henry's law

Horton Equation

hydraulic conductivity, measurement

Hydrostatics, 21

hysteresis, gypsum blocks

hysteretic

hysterisis

I

immiscible

independent domain assumptions

inertial forces

infiltration models

ink bottle effect

instantaneous profile method

internal energy

intrinsic permeability

irreducible

K

kinematic viscosity

Klinkenberg effect

L

Liquid Content

LNAPL

LNAPLs in wells

M

macropore

matric potential

mean free path length

microbe

Miller similarity

$\mathbf{N}$

NAPL

NAPLS

Navier Stokes Equation

negative pressure

neutron probe

neutron probe, errors

Newtonian fluid

non aqueous phase liquids

non-spreading NAPL

non-wetting fluid 
O

oil spill

Osmotic

osmotic potential

oven drying

$\mathbf{P}$

particle size distribution

pendular

permanent wilting point

phases

piezometer

pore size distribution

porosity

potential energy

pressure head

pressure potential

pressure potential, measurement

psychrometer

psychrometric equation

pyrite

R

rain-drop effect

reduced radius

relative humidity

Representative Elementary Volume

residual

REV

Reynolds number

Richards equation

$\mathbf{S}$

satiated

satuated

saturated liquid content

scaled conductivity

scaled pressure

scaled sorptivity

scaled time

scanning curve

similar media

similarity

similarity assumptions

soil diffusivity

sorptivity tube

specific flux

specific gravity

spreading NAPL

suction

surface tension

120 | Basic Relationships and the Soil Phases Under Equilibrium Conditions 


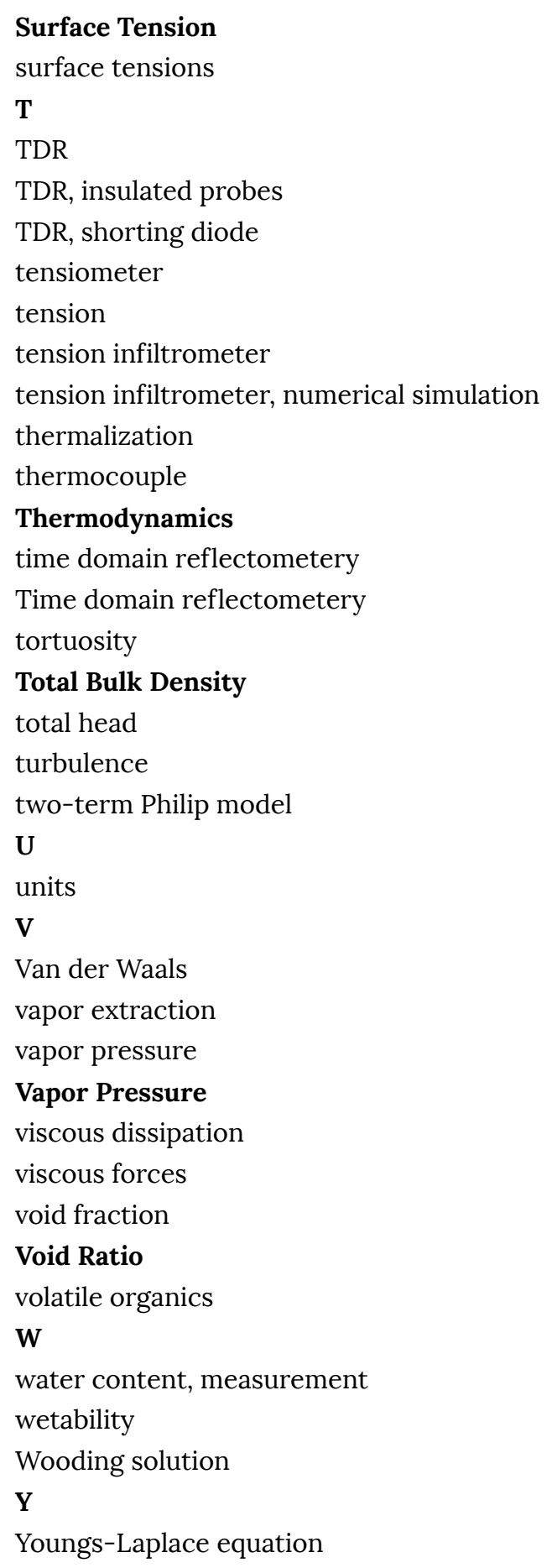

\section{References}

Brown and Montgomery, 1913, "Dehydration of Clays," Bureau of Standards Technologic Paper no. 21, Washington, D.C.: https://nvlpubs.nist.gov/nistpubs/nbstechnologic/nbstechnologicpaperT21.pdf Kirkham and Powers (1972) 
Revil, A. and A. Jardani, The Self potential Method: theory and applications in environmental geosciences, Cambridge University Press, ISBN 978-1-107-01927-0, 2013.

Sposito (1989)

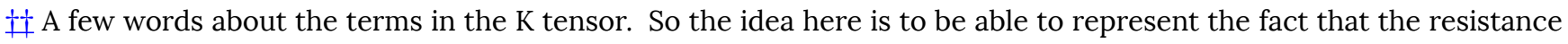
to flow might be different per direction due to orientation of the particles at a scale below that of the REV. So we will "take apart" the conductivity to explicitly represent the relationship between the gradient in potential in a particular direction, and the resulting flow that might happen in that same direction, or along a different axis. Writing Darcy's law

$$
\begin{aligned}
\mathrm{q} & =-\left[\begin{array}{c}
\mathrm{K}_{\mathrm{xx}} \mathrm{K}_{\mathrm{xy}} \mathrm{K}_{\mathrm{xz}} \\
\mathrm{K}_{\mathrm{yx}} \mathrm{K}_{\mathrm{yy}} \mathrm{K}_{\mathrm{yz}} \\
\mathrm{K}_{\mathrm{zx}} \mathrm{K}_{\mathrm{zy}} \mathrm{K}_{\mathrm{zz}}
\end{array}\right]\left[\frac{\partial \mathrm{h}}{\partial \mathrm{x}} \frac{\partial \mathrm{h}}{\partial \mathrm{y}} \frac{\partial \mathrm{h}}{\partial \mathrm{z}}\right] \\
& =-\left[\mathrm{K}_{\mathrm{xx}} \frac{\partial \mathrm{h}}{\partial \mathrm{x}}+\mathrm{K}_{\mathrm{xy}} \frac{\partial \mathrm{h}}{\partial \mathrm{y}}+\mathrm{K}_{\mathrm{xz}} \frac{\partial \mathrm{h}}{\partial \mathrm{z}} ; \mathrm{K}_{\mathrm{yx}} \frac{\partial \mathrm{h}}{\partial \mathrm{x}}+\mathrm{K}_{\mathrm{yy}} \frac{\partial \mathrm{h}}{\partial \mathrm{y}}+\mathrm{K}_{\mathrm{yz}} \frac{\partial \mathrm{h}}{\partial \mathrm{z}} ; \mathrm{K}_{\mathrm{zx}} \frac{\partial \mathrm{h}}{\partial \mathrm{x}}+\mathrm{K}_{\mathrm{zy}} \frac{\partial \mathrm{h}}{\partial \mathrm{y}}+\mathrm{K}_{\mathrm{zz}} \frac{\partial \mathrm{h}}{\partial \mathrm{z}}\right]
\end{aligned}
$$

To get a feel for the terms in the K-tensor, look at the terms in the $\mathrm{x}$-coordinate of flux. We see that gradients in each of the $\mathrm{x}, \mathrm{y}$, and $\mathrm{z}$ directions can give rise to flux in the $\mathrm{x}$ direction. In general we see that the term $\mathrm{K}_{\mathrm{ab}}$ is the proportionality constant relating gradients in potential in the b-direction to flux that results in the a-direction. How could such a thing happen? If the medium is aniostropic (i.e. permeability is a function of direction) then gradients that are not aligned with the directionality of permeability will give rise to flux not parallel with potential gradients. An easy analogy is movement on a slide in a playground. The direction of energy gradient due to gravity is vertically downward, but your resulting motion includes a lateral component, along the direction of the slide. This really is similar to the motion of water in a clay with oriented particles: the water will largely travel parallel to the sheet-structures of the clay rather than across the clay sheets because the resistance is so much lower, even if the gradient is not exactly in the same direction as the orientation of the clay sheets. If the coordinate system is aligned such that the coordinate system and primary directions of anisotropy are coincident the off diagonal terms in the permeability tensor will be zero (i.e., the $\mathrm{K}_{\mathrm{ab}}$ terms where $\mathrm{a} \neq \mathrm{b}$ ), leaving permeabilities in the three coordinate directions. If these are all equal, then the tensor collapses to a scalar. So the only reason to take the permeability as a tensor is to capture the effects of anisotropy.

$\ddagger$ Some of you may ask what is this up-side-down triangle all about? The $\nabla$ symbol stands for the "dell" operator. It is just mathematical short hand for calculating the three-directional derivative instead of the one directional derivative. It is defined as 


$$
\nabla=\frac{\partial}{\partial \mathrm{x}} \mathrm{i}, \frac{\partial}{\partial \mathrm{y}} \mathrm{j}, \frac{\partial}{\partial \mathrm{z}} \mathrm{k}
$$

where $\mathrm{i}, \mathrm{j}$, and $\mathrm{k}$ are the unit vectors in the $\mathrm{x}, \mathrm{y}$, and $\mathrm{z}$ directions. The result of "operating" on a function with $\nabla$ is to obtain a new vector which is the slope of the function. In practice, for any scalar valued function of $x, y, z, \nabla F$ tells us the magnitude and direction of the slope of $\mathrm{F}: \nabla \mathrm{F}$ points directly towards the steepest direction of uphill with a length proportional to the slope of the hill. Later you will also see this symbol in the notation $\nabla \cdot F$. The dot just tells us to take the dell and calculate the dot product of that and the function F (which needs to be a vector for this to make sense). This term "dell-dot-F" then tells us the divergence of the F function. If F were three directional flux content, this would be the amount of water leaving the point $(\mathrm{x}, \mathrm{y}, \mathrm{z})$. Notice that this is a scalar result! So $\nabla \mathrm{F}$ needs a scalar function $\mathrm{F}$ and gives a vector slope, while $\nabla \cdot \mathrm{F}$ uses a vector function $\mathrm{F}$ and gives a scalar result. 


\section{Liquid Flow in Soils (Hydrodynamics)}

\section{Hydrodynamics in Porous Media}

Having described how fluids and porous media interact when the situation is hydrostatic, we now push on to understand how the fluid gets around in media. We will start by investigating how fluids in porous media respond to local potential gradients (Darcy's Law), and then will add the conservation of mass to obtain the governing equation for motion in porous media (Richard's equation). 
Darcy's Law for saturated media 


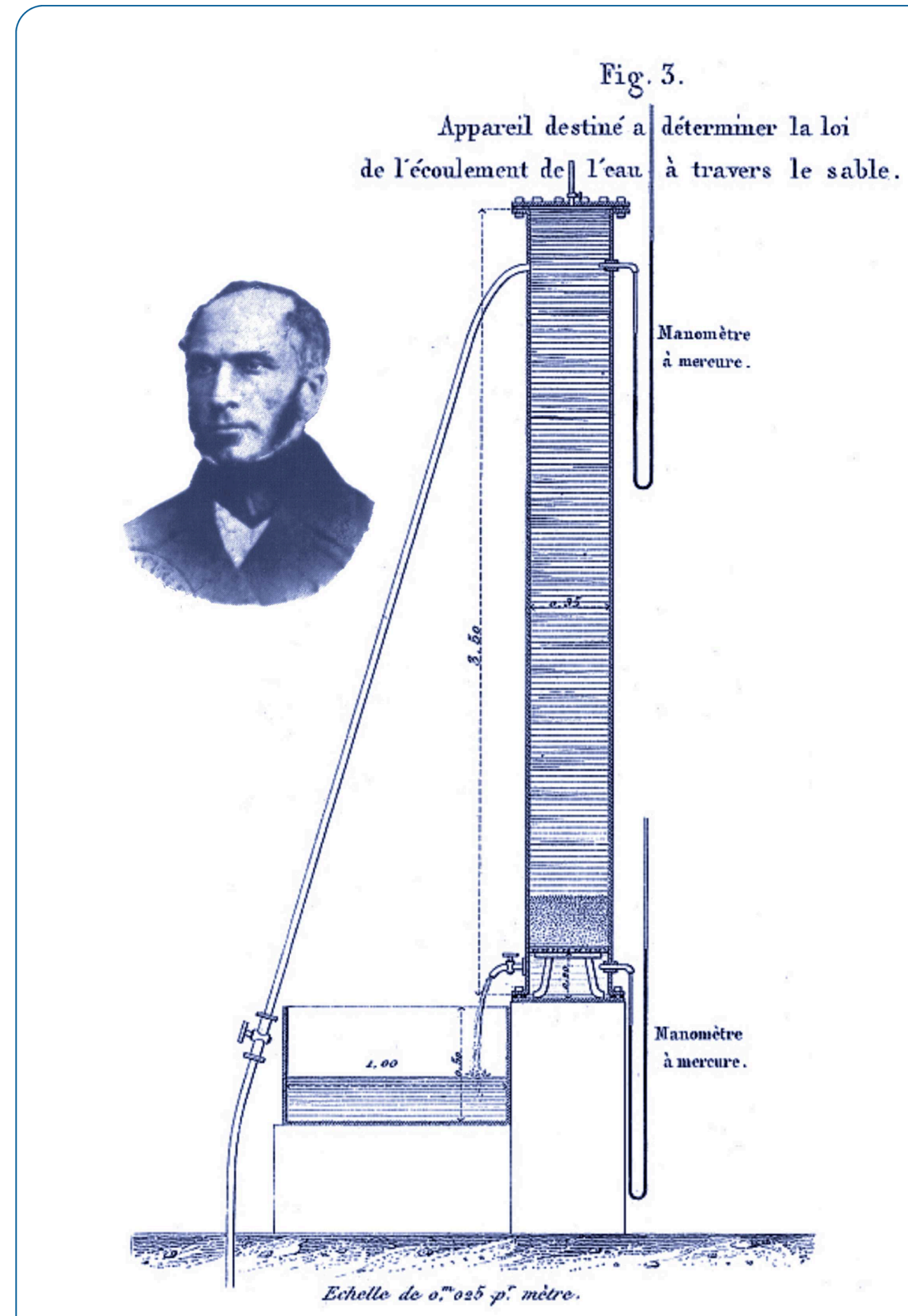

126 | Liquid Flow in Soils (Hydrodynamics) 
In the early 1850's, the Burgers of Dijon France employed Henry Darcy to assist in the calculation of how to provide water for the town's central fountain. Darcy experimentally determined that the flux of water through an isotropic porous medium could be expressed as the product of the resistance to flow which characterized the medium, and forces acting to "push" the fluid through the medium. Darcy defined the following properties:

$\mathrm{Q}$ - The rate of flow $\left(\mathrm{L}^{3} / \mathrm{T}\right)$ as the volume of water passed through a column per unit time.

$\mathrm{H}_{\mathrm{i}}$ - The total fluid potential in the medium at position i, measured in standing head equivalent. (see Figure 2.3a?) (L: force per unit area divided by $\rho g$ ).

$\mathrm{K}$ - The hydraulic conductivity of the medium. The proportionality between specific flux and imposed gradient for a given medium $(\mathrm{L} / \mathrm{T})$.

$\mathrm{L}$ - The length of vertical medium through which flow passes (L).

A - The cross-sectional area of the column $\left(\mathrm{L}^{2}\right)$.

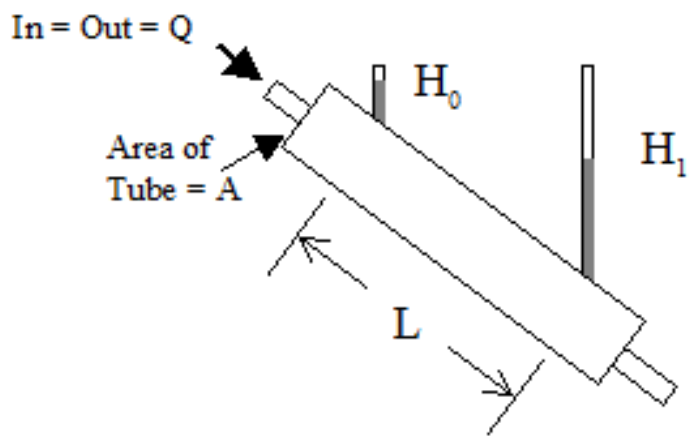

Figure 3.2 Set-up for Darcy's law. The two vertical tubes (called piezometers by hydrogeologists) labeled $\mathrm{H}_{0}$ and $\mathrm{H}_{1}$ provide measurements of the combined pressure and elevation potential, and the dimension $\mathrm{L}$ is the flow path length between the two pressure measurement points.

Darcy then observed that the flow of water in a column was well described by the equation

$$
\mathrm{Q}=-\mathrm{K} \frac{\mathrm{A}}{\mathrm{L}}\left(\mathrm{H}_{1}-\mathrm{H}_{0}\right)
$$

In words, [2.68] says that you will obtain a flow Q is proportional to the negative of the head loss (flow moves towards lower head), proportional to the area of flow, and inversely proportional to the distance over which the head is dissipated. Darcy's expression can be expressed for flow in multiple dimensions for isotropic media as

$$
\text { (3.2) } \mathrm{q}=\frac{\mathrm{Q}}{\mathrm{A}}=-\mathrm{K} \nabla \mathrm{H}
$$

where $\mathrm{q}$ is the specific flux vector ( $\mathrm{L} / \mathrm{T}$; volume of water per unit area per unit time), $\mathrm{K}$ is the saturated hydraulic conductivity tensor (second rank市) of the medium (L/T), and $\nabla H$ is the gradient in total hydraulic head. $\ddagger$ These parameters are fundamental, and it is worth getting a feel for their units. The specific flux, q, has units of length per time, or velocity. In the case of vertical flow, this may be thought of as speed at which the height of a pond of fluid would drop, as it infiltrated into the soil. BE CAREFUL. Even though q has the dimensions of velocity, it is not the velocity at which the average particle of water is moving through the soil; that is influenced by porosity of the medium and degree of saturation, as we shall soon see (Figure 3.3?). The specific flux is a vector, since flow has both a magnitude 
and direction. The total fluid potential is made up of two terms, as we discussed way back in section 2.2. First, there is the pressure potential, or head, which can be thought of as the height of a column of water which would be supported by the pressure measured at the point of interest. We typically denote this by a lower case $h$. Secondly, there is the elevation potential, which is simply the height measured from some standard reference level (often called the "datum," where elevation is taken to be zero). The elevation we denote with a $\mathrm{z}$, so we have the total potential $\mathrm{H}=\mathrm{h}+\mathrm{z}$. Both of the terms in the total head may be expressed to have units of length. 


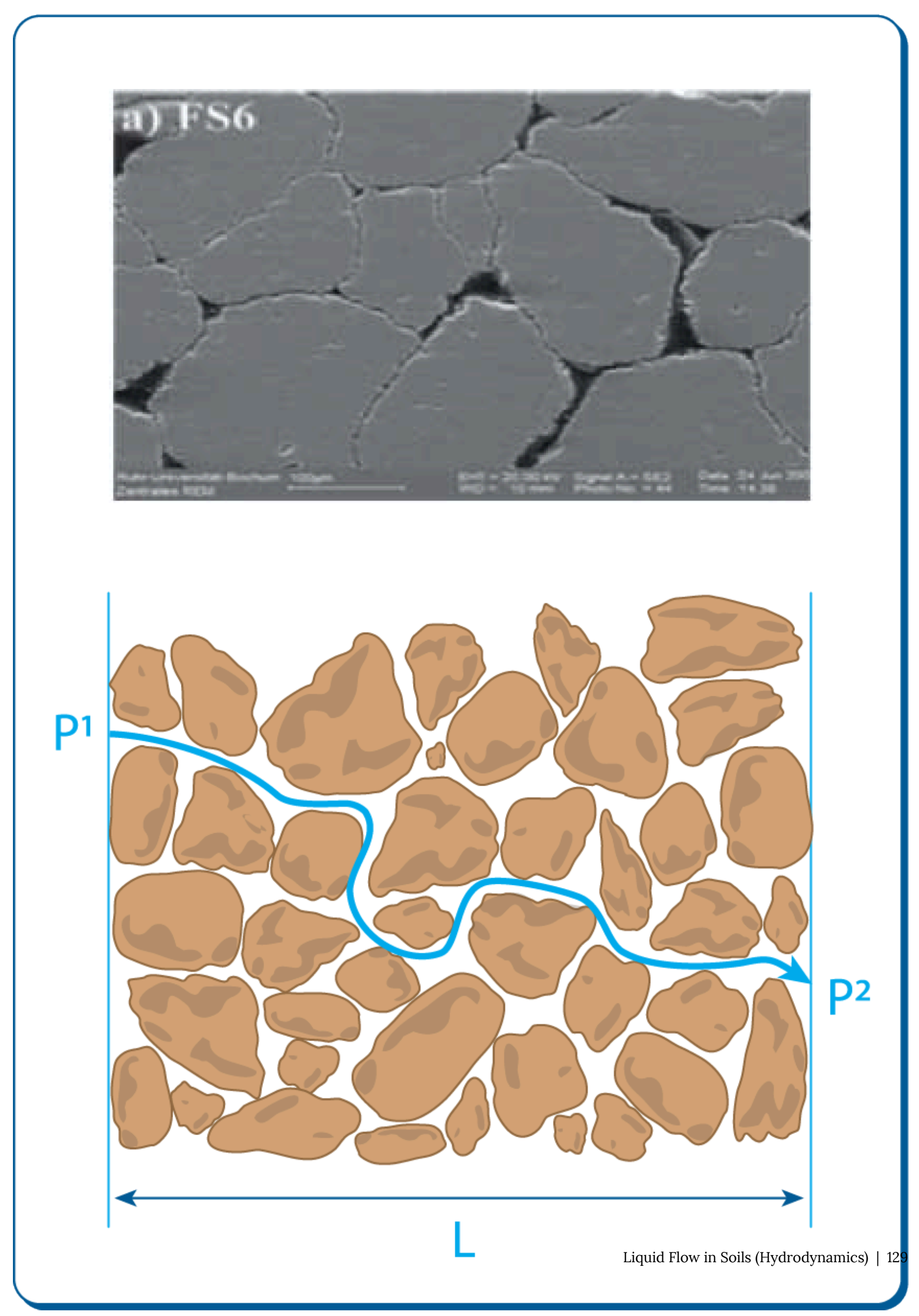


It is worth checking that the right hand side of [2.69] is indeed a vector: $\nabla \mathrm{H}$ is a vector, and a vector-tensor product is a vector, so that is as should be. Note that we could also obtain a vector on the right hand side by selecting $\mathrm{K}$ to be a scalar, which is often done (i.e., assuming that conductivity is independent of direction). Looking at the remainder of the parameters, clearly $Q=$ sq, and the magnitude of the gradient (ignoring direction, since the column only has flow in one direction)

$$
|\nabla \mathrm{H}|=\frac{\mathrm{H}_{1}-\mathrm{H}_{0}}{\mathrm{~L}}
$$

It is useful to observe the intuitively reasonable aspects of Darcy's expression. The rate of flow is directly related to the area of flow (e.g., put two columns in parallel under the same conditions and you will get twice the flow), inversely related to the length of flow (e.g., flow through twice the length with the same potential drop gives half the flux), and is directly related to the potential energy drop across the system (e.g., double the energy expended, head drop across the system, to obtain twice the flow). The expression is patently linear, in that all properties scale linearly with changes in system forces and dimensions.

Why should flow through porous media be a linear phenomenon? This really should be quite a surprise: most fluid mechanical processes are non-linear. For example, if you go twice as fast on your bicycle, the wind resistance will go up by a factor of four (proportional to velocity squared), not two (as would be true for a linear process). Is this linearity because the flow in porous media is not turbulent? While it is true that Darcy's law only applies to laminar flow, be careful, we require much more than laminar flow! You see, at velocities 100 times smaller than those required for turbulence there will be large local forces due to acceleration: these are called "inertial forces." Darcy's law is linear because most flow in porous media occurs at very low speeds where acceleration forces are negligibly small compared to the forces arising due to viscous shear. To see how this linearity arises, it is instructive to consider the Navier Stokes Equation for fluid flow, looking particularly at which terms must be very small in order to obtain a linear result. Written in one dimension, we recall that the $\mathrm{x}$-component of flow in a velocity field with velocities $\mathrm{u}, \mathrm{v}$, and $\mathrm{w}$ in the $\mathrm{x}, \mathrm{y}$, and $\mathrm{z}$ (vertical) directions, may be written

$$
\frac{\partial \mathrm{u}}{\partial \mathrm{t}}+\mathrm{u} \frac{\partial \mathrm{u}}{\partial \mathrm{x}}+\mathrm{v} \frac{\partial \mathrm{u}}{\partial \mathrm{y}}+\mathrm{w} \frac{\partial \mathrm{u}}{\partial \mathrm{z}}=\frac{-1}{\rho} \frac{\partial \mathrm{P}}{\partial \mathrm{x}}-\mathrm{g} \frac{\partial \mathrm{z}}{\partial \mathrm{x}}+\frac{\mu}{\rho} \nabla^{2} \mathrm{u}
$$

where P is the fluid pressure. Now let's impose the conditions for which Darcy's Law is considered valid; for "creeping flow," that is the frictional forces on the fluid phase are dominated by viscous dissipation. This requires that the local acceleration-deceleration (inertial) forces acting on parcels of water as they go through pores of differing size are small compared to the viscous and gravitational terms. In particular, the derivatives of velocities are taken to be small. So we take

$$
\frac{\partial u}{\partial x}=\frac{\partial u}{\partial y}=\frac{\partial u}{\partial z} \approx 0
$$

next let's assume steady flow,

$$
\frac{\partial \mathrm{u}}{\partial \mathrm{t}}=0
$$

With this somewhat drastic set of assumptions, Equation [2.71] becomes

$$
\frac{\partial}{\partial \mathrm{x}}(\mathrm{P}+\mathrm{rgz})=\mu \nabla^{2} \mathrm{u}
$$

On the left-hand-side of Equation [2.74] we have the familiar gradient of hydraulic potential, balancing the viscous 
dissipation due to flow on the right hand side. Noting that the expression is linear in so far as if you double the velocity you must double the gradient in head, we see that [2.74] has the same form as Darcy's Law. Is this a proof of Darcy's Law? It certainly does support the general form of the relationship between flow and gradient in potential. The idea is to show that the creeping flow assumption is sufficient to obtain linear flow behavior.

\section{I.2 Laminar Flow in Capillary Tubes}

An instructive and widely used model for flow through porous media is that of flow through a cylindrical capillary tube. This analysis reveals the fundamental relationships between pore size and permeability. Such a relationship have then be used to "construct" media as a collection of variously sized tubes, and is used as the motivation for many infiltration and flow formulations. We will derive the equation of flow through a capillary of radius $r_{o}$ with steady flow as shown in Figure 3.4. Considering a cylindrical control volume centered on the axis of the cylinder with radius $r$ and length $\Delta S$, the distribution of shear stress can be calculated by balancing the forces on the control volume. Since the system is steady (no acceleration), the net force in the direction of flow must be 0 , or

(3.8) $\Sigma \mathrm{F}=0$

The forces acting on the control volume are simply the end pressures and the shear force. Defining force to the right as positive (no politics, please!) we have:

end pressures:

$$
\text { at } \mathrm{S}=0 \quad \mathrm{~F}_{1}=\mathrm{P} \pi \mathrm{r}^{2}
$$

$$
\text { at } \mathrm{S}=\mathrm{S} \quad \mathrm{F}_{2}=\left(\mathrm{P}+\mathrm{S} \frac{\mathrm{dP}}{\mathrm{dS}}\right) \pi \mathrm{r}^{2}
$$

shear force:

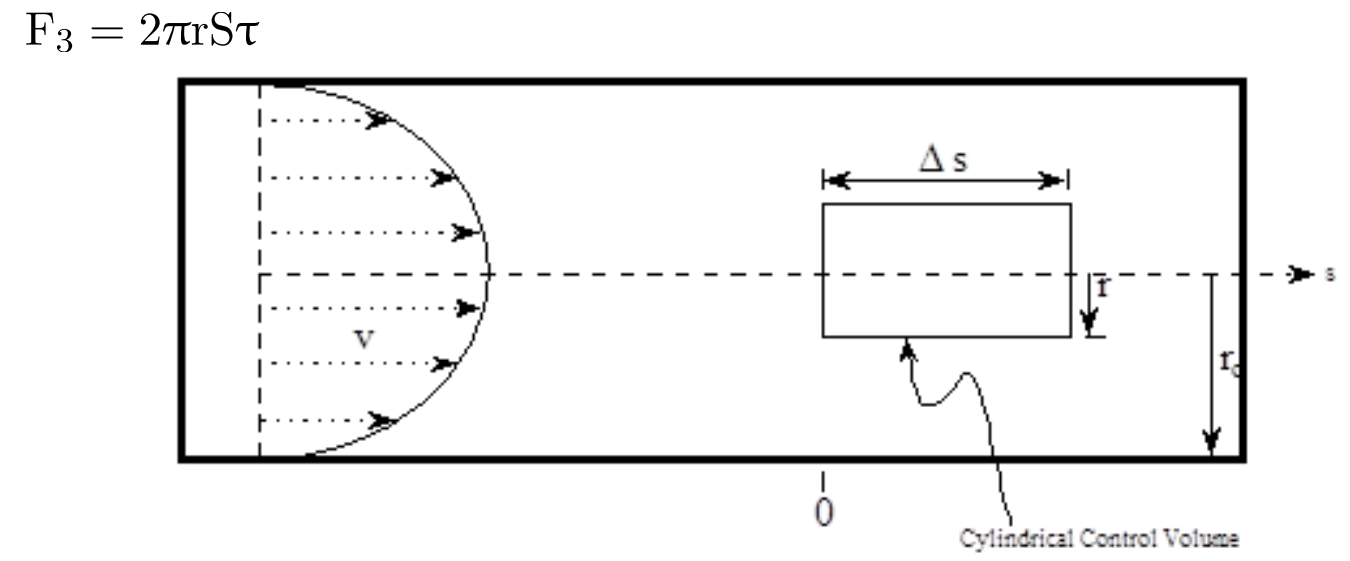

Figure 3.4. Definition sketch for a control volume for flow in a cylindrical tube.

where $\tau$ is the local shear stress. Putting these forces into [2.75], we obtain:

$$
P \pi r^{2}-\left(P+S \frac{d P}{d S}\right) \pi r^{2}-2 \pi r S \tau=0
$$


where we remember that $\mathrm{dP} / \mathrm{dS}$ is negative in sign (pressure drops along the direction of flow). Equation [2.76] immediately simplifies to

$$
\tau=-\frac{\mathrm{r}}{2} \frac{\mathrm{dP}}{\mathrm{dS}}
$$

Since $\mathrm{dP} / \mathrm{dS}$ is constant (the flow is steady and uniform), so we find the elegant result that shear stress varies linearly with radius (Figure 3.5).

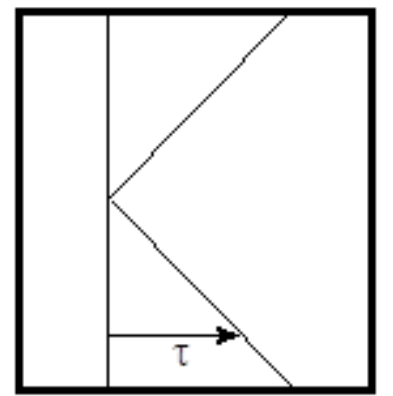

Figure 3.5 The shear stress increases linearly with radius in fluid flowing in the laminar regime in a cylindrical tube.

Now we may use definition of viscosity for a Newtonian fluid to put the shear stress [2.77] in terms of velocity and the fluid's dynamic viscosity $\mu .+$

$$
\tau=-\mu \frac{\mathrm{dV}}{\mathrm{dr}}
$$

Putting this into [2.77] we find the differential equation relating velocity to radial position and gradient in potential

$$
\frac{\mathrm{dV}}{\mathrm{dr}}=\frac{\mathrm{r}}{2 \mu} \frac{\mathrm{dP}}{\mathrm{dS}}
$$

This may be integrated

$$
\int_{V=0}^{V=V(r)} d V=\int_{r=r o}^{r} \frac{r}{2 \mu} \frac{d P}{d S} d r
$$

to obtain the velocity at any distance from the center of the capillary

$$
\mathrm{V}(\mathrm{r})=\frac{\left(\mathrm{r}^{2}-\mathrm{r}_{0}{ }^{2}\right)}{4 \mu} \frac{\mathrm{dP}}{\mathrm{dS}}
$$

which gives the velocity profile in a cylindrical pipe. To calculate the flux through the pipe we simply integrate over the area of the pipe:

$$
\mathrm{Q}=\int_{\text {Area }} \mathrm{VdA}
$$

in terms of cylindrical coordinates, $\mathrm{dA}=\mathrm{rd} \theta \mathrm{dr}$, and putting [2.81] in for $\mathrm{V}$, [2.82] becomes 


$$
\mathrm{Q}=\int_{\theta=0}^{0=2 \pi} \int_{\mathrm{r}=0}^{\mathrm{r}=\mathrm{r}_{0}} \frac{\left(\mathrm{r}^{2}-\mathrm{r}_{0}^{2}\right)}{4 \mu} \frac{\mathrm{dP}}{\mathrm{dS}} \mathrm{rdrd} \theta
$$

which can easily be evaluated to obtain

$$
\mathrm{Q}=-\frac{\pi}{\mu} \frac{\mathrm{r}_{0}}{8} \frac{\mathrm{dP}}{\mathrm{dS}}
$$

which is the well known Hagen-Poiseuille Equation. We are often interested in the flow per unit area (flux), for which we use the symbol $\mathrm{q}=\mathrm{Q} / \pi \mathrm{r}^{2}$

$$
\mathrm{q}=-\frac{1}{\mu} \frac{\mathrm{r}_{0}{ }^{2}}{8} \frac{\mathrm{dP}}{\mathrm{dS}}
$$

We commonly measure pressure in terms of hydraulic head, so we may substitute $\gamma \mathrm{h}=\mathrm{P}$, to obtain

$$
q=-\frac{r_{0}^{2}}{8} \frac{\gamma}{\mu} \frac{d h}{d S}
$$

Now we can identify the elements controlling capillary flow. The term $\mathrm{r}_{0}^{2} / 8$ is a purely geometric term describing the shape and size of the capillary, which is strictly a function of the solid medium (see Figure 3.6?). The coefficient 1/8 applies to a cylinder, whereas a square, or triangular capillary would be described by the same equation with a slight adjustment of this factor to reflect the impact of pore geometry. For example, had this been set up as flow between parallel plates (known as Couette flow, the basis for groundwater models based on the Hele-Shaw cell), we obtain the same result, but the number $1 / 8$ in the denominator is replaced by $1 / 12$. This will be referred to as the intrinsic permeability of the medium, denoted by the symbol $\kappa$. The second fraction on the right hand side of [2.86] $\gamma /$ $\mu$ is a function of the density and viscosity of fluid alone, while the third term is a function of the distribution of pressure alone. First and foremost notice that we have automatically recovered Darcy's law, and also been able to see why by pulling $\gamma / \mu$ out of the hydraulic conductivity we obtain an intrinsic property of the solid which can be applied to a range of fluids, such as predicting flow for oil versus water. 


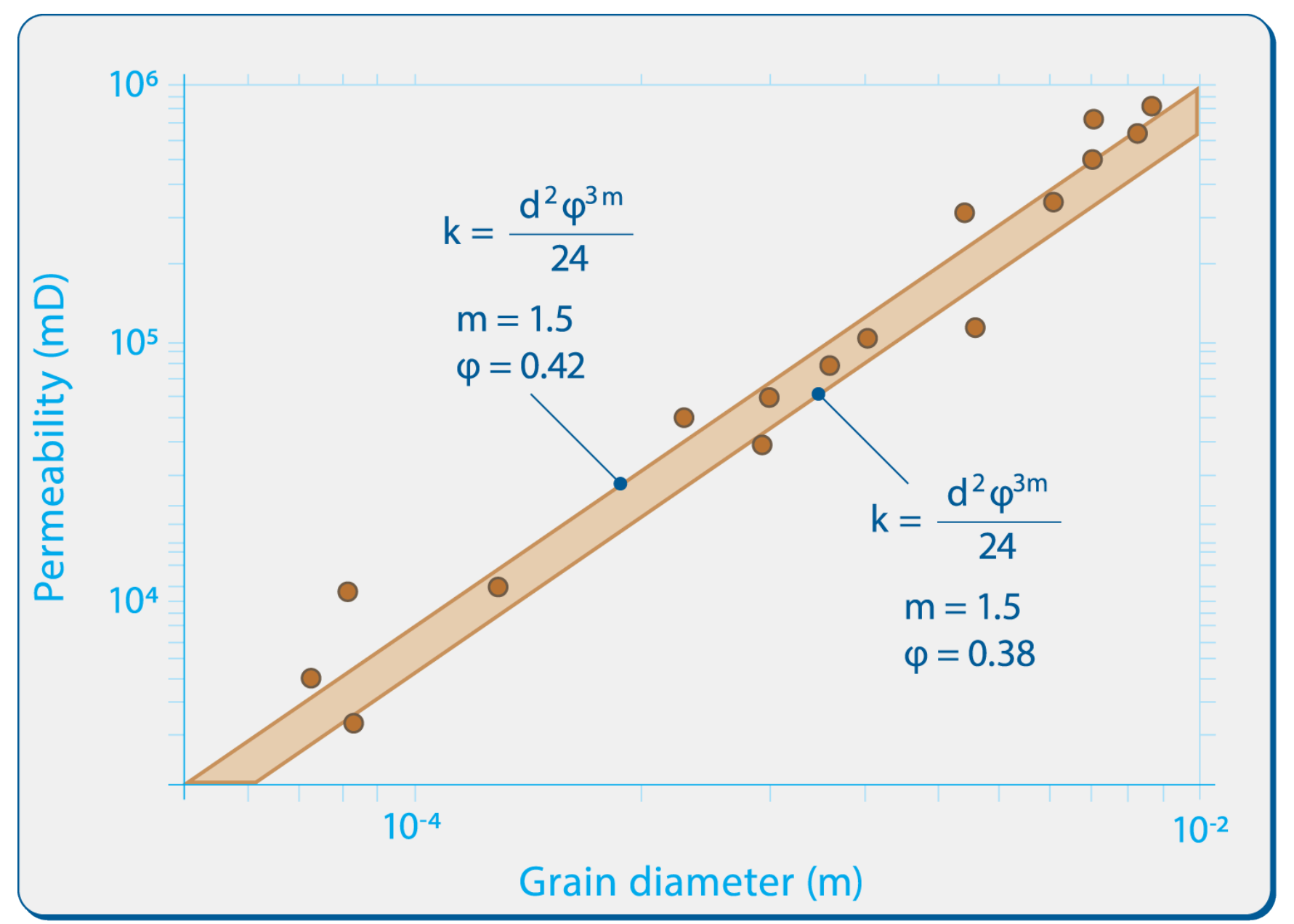

Figure 3.6

\section{Example 2-1: Laminar Flow in Tubes}

\section{Problem Statement:}

What is the average (laminar) flow velocity of water at $20^{\circ} \mathrm{C}$ through a $50 \mathrm{~m}$ long tube having diameter $\mathrm{d}=0.1$ $\mathrm{m}$ under a pressure difference of $100 \mathrm{~Pa}$ ?

\section{Solution:1}

We introduce into Eq.(XX) $\mathrm{L}=50 \mathrm{~mm}, \Delta \mathrm{P}=100$ pascal, $\mathrm{R}^{2}=(0.1 / 2)^{2} \mathrm{~m}^{2}$, and the viscosity of water at ${ }^{\circ} \mathrm{C}$ (taken from tabular values) is $0.001 \mathrm{~Pa}$ s. The resulting mean velocity is:

$$
\mathrm{v}=\frac{0.0025 \mathrm{~m}^{2}}{8 \times 0.001 \mathrm{Pas}} \frac{100 \mathrm{~Pa}}{50 \mathrm{~m}}=0.625 \mathrm{~m} / \mathrm{s}
$$


which is pretty fast! Let's check the Reynolds number to make sure that the Hagen-Poiseuille equation applies. The Reynolds number is given by

$$
\text { Re } \quad=\frac{\rho d v}{\mu}=\frac{1 \mathrm{~g} / \mathrm{cm}^{3} \times 10 \mathrm{~cm} \times 62.5 \mathrm{~cm} / \mathrm{s}}{0.01 \mathrm{~g} / \mathrm{cm}-\mathrm{s}}=62,500
$$

Whoops! This is WAY over the laminar to turbulent flow limit of $\operatorname{Re}<100$ which indicates the applicability of the Hagen-Poiseuille equation. Watch out: the result is WRONG!

\section{Darcy's Law at Reynolds Number Greater than I}

It is often stated that Darcy's Law breaks down at Reynolds numbers greater than 1. Since laminar flow holds in smooth capillary tubes with $\operatorname{Re}<2000$, suggesting that the Hagen-Poiseuille law would still be valid, why does Darcy's law break down so soon? First we should note that laminar flow breaks down in flow through natural media's at a Reynolds number closer to 100 due to the tortuosity of the flow paths (see Bear, 1972, pg. 178). But this is still far above the value required for deviations from Darcy's law. As we discussed above in the context of the Navier Stokes equation, the first source of difficulty for Darcy's law at high velocity arises from the magnitude of the local acceleration of fluids. In the derivation of the Hagen-Poiseuille equation, steady flow is assumed (i.e., no acceleration). But in natural media, even at steady-state, fluid must accelerate and decelerate as it passes around particles at the microscopic level. When the forces arising from these local accelerations are of the same order of magnitude as the viscous forces, they must be accounted for in the macroscopic flow equation. At such high flow rates, there is increased resistance to flow, so flux responds less to applied pressure gradients. To get a feel for this limitation on Darcy's law, it is worth going through a simple calculation of the relative magnitudes of the viscous and inertial forces. Li and Helm (1998) published a general analysis of this problem for the case of porous flow though possibly moving porous media. We will limit our analysis to the much simpler case of flow through stationary media.

To obtain an estimate of the magnitude of viscous forces, it is most direct to go back to the Hagen-Poiseuille solution for flow through a capillary tube. This solution considers only the viscous forces to obtain the solution for average flow velocity

$$
\mathrm{v}=\frac{\mathrm{r}^{2}}{8 \mu} \frac{\mathrm{dP}}{\mathrm{dx}}
$$

In a soil or aquifer material, the passages which carry flow have varying cross-sections, which is ignored in the derivation of this result. This oversight does not cause problems as long as the flow is "creeping" (the energy loss is dominated by viscous dissipation). Other forces which can dissipate energy are inertial forces (due to acceleration), and turbulent drag. For Re $<100$ (the turbulent threshold) it is reasonable to ignore turbulent dissipation. But if the passages have greatly differing cross-sections, then the fluid will accelerate and decelerate as it passes from large to small channels, which makes us wonder when inertial forces will be of a similar magnitude to the viscous forces which we have included in the Hagen Poiseuille solution.

To get an idea of the relative magnitudes of the inertial and viscous forces we consider the configuration shown below. 


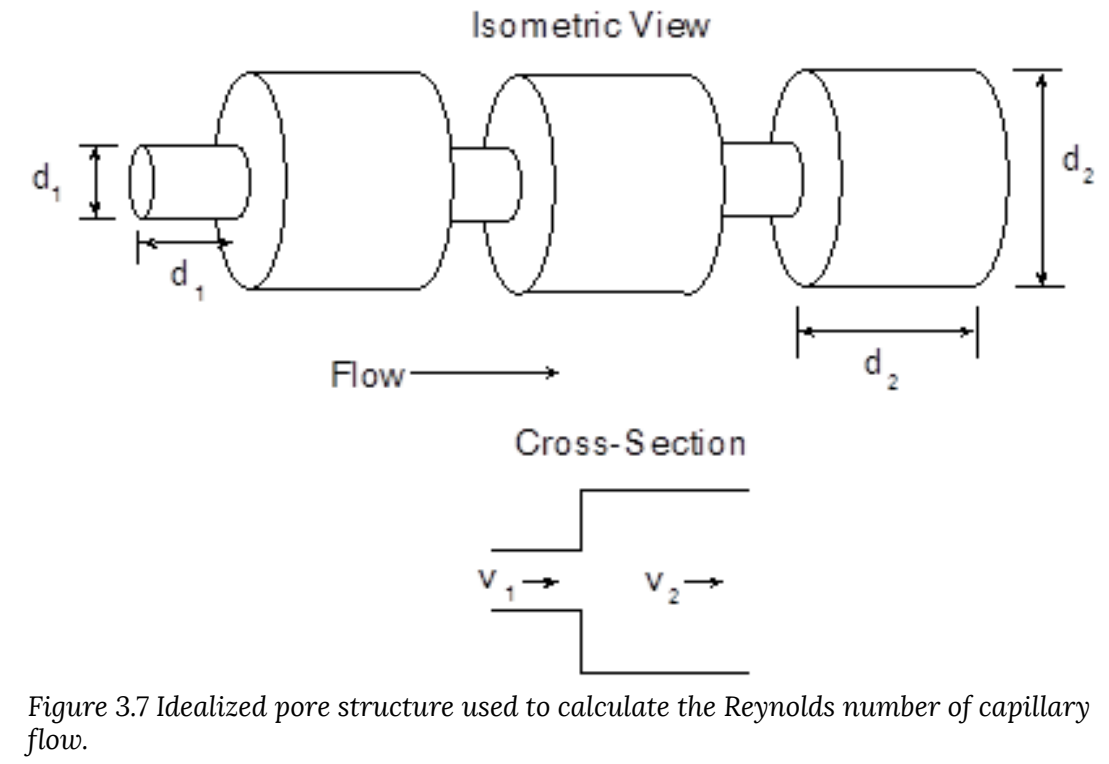

We would like to estimate the magnitude of the inertial and viscous dissipation, and develop a criteria which indicates when the forces are of similar magnitude. For the sake of allowing calculation, we will take $d_{2}=2 d_{1}$. By conservation of mass

(3.25) $\mathrm{v}_{2} \mathrm{~A}_{2}=\mathrm{v}_{1} \mathrm{~A}_{1}$

where $A_{2}$ and $A_{1}$ are the areas of the larger and smaller tubes respectively. This is simply

$$
\mathrm{v}_{2} \frac{\pi \mathrm{d}_{2}^{2}}{4}=\mathrm{v}_{1} \frac{\pi \mathrm{d}_{1}^{2}}{4}
$$

or

$$
\mathrm{v}_{2} \mathrm{~d}_{2}{ }^{2}=\mathrm{v}_{1} \mathrm{~d}_{1}{ }^{2}
$$

so

$$
\mathrm{v}_{1}=\frac{\mathrm{v}_{2} \mathrm{~d}_{2}^{2}}{\mathrm{~d}_{1}{ }^{2}}
$$

or

(3.26) $\mathrm{v}_{1}=4 \mathrm{v}_{2}$

Since the viscous forces increase drastically with decreasing radius, we can safely assume that the majority of the viscous dissipation occurs in the smaller tubes. From [2.87]

$$
\frac{\mathrm{dP}}{\mathrm{dx}}=\frac{8 \mathrm{v} \mu}{\mathrm{r}^{2}}
$$

but 


$$
\begin{aligned}
\frac{\mathrm{dP}}{\mathrm{dx}} & =\frac{\text { change in force per unit area }}{\text { unit length }} \\
& =\frac{\mathrm{F}_{\mathrm{v}} / \text { area }}{\text { length }} \\
& =\frac{\mathrm{F}_{\mathrm{v}} \mathrm{d} \frac{4}{\pi \mathrm{d}_{1}^{2}}}{\mathrm{~d}_{1}} \\
& =\frac{\mathrm{dP}}{\mathrm{dx}_{\mathrm{x}}}=\frac{8 \mathrm{v} \mu}{\mathrm{r}^{2}} \\
& =\frac{4 \mathrm{~F}_{\mathrm{v}}}{\pi \mathrm{d}_{1}{ }^{3}}
\end{aligned}
$$

so putting this into the Hagen-Poiseuille equation we obtain

$$
\frac{4 \mathrm{~F}_{\mathrm{v}}}{\pi \mathrm{d}_{1}^{3}}=\frac{32 \mathrm{v}_{1} \mu}{\mathrm{d}_{1}{ }^{2}}
$$

or

$$
\text { (3.30) } \mathrm{F}_{\mathrm{v}}=8 \pi \mathrm{v}_{1} \mathrm{~d}_{1} \mu
$$

Now the inertial force can be estimated by calculating the deceleration of the fluid passing from the small tube to the large tube. We know

(3.31) $\mathrm{F}_{\mathrm{I}}=\mathrm{ma}$

The mass involved can be estimated to be the volume of the fluid in deceleration, which is about half the volume of the large pore (in the second half of the large pore the water is accelerating into the next small neck). So 


$$
\begin{aligned}
\mathrm{m} & =\rho \frac{\mathrm{d}_{2}}{2} \frac{\pi \mathrm{d}_{2}{ }^{2}}{4} \\
& =\frac{\rho \pi \mathrm{d}_{2}{ }^{3}}{8} \\
& =\rho \pi \mathrm{d}_{1}{ }^{3}
\end{aligned}
$$

The acceleration may be estimated by considering the change in velocity over the time it takes to decelerate, which is approximately $\mathrm{d}_{2} / 2$.

$$
\begin{aligned}
& \mathrm{a}=\frac{\mathrm{dV}}{\mathrm{dt}} \\
& =\frac{\left[\mathrm{v}_{1}-\mathrm{v}_{2}\right]}{\left[\frac{\frac{\mathrm{d}_{2}}{2\left(\mathrm{v}_{1}+\mathrm{v}_{2}\right)}}{2}\right]}
\end{aligned}
$$

noting that $\mathrm{v}_{1}=4 \mathrm{v}_{2}$ and $\mathrm{d}_{2}=2 \mathrm{~d}_{1}$ this can be re-written as

$$
\mathrm{a}=\frac{15 \mathrm{v}_{2}{ }^{2}}{2 \mathrm{~d}_{1}} \approx \frac{8 \mathrm{v}_{2}{ }^{2}}{\mathrm{~d}_{1}}
$$


(a)

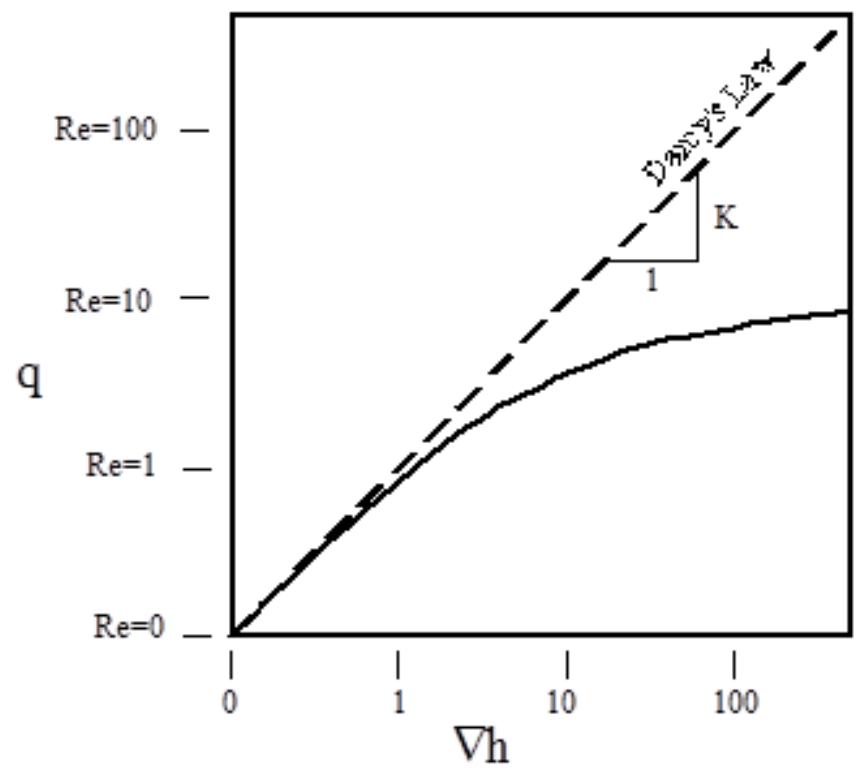

(b)

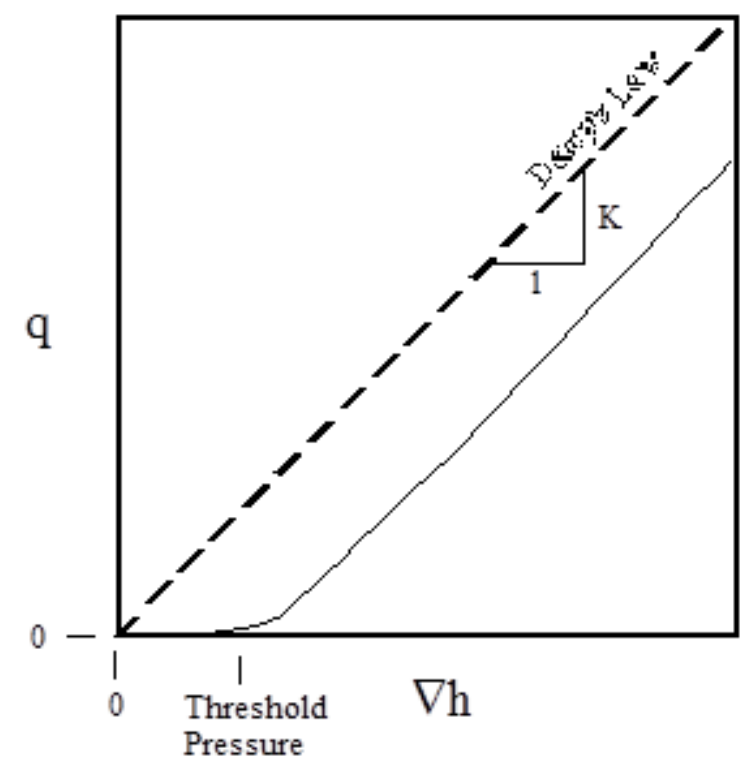

Figure 3.8 Illustration of the deviations from Darcy's law which may occur. (a) The effect of inertial terms becoming significant at Re>1. (b) The

somewhat more controversial assertion that at very low flow there may be a threshold gradient required to be overcome before any flow occurs at all due to hydrogen bonding of water. 


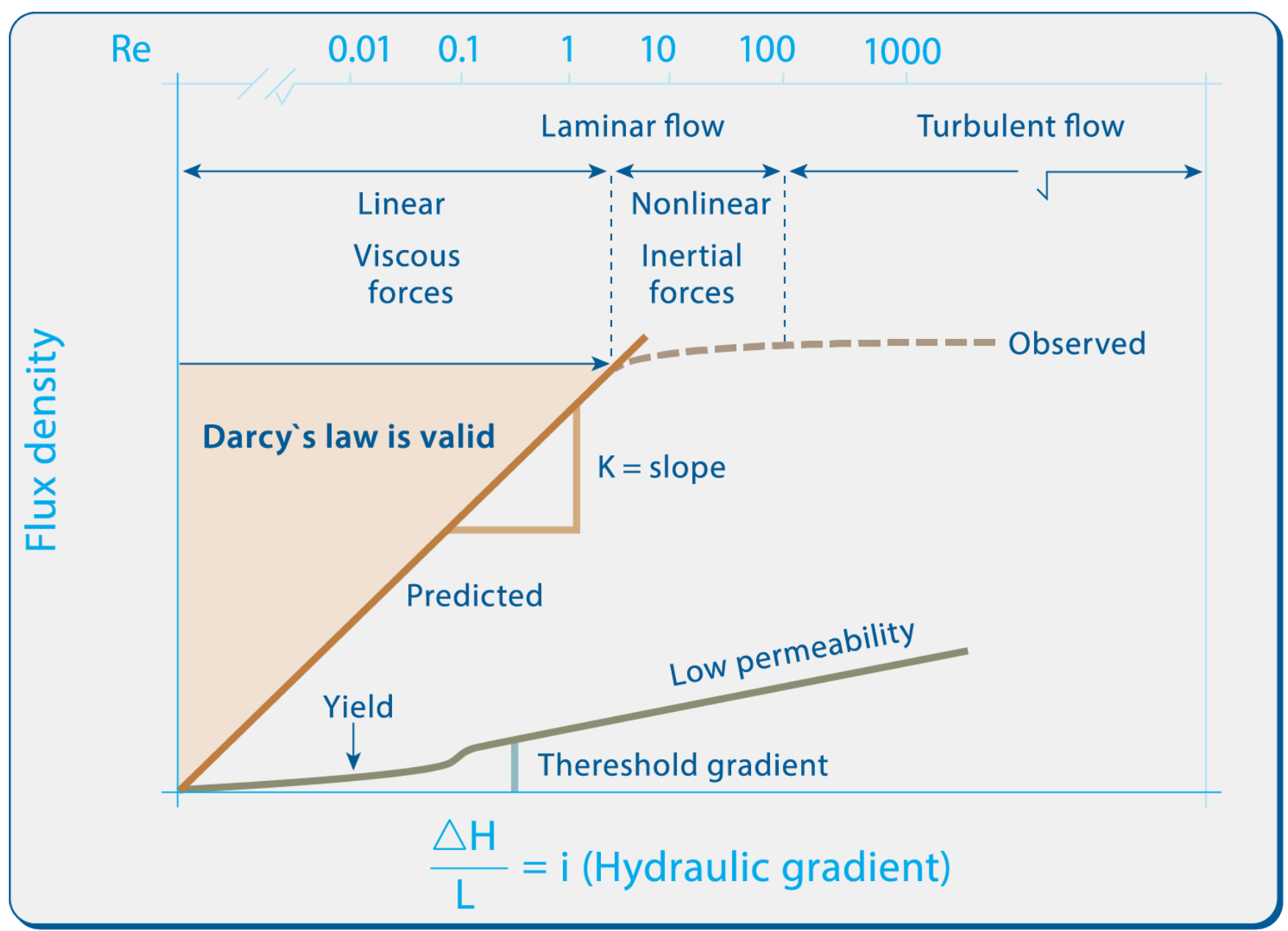

Figure 3.9

So the inertial force can be approximated by

$$
\begin{aligned}
\mathrm{F}_{1} & =\rho \pi \mathrm{d}_{1} 3 \frac{8 \mathrm{v}_{1}{ }^{2}}{\mathrm{~d}_{1}} \\
& =\frac{\rho \pi \mathrm{d}_{1}{ }^{2} \mathrm{v}_{1}{ }^{2}}{2}
\end{aligned}
$$

To obtain an estimate for when the assumption of creeping flow breaks down, we can calculate the Reynolds number which is obtained when viscous forces are equal to inertial forces. When this equality holds, it should be expected that Darcy's law will be in significant error. 


$$
\begin{aligned}
& \mathrm{F}_{\mathrm{I}}=\mathrm{F}_{\mathrm{v}} \\
& \frac{\rho \pi \mathrm{d}_{1}{ }^{2} \mathrm{v}_{1}{ }^{2}}{2}=8 \pi \mathrm{v}_{1} \mathrm{~d}_{1} \mu
\end{aligned}
$$

or

$$
\frac{\rho \mathrm{d}_{1} \mathrm{v}_{1}}{\mu}=16
$$

Which is to say that the inertial forces are approximately equal to the viscous forces when the Reynolds number is of the order of 10. Since the inertial forces go with the square of velocity, while the viscous forces go linearly with velocity, we see that at a Reynolds number of 1 the inertial forces are about one tenth the magnitude of viscous forces, which seems to be a reasonable cut-off point for the creeping flow approximation.

It is fine to acknowledge the break down of Darcy's law at very high flow regimes, but how does this relate to the vadose zone? How close do we come to this limit under typical flow conditions found in unsaturated soils? The maximum Reynolds number is obtained for the maximum particle diameter and maximum fluid velocity, assuming that density and viscosity are constant. Let's first consider typical water flow where $\mathrm{v}$ and $\mathrm{d}$ are maximized. This occurs during gravity driven flow near saturation in a coarse medium. For a coarse sandy soil, maximum neck diameter will be about $1 \mathrm{~mm}$, and vertical flux may be as high as $1 \mathrm{~cm} / \mathrm{min}$ (14 meters/day for saturated flow under full gravity gradient). The Reynolds number is then

$$
\begin{aligned}
\mathrm{R} & =\frac{\rho \mathrm{d}_{1} \mathrm{v}_{1}}{\mu} \\
& =\frac{1 \mathrm{~g} / \mathrm{cm}^{3} \times 0.1 \mathrm{~cm} \times 1 \mathrm{~cm} / \mathrm{min}}{0.01 \mathrm{~g} / \mathrm{cm}-\mathrm{s}} \\
& =0.167
\end{aligned}
$$

Typically conditions of water movement in the unsaturated zone will give rise to Reynolds numbers far below this value, but in coarse media (e.g. infiltration into pea gravel following a sudden spill), the creeping flow assumptions which are required for Darcy's law can be violated.

It is increasingly common to employ vapor extraction methods for removing volatile contaminants from unsaturated media. In these operations, air is pumped through a region of contaminated soil with the volatile contaminants diffusing into the air. The air is then extracted with the volatile contaminants and either vented to the atmosphere or captured, typically in an activated carbon filter. Air velocities in these processes can exceed $30 \mathrm{~m} /$ day $(0.035 \mathrm{~cm} / \mathrm{sec})$. Does Darcy's law apply? $\neq$ The Reynolds number for this air flow rate in the coarse soil used in the example considered above is 


$$
\begin{aligned}
\mathrm{R} & =\frac{\rho \mathrm{d}_{1} \mathrm{v}_{1}}{\mu} \\
& =\frac{0.001 \mathrm{~g} / \mathrm{cm}^{3} \times 0.1 \mathrm{~cm} \times 0.035 \mathrm{~cm} / \mathrm{s}}{1.8 \times} \\
(3.38) \quad & =0.02
\end{aligned}
$$

which is also well below the value required to maintain creeping flow. For a given soil and velocity, the Reynolds number for water flow is about 10 times that of air flow (the ratio of the kinematic viscosities). Note that immediately surrounding the air inlet and extraction pipes the flow could be much higher than the average bulk flow, and thus may violate the Reynolds number criteria. Similarly, immediately surrounding a pumping ground water well the local velocities could exceed the Reynolds number criteria. In such cases, the effective hydraulic conductivity will decrease with increasing flow velocity. Note that this makes Darcy's law non-linear, which greatly complicates analysis of flow. Fortunately, this is very rarely of concern in natural flow systems. Near wells where pumping is intense, the Reynolds Number criteria can be violated. This has been long noted, and non-linear flux-dependent adjustments to Darcy's law have been proposed by many, most notably Forschheimer (Forchheimer, P.: 1901, Wasserbewegung durch boden,Zeit. Ver. Deutsch. Ing.45, 1781-1788). So if you are working in high Reynolds Number flow, what do you do? Whitaker (Whitaker, S., The Forchheimer equation: A theoretical development, Transp Porous Med (1996) 25: 27. doi:10.1007/ BF00141261) showed that the correction was proportional to the square of velocity (as we saw above and proposed by Forschheimer), and the threshold for inclusion of these effects was indeed at Reynolds Number of about 1.

\section{Example 2-2: Darcy's Law}

Problem Statement - Case A:

A constant $20 \mathrm{~mm}$ of water is ponded on the surface of a $50 \mathrm{~mm}$ long saturated vertical sand column. What is the water flux from the bottom of the column if the saturated hydraulic conductivity is $50 \mathrm{~mm} /$ day? 
a

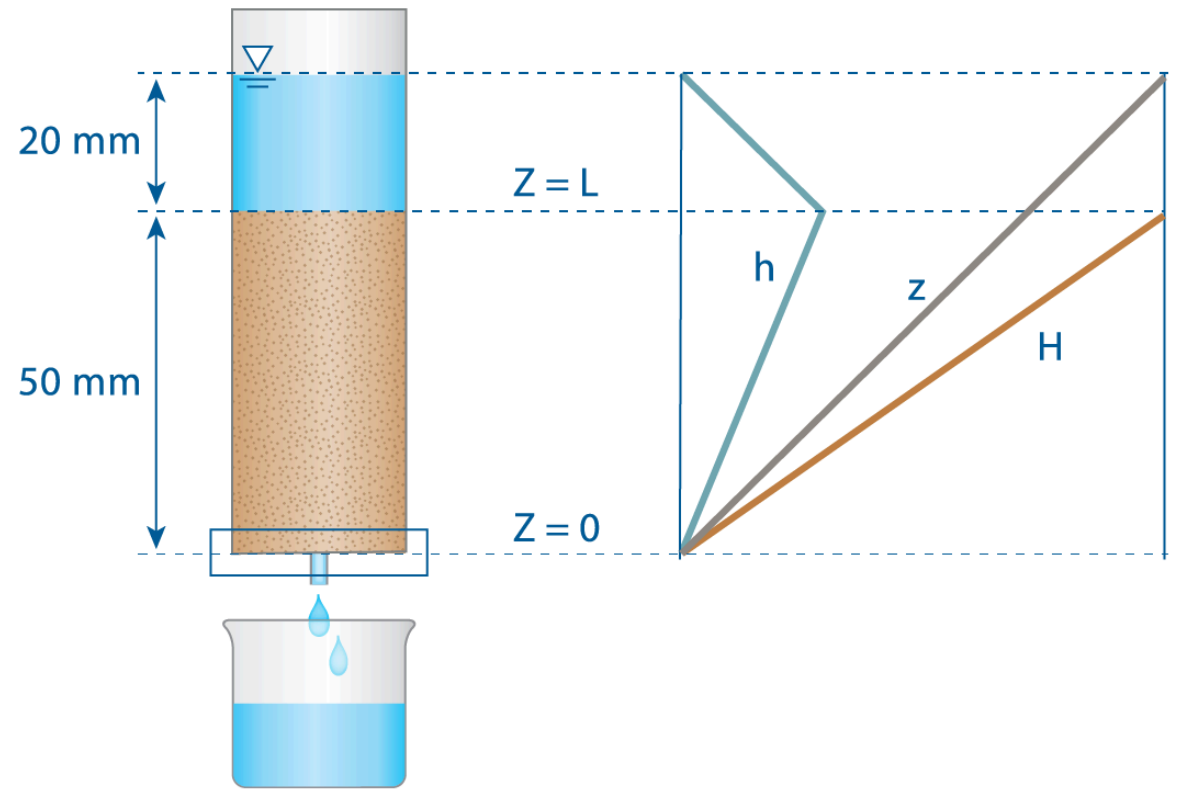

$\underline{b}$

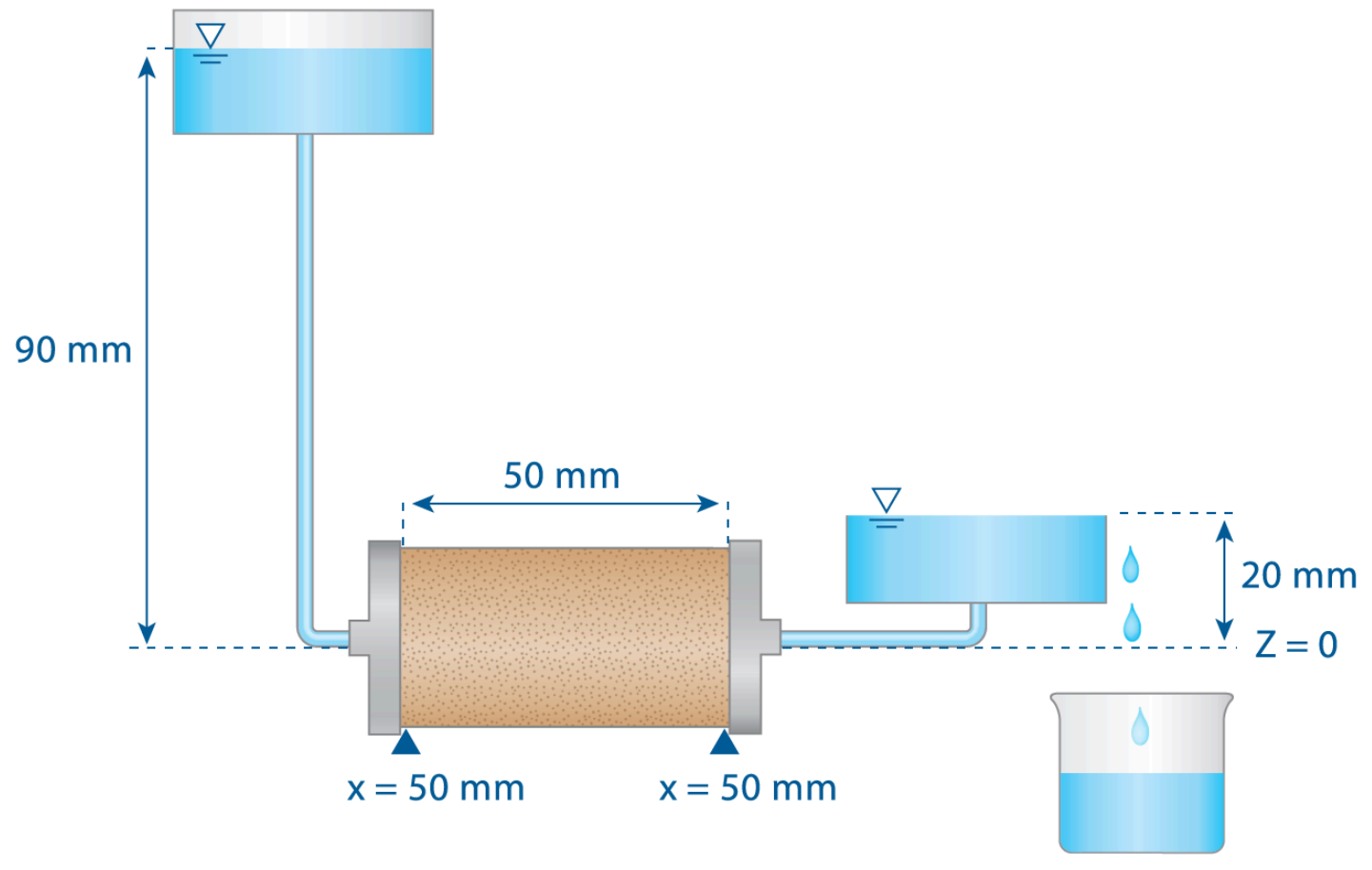

Figure 3.10 


\section{Solution:}

- (1) We define a reference (datum) at $\mathrm{z}=0$.

- (2) Since we are interested in the difference in hydraulic potential across the soil length only, we consider the potentials at the inlet and the outlet. The total hydraulic head at the inflow is the pressure potential at the top of the sand plus the elevation potential:

$\mathrm{H}_{\text {in }}=\mathrm{h}_{\text {in }}+\mathrm{z}_{\text {in }}=20 \mathrm{~mm}+50 \mathrm{~mm}=70 \mathrm{~mm}$; and at the bottom end we have

$\mathrm{H}_{\text {out }}=\mathrm{h}_{\text {out }}+\mathrm{z}_{\text {out }}=0+0=0 \mathrm{~mm}$. Note that the pressure head $\mathrm{h}_{\text {out }}$ is zero at the base of any soil column where water drips into air at atmospheric pressure.

- (3) The difference in head across the sand is therefore:

$\Delta \mathrm{H}=\mathrm{H}_{\mathrm{in}}-\mathrm{H}_{\mathrm{out}}=70 \mathrm{~mm}-0 \mathrm{~mm}=70 \mathrm{~mm}$, and the hydraulic gradient

$\Delta \mathrm{H} / \Delta \mathrm{z}=(70 \mathrm{~mm}-0 \mathrm{~mm}) /(50 \mathrm{~mm}-0 \mathrm{~mm})=1.4$ (dimensionless). [It is worth noting here that had no water been "ponded" on the sand surface that the gradient would be equal to one. This is just to say that if the pressure is the same on the top and bottom of the sand, then the only source of gradient is that of gravity.]

- (4) The flux is: $\mathrm{J}_{\mathrm{w}}=-\mathrm{K}(\Delta \mathrm{H} / \mathrm{z})=-50 \mathrm{~mm} / \mathrm{d} \times 1.4=-70 \mathrm{~mm} / \mathrm{d}$. The negative sign indicates downward flow.

\section{Problem Statement - Case B:}

The sand column from Case $a$ is now placed horizontally with $90 \mathrm{~mm}$ of water ponded on the left side and 20 $\mathrm{mm}$ on the right side. We will define our datum $\mathrm{z}=0$ at the center of the sand core.

\section{Solution:}

- (1) We are interested in the difference in hydraulic potential across the soil length only. The hydraulic head at the inflow is: $\mathrm{H}_{\text {in }}=\mathrm{h}_{\text {in }}+\mathrm{z}_{\text {in }}=90 \mathrm{~mm}+0 \mathrm{~mm}=90 \mathrm{~mm}$; and at the outlet we have $\mathrm{H}_{\text {out }}=\mathrm{h}_{\text {out }}+\mathrm{z}_{\text {out }}=20 \mathrm{~mm}+0=20 \mathrm{~mm}$. The difference is: $\Delta \mathrm{H}=\mathrm{H}_{\text {in }}-\mathrm{H}_{\text {out }}=90-20=70 \mathrm{~mm}$; and the hydraulic gradient $\Delta \mathrm{H} / \Delta \mathrm{z}=(90-20) /(0-50)=-1.4$

- (2) The flux is: $\mathrm{J}_{\mathrm{w}}=-\mathrm{K}(\Delta \mathrm{H} / \Delta \mathrm{x})=-50 \mathrm{~mm} / \mathrm{d} \times-1.4=70 \mathrm{~mm} / \mathrm{d}=2.917 \mathrm{~mm} / \mathrm{h}$, which is identical in magnitude to the flux in Case a (the sign is positive since the horizontal flow occurs in the direction of increasing $\mathrm{x}$ ).

- (3) To find the cumulative volume of water we use the definition of flux density as: $J_{w}=V /(A t)$, where $\mathrm{A}=1000 \mathrm{~mm}^{2}$ and $\mathrm{t}=12 \mathrm{~h}$. Thus $\mathrm{V}=\left(\mathrm{A} \mathrm{t} \mathrm{J}_{\mathrm{w}}\right)=1000 \mathrm{~mm}^{2} * 12 \mathrm{~h} * 2.917 \mathrm{~mm} / \mathrm{h}=35004 \mathrm{~mm}^{3}$, which to reasonable precision should be reported to no more than two digits ( $\mathrm{K}$ is rarely known to better than $1 \%$ accuracy), so should be given as $35000 \mathrm{~mm}^{3}$

\section{Example 2-3: Saturated Flow and Potential Diagram}

\section{Problem Statement:}


A constant water pressure of $20 \mathrm{kPa}$ was maintained at the bottom of a $0.5 \mathrm{~m}$ vertical saturated soil column, and the water height at the column's top was also kept constant at $20 \mathrm{~mm}$. Given the soils saturated hydraulic conductivity $\mathrm{K}_{\mathrm{s}}=5 \mathrm{~mm} / \mathrm{hr}$, find:

(1) The direction of flow; draw a system sketch and a potential diagram

(2) The water flux density $\mathrm{J}_{\mathrm{W}}$, and

(3) The height of ponded water on top of the column that causes a cessation of flow.

\section{Solution:}

[need to enter the material included in the word file "Example2-3 in insert in chapter 3" from Dani's class notes here. This will also require changing the greek symbol psi-h to H, psi-p to h, and psi-z to z.] 


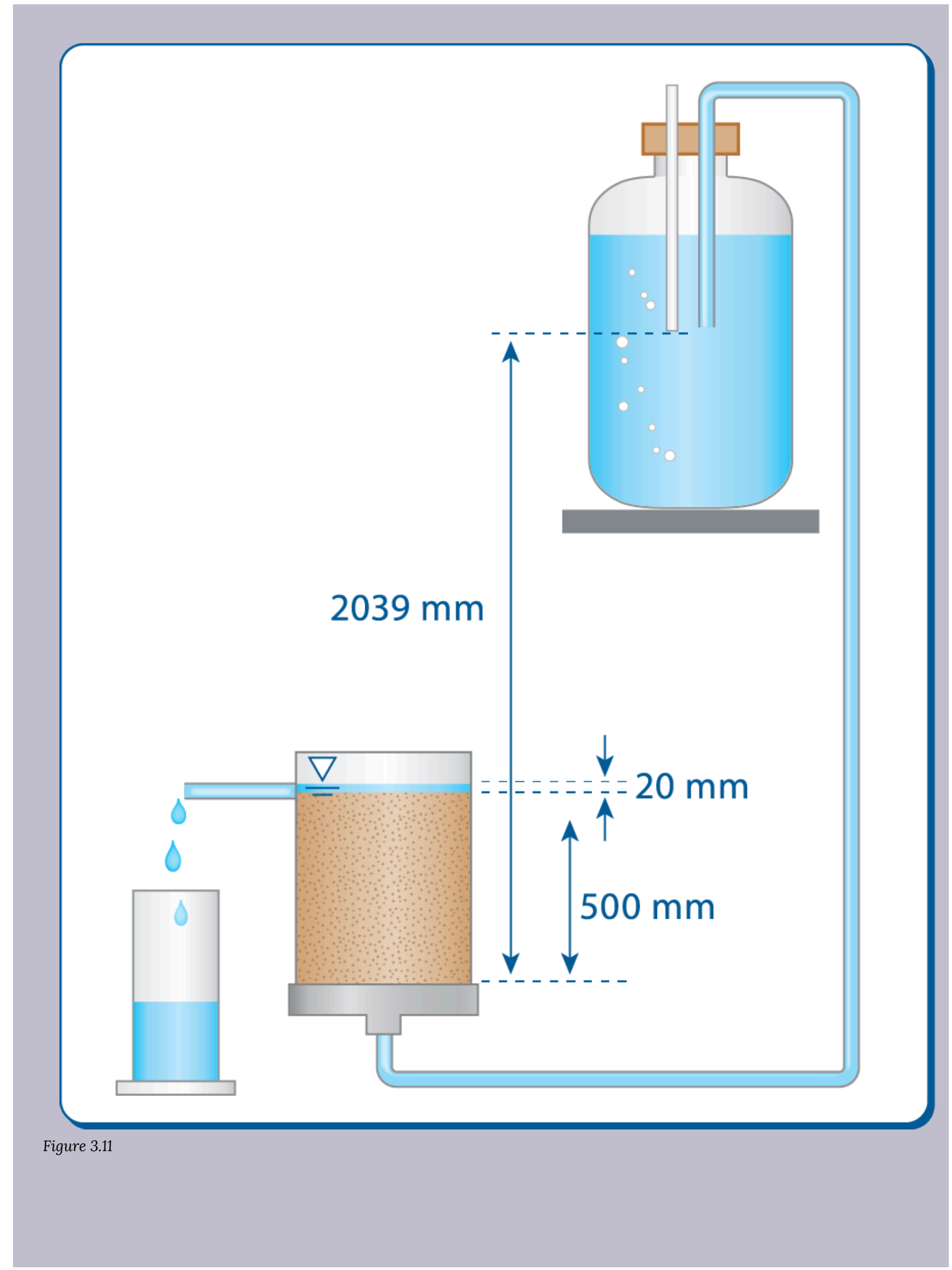

146 | Liquid Flow in Soils (Hydrodynamics) 
First we convert the pressure at the bottom of the column from potential $(\mathrm{kPa})$ to head $(\mathrm{m})$ :

$$
h=\frac{\Psi}{\rho_{w} g}=\frac{20000}{1000 \times 9.81}=2.039[\mathrm{~m}]
$$

where $\rho_{\mathrm{W}}$ is the density of water $\left(1000 \mathrm{~kg} / \mathrm{m}^{3}\right)$, and $\mathrm{g}$ is the acceleration of gravity $\left(9.81 \mathrm{~m} / \mathrm{s}^{2}\right)$. [Note:

$\left.\mathrm{Pa}=\mathrm{N} / \mathrm{m}^{2}=\mathrm{kg} / \mathrm{ms}^{2}\right]$.

Assuming the bottom of the column as reference level we then calculate the head at the top and the bottom as:

$$
\Psi_{h T O P}=\Psi_{p T O P}+\Psi_{z T O P}=20+500=520[\mathrm{~mm}]
$$

$$
\Psi_{h B O T}=\Psi_{p B O T}+\Psi_{z B O T}=2039+0=2039[\mathrm{~mm}]
$$

with known Ks we now calculate the flux density $\mathrm{J}_{\mathrm{W}}$ as:

$$
J_{w}=-K_{s} \frac{\Psi_{h T O P}-\Psi_{h B O T}}{Z_{T O P}-Z_{B O T}}=-5 \frac{520-2039}{500-0}=15.19[\mathrm{~mm} / \mathrm{h}]
$$

Positive $\mathrm{J}_{\mathrm{W}}$ means flow from bottom to top. The flow ceases when the hydraulic head at the top equals the head at the bottom:

$$
\Psi_{h T O P}=\Psi_{h B O T} \Rightarrow \Psi_{p T O P}=2039-500=1539[\mathrm{~mm}]
$$




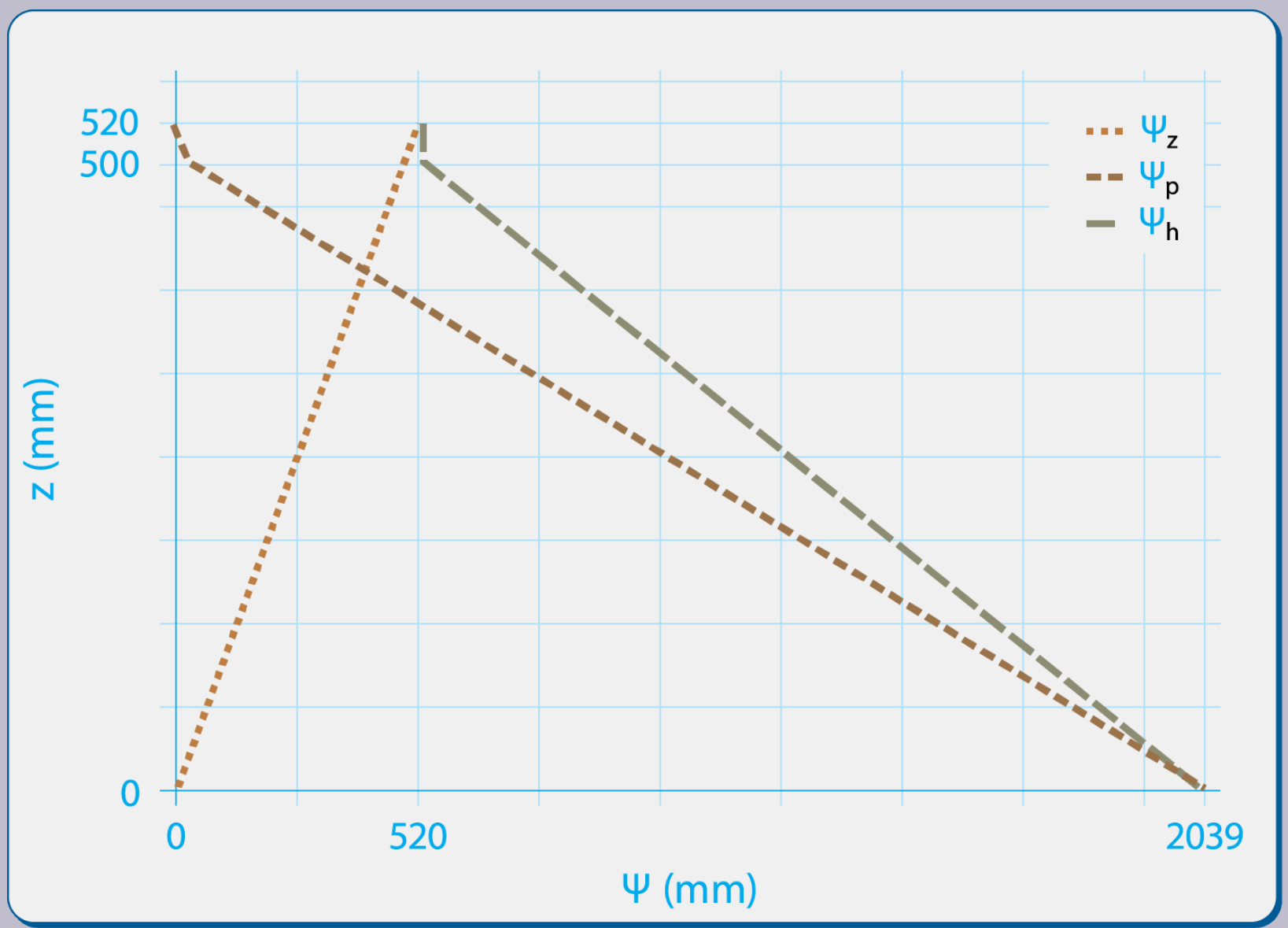

Figure 3.12

\section{Example 2-3): Saturated Flow and Potential Diagram}

\section{Problem Statement:}

A constant water pressure of $20 \mathrm{kPa}$ was maintained at the bottom of a $0.5 \mathrm{~m}$ vertical saturated soil column, and the water height at the column's top was also kept constant at $20 \mathrm{~mm}$. Given the soils saturated hydraulic conductivity $\mathrm{K}_{\mathrm{s}}=5 \mathrm{~mm} / \mathrm{hr}$, find:

(1) The direction of flow; draw a system sketch and a potential diagram

(2) The water flux density $J_{W}$, and

(3) The height of ponded water on top of the column that causes a cessation of flow.

\section{Solution:}




\section{(1) System Sketch}

First we convert the pressure at the bottom of the column from potential $(\mathrm{kPa})$ to head $(\mathrm{m})$ :

$$
h=\frac{\Psi}{\rho_{w} g}=\frac{20000}{1000 \times 9.81}=2.039[\mathrm{~m}]
$$

where $\rho_{\mathrm{w}}$ is the density of water $\left(1000 \mathrm{~kg} / \mathrm{m}^{3}\right)$, and $\mathrm{g}$ is the acceleration of gravity $\left(9.81 \mathrm{~m} / \mathrm{s}^{2}\right)$. [Note:

$\left.\mathrm{Pa}=\mathrm{N} / \mathrm{m}^{2}=\mathrm{kg} / \mathrm{ms}^{2}\right]$.

Assuming the bottom of the column as reference level we then calculate the head at the top and the bottom as:

$$
\begin{gathered}
\Psi_{h T O P}=\Psi_{p T O P}+\Psi_{z T O P}=20+500=520[\mathrm{~mm}] \\
\Psi_{h B O T}=\Psi_{p B O T}+\Psi_{z B O T}=2039+0=2039[\mathrm{~mm}]
\end{gathered}
$$

with known Ks we now calculate the flux density $\mathrm{J}_{\mathrm{W}}$ as:

$$
J_{w}=-K_{s} \frac{\Psi_{h T O P}-\Psi_{h B O T}}{Z_{T O P}-Z_{B O T}}=-5 \frac{520-2039}{500-0}=15.19[\mathrm{~mm} / \mathrm{h}]
$$

Positive $\mathrm{J}_{\mathrm{W}}$ means flow from bottom to top. The flow ceases when the hydraulic head at the top equals the head at the bottom:

$$
\Psi_{h T O P}=\Psi_{h B O T} \Rightarrow \Psi_{p T O P}=2039-500=1539[\mathrm{~mm}]
$$

(2) Potential Diagram

\subsection{Measurement of Saturated Hydraulic Conductivity}

Experiments may be designed to use Darcy's law for determining the saturated hydraulic conductivity (Ks) from measurements of hydraulic heads and water flux density (denoted as flux) from a soil column of a known geometry. We can use either the vertical or the horizontal setup shown in example 2-1. The constant head method (Klute and Dirksen, 1986) is based on maintaining constant heads across the soil sample, a similar procedure to that shown in example 2-1.

\section{Example 2-4): Measurement of Ks using the Constant Head Method}

\section{Problem Statement:}

A vertical $50 \mathrm{~mm}$ long saturated soil column with a constant $50 \mathrm{~mm}$ of water ponded on the top surface (Case (a) in example 2-1) was used to determine the saturated hydraulic conductivity of the soil. The area of the column was $1000 \mathrm{~mm}^{2}$ and the volume of water collected in $5 \mathrm{~h}$ was $25000 \mathrm{~mm}^{3}$; what was Ks of the soil?

\section{Solution:}


The first step is to rearrange Darcy's equation to solve for the hydraulic conductivity. For vertical flow we obtain:

$$
K_{s}=-J_{w} \frac{\Delta z}{\Delta \Psi_{h}}
$$

The hydraulic gradient is: $\Psi \mathrm{h} / \mathrm{z}=[(50+50)-(0+0)] /[50-0]=2.0$, and its inverse $\mathrm{z} / \Psi \mathrm{h}=0.5$. The flux can be calculated from the volume, time and area by:

$\mathrm{J}_{\mathrm{w}}=\mathrm{V} /(\mathrm{A} \mathrm{t})=-25000 /(1000 * 5)=-5 \mathrm{~mm} \mathrm{~h}^{-1}$ (negative sign indicates downward flow). Inserting these values in the equation above, we obtain:

$$
\mathrm{K}_{\mathrm{s}}=-\mathrm{J}_{\mathrm{w}}(\mathrm{z} / \Psi \mathrm{h})=-(-5) * 0.5=2.5 \mathrm{~mm} \mathrm{~h}^{-1} \text {. }
$$

An alternative method for measuring $\mathrm{K}_{\mathrm{S}}$, which does not require the maintenance of a constant head nor any outflow measurement, is called the falling head method. The device used for field measurement by this method is the falling head permeameter. The experiment in example 2-3 may be conducted such that only the initial and final depths of the ponded water are recorded, expressed as pressure head in length units. The rate of decrease in the depth of ponding is equal to the flux per unit area if the cross sectional area (A) of the column is constant:

$$
J_{w}=\frac{A d \Psi_{p}}{A d t}=-\frac{K_{s}}{\Delta z}\left[\left(\Psi_{p I N}(t)-0\right)+(L-0)\right]=-\frac{K_{s}}{L}\left(\Psi_{p I N}(t)+L\right)
$$

Rearranging and integrating between the measured values of $\Psi_{\mathrm{pIN}}$, denoted as $\Psi_{\mathrm{p} 0}$ and $\Psi_{\mathrm{p} 1}$, corresponding to initial $\left(\mathrm{t}_{0}\right)$ and final $\left(\mathrm{t}_{1}\right)$ times of measurement, respectively, yields:

$$
\int_{\Psi_{p 0}}^{\Psi_{p 0}} d \Psi_{p} \Psi_{p}+L=\ln \left[\frac{\Psi_{p 1}+L}{\Psi_{p 0}+L}\right]=-\int_{t_{0}=0}^{t_{1}} \frac{K_{s} d t}{L}=-\frac{K_{s} t_{1}}{L}
$$

The final result in terms of $\mathrm{K}_{\mathrm{s}}$, assuming the infiltration area for water entry is the same as the water reservoir area, is:

$$
K_{s}=\frac{L}{t_{1}} \ln \left[\frac{\Psi_{p 0}+L}{\Psi_{p 1}+L}\right]
$$

Otherwise the RHS of Eq.(55) must be multiplied by the ratio Areservoir/Aentry. Note that under the conditions of the falling head method, the flux is not constant, and it varies with the changes in the hydraulic head.

\subsection{Saturated Steady Flow Through Layered Soil}

Consider a soil column consisting of two distinct layers, each with its own thickness and hydraulic conductivity. Under steady-state flow conditions the flux through both layers is equal. To simplify the discussion let us adopt the simpler notation of: $\mathrm{H}=\mathrm{h}+\mathrm{z}$, where $\mathrm{H}$ is the hydraulic head, $\mathrm{h}$ is the matric head (in unsaturated soils the pressure potential is zero), and $\mathrm{z}$ is the gravitational head. Let $\mathrm{H}_{1}$ be the hydraulic head at inlet, $\mathrm{H}_{2}$ the hydraulic head at the interface between layer 1 (inlet) and layer 2 (outlet), and $\mathrm{H}_{3}$ the hydraulic head at the outlet end of the column. During steady flow with equal flux through both layers we have: 


$$
\mathrm{J}_{\mathrm{w}}=-\mathrm{K}_{\mathrm{s} 1} \frac{\mathrm{H}_{1}-\mathrm{H}_{2}}{\mathrm{~L}_{1}}=-\mathrm{K}_{\mathrm{s} 2} \frac{\mathrm{H}_{2}-\mathrm{H}_{3}}{\mathrm{~L}_{2}}
$$

and solving for $\mathrm{H}_{2}$ we obtain two equations:

$$
\mathrm{H}_{2}=\mathrm{H}_{1}+\mathrm{J}_{\mathrm{w}} \frac{\mathrm{L}_{1}}{\mathrm{~K}_{\mathrm{s} 1}} \quad \text { and } \quad \mathrm{H}_{2}=-\mathrm{J}_{\mathrm{w}} \frac{\mathrm{L}_{2}}{\mathrm{~K}_{\mathrm{s} 2}}+\mathrm{H}_{3}
$$

The two equations are combined to obtain a solution for the flux in a layered soil:

$$
\mathrm{K}_{\mathrm{s}-\mathrm{eff}} \frac{\mathrm{H}_{3}-\mathrm{H}_{1}}{\mathrm{~L}_{1}+\mathrm{L}_{2}}=\mathrm{J}_{\mathrm{w}}=\frac{-\left(\mathrm{H}_{1}-\mathrm{H}_{3}\right)}{\left(\frac{\mathrm{L}_{2}}{\mathrm{~K}_{\mathrm{s} 2}}+\frac{\mathrm{L}_{1}}{\mathrm{~K}_{\mathrm{s} 1}}\right)}
$$

where we have introduced an effective saturated hydraulic conductivity for the two-layer column.

The solution obtained in Eq.(58) can be generalized to a soil profile having multiple layers. It provides a practical definition for the effective hydraulic conductivity of a nonuniform soil profile consisting of $\mathrm{n}$ distinct layers, each having its own Ks and thickness L. Setting $\mathrm{J}_{\mathrm{w}}=\mathrm{K}_{\mathrm{s}-\mathrm{eff}}\left(\mathrm{H}_{\mathrm{n}}-\mathrm{H}_{1}\right) / \Sigma \mathrm{L}_{\mathrm{i}}$ in Eq.(58), we may obtain a definition of $\mathrm{K}_{\mathrm{s}-\text { eff }}$ for the layered soil profile in a direction normal to layering as:

$$
\mathrm{K}_{\mathrm{s}-\operatorname{eff}(\mathrm{N})}=\frac{\sum_{\mathrm{i}=1}^{\mathrm{n}} \mathrm{L}_{\mathrm{i}}}{\sum_{\mathrm{i}=1}^{\mathrm{n}} \frac{\mathrm{L}_{\mathrm{i}}}{\mathrm{K}_{\mathrm{si}}}}
$$

where i subscripts refer to depth layers, and Ks-eff(N) is a harmonic mean effective Ks for the flow normal (perpendicular) to layering. For flow parallel to the layering, we get:

$$
\mathrm{K}_{\mathrm{S}-\operatorname{eff}(\mathrm{N})}=\frac{\sum_{\mathrm{i}=1}^{\mathrm{n}} \mathrm{L}_{\mathrm{i}} \mathrm{K}_{\mathrm{i}}}{\sum_{\mathrm{i}=1}^{\mathrm{n}} \mathrm{L}_{\mathrm{i}}}
$$

where $\mathrm{K}_{\mathrm{S}-\mathrm{eff}(\mathrm{P})}$ is a thickness-weighted arithmetic mean effective $\mathrm{K}_{\mathrm{S}}$ for flow parallel to layering. The usefulness of Eqs.(59) and (60) is in replacing a nonhomogeneous soil column by an equivalent "homogeneous" one characterized by Ks-eff.

\section{Example 2-5): Effective Saturated Hydraulic Conductivity}

\section{Problem Statement:}

A $1 \mathrm{~m}$ long glass tube having a radius of $1 \mathrm{~mm}$ was inserted into a $1 \mathrm{~m}$ long saturated cylindrical soil column with diameter of $100 \mathrm{~mm}$, and Ks of $0.01 \mathrm{~mm} / \mathrm{min}$. The water head at the top of the column and at the tube's inlet was $0.25 \mathrm{~m}$ while the outlets where at atmospheric pressure. 
(1) What would be the total flux through the column tube system and what percentage is contributed by the tube?

(2) What is the effective Ks of the column-tube system

\section{Solution:}

(1) Hydraulic potential at the tube inlet

$$
\Psi_{k}=1000 \times 9.81 \times 1.25=12263[\mathrm{~Pa}]
$$

(2) Average velocity within the tube

$$
\overline{\mathrm{v}}=\frac{0.001^{2}}{8 \cdot 0.001}\left(\frac{12263}{1}\right)=1.5330[\mathrm{~m} / \mathrm{s}]=91620[\mathrm{~mm} / \mathrm{min}]
$$

(3) Tube flux

$$
\mathrm{Q}_{\text {tube }}=91620 \cdot 1^{2} \cdot \pi=287833\left[\mathrm{~mm}^{3} / \mathrm{min}\right]
$$

(4) Flux density soil column

$$
\mathrm{J}_{\mathrm{w}}=-0.01 \cdot \frac{1250}{1000}=-0.0125[\mathrm{~mm} / \mathrm{min}]
$$

(5) Column flux

$$
\mathrm{Q}_{\mathrm{c}}=0.0125 \cdot \pi \cdot\left(50^{2}-1^{2}\right)=98\left[\mathrm{~mm}^{3} / \mathrm{min}\right]
$$

(6) Total flux

$$
\mathrm{Q}_{\mathrm{T}}=287833+98=287931\left[\mathrm{~mm}^{3} / \mathrm{min}\right]
$$

(7) Contribution tube to total flux

$$
[\%]=\frac{Q_{\text {tube }}}{\mathrm{Q}_{\mathrm{T}}} \cdot 100=\frac{287833}{287931} \cdot 100=99.96[\%]
$$

(8) Total flux density

$$
\mathrm{J}_{\mathrm{T}}=\frac{287931}{50^{2} \cdot \pi}=36.7[\mathrm{~mm} / \mathrm{min}]
$$

(9) Effective saturated conductivity

$$
\mathrm{K}_{\mathrm{s}-\mathrm{eff}}=36.7 \cdot \frac{1000}{1250}=29.3[\mathrm{~mm} / \mathrm{min}]
$$

(1) To apply Poiseuille's law for average flow velocity within the tube we first have to convert hydraulic head $h$ (m) to hydraulic potential yh (Pa) using the following relationship:

(3.62) $\Psi_{\mathrm{h}}=\rho_{\mathrm{w}} \mathrm{gh}$

where $\mathrm{rw}$ is the density of water $(1000 \mathrm{~kg} / \mathrm{m} 3)$, and $\mathrm{g}$ is the acceleration of gravity $(9.81 \mathrm{~m} / \mathrm{s} 2)$. [Note: The hydraulic head is the sum of water head and tube length]. 
(2) With known hydraulic potential we now can solve Poiseuille's law:

$$
\overline{\mathrm{v}}=\frac{\mathrm{r}^{2}}{8 \eta}\left(\frac{\Delta \mathrm{P}}{\mathrm{L}}\right)
$$

where $r$ is the tube radius $(\mathrm{m}), \mathrm{h}$ is the viscosity of water at $20^{\circ} \mathrm{C}(0.001 \mathrm{~Pa} \mathrm{~s}), \mathrm{DP}$ is the pressure gradient $(\mathrm{Pa})$, and $\mathrm{L}$ is the tube length $(\mathrm{m})$. The tubes flux Qtube is simply the product of average velocity and the tubes cross-sectional area Atube.

The flux of the soil column QC is given as:

$$
\mathrm{Q}_{\mathrm{c}}=\mathrm{J}_{\mathrm{w}} \mathrm{A}_{\mathrm{c}}
$$

with the flux density Jw calculated as:

$$
\mathrm{J}_{\mathrm{w}}=-\mathrm{K}_{\mathrm{s}}(\Delta \mathrm{h} / \Delta \mathrm{z})
$$

The systems (tube \& column) flux density JT is simply the ratio of the total flux QT and the total crosssectional area AT. The system's effective saturated conductivity is calculated as:

$$
\mathrm{K}_{\mathrm{s}-\mathrm{eff}}=\mathrm{J}_{\mathrm{T}}(\Delta \mathrm{z} / \Delta \mathrm{h})
$$

\subsection{The Kozeny-Carman Predictive Model for Saturated Hydraulic Conductivity}

Efforts to predict hydraulic conductivity based on basic soil structural properties such as porosity or solid/pore geometry date back many years. The idea is extremely attractive because of the obvious connection between water flow and the distribution of solids and pore spaces in the soil medium. Kozeny introduced a model relating soil permeability to porosity in 1927. The relationship of permeability (k) to saturated hydraulic conductivity (Ks) is expressed as $\mathrm{K}_{\mathrm{s}}=\mathrm{k}(\rho \mathrm{g} / \eta)$, where $\rho$ is the liquid density, $\mathrm{g}$ the acceleration of gravity, $\eta$ the liquid dynamic viscosity (Pa s), and $\rho g / \eta$ is termed the fluidity (typical value for water at $20^{\circ} \mathrm{C}$ is: $9.81 \times 10^{6}[\mathrm{~m} \mathrm{~s}]^{-1}$ ). Note that $\mathrm{k}$ has dimensions of $\mathrm{L}^{2}$. Kozeny's model was based on application of Poiseuille's law to flow through granular porous media lacking structure or consolidation. Pores are represented as cylindrical tubes with a constant radius, R. Rearranging Poiseuille's law for laminar flow tubes [Eq.(50)] and considering that the fraction of pores is given by the porosity n, yields Kozeny's model in its simplest form:

$$
\mathrm{k}=\frac{\mathrm{n}}{2}\left(\frac{\mathrm{R}}{2}\right)^{2} \quad\left[\mathrm{~m}^{2}\right]
$$

where R/2 is the ratio of cylindrical pore volume to wetted surface area, the so-called hydraulic radius. Kozeny's approach was extended by Carman (Scheidegger, 1957; Lagerwerff et al., 1969) to take the form:

$$
\mathrm{k}=\frac{\mathrm{n}^{3}}{\mathrm{c} \tau^{2} \mathrm{~A}_{\mathrm{v}}{ }^{2}(1-\mathrm{n})^{2}} \quad\left[\mathrm{~m}^{2}\right]
$$

where $\mathrm{n}$ is porosity, $\mathrm{c}$ is an empirical coefficient to represent particle size and shape (and hence of pores), $\tau$ is tortuosity of the flow path, and $A_{v}$ is the particle surface area per unit mass of solids in porous medium (Section 1.5.4). For non-cubic particles, a value of 2 to 3 for $\mathrm{c}$ has been found to work well. If the mean flow direction is taken as $45^{\circ}$ from the vertical axis, then $\tau=2^{1 / 2}$. Eq. (62) may be simplified through definition of a "hydraulic radius", which is the 
surface exposed to the wetting solution per unit volume of porous medium. The hydraulic radius, $\mathrm{R}_{\mathrm{h}}$, is related to the specific surface area $\left(A_{v}\right)$ and the porosity as:

(3.69) $\mathrm{R}_{\mathrm{h}}=\mathrm{A}_{\mathrm{v}}(1-\mathrm{n})$

Substitution of Eq.(63) into (62) yields an equivalent expression:

$$
\mathrm{k}=\frac{\mathrm{n}^{3}}{\mathrm{c} \tau^{2} \mathrm{R}_{\mathrm{h}}{ }^{2}}
$$

The product $\mathrm{c \tau}^{2}$ is commonly about 5 . The pore shapes, surfaces, and tortuosity are interdependent, thus the product $c \tau^{2} \mathrm{R}_{\mathrm{h}}{ }^{2}$ is often lumped into a single "formation factor", $\mathrm{F}^{2}$. Eq.(64) is thus simplified to $\mathrm{k}=\mathrm{n}^{3} / \mathrm{F}^{2}$, and $\mathrm{K}_{\mathrm{s}}$ for water can be estimated as:

$$
\mathrm{K}_{\mathrm{s}}=\mathrm{k}\left(\frac{\rho \mathrm{g}}{\eta}\right)=\frac{\mathrm{n}^{3}}{\mathrm{~F}^{2}}\left(\frac{\rho \mathrm{g}}{\eta}\right)
$$

Unfortunately, this rather simplistic equation often provides unsatisfactory estimates of $\mathrm{K}_{\mathrm{S}}$ in many soils (Fig.2-5), partly because it is based on an assumed uniform pore size distribution. This is at variance with most natural soils, which exhibit a substantial range in particle and pore sizes, and with the effects of soil structure on effective hydraulic diameter. It does, however, often produce satisfactory results in sands and in porous materials used in engineering or industrial applications, which have characteristics more similar to those assumed in its derivation.

Recently, Revil and Cathles [1999] analyzed the problem of predicting permeability of porous media, working with shaly sands. They used concepts from electrical conductance in porous media; in particular, the concept of a formation factor, F, that is related to the porosity and pore geometry by:

(3.72) $\mathrm{F}=\mathrm{n}^{-\mathrm{m}}$

where $\mathrm{m}$ is the so-called cementation exponent that varies with pore geometry and takes on values between 1.5 and 2 for most granular materials including sands. In practice the formation factor describes the ratio of the pure solution electrical conductivity to that of the bulk solution-saturated porous medium. The derivations of Revil and Cathles [1999] yielded a very simple expression for permeability,

$$
\text { (3.73) } \mathrm{k}=\frac{\mathrm{d}^{2} \mathrm{n}^{3 \mathrm{~m}}}{24}
$$

which provided remarkable predictions for granular materials having known mean diameter $d$, as depicted in Fig.2-6. The results were later extended to deal with predictions for sand-clay mixtures as detailed in Revil and Cathles [1999].

\section{Extending Darcy's Law to Unsaturated Media}

Most flow processes in the field occur while the soil is unsaturated, or in the presence of an air-phase. This situation drastically modifies water flow channels. As the water content decreases, the flow in the water phase is constrained to narrower and more tortuous channels. Darcy's law, which was conceived for saturated flow only, was extended by Edgar Buckingham (1907) to unsaturated water flow. Buckingham's main assumption was that the unsaturated hydraulic conductivity is a function of the water content or matric potential, i.e. the degree of wetness. In the variablysaturated Buckingham-Darcy's law for flow the basic form is preserved as

(3.74) $\mathrm{q}=-\mathrm{K}(\theta) \nabla \mathrm{H}$

where the hydraulic conductivity, $\mathrm{K}(\theta)$, is now a function of the moisture content. Notice that we prefer not to write the conductivity as a function of pressure: the geometry of the water filled pores is the most direct control of resistance to liquid movement, which is largely dictated by $\theta$. Of course, it is possible to conceive of a soil that came to a particular water content by two different processes wherein the exact geometry of the liquid phase was different (for instance, 
think about starting with a saturated soil and drying it by applying a negative pressure to one boundary versus starting with a dry soil and wetting it by forcing a mist of water through it carried by moving air). In the same sense, the geometry of the water phase might well change in time, for example as an initially dry soil wets (for instance, see Weller, U., and H.-J. Vogel. 2011. Conductivity and hydraulic non-equilibrium across drainage and infiltration fronts. Vadose Zone J., doi:10.2136/vzj2011.0134). This consideration is especially important in the case of sharp, rapidly moving wetting fronts (e.g., infiltration in a desert following an intense rainstorm). Getting back to the question of representing conductivity in terms of pressure, here you would have to both take into account these dynamic effects and you must employ the hysteretic functional relationship between $\theta$ and $\mathrm{h}$ (see table 3.2 for common forms of $K(\theta)$ ).

So how does $\mathrm{K}$ vary with $\theta$ ? In a nutshell, conductivity drops very quickly as $\theta$ departs of saturation. Three factors are responsible for this behavior:

1. Large pores empty first. These are the pores with least resistance to flow, since they have the largest diameters (recall the $1 / \mathrm{r}^{4}$ dependence of hydraulic resistance in the Hagen-Poiseuille equation).

2. Flow paths increase in length. Instead of proceeding straight through a chunk of medium, the flow must avoid all the empty pores, making the path more tortuous.

3. There is less cross-section of flow. In any given area normal to flow, all the fluid must pass through a smaller portion of this area; for a given aerial flux the pore velocity must be higher.

To get a feel for how fast $\mathrm{K}$ drops, let's consider the effect of tortuosity alone (item 2 above). Thinking back to the capillary tube model we know that the resistance to flow will be a function of the true pore-fluid velocity and the true fluid path lengths. These must be distinguished from the "Darcian" flow length and the "Darcian" velocity, as shown in Figure 3.13.

So writing the true pressure gradient that acts on the fluid we have

$$
\frac{\mathrm{P}}{\mathrm{L}}=\left(\frac{\mathrm{L}_{\mathrm{e}}}{\mathrm{L}}\right) \frac{\mathrm{P}}{\mathrm{L}_{\mathrm{e}}}
$$

where we recognize $\mathrm{L}_{\mathrm{e}} / \mathrm{L}$ as the ratio of the Darcian length to the true path length. Next we write the equation for capillary flow velocity, $v_{f}$ (Hagen-Poiseuille)

$$
\mathrm{v}_{\mathrm{f}}=-\frac{\gamma}{8 \mu} \mathrm{r}^{2} \frac{\mathrm{P}}{\mathrm{L}_{\mathrm{e}}}
$$

Our objective here is to understand the dependence of $\mathrm{K}(\theta)$ on microscopic parameters, so translating into the Darcian velocity, q, we find 


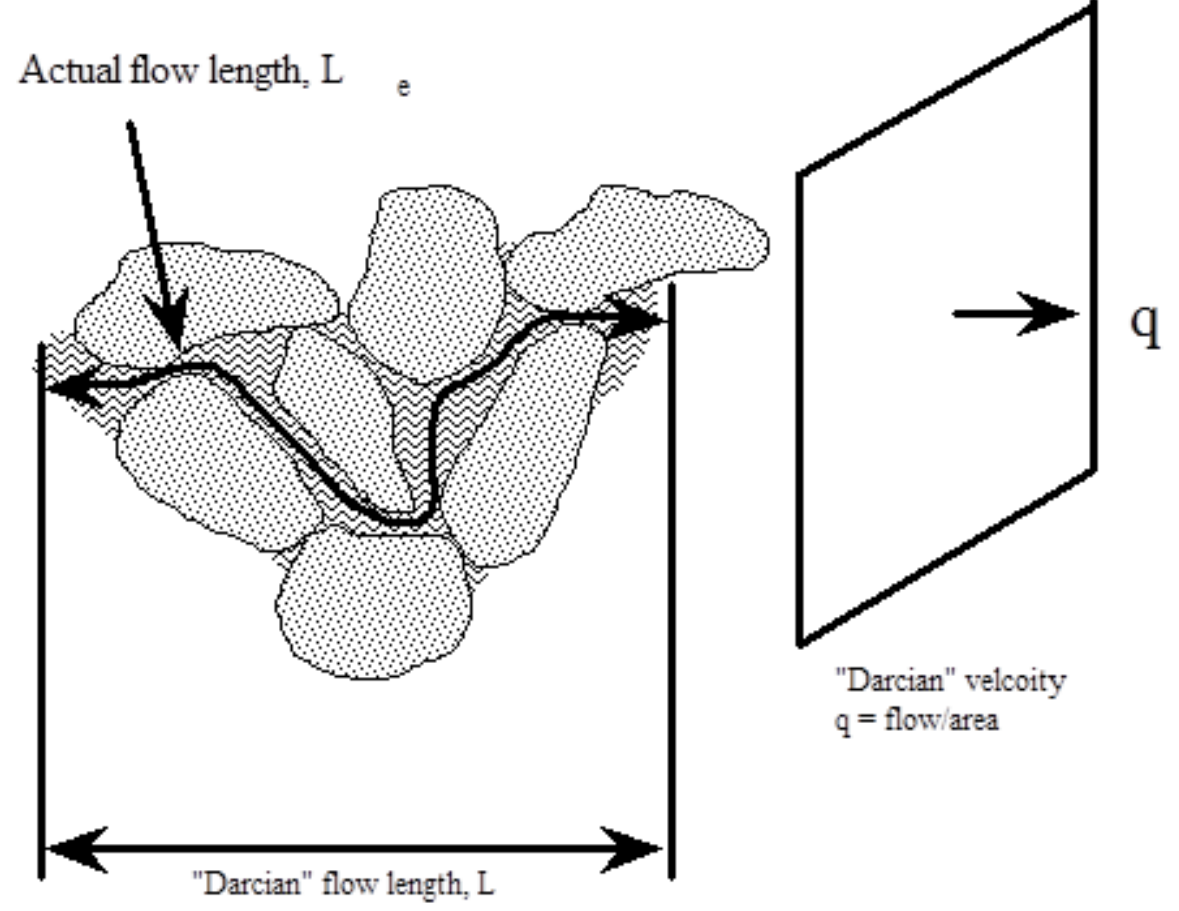

Figure 3.13 Illustration of the difference between the true microscopic fluid flow path length and flow velocity in comparison with the "Darcian" values, which are based on a macroscopic picture of the system.

$$
\mathrm{v}_{\mathrm{f}}=\frac{\mathrm{qL}_{\mathrm{e}}}{\mathrm{nL}}=-\frac{\gamma}{8 \mu} \mathrm{r}^{2} \frac{\mathrm{P}}{\mathrm{L}} \frac{\mathrm{L}}{\mathrm{L}_{\mathrm{e}}}
$$

where $\mathrm{n}$ is the porosity. Solving for q we find

$$
q=-\frac{\gamma n r^{2}}{8 \mu} \frac{P}{L}\left(\frac{L}{L_{e}}\right)^{2}
$$

or, after comparing to Darcy's law we see that

$$
\mathrm{K}=\mathrm{Cr}^{2}\left(\frac{\mathrm{L}}{\mathrm{L}_{\mathrm{e}}}\right)^{2}
$$

where $\mathrm{C}$ is some constant. It is evident that the conductivity goes down with the square of the tortuosity and the square of the typical radius of water filled pores. Considering these results in connection with the three effects of decrease in moisture content given above, it is clear that conductivity will drop precipitously with decreased moisture content. A diagram of the relationship between $\theta$ and $\mathrm{K}$ is shown in Figure 3.14. 


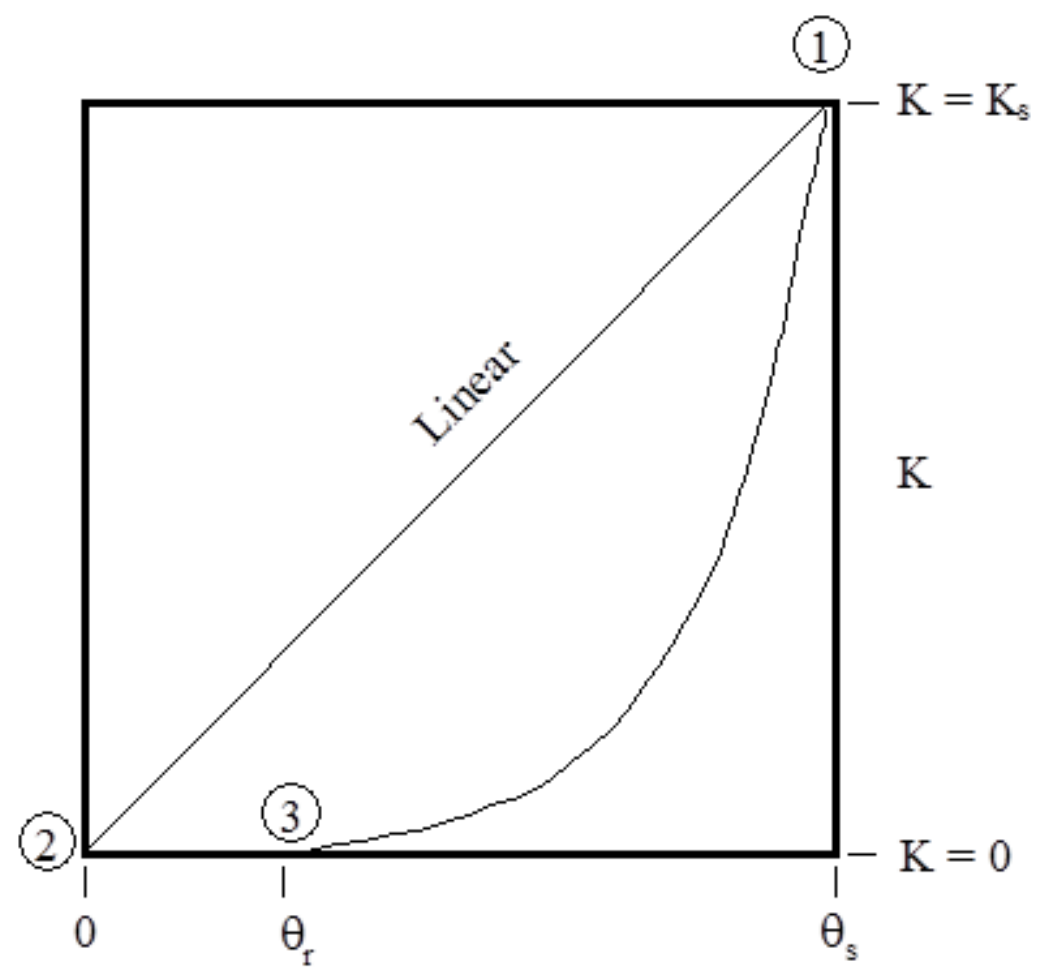

Figure 3.14 Characteristics of relationship between hydraulic conductivity and moisture content. The point labeled 1 indicates that at saturation, the conductivity is Ks. Between points 2 and $3, K(\theta)=0$ as the water is in disjoint pendular units which cannot respond to macro scale gradients in potential.

\subsection{A Comparison Between Saturated and Unsaturated Flow}

The driving force for flow in a saturated soil under constant gravitational potential is the gradient of positive pressures, whereas under unsaturated conditions the gradient is of negative matric potentials. The second and most important difference between saturated and unsaturated flow is the hydraulic conductivity. When the soil is completely watersaturated, the pores conduct at a maximal rate. When the soil desaturates, some of the water-filled pores become partially or completely air-filled, and the cross-sectional area available for mass flow decreases.

Because the soil water pressure becomes subatmospheric (negative), the first pores to evacuate, in accordance with the capillary law, are the largest, and thus there is a steep drop in conductivity of several orders of magnitude from its saturated value. To maintain continuous liquid flow, the empty pores must be by-passed, resulting in an increasingly tortuous pathway.

Finally, the saturated flow equations having a constant saturated hydraulic conductivity (Ks) are replaced with an unsaturated hydraulic conductivity function, $\mathrm{K}(\mathrm{h})$. This function is dependent on the flow path attributes of water content or matric potential, resulting in highly nonlinear and mathematically complicated flow equations.

\subsection{Unsaturated Hydraulic Conductivity Function}

The unsaturated hydraulic conductivity is a nonlinear function of water content, K(q), or matric potential, K(h). Some typical K(h) and K(q) curves for sandy and loamy soils are shown in Fig.2-7 and in Fig.2-8. Note that at saturation the 
sandy soil has higher conductivity because of the larger water-filled pore spaces. These large pores drain at low suction, however, causing a considerable decrease in conductivity. The loamy soil, having smaller (on average) pores, retains a greater number of water-filled pores and thus has higher conductivity than the sandy soil at these potentials. A number of alternative models of permeability based on basic soil properties have been formulated for Ks or K(h), and some approaches to estimating $\mathrm{K}(\mathrm{h})$ will be treated in more detail in the following section.

\subsubsection{Advanced Topic}

Predictive Models for Soil Hydraulic Conductivity - (Jury et al., 1991)

The relationships between the hydraulic conductivity and the degree of saturation or matric potential arouse interest in methods for predicting the soil hydraulic conductivity based on the relationship between water content and matric potential, i.e. soil water retention. The idea is to simplify the complicated soil geometry by representing the soil as if comprised of a bundle of continuous capillaries. Figure 2-9? depicts a hypothetical cross-section in such a bundle having various sizes of capillaries. The flow is assumed to occur only in these capillaries; no bypass flow. The length of the bundle of capillaries (Lc), is assumed to be larger than the length of the soil column it represents $(\mathrm{L})$; the ratio $\mathrm{L} / \mathrm{Lc}$ is the tortuosity factor $(\mathrm{T})$.

Application of Poiseuille's law to the bundle of capillaries with a gradient DH across its length enable computation of the total volume of flow through the column by:

$$
\mathrm{Q}_{\text {tot }}=\sum_{\mathrm{j}=1}^{\mathrm{M}} \mathrm{N}_{\mathrm{j}} \mathrm{Q}_{\mathrm{j}}=\frac{\pi \rho_{w} \mathrm{~g}}{8 \eta} \frac{\Delta \mathrm{H}}{\mathrm{L}_{\mathrm{c}}} \sum_{\mathrm{j}=1}^{\mathrm{M}} \mathrm{N}_{\mathrm{j}} \mathrm{R}_{\mathrm{j}}^{4}
$$

where $\mathrm{Nj}$ is the number of capillaries of radius $\mathrm{Rj}$ in the bundle, and $\mathrm{M}$ is the number of different (arbitrarily assigned) capillary size classes. The flux (Jw) through the column is:

$$
\mathrm{J}_{\mathrm{w}}=\frac{\mathrm{Q}_{\text {tot }}}{\mathrm{A}}=\frac{\pi \rho_{w} \mathrm{~g}}{8 \eta} \frac{\Delta \mathrm{H}}{\mathrm{L}_{\mathrm{c}}} \sum_{\mathrm{j}=1}^{\mathrm{M}} \mathrm{n}_{\mathrm{j}} \mathrm{R}_{\mathrm{j}}^{4}
$$

where $n j$ is the number of tubes with radius Rj per unit area $(n j=N j / A)$. The next step is to convert the soil water characteristic curve into a soil water content vs. equivalent pore radii curve. This is done by using the capillary-rise equation to convert a matric potential value to an equivalent radius of capillarity (Fig.2-10a). The total (saturation) water content is divided into a certain number (M) of equal increments (Dqv), where the matric potential hj corresponds to a water content at the left-hand-side of the increment, $\mathrm{qLHS}=\mathrm{qs}-\mathrm{i} *$ Dqv (see Fig. 2-10b).

It is assumed that all tubes having radii greater than $\mathrm{Rj}$ have drained at a matric potential of $\mathrm{h}_{\mathrm{j}}$

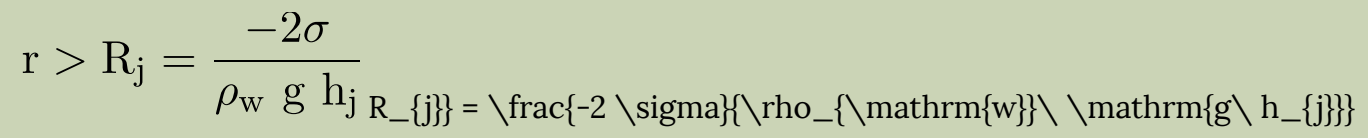

\section{$\backslash$ end $\{$ equation*\}" title="Rendered by QuickLaTeX.com">}

Note that we are assuming a zero contact angle between soil and water, thus cosg $=1.0$. The number of 
capillaries having a radius Rj per unit area in each water content interval is: $n j=D q v / p R j 2$ (where Dqv is interpreted as the fraction of the water-filled cross sectional area that is reduced when all capillaries having a radius of Rj drain).

Fig.2-10: a) Conversion of a SWC to equal increments of qi vs. Ri curve. b) Illustration of the summation of "conducting" pores of radii, Rj, for any given water content increment, Dqv. Approximations may target left hand side (LHS), midpoint (MP) or right hand side (RHS) of each increment depending on the scheme applied.

Inserting Eq. (71) and nj into Eq.(70) yields:

$$
\mathrm{J}_{\mathrm{w}}=\frac{\pi \rho_{\mathrm{w}} \mathrm{g}}{8 \eta} \frac{\Delta \mathrm{H}}{\mathrm{L}_{\mathrm{c}}} \sum_{\mathrm{j}=1}^{\mathrm{M}} \frac{\Delta \theta_{\mathrm{v}}}{\pi \mathrm{R}_{\mathrm{j}}{ }^{2}} \mathrm{R}_{\mathrm{j}}{ }^{4}=-\left[\frac{\sigma^{2} \Delta \theta_{\mathrm{v}}}{2 \eta \rho_{\mathrm{w}} \mathrm{g}} \sum_{\mathrm{j}=1}^{\mathrm{M}} \frac{1}{\mathrm{~h}_{\mathrm{j}}{ }^{2}}\right] \frac{\mathrm{L}}{\mathrm{L}_{\mathrm{c}}} \frac{\Delta \mathrm{H}}{\Delta \mathrm{z}}
$$

In Eq.(72) we introduced a multiplication by $-\mathrm{L} / \mathrm{Dz}=1$ (where $) \mathrm{z}=0-\mathrm{L}$ ) to bring it to a form similar to Darcy's law (Eq.(51)). Recall that $\mathrm{L} / \mathrm{Lc}$ is defined as the tortuosity factor (T), the ratio of the column and flow path lengths. We may associate all the factors in front of the hydraulic gradient DH/Dz with the saturated hydraulic conductivity (Ks) of the bundle of capillaries:

$$
\mathrm{K}_{\mathrm{s}}=\frac{\tau \sigma^{2} \Delta \theta_{\mathrm{v}}}{2 \eta \rho_{\mathrm{w}} \mathrm{g}} \sum_{\mathrm{j}=1}^{\mathrm{M}} \frac{1}{\mathrm{~h}_{\mathrm{j}}^{2}}
$$

As water content decreases from qs to $q \mathrm{~s}^{-} \mathrm{i}^{\star} \mathrm{Dqv}$ the tubes larger than Ri drain and do not contribute to water flow. The effect on the unsaturated hydraulic conductivity, which is a function of the water content, is given by:

$$
\mathrm{K}\left(\theta_{\mathrm{s}}-\mathrm{i} \Delta \theta_{\mathrm{v}}\right)=\frac{\tau \sigma^{2} \Delta \theta_{\mathrm{v}}}{2 \eta \rho_{\mathrm{w}} \mathrm{g}} \sum_{\mathrm{j}=\mathrm{i}+1}^{\mathrm{M}} \frac{1}{\mathrm{~h}_{\mathrm{j}}^{2}}
$$

Equation (74), originally developed by Childs and Collis-George (1950), serves as the basis for many predictive models of unsaturated hydraulic conductivity, most of which are based on SWC information and assume a capillary bundle geometry. Even with a detailed SWC, there is still an unknown in Eq.(74), i.e. the tortuosity (T). One way to circumvent this problem is to measure the saturated hydraulic conductivity (which is relatively easy to measure) and use it to solve for $\mathrm{T}$, assuming it is constant. One such formulation was proposed by Jackson (1972). Because we want to plot Ks against qs and we have a division by hj2, in eq. (74) (i.e., $\mathrm{h}=0$ at qs) we compute qi and hj at the Dqv increment's midpoint, qiMP.

$$
K\left(\theta_{i}^{M P}\right)=K_{s}\left(\frac{\theta_{i}^{M P}}{\theta_{s}}\right)^{c} \frac{\sum_{j=i}^{M} \frac{2 j+1-2 i}{h_{j}{ }^{2}}}{\sum_{j=1}^{M} \frac{2 j-1}{h_{j}{ }^{2}}}
$$


where qiMP $=$ qiLHS $+\mathrm{Dqv} / 2$, $\mathrm{c}$ is a constant usually taken as $\mathrm{c}=1$, and the water content is divided to $\mathrm{M}$ equal increments of Dqv.

This scheme computes an approximate hydraulic conductivity value for each given increment of water content. By plotting the computed K(qiMP) values against the RHS (qiRHS = qiLHS + Dqv) of the Dqv increment rather than qiMP or qiLHS, we satisfy the saturated hydraulic conductivity condition. The error introduced with these adjustments grows as the number of increments, M, is smaller. Jackson's approximation gives reasonable results with $M=10$ for example when compared with semi-empirical hydraulic conductivity models derived from soil water retention curves. This is illustrated using the VG model in the following example.

\section{Example 2-2): Prediction of Unsaturated Hydraulic Conductivity}

A matric potential-soil water content function (SWC) is given by the VG model expressed here in terms of the matric potential (h) as:

$$
\mathrm{h}=\frac{1}{\alpha}\left[\Theta^{-\frac{1}{m}}-1\right]^{\frac{1}{n}}
$$

where $\mathrm{Q}=(\mathrm{q}-\mathrm{qr}) /(\mathrm{qs}-\mathrm{qr})$ ranges between 0 and 1. Use Jackson's (1972) formula (Eq.(75)) to calculate the unsaturated hydraulic conductivity, $\mathrm{K}(\mathrm{q})$, given the following parameters: $\mathrm{a}=2.79 \mathrm{~m}-1$, $\mathrm{n}=1.6(\mathrm{~m}=1-1 / \mathrm{n}=0.375)$, qs $=0.55 \mathrm{~m}^{3} / \mathrm{m}^{3}, \mathrm{qr}=0.05 \mathrm{~m}^{3} / \mathrm{m}^{3}, \mathrm{~K}_{\mathrm{s}}=50 \mathrm{~mm} / \mathrm{h}$, and $\mathrm{M}=10$. We will assume that $\mathrm{c}=1$ in Eq. (75).

Solution: We can obtain $M$ equal increments of soil water content, qi, between qs and qr and the corresponding matric potential, hj, calculated at the midpoint using the following expressions:

$$
\theta_{i}^{M P}=\theta_{s}-\left(\theta_{s}-\theta_{T}\right) \frac{2 i=1}{2 M}, \quad h_{j}{ }^{M P}=\frac{1}{\alpha}\left[\left(1-\frac{2 j-1}{2 M}\right)^{-\frac{1}{m}}-1\right]^{\frac{1}{n}}
$$

where the terms (2i-1)/2M and 1-(2j-1)/2M associate the corresponding Dqv increment on the SWC curve in Fig. 2-10a. Note the decreasing qv values from saturation to the residual water content for $\mathrm{i}=\mathrm{j}$ from 1 to $\mathrm{M}$. For example,

$$
\left.\mathrm{h} 8 \mathrm{MP}=(1 / 2.79) *\left((1-(16-1) / 20)^{(}-2.667\right)-1\right)^{0} .625=3.56 \mathrm{~m}
$$

and the corresponding water content is

$$
\mathrm{q} 8 \mathrm{MP}=0.55-(0.55-0.05) *(16-1) / 20=0.175 \mathrm{~m}^{3} / \mathrm{m}^{3}
$$


(see Table 3.1).

The calculations of K(qiMP) can be performed easily on a computer spreadsheet as shown in Table 3.1. If follow on with the previous example we can calculate K(q8) using eq. (75):

$$
K\left(\theta_{s}^{M P}\right)=K_{s}\left(\frac{\theta_{8}^{M P}}{\theta_{s}}\right)^{c} \frac{\left[\frac{2 \cdot 8+1-2 \cdot 8}{h_{8}^{2}}+\frac{2 \cdot 9+1-2 \cdot 8}{h_{9}^{2}}+\frac{2 \cdot 10+1-2 \cdot 8}{h_{10}{ }^{2}}\right]}{\left[\frac{2 \cdot 1-1}{h_{1}^{2}}+\frac{2 \cdot 2-1}{h_{2}{ }^{2}}+\frac{2 \cdot 3-1}{h_{3}{ }^{2}}+\ldots+\frac{2 \cdot 10-1}{h_{10}{ }^{2}}\right]}
$$

The resulting calculations for K(qiMP) are plotted against qiRHS in Fig.2-11 and show very good agreement with the analytically derived van Genuchten (VG) K(qv) function which we will discuss in the next section.

Table 3.1: Calculation of the K(q) function for Sarpy loam from SWC information.

\begin{tabular}{llllll}
\hline $\mathbf{j}, \mathbf{i}$ * & $\mathbf{h j}(\mathbf{m})$ & qiMP & qiRHS ** & $\mathbf{S}(2 \mathbf{j + 1 - 2 i ) h j - 2}$ & $\mathbf{K}(\mathbf{q i M P})(\mathbf{m m} / \mathbf{h r})$ \\
1 & 0.108 & 0.525 & 0.55 & 213.000 & 47.7 \\
2 & 0.245 & 0.475 & 0.50 & 70.8 & 14.4 \\
3 & 0.392 & 0.425 & 0.45 & 30.3 & 5.50 \\
4 & 0.579 & 0.375 & 0.40 & 13.0 & 2.09 \\
5 & 0.842 & 0.325 & 0.35 & 5.28 & 0.732 \\
6 & 1.25 & 0.275 & 0.30 & 1.90 & 0.223 \\
7 & 1.98 & 0.225 & 0.25 & 0.564 & 0.0542 \\
8 & 3.56 & 0.175 & 0.20 & 0.123 & 0.0092 \\
9 & 8.43 & 0.125 & 0.15 & 0.0151 & 0.00081 \\
10 & 52.8 & 0.075 & 0.10 & 0.00036 & 0.000011 \\
\hline
\end{tabular}

* i points to each Dqv increment while $\mathrm{j}$ counts all water filled pores, $\mathrm{Rj}$, from $\mathrm{j}=\mathrm{i}$ to $\mathrm{M}$ (eq. (75)).

** K(qiMP) is plotted against qiRHS in order to match qs with KS

Fig.2-11: Predictions of unsaturated hydraulic conductivity from SWC information based on Jackson's method (symbols) and the van Genuchten analytical model (line).

\subsubsection{Parametric Models for Unsaturated Hydraulic Conductivity}

Some researchers have extended the concepts discussed in the previous section and derived closed-form analytical predictions of the unsaturated hydraulic conductivity. The basic step is replacing the summations with integrals of SWC analytical (parametric) expressions.

The two parametric SWC models that were presented in Section 1 are amenable to such integration. Van Genuchten (1980) derived an expression for the unsaturated hydraulic conductivity based on similar parameters to those proposed for expressing SWCs and on a modification to Eq. (75) proposed by Mualem (1976). The resulting water contentdependent unsaturated hydraulic conductivity $\mathrm{K}(\mathrm{q})$ is given by: 


$$
\frac{K(\Theta)}{K_{s}}=\Theta^{\frac{1}{2}}\left[1-\left(1-\Theta^{\frac{1}{m}}\right)^{m}\right]^{2}
$$

where $\mathrm{Q}=(\mathrm{q}-\mathrm{qr}) /(\mathrm{qs}-\mathrm{qr})$ is effective saturation, and $\mathrm{m}=1-1 / \mathrm{n}$ is a dimensionless parameter of the SWC. An example of a K(q) function is shown in Fig.2-11 (Example 2-6). The unsaturated hydraulic conductivity can also be expressed in terms of matric head (h):

$$
\frac{K(h)}{K_{s}}=\frac{\left[\left(1-(\alpha h)^{n-1}\left[1+(\alpha h)^{n}\right]^{m}\right)\right]^{2}}{\left[1+(\alpha h)^{n}\right]^{\frac{m}{2}}}
$$

Similarly, expressions for Brook's and Corey's (1964) model may be derived. For K(h) they obtained:

$$
\frac{K(h)}{K_{s}}=\left(\frac{h}{h_{b}}\right)^{-2-3 \lambda}
$$

where hb is the matric head at air-entry value or "bubbling pressure", and 1 is the pore distribution index parameter (see Section 1). The corresponding expression for $\mathrm{K}(\mathrm{q})$ is given by:

$$
\frac{K(\Theta)}{K_{s}}=\Theta^{3+\frac{2}{\lambda}}
$$

where $\mathrm{Q}=(\mathrm{q}-\mathrm{qr}) /(\mathrm{qs}-\mathrm{qr})$ is the effective saturation.

Another very useful and widely used parametric model for unsaturated hydraulic conductivity was proposed by Gardner (1958):

$$
K(h)=K_{s} e^{\beta h}
$$

where $b$ (dimensions of $\mathrm{L}^{-1}$ ) is a parameter related to typical pore size of the medium. The primary advantage of this form of $\mathrm{K}(\mathrm{h})$ is that it allows solutions to some flow problems to be obtained analytically. For example, Wooding's (1968) solution to unconfined 3-dimensional infiltration from a shallow cylindrical pond which is commonly utilized in measurement of soil hydraulic properties using disk permeameters, incorporates this formulation of the hydraulic conductivity.

Summarizing, analytical expressions for the unsaturated hydraulic conductivity may be derived from soil water retention information, e.g. VG and BC. Some expressions are based on empirical relationships which seem to fit observed conductivity data (or at least portions of it), and facilitate easy solutions to flow problems (Gardner, 1958). Parametric expressions for unsaturated hydraulic conductivity are particularly useful for purposes of modeling, for obtaining analytical solutions to flow and transport problems (see Example 2-7 in the next section), and for comparison between different types of soils or other porous media.

\section{Gas Flow in Porous Media}

On the surface, we might expect that the movement of gases in porous media would be a simple extension of the movement of liquids: just put in the right density and viscosity into the intrinsic permeability, and use Darcy's law. Well, it isn't quite that simple. Looking back at the derivation of the Hagen-Poiseuille equation you will notice that the velocity of the fluid at the walls of the capillary were assumed to be zero, satisfying the well known "no-slip" boundary condition which is quite typically invoked in the case of liquid flow. The idea behind the no-slip boundary is that collisions between molecules of liquid are so frequent that near a fixed surface the closest water molecules will be constantly losing all of their wall-parallel velocity to the wall, rendering them motionless from the macroscopic perspective. This is equivalent to observing that the mean free path of travel of the molecules in the liquid is very short compared to the aperture 
through which the liquid is moving. With gases the mean free length of travel may well be of the order of the pore size of our media. This being the case, the no-slip condition does not apply, rendering any results found using liquids to be inapplicable. This phenomena is referred to as the Klinkenberg effect due to the 1941 paper by L.J. Klinkenberg.

What we find for gases, then, is that the permeability of the media is a function of the gas pressure, which dictates the mean free path length for the gas molecules. The permeability to gases, $\mathrm{kg}$, is greater than that of liquids, and for flow through capillaries is given by

$$
\mathrm{k}_{\mathrm{g}}=\mathrm{k}\left(1=\frac{4 \mathrm{c} \lambda}{\mathrm{r}}\right)
$$

where $\mathrm{k}$ is the intrinsic permeability to liquid flow, $\mathrm{r}$ is the characteristic radius of the capillary apertures of the media, $\lambda$ is the mean free path length of the gas molecules, and $\mathrm{c}$ is the proportionality factor between the mean free path for the gas compared to the mean free path for gas which just collided with the capillary wall (c is just slightly less than 1, Klinkenberg, 1941). As we expected, if the capillary is much larger than the mean free path length, the intrinsic permeability for liquids is recovered, whereas, in very small aperture media, gas permeability can vastly exceed that of liquid permeability. The ratio of $\mathrm{kg} / \mathrm{k}$ provides a measure of the size of the typical conducting pathways, and has been used as a diagnostic parameter (Reeve, 1953). It is interesting to note that as pressure goes to zero the mean free path length becomes constrained by the capillaries to be a few times r, yielding an upper limit for kg of about 10 times the liquid permeability. In Klinkenberg's paper the upper limit of this ratio was observed to be between 5 and 20 for three media.

For practical purposes, the correction for pressures in the vicinity of 1 bar was found to be from 1.2 to 1.8 over the liquid permeability, depending upon the pore size of the medium (Klinkenberg, 1941). Methods of measurement of gas permeability are provided by Corey (1986), with some interesting new methods being discussed in the literature (e.g. Moore and Attenborough, 1992). The gas permeability is strongly dependent on the liquid content of the medium, analogous to the dependence of hydraulic conductivity upon moisture content (see Corey, 1986).

\section{Adding the Conservation of Mass: the Richards'on Equation}

So far we have developed the equation of state that relates flux to gradients in pressure (Darcy's law). We now need to add the constraint imposed by the conservation of mass to obtain the governing equation for unsaturated fluid flow in porous media, generally known as Richards equation after L.A. Richards, who derived and published this result in 1931, but this actually followed the 1922 publication of the result by Richardson (1922), so we compromise and call it the Richards'on equation. There are many ways to obtain this result; here we will simply follow a concise derivation used by J.-Y. Parlange in his teaching.

Consider an arbitrary volume of partially saturated medium, as shown below. Let's now do some bookkeeping of fluid going into and out of this volume. We know that whatever net flux through this surface there is will lead to corresponding changes in total moisture content in time. This may be written formally as

$$
\frac{\partial}{\partial \mathrm{t}} \iiint_{\text {volume }} \mathrm{qdV}=-\iint_{\text {surface }} \mathrm{q} \bullet \mathrm{ndS}
$$

where $\mathrm{n}$ is the unit normal vector and $\mathrm{q}$ is the vector flux, $\mathrm{dV}$ and $\mathrm{dS}$ are volume and surface elements, as shown below in Figure 3.15 


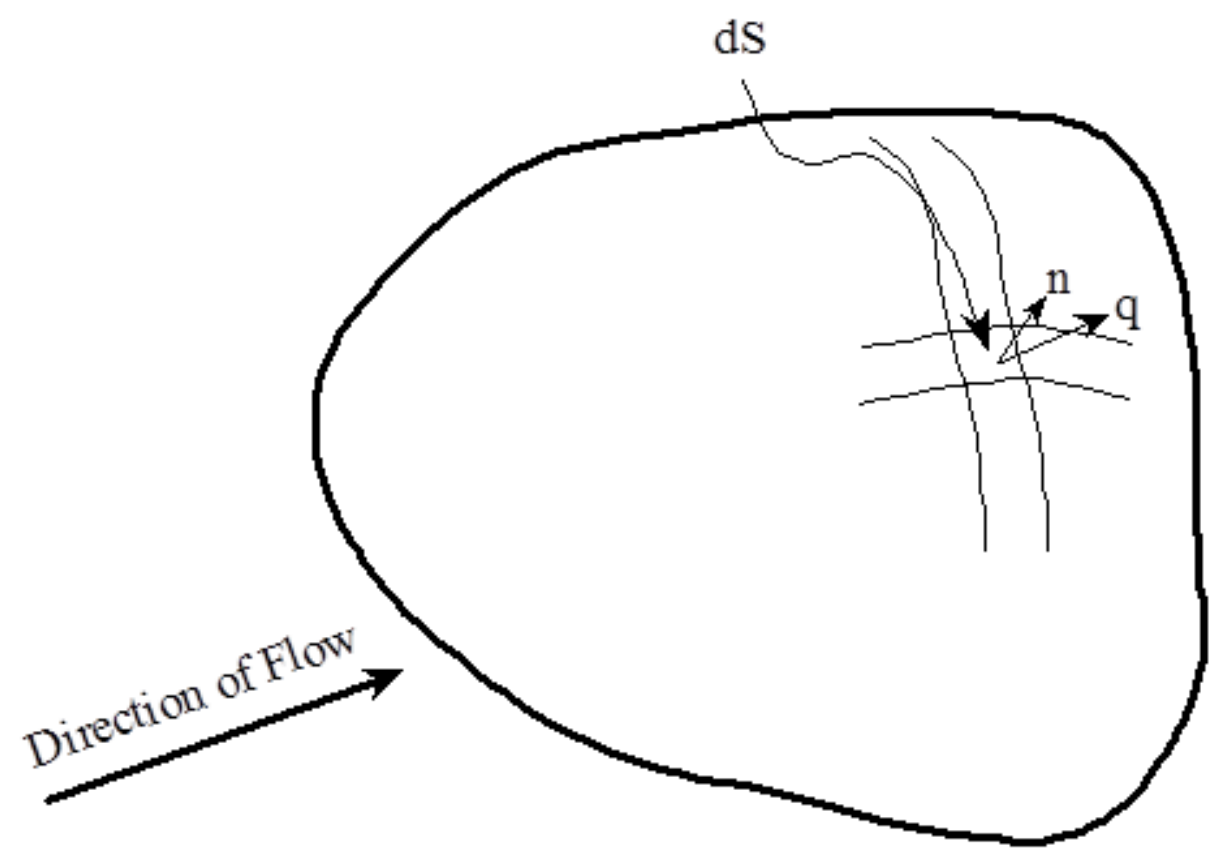

Figure 3.15 Definition sketch for the equation [2.109].

Since the volume is independent of time, we can bring the time derivative inside the integral on the left hand side of [2.109]

$$
\int_{\text {volume }} \iint \frac{\partial}{\partial t} \mathrm{qdV}=-\iint_{\text {surface }} \mathrm{q} \bullet \mathrm{nd} \mathrm{S}
$$

Now we can apply the divergence theorem to the right side of [2.110]

$$
\iint_{\text {surface }} \mathrm{q} \bullet \mathrm{ndS}=\iiint_{\text {volume }} \nabla \bullet \mathrm{qdV}
$$

Combining [2.110] and [2.111] we obtain

$$
\iiint_{\text {volume }}\left(\frac{\partial \theta}{\partial t}+\nabla \bullet q\right) d V=0
$$

Notice that since [2.112] is true for any volume element that the integrand must be zero for all points. To convince yourself of this, consider that if there was any point for which the integrand was not zero we could put an arbitrarily small volume around that point and the integral would also become non-zero, which would contradict [2.112]. So we have

$$
\frac{\partial \theta}{\partial t}+\nabla \bullet q=0
$$

We can now replace q using Darcy's law to obtain Richards equation

$$
\frac{\partial \theta}{\partial t}+\nabla \bullet(\mathrm{K}(\theta) \nabla \mathrm{H})
$$


where $\mathrm{H}$ is the total potential. This is also referred to as the Fokker-Planck equation.

Writing the total potential as the sum of the gravity potential and the pressure potential, $\mathrm{H}=\mathrm{h}+\mathrm{z}$, we notice that (3.104) $\nabla \mathrm{H}=\nabla \mathrm{h}+\nabla \mathrm{z}$

but

$$
\nabla \mathrm{z}=\frac{\partial \mathrm{z}}{\partial \mathrm{x}} \mathrm{i}+\frac{\partial \mathrm{z}}{\partial \mathrm{y}} \mathrm{j}+\frac{\partial \mathrm{z}}{\partial \mathrm{z}} \mathrm{k}_{\mathrm{i}}=0_{\mathrm{i}}+0_{\mathrm{j}}+1_{\mathrm{k}}=1_{\mathrm{k}}
$$

where $\mathrm{i}, \mathrm{j}$, and $\mathrm{k}$ are unit vectors in the $\mathrm{x}, \mathrm{y}$, and $\mathrm{z}$ directions. So the right side of Richards equation becomes

$$
\nabla \bullet[\mathrm{K}(\theta) \nabla \mathrm{H}]=\nabla \bullet\left[\mathrm{K}(\theta)\left(\nabla \mathrm{h}+1_{\mathrm{k}}\right)\right]
$$

$$
=\nabla \bullet[\mathrm{K}(\theta)(\nabla \mathrm{h})]+\nabla \bullet\left[\mathrm{K}(\theta) 1_{\mathrm{k}}\right]
$$

Equation [2.117] can be simplified by noticing that

$$
\begin{aligned}
\nabla \bullet\left[\mathrm{K}(\theta) 1_{\mathrm{k}}\right] & =\left(\frac{\partial \mathrm{z}}{\partial \mathrm{x}} \frac{\partial \mathrm{z}}{\partial \mathrm{y}} \frac{\partial \mathrm{z}}{\partial \mathrm{z}}\right) \bullet(0,0, \mathrm{~K}((\theta)) \\
& =\partial \mathrm{K}(\theta) \partial \mathrm{z}
\end{aligned}
$$

so Richards equation may be rewritten as

$$
\frac{\partial \theta}{\partial \mathrm{t}}=\nabla \bullet[\mathrm{K}(\theta)(\nabla \mathrm{h})]+\frac{\partial \mathrm{K}(\theta)}{\partial \mathrm{z}}
$$

which is a common form of this result. Equation [2.119] includes $\theta, K(\theta)$, and $h$ which is a functional of $\theta$. So before we can get anywhere we need the relationships, $\mathrm{K}(\theta)$ and $\mathrm{h}\{\theta\}$, at which point it is at least possible to solve [2.119]. These relationships are discussed in some detail in the following section. Since Richards equation is first order in time and second order in space, a well posed problem will require an initial condition (e.g., you need to specify the initial moisture distribution, but not the time rate of change of moisture content) and two boundary conditions (e.g., the pressure on the upper boundary will be $\mathrm{h}$ and the water table is at position $\mathrm{z}=0$ ).

Whenever you write a differential equation down, you should look and see if it can be put into a "familiar" form, to be able to take advantage of solutions that have been obtained to that same equation in some other context. In the case of Richards equation, it can be put in the form of a diffusion equation, which fits this description. To do so, we introduce the function

$$
\mathrm{D}(\theta)=\mathrm{K}(\theta) \frac{\partial \mathrm{h}}{\partial \mathrm{q}}
$$

which is called the soil diffusivity. We now note that the quantity in Richards equation which depends explicitly on pressure can be rewritten 


$$
\begin{aligned}
\mathrm{K} \nabla \mathrm{h} & =\mathrm{K}\left(\frac{\partial \mathrm{h}}{\partial \mathrm{x}} \frac{\partial \mathrm{h}}{\partial \mathrm{y}} \frac{\partial \mathrm{h}}{\partial \mathrm{z}}\right) \\
& =\mathrm{K}\left(\frac{\partial \mathrm{h}}{\partial \theta} \frac{\partial \theta}{\partial \mathrm{x}} \frac{\partial \mathrm{h}}{\partial \theta} \frac{\partial \theta}{\partial \mathrm{y}} \frac{\partial \mathrm{h}}{\partial \theta} \frac{\partial \theta}{\partial \mathrm{z}}\right) \\
& =\mathrm{K} \frac{\partial \mathrm{h}}{\partial \theta}\left(\frac{\partial \theta}{\partial \mathrm{x}} \frac{\partial \theta}{\partial \mathrm{y}} \frac{\partial \theta}{\partial \mathrm{z}}\right) \\
& =\mathrm{D} \nabla \theta
\end{aligned}
$$

which if put into Richards equation gives us an equation reminiscent of the classical diffusion equation with the gravity driven flux term added

$$
\frac{\partial \theta}{\partial \mathrm{t}}=\nabla \bullet[\mathrm{D}(\theta) \nabla \theta]+\frac{\partial \mathrm{K}(\theta)}{\partial \mathrm{z}}
$$

You may recall that the favorite trick amongst people solving diffusion problems is to assume that D is fairly constant over space and pull it outside the derivative. Well this trick is well justified in many heat diffusion problems, but it is generally way off base here! The soil water diffusivity is a strongly non-linear function of $\theta$ and varies drastically as a function of space. This makes Richards equation quite an interesting challenge in terms of finding tidy analytical solutions, and even makes numerical modelers wince a bit due to the very rapid changes in both $\mathrm{D}$ and $\theta$.

\section{IO.2 Advanced Topic: Numerical Solutions}

The high nonlinearity of the Richards equation for unsaturated flow makes it much more difficult to solve than other problems in classical physics involving flow of heat or electricity. Except for a few special cases, the only way to obtain solutions to practical flow problems represented by the Richards equation is by means of successive numerical approximations, generally using computers.

The basis for many numerical solutions to the flow equation involves the following steps: (i) discretization of the flow domain into a grid: "layers" for one dimensional flow or a mesh for two-dimensional flow; (ii) discretization of the continuous partial differential equation into a series of approximated difference equations, one for each of the spatial nodes (see the scheme in Fig.2-15); and (iii) formulation of the problem into a computer code which is able to solve the equations for the entire flow domain at various time steps. 


\section{Example 2-10): A Numerical Approximation to the Richards Equation}

Find: A numerical approximation to changes in water content with time under vertical unsaturated and nonsteady flow into a soil with a known initial water content, and known hydraulic functions $\mathrm{q}(\mathrm{y})$ and $\mathrm{K}(\mathrm{y})$.

Solution: We first discretize the soil profile (the spatial domain) into units of $\mathrm{Dz}$, and the time into units of Dt. We denote the water content at the $\mathrm{i}$-th depth increment and at the $\mathrm{j}$-th time step as qij; similarly we express the corresponding matric head as hij. The hydraulic conductivity, which is a function of the matric head (or of the water content), relates transfer between two soil layers and thus must be averaged to represent both layers as: $\mathrm{K}([\mathrm{hij}+\mathrm{hji}+1] / 2)=\mathrm{Kji}+1 / 2$. The resulting numerical approximation to Eq. (92) for vertical flow is then given by

Combining terms and rearranging allows us to solve for the only unknown in Eq.101, which is qij +1 , the water content for the i-th depth increment at the next (future) time step:

Because this is a discrete approximation of a continuous process, increments Dz and Dt should be kept small; the actual selection criteria are beyond the scope of this course. In addition, initial soil water contents with respect to depth and the conditions at the boundaries of the flow domain need to be specified.

\section{Expressions for Conductivity and Retention}

As was pointed out above, to begin to solve Richards equation we need the mathematical relationships between pressure, moisture content and conductivity. Conductivity is generally regarded as a non-hysteretic function of moisture content, while moisture content and pore pressure are related through a hysteretic functional. Very often this hysteretic nature is ignored to simplify the analysis, leading to the use of expressions of conductivity directly in terms of pressure potential. To obtain an analytical solution to Richards equation, these relationships must be in the form of analytical expressions. For numerical solutions to Richards equation, the relationships may be either analytical expressions, or constructed as interpolations from tables of laboratory data. The independent domain model for the hysteretic relationship between pressure and moisture content is easily implemented in numerical formulations.

With this motivation, a host of mathematical relationships for $K(\theta)$ and $h(\theta)$ have been introduced, which are summarized in Table 3.2. As pointed out by Fuentes et al. (1992), some of these relationships will provide non-physical results in certain soils, thus they must be used with care, and the expressions obtained must be evaluated for their quality of description on a case-by-case basis. A full discussion of the pros and cons of each of these expressions is beyond the scope of this book. Generally, the more complex expressions are favored for their ability to fit experimental data, while the simpler results (such as Gardner's) are often employed as they can be handled analytically, yielding useful closed form results.

Table 3.2 Selected expressions for $\mathrm{K}(\theta), \mathrm{h}(\theta), \theta(\mathrm{h})$ and $\mathrm{D}(\theta)$. 


$$
\begin{aligned}
& \mathrm{D}\left(\frac{\theta-\theta_{0}}{\theta_{\mathrm{s}}-\theta_{0}}\right)=\frac{\mathrm{K}_{\mathrm{s}}\left|\mathrm{h}_{\mathrm{c}}\right|(1-\beta)}{\left(\theta_{\mathrm{s}}-\theta_{\mathrm{I}}\right)\left(1-\beta\left(\mathrm{d} \frac{\theta-\theta_{0}}{\theta_{\mathrm{s}}-\theta_{0}}\right)^{2}\right)} \quad \text { with } 0<\beta<1^{\text {Fujita (1952) }} \\
& \mathrm{K}(\mathrm{h})=\mathrm{K}_{\mathrm{s}} \exp (\alpha \mathrm{h}) \\
& \mathrm{K}(\mathrm{h})=\frac{\mathrm{K}_{\mathrm{s}}}{\mathrm{h}^{\mathrm{n}}+\mathrm{b}} \quad \text { with } 1<\mathrm{n}<4 \\
& \frac{\theta-\theta_{0}}{\theta_{\mathrm{s}}-\theta_{0}}=\frac{1}{1+\left(\mathrm{d} \frac{\mathrm{h}}{\mathrm{h}_{\mathrm{b}}}\right)^{\mathrm{b}}} \\
& \mathrm{D}(\theta)=\frac{\mathrm{D}_{0}(\mathrm{n}+1) \theta^{\mathrm{n}}}{\mathrm{n}}\left(1-\frac{\theta^{\mathrm{n}}}{\mathrm{n}+1}\right) \quad \text { where } \mathrm{D}_{0}=\mathrm{D}\left(\theta_{\mathrm{s}}\right) \\
& \mathrm{K}(\mathrm{h})=\mathrm{K}_{\mathrm{s}} \exp \left[\alpha\left(\mathrm{h}-\mathrm{h}_{0}\right)\right] \quad \text { for } \mathrm{h}<\mathrm{h}_{0} \\
& \text { and } \mathrm{K}(\mathrm{h})=\mathrm{K}_{\mathrm{s}} \quad \text { for } \mathrm{h} \geq \mathrm{h}_{0} \\
& \frac{\theta-\theta_{0}}{\theta_{\mathrm{s}}-\theta_{0}}=\left(\frac{\mathrm{h}_{\mathrm{cr}}}{\mathrm{h}}\right)^{\lambda} \quad \text { for } \mathrm{h}<\mathrm{h}_{\mathrm{cr}} \\
& \theta=\theta_{\mathrm{s}} \quad \text { for } \mathrm{h} \geq \mathrm{h}_{\mathrm{cr}} \\
& \mathrm{K}(\theta)=\mathrm{K}_{\mathrm{s}}\left(\frac{\theta-\theta_{0}}{\theta_{\mathrm{s}}-\theta_{0}}\right)^{\eta} \text { for } \mathrm{h}<\mathrm{h}_{\mathrm{cr}} \\
& \mathrm{K}(\theta)=\mathrm{kK}_{\mathrm{s}} \quad \text { for } \mathrm{h} \geq \mathrm{h}_{\mathrm{cr}} \\
& \text { where } \eta=\frac{2}{\lambda}+3
\end{aligned}
$$


where $\eta=\frac{2}{\lambda}+3$

Brooks and Corey (1964)

$K(\theta)=K_{s}\left(\frac{\theta-\theta_{0}}{\theta_{s}-\theta_{0}}\right)^{1 / 2}\left[\frac{\int_{\theta}^{\theta} d \frac{d x}{h}}{\int_{\theta} d \frac{d y}{h}}\right]^{2}$ for any retention function $\theta(h)$

Mualem (1976)

where $\mathrm{x}$ and $\mathrm{y}$ are dummy variables of integration

$\left.\frac{\theta-\theta_{0}}{\theta_{\mathrm{s}}-\theta_{0}}=\frac{1}{(} 1+(\alpha \mathrm{h})^{\mathrm{n}}\right)^{\mathrm{m}}$

which yields, using Mulaem's 1976 expression given above,

$K(\theta)=K_{\mathrm{s}}\left(\frac{\theta-\theta_{0}}{\theta_{\mathrm{s}}-\theta_{0}}\right)^{1 / 2}\left\{1-\left[\left(\frac{\theta-\theta_{0}}{\theta_{\mathrm{s}}-\theta_{0}}\right)^{1 / \mathrm{m}}\right]^{\mathrm{m}}\right\}^{2}$

Van Genuchten

where $\alpha>0, m=1-1 / \mathrm{n}$ with $\mathrm{n}>1$.

$K(\theta)=K_{s}\left(\frac{\theta-\theta_{0}}{\theta_{s}-\theta_{0}}\right)^{2}\left[1-\gamma \frac{\int_{\theta_{s}}^{\theta_{s}} \mathrm{D}(x) d x}{\int_{0}^{\theta_{s}} \mathrm{D}(\mathrm{y}) \mathrm{dy}}\right]$ where $0 \leq \gamma \leq 1$

Parlange et al. (1985)

where $\mathrm{x}$ and $\mathrm{y}$ are dummy variables of integration

$\theta=\theta_{0}+\left(\frac{\theta_{\mathrm{s}}-\theta_{0}}{2}\right) \operatorname{erfc}\left(\frac{\ln \left(\mathrm{h}_{\mathrm{c}}-\mathrm{h}\right) /\left(\mathrm{h}_{\mathrm{c}}-\mathrm{h}_{0}-\sigma^{2}\right.}{2^{1 / 2} \sigma}\right) \quad$ for $\mathrm{h}<\mathrm{h}_{\mathrm{c}}$

Kosugi (1994)

for $\mathrm{h} \varepsilon \mathrm{h}_{\mathrm{c}}$

$\theta=\theta_{\mathrm{s}}$

Kosugi (1994) which can be put into Mualem's equation to yield a conductivity function

$$
\mathrm{K}(\mathrm{h})=\mathrm{K}_{\mathrm{s}}\left(\frac{\theta-\theta_{0}}{\theta_{\mathrm{s}}-\theta_{0}}\right)^{1}\left[\frac{1}{2} \operatorname{erfc}\left\{\frac{1}{2^{1 / 2} \sigma} \ln \left(\frac{\mathrm{h}}{\mathrm{h}_{0}}\right)\right\}\right]^{2}
$$

Das and Kluitenberg (1996)

The matric potential will be continuous across layers, while the water content profile exhibits a sharp discontinuity. A further problem here is that it is not possible to take the required derivative in Eq.(98), , across the discontinuity. Note that it may be permissible to use this equation in homogenous soils for conditions where the vertical gravitational 
component is very small in comparison with matric influences (translated into a water content "gradient"). Examples of this are the early phases of infiltration into relatively dry soil, and evaporation of water from the soil surface.

In some special cases the diffusivity may be assumed constant over a small range of water content. A constant diffusivity facilitates analytical solutions to Eq.(98) for certain boundary conditions. In many cases analytical solutions to flow problems are favored over numerical solutions due to their mathematical simplicity, the information they provide concerning the physical process, and the physical significance of their parameters.

Fig.2-14: Continuous matric potential profile vs. discontinuous water content profile across a textural boundary in layered soil

\section{Example 2-9): Parametric Forms of the Diffusivity Function}

Develop parametric forms of the soil water diffusivity function based on the van Genuchten and BrooksCorey models.

Solution: The diffusivity function is defined as $\mathrm{D}(\theta)=\mathrm{K}(\theta) \mathrm{dh} / \mathrm{d} \theta$. Substituting Eq.(79) and Eq.(39) for the VG model results in (van Genuchten, 1980):

$$
\mathrm{D}(\Theta)=\frac{(1-\mathrm{m}) \mathrm{K}_{\mathrm{s}}}{\alpha \mathrm{m}\left(\theta_{\mathrm{s}}-\theta_{\mathrm{r}}\right)} \Theta^{\frac{1}{2}-\frac{1}{\mathrm{~m}}}\left[\left(1-\Theta^{\frac{1}{\mathrm{~m}}}\right)^{-\mathrm{m}}+\left(1+\Theta^{\frac{1}{\mathrm{~m}}}\right)^{\mathrm{m}}-2\right]
$$

where $\Theta$ is effective saturation (see Eq.(39)). Taking the same approach for the Brooks-Corey model, we substitute Eqs.(81) and (40) to get:

$$
\mathrm{D}(\Theta)=\frac{\mathrm{K}_{\mathrm{s}} \mathrm{h}_{\mathrm{b}}}{\lambda\left(\theta_{\mathrm{s}}-\theta_{\mathrm{r}}\right)} \Theta^{2+\frac{1}{\lambda}}
$$

\section{Miller Similarity and Scaling of Capillary Properties}

The basic question we seek to address in this section is how can we take information about the hydraulic properties of one medium to make quantitative predictions of the properties of another medium. This effort arose following a canoe trip between the brothers Ed and Bob Miller in the early 1950's (personal communication, Bob Miller to John Selker in about 1990). Apparently, in the many hours of paddling in the Wisconsin Boundary Waters Bob was explaining his extensive testing of soil properties. His brother Ed thought Bob was working way too hard. Wouldn't it be great if he only had to measure the hydraulic conductivity of one soil, and then could predict the conductivity of other soils in some simple way? In the same way, it would be very useful to be able to quantitatively extend observations of a particular flow pattern in one medium to other medium. After a collaboration which spanned what Bob found to be an interminably long time as Ed polished the presentation, in 1956 Miller and Miller presented a comprehensive methodology to carry out such extensions of information between pairs of porous media systems. As you might surmise from the introduction, these tools allow us to greatly extend the applicability of experimental and theoretical results, and thus are worth taking the time to understand. In this section we follow the presentation of Miller and Miller quite closely.

Before getting drawn into the particulars, it is worth putting a few bounds on our expectations as to what should be possible. We should not, for instance, expect to make measurements in a sandy material and in hopes of learning about the behavior of clays, as these media are fundamentally differing in their chemical properties and pore-scale geometric 
configuration. The features of clay that control its interaction with water are the expansive charged surface area and platy geometry, while for sands, the particle size distribution and angularity of the grains are the relevant parameters. Thus, to extrapolate from one medium to another, the two systems must at least be similar in the geometric sense. This property is shown conceptually in Figure 3.16.
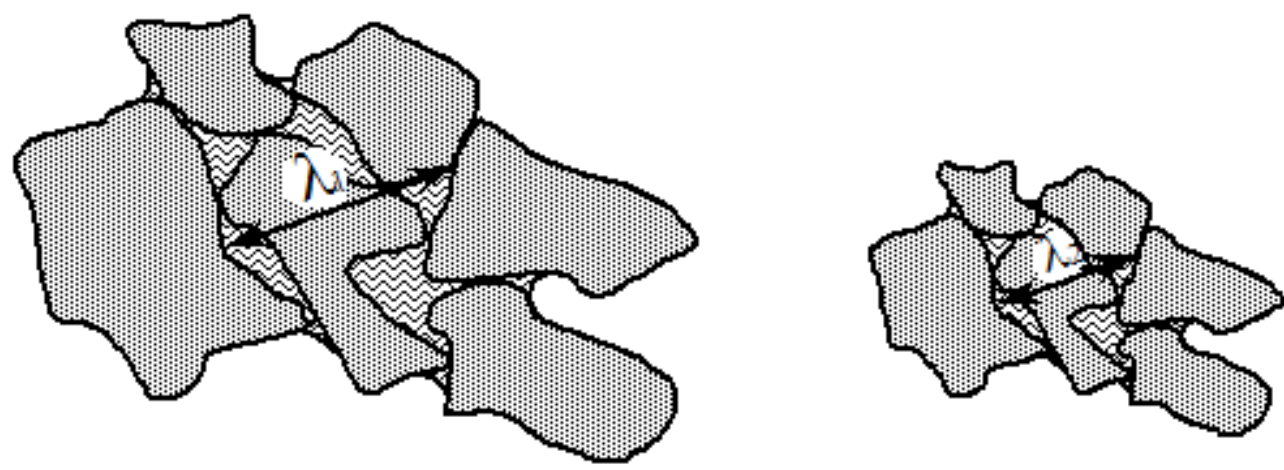

Figure 3.16 Geometrically similar media with their characteristic microscopic length scales, $\lambda_{1}$ and $\lambda_{2}$ (After Miller and Miller, 1956).

In Figure 3.16 we have selected identical geometric shapes, and have just magnified (scaled) one of the media. We have then chosen a particular geometric feature, and measured it to find it had lengths $\lambda_{1}$ and $\lambda_{2}$ in the two media. We will refer to these as the "characteristic microscopic length scales" of the two media. All we are looking for in these $\lambda$ 's is some dimension which can be identified readily and reflects the typical dimensions at the grain scale. In practice, people often use the $d_{50}$ as their $\lambda$ as it is easy to measure and therefore widely reported. Any other measure would be fine so long as you are consistent in using the same measure for each of the media.

Enough of the generalities, lets see how this works! We first need to delineate our assumptions required of our media, liquid, gas and pore system.

Assumptions for similarity

1. Media. The media is assumed to be uniform with regards to position, orientation and time. That is to say the media must be homogeneous, isotropic, and permanent.

2. Liquid. The liquid must be uniform in surface tension, contact angle, viscosity, and density. A key point is that to maintain geometric similarity, the contact angle must be the same in the two systems, although surface tension and viscosity may differ.

3. Gas. The gas phase is assumed to move freely in comparison to the liquid phase, and is assumed to be at a uniform pressure.

4. Connectivity. This analysis will be limited to funicular states in both the air and water phases. There will be no isolated bubbles or droplets, and pressures can communicate freely.

We will further assume that in each system the surface tension, pore geometry and contact angle are fixed in time. The only variable property will be the pressure of the liquid relative to the gas. What about hysteresis? No problem - this can be included in similarity analysis. As usual, we simply need to do some bookkeeping to keep track of the sequence of end-points of changes in pressure for each location (see section 2.3.3). As before, the moisture content at any point will be a function of the final pressure and this sequence of end-points, or a functional of the present pressure. We require that for two systems to be in similar states they must have followed the same sequence of scaled pressures (we will soon see what is meant by scaled pressure).

We need also provide a more rigorous definition of what is meant by geometric similarity. Figure 3.16 shows two media that are precisely scaled duplicates of each other, where the distance between any two geometric features of media 1 is $\lambda_{1} / \lambda_{2}$ times that found in medium 2 . This exact similarity is akin to that of similar triangles, and has probability zero 
of occurring in any two natural media. Although exact similarity is certainly sufficient for our needs, it is not necessary, and is therefore overly restrictive. The necessary and sufficient conditions are that media 1 and 2 are similar if, and only if, there exists a constant $\alpha=\lambda_{1} / \lambda_{2}$ such that if all length dimensions in medium 1 are multiplied by $\alpha$, the probability of any given geometric shape to be seen in the scaled medium 1 and medium 2 is identical. In this same way, one could characterize the degree of similarity by computing the optimal value of $\alpha$ then compute the mean deviation between the two particle size distributions when scaled by $\alpha$.

If the particle size distributions are linearly related (that is, two particle size distributions will fall on one line if the particle dimensions of one is multiplied by the appropriate constant), and the porosity's are the same, then the two media are similar in the sense of Miller and Miller. If you want to be even more careful you can require the particles to have similar shape, for example, as measured by their sphericity. Throughout this presentation it is easiest to visualize media which are exactly similar (Figure 3.16), while keeping the less restrictive definition in mind for applications.

Rather than being restricted to considering pairs of media, it is most convenient to define a "scaled medium" which can then be compared to any other similar media. To do this we simply divide all geometric dimensions in the media by the characteristic microscopic length scale for that medium, $\lambda$. Any quantity which has been scaled will be noted by putting a dot suffix, e.g., K• would denote the scaled conductivity.

Our goal is to be able to relate the pressure, moisture content, hydraulic conductivity, sorptivity, and any other hydraulic properties, between similar media. We will take it step by step, first considering the moisture content of two media with geometrically similar emplacement of water. We first need to recognize that if the position of the water is geometrically similar, then the volumetric moisture content will be the same in the two systems. Think of using a magnifying glass to look at a wet soil; no matter what magnification you looked at, the moisture content would be the same. So now let's move on to scaling pressure.

How can we relate the water pressure between two similar systems? For this analysis we will be considering two different textures of soil that are at the same volumetric water content. If we consider any particular gas/liquid interface we recall that Laplace's equation gives us the pressure in terms of the reduced radius $\mathrm{R}$

Where $\gamma$ is the contact angle, $\sigma$ is the surface tension, and $p$ is the difference in pressure between the gas and liquid (remember that although geometrically similar media must have the same contact angle, that angle is not restricted to any particular value). Multiplying both sides of $[2.123]$ by $\lambda / \sigma$ yields

$$
2 \cos \gamma \frac{\lambda}{\mathrm{R}}=\frac{\lambda \mathrm{p}}{\sigma}
$$

Here is the crux of identifying similarity relationships: the stuff on the left of this equation is constant for any similar medium, thus the terms on the right side must be constant as well. In this way we have obtained a method for scaling the pressure!

Notice that we could have put the cos $\gamma$ term on either side of this equation since it must be constant between similar media. To make sure you don't forget that it must be constant, it is safest to just leave it out of the scaling relationship so you don't try to compare situations which have different contact angle. It is natural then to define the scaled pressure as

$$
\mathrm{p}_{\bullet}=\frac{\lambda \mathrm{p}}{\sigma}
$$

Although we have kept the pressure notation, but this could just as well be written in units of head, which we would write as h. To see how this scaling of pressure works, it is useful to consider a simple example. Let's calculate the pressure in medium 1 at some moisture content $\theta$ given that we know the pressure in medium 2 at the same value $\theta$. From above, we note that the pressure potentials are related simply as 


$$
\mathrm{h}_{\bullet}(\theta)=\frac{\lambda_{1} \mathrm{~h}_{1}(\theta)}{\sigma_{1}}=\frac{\lambda_{2} \mathrm{~h}_{2}(\theta)}{\sigma_{2}}
$$

so we can obtain the pressure potential of the second medium as

$$
\mathrm{h}_{2}(\theta)=\frac{\lambda_{1} \sigma_{2}}{\lambda_{2} \sigma_{1}} \mathrm{~h}_{1}(\theta)
$$

An application of this formula to four similar sands is shown in Figure 3.17.

\section{[this figure needs to be inserted]}

Figure 3.17 Illustration of scaling of pressure potential of Miller similar media to compare characteristic draining curves for four sands. In this case the Sands are four grades of Accusand (from Schroth et al., 1996).

So far so good, but how about scaling hydraulic conductivity? Our strategy will be the same: we will try to write down some basic physically based equations describing flow, then try to solve for a product of geometric and measured parameters which is constant for any two samples from similar media. So, as in the case of pressure potential, we need to go back to the underlying physical equations to derive the correct expression for scaling. In the case of pressure, we needed to go to Laplace's equation. For conductivity, we need to understand the physical basis for conductivity as defined in Darcy's law. The best way to do this is to identify the terms which make up K in Darcy's law in the NavierStokes equation for creeping flow (i.e., where inertia and turbulence are insignificant: see discussion of Darcy's law). For these conditions, the Navier-Stokes equation may be written

(3.119) $(\mathrm{g}-\nabla \mathrm{p})=\eta \nabla^{2} \mathrm{v}$

where $g$ is the acceleration of gravity, $\eta$ is the liquid viscosity and $v$ is the microscopic local velocity. This may be compared to Darcy's law

$$
\text { (3.120) } \mathrm{v}=\mathrm{K}(\mathrm{p})(\mathrm{g}-\nabla \mathrm{p})
$$

which can be written

$$
(\mathrm{g}-\nabla \mathrm{p})=\frac{\mathrm{v}}{\mathrm{K}(\mathrm{p})}
$$

Equating the two and solving for $\mathrm{K}$ we find

$$
\mathrm{K}(\mathrm{p})=\frac{\mathrm{v}}{\eta \nabla^{2} \mathrm{v}}
$$

To simplify the form of the equation, without any change in the generality, we consider a one-dimensional case, where we see that

$$
\mathrm{v}=\frac{\partial \mathrm{l}}{\partial \mathrm{t}}=\lambda \frac{\partial \mathrm{l} \bullet}{\partial \mathrm{t}}=\lambda \alpha \frac{\partial \mathrm{l} \bullet}{\partial \alpha \mathrm{t}}=\lambda \alpha \mathrm{v}_{\bullet}
$$

where 1 is a microscopic unit of length, and $\alpha$ is the scaling parameter for time, such that $t_{\bullet}=\alpha$, which we shall determine shortly. For one-dimensional problems we can further write that 


$$
\begin{aligned}
\nabla^{2} \mathrm{v} & =\frac{\partial^{2}\left(\mathrm{~d} \frac{\partial \mathrm{l}}{\partial \mathrm{t}}\right)}{\partial \mathrm{l}^{2}} \\
& =\frac{\partial^{2}\left(d \frac{\partial \mathrm{l}_{\bullet}}{\partial \mathrm{t}}\right)}{\partial \lambda^{2} \mathrm{l}_{\bullet}^{2}} \\
& =\frac{\alpha}{\lambda} \nabla_{\bullet}{ }^{2} \mathrm{v}_{\bullet}
\end{aligned}
$$

substituting [2.132] and [2.133] into [2.131] yields

$$
\mathrm{K}(\mathrm{p})=\lambda^{2} \frac{\mathrm{v} \bullet}{\eta \nabla_{\bullet}^{2} \mathrm{v} \bullet}
$$

Putting all of the unscaled variables on the left we see that

$$
\frac{\eta \mathrm{K}(\mathrm{p})}{\lambda^{2}}=\frac{\mathrm{v}_{\bullet}}{\nabla_{\bullet}^{2} \mathrm{v}_{\bullet}}
$$

The right-hand side of [2.135] is only dependent on the properties of the scaled medium, implying that the left-hand side must be as well. Therefore $\eta \mathrm{K}(\mathrm{p}) / \lambda^{2}$ is the scaled conductivity, accounting for both viscosity and length scale.

$$
K_{\bullet}(p)=\frac{\eta K(p)}{\lambda^{2}}
$$

We have to be careful here because the $\mathrm{p}$ is not the scaled pressure, so the left-hand side is a bit of a mixture between scaled and unscaled values. To be just that much more complete in our scaling, we should write this in the scaled terms we derived above:

$$
\mathrm{K}_{\bullet}\left(\mathrm{p}_{\bullet}\right)=\frac{\eta \mathrm{K}\left(\frac{\mathrm{p} \lambda}{\sigma}\right)}{\lambda^{2}}=\frac{\eta \mathrm{K}(\theta)}{\lambda^{2}}
$$

To see how this would be applied, let us go back to the two similar media at moisture content $\theta$. We know that their scaled conductivities will be identical, so

$$
K_{\bullet}\left(p_{\bullet}\right)=\frac{\eta_{1} K_{1}(\theta)}{\lambda_{1}^{2}}=\frac{\eta_{2} K_{2}(\theta)}{\lambda_{2}^{2}}
$$

or, solving for $\mathrm{K}_{2}$ in terms of $\mathrm{K}_{1}$ we find

$$
K_{2}(\theta)=K_{1}(\theta) \frac{\eta_{1} \lambda_{2}^{2}}{\eta_{2} \lambda_{1}^{2}}
$$

or we can gather this with our pressure scaling results to put this in terms of pressure

$$
\mathrm{K}_{2}(\Psi)=\mathrm{K}_{1}\left(\frac{\lambda_{1} \sigma_{2}}{\lambda_{2} \sigma_{1}}\right) \frac{\eta_{1} \lambda_{2}^{2}}{\eta_{2} \lambda_{1}^{2}}
$$


So far we have only worked with the information we have about the microscopic life of the system (e.g., Laplace equation and the Navier-Stokes equation). By looking to the macroscopic properties of the system, we can obtain the last scaling parameters we require, namely the scaling of time. To do this we will need to consider Darcy's law and the conservation of mass. In the absence of gravity Darcy's law states

(3.132) $\mathrm{v}=-\mathrm{K}(\mathrm{p}) \nabla \mathrm{p}$

Multiplying both sides by $\eta / \lambda \sigma$, we find

$$
\frac{\eta}{\lambda \sigma} \mathrm{v}=\frac{\eta}{\lambda^{2}} \mathrm{~K}(\mathrm{p}) \nabla\left(\frac{\lambda \mathrm{p}}{\sigma}\right)
$$

From a macroscopic viewpoint, both $\mathrm{v}$ and $\nabla$ are functions of the macroscopic length scale, say $\mathrm{L}$. The product $\mathrm{L} \nabla$ is the reduced form of the gradient operator. So we can multiply both sides by L to put the right side of this equation in the reduced form

$$
\frac{\mathrm{L \eta}}{\lambda \sigma} \mathrm{v}=\frac{\eta}{\lambda^{2}} \mathrm{~K}(\mathrm{p}) \mathrm{L} \nabla\left(\frac{\lambda \mathrm{p}}{\sigma}\right)=\mathrm{K}_{\bullet} \nabla \bullet \mathrm{p} \bullet
$$

So we can identify that the left side of this equation must also be in reduced form, thus the reduced macroscopic velocity is given by:

$$
\mathrm{v}_{\bullet}=\frac{\mathrm{L \eta}}{\lambda \sigma} \mathrm{v}
$$

From this we can also obtain the scaling relationship for time. We would like to obtain the scaling parameter for time, say $\alpha$, such that $\alpha \mathrm{t}=\mathrm{t}$. Using the definition of velocity we may write

$$
\mathrm{v}_{\bullet}=\frac{\mathrm{dx}_{\bullet}}{\mathrm{dt}_{\bullet}}
$$

which, using the fact that $\mathrm{dx} \cdot=\mathrm{dx} / \mathrm{L}$ and $\mathrm{dt} \cdot=\alpha \mathrm{dt}$, may be rewritten

$$
\mathrm{v}_{\bullet}=\frac{\mathrm{dx} / \mathrm{L}}{\mathrm{d} \alpha \mathrm{t}}=\frac{1}{\alpha \mathrm{L}} \frac{\mathrm{dx}}{\mathrm{dt}}=\frac{1}{\alpha \mathrm{L}} \mathrm{v}
$$

Now solving for $\alpha$ we find

$$
\alpha=\frac{\mathrm{v}}{\mathrm{v}_{\bullet} \mathrm{L}}=\frac{\lambda \sigma}{\mathrm{L}^{2} \eta}
$$

or

$$
\mathrm{t}_{\bullet}=\frac{\lambda \sigma}{\mathrm{L}^{2} \eta} \mathrm{t}
$$

So there we are! We can now scale any process of capillary flow in similar media. To see how this system works, let's scale one more soil-water parameter, the sorptivity, S. From the definition of $\mathrm{S}$ we know that 


$$
\begin{aligned}
S(\theta) & =2 D(\theta) \frac{d \theta}{d \phi} \\
& =2 K(\theta) \frac{d \Psi}{d \phi} \\
& =2 K(\theta) \frac{d \Psi}{d\left(d \frac{x}{t^{1 / 2}}\right)}
\end{aligned}
$$

where $\mathrm{S}$ is the sorptivity, $\mathrm{D}$ is the diffusivity, $\mathrm{K}$ is the conductivity, $\theta$ is the moisture content and $\varphi$ is the Boltzman transform variable, $\varphi=\mathrm{xt}^{-1 / 2}$. To derive the scaled value of sorptivity we must replace the variables with the appropriate scaled quantities. Drawing together the scaled variables from above

$$
\begin{aligned}
& x_{\bullet}=\frac{x}{L} \\
& t_{\bullet}=\frac{\lambda \sigma t}{\eta L^{2}}
\end{aligned}
$$

$\mathrm{K}_{\bullet}=\frac{\eta}{\lambda^{2}} \mathrm{~K}\left(\frac{\lambda \Psi}{\sigma}\right)$

and

$$
\Psi_{\bullet}=\frac{\lambda \Psi}{\sigma}
$$

where $\mathrm{L}$ is the macroscopic length scale of the process. We find 


$$
\begin{aligned}
S & =\frac{2 \lambda^{2}}{\eta} K_{\bullet} \frac{d\left(d \frac{\sigma}{\lambda} \Psi_{\bullet}\right)}{d\left(d \frac{L x_{\bullet}}{\sqrt{d \frac{\eta L^{2} \mathrm{t}_{\bullet}}{\lambda \sigma}}}\right)} \\
& =\sqrt{\frac{\lambda \sigma}{\eta}} 2 K_{\bullet} \frac{d \Psi \bullet}{d \phi} \\
& =\sqrt{\frac{\lambda \sigma}{\eta}} S_{\bullet}
\end{aligned}
$$

that $\mathrm{S}$ scales with $\lambda^{1 / 2}$, and as a little bonus, we see what the effect of changing fluid properties would produce in the value of sorptivity. An additional application of scaling is given in chapter 3 in the discussion of fingered flow where we use the scaled sorptivity.

Well all of this theory is fine, but how does it work in the real world? Quite well actually. Considering the scaling of conductivity, Bear (1972) Figure 5.5.1? illustrates that on a log-log scale that the slope of the permeability particle size plot is two, indicating that $\mathrm{K}_{\mathrm{S}} \propto \lambda^{2} \mathrm{~K}_{\mathrm{S}}$, as predicted by Miller and Miller scaling theory. This is further supported by the data from Warrick (1990) shown in Figure 3.18, which illustrates how the apparent variability in conductivity can almost entirely be explained by Miller and Miller scaling theory. Regarding the scaling of the characteristic draining curves, Figure 3.20 shows examples of scaling used on very large data sets, where again, most of the variability in characteristic draining properties was explained by Miller and Miller scaling concepts.

Figure 3.18 Plot of dependence of Ks on grain size illustrating the 12 dependence predicted by Miller and Miller scaling theory (copied with permission from Bear, 1972).

Figure 3.19 Figure from Warrick (1990) showing the results of scaling conductivity.

Figure 3.20 Figure 4-2 from Warrick (1990) showing the efficacy of scaling pressures.

To summarize, Miller and Miller scaling theory allows quantitative extension of knowledge of hydrodynamic properties of one medium to be extrapolated to any similar medium. Further, by writing the equations of flow in terms of scaled variables, the influence of grain size, viscosity and surface tension can all be explicitly identified. To see how handy this is, consider the following: say you had solved an infiltration problem for one temperature, and you wanted to see how changing the temperature would effect the solution. Simply write the solution in terms of scaled variables, and explicitly show the effects of changes in surface tension and viscosity without having to re-solve the problem.

\section{II Infiltration}

An important class of flow events involves water entry through the soil surface in a process known as infiltration. The rate of this process relative to the rate of water supply to the surface determines how much water will enter the soil, 
and how much, if any, will pond and perhaps create overland flow (runoff). The leading edge of the wetted soil volume, the wetting front, advances into the drier soil region in response to matric potential and gravitational gradients.

Based on field observations, the infiltration rate denoted as $i$ is found to be dependent upon the initial soil water content, the hydraulic conductivity of the surface soil, the elapsed time since the onset of water application, and the presence of impeding layers and other heterogeneities within the soil profile. In most situations the infiltration rate (i) is highest when water first enters the soil, and gradually decreases with time until a constant final rate (if) is attained (Fig.2-16). This behavior is also reflected in the cumulative infiltration (I) showing a rapid increase in the volume of infiltration at short times, which decreases gradually to a nearly linear rate of cumulative infiltration at large times.

In many natural situations the initial rate of water application such as the rainfall rate or sprinkler irrigation rate is less than the soil's initial infiltration capacity (i), but higher than the potential final rate (if) for the soil under the given conditions. This common situation means that a transition must occur in which water application rate will eventually exceed the soil's infiltration rate, and ponding possibly followed by runoff is likely to occur. Given an application rate (P) we may use the infiltration equation presented in the next section to predict the time to onset of ponding.

\section{II.I Empirical Infiltration Equations}

Based on a predictable and well-behaved shape of the infiltration rate vs. time relationship, several functions having shapes similar to the expected behavior have been proposed as predictive equations. Attempts were sometimes later made to attach physical significance to the various parameters of these empirical equations.

The Lewis (Kostiakov) Equation: One of the most widely used empirical expressions was originally proposed by Lewis (1937) but was erroneously attributed to Kostiakov (1932; see discussion by Swartzendruber, 1993):

$$
\text { cumulative : } \mathrm{I}=\mathrm{kt}^{\mathrm{a}} \quad \text { rate }: \mathrm{i}=\frac{\mathrm{dI}}{\mathrm{dt}}=\mathrm{akt}^{\mathrm{a}-1}
$$

where I is the cumulative depth of infiltration or the volume of water per unit soil surface area, $t$ is the elapsed time, $\mathrm{k}$ and a are empirical parameters, and $\mathrm{i}=\mathrm{dI} / \mathrm{dt}$ is the infiltration rate. The main disadvantages of this equation are: (1) its disregard for different initial water contents; and (2) for long times it erroneously predicts a zero infiltration rate. The latter problem can be fixed by adding a parameter, f0, to Eq.(103) representing a final infiltration rate for long times resulting in the following modified equations: $\mathrm{I}=\mathrm{kta}+\mathrm{tf} 0$, and $\mathrm{i}=\operatorname{akt}(\mathrm{a}-1)+\mathrm{f} 0$.

The Horton Equation: Horton (1940) proposed another empirical equation based on an exponential form:

$$
\text { cumulative : } \quad \mathrm{I}=\mathrm{i}_{\mathrm{f}} \mathrm{t}+\frac{\mathrm{i}_{0}-\mathrm{i}_{\mathrm{f}}}{\delta}\left[1-\mathrm{e}^{-\delta \mathrm{t}}\right] \quad \text { rate }: \quad \mathrm{i}=\mathrm{i}_{\mathrm{f}}+\left(\mathrm{i}_{0}-\mathrm{i}_{\mathrm{f}}\right) \mathrm{e}^{-\delta \mathrm{t}}
$$

where $\mathrm{i} 0$ and if are the initial and final infiltration rates, respectively, and $\mathrm{d}$ is an empirical parameter.

\section{II.2 Physically-Based Infiltration Equations}

In this section we will develop some of the basic equations and solutions for infiltration in homogeneous isotropic soils. Although these solutions are developed in the context of somewhat idealized conditions, their utility comes from the fact that we can actually find solutions! 


\section{Philip's Solution}

Philip $(1957,1969)$ presented the first analytical solution to the Richards equation for vertical and horizontal infiltration. For horizontal infiltration Philip showed that the cumulative and instantaneous infiltration rates are given by:

$$
\text { cumulative : } \mathrm{I}=\mathrm{S} \mathrm{t}^{\frac{1}{2}} \quad \text { rate }: \mathrm{i}=\frac{1}{2} \mathrm{~S} \mathrm{t}^{-\frac{1}{2}}
$$

with $\mathrm{S}$ as the sorptivity which is a function of initial and boundary water contents, $\mathrm{S}=\mathrm{S}(\mathrm{q} 0$, qi), and $\mathrm{t}$ is time since water application. When a sharp wetting front exists, the sorptivity may be approximated by:

$$
\mathrm{S}\left(\theta_{0}, \theta_{\mathrm{i}}\right)=\frac{\left(\theta_{0}-\theta_{\mathrm{i}}\right) \mathrm{L}_{\mathrm{f}}}{\mathrm{t}^{1 / 2}}
$$

where $L_{f}$ is the distance from the surface to the wetting front.

For vertical infiltration, Philip's solution to Eq.(92) describes the time dependence of cumulative infiltration as an infinite series in powers of $\mathrm{t}^{1 / 2}$ :

$$
\mathrm{I}=\mathrm{S} \mathrm{t}^{\frac{1}{2}}+\mathrm{A}_{1} \mathrm{t}^{\frac{2}{2}}+\mathrm{A}_{2} \mathrm{t}^{\frac{3}{2}}+\ldots
$$

where the parameters $A_{1}, A_{2}, \ldots$ are dependent upon the soil properties and on initial and boundary water contents. In practice, the series is truncated and only the first two terms are retained, resulting in the following equations, which are valid for short times:

$$
\text { cumulative : } \mathrm{I}=\mathrm{S} \mathrm{t}^{\frac{1}{2}}+\mathrm{A}_{1} \mathrm{t} \quad \text { rate }: \mathrm{i}=\frac{1}{2} \mathrm{~S} \mathrm{t}^{-\frac{1}{2}}+\mathrm{A}_{1}
$$

The first term in each describes the influence of the sorptive forces of (relatively) dry soil, and the second term the contribution of gravity. The influence of the first term diminishes with time and reflects the reduction in the hydraulic gradient as the soil becomes more saturated. For long infiltration times when water is ponded on the soil surface (q0 =qs at the surface), the final infiltration rate approaches $K(q s)$, and thus the ratio $A_{1} / K_{s}$ is bounded by $1 / 3 \leq A_{1} / K_{s} \leq 2 / 3$.

For flux-limited infiltration rate such as low intensity rainfall, $\mathrm{P}$, we may approximate the time to ponding tp from the time at which $\mathrm{i}=\mathrm{P}$ (note that $\mathrm{P}$ is a flux), which is given by:

$$
\mathrm{t}_{\mathrm{p}}=\frac{\mathrm{S}^{2}}{4\left(\mathrm{P}-\mathrm{A}_{1}\right)^{2}}
$$

This is particularly useful for predicting whether and when surface runoff will occur, for example. However, this matching method ignores the fact that the original infiltration curve is modified by the rainfall and a correction known as "time compression" (Kim et al., 1996) is required:

$$
\mathrm{t}_{\mathrm{c}}=\frac{\mathrm{S}^{2}\left(2 \mathrm{P}-\mathrm{A}_{1}\right)}{4 \mathrm{P}\left(\mathrm{P}-\mathrm{A}_{1}\right)^{2}}
$$

and the actual infiltration curve is now shifted by the amount: tshift $=\mathrm{tc}-\mathrm{tp}$. Hence calculations of infiltration rates for times $\mathrm{t}>\mathrm{tc}$ should consider the "tshift" correction.

\section{Example 2-1): Philip's Solution to Vertical Infiltration}

During horizontal infiltration into a sandy soil with $\theta_{\mathrm{i}}-0.1 \mathrm{~m}^{3} / \mathrm{m}^{3}$ and $\theta_{\mathrm{f}}=\theta_{\mathrm{s}}=0.4 \mathrm{~m}^{3} / \mathrm{m}^{3}$, a 
sharp wetting front was observed $100 \mathrm{~mm}$ from the source after $36 \mathrm{~min}$. Given that saturated hydraulic conductivity $\left(\mathrm{K}_{\mathrm{S}}\right)$ for the soil is $2 \mathrm{~mm} / \mathrm{min}$, find: (i) the vertical and horizontal cumulative infiltration (I) into the soil at 10, 60, 1440, $43200 \mathrm{~min}$, and (ii) the ratio $\left(\mathrm{A}_{1} \mathrm{t}\right) /\left(\mathrm{St}^{1 / 2}\right)$ as a function of time.

Solution:

The first step is to estimate the sorptivity (S) for Philip's solution from the sharp wetting front information using Eq.3.XX: $\mathrm{S}=(0.4-0.1) * 100 /\left(36^{1 / 2}\right)=30 / 6=5 \mathrm{~mm} \mathrm{~min}^{-1 / 2}$. Next we estimate $\mathrm{A}_{1}=\mathrm{K}_{\mathrm{s}} / 2=1 \mathrm{~mm} / \mathrm{min}$. Finally, we apply Philip's Eqs. 3.XX. and 3.XX. for horizontal and vertical cumulative infiltration, respectively.

\begin{tabular}{|c|c|c|c|}
\hline \multirow[t]{2}{*}{$\begin{array}{l}\text { Time } \\
\text { [min] }\end{array}$} & \multicolumn{2}{|c|}{ Cumulative Infiltration } & \multirow[t]{2}{*}{$\left(\mathrm{A}_{1} \mathrm{t} / \mathrm{St}^{1 / 2}\right)$} \\
\hline & Horizontal [mm] & Vertical [mm] & \\
\hline 10 & 15.8 & 25.8 & 0.633 \\
\hline 60 & 38.7 & 98.7 & 1.55 \\
\hline 1440 & 189.7 & 1629.7 & 7.59 \\
\hline 43200 & 3286.3 & 46486.3 & 13.4 \\
\hline
\end{tabular}

The ratio $\left(\mathrm{A}_{1} \mathrm{t}\right) /\left(\mathrm{St}^{1 / 2}\right)$ represents the relative contribution to the total flow resulting from gravity as compared with the contribution due to capillary forces. In this example we see that the gravity-induced contribution becomes dominant after only a relatively short time. For finer-textured soils we can expect $\mathrm{K}_{\mathrm{s}}$ to be smaller, while $\mathrm{S}$ is likely to increase (for similarly dry conditions) resulting in an extended period of capillarydominant infiltration.

\section{Example 2-2): Philip's Solution to Vertical Infiltration}

Problem Statement:

An infiltration experiment into a horizontal soil column resulted in a sharp wetting front at $50 \mathrm{~mm}$ from the inlet after $25 \mathrm{~min}$. Given: $\mathrm{K}_{\mathrm{s}}=0.01 \mathrm{~mm} / \mathrm{min}, \theta_{\mathrm{i}}=0.1 \mathrm{~m}^{3} / \mathrm{m}^{3}, \theta_{\mathrm{s}}=0.45 \mathrm{~m}^{3} / \mathrm{m}^{3}$, and the diameter of the column was $60 \mathrm{~mm}$. Use Philip's solution to find:

1. The soil's sorptivity.

2. The wetting front advance after $2 \mathrm{hr}$ if the column was rotated to a vertical position $1 \mathrm{hr}$ after the beginning of the experiment.

3. The volume of water that infiltrated during the horizontal and the vertical phases during the $2 \mathrm{hr}$ 
experiment.

Solution:

1. First we use Philip's solution to determine the soil's sorptivity S:

$$
\mathrm{S}\left(\theta_{0}, \theta_{\mathrm{i}}\right)=\frac{\left(\theta_{0}-\theta_{\mathrm{i}}\right) \mathrm{L}_{\mathrm{f}}}{\mathrm{t}^{1 / 2}} \quad \mathrm{~S}=\frac{(0.45-0.1) \cdot 50}{25^{1 / 2}}=3.5\left[\mathrm{~mm} / \mathrm{min}^{1 / 2}\right]
$$

where $\theta_{\mathrm{i}}$ is the initial water content, $\theta_{0}$ is the boundary water content at the wetting front (equals $\theta_{\mathrm{S}}$ ), $\mathrm{Lf}_{\mathrm{f}}$ is the distance from the boundary to the wetting front after advance time $t$.

2. The wetting front depth $\mathrm{L}_{\mathrm{f}}$ after 2 hours ( $1 \mathrm{hr}$ horizontal, $1 \mathrm{hr}$ vertical) is obtained by combining Philip's solution for horizontal and vertical cumulative infiltration over the appropriate time periods and dividing by $\Delta \theta$ :

$$
\mathrm{L}_{\mathrm{f}}=\frac{\mathrm{I}}{\Delta \theta} \quad \text { with: } \mathrm{I}=\mathrm{St}^{\frac{1}{2}}+\mathrm{A}_{1} \mathrm{t}
$$

where $A_{1}$ is a parameter dependent on initial and boundary water contents, and soil properties. The sorptivity component (first term) is acting over the entire period of two hours, while the gravity component (second term) is acting only for the vertical case (second hour of the experiment).

$$
\mathrm{L}_{\mathrm{f}}=\frac{3.5 \cdot 120^{1 / 2}+\frac{0.01}{2} \cdot 60}{(0.45-0.1)}=110.4[\mathrm{~mm}]
$$

3. The volume of infiltrating water is simply calculated by multiplying the column's cross sectional area with cumulative infiltration for the horizontal $\mathrm{I}_{\mathrm{H}}$ and vertical $\mathrm{IV}_{\mathrm{V}}$ cases:

(3.155) $\mathrm{I}_{\mathrm{H}}=\mathrm{St}^{\overline{2}} \quad \mathrm{I}_{\mathrm{V}}=\mathrm{St}^{\overline{2}}+\mathrm{A}_{1} \mathrm{t}$

Note that $\mathrm{A}_{1}$ may be approximated by $\mathrm{K}_{\mathrm{s}} / 2$

$V_{H}=30^{2} \cdot \pi \cdot 3.5 \cdot 60^{1 / 2}=76654\left[\mathrm{~mm}^{3}\right]$

$V_{V}=30^{2} \cdot \pi \cdot 3.5 \cdot\left(120^{1 / 2}-60^{1 / 2}\right)+\frac{0.01}{2} \cdot(120-60)=31752\left[\mathrm{~mm}^{3}\right]$

Total Volume

$$
V_{T}=76654+31752=108406\left[\mathrm{~mm}^{3}\right]
$$




\section{The Brutsaert Infiltration Model}

Brutsaert (1977) resulting from an approximate solution to the Richards equation for vertical infiltration. The result for cumulative infiltration was estimated as (Brutsaert, 1977; eq. 73):

$$
Q=n L=t K_{s}+\frac{S^{2}}{\beta K_{s}}\left\{1-\left[1+\beta\left(\frac{t^{1 / 2} K_{s}}{S}\right)\right]^{-1}\right\}
$$

while the rate of infiltration was estimated as:

$$
q=K_{s}+\frac{S}{2 t^{1 / 2}}\left[1+\beta\left(\frac{K_{s} t^{1 / 2}}{S}\right)\right]^{-2}
$$

where $\mathrm{b}$ is about $(2 / 3)$ and $\mathrm{S}$ is the soil sorptivity from initial to final saturation.

\section{The Green and Ampt model}

The mark of a successful engineering model is that the approximations which are employed give rise to a method which is robust under a broad range of situations, while being simple to understand and apply. The one-dimensional infiltration model developed by Green and Ampt in 1911 is unusually successful from this perspective, wherein they simplified the gradual transition for dry to wet soil as a single abrupt wetting process (Figure 3.21. It has proven to be a very useful as a conceptual model for the physical processes of infiltration, reproducing the key temporal properties of horizontal and vertical infiltration. 


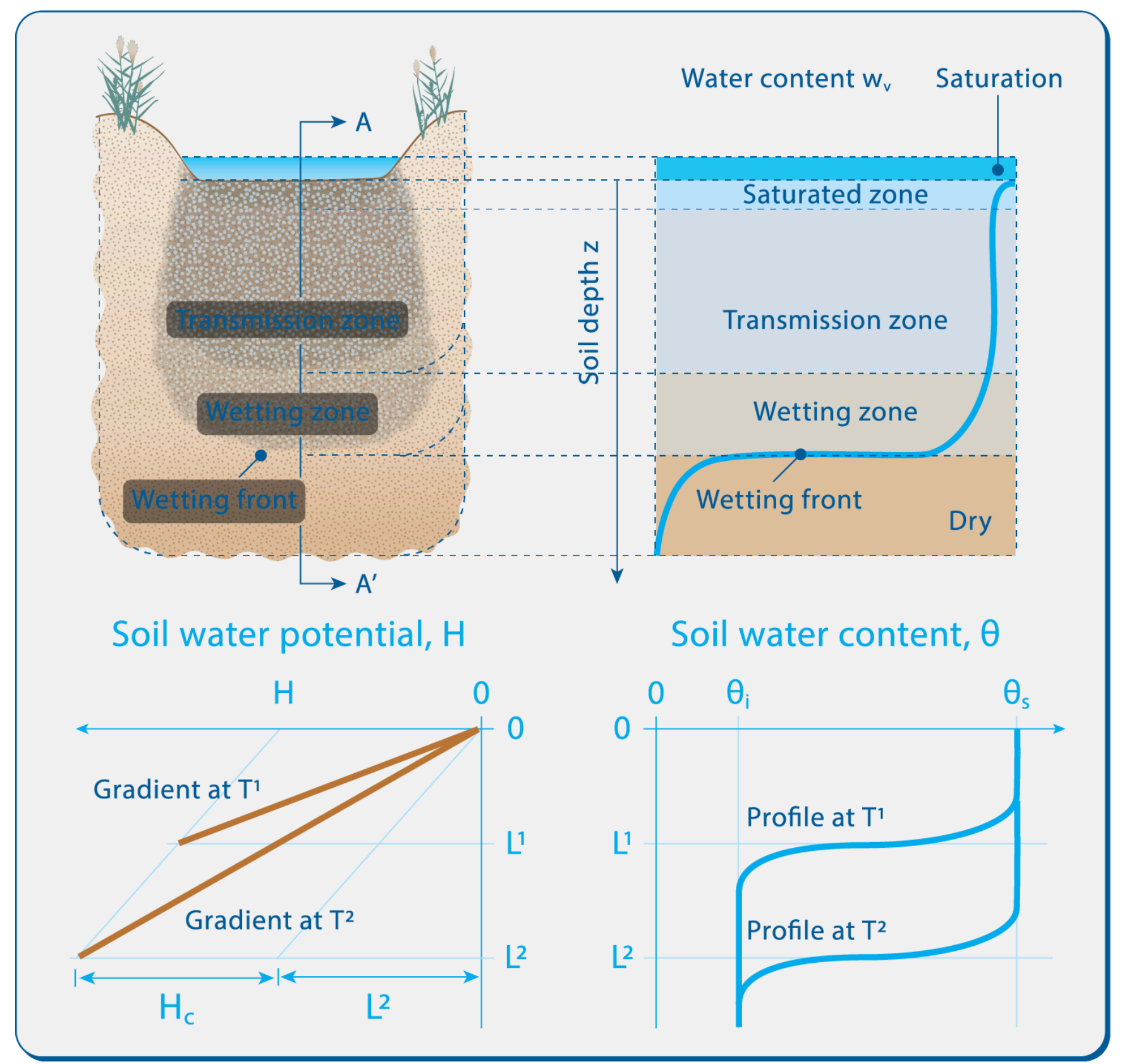

Figure 3.21 Illustration of simplified conceptualization of infiltration, with a wetting front, wetting zone, and saturated "transmission zone." (upper two panels, modified from Hillel 1980). Green and Ampt's approximation for the infiltration of water is shown in the lower two panels where the wetting zone and transmission zone are both taken to be saturated, separated from the dry soil by an abrupt wetting front, which exerts a capillary pull which combined with the gravitational potential provides the energetic drivers of infiltration. (bottom two panels modified from Smith and Williams, 1980).

The Green and Ampt model was first conceived of in the framework of a capillary tube bundle concept, and this representation is useful in relating soil texture with infiltration, so we will employ this representation here for its pedagogical utility. The soil is modeled as a bundle of capillary tubes oriented in the direction of infiltration. It is further assumed that the tubes fill in parallel to the same depth (i.e., the wetting front is at a well-defined position which is the same in all tubes). The model may be employed in calculating the infiltration into soils which proceed at any angle relative to vertical by simply adjusting the magnitude of gravity. We will first calculate the horizontal rate of infiltration into such a dry soil, then consider vertical infiltration. In both cases we will solve one-dimensional problems, but we 
hasten to point out that this conceptual approach can be applied to multi-dimensional problems. The basic notion is that infiltration is driven by two forces: first, there is the suction produced by a sharp wetting front, and second there is the force of gravity acting on the water.

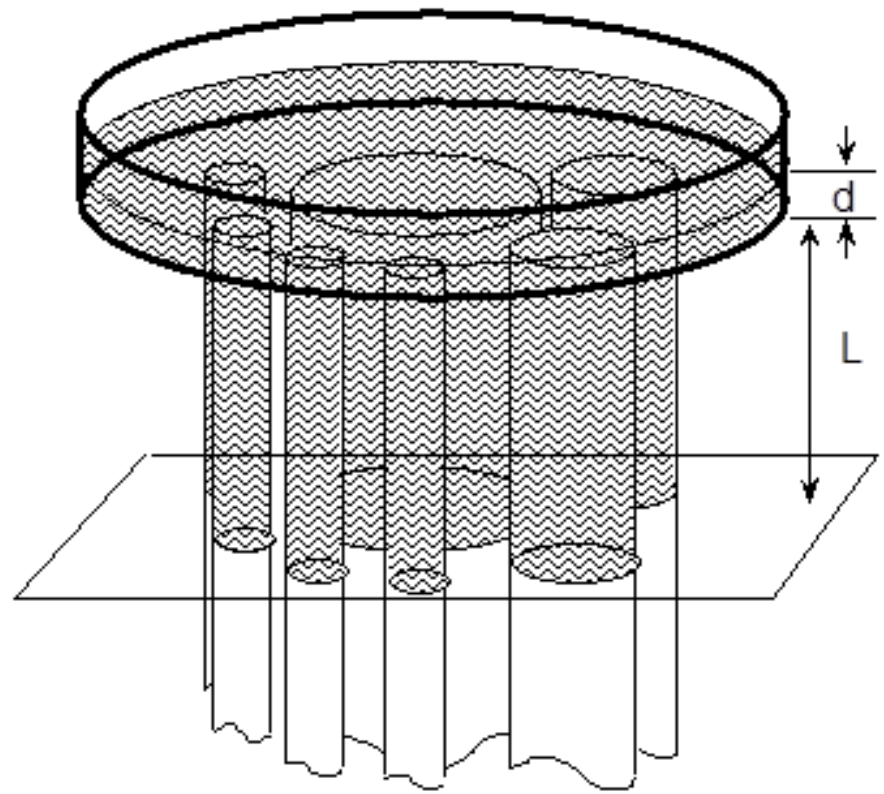

Figure 3.22 Illustration of conceptual model for vertical Green and Ampt infiltration. Water is infiltrating through a set of parallel capillary tubes of various diameters with a ponding depth $d$ and has advanced with a level wetting front to a depth $\mathrm{L}$.

We will first discuss the form of the wetting front potential term. Keep in mind that here we are just trying to build some physical intuition, so we do not suggest that real soils should be considered in the idealized fashion we employ below, but it is useful to show the basic relationship between pore size and infiltration. So imagine a bundle of capillary tubes of $\mathrm{N}$ sizes, where each of the sizes makes up a fraction ai of the total cross-sectional pore area (i.e., $\sum_{i=1}^{\mathrm{N}} \mathrm{a}_{\mathrm{i}}=\mathrm{n}$, where $\mathrm{n}$ is the soil porosity). To calculate the infiltration rate, we will simply calculate the capillary force that draws the water into the soil, and the dissipation of energy resulting from the viscous flow through the capillary pores. The driving force for infiltration is the surface tension of the water/pore-wall interface. For one unit of cross-sectional area, what will the total length, la, of water/solid contact be? Well, we can just add it up. Summing the wall length per unit area times the fraction of area for that pore group we find 


$$
\begin{aligned}
& 1_{\mathrm{a}}=\sum \mathrm{i}=1^{\mathrm{N}} \frac{\text { area of tube } \mathrm{i} \text { wall length of } \mathrm{i}}{\text { unit area }} \frac{\text { area of tube } \mathrm{i}}{1_{\mathrm{a}}}=\sum \mathrm{i}=1^{\mathrm{N}} \frac{\text { area of tube } \mathrm{i}}{\text { wnit area }} \frac{\text { area of tube } \mathrm{i}}{\text { areath of } \mathrm{i}} \\
& 1_{\mathrm{a}}=\sum \mathrm{i}=1^{\mathrm{N}} \mathrm{a}_{\mathrm{i}} \frac{2}{\mathrm{r}_{\mathrm{i}}}
\end{aligned}
$$

To calculate the force which will be exerted on infiltrating water, we can assume a contact angle of $\gamma$, and calculate the pressure per unit area caused by the surface tension, $\mathrm{P}_{\mathrm{f}}$, at the water/solid interface as

$$
\mathrm{P}_{\mathrm{f}}=\sigma \mathrm{l} \cos \gamma
$$

$$
\mathrm{P}_{\mathrm{f}} \quad=\sigma \cos \gamma \sum \mathrm{i}=1^{\mathrm{N}} \mathrm{a}_{\mathrm{i}} \frac{2}{\mathrm{r}_{\mathrm{i}}}
$$

Now what would the resistance to flow be in such a system? Well, let's just use Hagen-Poiseuille's capillary flow law. For the $\mathrm{i}^{\text {th }}$ tube, we have the flow per unit area, $\mathrm{q}_{\mathrm{i}}$, of

$$
\mathrm{q}_{\mathrm{i}}=\frac{\mathrm{r}_{\mathrm{i}}^{2} \mathrm{P}}{8 \mathrm{~L} \mu}
$$

Where $\Delta \mathrm{P}$ is the pressure drop across a capillary tube of length $\mathrm{L}$ with a fluid flowing with dynamic viscosity $\mu$. Notice that we assume the pressure drop across each tube is equal in that there is assumed to be a well defined pressure at the wetting front which is equal everywhere. For our capillary bundle the total flux would be

$$
\begin{aligned}
& \mathrm{q}=\sum \mathrm{i}=1^{\mathrm{N}} \mathrm{a}_{\mathrm{i}} \frac{\mathrm{r}_{\mathrm{i}}{ }^{2} \mathrm{P}}{8 \mathrm{~L} \mu} \\
& \mathrm{q}=\frac{\mathrm{P}}{8 \mathrm{~L} \mu} \sum \mathrm{i}=1^{\mathrm{N}} \mathrm{a}_{\mathrm{i}} \mathrm{r}_{\mathrm{i}}{ }^{2}
\end{aligned}
$$

If the flow is horizontal, then the driving force is simply the surface tension, so the pressure drop in the tubes is $\mathrm{P}_{\mathrm{f}}$ and the flux into the system is given by

$$
\mathrm{q}=\frac{\mathrm{P}_{\mathrm{f}}}{8 \mathrm{~L} \mu} \sum \mathrm{i}=1^{\mathrm{N}} \mathrm{a}_{\mathrm{i}} \mathrm{r}_{\mathrm{i}}^{2}
$$

We may express this in the customary units of pressure head by defining $\rho g h_{f}=P_{f}$. For convenience, let's define the system constant 


$$
\mathrm{K}=\sum \mathrm{i}=1^{\mathrm{N}} \frac{\rho \operatorname{ga}_{\mathrm{i}} \mathrm{r}_{\mathrm{i}}^{2}}{8 \mu}
$$

where we recognize K as the saturated hydraulic conductivity of the soil. Now (‥165) becomes

$$
q=\frac{h_{f} K}{L}
$$

The porosity of the soil is $\mathrm{n}$, and the soil is going from dry to saturation, we can relate the flux to the position of the sharp wetting front

$$
\mathrm{q}=\frac{\mathrm{d}}{\mathrm{dt}} \mathrm{nL}
$$

Combining (3.166) and (3.167) we obtain a differential equation for the position of the wetting front in time

$$
\mathrm{n} \frac{\mathrm{dL}}{\mathrm{dt}}=\frac{K \mathrm{~h}_{\mathrm{f}}}{\mathrm{L}}
$$

which can be integrated to find that

$$
\frac{\mathrm{nL}^{2}}{2}=K \mathrm{~h}_{\mathrm{f}} \mathrm{t}
$$

or, solving for the length of infiltration

$$
\mathrm{L}=\sqrt{\frac{2 K h_{\mathrm{f}} \mathrm{t}}{\mathrm{n}}}
$$

Here we have the first major result from the Green and Ampt model. Apparently the wetting front advances with the square root of time, depends on the square root of the saturated conductivity of the soil, and goes with the square root of the soil "capillary pull." This is the equation you would use to predict the horizontal movement of a wetting front, for instance from the sides of an irrigation canal or through a dike. Notice that (‥170) indicates that the total infiltration is also proportional to the square root of time. To see how the flux varies in time we can use (3.170) and (3.167) to obtain

$$
\int_{0}^{\mathrm{t}} \frac{\mathrm{qdt}}{\mathrm{n}}=\sqrt{\frac{2 K h_{\mathrm{f}} \mathrm{t}}{\mathrm{n}}}
$$

Carrying out the calculation of the integral, we find

$$
\begin{aligned}
\mathrm{q} & =\sqrt{\frac{K h_{\mathrm{f}} \mathrm{n}}{2 \mathrm{t}}} \\
& =1 / 2 \mathrm{~S}_{\mathrm{GA}} \mathrm{t}^{-1 / 2}
\end{aligned}
$$

where we have introduced the term $\mathrm{S}_{\mathrm{GA}}$ (referred to as the sorptivity) which is a constant dependent on the media, and equal to $(2 \mathrm{nKh})^{1 / 2}$. The key observation from (3.172) is that the horizontal flux into a soil decreases proportionally to one over the square root of time. A simple, yet powerful result.

We can get some idea of how the soil texture will affect infiltration by looking at this in terms of the tube radii. From our earlier definitions we find 


$$
\mathrm{S}_{\mathrm{GA}}{ }^{2}=\frac{\mathrm{n} \sigma \cos \gamma}{2 \mu}\left(\sum_{\mathrm{i}=1}^{\mathrm{N}} \rho g \mathrm{a}_{\mathrm{i}} \mathrm{r}_{\mathrm{i}}{ }^{2}\right)\left(\sum_{\mathrm{i}=1}^{\mathrm{N}} \frac{\mathrm{a}_{\mathrm{i}}}{\rho g \mathrm{r}_{\mathrm{i}}}\right)
$$

which is a constant for the media and fluid. To help "see" how $\mathrm{S}_{\mathrm{GA}}$ will relate to soil characteristics, we can make the vastly simplifying assumption that the soil has only one pore radius. In this case, $\mathrm{S}_{\mathrm{GA}}$ becomes

$$
\begin{aligned}
\mathrm{S}_{\mathrm{GA}}{ }^{2} & =\frac{\sigma \mathrm{r} \cos \gamma}{2 \mu} \\
& =\mathrm{Cr}
\end{aligned}
$$

where $\mathrm{C}$ is a constant which depends only on the fluid. (3.174) then tells us that for our simplified soil that the infiltrating flux will be given by

$$
q=\sqrt{\frac{\mathrm{Cr}}{\mathrm{t}}}
$$

Thus, for finer soils the infiltrating flux will go down with the square root of $r$, the characteristic radius of the soil pores. This is in stark contrast to the conductivity which decreases with $r^{2}$. Why the difference? Because the huge surface area of the particles in the finer soil provides a large capillary "pull" into the soil, which is almost enough to overcome the lower hydraulic conductivity. This result explains much of the success of early strategies of irrigated agriculture. If you had supposed that infiltration was controlled primarily by hydraulic conductivity, then where soils had grains that were twice as large as the average for the field, the infiltration would be four times higher, while locations where the pores were half as large, the infiltration would be four times smaller. So across this field we would have a factor of 16 difference in infiltration. This would be hugely wasteful of water, requiring massive (16 times) overirrigation of the coarser areas just to get the required water into the finer sections of the field. Beyond wasting water, this would lead to washing out of the critical nutrient nitrate in the areas of high infiltration, which would further suppress productivity. Fortunately, because the infiltration goes with $\mathrm{r}^{1 / 2}$, the difference in water applied across this field would be just a factor of two, so the required over-irrigation is reduced by a factor of eight! This allowed for good (but certainly not optimal) productivity with simple flood irrigation.

Getting back to the Green and Ampt model, how can these results be extended to vertical infiltration? In fact, solving for vertical infiltration is essentially as easy as solving for horizontal infiltration. This is one of the strengths of the Green and Ampt approach. We shall start by considering the case of ponded infiltration. Darcy's law for the system is simplified by the fact that the wetting front is assumed to be sharp, with saturation behind the front. We obtain

$$
\mathrm{q}=-\mathrm{K}_{\text {sat }}\left[\frac{\text { head loss }}{\text { length of travel }}\right]
$$

The head loss across the system is the sum of the potential at the wetting front, $\mathrm{h}_{\mathrm{f}}$, the depth of ponding, $\mathrm{d}$, plus the depth of infiltration, $\mathrm{L}$, while the length of travel is L. Using (3.167), this gives us

$$
\frac{\mathrm{d}}{\mathrm{dt}} \mathrm{nL}=\mathrm{K}_{\mathrm{sat}}\left[\frac{\mathrm{h}_{\mathrm{f}}+\mathrm{d}+\mathrm{L}}{\mathrm{L}}\right]
$$

\footnotetext{
Separating variables,
} 


$$
\begin{aligned}
\mathrm{dt} & =\frac{\mathrm{n}}{\mathrm{K}_{\mathrm{sat}}}\left[\frac{\mathrm{LdL}}{\mathrm{h}_{\mathrm{f}}+\mathrm{d}+\mathrm{L}}\right] \\
& =\frac{\mathrm{n}}{\mathrm{K}_{\mathrm{sat}}}\left[1-\frac{\mathrm{h}_{\mathrm{f}}+\mathrm{d}}{\mathrm{h}_{\mathrm{f}}+\mathrm{d}+\mathrm{L}}\right] \mathrm{dL}
\end{aligned}
$$

which may be integrated to obtain

$$
\mathrm{t}=\frac{\mathrm{n}}{\mathrm{K}_{\mathrm{sat}}}\left\{\mathrm{L}-\left(\mathrm{h}_{\mathrm{f}}+\mathrm{d}\right) \ln \left[\frac{\mathrm{h}_{\mathrm{f}}+\mathrm{d}+\mathrm{L}}{\mathrm{h}_{\mathrm{f}}+\mathrm{d}}\right]\right\}
$$

So we find that the square root of time behavior found for horizontal infiltration is replaced with a more complex logarithmic relationship. With (3.179) you may compute the time required to obtain any given amount of infiltration for a given soil and depth of ponding (the total infiltration is just $\mathrm{nL}$ ).

To see how the result shown in (3.179) relates to the horizontal case, let's consider the case when the wetting front has not proceeded very far. In this case, L is very small, so we can simplify (‥179) by keeping just the first three terms in the Taylor expansion of the logarithm. We recall the general form of the Taylor expansion

$$
f(x+x)=f(x)+x f^{\prime}(x)+\frac{x^{2}}{2 !} f^{\prime \prime}(x)+\frac{x^{3}}{3 !} f^{\prime \prime \prime}(x)+\cdots
$$

To put (․179) in the form which can be calculated using a Taylor series we will let $\Delta \mathrm{x}=\mathrm{L} /\left(\mathrm{h}_{\mathrm{f}}+\mathrm{d}\right)$, we can expand the logarithm, keeping the first two terms of the expansion

$$
\begin{aligned}
\operatorname{Ln}\left[\frac{\mathrm{h}_{\mathrm{f}}+\mathrm{d}}{\mathrm{h}_{\mathrm{f}}+\mathrm{d}}+\frac{\mathrm{L}}{\mathrm{P}_{\mathrm{f}}+\mathrm{d}}\right] & =\operatorname{Ln}\left[\frac{\mathrm{h}_{\mathrm{f}}+\mathrm{d}}{\mathrm{h}_{\mathrm{f}}+\mathrm{d}}\right]+\frac{\mathrm{L}}{\mathrm{h}_{\mathrm{f}}+\mathrm{d}}-\frac{\left[\mathrm{d} \frac{\mathrm{L}}{\mathrm{h}_{\mathrm{f}}+\mathrm{d}}\right]^{2}}{2 !}+\cdots \\
& \approx \frac{\mathrm{L}}{\mathrm{h}_{\mathrm{f}}+\mathrm{d}}-\frac{1}{2}\left[\frac{\mathrm{L}}{\mathrm{h}_{\mathrm{f}}+\mathrm{d}}\right]^{2}
\end{aligned}
$$

Hence for the very early infiltration (that is, when $\mathrm{L}$ is much less than $\mathrm{d}$ or $\left.\mathrm{h}_{\mathrm{f}}\right)(\underline{3.179})$ becomes

$$
\mathrm{t}=\frac{\mathrm{nL}^{2}}{2 \mathrm{~K}_{\mathrm{sat}}\left(\mathrm{h}_{\mathrm{f}}+\mathrm{d}\right)}
$$

or, solving for $\mathrm{L}$ we find

$$
L=\sqrt{\frac{2 K_{s a t}\left(h_{f}+d\right) t}{n}}
$$

which is precisely the result obtained for horizontal infiltration in equation ( $\underline{3.170})$, with the added head loss due to the ponding. So we see that for short times, horizontal infiltration follows the same pattern as vertical infiltration. This indicates that the capillary forces pulling the liquid into the soil (which are the same for vertical and horizontal cases) are 
much greater than the gravitational forces that develop as the column of water grows in height. It is also worth noting that the solution for forced horizontal infiltration, injected with head d, is given by (3.183).

Using the same procedure, we can solve for infiltration under a falling head. In this case, we replace the depth of ponding, d, with this depth minus the depth of water which has infiltrated, nL. Using ( $\underline{3.176})$ as before, we obtain

$$
\mathrm{t}=\frac{\mathrm{n}}{\mathrm{K}_{\mathrm{sat}}(1-\mathrm{n})^{2}}\left\{\mathrm{~L}(1-\mathrm{n})-\left(\mathrm{h}_{\mathrm{f}}+\mathrm{d}\right) \ln \left[\frac{\mathrm{h}_{\mathrm{f}}+\mathrm{d}+\mathrm{L}(1-\mathrm{n})}{\mathrm{h}_{\mathrm{f}}+\mathrm{d}}\right]\right\} \text { for } \mathrm{nL}<\mathrm{d}
$$

This is a very handy result for a number of problems, and was first published by Polubarinova-Kochina (1962). In the agricultural arena, this allows estimation of the time required for a desired depth of irrigation water to infiltrate. In the area of hazardous waste, this would allow estimation of the time for a contaminated spill to soak in. The comparison between the three Green and Ampt solutions (horizontal, fixed head vertical, and falling head vertical) are shown in the figure below.

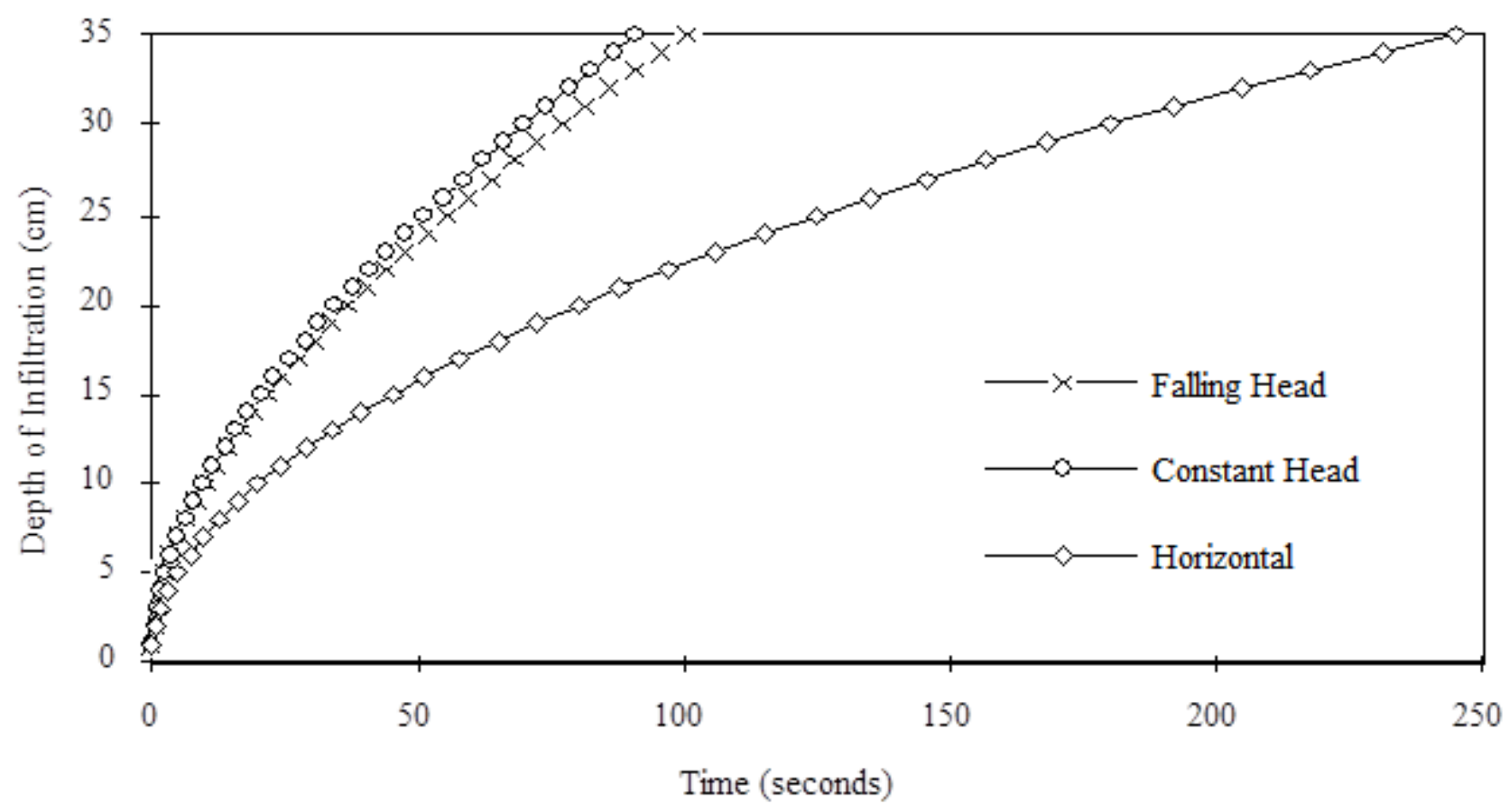

Figure 3.23 Green and Ampt model predictions for horizontal, fixed head vertical, and falling head vertical infiltration in a soil with $30 \%$ pore space, $K_{\mathrm{s}}=0.03 \mathrm{~cm} / \mathrm{sec}, h_{\mathrm{f}}=25 \mathrm{~cm}$ and $d=20 \mathrm{~cm}$.

The results obtained from the Green and Ampt model are easily converted to apply to different fluids. The results will simply be scaled by viscosity, contact angle, and surface tension in the calculation of $\mathrm{h}_{\mathrm{f}}$. This then may be applied to nonaqueous phase fluids (oils). Density effects may be included in a similar fashion.

While the exact formulation allows direct computation of the time it takes to infiltrate to a certain depth, hydrologists typically would rather obtain the depth of wetting in terms of $t$, which would also provide solutions for flux, $q(q=$ $n \mathrm{dL} / \mathrm{dt})$ and cumulative infiltration, $\mathrm{Q}(\mathrm{Q}=n \mathrm{~L})$. This has been addressed by a number of authors (Parlange et al., 2002; Barry et al., 2005; Assouline, 2013; Ali et al., 2016), and recently Assouline and Selker (2017 - in press) introduced an approach that was both accurate and in keeping with the simplicity of the Green and Ampt approach.

The Assouline and Selker's (2017 - in press) strategy was to recognize that the first term on the right of (3.XXX) is the "long-time" component (dominant when $t>>$ ) and the logarithmic term is only significant in the early phases of 
infiltration when the wetting front has progressed to a depth of the order of the ponding depth plus the wetting front potential (in hydraulic head). To keep the math simple we will take $d=0$, but the approach works equally well for nonzero d. We seek a "reasonable" estimate for $L$ in the logarithmic term of (2.XXX), Denoted $\mathrm{L}$, the estimate represents the sum of gravitational flow and the solution for capillary imbibition based on Philip's simplified solution for infiltration presented above

$$
E=A \frac{t K_{s}}{n}+\sqrt{\frac{2 t K_{s} h_{f}}{n}}
$$

where $\mathrm{A}$ is an empirical factor modulating the gravitational component which is typically taken to be between $1 / 3$ and 1, depending on the soil properties and the duration of the infiltration process, but here can be set to $2 / 3$ without significant loss of precision (Philip, 1957; Youngs, 1968; Talsma and Parlange, 1972; Turner, 2006). We then may solve explicitly for $L$ :

$$
L=\frac{t K_{s}}{n}+h_{f} \ln \left[1+A \frac{t K_{s}}{n h_{f}}+\sqrt{\frac{2 t K_{s}}{n h_{f}}}\right]
$$

We can differentiate this to obtain infiltration flux, $q$ :

$$
q=n \frac{d L}{d t}=K_{s}+\frac{A K_{s}+\sqrt{\frac{n K_{s} h_{f}}{2 t}}}{1+A \frac{K_{s} t}{n h_{f}}+\sqrt{\frac{2 K_{s} t}{n h_{f}}}}
$$

With such expressions, the usual quantities such as time to ponding can be obtained with little difficulty (Assouline et al., 2007). Assouline and Selker (2017 - in press) found that the solution was accurate to $1 \%$ of the exact Green and Ampt solution for a variety of soils, so little accuracy is lost using this result.

We see that the Green and Ampt approach allows for easily applied equations, but is the crude estimate obtained using this sharp-interface assumption accurate enough to justify its use? The short answer is that yes, in many cases the error introduced by using this very drastic assumption introduces little error compared to our lack of accurate information about the average, and worse yet spatially variable properties of soils.

\section{Determination of Green and Ampt Parameters}

Of course the utility of the Green and Ampt model is limited by our ability to obtain the parameters required. Very useful tabulated values based on soil texture may be found in Rawls and Brakensiek (1983), and are reproduced in the widely available Handbook of Hydrology (Maidment, 1992, page 5.34-5.38). If possible, it is preferable to measure the values directly. Bouwer (1966) provided a description of a simple field measurement system which may be used to get the two parameters required to apply the Green and Ampt approach. The setup of the equipment is shown below in Figure 3.24 It consists of an elevated reservoir, which upon opening a valve, may be drained into a ring which is pounded about 10 $\mathrm{cm}$ into the ground. After letting all of the air out of the system, the wetting front is allowed to proceed to about the bottom of the ring. At this time a measurement is made of the rate of reservoir depletion. Immediately following this, the supply valve is closed, and a vacuum gauge is read to determine the maximum suction generated by the wetting front. (Note that the measurement must be made in a location which is free of cracks and worm holes to avoid breaking the tension provided by the wetting front). Immediately following this measurement, the device is pulled out of the soil, and you measure the depth of the wetting front, L. A sample of soil is taken back to the laboratory for the determination of the porosity, $\mathrm{n}$.

The measured value of maximum tension (being careful to subtract the static pressure generated by the column of 
water of height $\mathrm{G}$ ) is assumed to be the air entry pressure of the soil, which is taken to be twice the water entry pressure. The water entry pressure is taken as the Green and Ampt wetting front potential hf. Since the change in head over the period of the measurement of the intake rate is small, the constant head solution for infiltration (equation (3.179)) is used to solve for $\mathrm{K}_{\mathrm{s}}$. With this, you have the two parameters required to carry out calculations using the Green and Ampt model.

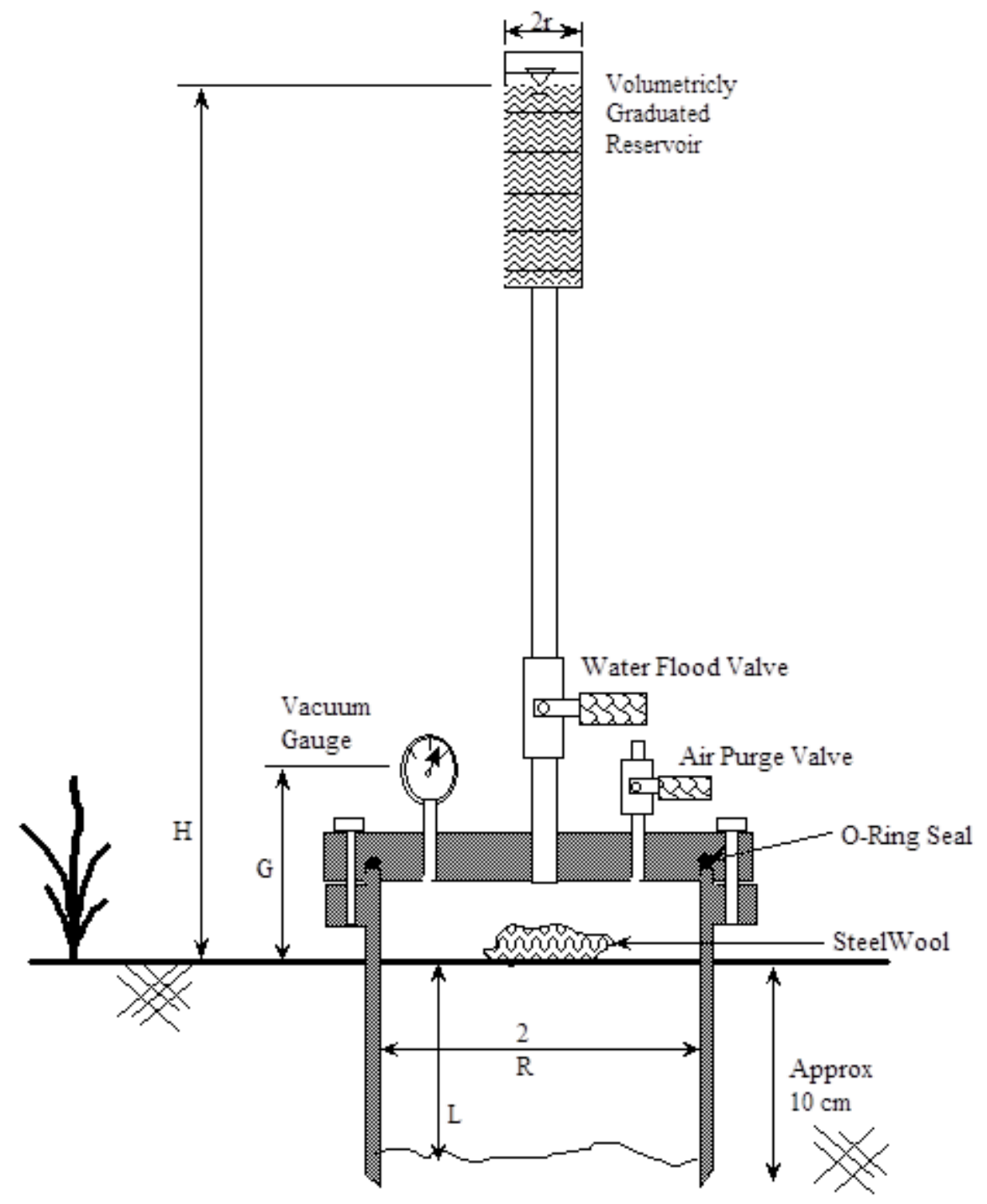

Figure 3.24 Bouwer falling head ring infiltrometer.

Solutions using Richards equation.

The Bruce and Klute equation for horizontal infiltration

The results obtained using the Green and Ampt model may be compared to the solution to Richards Equation for 
horizontal infiltration. Richards Equation is easy to derive, so let's do it here for one-dimensional horizontal flow. For horizontal flow Darcy's law says that

$$
q=-K \frac{\partial h}{\partial z}
$$

The conservation of mass further indicates that the time rate of change in stored water is equal to the negative of the change in flux with distance (i.e., an increase or decrease in flux with distance results in respective depletion or accumulation of stored water), e.g.,

$$
-\frac{\partial q}{\partial t}=\frac{\partial q}{\partial z}
$$

Taking the first derivative of [3.29] with respect to position, and substituting [3.30] in for the left hand side, we obtain

$$
\frac{\partial \mathrm{q}}{\partial \mathrm{t}}=\frac{\partial}{\partial \mathrm{z}}\left[\mathrm{K}\left(\frac{\partial \mathrm{h}}{\partial \mathrm{z}}\right)\right]
$$

If we use the definition of soil-water diffusivity, $\mathrm{D} \partial \theta=\mathrm{K} \partial \Psi$, [3.31] may be re-written as

$$
\frac{\partial q}{\partial t}=\frac{\partial}{\partial z}\left[D\left(\frac{\partial q}{\partial z}\right)\right]
$$

which is the governing equation for the system. For well-defined boundary and initial conditions, if we find a solution to [3.32] which satisfies these conditions, then we have the unique solution. Taking a look at the Green and Ampt solution, and at equation [3.16], we see a one-to-one relationship between the square root of time and position. With that motivation, we will introduce a "similarity variable" which codifies this observation. Referred to as either the Boltzman or the Buckingham transform, we define a new variable $\phi$ as

$$
\phi=\frac{\mathrm{x}}{\sqrt{\mathrm{t}}}
$$

We want to write [3.32] in terms of $\phi$. To carry out this substitution, we calculate

$$
\frac{\partial \phi}{\partial \mathrm{x}}=\frac{1}{\sqrt{\mathrm{t}}}
$$

or

$$
\partial \mathrm{x}=\partial \phi \sqrt{\mathrm{t}}
$$

and to obtain the substitution for the time derivatives

$$
\begin{aligned}
\frac{\partial \phi}{\partial \mathrm{t}} & =\frac{-\mathrm{x}}{2 \mathrm{t}^{3 / 2}} \\
& =\frac{-\phi}{2 \mathrm{t}}
\end{aligned}
$$

or

$$
\partial \mathrm{t}=\frac{-2 \mathrm{t}}{\phi} \partial \phi
$$

Substituting the expressions for $\partial \mathrm{x}$ and $\partial \mathrm{t}$ from [3.34] and [3.36] into [3.32], we see that the left side is given by 


$$
\frac{\partial \phi}{\partial \mathrm{t}}=\frac{\partial \theta}{\partial \phi} \frac{\partial \phi}{\partial \mathrm{t}}=\frac{\mathrm{d} \theta}{\partial \phi} \frac{-\phi}{2 \mathrm{t}}
$$

Notice that the partial derivatives are now simple derivatives since there is only one variable in the similarity version of the equation. In the same way, the right side can be put in terms of the similarity variable

$$
\begin{aligned}
\frac{\partial}{\partial \mathrm{x}}\left[\mathrm{D}\left(\frac{\partial \theta}{\partial \mathrm{x}}\right)\right] & =\frac{\partial \phi}{\partial \mathrm{x}} \frac{\partial}{\partial \phi}\left[\mathrm{D}\left(\frac{\partial \phi}{\partial \mathrm{x}} \frac{\partial \mathrm{q}}{\partial \phi}\right)\right] \\
& =\frac{1}{\sqrt{\mathrm{t}}} \frac{\mathrm{d}}{\mathrm{d} \phi}\left[\mathrm{D}\left(\frac{1}{\sqrt{\mathrm{t}}} \frac{\mathrm{d} \theta}{\mathrm{d} \phi}\right)\right] \\
& =\frac{1}{\mathrm{t}} \frac{\mathrm{d}}{\mathrm{d} \phi}\left[\mathrm{D} \frac{\mathrm{d} \theta}{\mathrm{d} \phi}\right]
\end{aligned}
$$

Now using [3.38] and [3.41] in [3.32], we find

$$
\frac{-\phi}{2 \mathrm{t}} \frac{\mathrm{d} \theta}{\mathrm{d} \phi}=\frac{1}{\mathrm{t}} \frac{\mathrm{d}}{\mathrm{d} \phi}\left[\mathrm{D} \frac{\mathrm{d} \theta}{\mathrm{d} \phi}\right]
$$

or

$$
\frac{-\phi}{2} \frac{\mathrm{d} \theta}{\mathrm{d} \phi}=\frac{\mathrm{d}}{\mathrm{d} \phi}\left[\mathrm{D} \frac{\mathrm{d} \theta}{\mathrm{d} \phi}\right]
$$

Multiplying each side by $\mathrm{d} \phi$ and integrating from $\theta=\theta_{\mathrm{i}}$ to $\theta$ we obtain

$$
\int_{\theta_{i}}^{\theta} \frac{-\phi}{2} d \theta^{\prime}=D(\theta) \frac{d \theta}{d \phi}
$$

where $\theta^{\prime}$ is the dummy variable of integration. This may be rearranged to obtain

$$
\mathrm{D}(\theta)=\frac{-1}{2} \frac{\mathrm{d} \phi}{\mathrm{d} \theta} \int_{\theta_{\mathrm{i}}}^{\theta} \phi \mathrm{d} \theta^{\prime}
$$

Which is known as the Bruce and Klute Equation (Bruce and Klute, 1956). If $\theta_{\mathrm{i}}$ is zero (initially dry soil), the integral in [3.45] is identified as a soil-water parameter

$$
\mathrm{S}(\theta) \equiv \int_{0}^{\theta} \phi \mathrm{d} \theta
$$


which is referred to as the soil sorptivity. For infiltration into initially dry soil, [3.46] then becomes

$$
\mathrm{D}(\theta)=\frac{-\mathrm{S}}{2} \frac{\mathrm{d} \phi}{\mathrm{d} \theta}
$$

Clearly solutions to [3.47] will depend on the particular form of $D(\theta)$. What forms of the function $\mathrm{D}(\theta)$ allow for analytical solutions? Philip (1960 a, b) developed a broad set of forms of $D(\theta)$ which produce exact solutions to [3.47]. Brutsaert (1968) then provided an expression for diffusivity which fits well to natural soils and allows solution of [3.43], which we will denote $\mathrm{D}_{\mathrm{B}}$

$$
\mathrm{D}_{\mathrm{B}}=\frac{\mathrm{D}_{0}(\mathrm{n}+1)}{\mathrm{n}} \theta^{\mathrm{n}}\left(1-\frac{\theta^{\mathrm{n}}}{\mathrm{n}+1}\right)
$$

Where $\mathrm{n}$ and $\mathrm{D}_{\mathrm{o}}$ are parameters determined experimentally for each soil. Typically $\mathrm{n}$ is between 1 and 10 , depending on the soil's pore size distribution, and $D_{0}$ is the diffusivity at saturation. Using this expression for $D(\theta)$, [3.45] is, in general, solved by

$$
\phi=\left(1-\theta^{\mathrm{n}}\right)\left(\frac{2 \mathrm{D}_{0}(\mathrm{n}+1)}{\mathrm{n}^{2}}\right)^{1 / 2}
$$

which may be easily checked by putting [3.48] and [3.49] into [3.43]. This equation gives the exact solution for the shape of the wetting front as a function of time. Notice that this is exactly the information that we could not get out of the Green and Ampt approach. The dependence of the solution upon $\mathrm{n}$ is shown below.

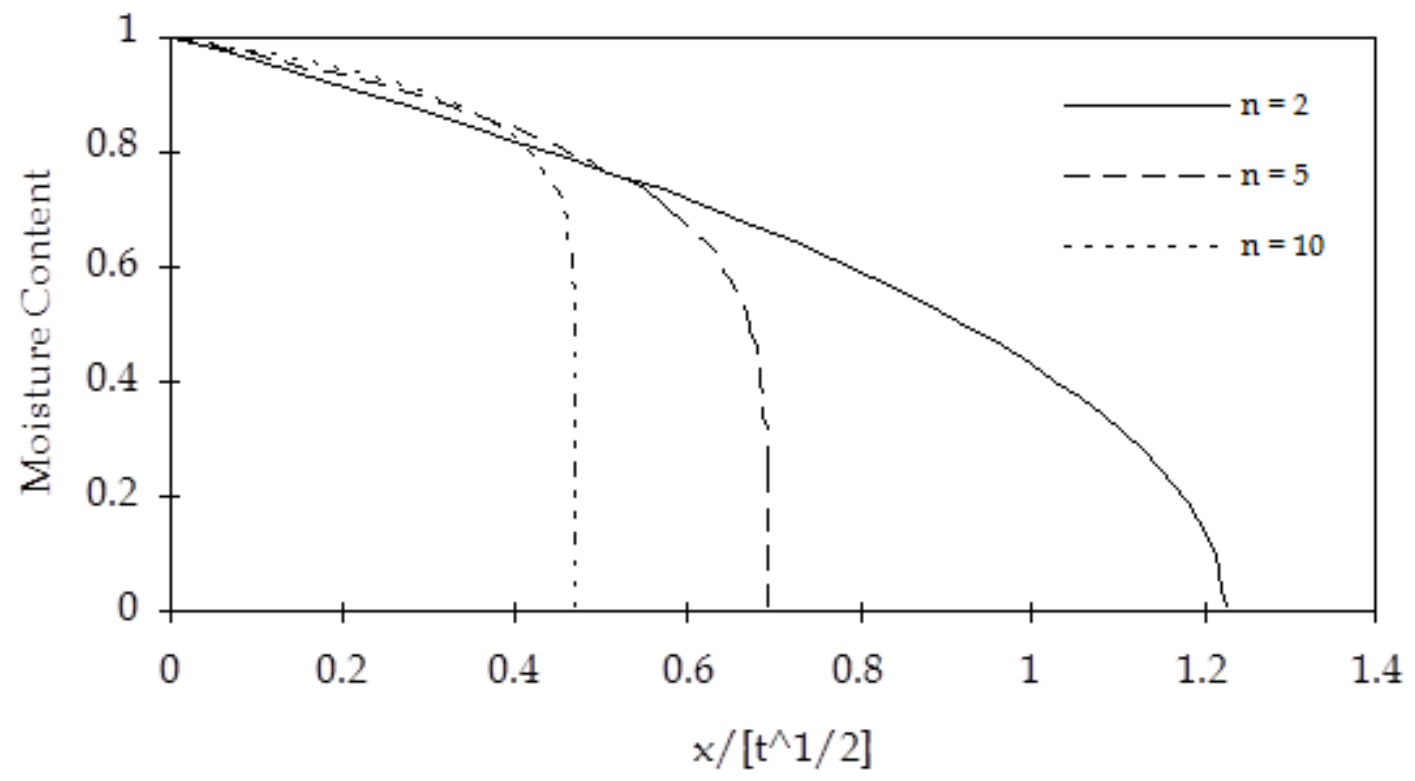

Figure 3.25 Horizontal infiltration as a function of $n$ for a Brutsaert soil with $\mathrm{D}_{\mathrm{o}}=1$, and $n=2,5$, and 10. The wetting front becomes increasingly sharp as $n$ increases, making the pore size distribution narrower.

We are also in a position to solve for the sorptivity. Using [3.49], [3.46] becomes 


$$
\begin{aligned}
\mathrm{S}(\theta) & =\int_{0}^{\theta} \phi \mathrm{d} \theta \\
& =\int_{0}^{\theta}\left(1-\theta^{\prime \mathrm{n}}\right)\left(\frac{2 \mathrm{D}_{0}(\mathrm{n}+1)}{\mathrm{n}^{2}}\right)^{1 / 2} \mathrm{~d} \theta^{\prime}
\end{aligned}
$$

and defining

$$
\mathrm{C}=\left(\frac{2 \mathrm{D}_{0}(\mathrm{n}+1)}{\mathrm{n}^{2}}\right)^{1 / 2}
$$

[3.50] becomes

$$
\mathrm{S}(\theta)=\mathrm{C} \int_{0}^{\theta}\left(1-\theta^{\prime \mathrm{n}}\right) \mathrm{d} \theta^{\prime}
$$

which is easily integrated to get a neat closed form solution for $S(\theta)$

$$
\mathrm{S}(\theta)=\mathrm{C}\left(\theta-\frac{\theta^{\mathrm{n}+1}}{\mathrm{n}+1}\right)
$$

Most often the sorptivity is reported for a saturated soil, for which $\theta=1$, and we find

$$
\begin{aligned}
\mathrm{S}(1) & =\frac{\mathrm{nC}}{(\mathrm{n}+1)} \\
& =\left(\frac{2 \mathrm{D}_{0}}{\mathrm{n}+1}\right)^{1 / 2}
\end{aligned}
$$

So why do we bother with this sorptivity term in the first place? If we try to answer one of our first questions this becomes clear. Suppose you want to know the cumulative infiltration as a function of time, how can you calculate this? It can be computed by integrating the moisture content over all positions at a given time. That is the cumulative infiltration, I, may be written as

$$
\mathrm{I}=\int_{0}^{\infty} \theta \mathrm{dx}
$$


We can evaluate the same integral by switching the bounds of integration so that we integrate all positions over the moisture content. Hence

$$
\mathrm{I}=\int_{0}^{1} \mathrm{xd} \theta
$$

But this may be written in terms of our similarity variable as

$$
\mathrm{I}=\mathrm{t}^{1 / 2} \int_{0}^{1} \phi \mathrm{d} \theta
$$

or

$$
\text { (3.215) } \mathrm{I}=\mathrm{St}^{1 / 2}
$$

which is exactly the same result we obtained by using the Green and Ampt approach! This shows that the approximations involved in the Green and Ampt provide quite a good estimate of flux into the soil, even though the description of the distribution of water within the soil profile is very crude. As we did for the Green and Ampt result, we can calculate the rate of infiltration as the time rate of change of cumulative infiltration

$$
\mathrm{q}=\frac{\mathrm{dI}}{\mathrm{dt}}=\frac{1}{2} \mathrm{St}^{-1 / 2}
$$

which is again identical to equation [3.16] derived using Green and Ampt.

So what is sorptivity? It is a parameter which expresses the macroscopic balance between the capillary forces and the hydraulic conductivity which act in the process of infiltration. We recall from the discussion of the Green and Ampt results that $\mathrm{S}_{\mathrm{GA}}=\sqrt{2 \mathrm{~K}_{\mathrm{sat}} \mathrm{P}_{\mathrm{f}} \mathrm{n}}$, and that since $\mathrm{K}_{\text {sat }}$ goes up with $\lambda^{2}$ and $\mathrm{P}$ goes with $1 / \lambda$ (where $\lambda$ is the characteristic microscopic length scale, for instance $\mathrm{d}_{50}$ ), then we can guess that sorptivity will get larger for coarser soils, but only with $\lambda^{1 / 2}$. For a more rigorous approach to this argument, see section 2.7 which explains Miller and Miller (1956) scaling and Hillel and Elrick (1990, SSSA Special Pub. \# 25) where the theory of "scaling" soil properties between various soil textures is developed in the more general sense.

We have only solved Richards equation for horizontal infiltration. Obtaining exact solutions for vertical infiltration is much more challenging, although restrictive classes of exact solutions are available, as discussed recently by Broadbridge (1988).

["Evaporation from water tables 19 June 2017" added below]

\section{Evaporation from water tables}

To quantify water balances we must estimate all the major contributors to flux. For example, to track soil water storage and aquifer recharge, we must identify that precipitation which infiltrates versus that which runs off. Once water enters a soil, it may leave by percolating to an aquifer, being taken up by roots, or evaporate directly from the surface. In this section we will quantify the magnitudes of loss through evaporation, looking at the impact of atmospheric demand, depth to the aquifer, and soil texture. We will start with estimation of evaporative flux at steady-state from a water table relatively close to the surface (under $10 \mathrm{~m}$ ) following the work of Gardner (1958). We then extend this calculation to estimate the time required for a water table to drop to a particular level. Finally, we will estimate the steady-state flux from very deep water tables in arid environments, showing the unique aspects of geothermally driven fluxes. 
Steady-state Evaporation from a Shallow Water Table.

In 1958 Gardner published an elegant solution for evaporation from a water table under steady-state conditions. This solution is of interest for several reasons: it is instructive in terms of how to solve simple unsaturated flow problems; it provides very handy results; and it introduced a family of useful and widely used forms of the hydraulic conductivity function.

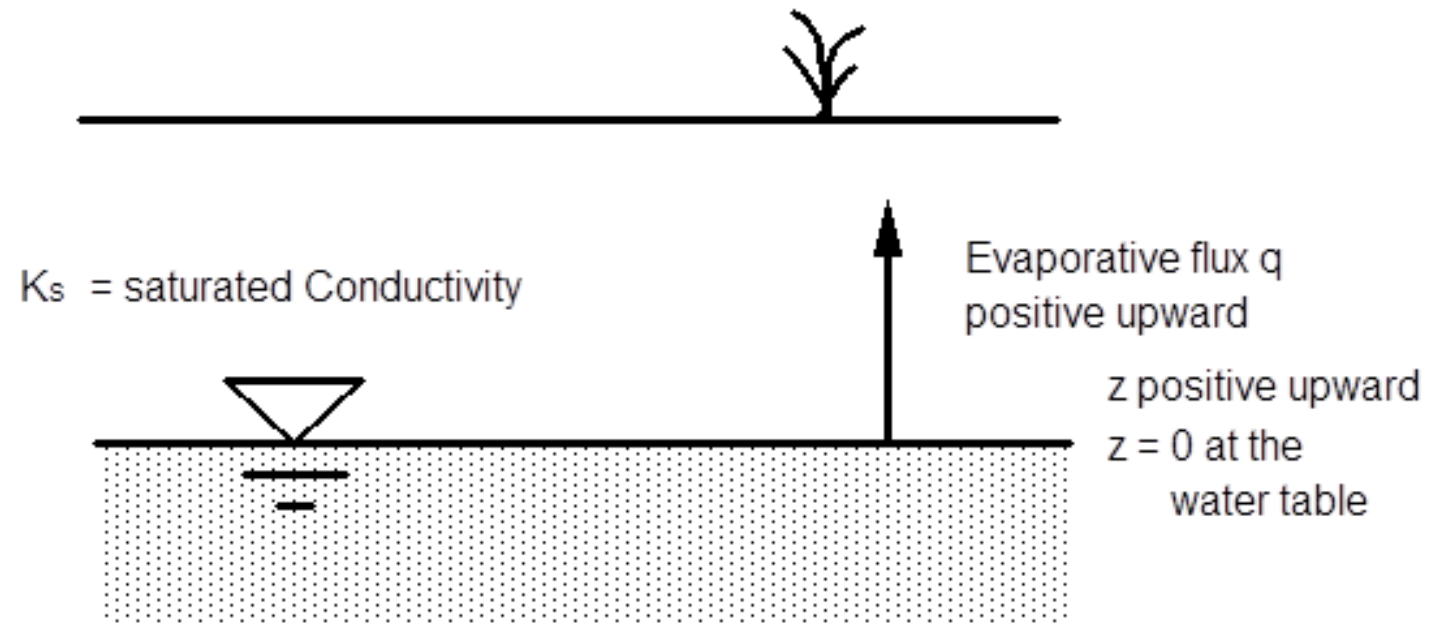

Figure 3.26 Definition sketch for evaporation from water table problem.

The problem we will consider is that of evaporation from a broad land surface. We will assume that the soil is uniform, and that the process is one-dimensional, with water moving only in the vertical direction. As shown above, the coordinates will be defined as positive upward from the water table. Notice that since the system is at steady state, the flux must be constant with elevation, i.e. $\mathrm{q}(\mathrm{z})=\mathrm{q}$ (otherwise, water would either build up or be depleted at particular depths).

For this set of conditions, Richards equation is simply

$$
\frac{\partial \theta}{\partial t}=\frac{\partial}{\partial z}\left[K\left(\frac{\partial h}{\partial z}\right)\right]+\frac{\partial K}{\partial z}
$$

At steady state the moisture content is constant in time, thus $\partial \theta / \partial \mathrm{t}=0$, and equation (‥217) becomes a differential equation in $\mathrm{z}$ alone

$$
-\frac{d K}{d z}=\frac{d}{d z}\left[K\left(\frac{d h}{d z}\right)\right]
$$

Since both sides are first derivatives in z, equation (3.218) may be integrated to recover the Buckingham-Darcy Law for unsaturated flow

$$
-K=K \frac{d h}{d z}+q
$$

or

$$
q=-K\left[\frac{d h}{d z}+1\right]
$$


where the constant of integration $\mathrm{q}$ is the vertical flux through the system. Notice that we could assign the sign of $\mathrm{q}$ to be either positive or negative, and have selected the sign convention to correspond to the definition sketch.

We would like to solve for the potential (head) as a function of elevation. Solving (??) for dz we find

$$
\mathrm{dz}=\frac{-\mathrm{dh}}{\frac{\mathrm{q}}{\mathrm{K}}+1}
$$

which may be integrated to obtain

(3.222) 0

$$
\int_{0}^{\mathrm{z}} \mathrm{dz}=\mathrm{z}=\int_{0}^{\mathrm{h}(\mathrm{z})} \frac{-\mathrm{dh}^{\prime}}{\frac{\mathrm{q}}{\mathrm{K}}+1}
$$

where $h^{\prime}$ is the dummy variable of integration and $h(z)$ is the potential at the elevation $\mathrm{z}$, and will be simply noted as $\mathrm{h}$ from here on. The lower bound of this integral is taken at the water table where $h(0)=0$. To solve this integral requires a relationship between conductivity and pressure. For this problem Gardner introduced several conductivity functions, including the exponential relationship

(3.223) $\mathrm{K}(\mathrm{h})=\mathrm{K}_{\mathrm{s}} \exp (\alpha \mathrm{h})$

Putting this into (‥222) we obtain

$$
\mathrm{z}=\int_{0}^{\mathrm{h}} \frac{-\mathrm{dh}^{\prime}}{\frac{\mathrm{q}}{\mathrm{K}_{\mathrm{s}} \exp \left(\alpha \mathrm{h}^{\prime}\right)}+1}
$$

which may be re-arranged as

$$
\mathrm{z}=\int_{0}^{\mathrm{h}} \frac{-\mathrm{K}_{\mathrm{s}} \exp \left(\alpha \mathrm{h}^{\prime}\right) \mathrm{dh}^{\prime}}{\mathrm{q}+\mathrm{K}_{\mathrm{s}} \exp \left(\alpha \mathrm{h}^{\prime}\right)}
$$

To solve this we change variables and let

$$
\mathrm{x}=\mathrm{K}_{\mathrm{s}} \exp (\alpha \mathrm{h})
$$

so

$$
\mathrm{dx}=\alpha \mathrm{K}_{\mathrm{s}} \exp (\alpha \mathrm{h}) \mathrm{dh}
$$

$$
\mathrm{dx}=\alpha \mathrm{xdh}
$$

Substituting $(\underline{3.226})$ into $(\underline{3.225})$ we obtain 


$$
\begin{aligned}
& z=-\int_{0}^{h} \frac{x_{d h^{\prime}}}{q+x} \\
& z=-\frac{1}{\alpha} \int_{x(0)}^{x(h)} \frac{d x}{q+x}
\end{aligned}
$$

which may be integrated to obtain

$$
\begin{aligned}
\mathrm{z}=-\left.\frac{1}{\alpha} \operatorname{Ln}(\mathrm{q}+\mathrm{x})\right|_{\mathrm{x}=\mathrm{x}(0)} ^{\mathrm{x}=\mathrm{x}(\mathrm{h})} \\
=-\frac{1}{\alpha} \operatorname{Ln}\left(\mathrm{q}+\mathrm{K}_{\mathrm{s}} \exp (\alpha \mathrm{h})\right)+\frac{1}{\alpha} \operatorname{Ln}\left(\mathrm{q}+\mathrm{K}_{\mathrm{s}}\right) \\
=-\frac{1}{\alpha} \operatorname{Ln}\left[\frac{\mathrm{q}+\mathrm{K}_{\mathrm{s}} \exp (\alpha \mathrm{h})}{\mathrm{q}+\mathrm{K}_{\mathrm{s}}}\right]
\end{aligned}
$$

which is the relationship between pressure and elevation for evaporation from the water table for a soil with exponential conductivity given by (3.223). Gardner (1958) notes that (3.222) may also be solved in closed form for conductivity's of the form: $\mathrm{K}=\mathrm{a} /\left(\mathrm{h}^{\mathrm{n}}+\mathrm{b}\right)$ for $\mathrm{n}=1,3 / 2,2,3$, and 4 .

We can put (3.228) into a more easily understood form through some simple manipulations. Note that we may write: $\mathrm{h}=(1 / \mathrm{a}) \operatorname{Ln}[\exp (\mathrm{ah})]$, so adding and subtracting $\mathrm{h}$ to $(\underline{3.228})$ we may write

$$
\mathrm{z}=-\mathrm{h}+\frac{1}{\alpha} \operatorname{Ln}[\exp (\alpha \mathrm{h})]-\frac{1}{\alpha} \operatorname{Ln}\left[\frac{\mathrm{q}+\mathrm{K}_{\mathrm{s}} \exp (\alpha \mathrm{h})}{\mathrm{q}+\mathrm{K}_{\mathrm{s}}}\right]
$$

or

$$
\mathrm{z}=-\mathrm{h}-\frac{1}{\alpha} \operatorname{Ln}\left[\frac{\mathrm{q} \exp (-\alpha \mathrm{h})+\mathrm{K}_{\mathrm{s}}}{\mathrm{q}+\mathrm{K}_{\mathrm{s}}}\right]
$$

which shows both the contribution of the potential and the flux terms separately. That is, if the flux is zero, you see that the second term on the right hand side goes to zero, and the potential is simply the elevation above the water table, as we would expect. As the flux increases, the second term increases in magnitude, indicating that at a given elevation, the potential decreases (i.e., the soil gets drier), as we would expect for increasing evaporative flux. 
Equation (‥228) may also be solved for pressure in terms of elevation to obtain the pressure profile

$$
\mathrm{h}=\frac{1}{\alpha} \operatorname{Ln}\left[\exp (-\alpha \mathrm{z})\left(\frac{\mathrm{q}}{\mathrm{K}_{\mathrm{s}}}+1\right)-\frac{\mathrm{q}}{\mathrm{K}_{\mathrm{s}}}\right]
$$

Although we are primarily interested in upward flux, it is interesting to note that if the flux is $-\mathrm{K}_{\mathrm{s}}$ that the pressure is zero everywhere, which is as we would expect for steady infiltration at $\mathrm{K}_{\mathrm{s}}$. Thus, this solution may be used for either steady infiltration or evaporation.

Another calculation of interest is the theoretically maximum evaporative flux, $\mathrm{q}_{\max }$. At the maximum flux, the matric potential at the soil surface is $-\infty$, so the argument of the logarithm must go to zero. This implies that

$$
\frac{q_{\max }}{K_{\mathrm{s}}}=\exp (-\alpha z)\left(\frac{q_{\max }}{K_{\mathrm{s}}}+1\right)
$$

solving for $\mathrm{q}_{\max }$, we find

$$
\mathrm{q}_{\max }=\frac{\mathrm{K}_{\mathrm{s}}}{\exp (\alpha z)-1}
$$

where $\mathrm{z}$ is the depth from the water table to the soil surface. Considering successive depths of $\mathrm{z}=1 / \alpha, 2 / \alpha, 3 / \alpha$ we find that $\mathrm{q}_{\max }(\mathrm{z}) / \mathrm{K}_{\mathrm{s}}=0.58,0.16$, and 0.05 , pointing out the very rapid decrease in evaporative flux as the depth to the water table increases. Figures 3.36 and 3.37 illustrate the sharpness of definition of the maximum flux, and the dependence on soil type and depth to the water table.

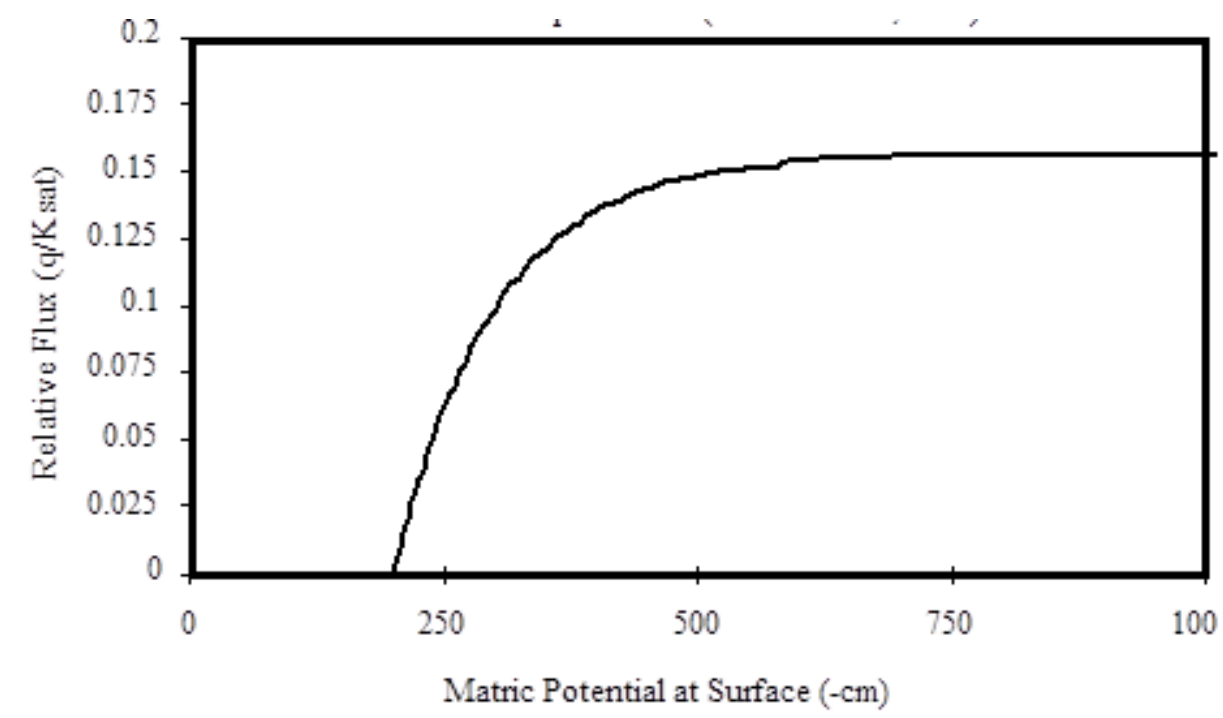

Figure 3.27 Dependence of evaporation rate upon head at soil surface. Here the water table was taken to be at 2 meters depth, and $\alpha=0.01$. 


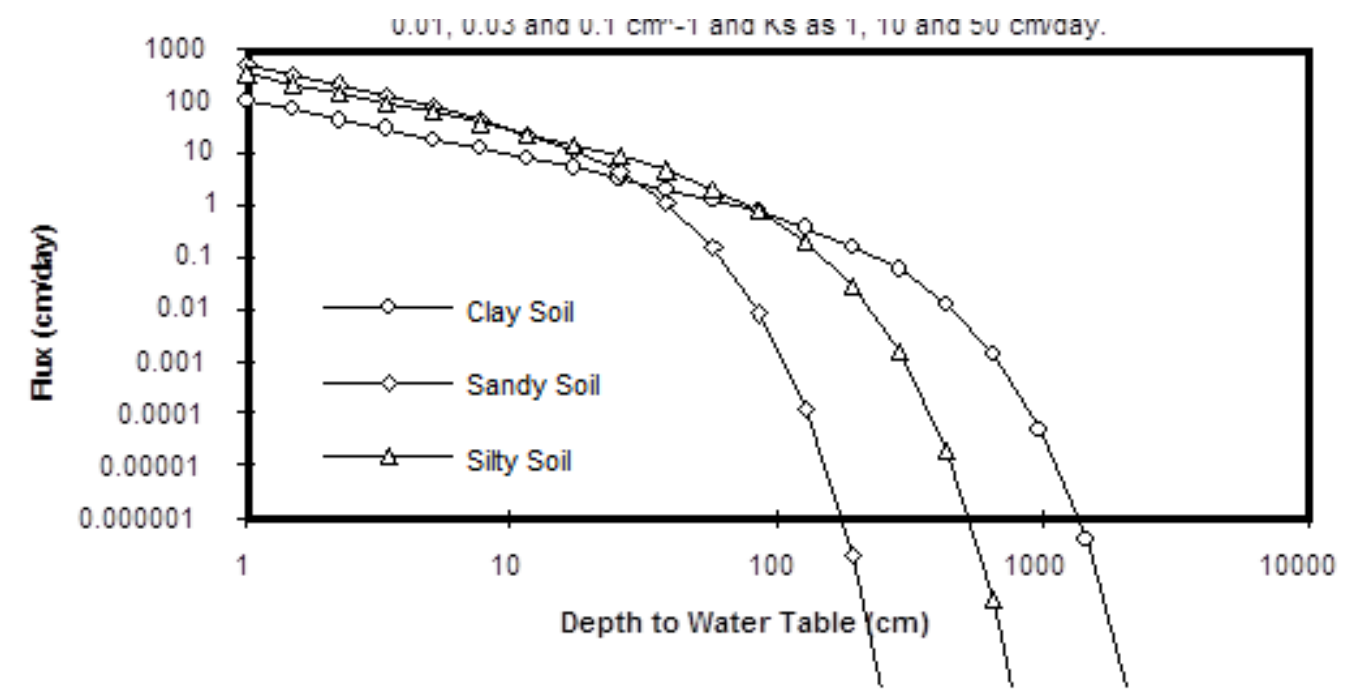

Figure 3.28 Maximum evaporative loss from a water table as a function of depth to water table and soil type. Here the values for $\alpha$ and $K$ were taken to be $0.01 / \mathrm{cm} .0 .03 / \mathrm{cm}$, and $0.1 / \mathrm{cm}$ and $1 \mathrm{~cm} /$ day, $10 \mathrm{~cm} /$ day and $50 \mathrm{~cm} /$ day for the clay, silt and clay soils.

\section{Estimate of Time to achieve a particular water table depth}

So we have an estimate of the steady-state flux from a water table at a fixed depth, but can we use this to estimate the rate of decline of the water table, and estimate how long we should expect it to take for a water table to arrive at a particular depth? In this case we will assume that there is not lateral contribution to the gain/loss of water in the system (e.g., one-dimensional flow). The initial interest in this computation came from a visit to El Bur Somalia, a place a few hundred miles from the hottest inhabited place on earth. It was surprising that the water table was only about $3.5 \mathrm{~m}$ below the surface. There was the possibility of lateral flow, but I wanted to see how long it might take for the water table to drop to this level if the site started with the water table at the surface and the only water loss was due to evaporation. Officially Gardner's result is for a steady-state system, but let's just assume that as the water table declines that the distribution of water in the system changes slowly enough that this assumption remains satisfied despite the slight deviation from steady state. What will the maximum rate of water table decline? If the average loss of water in the profile is $\theta_{\mathrm{e}}$, then the rate of water table decline will be $\mathrm{q}_{\mathrm{max}} / \theta_{\mathrm{e}}$. We then may write

$$
\frac{\mathrm{dz}}{\mathrm{dt}}=\frac{\mathrm{q}_{\max }}{\theta_{\mathrm{e}}}=\frac{\mathrm{K}_{\mathrm{s}}}{\theta_{\mathrm{e}}[\exp (\alpha \mathrm{z})-1]}
$$

Solving for dt and integrating, we find the time for the water table to reach a depth $\mathrm{z}$ is approximately

$$
\mathrm{t}=\frac{\theta_{\mathrm{S}}}{\mathrm{K}_{\mathrm{s}}}\left[\frac{\exp (\alpha \mathrm{z})-1}{\alpha}-\mathrm{z}\right]
$$

For typical values of these parameters for a silty soil (say $\alpha=5 / \mathrm{m}, \mathrm{K}_{\mathrm{s}}=1 \mathrm{~m} / \mathrm{d}$ and $\theta_{\mathrm{e}}=0.2$ ) the time to reach a depth of $3.5 \mathrm{~m}$ is predicted to be 4,400 years, which matches the Somali observations pretty well. It is interesting to note that to drop another $0.5 \mathrm{~m}$ would take 50,000 years! It is further instructive to think about how shallow water tables might be water that has been in situ since the last ice-age: in a desert society which relies on a shallow depth to groundwater is effected by the introduction of mechanical pumps which can drop the water table by up to a meter per year which would likely never recover within a human lifespan. 


\section{Rate of Evaporative Flux from a Very Deep Water Table}

It is also interesting to estimate how much water will be lost from a deep water table to the atmosphere. From the calculations just above we know that these fluxes will be very small, but can we put numbers to this flux? Is the physics just an expansion of Gardner's approach, or are there other processes involved? There are many arid places on earth where there is no net recharge, but at depth there is a water table. So suppose that we are in a very dry place (for example, the Atacama Desert) where there is essentially no precipitation or percolation. Going back to our thinking with Gardner's approach, we know that if the water table were at the surface, the rate of evaporation will be controlled by the energy delivery to the surface. Then, as the upper soil dries, the rate of evaporation becomes limited by the rate at which soil can deliver water to the surface through capillary transport. As we discussed above, this can lead to a water table dropping to on the order of $5 \mathrm{~m}$ over the course of a few thousand years, depending on the soil texture. But let's suppose that you came to the desert and found that the water table was over $20 \mathrm{~m}$ below the surface. How much water might be lost from such a deep aquifer? One thing we can be sure is that the water which does go all the way from the phreatic surface to the atmosphere must move in the vapor phase, as Gardner (1958) showed that the liquid permeability is not sufficient to supply any appreciable water from such a depth. So the processes of interest are the phase change from liquid to vapor phase, which will occur primarily near the phreatic surface, and the transport of the water vapor from its source to the surface.

At these depths we first must identify if there is a source of energy sufficient to generate a vapor flux. Here the logical source of energy is the geothermal heat flux from the center of the earth, which is generally taken to be about $0.1 \mathrm{~W} / \mathrm{m}^{2}$, or in a year about $3 \mathrm{MJ} / \mathrm{yr}^{-\mathrm{m}^{2}}$ (Pollack et al., 1993). Since the latent heat of water is about $2 \mathrm{MJ} / \mathrm{kg}$, this energy, if all this energy were employed in the vaporization of water, this could convert $1.5 \mathrm{~kg} / \mathrm{yr}-\mathrm{m}^{2}$, a flux of about $1.5 \mathrm{~mm} / \mathrm{yr}$. This is close to the value reported by Li et al., $(2010,2016)$. There are two problems with this number: 1 . If all the energy went to evaporation, why would the geothermal gradient in temperature be seen to be linear as it crosses the phreatic surface?; and, 2. if this much vapor was being created, what mechanism would transport the vapor?

While the first issue certainly suggests that something is not right with this model (we don't see such breaks in slope of the geothermal gradient), it is the second issue which makes the non-physical nature of this concept clear. Since there is no net advection vertically in the deep subsurface, we know that diffusion is the mechanism that would transport the water vapor. Considering Li et al's site for the moment, suppose that the water table were $200 \mathrm{~m}$ below the surface. Now, even though this is completely out of keeping with what is seen in the field, let's take the biggest possible water vapor gradient: with a vapor concentration of $0 \mathrm{~kg} / \mathrm{m}^{3}$ at the surface, and saturated with water vapor at the water table. [We will later show that this assumption vastly over-estimates the potential flux, since through most of the profile the atmosphere will be essentially saturated with water vapor.] That said, we will allow for this over-estimate just to make sure the vapor flux is not able to carry the vapor flux that would result from complete consumption of the geothermal energy flux. For a water table at $30^{\circ} \mathrm{C}$, the saturated water vapor concentration would be about $0.03 \mathrm{~kg} / \mathrm{m}^{3}$. Using a typical diffusion coefficient for water vapor of $1,000 \mathrm{~m}^{2} / \mathrm{yr}$, Fick's law tells us that the flux is equal to the diffusion coefficient times the gradient in concentration. For the moment we can ignore the fact that much of the pore space in the vadose zone is filled with solids, and just compute the upper-bound on flux if this were taking place in pure air:

$$
q=-D \frac{d C}{d x}=-1.000 \mathrm{~m}^{2} / \frac{-30 \frac{\mathrm{g}}{\mathrm{m}^{3}}}{200 \mathrm{~m}}=150 \mathrm{~g} / \mathrm{m}^{2} \mathrm{y}
$$

This biggest-physically-conceivable diffusive flux is 10 times smaller than the upper bound on flux dictated by energetic consideration. Thus, Li et al.'s values are simply not in keeping with physical constraints on transport.

An alternate approach to this estimation was provided by Ross (1988). Ross posited that if the water in the vadose zone were hydrostatic (after all, we are assuming that there has been no net recharge in many hundreds or thousands of years), then the pressure potential would be linear with depth from the water table, and we can use the psychrometric equation to compute the resulting vapor pressure gradient. With this and Fick's law, he computed the flux to be 0.0001 
$\mathrm{mm} / \mathrm{yr}$ for a $200 \mathrm{~m}$ deep aquifer, 50,000 times smaller than that suggested by Li et al., and 3,000 times smaller than would be obtained from a linear water vapor gradient.

The key to getting the right answer to this question is to take seriously Gardner's 1958 finding: capillary communication along the profile essentially stops when pressures are more than about $-10 \mathrm{~m}$. This is also the depth where annual fluctuations in temperature are no longer significant. So below this level we can take the temperature gradient to be constant, and equal to the geothermal gradient. From the psychrometric equation we know that the humidity is over 99\% at such small negative pressures as are found at "field capacity" such as would be expected in a deep vadose zone. So let's start by recognizing the well-known fact that the humidity of deep unsaturated soil profiles are essentially saturated with water vapor below about 10 m depth (e.g., see Walvoord et al., 2002; Scanlon et al., 2003). Now we know from our consideration of Li et al.'s work that there is more than enough energy to drive vapor flow, and that vapor diffusion provides the key limitation. It turns out that the water vapor gradient is provided by the strong temperature dependence of saturated water vapor content, and the gradient in temperature is dictated by the geothermal gradient.

We start with the assumption employed by our predecessors, that the water table is over $200 \mathrm{~m}$ below the surface, though we will later see that this does not strongly influence the computed flux. All of the water vapor that moves from the water table must diffuse through this $200 \mathrm{~m}$ section of earth, where we may take the geothermal gradient to be $25^{\circ} \mathrm{C} / \mathrm{km}$, so a $200 \mathrm{~m}$ section will have about $5^{\circ} \mathrm{C}$ in thermal gradient. Per our discussion above, and the data of Scanlon et al. (2003), the atmosphere more than $20 \mathrm{~m}$ below the soil surface is essentially saturated in water vapor, so the gradient in absolute humidity, which via Fick's law will drive vapor transport, will be due to the temperature dependence of the saturated vapor pressure of water. Looking to a standard psychrometric chart we see that the water vapor density will be about $0.0275 \mathrm{~g} \mathrm{H}_{2} \mathrm{O} / \mathrm{g}$ of dry air at $30^{\circ} \mathrm{C}$, and $0.020 \mathrm{~g} \mathrm{H}_{2} \mathrm{O}$ per gr air at $25^{\circ} \mathrm{C}$. Since a $\mathrm{m}^{3}$ of dry air is $1000 \mathrm{~g}$, we can convert this to volumetric units (here denoted $\vartheta$ ) as being

$$
\vartheta=(0.0275-0.02) \frac{\mathrm{gH}_{2} \mathrm{O}}{\mathrm{g} \text { dry air }} \frac{1000 \mathrm{~g} \text { dry air }}{\mathrm{m}^{3} \text { dry air }}=7.5 \mathrm{gH}_{2} \mathrm{O} / \mathrm{m}^{3}
$$

As before, the diffusion coefficient for water vapor in pure air at these temperatures is approximately $1,000 \mathrm{~m}^{2} / \mathrm{yr}$. Being a bit more careful now, we should reduce this by a factor of three due to porosity, and a factor of two due to tortuosity, but multiply it by about two to account for enhanced diffusion, per Philip and de Vries (1957). These values would be easily adjusted in case site-specific information is available, but these values will satisfy our requirements to identify the approximate value of desert fluxes from water tables, wherein we find the effective diffusion coefficient is $0.10 \mathrm{~cm}^{2} / \mathrm{s}$, which is $1 \times 10^{-5} \mathrm{~m}^{2} / \mathrm{s}$, or since there are $3.1 \times 10^{7} \mathrm{~s} /$, so D may be taken as $300 \mathrm{~m}^{2} /$. Applying Fick's law, denoting the vapor flux as q, we thus obtain:

$$
q=-D \frac{d C}{d x}=-300 \mathrm{~m}^{2} / \frac{-7.5 \frac{\mathrm{g}}{\mathrm{m}^{3}}}{200 \mathrm{~m}}=11 \mathrm{~g} / \mathrm{m}^{2} \mathrm{y}
$$

Which in units of velocity is $0.011 \mathrm{~mm} /$. As one should expect given the common underlying physical basis and considerations, this is almost exactly what Walvoord et al. (2002) and Scanlon et al. (2003) found in their numerical simulations of sites where water tables were over $100 \mathrm{~m}$ below the surface. On the other hand, Ross (1984) estimated this flux to be $0.0001 \mathrm{~mm} /$, which is 100 times lower than this value. As we now see, this discrepancy arose due to his ignoring thermal effects of the earth's geothermal gradient on vapor pressure, instead assuming that a hydrostatic pore pressure was responsible for the potential gradient. At the same time, the value we obtain is 500 times less than the experimentally estimated flux suggested by Li et al. (e.g., 2014) which we showed were out of keeping with the underlying transport physics of the problem.

An important feature of this calculation is the fact that the vapor flux depends on the geothermal gradient rather than the boundary conditions. This implies that the vapor flux would remain at essentially this same value regardless of the depth of the water table, so long as the geothermal gradient was linear (which is generally true), and that the temperature versus saturated vapor pressure could be reasonably estimated by a straight line in the range of temperatures under consideration. Thus, for phreatic surfaces below on the order of $20 \mathrm{~m}$ (that is, getting beyond the first $10 \mathrm{~m}$ of the 
vadose zone where the annual cycles of temperature are seen) the rate of vapor flux due to geothermal gradients is independent of the depth to the water table.

To give a longer-timescale interpretation, in 1000 years we predict about $10 \mathrm{~mm}$ of vapor loss from the water table, which for an aquifer with 0.25 drainable porosity, would lead to a decline of about $25 \mathrm{~mm}$ per millennium, or $25 \mathrm{~m}$ per million years, suggesting that unless the system had been under arid conditions for many million years, the vapor flux from the aquifer would likely not be the key factor in establishing the depth to groundwater. Rather, if you find a water table well below $20 \mathrm{~m}$ depth, it is almost certain that its depth is controlled either by someone pumping a well in the area, or by the elevation of the nearest drainage point for water from this aquifer.

\section{Example 2-8): Unsaturated Steady State Flow to a Water Table}

Problem Statement:

(1) Compute and plot the vertical distributions of the soil matric potential under steady flow into a water table at a depth of $3 \mathrm{~m}$ from the surface for the flow rates of $\mathrm{J}_{\mathrm{W}} / \mathrm{K}_{\mathrm{s}}=-0.001,-0.01,-0.1$, and -0.5 . Note that the sign of the downward flux is negative. The unsaturated hydraulic conductivity of the soil is based on Gardner's (1958) model, and is characterized by $\mathrm{b}=5 \mathrm{~m}-1$.

(2) Estimate the hydraulic gradient $(\mathrm{dH} / \mathrm{dz})$ from 0 to $1 \mathrm{~m}$ below the soil surface.

Solution:

(1) The depth dependent matric potential may be calculated according to the following relationship:

$$
h(z)=\frac{1}{\beta} \ln \left[-\frac{J_{w}}{K_{s}}+e^{-\beta(L+z)}+\frac{J_{w}}{K_{s}} e^{-\beta(L+z)}\right]
$$

with $\mathrm{z}$ as the actual depth, $\mathrm{L}$ as the depth of the entire profile from surface to water table, Jw/Ks as the flow rate, and $\mathrm{b}$ as Gardner's K(q) parameter. 


\begin{tabular}{ccccc}
\hline $\mathrm{Jw} / \mathrm{Ks}$ & -0.001 & -0.01 & -0.1 & -0.5 \\
$\mathrm{Z}[\mathrm{m}]$ & $\mathrm{h}(\mathrm{z})[\mathrm{m}]$ & $\mathrm{h}(\mathrm{z})[\mathrm{m}]$ & $\mathrm{h}(\mathrm{z})[\mathrm{m}]$ & $\mathrm{h}(\mathrm{z})[\mathrm{m}]$ \\
0.00 & -1.3815 & -0.9210 & -0.4605 & -0.1386 \\
-0.25 & -1.3813 & -0.9210 & -0.4605 & -0.1386 \\
-0.50 & -1.3808 & -0.9210 & -0.4605 & -0.1386 \\
-0.75 & -1.3790 & -0.9208 & -0.4605 & -0.1386 \\
-1.00 & -1.3727 & -0.9201 & -0.4604 & -0.1386 \\
-1.25 & -1.3522 & -0.9179 & -0.4602 & -0.1386 \\
-1.50 & -1.2936 & -0.9104 & -0.4595 & -0.1385 \\
-1.75 & -1.1667 & -0.8861 & -0.4571 & -0.1382 \\
-2.00 & -0.9725 & -0.8188 & -0.4487 & -0.1373 \\
-2.25 & -0.7419 & -0.6805 & -0.4221 & -0.1340 \\
-2.50 & -0.4978 & -0.4788 & -0.3499 & -0.1229 \\
-2.75 & -0.2495 & -0.2451 & -0.2055 & -0.0882 \\
-3.00 & 0.0000 & 0.0000 & 0.0000 & 0.0000 \\
\hline
\end{tabular}

(2) To estimate the hydraulic gradient we have to solve:

$$
\begin{aligned}
& \frac{d H}{d z}=\frac{d(h+z)}{d z}=\frac{d h}{d z}+1 \\
& J_{w} / K_{s}=-0.001 \\
& \frac{d H}{d z}=\frac{-1.38149+1.37268}{1}+1=0.99119 \\
& J_{w} / K_{s}=-0.01 \Rightarrow d H / d z=0.99911 \\
& J_{w} / K_{s}=-0.1 \Rightarrow d H / d z=0.99992 \\
& J_{w} / K_{s}=-0.5 \Rightarrow d H / d z=0.99999
\end{aligned}
$$

Solutions for Capillary Barriers

One of the most basic geologic features is that of bedding of sedimentary materials. Typically, such beds are made up of lamina which are extensive in the horizontal direction $(10-1,000 \mathrm{~m})$ and much smaller in vertically dimension (homogeneous over $0.01-10 \mathrm{~m}$ ). These strata are commonly locally inclined. Another common geologic configuration is that of fractured rock, where large elements of uniform material are separated by fissures. How these geologic features 
influence infiltration if they are found in the vadose zone? It turns out that we can obtain quantitatively predictive analytical solutions for this flow regime, and the process of obtaining the solution is quite instructive.

First, it is important to think carefully about the manner in which moisture content and pressure transition across a textural interface.

The matric potential will be continuous across layers, while the water content profile exhibits a sharp discontinuity. A further problem here is that it is not possible to take the required derivative in Eq.(98), , across the discontinuity. Note that it may be permissible to use this equation in homogenous soils for conditions where the vertical gravitational component is very small in comparison with matric influences (translated into a water content "gradient"). Examples of this are the early phases of infiltration into relatively dry soil, and evaporation of water from the soil surface.

In some special cases the diffusivity may be assumed constant over a small range of water content. A constant diffusivity facilitates analytical solutions to Eq.(98) for certain boundary conditions. In many cases analytical solutions to flow problems are favored over numerical solutions due to their mathematical simplicity, the information they provide concerning the physical process, and the physical significance of their parameters.

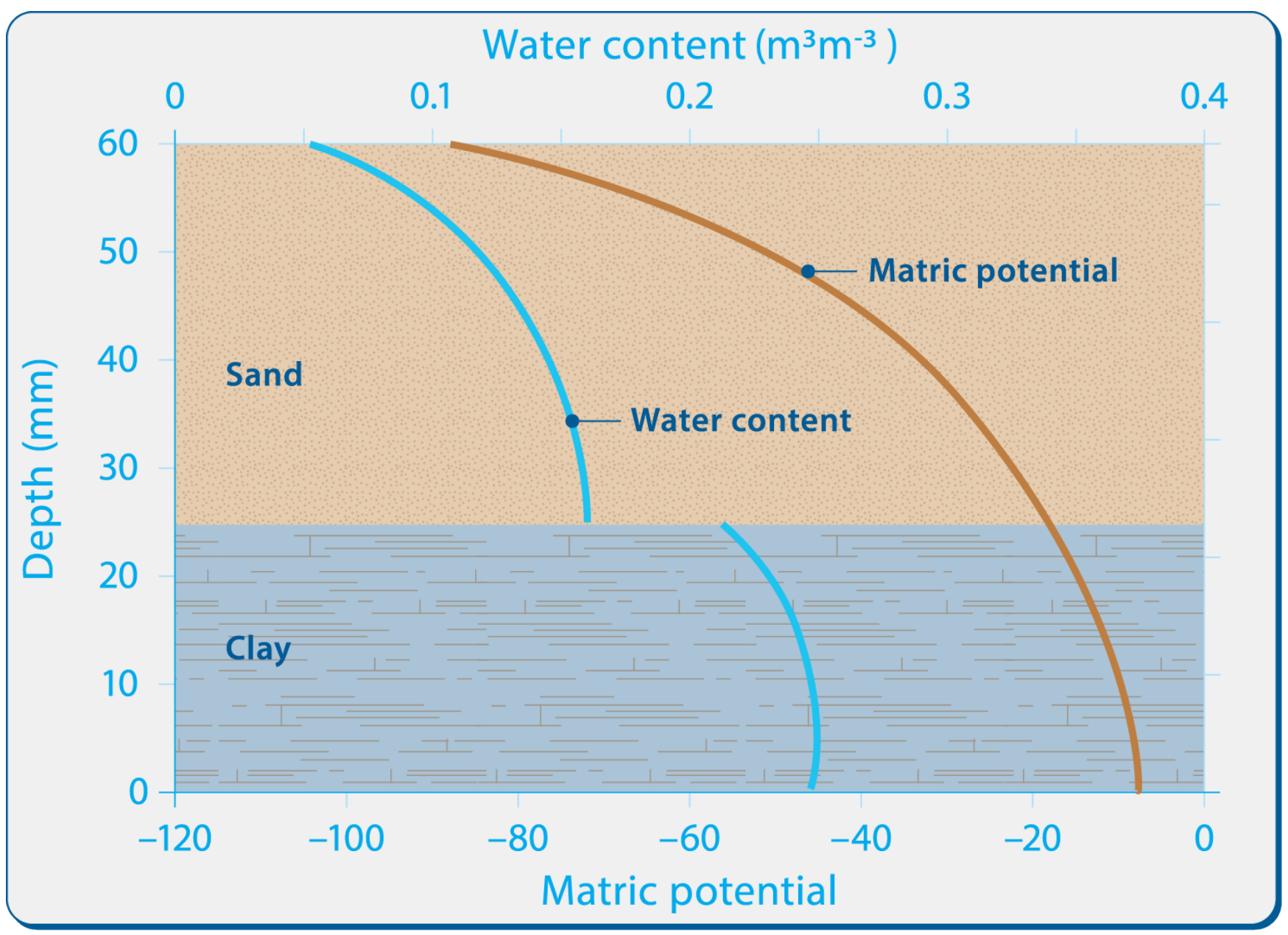

Figure 3.29: Continuous matric potential profile vs. discontinuous water content profile across a textural boundary in layered soil.

As the basic building block for sloping layered media, let us consider a single interface at an inclination $\phi$, as shown in Figure 3.30. From a hydrologic perspective, there are three possible configurations which could arise in this system: (1) medium 1 is more permeable (coarser) 


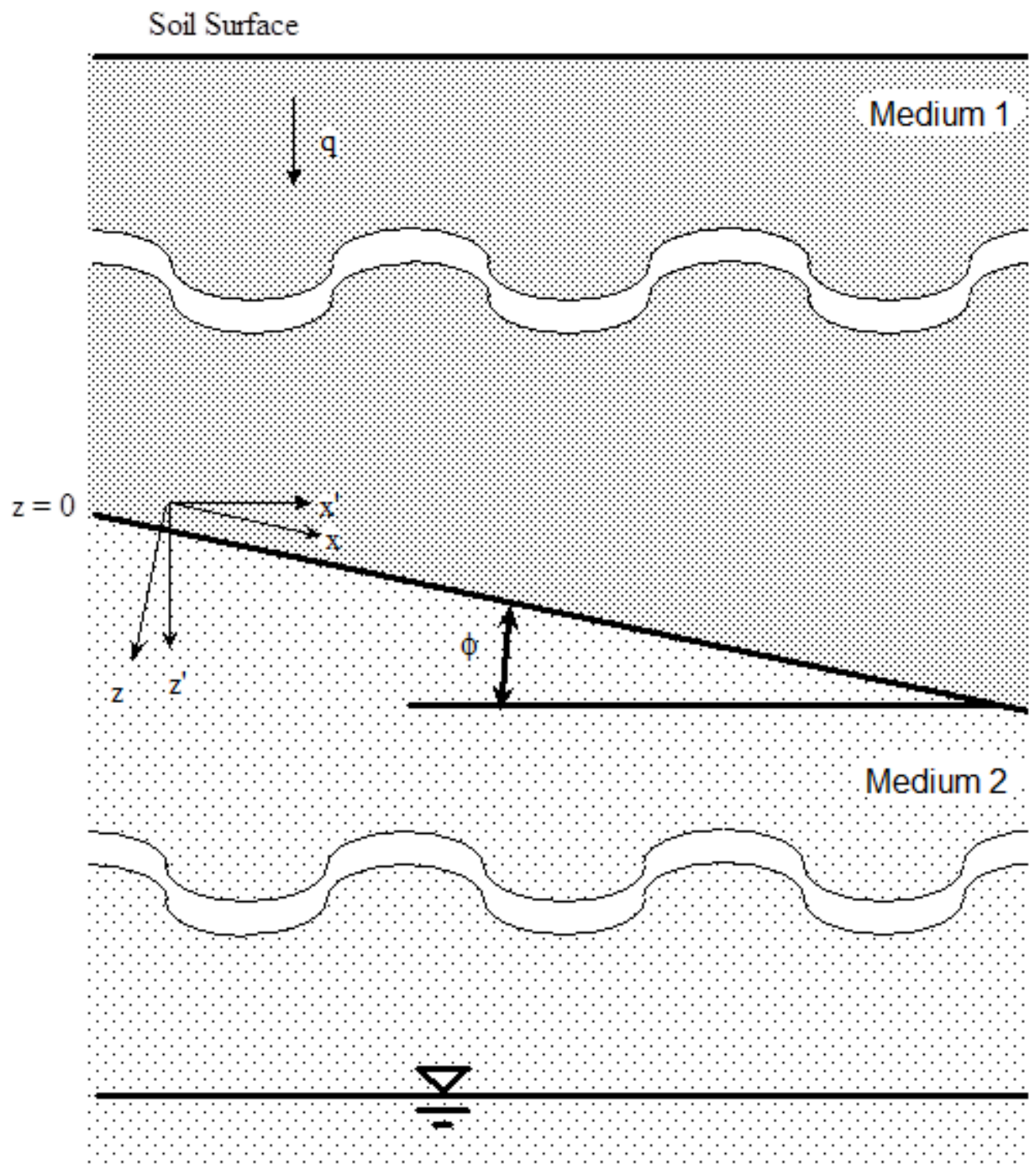

Figure 3.30 Most basic form of layered geologic materials, where two extensive layers meet at an angle $\phi$ well below the soil surface, and well above the water table. Sketch defines primary variables (following Ross, 1990).

than medium 2; (2) the two media are hydraulically identical, and (3) medium 1 is less permeable (finer) than medium 2. When we speak of more and less permeable, this is in the sense of the saturated conductivities being larger or smaller. As we shall see, the roles of more and less permeable can be switched under unsaturated flow conditions.

We will consider the influence of this situation on vertical infiltration. Case 2 can be dispensed with simply by applying the theory for homogeneous media developed in Chapter 2. Case 1 is pretty similar, with the system basically showing vertical infiltration. $\neq$ The most interesting case is 3 , where a fine media overlies a coarser media.

To get a feel for what will happen with a fine media, over a coarse media it is instructive to consider the most extreme example where the lower media is infinitely coarse, that is, simply a gap. This would be a reasonable image for fractured rock. Water will only go into this gap when the water pressure at the crack is greater than or equal to zero. Hence, the crack acts like an impermeable barrier until there is a sufficient amount of water flowing parallel to the crack to generate positive pressure at the boundary at which time water will drip off into the crack.

So how much water will flow parallel to the crack prior to water dripping off into the gap? This amount is what we will 
call the "diversion capacity" of the crack, being the amount of water which the crack can divert laterally through the fine media. We can carry out a crude calculation to get a feel for the strategy we will use in the more detailed calculation. As mentioned, water won't drip into the fracture until the pore pressure is zero at the face of the crack. Thinking of the characteristic curve for the media, the pressure at the crack won't get to zero until it is essentially saturated for a depth of at least $\left|h_{\mathrm{ae}}\right|$ above the crack. So before water traverses the crack there will be a layer $\left|h_{\mathrm{ae}}\right|$ where the conductivity is $K_{\text {sat. }}$. Above this height, the conductivity will be much smaller, and the influence of the crack less pronounced, so we will assume that all of the diversion occurs in the layer of thickness $\left|h_{a}\right|$ flowing parallel to the crack. To simplify this analysis, let's make a few assumptions:

1) The flow is steady.

2) The primary lateral driving force for flow is gravity (the gradients in pressure head parallel to the crack are minimal).

3) The flow is parallel to the crack in the capillary fringe region.

4) Above the capillary fringe the flow is vertical.

With these assumptions, Darcy's law tells us that the total flux in the x-direction, Q, will be the area of flow (in this case the height of the capillary fringe) times the conductivity (here assumed to be $\mathrm{K}_{\mathrm{S}}$ ) times the hydraulic gradient (purely gravity)

$$
\begin{aligned}
\mathrm{Q} & =A K_{\mathrm{s}} \frac{\mathrm{dH}}{\mathrm{dx}} \\
& =\left|\mathrm{h}_{\mathrm{ae}}\right| \mathrm{K}_{\mathrm{s}} \sin \phi
\end{aligned}
$$

We could define lateral flux, Q', as the component of flow in the horizontal direction (that is the x' direction) which would be simply

$$
\mathrm{Q}^{\prime}=\left|\mathrm{h}_{\mathrm{ae}}\right| \mathrm{K}_{\mathrm{s}} \tan \phi
$$

To get a feel for the practical significance of these results, we can consider some typical values for a permeable sandstone, where $h_{\mathrm{ae}}$ might be $100 \mathrm{~cm}, \mathrm{~K}_{\mathrm{s}}$ might be as high as $1 \mathrm{~cm} / \mathrm{hr}$ and $\phi$ might be 15 degrees. This would yield a Q of about $26 \mathrm{~cm} 2 / \mathrm{hr}$. If this were on a site which had an annual percolation of $50 \mathrm{~cm} / \mathrm{yr}$ which all occurred at a constant rate over a two-month period, this would indicate an infiltration rate of $0.035 \mathrm{~cm} / \mathrm{hr}$, which means this crack could divert water about 10 meters before water would go into the crack. Clearly, a tiny crack can have a pronounced influence on the routing of water through unsaturated material.

If we put on the hat of an engineer, this little capillary diversion could be quite handy. The interface provides a nondegradable barrier to water entry, which could be used over landfills and other areas where it is necessary to protect from infiltration. From a design perspective, it would be very useful to have a formula which would be able to more accurately predict the diversion capacity for a capillary barrier based on the properties of the two porous media found at the interface. This is precisely what Benjamin Ross provided in his 1990 article on this topic (Ross, 1990). We will go through this calculation in detail both because it provides a useful result, and because it demonstrates some nice techniques for approaching problems in the unsaturated zone. 


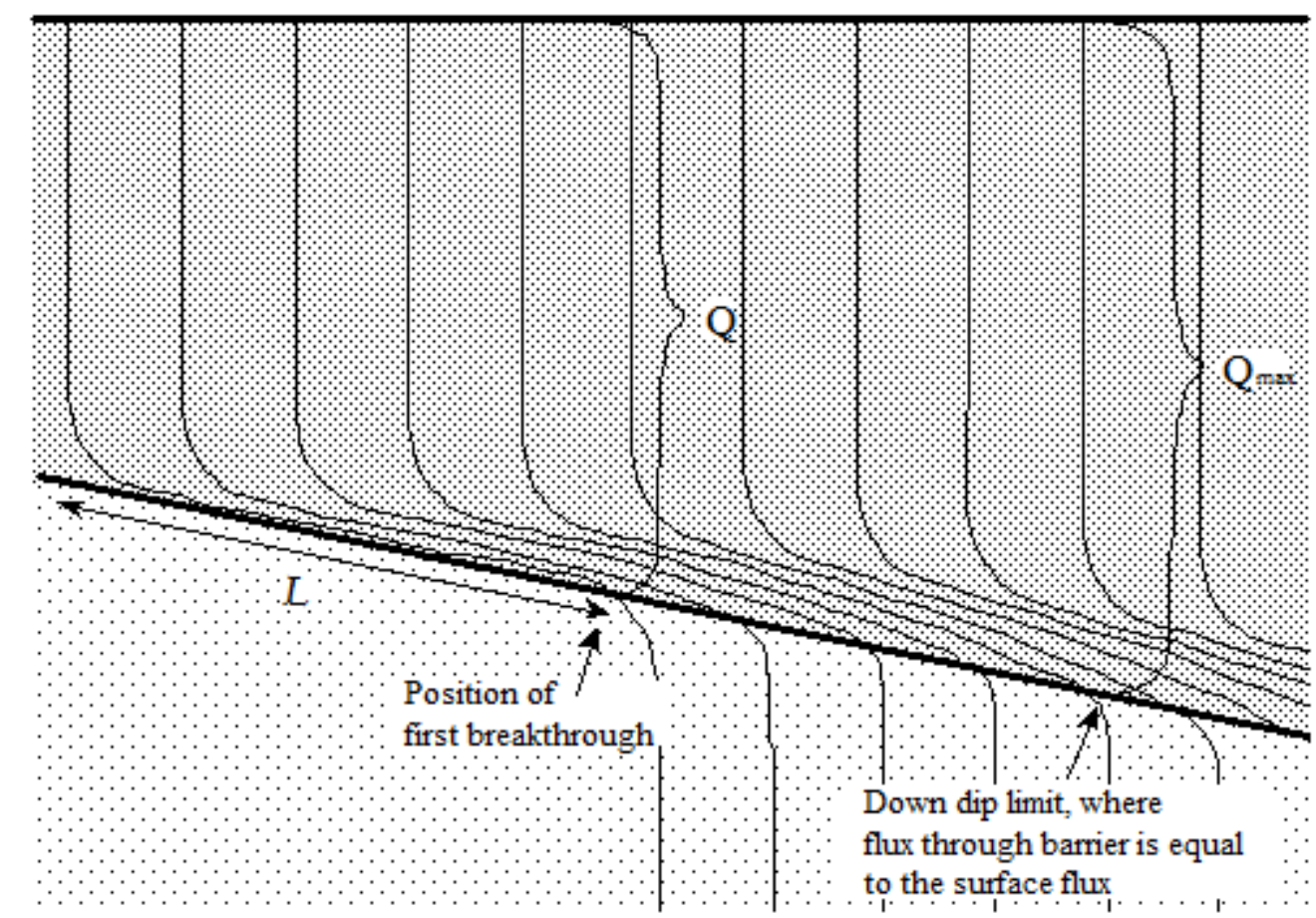

Figure 3.31 Illustration of diversion length (L), diversion capacity (Q) and the down dip limit.

Following Ross (1990), we will approach the solution to this problem in two steps. First, we will develop the governing equations. Then we will solve for the pressure profile for steady infiltration into a system with a single, horizontal, fineover-coarse bedding. Finally we will extend this result to a special case of the sloping layer problem. The trick here is to formulate the intrinsically two-dimensional problem into a one-dimensional setup, which is much easier to deal with.

In both the horizontal and sloping cases we will take the interface to be "far" below the surface, and "far" above the water table. We will discuss in quantitative terms how far "far" is at the end of the section. Further, we will assume that the flow is steady. Finally, we will solve the problem at the "down dip" limit, where there is no variation in the flow along the length of the interface. This last condition may seem restrictive, but it turns out that the down dip limit occurs very soon after breakthrough, given the much higher permeability of the lower material. The diversion flux we calculate will be an upper limit for diversion, but a tight upper limit given the rapid attainment of this down dip limit condition. This condition on the calculation must be kept in mind: if you are designing a capillary barrier to protect some waste material, the results we obtain will not be conservative in the sense that they predict that the flow will break through before a certain position. We might prefer a calculation which gave us a length where we were sure not to have flow, but in truth the uncertainty in parameter values and local deviations in geometry from that shown is a greater source of uncertainty than the assumptions required in this calculation.

Getting down to business, let's put Richards equation into the required form to solve the problem (note the sign convention of $\mathrm{z}$ being positive downward). 


$$
\begin{aligned}
\frac{\partial \theta}{\partial \mathrm{t}} & =-\nabla \cdot \mathrm{K} \nabla \mathrm{H} \\
& =-\nabla \cdot\left[\mathrm{K} \nabla\left(\mathrm{h}-\mathrm{z}^{\prime}\right)\right] \\
& =-\nabla \cdot[\mathrm{K} \nabla \mathrm{h}]-\nabla \cdot\left[\mathrm{K} \nabla \mathrm{z}^{\prime}\right]
\end{aligned}
$$

But from the geometry of the setup we see that

(3.244) $\mathrm{z}^{\prime}=\mathrm{x} \sin \phi+\mathrm{z} \cos \phi$

Hence

$$
\begin{aligned}
\nabla \mathrm{z}^{\prime} & =\nabla(\mathrm{x} \sin \phi+\mathrm{z} \cos \phi) \\
& =\frac{\partial}{\partial \mathrm{x}}(\mathrm{x} \sin \phi+\mathrm{z} \cos \phi) \text { hat }_{\mathrm{x}}+\frac{\partial}{\partial \mathrm{z}}(\mathrm{x} \sin \phi+\mathrm{z} \cos \phi) \text { hat }_{\mathrm{z}} \\
& =\sin \phi \text { hat }_{\mathrm{x}}+\cos \phi \text { hat }_{\mathrm{z}}
\end{aligned}
$$

so [3.81] may be written

$$
\text { (3.246) } \frac{\partial \mathrm{q}}{\partial \mathrm{t}}=-\nabla \cdot[\mathrm{K} \nabla \mathrm{h}]-\sin \phi \frac{\partial \mathrm{K}}{\partial \mathrm{x}}-\cos \phi \frac{\partial \mathrm{K}}{\partial \mathrm{z}}
$$

We are interested in the steady state, so $\partial \theta / \partial \mathrm{t}=0$, which gives us

$$
\nabla \cdot \mathrm{K} \nabla \mathrm{h}=-\sin \phi \frac{\partial \mathrm{K}}{\partial \mathrm{x}}-\cos \phi \frac{\partial \mathrm{K}}{\partial \mathrm{z}}
$$

To have any hope of solving this problem, we need a functional relationship between pressure and conductivity. For reasons of mathematical convenience, we will use the Gardner (1958) exponential conductivity function,

$$
\begin{aligned}
\mathrm{K} & =\mathrm{K}_{\mathrm{sat}} \exp (\alpha \mathrm{h}) \\
& =\mathrm{K}_{\mathrm{sat}} \mathrm{K}_{\mathrm{rel}}
\end{aligned}
$$

where for convenience we have defined the relative conductivity, $\mathrm{K}_{\text {rel }}$, as

(3.249) $\mathrm{K}_{\mathrm{rel}}=\exp (\alpha \mathrm{h})$

Using [3.86] and [3.87] in [3.85], after factoring $\mathrm{K}_{\text {sat }}$ out from both sides, we find 


$$
\nabla \cdot \mathrm{K}_{\mathrm{rel}} \nabla \mathrm{h}=\sin \phi \frac{\partial \mathrm{K}_{\mathrm{rel}}}{\partial \mathrm{x}}+\cos \phi \frac{\partial \mathrm{K}_{\mathrm{rel}}}{\partial \mathrm{z}}
$$

Now we employ the little trick that [3.87] can be solved for $\mathrm{h}$

(3.251) $\mathrm{h}=(1 / \alpha) \mathrm{LnK}_{\text {rel }}$

Using this identity, the left hand side of [3.88] may be simplified

$$
\begin{aligned}
\nabla \cdot \mathrm{K}_{\mathrm{rel}} \nabla \mathrm{h} & =\nabla \cdot\left[\mathrm{K}_{\mathrm{rel}} \nabla(1 / \alpha) \mathrm{LnK}_{\mathrm{rel}}\right] \\
& =(1 / \alpha) \nabla \cdot\left[\mathrm{K}_{\mathrm{rel}} \nabla \mathrm{LnK}_{\mathrm{rel}}\right] \\
& =(1 / \alpha) \nabla \cdot\left[\mathrm{K}_{\mathrm{rel}} \frac{1}{\mathrm{~K}_{\mathrm{rel}}} \nabla \mathrm{K}_{\mathrm{rel}}\right] \\
& =(1 / \alpha) \nabla^{2} \mathrm{~K}_{\mathrm{rel}}
\end{aligned}
$$

$\ddagger$

Putting this into [3.88], we obtain the governing equation for the problem

$$
\nabla^{2} K_{\mathrm{rel}}-\alpha \sin \phi \frac{\partial \mathrm{K}_{\mathrm{rel}}}{\partial \mathrm{x}}-\alpha \cos \phi \frac{\partial \mathrm{K}_{\mathrm{rel}}}{\partial \mathrm{z}}=0
$$

which holds both above and below the textural interface.

We know that pressure must vary continuously at all points, which holds across the interface. Using subscripts 1 to indicate the upper media, and subscripts 2 to indicate the lower media, at $\mathrm{z}=0$ we know that

(3.254) $\mathrm{h}_{1}(\mathrm{z}=0)=\mathrm{h}_{2}(\mathrm{z}=0)$

(Since $z$ is our only variable, from here on out we will simply put 0 instead of $z=0$ to simplify the equations.) Using [3.89] we then have

$$
\frac{1}{\alpha_{1}} \operatorname{LnK}_{\mathrm{rel} 1}(0)=\frac{1}{\alpha_{2}} \operatorname{LnK}_{\mathrm{rel} 2}(0)
$$

Solving for $\mathrm{K}_{\mathrm{rel1}}(0)$ we find

$$
\mathrm{K}_{\mathrm{rel} 1}(0)=\left[\mathrm{K}_{\mathrm{rel} 2}(0)\right]^{\alpha_{1} / \alpha_{2}}
$$

This will be a useful identity later.

Now, as a stepping stone, let's calculate the pressure profile for this two media system where the interface is horizontal. That is, let's take $\phi=0$. In this special case, the governing equation [3.91] reduces to

$$
\frac{\partial^{2} \mathrm{~K}_{\mathrm{rel}}}{\partial \mathrm{z}^{2}}-\alpha \frac{\partial \mathrm{K}_{\mathrm{rel}}}{\partial \mathrm{z}}=0
$$

Which may be integrated once to yield

$$
\frac{\partial \mathrm{K}_{\mathrm{rel}}}{\partial \mathrm{z}}=\alpha \mathrm{K}_{\mathrm{rel}}-\alpha \mathrm{c}_{0}
$$


where $c_{o}$ is a constant of integration. Separating terms in $\mathrm{K}_{\mathrm{rel}}$ and $\mathrm{z}$

$$
\frac{\partial K_{\text {rel }}}{K_{\text {rel }}-c_{0}}=\alpha \text { partialz }
$$

this may be integrated to yield

$$
\operatorname{Ln}\left(K_{\mathrm{rel}}-\mathrm{c}_{0}\right)=\alpha \mathrm{z}+\operatorname{Ln}\left(\mathrm{c}_{1}\right)
$$

where $c_{1}$ is the second constant of integration. This can be rewritten as

$$
\mathrm{K}_{\mathrm{rel}}=\mathrm{c}_{1} \exp (\alpha \mathrm{z})+\mathrm{c}_{0}
$$

which is the general solution for the system, holding for either the fine or coarse material. Although the solution is written in terms of the relative permeability, it could just as easily be written in terms of pressure, using [3.87]. In each of the layers the two constants may have different values, so we have the values of four constants to determine.

To evaluate the constants we simply need to apply the boundary conditions. Let's start by solving for the medium below the interface. At the interface between the upper and lower media $\mathrm{z}=0$, so we have

$$
\mathrm{K}_{\mathrm{rel} 2}(0)=\mathrm{c}_{2}+\mathrm{c}_{3}
$$

The lower boundary condition is provided by the water table, at $z_{w t}$, where $\Psi=0$ by definition, and thus $K_{\text {rel }}=1$. Here [3.98] becomes

$$
\mathrm{K}_{\mathrm{rel} 2}\left(\mathrm{z}_{\mathrm{wt}}\right)=\mathrm{c}_{2} \exp \left(\alpha_{2} \mathrm{z}_{\mathrm{wt}}\right)+\mathrm{c}_{3}
$$

or

(3.264) $\mathrm{c}_{2}=\left(1-\mathrm{c}_{3}\right) \exp \left(-\alpha_{2} \mathrm{z}_{\mathrm{wt}}\right)$

Putting these results together yields

$$
c_{2}=\left[\exp \left(-\alpha_{2} z_{w t}\right)-K_{\text {rel2 }}(0)\right]\left[\exp \left(\alpha_{2} z_{w t}\right)-1\right]
$$

and

(3.266) $\mathrm{c}_{3}=\mathrm{K}_{\mathrm{rel} 2}(0)-\left[\exp \left(-\alpha_{2} \mathrm{z}_{\mathrm{wt}}\right)-\mathrm{K}_{\mathrm{rel} 2}(0)\right]\left[\exp \left(\alpha_{2} \mathrm{Z}_{\mathrm{wt}}\right)-1\right]$

Looking at [3.101] we see that since the water table is at some large depth, $\mathrm{c}_{2} \approx 0$. Thus what happens is that $\mathrm{K}_{\mathrm{rel}}$ goes from a value of 1 at the water table asymptotically to a value of $K_{\text {rel2 }}(0)$ at the interface. Since the decrease from the water table is exponential, the asymptotic value is reached quite quickly; i.e., within a distance of a few times $1 / \alpha_{2}$. Above this height the value of $K_{r e l}$ is constant. Since $K_{\text {rel }}$ is a function of pressure (see [3.78]), this means that the pressure is also constant. So the only potential gradient driving the flow is gravity. This is often referred to as "unit gradient" flow, since $\partial \mathrm{H} / \partial \mathrm{z}=1$ under these conditions. Unit gradient flow is the norm if the flow is steady and you are considering a spot which is not too close to a water table or change in media (see Figure 3.32). 


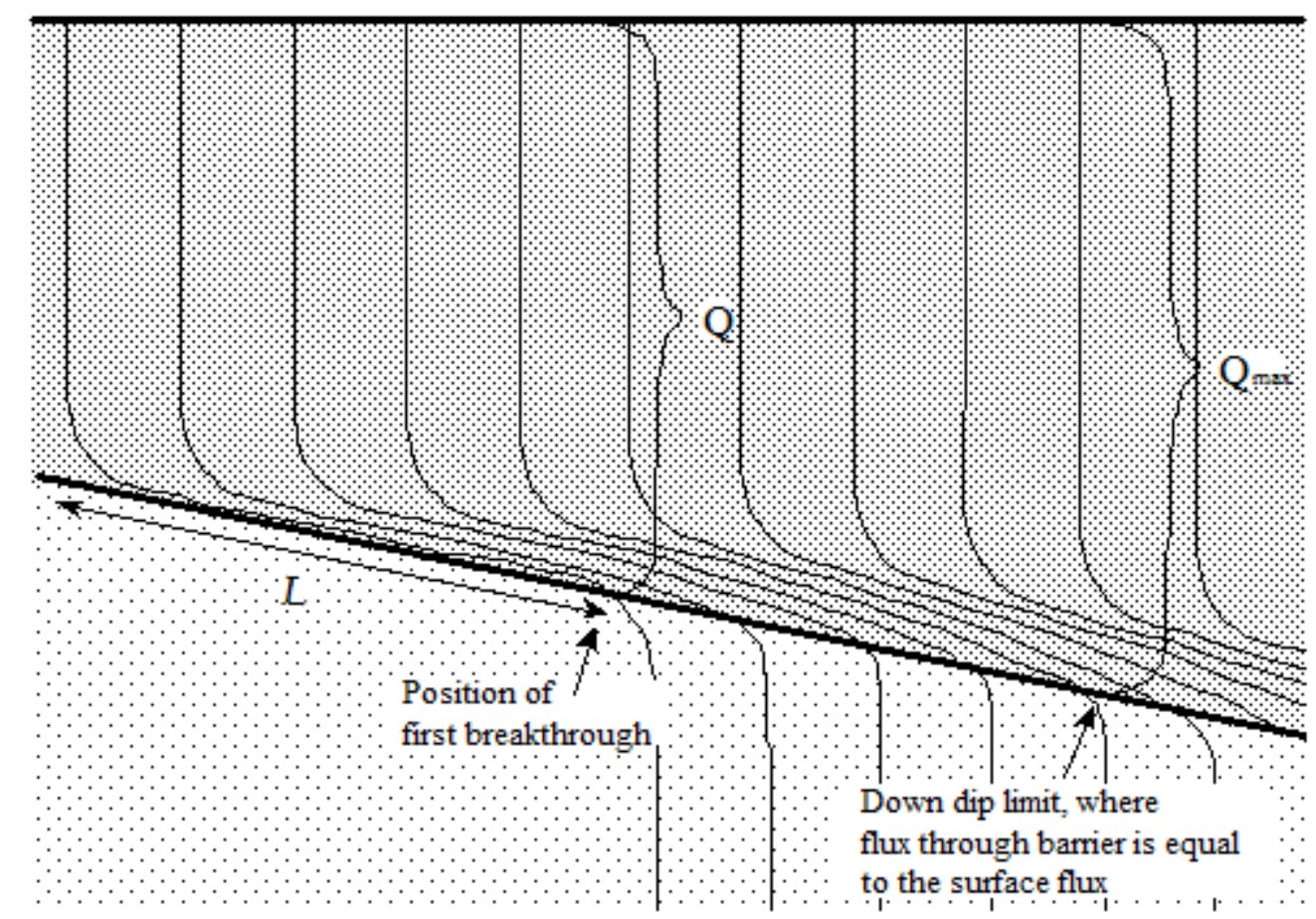

Figure 3.32 Illustration of the transition between the water table and the unit gradient region for the lower media.

So since we are considering steady, one-dimensional flow, the fluxes at all elevations are simply q in the downward direction (otherwise there would be some place where it was either getting wetter or drier in time, which would not qualify as steady-state). We can apply the unit gradient feature and the known flux to solve for the permeability of the coarse material at the interface, $K_{\mathrm{rel} 2}(0)$. From Darcy's Law for the coarse material directly below the interface we then have

$$
\begin{aligned}
\mathrm{q} & =\mathrm{K}_{\mathrm{sat} 2} \mathrm{~K}_{\mathrm{rel} 2}(0) \frac{\partial \mathrm{H}}{\partial \mathrm{z}} \\
& =\mathrm{K}_{\mathrm{sat} 2} \mathrm{~K}_{\mathrm{rel} 2}(0)
\end{aligned}
$$

(noting that we drop the usual minus sign since we have defined $\mathrm{z}$ to be positive in the downward direction) or

$$
\mathrm{K}_{\mathrm{rel} 2}(0)=\frac{\mathrm{q}}{\mathrm{K}_{\mathrm{sat} 2}}
$$

We still need to solve for the constants above the interface. By the same argument as before, the conductivity far above the interface will be constant and in a unit gradient flow regime, so we will have

$$
\mathrm{K}_{\mathrm{rel1}}(\mathrm{zll0})=\frac{\mathrm{q}}{\mathrm{K}_{\mathrm{sat} 1}}
$$

The governing equation for this region is

$$
\mathrm{K}_{\mathrm{rel}}(\mathrm{z})=\mathrm{c}_{1} \exp (\alpha \mathrm{z})+\mathrm{c}_{0}
$$

so from [3.106] we have 


$$
\mathrm{c}_{0}=\frac{\mathrm{q}}{\mathrm{K}_{\mathrm{sat} 1}}
$$

At the interface, $\mathrm{z}=0$, and we find

$$
\mathrm{K}_{\mathrm{rel}}(0)=\mathrm{c}_{1}+\frac{\mathrm{q}}{\mathrm{K}_{\mathrm{sat} 1}}
$$

We are finally ready to use [3.94], which with [3.105] gives us

$$
\mathrm{K}_{\mathrm{rel} 1}(0)=\left[\frac{\mathrm{q}}{\mathrm{K}_{\mathrm{sat} 2}}\right]^{\alpha_{1} / \alpha_{2}}
$$

Putting this into [3.108], we find

$$
c_{1}=\left[\frac{q}{K_{\mathrm{sat} 2}}\right]^{\alpha_{1} / \alpha_{2}}-\frac{q}{K_{\mathrm{sat} 1}}
$$

and thus that for the solution for the fine soil is given by

$$
\mathrm{K}_{\mathrm{rel1}}(\mathrm{z})=\exp \left(\alpha_{1} \mathrm{z}\right)\left[\left(\frac{\mathrm{q}}{\mathrm{K}_{\mathrm{sat} 2}}\right)^{\mathrm{a}_{1} / \mathrm{a}_{2}}-\frac{\mathrm{q}}{\mathrm{K}_{\mathrm{sat} 1}}\right]+\frac{\mathrm{q}}{\mathrm{K}_{\mathrm{sat}}}
$$

Right at the interface, $\backslash \exp \left(\alpha_{1} z\right)=1$, but as you go up, this term dies off quickly (within a few times $1 / \alpha_{1}$ ), after which you are in the upper unit gradient region. Of course, since $\mathrm{K}_{\text {rel }}$ is a one-to-one function of pressure, ( $\underline{3.167)}$ gives an exact solution for pressure above the interface as well.

By now you are probably thinking "we went through all that just to solve for the horizontal case?!?" Fortunately, most of our work is done, since adding the slope is easy. Since we are solving for the down dip limit, there will be no gradients acting in the $\mathrm{x}$-direction, and the vertical component of flux will be q everywhere. This means that our calculations for the lower region are still valid, including the result $\mathrm{K}_{\text {rel2 }}=\mathrm{q} / \mathrm{K}_{\mathrm{s} 2}$. Now looking to the upper layer, the governing equation becomes (from [3.91] where the $\partial() / \partial x$ term is zero in the down-dip limit)

$$
\nabla^{2} \mathrm{~K}_{\mathrm{rel} 2}-\alpha_{1} \cos \phi \frac{\partial \mathrm{K}_{\mathrm{rel} 2}}{\partial \mathrm{z}}=0
$$

by defining a new constant $\alpha^{\prime}=\alpha \cos \phi$, we see that this equation is exactly the equation we solved for the horizontal case! (see [3.95] So we can just use that solution, substituting $\alpha_{1} \cos \phi$ for $\alpha_{2}$ :

$$
K_{\text {rel1 }}(z)=\exp \left(\alpha_{1} \cos \phi z\right)\left[\left(\frac{q}{K_{\text {sat } 2}}\right)^{\alpha_{1} / \alpha_{2}}-\frac{q}{K_{\text {sat } 1}}\right]+\frac{q}{K_{\text {sat1 }}}
$$

So we have the exact solution for conductivity and pressure for the sloping interface. Now we are in a position to calculate the diverted flux. 


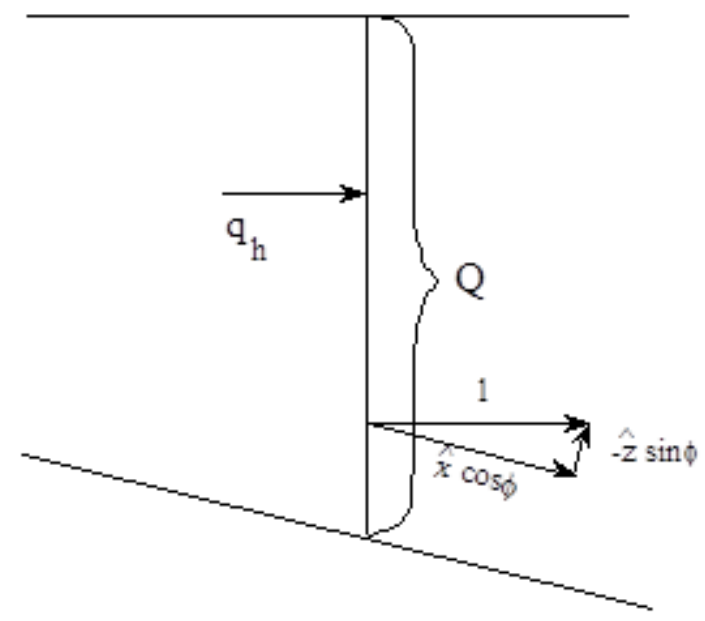

Figure 3.33 Sketch of the vector analysis of the horizontal flux terms

We are interested in the amount of horizontal flux, and will simply apply Darcy's law. The potential gradient is only due to variation in capillary pressure, so we have

$$
\begin{aligned}
\mathrm{qh} & =-\mathrm{K} \nabla\left[\mathrm{y}\left(\mathrm{hat}_{\mathrm{x}} \cos \phi-\mathrm{hat}_{\mathrm{z}} \sin \phi\right)\right] \\
& =-\mathrm{K}_{\mathrm{s} 1} \mathrm{~K}_{\mathrm{rel} 1}(\mathrm{z})\left[\frac{\partial \mathrm{h}}{\partial \mathrm{x}} \cos \phi-\frac{\partial \mathrm{h}}{\partial \mathrm{z}} \sin \phi\right]
\end{aligned}
$$

But we know that there are no gradients in the $\mathrm{x}$-direction, so we have

$$
\mathrm{qh}=\mathrm{K}_{\mathrm{s} 1} \mathrm{~K}_{\mathrm{rel} 1}(\mathrm{z})\left[\frac{\mathrm{dh}}{\mathrm{dz}} \sin \phi\right]
$$

To obtain the total flux, we simply integrate this from $\mathrm{z}=0$ to $\mathrm{z}=-\infty$ where the vertical length differential is $\partial \mathrm{z} / \partial \cos \phi$, or

$$
\mathrm{Q}={ }_{\mathrm{z}=0}^{\mathrm{z}=-\infty} \mathrm{K}_{\mathrm{s} 1} \mathrm{~K}_{\mathrm{rel} 1}(\mathrm{z}) \frac{\mathrm{dh}}{\mathrm{dz}} \sin \phi \frac{\mathrm{dz}}{\cos \phi}
$$

Canceling the dz's and integrating over pressure we obtain

$$
\mathrm{Q}={ }_{\mathrm{h}(-\infty)}^{\mathrm{h}(0)} \mathrm{K}_{\mathrm{s} 1} \mathrm{~K}_{\mathrm{rel1}}(\mathrm{z}) \tan \phi \mathrm{dh}
$$

We now employ the relative conductivity in terms of the capillary pressure

$$
\mathrm{Q}=\mathrm{K}_{\mathrm{s} 1} \tan \phi_{\mathrm{h}(-\infty)}^{\mathrm{h}(0)} \exp \left(\alpha_{1} \mathrm{~h}\right) \mathrm{dh}
$$

which is easily evaluated to yield 


$$
\begin{aligned}
\mathrm{Q} & =\frac{\mathrm{K}_{\mathrm{s} 1} \tan \phi}{\alpha_{1}}\left[\exp \left(\alpha_{1} \mathrm{~h}(0)\right)-\exp \left(\alpha_{1}(0)\right)\right] \\
& =\frac{\mathrm{K}_{\mathrm{s} 1} \tan \phi}{\alpha_{1}}\left[\mathrm{~K}_{\mathrm{rel} 1}(0)-\mathrm{K}_{\mathrm{rel} 1}(-\infty)\right]
\end{aligned}
$$

Using the previously established facts that $\mathrm{K}_{\mathrm{rel1}}(-\infty)=\mathrm{q} / \mathrm{K}_{\mathrm{s} 1}$ and $\mathrm{K}_{\mathrm{rel1}}(0)=\left(\mathrm{q} / \mathrm{K}_{\mathrm{s} 2}\right)^{\alpha_{1} / \alpha_{2}}$, the exact solution of the downdip diversion capacity can be written algebraically in terms of the slope of the interface, the incident flux, and the hydraulic properties of the two soils as

$$
\mathrm{Q}=\frac{\mathrm{K}_{\mathrm{s} 1} \tan \phi}{\alpha_{1}}\left[\left(\mathrm{q} / \mathrm{K}_{\mathrm{s} 2}\right)^{\alpha_{1} / \alpha_{2}}-\mathrm{q} / \mathrm{K}_{\mathrm{s} 1}\right]
$$

A reasonable approximation of this can be obtained by noting that $\mathrm{q}<<\mathrm{K}_{\mathrm{s} 1}$ and that $\mathrm{K}_{\mathrm{rel}}(0) \approx 1$ (that is to say that the upper layer is essentially saturated at the boundary when breakthrough occurs). With these simplifications, [3.120] becomes

$$
\mathrm{Q} \approx \frac{\mathrm{K}_{\mathrm{s} 1} \tan \phi}{\alpha_{1}}
$$

Noting further than $1 / \alpha_{1} \approx\left|h_{\mathrm{a}} \mathrm{e}\right|$, we find

$$
\mathrm{Q} \approx\left|\mathrm{h}_{\mathrm{ae}}\right| \mathrm{K}_{\mathrm{s}} \tan \phi
$$

which is exactly the result we came up with using Darcy's law and a little intuition way back at the beginning of this section (see [3.80])! So why the painful derivation? Well, the various results obtained in the derivation tell us far more about the system, including the lateral flux as a function of height, which is useful in designing capillary barriers, and provides a rigorous foundation for our earlier result. In addition, it should give some confidence that by considering the basic physics of a problem, you will get a reasonable approximate result for such unsaturated flow systems.

From a design perspective, we might want to know the length over which a capillary barrier would be effective at diverting flux. Since the vertical flux is simply q, the total flux diverted over a distance L is simply qL. At the point of breakthrough, $\mathrm{Q}=\mathrm{qL}$, so [3.122] gives us

$$
\mathrm{L} \approx \frac{\left|\mathrm{h}_{\mathrm{ae} 1}\right| \mathrm{K}_{\mathrm{s} 1} \tan \phi}{\mathrm{q}}
$$

and for small angles $\tan \phi \approx \phi$, which gives

$$
\mathrm{L} \approx \frac{\left|\mathrm{h}_{\mathrm{ae} 1}\right| \mathrm{K}_{\mathrm{s} 1} \phi}{\mathrm{q}}
$$

In words, then, the diversion length is inversely related to incident flux, and directly related to the angle of the interface, the water entry pressure, and the saturated conductivity of the upper material. So if we want to design a capillary barrier, how fine should the fine soil be? Do we get more diversion by choosing very fine material, which has a deep saturated region, or coarser material which has a higher conductivity? We can easily determine this using Miller scaling. We know that if $\lambda$ is the characteristic microscopic length scale, that $K_{\mathrm{s} 1}=C \lambda^{2}, h_{\mathrm{ae} 1}=\mathrm{C}^{\prime} / \lambda$, where $\mathrm{C}$ and $\mathrm{C}^{\prime}$ are constants. So for a given flux and angle we have

(3.289) $\mathrm{L}=\mathrm{C}^{\prime \prime} \lambda$

Where C" is a constant. This tells us that choosing a soil with large characteristic particles size (coarse soil) will give proportionally larger diversion. Of course this only holds as long as our simplifying assumptions are valid. One unstated assumption was that the grain size is smaller than $\left|\mathrm{h}_{\mathrm{ae} 1}\right|$, since if this is not true, the water will drain from the media 
rather than flow over even a single grain. So don't let [3.126] make you try using pea gravel for the top layer. We can be fairly sure, however, that coarse sand will give greater diversion than silty soil.

So let's consider a few extensions of Ross' result. First, what about this Gardner conductivity function. We know that while the conductivity does drop off quickly with moisture content, that in terms of pressure, the conductivity should stay near $\mathrm{K}_{\text {sat }}$ until the pressure exceeds the air entry pressure. Based on this reasoning, Ritjema (1965) introduced a modified exponential conductivity function

$$
\begin{array}{ll}
K=K_{\text {sat }} & |h|<\left|h_{\mathrm{ae}}\right| \\
\mathrm{K}=\mathrm{K}_{\mathrm{sat}} \exp \left[\alpha\left(\mathrm{hh}-\mathrm{h}_{\mathrm{ae}}\right)\right. & |\mathrm{h}| \geq\left|\mathrm{h}_{\mathrm{ae}}\right|
\end{array}
$$

Steenhuis et al. (1991) recalculated the Ross result using this conductivity function. Steenhuis et al also added the concept from Kung (1990) that the water will not enter the lower layer until it exceeds the water entry pressure of that layer, $\mathrm{h}_{\mathrm{we} 2}$, and obtain the result

$$
\mathrm{L}<\tan \phi\left[\frac{\left(\mathrm{K}_{\mathrm{s}} / \mathrm{q}\right)-1}{\alpha_{1}}+\frac{\mathrm{K}_{\mathrm{s}}}{\mathrm{q}}\left|\mathrm{h}_{\mathrm{ae} 1}-\mathrm{h}_{\mathrm{we} 2}\right|\right]
$$

Which for $\mathrm{q}<<\mathrm{K}_{\mathrm{s} 1}$ reduces to

$$
\mathrm{L}<\frac{\mathrm{K}_{\mathrm{s}}}{\mathrm{q}} \tan \phi\left[\frac{1}{\alpha_{1}}+\left|\mathrm{h}_{\mathrm{ae} 1}-\mathrm{h}_{\mathrm{we} 2}\right|\right]
$$

By adding the effect of the air entry pressure, the result of Steenhuis et al. (1991) significantly increases the predicted length of capillary diversion (LSteenhuis $\approx 2$ L Ross). Neither of these results has been rigorously tested, although preliminary laboratory results are in agreement with the magnitude of the predictions (Selker, unpublished). The numerical study of Oldenberg (1993) shows earlier breakthrough than predicted by Ross, however the lower boundary condition employed had the water table close to the capillary barrier, which should be expected to reduce the diversion capacity.

The subject of capillary barriers has received increasing attention in recent years. Warrick et al. (1997) extend the analytical solutions beyond simple down-dip limit cases, Webb (1997) extends Ross's result to more general retention functions, Selker (1997) provides optimal capillary barrier solutions based on parabolic barrier interfaces for arbitrary soil retention and conductivity functions, and finally Ho and Webb (1998) provide numerical simulations of heterogeneous capillary barriers.

\section{Preferential Flow Processes}

The term "preferential flow" describes a variety of processes which give rise to a rate of flow which is highly variable within units of media which are homogeneous at the meter-scale. The two classes of preferential flow are macropore flow and fingered flow. Macropore flow refers to movement along pathways such as cracks and wormholes, which are larger than would be suggested by the particle size distribution of the soil. Fingered flow encompasses processes in which flow is broken into isolated pathways of movement through a media which is homogeneous. Fingered flow most often arises due to fundamental instabilities in the process of infiltration, analogous to the dancing tongues of flames coming off a burning log. At present we are not able to predict a priori the occurrence or magnitude of preferential flow processes with significant accuracy using analytical or numerical models. On the other hand, with an understanding of the basis of these flow processes one can reliably identify the sites where each preferential flow processes could contribute to flow and transport. We therefore limit our discussion here to the level of conceptual understanding of the 
processes, providing only limited discussion of the extensive, but as yet still inconclusive, literature which has developed in attempts to predict the effects of preferential flow (e.g., Beven and Germann, 1981; Germann and Beven, 1986; Gish and Shirmohammadi, 1991; Glass and Yarrington, 1996; Hoogmoed and Bouma, 1980; Hutson, and Wagenet, 1995; Mallants et al., 1996; Mohanty, et al., 1997; Ogden et al., 1992; Scotter, 1978; Webb and Anderson, 1996; White et al. , 1984).

The importance of preferential flow is its acceleration of water and contaminant transport through vadose materials. By isolating flow to a small fraction of the volume of the media, the rate of vertical movement can be vastly increased, leading to less sorption, less time for degradation, and bypassing of plant roots. Such processes can make water unavailable for plant utilization, and particularly in the case of pesticides and other man-made contaminants, can lead to contamination of aquifers.

\section{Macropore Flow}

For macropore flow to occur water must enter and flow along pores which are much larger than those created by inter granular pores in freshly packed media. Such flow paths are created in expansive soils which are subjected to cyclic wetting and drying, along the paths of decaying plant roots, by animal activity such as worms, crawfish, and gophers, or by landscape altering events such as subsidence, earthquakes, etc. which may lead to fracturing of the soil and geologic environment (see Luxmore, 1981, and Skopp, 1981 for attempts to rigorously define what macropores are). Clearly most geologic media contain such features. Why are they important to flow? Consider the results for Hagan-Poiseuille flow in capillary tubes derived in Chapter 2. We found that for a given gradient in potential, such as generated by the force of gravity, that the velocity of flow in a tube varied with the square of the tube's diameter, while the total discharge went with the fourth power of tube diameter. Thus if a worm hole is 100 times the size of an inter granular pore, it would carry 10,000 times the flow per unit area as the surrounding soil under gravity driven flow.

So why doesn't all flow in structured soils follow macropore flow paths? For water to flow along these large open channels it must not be pulled by capillary forces into the finer pores surrounding it. This can occur under two conditions: either the surrounding pores are already water filled (i.e., the local pore pressure is near zero); or the flow through the macropore exceeds the rate of loss to the surrounding soil. The first case is common in humid regions during the wet season, when soils are almost saturated for extended periods. The second case requires a very intense water application, which could occur under natural conditions, but is most common in irrigated systems. Thus, macropore flow can occur in a wide variety of settings, but only under specific conditions. The situation is further complicated by the special surface features of macropores. Since the aperture of flow is much larger than the size of the grains that make up the matrix, the macropores are typically conduits for movement of soil particles (translocation). The walls of the macropores will then be lined with layers of particles which entered the macropore and became attached when water was drawn out of the surface into the surrounding media. This layered lining limits the rate of loss of water from the macropore, and creates a unique biological environment for solute sorption and degradation (Pivetz and Steenhuis, 1995).

\section{Fingered Flow}

Hill and Parlange (1972) noted that in when water entered dry coarse-textured soils it tended to move in isolated regions, or fingers of flow. This process was recognized as an instability in the wetting front, and was soon put into the mathematical framework of linear instability theory (Parlange and Hill, 1976), which has since been shown to provide reasonable estimates of the physical dimensions (i.e. width) of these fingers of flow (Glass et al., 1989; Selker et al., 1992; Selker and Schroth, 1998). Our ability to predict finger width is useful in that it confirms that our conceptual model for fingered flow is capturing the physical basis of the process. From this position of confidence, we then can utilize this result to see where fingers are likely to be prominent. The size of fingers is related inversely to the characteristic grain 
size of a medium (Figure 3.34): when the soil has a texture of silt or finer, the finger dimensions are predicted to be $>1 \mathrm{~m}$. Fingered flow occurs only in unstructured soils. Hence, fingered flow is expected only in soils which are predominantly sand.

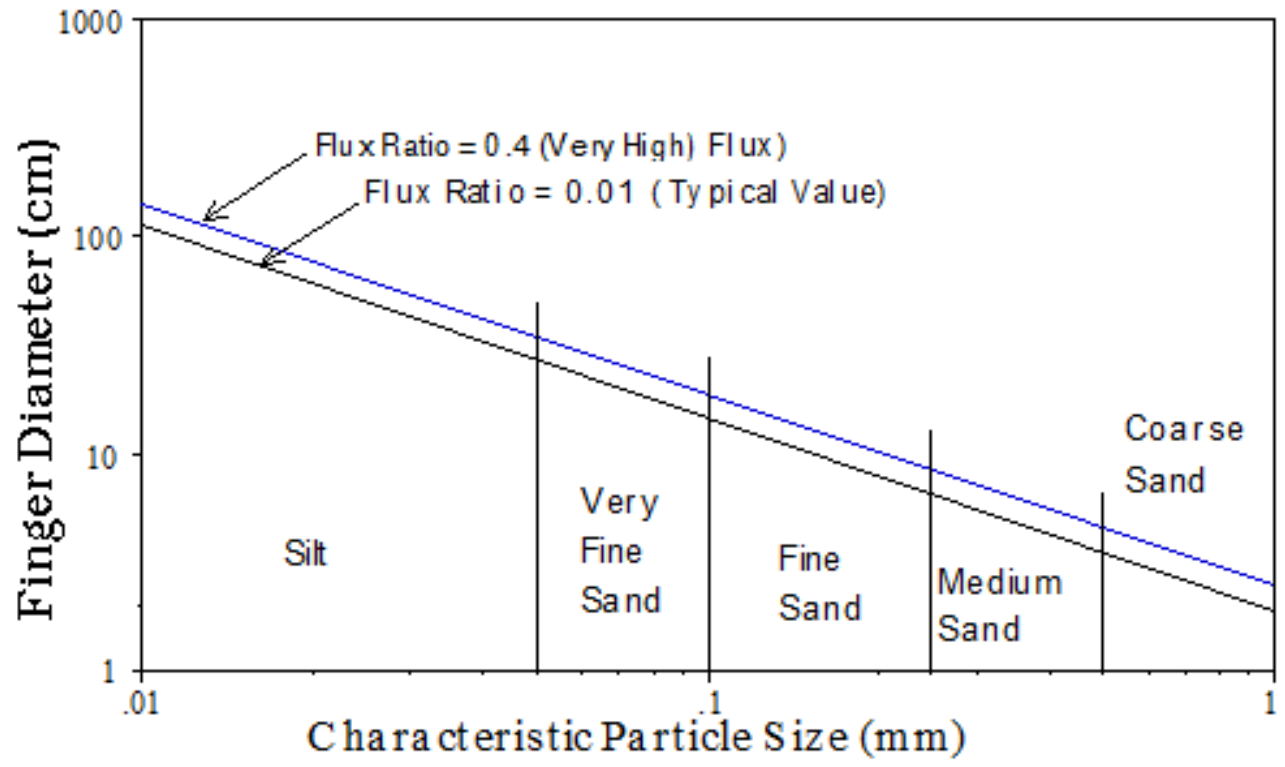

Figure 3.34 Dependence of finger width on the characteristic grain size of a soil and the flux ratio $(q / \mathrm{Ks})$ into the system (using the results of Parlange et al., 1990).

Finger width is not strongly affected by the flux through the system so long as the rate of infiltration is well below the saturated conductivity of the system (Figure 3.34). Rather, the flux through the system typically affects the number of fingers which form. When the flux is increased up to the rate of the saturated conductivity of the soil, the fingers will grow in width and frequency to the point where they finally merge to yield a flat wetting front without fingered properties. Since most sandy soils have extremely high conductivity compared to naturally occurring infiltration rates, this rarely occurs in natural conditions.

Understanding effects of prior moisture content on finger formation and finger persistence is important in managing production on sandy soils. As shown by Lui et al. (1993), when soil is at field capacity, fingers will be approximately 10-fold wider than those developing in dry conditions. Thus, if a sandy soil is not allowed to dry completely, the bypassing effect will be reduced, if not entirely eliminated.

Once a finger has formed in a particular location, it will remain (persist) until the soil has either dried entirely, or has been completely saturated (Glass et al., 1989). Given the very high conductivity of sandy soils, elimination of persistent fingers requires either drying from the surface, which is effective to a maximum depth of about 1 meter, or raising the water table. A striking example of the impact of persistence was seen in Laffeyette, Indiana (Figure 3.35). Distinct fingers of flow were made visible in the coarse material due to the chemical weathering of the calcareous material. Within the fingers, pebbles were readily crushed manually, while outside of the fingers the gravel strength was typical of unweathered material. Clearly these fingers had persisted for many decades.

Figure 3.35 Dr. R. Bryant stands next to glossic features in a soil believed to have been formed as a result of fingered flow which persisted for periods of hundreds, if not thousands, of years. The apparent difference in coloration in the fingers is not due to moisture content, but results from the extensive chemical weathering of the material within the fingers. 


\section{Solution Sampling and Flux Measurement}

Monitoring of water and contaminant movement in the vadose zone requires instrumentation capable of measuring flux and solute concentration. We will briefly discuss the methods which have been employed, and their advantages and limitations. Three methods have been used widely in this capacity: weighing lysimetry, pan lysimetry, and suction cup lysimetry.

The most precise measurements of rates of evapotranspiration and percolation are achieved using weighing lysimeters. Weighing lysimeters consist of a block of soil contained in an impermeable and mechanically isolated housing which is placed on a weighing device (mechanical scale or load cell) (Figure 3.35). A drainage port is typically located in the bottom of the housing to allow monitoring of percolation volume and quality. Drainage can be enhanced through application of negative water potentials at the lower boundary using vacuum applied to porous tubes, or capillary wicks. The size of the soil block employed in such a setup depends upon the application, with many of those in present use being large enough to contain an entire fruit tree without undue restriction to its roots (Phene et al., 1991). For many applications the cost of weighing lysimeters is prohibitive, typically being on the order of $\$ 25,000$ per installation. Significant cost savings, reducing the per-unit cost to a few thousand dollars, can be achieved through use of load cells in non-accessible mountings (as opposed to set-ups where the scale system is accessible for maintenance).

A typical weighing lysimeter is sufficiently sensitive to be able to detect changes in water content equivalent to a gain or loss of $0.01 \mathrm{~mm}$ of water depth over the soil surface. This makes these devices ideally suited to monitoring of the temporal characteristics of consumptive water use as required to generate crop water use curves. Lysimeters have also been built which are too large to be weighed, but instead are interrogated for water distribution by means of neutron probes or other water content measurement tools. The precision of such systems is far lower than that of the weighing lysimeter.

In many cases weighing lysimeters are impractical, but one still requires knowledge of water and solute flux passing a certain elevation within the vadose zone. In this case pan lysimeters are often the method of choice. A pan lysimeter consists of an impermeable horizontal sheet inserted into a vadose zone which intercepts percolating water. The pan is drained to a collection vessel. By monitoring the time rate of water collection, water movement can be documented, and through analysis of the collected sampler one may obtain information regarding the presence and concentration of dissolved compounds.

Figure 3.35 Typical weighing lysimeter setup (taken with permission from Phene et al., 1991)

Until recently, pan lysimeters have been used in either a zero-tension passive collection mode, or in an active-tension collection mode using a porous plate. The zero-tension mode has been shown to significantly under-sample vadose water (e.g., Jamison and Fox, 1992), and that the collected water misrepresent the quality and quantity of the true recharge due to under-sampling of matrix flow (Steenhuis et al., 1995). The active collection mode provides continuous vacuum to the device, but the magnitude of the vacuum needs continuous adjustment to match temporal variations in soil water pressure potential. In many circumstance, this degree of monitoring and control prohibits its use in many field settings, and reliability is a major difficulty.

The recent introduction of wick pan samplers, whereby soil water is extracted through the application of tension through a hanging wick, has provided a cost-effective and alternative method of soil solution sampling. The concept of the technique was introduced by Brown et al. (1986), and has been refined through extensive laboratory and field experiments in the past few years. Key advantages are that the samplers operate in a passive mode, and that the applied tensions are designed to match a broad range of soil water fluxes. Recent advances in the wick sampler technique include wick selection design equations (Boll et al. 1992; Knutson and Selker, 1994; Rimmer et al., 1995a), wick preparation methods (Knutson et al., 1994), wick influence on sample concentration due to dispersion (Boll et al., 1992; Polletica et al., 1992; Knutson and Selker, 1996), and laboratory experiments evaluating wick performance (Rimmer et al. 1995b). Field evaluations of wick samplers have shown that they are most effective in soils with significant transport occurs with water at pressures of -0.1 to $-1.5 \mathrm{~m}$ pressure, which covers a broad range of soil types from sandy to silt loam (Boll et al., 1991; Holder et al., 1991; Brandi-Dohrn et al., 1996b). Sampling from soils of clay texture is not improved through use of 
wicks (Steenhuis et al., 1995) in comparison to zero-tension samplers. Although the development of wick samplers adds an important new set of capabilities, they are still expensive to build and install (about $\$ 500$ per sampler). There is still a great need for additional reliable and cost-effective methods for the measurement of solute concentration and water flux in the vadose zone.

\section{Methods of Media Characterization}

Throughout this chapter we have discussed the parameters which are used to describe the static and dynamic character of fluid movement in porous media. To apply these concepts to real soils, the first step is to obtain a reliable measurement of these parameters. Quantities of interest include fluid content, sorptivity, conductivity, porosity, and possibly issues of electrical conductivity and dielectric. Such measurements are often challenging to carry out, and the development of more robust and reliable techniques is an area of active research. In the following sections we present a brief introduction to several of the more important methods. For a comprehensive, if some what dated, discussion of these and other techniques, one may refer to several handbooks available (e.g., Klute, 1986; Carter, 1993, and the series of publications by the ASTM).

\section{Measurement of Pore Water Potential}

Water movement through unsaturated media is controlled by gradients in potential, which are typically dominated by gravity and hydraulic pressure. While determination of the gravitational potential is simply a matter of measuring elevation, measurement of soil water pressure potential in unsaturated media continues to present significant practical difficulties. Three pressure measurement approaches have been implemented widely: (1) direct measurement in a body of water isolated from the soil by a rigid porous membrane (tensiometer), (2) indirect measurement of pressure via measurement of the energy state of a calibrated medium (e.g., gypsum block), and (3) indirect measurement of pressure via measurement of relative humidity of the vapor phase (psychrometer). We will briefly describe each of these methods, and present some of the recent advances that have taken place in these technologies.

The tensiometer is a device which creates a continuous water film between the soil water and a pressure gauge (Figure 2.36?). The water within the gauge and that in the soil are separated by a rigid porous membrane that allows water to pass through unobstructed, but with pores sufficiently fine that gas cannot enter (recall Laplace's equation for the pressure across gas-liquid interfaces).

(a) (b)

Figure 3.36 Components of (a) tensiometer and (b) porous cup sampler.

Some of the interesting recent improvements in tensiometers have been directed toward reduction of the response time and increasing the range of pressures over which they can be used. Reduction of response time has been achieved through careful de-airing of the filling solution (water), and the application of solid-state pressure transducers, which both have reduced response times to one second or less (Selker et al., 1991). It has long been known that pure water can support negative pressures in excess of 1 bar while remaining in the liquid state. Recent experimental studies have shown that the maximum attainable value is greater than 140 Bar (Zheng et al., 1991), yet liquid-filled tensiometers are widely assumed to fail at $1 \mathrm{bar}$, due to the dissolution of gas from the water in the tensiometer filing solution. Recent studies by Miller and Salehzadeh (1993) and Tamari et al. (1993) present tensiometers which measure pressures in excess of 1 bar through removal of dissolved gas. These techniques are feasible for both laboratory and field measurements, as shown by Morrison and Szecsody (1987).

If one were to remove the gauge from the tensiometer and apply a strong negative pressure at this port, water from the soil would be drawn into the device, providing a soil water sampling tool. Such devices are widely use, and are 
called porous cup samplers (Figure 2.36?). When tensiometric measurements are required, soil solution samples are also often of interest. Morrison and Szecsody (1987) introduced the notion of utilizing a single porous cup device for the joint application of solution sampling and soil water potential measurement. Tokunaga (1992) explored the same concept further and provided an explicit method to determine the time required for each measurement to maintain independence, and demonstrated the application of such a device under several field conditions. Essert and Hopmans (1994) present yet another device, where a single probe allows the simultaneous measurement of soil water potential and extraction of soil solution. This was achieved by divided the traditional tensiometer into two separate compartments. The concept of combined solution sampling and pressure measurement was pushed one step further by Baumgartner et al. (1994), who devised a porous stainless steel Time Domain Reflectometry (TDR) probe which allowed the simultaneous measurement of pressure and moisture content, and can be used for the extraction of solution samples as well.

Indirect measurement of low soil water pressure potentials can be achieved through observation of the energy state of a calibrated medium such as a gypsum block. The notion here is that if a stable porous medium is put into contact with a natural porous medium, the water pressure of the two media will come into equilibrium. By measuring the water content of the stable porous medium, and relating this to a known characteristic curve for this medium, one can infer the pressure of the system. The moisture content of the instrument medium is inferred by measuring its thermal conductivity, electrical conductivity, or heat capacity. This approach suffers from several difficulties which render it only useful for qualitative observation of soil moisture. Most fundamentally, all capillary media is subject to hysteresis in the water content pressure characteristic relationship. Therefore, even if one were able to exactly measure the moisture content of the instrument medium, the pressure of water within the device (and therefore in the surrounding soil) are only known within the span of the hysteretic retention curve of the medium. Other difficulties include fouling of the medium due to clay translocation and microbial activity, each of which will alter the instrument's pressure water content characteristic function. Despite these difficulties, innovation in this area continues to yield useful tools. For instance, a novel optical sensing method was introduced by Cary et al. (1989a) in which the opacity of a porous block was measured using a light emitting diode-phototransistor pair. This technique is based on the dependence of light transmission of the block on fluid saturation (Hoa, 1981). The method was shown to be useful in the detection of both water (in fritted glass) and oil (in porous polyethylene).

Although in theory tensiometers may be used to measure pressures beyond one Bar, as discussed above, the practical difficulties become severe. The most widely adopted method for monitoring pressures <-1 Bar is the Psychrometer. Recall the psychometric Equation [2.52] which relates the measured vapor pressure to water potential. This relationship can be exploited experimentally by observing the rate of decline in temperature of a Peltier cooled junction inserted into a medium (Spanner, 1951; Rawlins and Campbell, 1986). When the temperature passes through the dew point, the rate of cooling decreases as local water vapor condenses on the probe. Comparing the dew point temperature to the medium temperature allows immediate calculation of the relative humidity. The many practical issues surrounding implementation of such a measurement are presented by Rawlins and Campbell (1986). Recently Gee et al. (1992)

introduced the use of a chilled mirror psychrometer which greatly expedites the measurement of humidity for laboratory samples.

\section{I2 Field Methods for Estimating Soil Hydraulic Functions}

It is imperative to measure soil hydraulic properties in the field. The main reason is that small samples used in the lab may not represent the conditions in the field accurately, particularly for hydraulic conductivity determination. Soil cores may not adequately represent the true macroporosity of the bulk soil, for example because of dead-end pores resulting from the finite sample volume, and the sampling may alter the structure of the soil matrix, resulting in biased estimates of conductivity. Realistic modeling and prediction of water flow and solute transport is therefore dependent upon reliable and representative estimates of soil hydraulic properties inferred from field experiments and measurements 
made at scales larger than that of small lab cores. In the following section we discuss several methods for inferring soil hydraulic properties, with emphasis on saturated and unsaturated soil hydraulic conductivity.

\section{I2.I The Instantaneous Profile Method}

The instantaneous profile method (Watson, 1966) is based on simultaneous monitoring of changes in soil water content and matric potential within a soil profile that was initially saturated and is undergoing internal drainage. The soil surface which represents the top boundary of the soil profile is covered and insulated to prevent evaporation and thermal gradients. The measurements and the controlled boundary conditions allow the application of the mixed form of the Richards equation in a discrete form. The equation is rearranged to provide direct estimates of the unsaturated hydraulic conductivity using measurements of other attributes:

$$
\int_{0}^{-\mathrm{L}} \frac{\partial \theta}{\partial \mathrm{t}} \mathrm{dz}=\left.\mathrm{K} \frac{\partial \mathrm{H}}{\partial \mathrm{z}}\right|_{\mathrm{z}=-\mathrm{L}}-\left.\mathrm{K} \frac{\partial \mathrm{H}}{\partial \mathrm{z}}\right|_{\mathrm{z}=0}
$$

Since there is no flow across the plastic cover, the second term on the right hand side (RHS) is zero. The changes in water content between two consecutive measurements taken at two times, $\mathrm{t} 1$ and $\mathrm{t} 2$, are integrated or algebraically summed from the soil surface $\mathrm{z}=0$ to a desired depth, $\mathrm{z}=-\mathrm{L}$, and the corresponding changes in the matric potential are measured (see Fig.2-18 for the measurement scheme). The working equation is:

$$
\frac{\int_{0}^{-\mathrm{L}} \theta\left(\mathrm{z}, \mathrm{t}_{2}\right) \mathrm{dz}-\int_{0}^{-\mathrm{L}} \theta\left(\mathrm{z}, \mathrm{t}_{1}\right) \mathrm{dz}}{\mathrm{t}_{2}-\mathrm{t}_{1}}=\left.\mathrm{K}(\bar{\theta}) \frac{\partial \mathrm{H}}{\partial \mathrm{z}}\right|_{\mathrm{z}=-\mathrm{L}}
$$

where the hydraulic gradient is an average gradient calculated at two different times across the control plane $\mathrm{z}=$ $-\mathrm{L}$, and $\mathrm{K}(\mathrm{q})$ is related to the average water content between $\mathrm{t} 1$ and $\mathrm{t} 2$ at the profile between 0 and $-\mathrm{L}$, or between two control planes in the profile with known boundary conditions. Hence a tensiometer should be installed at known depths above and below the plane $\mathrm{z}=-\mathrm{L}$ to obtain the mean hydraulic potential gradient. The term on the RHS of Eq.(122) is an approximation of the flux that flows through $\mathrm{z}=-\mathrm{L}$. The flux is divided by the hydraulic gradient on the LHS to obtain the unsaturated hydraulic conductivity K(q):

$$
K(\bar{\theta})=\frac{\int_{0}^{-\mathrm{L}}\left[\theta_{\mathrm{i}+1}(\mathrm{z})-\theta_{\mathrm{i}}(\mathrm{z})\right] \mathrm{dz}}{\left(\mathrm{t}_{\mathrm{i}+1}-\mathrm{t}_{\mathrm{i}}\right)\left(\frac{\partial \overline{\mathrm{h}}}{\partial \mathrm{z}}+1\right)}
$$

where $\mathrm{q}=[\mathrm{qi}+1(-\mathrm{L})+\mathrm{qi}(-\mathrm{L})] / 2$, and the hydraulic gradient is averaged similarly. 


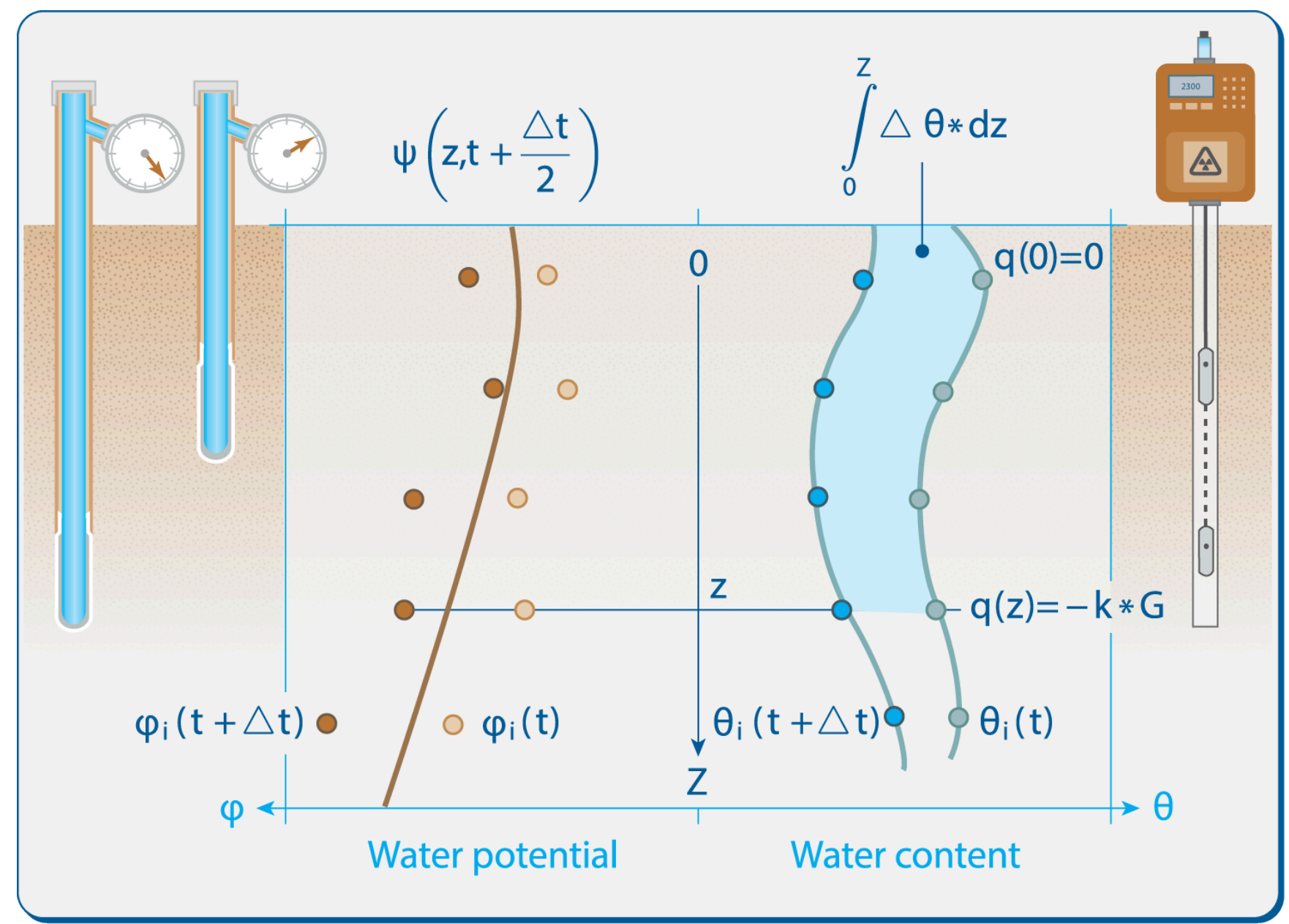

Figure 3.37: A scheme of the Instantaneous Profile method calculations (modified from Flühler et al., 1976).

An experimental plot for application of the instantaneous profile method should be large enough to ensure that measurements made at its center are unaffected by the conditions at the lateral boundaries; a plot of a few m on each side should suffice. There are several limitations to this method: (i) the soil should be quite homogeneous; otherwise the use of "noisy" data with the Richards equation for an assumed uniform soil may result in unreasonable estimates of K(q), (ii) the burden of measurements is heavy, especially at the initial stages where measurements should be taken frequently enough to capture the rapid changes in water status. A study to analyze and quantify the errors associated with the method was presented by Flühler et al. (1976).

\section{Unit Gradient Internal Drainage}

A simpler version of the instantaneous profile method is based on the assumption of a unit hydraulic gradient, i.e., , which may develop when the soil profile is homogeneous and water content is uniform. The matric potential under these conditions is expected to be similar everywhere, and the only contribution to a gradient in hydraulic head is gravitational and thus $\mathrm{dH} / \mathrm{dz}=\mathrm{dz} / \mathrm{dz}=1$. Under such conditions we may write the following equation:

$$
\mathrm{K}(\bar{\theta})=\mathrm{L}\left(\frac{\mathrm{d} \bar{\theta}}{\mathrm{dt}}\right)
$$


where is the average water content in the profile above $\mathrm{z}=-\mathrm{L}$ (during $\mathrm{t}$ and $\mathrm{t}+\mathrm{dt}$ ). Other methods based on the assumption of a unit hydraulic gradient have also been developed (Klute, 1986).

\section{I2.2 Infiltrometers and Permeameters}

Many field methods for measurement of Ks and $\mathrm{K}(\mathrm{q})$ or $\mathrm{K}(\mathrm{h})$ are based on conducting controlled infiltration experiments with known flow geometry, intake area, flow rate, and boundary conditions. Knowledge of these flow variables enables the application of various solution techniques to infer the unknown hydraulic properties Ks and $\mathrm{K}(\mathrm{q}, \mathrm{h})$ from the results of the experiment, e.g. from the changes in flux with time, or from final flux rate. Devices that are designed to facilitate such experiments by confining the flow to a certain geometry, or boundary conditions, or provide other information on the various flow attributes are called infiltrometers, or permeameters if designed specifically for measuring hydraulic conductivity. One of the most common infiltrometer designs for one-dimensional flow is the Double-ring Infiltrometer.

The double-ring infiltrometer (Swartzendruber and Designs, 1961) consists of two thin-walled metal cylinders. The two cylinders are concentrically placed and driven into the soil to a depth of 5 to $10 \mathrm{~cm}$ (Fig.2-19). Water is ponded to the same shallow depth in both the inner and outer rings. The flow in the inner ring is presumed to be one dimensional with vertical streamlines. Flow from the outer ring may diverge laterally due to "edge effects" (Bouwer, 1986). The idea behind such a design is to establish 1-D flow conditions for the inner ring, while satisfying edge effects and lateral flow by the presence of the outer ring. 


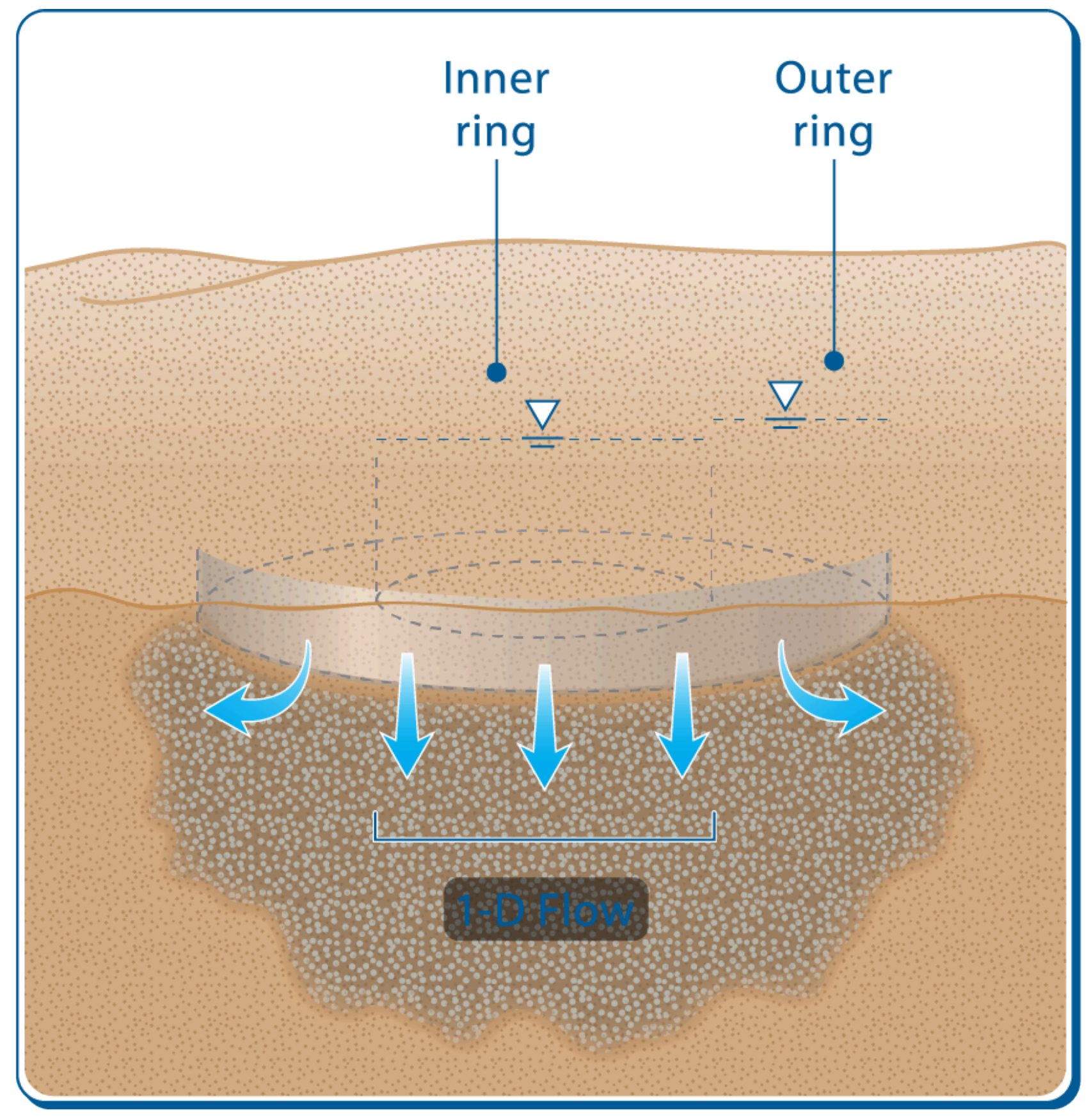

Figure 3.38: Sketch of a double-ring infiltrometer

Water flow rate vs. time from the inner ring is monitored by means of a Mariotte flask or other constant head water supply until it reaches a constant value. The assumption is that the soil layer immediately below the ponded area is fully saturated and thus the matric potential is essentially zero. Under these conditions the hydraulic gradient is unity which presumably renders the final infiltration rate (final flux) equal to the soil's saturated hydraulic conductivity, Ks:

$$
\mathrm{i}_{\mathrm{f}}=-\mathrm{K}_{\mathrm{s}} \frac{\Delta(\mathrm{h}+\mathrm{z})}{\Delta \mathrm{z}} \approx-\mathrm{K}_{\mathrm{s}} \frac{\Delta \mathrm{z}}{\Delta \mathrm{z}} \approx-\mathrm{K}_{\mathrm{s}}
$$


However, as we have seen from Philip's analyses (Eq.(117)) the long-time flux if is actually a fraction of Ks, bounded by $1 / 3 \leq$ if $/ \mathrm{Ks} \leq 2 / 3$; a value of if $/ \mathrm{Ks}=0.5$ is usually a good assumption for practical application of this technique. 
Disc Permeameter and the Dripper Method:

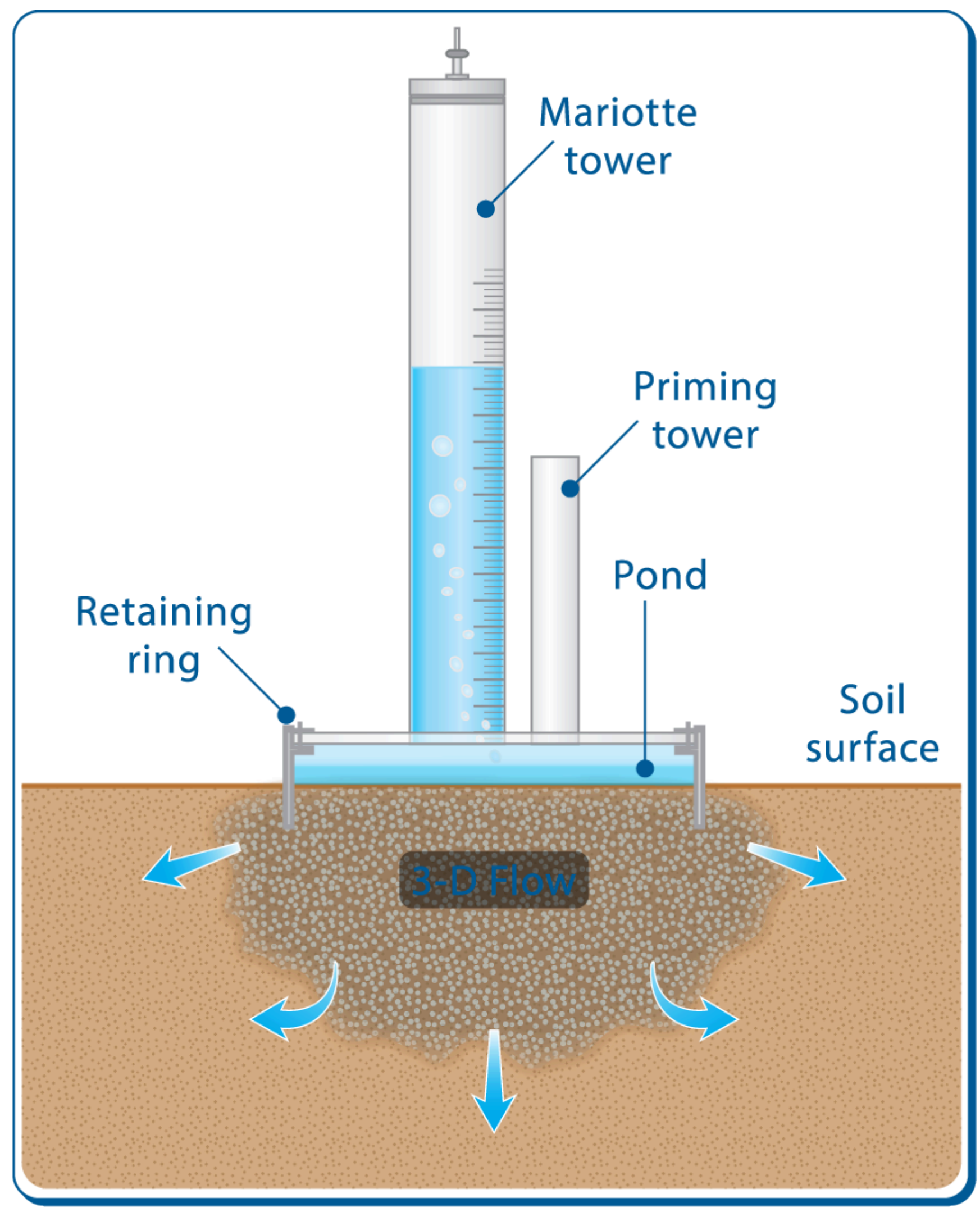

Figure 3.39: Sketch of a disc permeameter based on Wooding's (1968) solution 
Wooding's (1968) approximate solution to three-dimensional infiltration from a circular shallow pond facilitated infiltration experiments which use a single disk-shaped water source to infer the soil's Ks and K(h). Disc-shaped permeameters (Perroux and White, 1988) allow for application of water under saturated and unsaturated (i.e., negative pressure at the supply inlet) conditions for field application of Wooding's solution. In its simplest design (Fig.2-20), the disk permeameter consists of a metal ring having a radius of 10 to $15 \mathrm{~cm}$, and about 3 to $5 \mathrm{~cm}$ in height. The ring is pushed into the soil to a depth of slightly less than $1 \mathrm{~cm}$. A graduated Mariotte tower is placed over the metal ring to supply water to the soil surface inside the ring at a constant head and at measurable rates. The time-dependent infiltration rate $\mathrm{i}(\mathrm{t})$ is determined from the time-dependent changes in the height $(\mathrm{h})$ of water in the graduated Mariotte tower, and the cross-sectional soil area Asoil, as $\mathrm{i}(\mathrm{t})=$ Vwater $/$ Asoil $=(\mathrm{Dh} \cdot$ Atower $) /$ Asoil. In addition, initial and final water contents (qi, and $\mathrm{qf}=\mathrm{qs}$ ) at the soil surface must be measured or estimated.

Analysis of the results involve a series of assumptions and steps. A primary assumption is that the infiltration rate at very short times may be approximated by Philip's solution for 1-D infiltration at short times, i.e., I = St1/2. The main steps in the analysis of $i$ vs. $t$ include: (1) approximating the sorptivity (S) from the slope of I vs. $t 1 / 2$ for the first several measurements over a short time; then (2) approximating the ratio Ks/b using:

$$
\frac{\mathrm{K}_{\mathrm{s}}}{\beta} \approx \frac{\mathrm{bS}^{2}}{\left(\theta_{\mathrm{f}}-\theta_{\mathrm{i}}\right)}
$$

where $b$ is a shape parameter bounded between $1 / 2$ and $p / 4$. A value of $b=0.55$ is suitable for many field soils; (3) Ks is estimated using Eq.(120):

$$
\mathrm{K}_{\mathrm{s}}=\mathrm{i}_{\mathrm{f}}-\frac{4 \mathrm{bS}^{2}}{\pi \mathrm{r}_{\mathrm{s}}\left(\theta_{\mathrm{f}}-\theta_{\mathrm{i}}\right)}
$$

Finally, b is estimated from Eq.(126) using the value of Ks resulting from Eq.(127) as:

$$
\beta=\frac{\left(\theta_{\mathrm{f}}-\theta_{\mathrm{i}}\right) \mathrm{K}_{\mathrm{s}}}{\mathrm{bS}^{2}}
$$

Another method for estimating soil hydraulic properties based on Wooding's approximation was developed by Shani et al. (1987), in which the flux (if) rather than the saturated radius (rs) is maintained constant. This is known as the dripper method. A dripper having a fixed discharge rate $(\mathrm{Q})$ is used while monitoring changes in the saturated radius of ponding on the soil surface. After a period of time, which is dependent upon the soil type, initial conditions, and dripper discharge, rs reaches a final size corresponding to its steady state value. The measurement is then repeated with a different discharge rate (Q). Knowledge of several values of rs vs. if (=Q/prs2) enables solution of Eq.(120) by linear regression of if vs. 1/rs. The intercept of the regression line is Ks, and b is computed from the slope of the line (s) as: $\mathrm{b}=4 \mathrm{Ks} / \mathrm{sp}$.

\section{Example 2-13): Disc Permeameter (Measurement and Analysis)}

\section{Problem Statement:}

A disc permeameter was used to measure the surface hydraulic properties of a sandy soil in Goshute Valley in eastern Nevada. The diameter of the metal ring at the soil surface was $210 \mathrm{~mm}$, and that of the cylindrical water supply tower was $57 \mathrm{~mm}$. The initial and saturated water contents were qi $=0.07 \mathrm{~m} 3 / \mathrm{m} 3$, and qs $=0.38 \mathrm{~m} 3 / \mathrm{m} 3$. The following measurements of water height in the supply tower versus elapsed time where acquired: 


\begin{tabular}{llllll}
\hline Time $[\mathrm{sec}]$ & Height $[\mathrm{cm}]$ & Time $[\mathrm{sec}]$ & Height $[\mathrm{cm}]$ & Time $[\mathrm{sec}]$ & Height $[\mathrm{cm}]$ \\
0 & 0.0 & 1846 & 5.0 & 3952 & 9.0 \\
22 & 1.0 & 2040 & 5.5 & 4480 & 10.0 \\
140 & 2.0 & 2362 & 6.0 & 5044 & 11.0 \\
810 & 3.0 & 2609 & 6.5 & 5623 & 12.0 \\
1068 & 3.5 & 2877 & 7.0 & 6210 & 13.0 \\
1326 & 4.0 & 3126 & 7.5 & 6813 & 14.0 \\
1588 & 4.5 & 3407 & 8.0 & 7404 & 15.0 \\
\hline
\end{tabular}

(1) Find the sorptivity assuming I = St1/2 for short times (use the first few measurements)

(2) Determine the steady state infiltration rate (use the last 5-8 measurements)

(3) Determine Ks and b for this soil.

Solution:

(1) First we calculate the cumulative infiltration volume I for each time step according to the following equation:

$$
I=\frac{\left(\frac{d_{\mathrm{s}}}{2}\right)^{2} \pi \mathrm{h}}{\left(\frac{\mathrm{d}_{\mathrm{r}}}{2}\right)^{2} \pi}
$$

where ds is the diameter of the supply tower, $\mathrm{dr}$ is the diameter of the metal ring, and $\mathrm{h}$ is the water height in the supply tower.

(2) For each time step we take the square root of the elapsed time (t1/2) and plot the values against the cumulative infiltration. This data set is the basic input for the determination of sorptivity.

(3) To find the sorptivity we take the first three data pairs (22, 140, and $810 \mathrm{sec})$ and perform a regression analysis using the statistical tools provided in most computer spreadsheets (e.g. Excel, Quattro Pro). Since we have a linear relationship between I and $\mathrm{t} 1 / 2$ the sorptivity is simply the coefficient (slope) of the regression line (the intercept is zero in this case).

$$
\mathrm{I}=\mathrm{St}^{1 / 2} \Rightarrow \mathrm{y}=\overbrace{a}^{\text {coefficient }} \mathrm{x}+\overbrace{b}^{\text {constant }}
$$

(4) To determine the steady state infiltration rate we perform a regression analysis with the last 8 data pairs of I versus time ( $\mathrm{t}$ ). The regression coefficient gives the steady state infiltration rate.

(5) With known steady state infiltration rate if and sorptivity $\mathrm{S}$ we now can calculate Ks and b using the following relationships:

$$
\mathrm{K}_{\mathrm{s}}=\mathrm{i}_{\mathrm{f}}-\frac{4 \mathrm{bS}}{\pi \mathrm{r}_{\mathrm{s}}\left(\theta_{f}-\theta_{\mathrm{i}}\right)}
$$


where $b$ is a shape parameter ( $b=0.55$ is suitable for most field soils), rs is the saturated radius (radius of the metal ring), and qi and qf are the initial and saturated water contents

\section{Tabulated Calculation:}




\begin{tabular}{|c|c|c|c|c|c|c|c|}
\hline $\begin{array}{l}\text { Measureme } \\
\text { nts }\end{array}$ & on & & & & & & \\
\hline $\begin{array}{l}\text { Time } \\
{[\mathrm{sec}]}\end{array}$ & $\begin{array}{l}\text { Height h } \\
{[\mathrm{cm}]}\end{array}$ & $\mathrm{e}_{\mathrm{n}]^{[\mathrm{mi}}}^{\operatorname{Tim}}$ & $\begin{array}{l}\mathrm{e}^{1 / 2} \operatorname{Tim}^{\operatorname{Tim}} \\
\left.{ }^{[/ 2}\right]\end{array}$ & $\begin{array}{l}\text { Hei } \\
\text { ght h } \\
]^{[\mathrm{mm}}\end{array}$ & $\begin{array}{c}\text { Cumulative } \\
\text { infiltration [mm] }\end{array}$ & $\begin{array}{l}\text { Regression line } \\
\text { for sorptivity }\end{array}$ & $\begin{array}{l}\text { Regression line for } \\
\text { infiltration rate }\end{array}$ \\
\hline 0 & 0.0 & $0^{0.0}$ & $0^{0.0}$ & 0 & 0.000 & 0.000 & \\
\hline 22 & 1.0 & $7^{0.3}$ & 0.61 & 10 & 0.737 & 0.404 & \\
\hline 140 & 2.0 & $3^{2.3}$ & 1.53 & 20 & 1.473 & 1.020 & \\
\hline 810 & 3.0 & $0^{13.5}$ & 3.67 & 30 & 2.210 & 2.454 & \\
\hline 1068 & 3.5 & $0^{17.8}$ & 4.22 & 35 & 2.579 & 2.817 & \\
\hline 1326 & 4.0 & $0^{22.1}$ & 4.70 & 40 & 2.947 & 3.139 & 3.271 \\
\hline 1588 & 4.5 & $47^{26 .}$ & 5.14 & 45 & 3.315 & 3.435 & 3.609 \\
\hline 1846 & 5.0 & $7^{30.7}$ & 5.55 & 50 & 3.684 & 3.704 & 3.941 \\
\hline 2040 & 5.5 & $00^{34 .}$ & 5.83 & 55 & 4.052 & 3.894 & 4.190 \\
\hline 2362 & 6.0 & $37^{39 .}$ & 6.27 & 60 & 4.420 & 4.190 & 4.605 \\
\hline 2609 & 6.5 & $48^{43 .}$ & 6.59 & 65 & 4.789 & 4.403 & 4.923 \\
\hline 2877 & 7.0 & $5^{47.9}$ & 6.92 & 70 & 5.157 & 4.624 & 5.268 \\
\hline 3126 & 7.5 & $0^{52.1}$ & 7.22 & 75 & 5.526 & 4.820 & 5.588 \\
\hline 3407 & 8.0 & $8^{56.7}$ & 7.54 & 80 & 5.894 & 5.032 & 5.950 \\
\hline 3952 & 9.0 & $87^{65 .}$ & 8.12 & 90 & 6.631 & 5.419 & 6.652 \\
\hline 4480 & 10.0 & $7^{74.6}$ & 8.64 & 100 & 7.367 & 5.770 & 7.331 \\
\hline 5044 & 11.0 & $07^{84 .}$ & 9.17 & 110 & 8.104 & 6.123 & 8.057 \\
\hline 5623 & 12.0 & $2^{93.7}$ & 9.68 & 120 & 8.841 & 6.464 & 8.802 \\
\hline 6210 & 13.0 & $50^{103 .}$ & 10.17 & 130 & 9.578 & 6.793 & 9.558 \\
\hline 6813 & 14.0 & $55^{113 .}$ & $6^{10.6}$ & 140 & 10.314 & 7.116 & 10.334 \\
\hline 7404 & 15.0 & $40^{123 .}$ & 11.11 & 150 & 11.051 & 7.418 & 11.095 \\
\hline
\end{tabular}


(6) Sorptivity

\section{Regression Analysis}

Constant: 0.0

Coefficient (Sorptivity)

$\mathrm{S}=0.6678 \mathrm{~mm} / \mathrm{min} 1 / 2$

$\mathrm{r} 2=0.6543$

(7) Steady State Infiltration Rate

\section{Regression Analysis}

Constant: 1.5644

Coefficient(Infiltration rate)

if $=0.0772 \mathrm{~mm} / \mathrm{min}$

$\mathrm{r} 2=0.9995$

(8) Saturated Conductivity and b

$$
\begin{aligned}
& K_{s}=0.0772-\frac{4 \cdot 0.55 \cdot 0.668^{2}}{\pi \cdot 105 \cdot(0.38-0.07)}=0.0676[\mathrm{~mm} / \mathrm{min}] \\
& \beta=\frac{(0.38-0.07) \cdot 0.0676}{0.55 \cdot 0.668^{2}}=0.0854\left[\mathrm{~mm}^{-1}\right]
\end{aligned}
$$

\section{Review Questions}

1. What are the two basic assumptions of the Green and Ampt infiltration model?

2. What is the time dependence of position of the wetting front for horizontal infiltration using the Green and Ampt media? The time dependence of infiltration rate? Cumulative infiltration?

3. For vertical infiltration, what is the time dependence of infiltration at very short time? What about at very long time? How do we define short and long time?

4. If we have made measurements of moisture content in a horizontal unit of soil which experienced infiltration for 2 hours, how would we extrapolate the moisture profile at later time? As an example, at a time of 16 hours, where would I look for soil of the same moisture content that was seen $5 \mathrm{~cm}$ from the water source after 2 hours?

5. Why did Gardner introduce the exponential conductivity model? What did it allow?

6. About how deeply would you expect evaporation to draw down the water table in a silt soil with no vegetation after a few hundred years? 
7. How does the diversion length of a capillary barrier system depend upon flux? Slope?

8. What is the "down dip limit"?

9. Define "unit gradient" flow.

10. Why a curved interface with slope proportional to distance from the crest makes sense?

11. What is the dependence between soil particle size and finger cross-section.

12. After pressure equilibrium has been achieved, why are fingered flow paths wetter than the surrounding soil? How does this relate to finger persistence.

\section{Problems}

1. An engineer was running infiltration tests on identical soil cores to design an liner for a waste disposal pond. She ran both vertical (downward) and horizontal tests, with negligible ponding depth. When she came back the next day after the janitor cleaned up the lab, she realized that she had not labeled which test was which. Here's the data which shows the wetting front position as a function of time.

\begin{tabular}{lll}
\hline Time (hr) & Infil. \#1 (cm) & Infil. \#2 (cm) \\
0.25 & 11.2 & 12.0 \\
1 & 22.4 & 25.8 \\
2 & 31.6 & 38.6 \\
3 & 38.7 & 38.6 \\
4 & 44.7 & 49.3 \\
5 & 50.0 & 58.9 \\
6 & 54.8 & 67.9 \\
7 & 59.2 & 76.4 \\
8 & 63.2 & 84.5 \\
\hline
\end{tabular}

1. Which is the data column is for vertical infiltration and which is for horizontal infiltration?

2. What is the sorptivity for this material?

3. Given that the wetting front pressure was found to be $50 \mathrm{~cm}$ and the porosity is 0.4 , what is the saturated conductivity predicted using a Green and Ampt model?

4. How long would it take for the wetting front to pass horizontally through a 3 meter thick liner? Vertically?

2. This same engineer took some data on the moisture profile of the system. For horizontal infiltration, she took the data at the $50 \mathrm{~cm}$ position, and recorded the moisture content in time, which is given below. Calculate the moisture contents at the positions listed below 


\begin{tabular}{ll}
\hline Time $(\mathrm{hr})$ & Deg. of Sat. \\
0.50 & 0.10 \\
0.51 & 0.40 \\
0.59 & 0.60 \\
0.72 & 0.70 \\
1.00 & 0.78 \\
2.78 & 0.90 \\
\hline
\end{tabular}

Position $(\mathrm{cm}) \quad$ Deg. of Sat.

0

30

50

65

80

100

3. During a hard rain at the end of a dry summer, a small pond instantly fills up with $10 \mathrm{~cm}$ of water. Using the Green and Ampt solution for falling head infiltration given below, calculate the time required for the water to all soak in. Use the data given below. Characteristic wetting curve for the soil:

\begin{tabular}{ll}
\hline$\theta\left(\mathrm{cm}^{\wedge} 3 / \mathrm{cm}^{\wedge} 3\right)$ & $\Psi(-\mathrm{cm})$ \\
0.35 & 0 \\
0.34 & 10 \\
0.32 & 20 \\
0.30 & 30 \\
0.15 & 40 \\
0.10 & 50 \\
0.09 & 75 \\
0.085 & 100 \\
\hline
\end{tabular}

4. Media \#1 has a mean grain size of $1 \mathrm{~mm}, \mathrm{Ks}=2 \mathrm{~cm} / \mathrm{hr}$ and $\mathrm{H}_{\mathrm{ae}}$ of $-10 \mathrm{~cm}$.

1. For a Miller similar media \#2 with mean grain size of $2 \mathrm{~mm}$, what would the values of $\mathrm{Ks}$ and $\mathrm{H}_{\mathrm{ae}}$ be?

2. Using a Green and Ampt approach for horizontal infiltration, how far would the wetting front move in after $1 \mathrm{hr}$ in media \#1? In media \#2? How does this distance scale with the mean grain size?

5. A transformer at an electric distribution substation explodes releasing 20,000 liters of DOW 710 
silicone heat exchange fluid (see attached data sheets). The fluid is at $25^{\circ} \mathrm{C}$ when it enters the soil. The spill is confined to a $200 \mathrm{~m}^{2}$ capture pond and starts to soak into the a Siani sand soil which underlies the site (see attached sheet). Assuming a oil-sand contact angle of $25^{\circ}$, how long do you predict it will take for the oil to completely soak in (use the Green and Ampt infiltration model)?

6. Derive Richards Equation by considering the flow through and storage in the cubic control volume shown below. The derivation should be based on substituting Darcy's law into the conservation of mass for the water in the control volume (i.e., $\Delta$ storage $=$ inflow-outflow).

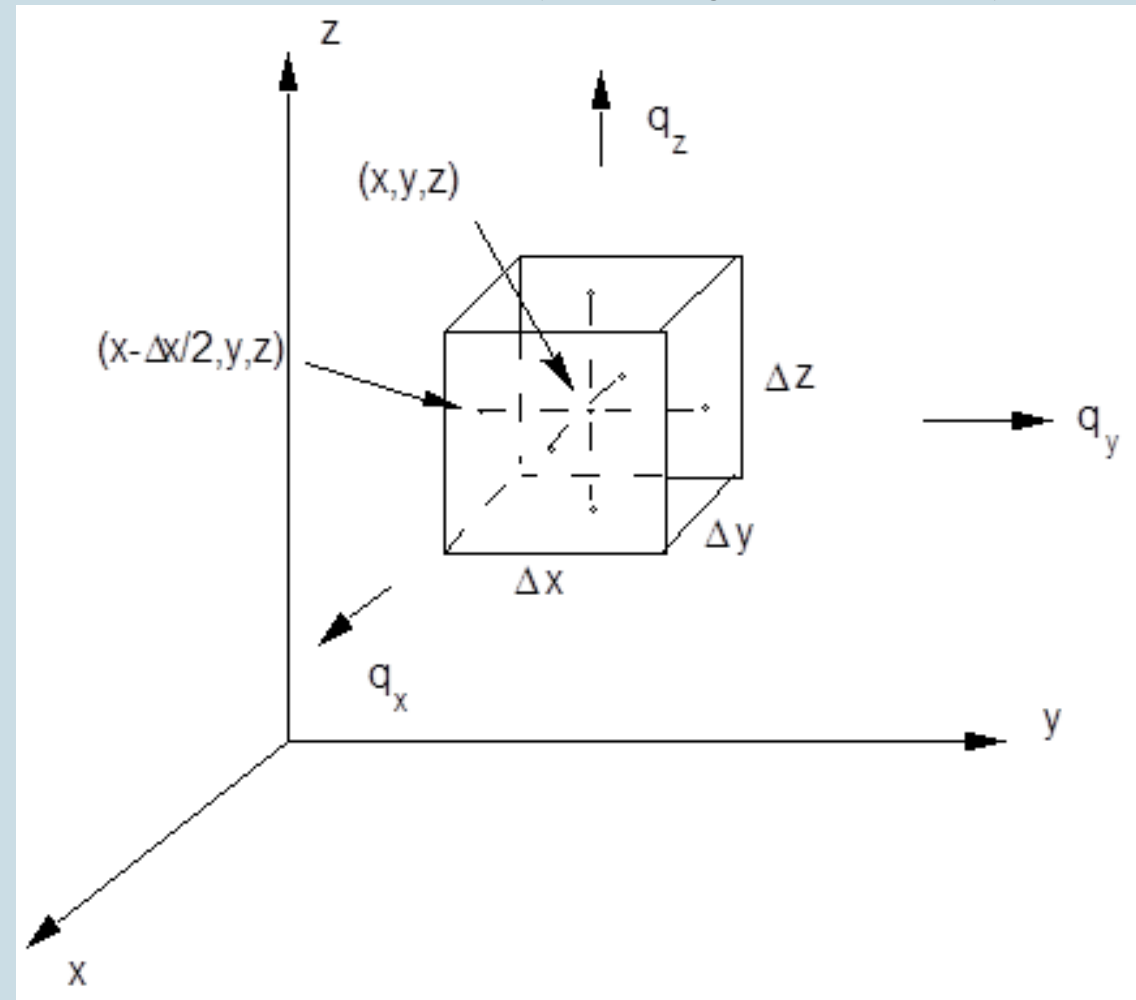

7. Derive the Green and Ampt solution for falling head infiltration given below. (Just follow the method used for the constant head case.)

$$
\mathrm{t}=\frac{\mathrm{n}}{\mathrm{K}_{\mathrm{sat}}(1-\mathrm{n})^{2}}\left\{\mathrm{~L}(1-\mathrm{n})-\left(\Psi_{\mathrm{f}}+\mathrm{d}\right) \ln \left[\frac{\Psi_{\mathrm{f}}+\mathrm{d}+\mathrm{L}(1-\mathrm{n})}{\Psi_{\mathrm{f}}+\mathrm{d}}\right]\right\} \text { for } \mathrm{nL}<\mathrm{d}
$$

8. Calculate how much run off (in terms of total depth of rainfall) which would result from the following $8.3 \mathrm{~cm}$ storm falling on a soil with porosity of $0.3, \mathrm{~K}_{\mathrm{s}}=0.02 \mathrm{~cm} / \mathrm{sec}, \Psi_{\mathrm{f}}=25 \mathrm{~cm}$. Assume that the ponding depth is minimal. Use the Green and Ampt infiltration model for vertical infiltration. 


\begin{tabular}{rr}
\hline \multicolumn{1}{c}{$\begin{array}{l}\text { Time } \\
(\mathrm{hr})\end{array}$} & \multicolumn{2}{c}{ Rainfall Rate } \\
$(\mathrm{cm} / \mathrm{hr})$ & \\
1 & 2 \\
2 & 1 \\
3 & 1 \\
4 & 2 \\
5 & 0.3 \\
6 & 0.5 \\
7 & 0.2 \\
8 & 0.1 \\
9 & 0.4 \\
10 & 0.3 \\
11 & 0.5 \\
12 & 0.1 \\
\hline
\end{tabular}

9. Capillary Barriers.

1. Using the results of Ross for capillary barrier diversion, and for a site with an upper fine soil with $\mathrm{K}_{\mathrm{S}}=0.0001 \mathrm{~m} / \mathrm{s}, \phi=10 \mathrm{deg}$ and $\alpha=10 \mathrm{~m}^{-1}$, plot L vs. q. What would $\mathrm{L}$ be for western Oregon where in the rainiest month we might have $10 \mathrm{~cm}$ of percolation?

2. Using the results

$$
\mathrm{K}_{\mathrm{rel} 1}(\mathrm{z})=\exp \left(\alpha_{1} \cos \phi \mathrm{z}\right)\left[\left(\frac{\mathrm{q}}{\mathrm{K}_{\mathrm{sat}}}\right)^{\alpha_{1} / \alpha_{2}}-\frac{\mathrm{q}}{\mathrm{K}_{\mathrm{s}} \mathrm{at}}\right]+\frac{\mathrm{q}}{\mathrm{K}_{\mathrm{sat} 1}}
$$

and

$$
\mathrm{q}_{\mathrm{h}}=\mathrm{K}_{\mathrm{s} 1} \mathrm{~K}_{\mathrm{rel1}}(\mathrm{z})\left[\sin \phi \frac{\mathrm{d} \Psi}{\mathrm{dz}}\right]
$$

calculate the horizontal flux as a function of $z$. Using the values from question (a), with $\mathrm{K}_{\mathrm{s} 2}=0.001$ $\mathrm{m} / \mathrm{s}$ and $\alpha_{2}=100 \mathrm{~m}^{-1}$, plot qh as a function of z. How thick should the fine soil cap be? Why?

\section{Index}

A

air entry pressure

B

Boltzman

Bouwer falling head ring infiltrometer 
Bruce and Klute equation

Bruce and Klute Equation

Buckingham transform

C

capillary

capillary diversion

capillary tube bundle

characteristic grain size

conservation of mass

crack diversion

cumulative infiltration

D

Darcy's law

degradation

depth of ponding

diffusivity

diversion length

down dip diversion capacity

down dip limit

dynamic viscosity

E

evaporation

evaporative flux

evaporative flux, maximum

evaporative flux, maximum

exponential conductivity

exponential conductivity function

F

falling head infiltration

finger persistence

finger width

fingered flow

fractured rock

G

Gardner conductivity

gravity

Green

Green and Ampt

Green and Ampt parameters

H

Hagan Poisuille

Hagen-Poiseuille

horizontal flux

horizontal infiltration

I

infiltration

instability

L

238 | Liquid Flow in Soils (Hydrodynamics) 


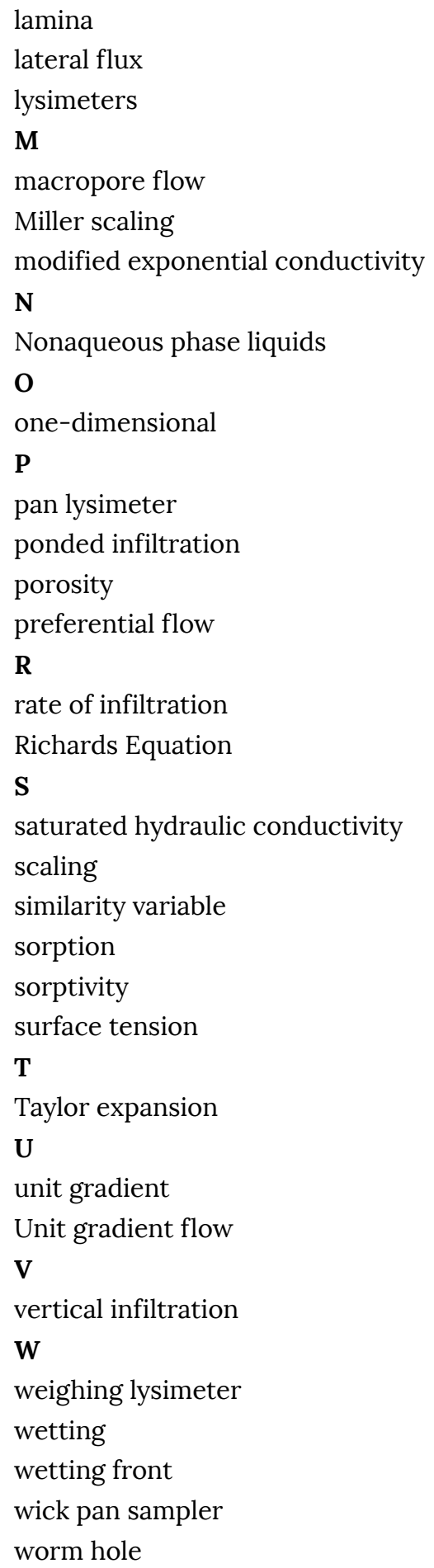

. Gardner left things here, but it is interesting to push the calculation one step further. When in Somalia I went to a place a few hundred miles from the hottest inhabited place on earth. I was surprised to learn that the water table was only about $3.5 \mathrm{~m}$ below the surface, despite the fact that the most recent major recharge event occurred thousands of years ago. Can we estimate the time it takes a water table to drop from the surface in time using Gardner's result? Well, officially Gardner's result is for a steady-state system, but let's just assume that the system changes slowly enough that 
this assumption is satisfied. What will the maximum rate of water table decline? If the average loss of water in the profile is $\theta_{\mathrm{e}}$, then the rate of water table decline will be $\mathrm{q}_{\max } / \theta_{\mathrm{e}}$. We then may write

$$
\frac{\mathrm{dz}}{\mathrm{dt}}=\frac{\mathrm{q}_{\max }}{\theta_{\mathrm{e}}}=\frac{\mathrm{K}_{\mathrm{s}}}{\theta_{\mathrm{e}}[\exp (\alpha \mathrm{z})-1]}
$$

Solving for $\mathrm{dt}$ and integrating, we find the time for the water table to reach a depth $\mathrm{z}$ is approximately

$$
\mathrm{t}=\frac{\theta_{\mathrm{e}}}{\mathrm{K}_{\mathrm{s}}}\left[\frac{\exp (\alpha \mathrm{z})-1}{\alpha}-\mathrm{z}\right]
$$

For typical values of these parameters for a silty soil (say $\alpha=5 / \mathrm{m}, \mathrm{K}_{\mathrm{s}}=1 \mathrm{~m} / \mathrm{d}$ and $\theta_{\mathrm{e}}=0.2$ ) the time to reach a depth of $3.5 \mathrm{~m}$ is predicted to be 4,400 years, which matches my observations pretty well. It is interesting to note that to drop another $0.5 \mathrm{~m}$ would take 50,000 years! It is further instructive to think about how a desert society which relies on a shallow depth to groundwater is effected by the introduction of mechanical pumps which can drop the water table by up to a meter per year.

$\ddagger$ To see this takes a bit of thinking. For case 1, there are two sub-cases: first, if the flow is above the saturated conductivity of the lower layer, and second, if the flux is less than this. If the flux exceeds the saturated hydraulic conductivity, then the pressure will be positive in the lower media, and a zero pressure saturated boundary will form in the upper media. Thus, the vertical infiltration will be that of uniform media above the interface, with a water table at some location, and will be one-phase saturated flow below the water table. For the second sub-case, the lower layer will be unsaturated, as will the upper layer. As we see in the following section, the finer material will have a more negative asymptotic pressure (far from the interface) so there will be a slight bending of flow lines "up slope" in the finer media, but the infiltration will essentially remain vertical (by "up slope" we mean toward the uphill direction).

$\ddagger$ This step may throw you if you haven’t been thinking about your introductory calculus recently. It is easy to illustrate for one-dimensional derivatives, and extends directly to the Doperator. By the chain rule we recall

$$
\frac{\partial \mathrm{f}(\mathrm{g}(\mathrm{x}))}{\partial \mathrm{x}}=\mathrm{f}^{\prime}(\mathrm{g}(\mathrm{x})) \mathrm{g}^{\prime}(\mathrm{x})
$$

for our case, $\mathrm{f}(\mathrm{x})=\ln (\mathrm{x})$, and $\mathrm{g}(\mathrm{x})=\mathrm{K}_{\text {rel }}$. Remembering that $\mathrm{d}(\operatorname{Lnx}) / \mathrm{dx}=1 / \mathrm{x}$, we find

$$
\frac{\partial \operatorname{Ln}\left(\mathrm{K}_{\mathrm{rel}}\right)}{\partial \mathrm{x}}=\frac{1}{\mathrm{~K}_{\mathrm{rel}}} \frac{\partial \mathrm{K}_{\mathrm{rel}}}{\partial \mathrm{x}}
$$

\section{Citations}

Li, H., W.F. Wang, G.B. Zhang, L.Y. Zhao, Measurement of deep buried phreatic water evaporation in extremely arid area, Acta Ecologica Sinica, 30(24):6798-6803, 2010.

Li, H., W. Wang, and B. Liu, The daily evaporation characteristics of deeply buried phreatic water in an extremely arid region, J. Hydro 514:172-179, 2014a.

Li, H. and W. Wang, Determination and analysis of phreatic water evaporation in extra-arid dune region, Acta Ecologica Sinica 34:116-122, 2014b.

Li, H.S., F.S. Wu, H.T. Zhan, F. Q., Wan, F. Wang, The Effect of Precipitation Pulses on Evaporation of Deeply Buried Phreatic Water in Extra-Arid Areas 10.2136/vzj2015.09.0127, 2016

Philip, J. R., and D. A. De Vries, Moisture movement in porous materials under temperature gradients, Eos Trans. AGU, 38(2), 222-232, doi:10.1029/TR038i002p00222, 1957.

Pollack, H.N., S.J. Hurter, and J.R. Johnson, Heat flow from the Earth's interior: Analysis of the global data set, Reviews of Geophysics, 31: 267-280, 1993, doi:10.1029/93RG01249 
Richardson, Lewis Fry (1922). Weather prediction by numerical process. Cambridge, The University press. p. 262.

Ross, B., A conceptual model of deep unsaturated zones with negligible recharge, Water Resour. Res., 20(11), 1627-1629, doi:10.1029/WR020i011p01627, 1984.

Scanlon, B. R., K. Keese, R. C. Reedy, J. Simunek, and B. J. Andraski, Variations in flow and transport in thick desert vadose zones in response to paleoclimatic forcing (0 - $90 \mathrm{kyr})$ : Field measurements, modeling, and uncertainties, Water Resour. Res., 39(7), 1179, doi:10.1029/2002WR001604, 2003.

Walvoord, M. A., M. A. Plummer, F. M. Phillips, and A. V. Wolfsberg, Deep arid system hydrodynamics, 1, Equilibrium states and response times in thick desert vadose zones,

Water Resour. Res., 38(12), 1308, doi:10.1029/2001WR000824, 2002. 


\section{Land Atmosphere Interactions}

- 1 Solar Radiation

- 2 Radiation Balance

- 2.1 Greenhouse gases impact on radiation

- 3 Soil Surface Energy Balance - local and global

- 4 Introduction to the structure of the boundary layer

- 5 Estimation of Evapotranspiration (Et)

- 5.1 Defining reference evaporation: potential, reference, and actual evaporation,

- 5.2 Water balance and lysimeter methods ("direct")

- 5.3 Aerodynamic Profile Method (Hanks, 1992)

- 5.4 Bowen Ratio - Energy Balance Method

- 5.5 Penman's Combination Method

- 5.6 Eddy covariance

- 5.7 Scintillometer

- 5.8 Based on remote sensing: SEBAL and METRIC

- 5.9 Empirical Methods: Priestly Taylor; Blaney-Criddle; Thornwaite; Palmer drought index

- 6 Plant Transpiration and Evapotranspiration

- 6.1 Bare Soil Evaporation

- 6.2 Evaporation from a Drying Soil Surface

- 7 Land surface models, climate models, and how to upscale.

- 7.1 CLM, CABLE, COSMOS, WARF, JULES

- 8 SUMMARY

- 9 Review questions

- 10 PROBLEMS

- 11 REFERENCES 


\section{Heat Flow and Thermal Effects in Soils}

\section{Heat Transport in Soils}

There are several pathways for the transport of energy in the form of heat through soils. [Cite figure 121 from chapter 3 here]. Heat may be transported in soils by conduction, radiation, and by convection with air or water flow (Figure 5.1). While conduction is generally considered to be the dominant mechanism for heat transport, and thus the entire subject of section 5.2 below, convection and radiation are yet worthy of careful consideration.

We recall from our discussion of the energy balance that radiative transfer in the subsurface would be due to black body radiation (also called longwave exchange). Thinking back, you will remember that black-body exchange is proportional to the fourth power of the temperature difference between the two bodies. Your toaster has glowing red wires that are at about $1300{ }^{\circ} \mathrm{K}$, while the toast is at about $300{ }^{\circ} \mathrm{K}$, so the difference is $900{ }^{\circ} \mathrm{K}$, and the rate of energy exchange will be proportional to $\left(900^{\circ} \mathrm{K}\right)^{\wedge} 4$ times the emissivities of the toast and wire (not far from one, especially for whole wheat bread). Now in the soil there might be 20 grains of sand per $\mathrm{cm}$, and the temperature gradient might be $1^{\circ} \mathrm{C}$ per $\mathrm{cm}$ (in a highest case), so the temperature difference between adjacent grains will be something like $1 / 20 \mathrm{x}$ $1^{\circ} \mathrm{C}=0.05^{\circ} \mathrm{C}$. So the rate of energy exchange between the grains will be $(0.05 / 900)^{\wedge} 4=10^{\wedge}-17$ times smaller than the exchange your bread feels. As you may have surmised, radiative transfer is insignificant under these conditions. One less thing to worry about!

You might be thinking that we will similarly dispense with convection, after all, how much heat can really be carried by the slow-moving water and air in soil? While in many cases, especially natural soils within a meter or two of the surface, your intuition is correct in figuring that convection generally plays a small role. However, the story is more interesting in cases of high flux (irrigated agriculture) or deep unsaturated zones. Especially far from the surface, where there are very low thermal gradients to drive conduction, convection of percolating water can strongly effect the subsurface temperature. An extreme case of convection in the subsurface are springs, where water typically emerges at a temperature close to that of the regional aquifer temperature rather than the soil temperature. So next time you are either cooling yourself in a mountain stream in heat of the summer, or warming in a thermal hot spring in the winter, you can thank convection for your pleasure.

Another very interesting case of energy transport is via gas movement in the form of water vapor transport. In this case the energy transfer is in the form of "latent" heat, in that the energy will be consumed turning liquid water into water vapor, and released when the water vapor condenses. Because the latent heat is so large $(2257 \mathrm{~J} / \mathrm{g})$ compared to the specific heat of water which is just $\mathrm{XX} \mathrm{J} /{ }^{\circ} \mathrm{K}-\mathrm{g}$. As we will discuss below, this latent heat transfer is typically driven by diffusive vapor movement, where water vapor moves from regions of high water vapor concentration to low concentration (which in damp soils means from warm areas to cooler areas). Technically we would refer to this as a diffusive flux, but it does give rise to a net flux of water vapor which carries energy through a soil profile, so it must be kept front and center as we consider heat flow processes.

Having touched on the lesser players, it is time to turn to the most important process for heat flow in soils. Conduction of heat through matter involves transfer of kinetic energy at the molecular level, where molecules in warmer regions vibrate rapidly resulting in collisions with, or excitation of, their colder "neighbors." The flow of heat in soil and the exchange of heat with the atmosphere determine a soil's thermal regime. This regime is characterized mainly by the soil temperature with its temporal and spatial variations. The soil thermal regime affects many biological, chemical and physical processes including above and below ground plant growth development, microbial activity, the kinetics and transformation of nutrients, frost heaving, and many other processes. 


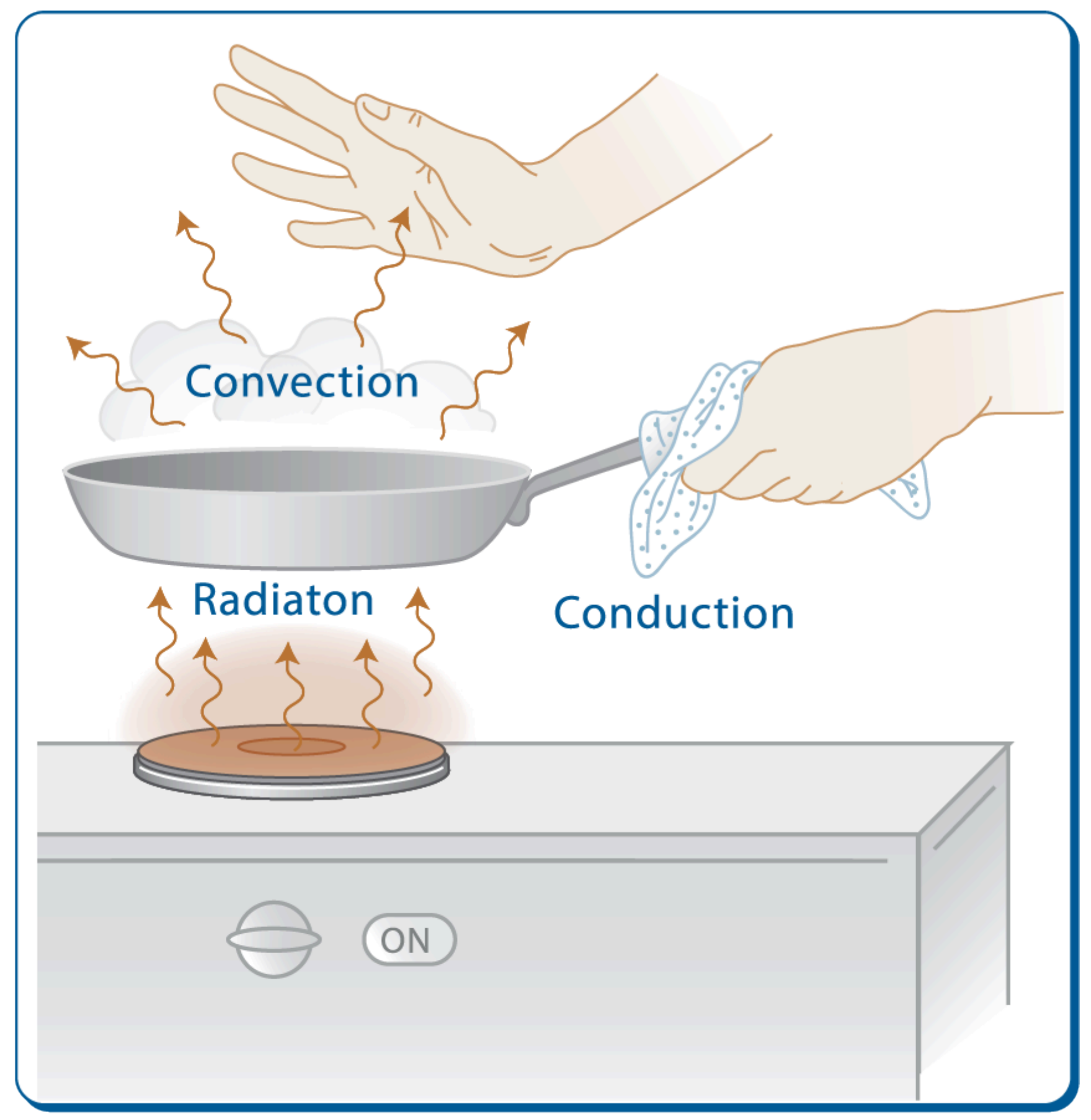

Figure 5.1 Cook stove illustration of the role of radiation, convection, and conduction in heat transfer.

Considering the soil-atmosphere interface, the heat capacity of the first $25 \mathrm{~cm}$ of soil buffers the air temperature, yielding warmer nights and cooler days (Figure 5.3). This also works on a seasonal basis, where the top two meters of soils change temperature. Of course, from the nuclear reactions taking place in the center of the earth there is about 0.1 $\mathrm{W} / \mathrm{m}^{2}$ of thermal energy leaving the surface, which gives rise to an average $25^{\circ} \mathrm{C} / \mathrm{km}$ thermal gradient in the earth. This "ground heat flux" is truly essential to understanding the dynamics of local conditions and global climate. 


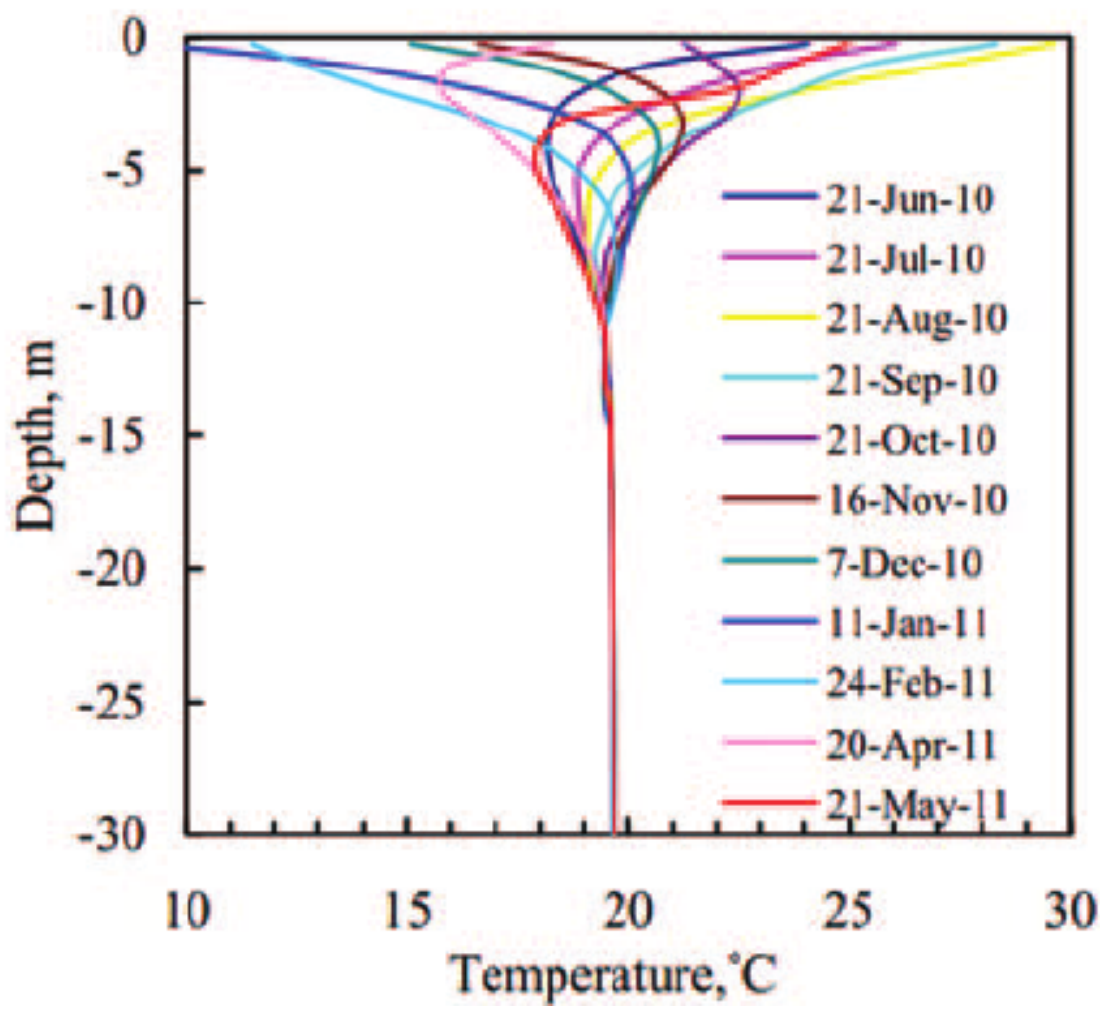

Figure 5.2 Simulated seasonal temperature profiles in Malaysia from Alam et al., 2015. It is noteworthy that the same depth dependence is seen around the world, almost universally showing a loss of seasonal variation at about $10 \mathrm{~m}$. 


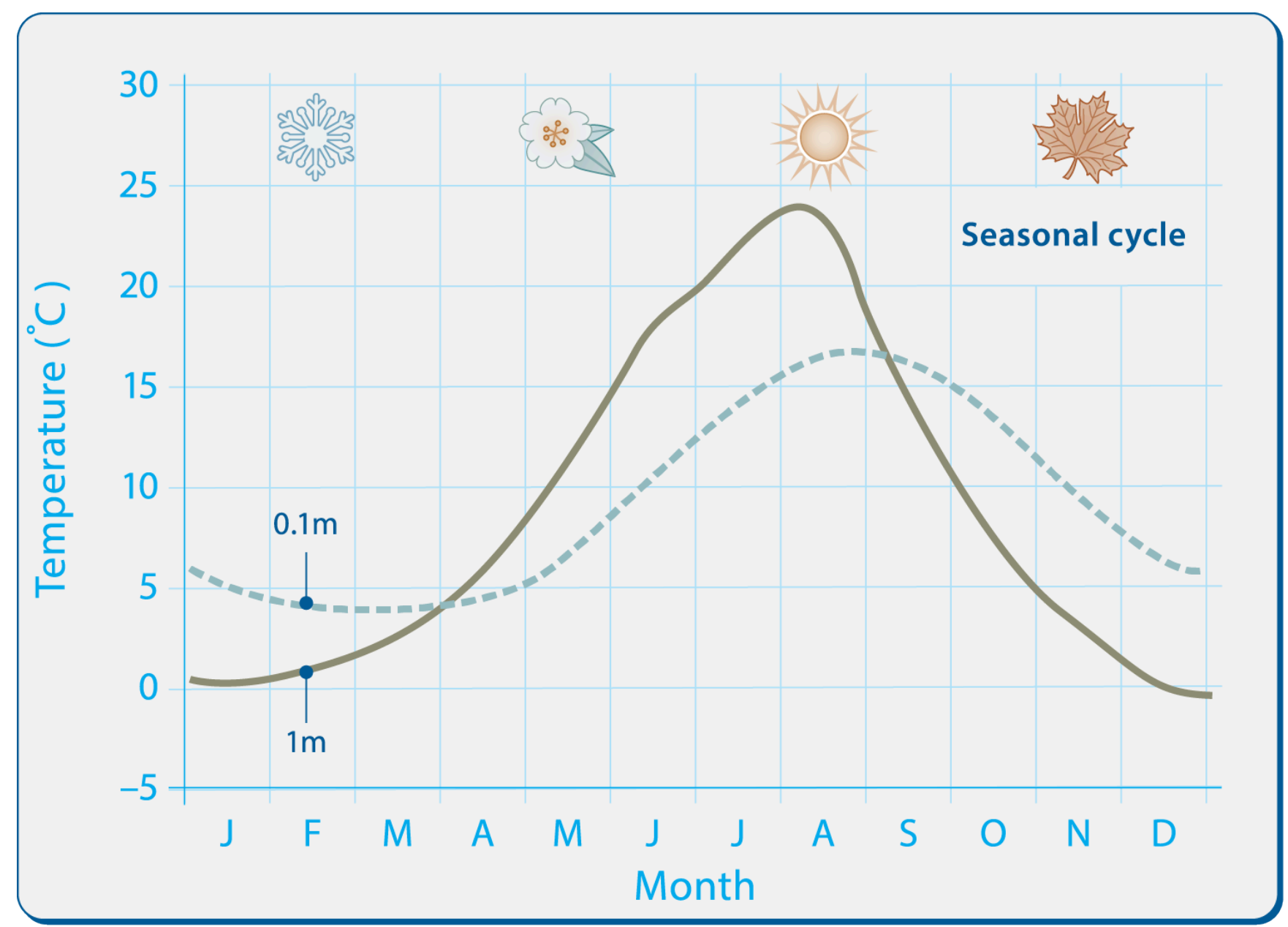

Figure 5.3 Plots of the penetration of diurnal (a) and seasonal (b) thermal signals at various depths in a typical soil profile. Note that diurnal variations essentially lost by $50 \mathrm{~cm}$ depth, while seasonal variations in temperature penetrate to about $10 \mathrm{~m}$, as seen in Figure 5.2 .

Thermal heat conduction can also drive water movement near soils surfaces, especially in arid environment's. Fundamentally this reflects the fact that near the surface of the soil much of the water is lost in the form of water vapor, and the phase change from liquid to vapor forms required significant energy (think of how cold your hands feel after washing them when you are in dry air). To make this a bit more concrete, let's consider a morning when the atmosphere is at $15^{\circ} \mathrm{C}$ and $40 \%$ relative humidity (RH). We can see from the psychrometric chart (Figure 5.4) that the absolute humidity would be about $0.0075 \mathrm{gr} \mathrm{H}_{2} \mathrm{O} / \mathrm{gr}$ dry air. Let's suppose that the surface soil is dry, and that $5 \mathrm{~cm}$ below the surface the soil is moist, so has $\mathrm{RH}=100 \%$, and has a temperature of $20^{\circ} \mathrm{C}$. Again, from the psychrometric chart, the absolute humidity $5 \mathrm{~cm}$ into the soil will be $0.015 \mathrm{gr} \mathrm{H}_{2} \mathrm{O} / \mathrm{gr}$ dry air. So we see there will be a gradient in humidity ( 0.15 gr $\mathrm{H}_{2} \mathrm{O} / \mathrm{gr}$ dry air/m), which will lead to a flux of water vapor toward the surface (Fick's law - we'll play with that later when we work on transport in soils). This will then lead to the lost water vapor to be replaced by evaporation of the soil water at the 5-cm depth, which will cool the soil locally. This energy may well be replaced by thermal conduction of heat stored from the previous day from deeper in the soil profile. These linked processes of vapor and thermal energy transport are subjects of much current research (e.g., Assouline et al., 2010, 2013). 


\section{Psychrometric Chart}

Barometric Pressure 101.325 $\mathrm{kPa}$ (Sea level)

Carrier Corporation Cat. No. 794-001, dated 1975

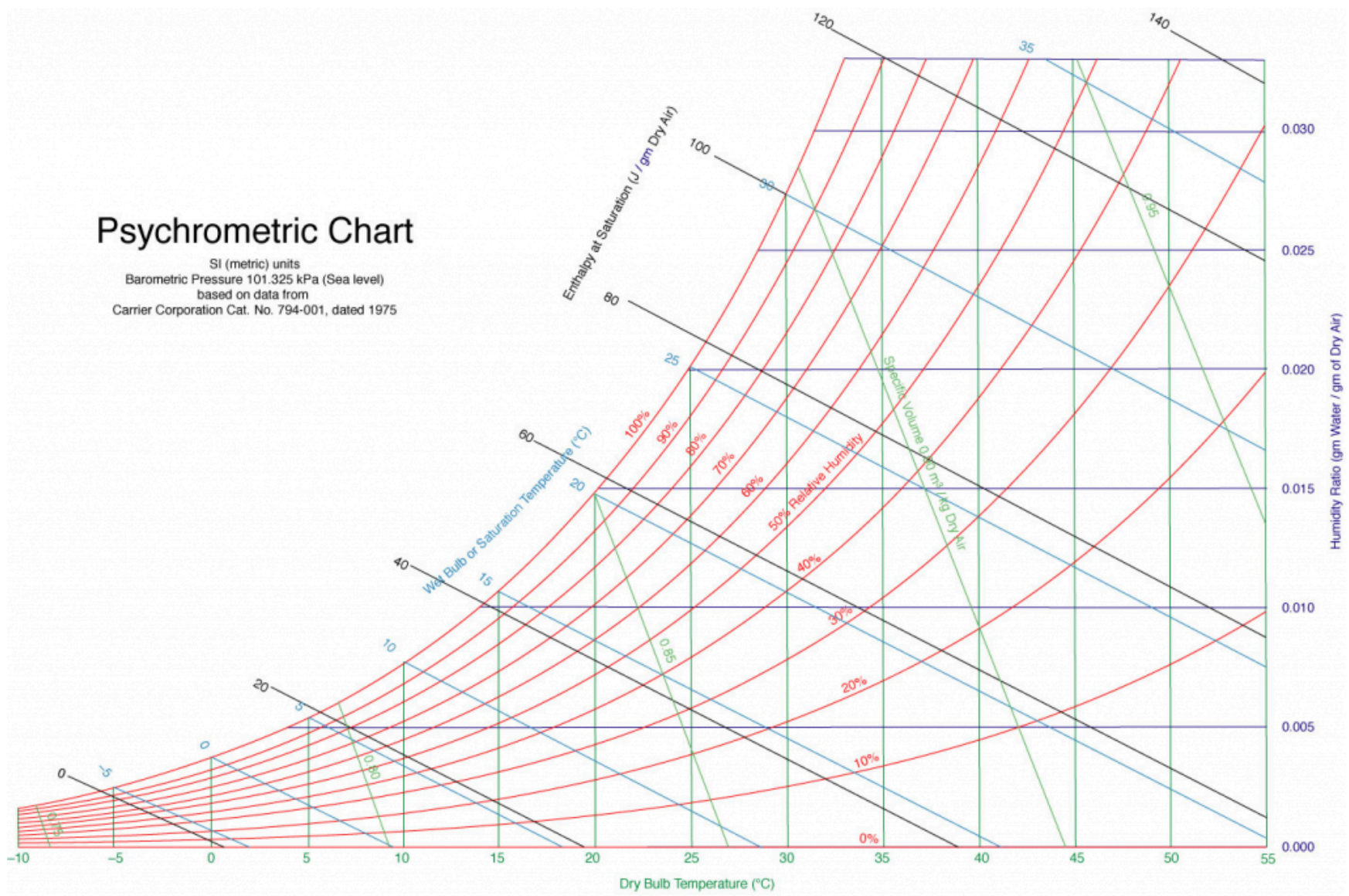

Figure 5.4 The Psychrometric chart (with permission, Carrier Corporation). Notice that you must follow the gridlines of the color that matches the parameter of interest.

\section{Heat Conduction in Soil}

The most important features of heat transfer in soils may be cast in a form of heat conduction, which we will use as the first step in "building" the heat transfer equation. In as soil without mass movement (of water or gas), the vertical one dimensional flux density of heat $\mathrm{J}_{\mathrm{H}}\left[\mathrm{W} \mathrm{m}^{-2}\right]$ ) in soil is described by Fourier's law:

$$
\mathrm{J}_{\mathrm{H}}=-\lambda \frac{\mathrm{dT}}{\mathrm{dz}}
$$




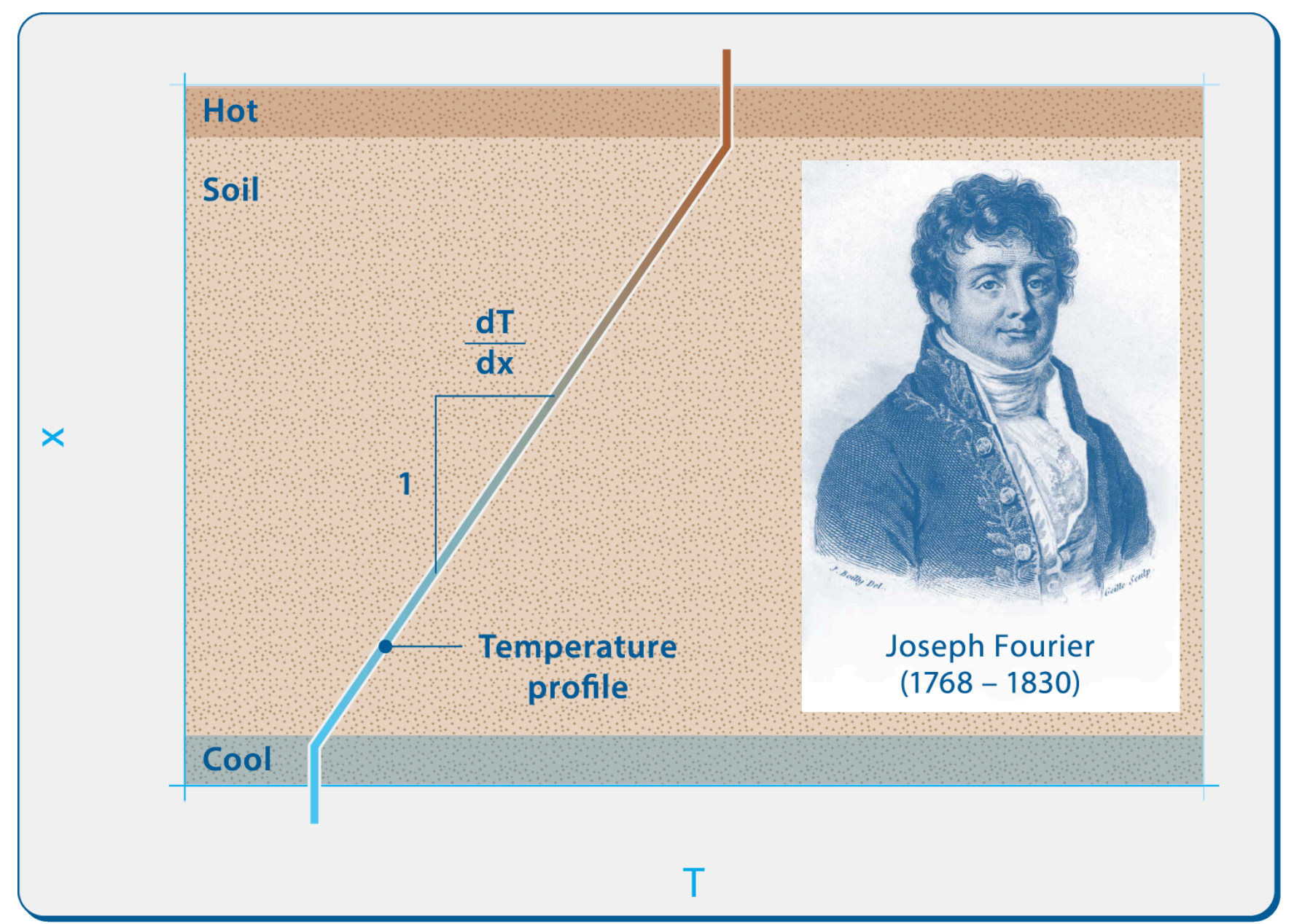

Figure 5.5 Joseph Fourier formulated in 1822 an empirical law for heat transport (considering heat conduction only). On the left we illustrate a steady-state flow of heat through a uniform media, this results in a linear gradient in temperature along the path of conduction.

relating the energy flux $\mathrm{J}_{\mathrm{H}}$ to the soil thermal conductivity $\lambda\left(\mathrm{J} \mathrm{m}^{-1} \mathrm{~s}^{-1{ }^{\circ}} \mathrm{C}^{-1}\right)$, and the gradient in temperature $\mathrm{T}\left({ }^{\circ} \mathrm{C}\right)$, with distance $z$, which could be the vertical spatial coordinate or soil depth. While thermal conductivity in soils is in principle independent of water vapor movement, the $\lambda$ in Eq. 165 should be considered as the apparent soil thermal conductivity, as latent heat transfer in the form of water vapor (that is energy liberation or consumption due to water changing between liquid and vapor states) cannot in practice be separated from conduction in moist soils; i.e., $\lambda=\lambda^{*}+$ $D_{\text {vapor }} * \mathrm{~L}$, where $\lambda *$ is the instantaneous thermal conductivity, $D_{\text {vapor }}$ is thermal vapor diffusivity, and $\mathrm{L}$ is latent heat of vaporization (Hiraiwa and Kasubuchi, 2000). We will discuss this more when we add mass movement to the equation.

A key observation is that $\lambda$ depends on the minerals which make up the soil, the porosity of the soil, and the water content of the sample (Figure 5.6). The thermal conductivity of the constituents of soil vary by 400 -fold, with quartz having fifteen times the conductivity of water, which in turn is about twenty times as conductive as air (Table 5.1). Analogous to the weakest link controlling the strength of a chain, flow of heat is controlled largely by the contacts between the mineral particles. Water can act as bridge between grains, so there is often a great change in conductivity with the transition from completely dry to residual moisture content, facilitating the movement of water through the minerals. Conversely, the same amount of water added near saturation change thermal conductivity very little, since the minerals are carrying the bulk of the heat (Figure 5.7). 


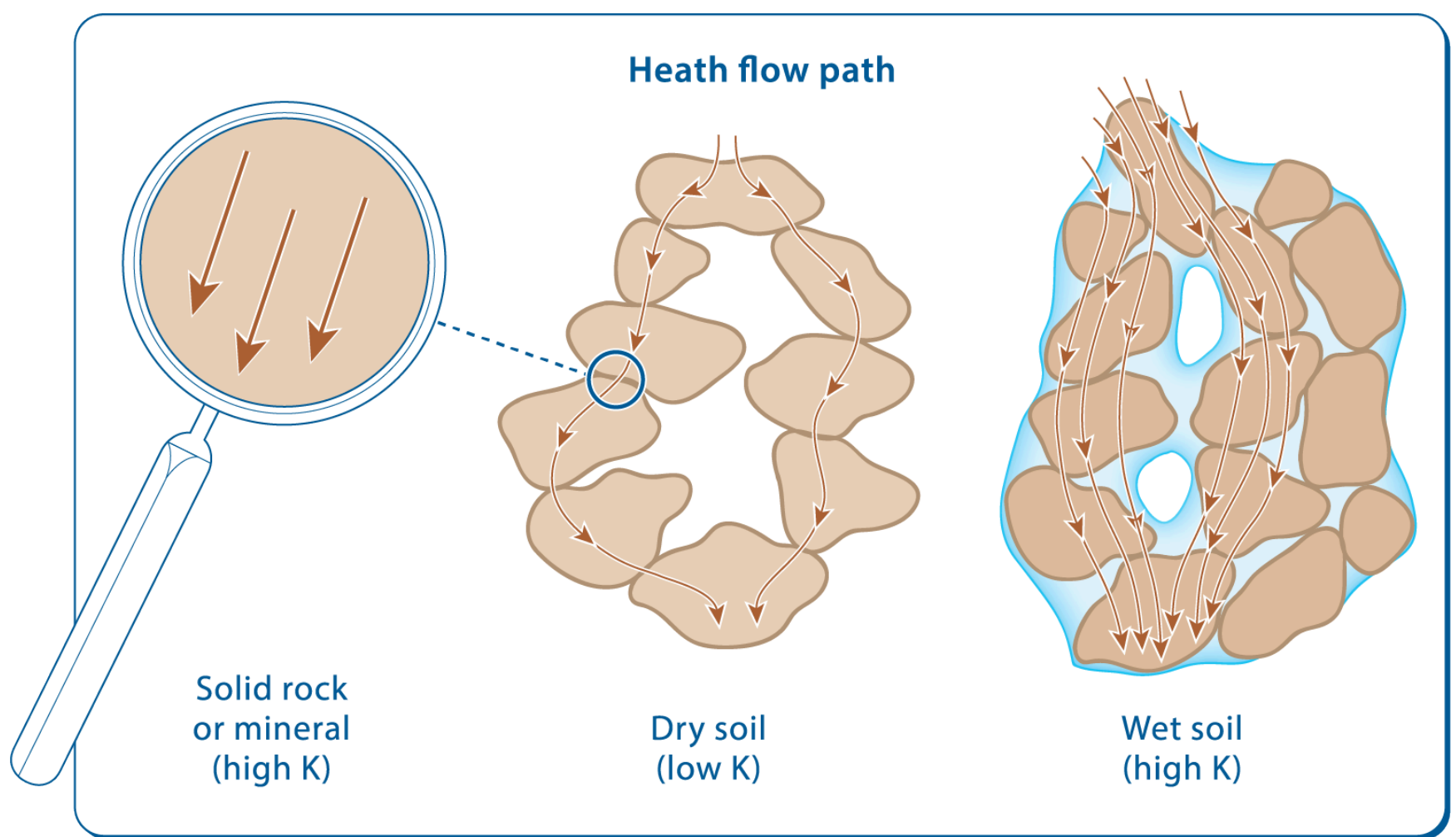

Figure 5.6 Illustration of heat flow in soils, emphasizing the role of mineral composition, porosity, and water content.

Table 5.1 Thermal conductivity of some of the typical constituents of soils (Source: XXX).

\begin{tabular}{lc}
\hline Constituent & {$\left[\mathrm{W} \mathrm{m}^{-1}{ }^{\circ} \mathrm{C}^{-1}\right]$} \\
Quarts & 8.8 \\
Soil minerals (avg.) & 2.9 \\
Soil organic matter & 0.25 \\
Water (liquid) & 0.57 \\
Ice (at $\left.0^{\circ} \mathrm{C}\right)$ & 2.2 \\
Air & 0.025 \\
\hline
\end{tabular}




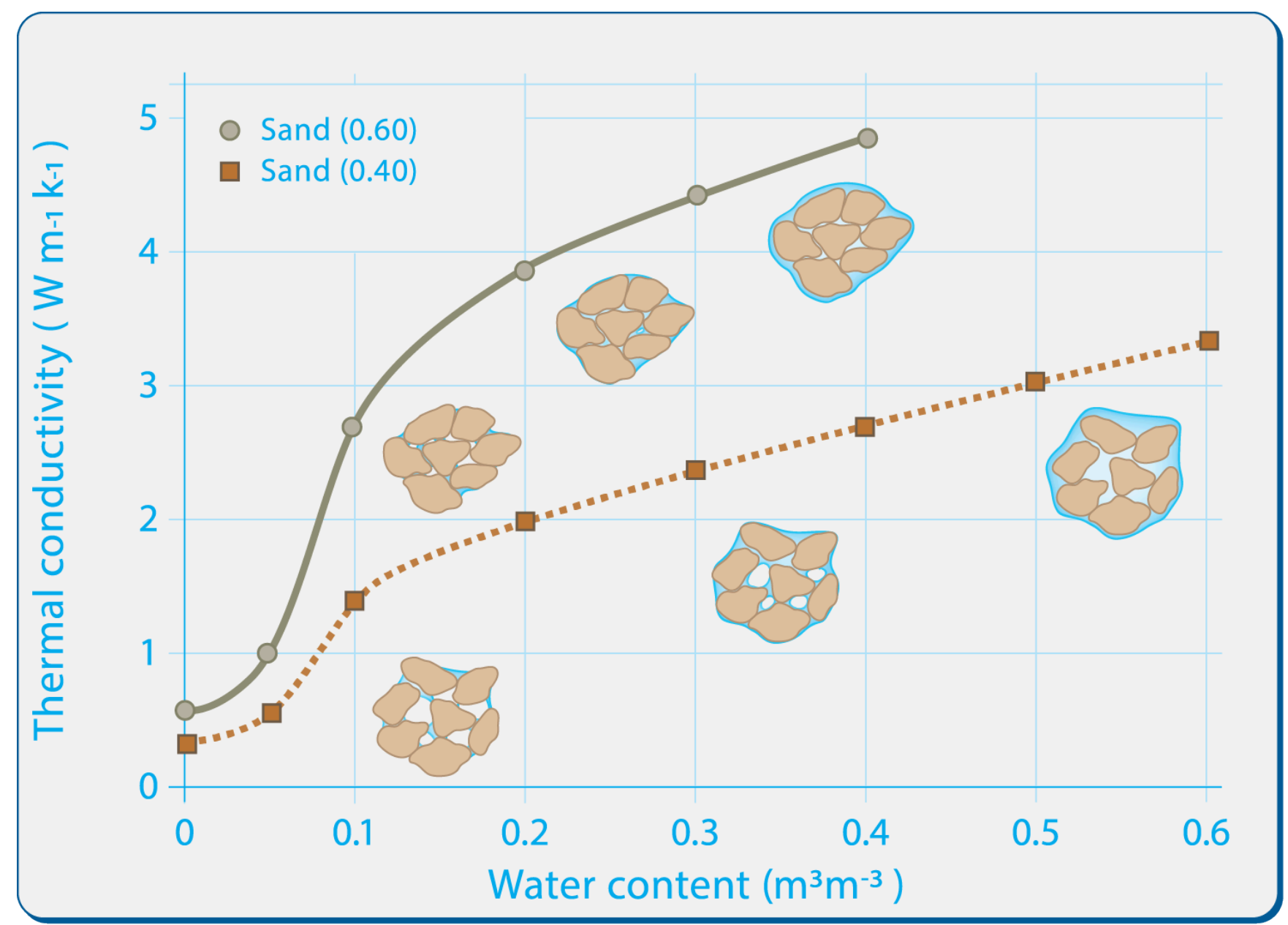

Figure 5.7 Typical valued of thermal conductivity of sand and clay soils as a function of water content. Note the disproportionately large impact of the first $10 \%$ of volumetric water content which bridges between grains, providing the key pathways for heat flow.

Combining the heat flux Eq.165) with the equation for conservation of heat energy results in a general expression for conductive soil heat flow where soil temperature may vary in time and space (Figure 5.8a and b). 


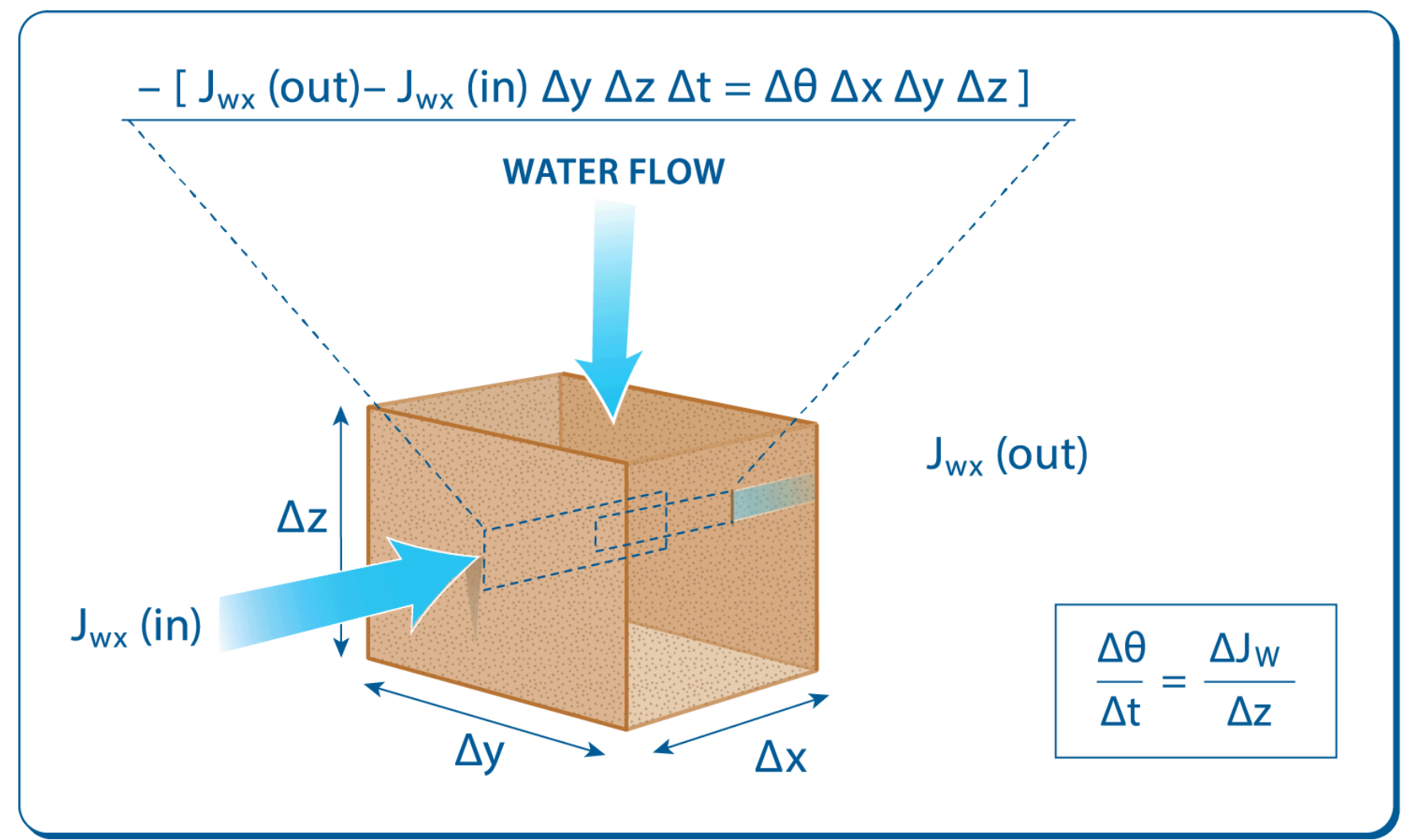

Figure 5.8a. The conservation of energy follows exactly the same reasoning as the conservation of mass employed in deriving Richards equation. 


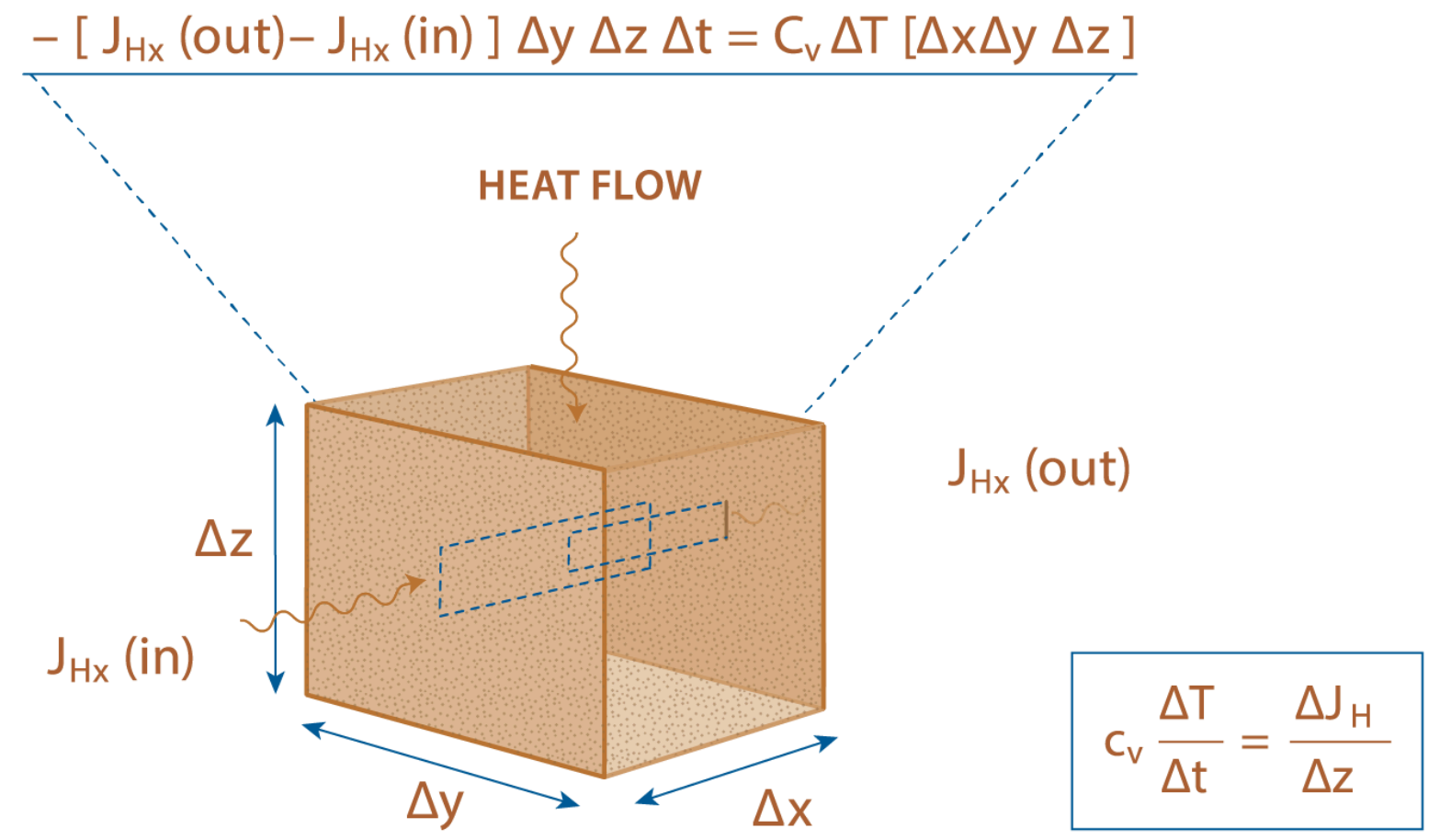

Figure 5.8b. The energy conservation equation if there were flow of heat in the $x$-direction only.

Using the control volume approach as we did in the derivation of Richards Equation, we consider an infinitesimally small cube with sides of length $\Delta x, \Delta y, \Delta z$ (Fig 147b). Let's take the center of the cube to be at point (x, y, z), so the sides of the cube will be above, below, in front, behind, left or right of this point adistances $\Delta x / 2, \Delta y / 2$, and $\Delta z / 2$. The basic concept is that

(5.2) Energy in - energy out = change in energy

So if the energy flux vector (has both magnitude and direction) is given by $\mathbf{J}_{\mathrm{H}}(\mathrm{x}, \mathrm{y}, \mathrm{z})$, and we will denote the component in the $\mathrm{x}$-direction (this is a scalar) to be $\mathrm{J}_{\mathrm{Hx}}(\mathrm{x}, \mathrm{y}, \mathrm{z})$. The overall plan is to estimate the flux at the surface of the cube as this central value adjusted by the gradient in flux times the distance the side of the cube is from the center (e.g., $\Delta \mathrm{x} / 2$ ). Let's start with just the component of flux along the $\mathrm{x}$-direction. The net flux in the $\mathrm{x}$-direction through the front and back sides of size $\Delta \mathrm{y} \Delta \mathrm{z}$ is given by:

$$
\begin{array}{r}
{\left[\left(J_{H x}(x, y, z)-\frac{x}{2}\left[\frac{\partial J_{H x}(x, y, z)}{\partial x}\right]\right)-\left(J_{H x}(x, y, z)+\frac{x}{2}\left[\frac{\partial J_{H x}(x, y, z)}{\partial x}\right]\right)\right] y z} \\
=-x y z\left[\frac{\partial J_{H x}(x, y, z)}{\partial x}\right]
\end{array}
$$

Let's think about Eq. 165c a bit. What is says is that if the flux of heat is increasing with distance along the $\mathrm{x}$-axis that the net energy due to flux in the $\mathrm{x}$-direction in our little cube will be negative. Well, if heat flux is increasing along the $\mathrm{x}$-axis, the energy needs to come from somewhere, so indeed, the energy stored in our cube due to $\mathrm{x}$-axis flow is dropping.

So the net rate of heat delivered to our cube taking into account $\mathrm{x}, \mathrm{y}$, and $\mathrm{z}$ directions is just 
Rate of change in stored heat

$$
\begin{array}{r}
=-x y z\left(\left[\frac{\partial J_{H x}(x, y, z)}{\partial x}\right]+\left[\frac{\partial J_{H y}(x, y, z)}{\partial x}\right]+\left[\frac{\partial J_{H z}(x, y, z)}{\partial x}\right]\right) \\
=-x y z \nabla \cdot \mathrm{J}_{\mathrm{H}}
\end{array}
$$

The resulting change in temperature will the thermal mass of the material in our little cube times the rate of change in temperature, which is just

$$
\text { Rate of change in stored heat }=x y z \rho_{s} c_{s} \frac{\partial T}{\partial t}
$$

Which when put together, canceling $\Delta \mathrm{x} \Delta \mathrm{y} \Delta \mathrm{z}$ from both sides, gives us the conservation of energy equation

$$
\rho_{s} c_{s} \frac{\partial T}{\partial t}=-\nabla \cdot J_{H}
$$

where $\rho_{\mathrm{S}}$ is soil bulk density, $\mathrm{c}_{\mathrm{S}}$ is the soil specific heat capacity on a mass basis (i.e., the amount of heat required to raise the temperature of a unit mass of moist soil by $\left.1^{\circ} \mathrm{C}\right)$. When combined with Fourier's law gives us in the general three-dimensional form

$$
\rho_{s} c_{s} \frac{\partial T}{\partial t}=-\nabla \cdot \lambda \nabla T
$$

or for the special case of there being only gradients in the z-direction (which is not so unusual in soils, which tend to be at the same temperature at any particular depth)

$$
\rho_{s} c_{s} \frac{\partial T}{\partial t}=-\frac{\partial T}{\partial z}\left(\lambda \frac{\partial T}{\partial z}\right)
$$

Note that the relationship expressed by Eq.166) is exactly analogous to that for unsaturated non-steady water flow (refer to Fig. 2-13?), where the change in heat flux $\left(\Delta \mathrm{J}_{\mathrm{Hz}}\right) \Delta \mathrm{x} \Delta \mathrm{y} \Delta \mathrm{t}=$ the change in heat content $\left(\mathrm{c}_{\mathrm{v}} \Delta \mathrm{T}\right) \Delta \mathrm{x} \Delta \mathrm{y} \Delta \mathrm{z}$, thus $\Delta \mathrm{J}_{\mathrm{Hz}} / \Delta \mathrm{z}=\mathrm{c}_{\mathrm{V}} \Delta \mathrm{T} / \Delta \mathrm{t}$. Now we see that we have three parameters to describe the essential physical properties that define the soil thermal response. As long as the properties of the soil do not change in space and time, we can collect them into a single value. Here we will follow the approaches commonly found in the literature. The expression may be simplified by using the soil's volumetric heat capacity $\left(c_{\mathrm{V}}=\rho_{\mathrm{S}} \mathrm{c}_{\mathrm{S}}\right)$, which may be approximated as (Brutsaert, 1982):

(5.9) $\mathrm{c}_{\mathrm{v}} \simeq 1.94 \cdot(1-\mathrm{n}-\varphi)+4.189 \cdot \theta_{\mathrm{v}}+2.50 \cdot \varphi \quad\left[\mathrm{MJ} \mathrm{m}^{-3} \mathrm{C}^{-1}\right]$

where $\mathrm{n}$ is the soil porosity, $\theta_{\mathrm{v}}$ is the volumetric water content, and $\varphi$ is the volume fraction of soil organic matter. The terms on the right-hand side of Eq.167) account for the contributions to volumetric heat capacity due to soil solids, water, and organic matter, respectively. For homogeneous soils $c_{V}$ and $\lambda$ may be considered as independent of $z$ (constant with depth), resulting in a simplified form of Eq.166) given as :

$$
\frac{\partial T}{\partial t}=-D_{H} \frac{\partial^{2} T}{\partial z^{2}}
$$

where $D_{H}=\lambda / c_{V}$ is the soil thermal diffusivity $[\mathrm{L} 2 / \mathrm{t}]$.

Analytical solutions to Eq.168) are available for a variety of heat sources, flow geometries, and for different boundary conditions. A definitive book on mathematical solutions to heat flow problems was written by Carslaw and Jaeger (1959). 


\section{Soil Thermal Properties}

In addition to being requisite for calculation of heat flow, a discussion of individual soil thermal properties aids in understanding how the solid, liquid, and gas phases interact to influence the dynamics of heat energy in soils.

\section{Heat Capacity}

The heat capacity per unit volume of soil is the quantity of heat needed to raise the temperature of a unit volume of soil by one degree $\mathrm{C}$. Knowledge of the volume fractions $\theta_{\mathrm{i}}$ of the soil constituents, which we will denote: minerals-m, water$\mathrm{w}$, air-a, and organic matter-om, with densities $\left(\rho_{\mathrm{i}}\right)$, and specific heat capacities per unit mass $\left(\mathrm{c}_{\mathrm{i}}\right)$, allows determination of volumetric heat capacity $\left(\mathrm{c}_{\mathrm{v}}\right)$ for moist soils as the sum of all of these constiuents:

(5.11) $\mathrm{c}_{\mathrm{v}}=\rho_{\mathrm{m}} \theta_{\mathrm{m}} \mathrm{c}_{\mathrm{m}}+\rho_{\mathrm{w}} \theta_{\mathrm{w}} \mathrm{c}_{\mathrm{w}}+\rho_{\mathrm{a}} \theta_{\mathrm{a}} \mathrm{c}_{\mathrm{a}}+\rho_{\mathrm{om}} \theta_{\mathrm{om}} \mathrm{c}_{\mathrm{om}}$

Using typical values of these components (Table 5.2) yields the approximation given in Eq.167).

Table 5.2 Specific Heat Capacities and Typical Densities of Soil Constituents (Brutsaert, 1982)

\begin{tabular}{cccc}
\hline Constituent & \multicolumn{2}{c}{ Specific Heat, $\mathrm{c}_{\mathrm{i}}$} & Density \\
& $\mathrm{J} \mathrm{kg}^{-1}{ }^{\circ} \mathrm{C}^{-1}$ & $\mathrm{c}_{\mathrm{V}}$ \\
Soil minerals & 733 & 2650 & 1.94 \\
Soil organic matter & 1926 & 1300 & 2.5 \\
Water & 4182 & 1000 & 4.18 \\
Air & 1005 & 1.2 & 0.0012 \\
\hline
\end{tabular}

\section{Thermal Conductivity}

Thermal conductivity is defined as the amount of heat transferred through a unit area in unit time (heat flux density) under a unit temperature gradient. The soil thermal conductivity $(\lambda)$ is dependent primarily upon the bulk density of the soil and its water content. Increasing soil bulk density hence the contacts between solid particles increases the thermal conductivity. Similarly, the thermal conductivity increases with increasing water content (Figure 5.11). Soil water improves the thermal contact between the soil particles, and replaces air which has 20 times lower thermal conductivity than does water (Table 5.3).

Table 5.3 Thermal Conductivities of Soil Constituents at $10^{\circ} \mathrm{C}$ (Hillel, 1980)

\begin{tabular}{ccc}
\hline Constituent & $\lambda\left[\mathrm{mcal}\left(\mathrm{cm} \mathrm{s}^{\circ} \mathrm{C}\right)^{-1}\right]$ & $\lambda\left[\mathrm{W} \mathrm{m}^{-1}{ }^{\circ} \mathrm{C}^{-1}\right]$ \\
Quartz & 21 & 8.8 \\
Soil minerals (avg.) & 7 & 2.9 \\
Soil organic matter & 0.6 & 0.25 \\
Water (liquid) & 1.37 & 0.57 \\
Ice (at 0 $\left.{ }^{\circ} \mathrm{C}\right)$ & 5.2 & 2.2 \\
Air & 0.06 & 0.025 \\
\hline
\end{tabular}


The thermal conductivity of a soil may be measured by means of a transient-heat probe consisting of a needle encasing a heater and thermocouple temperature sensor (call out NEW FIGURES HERE - see below). The sensor is embedded in the presumed homogenous soil where the needle approximates an infinitely long linear source of heat.

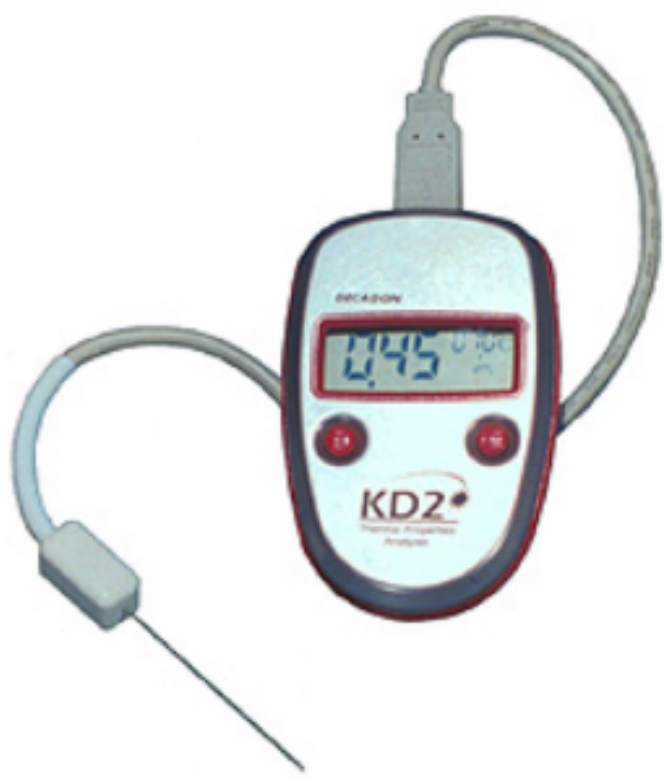

Figure 5.9 A commercially available single-probe meter to measure thermal properties of media which allow the insertion of a needle. (courtesy of METER, Pullman Washington) 


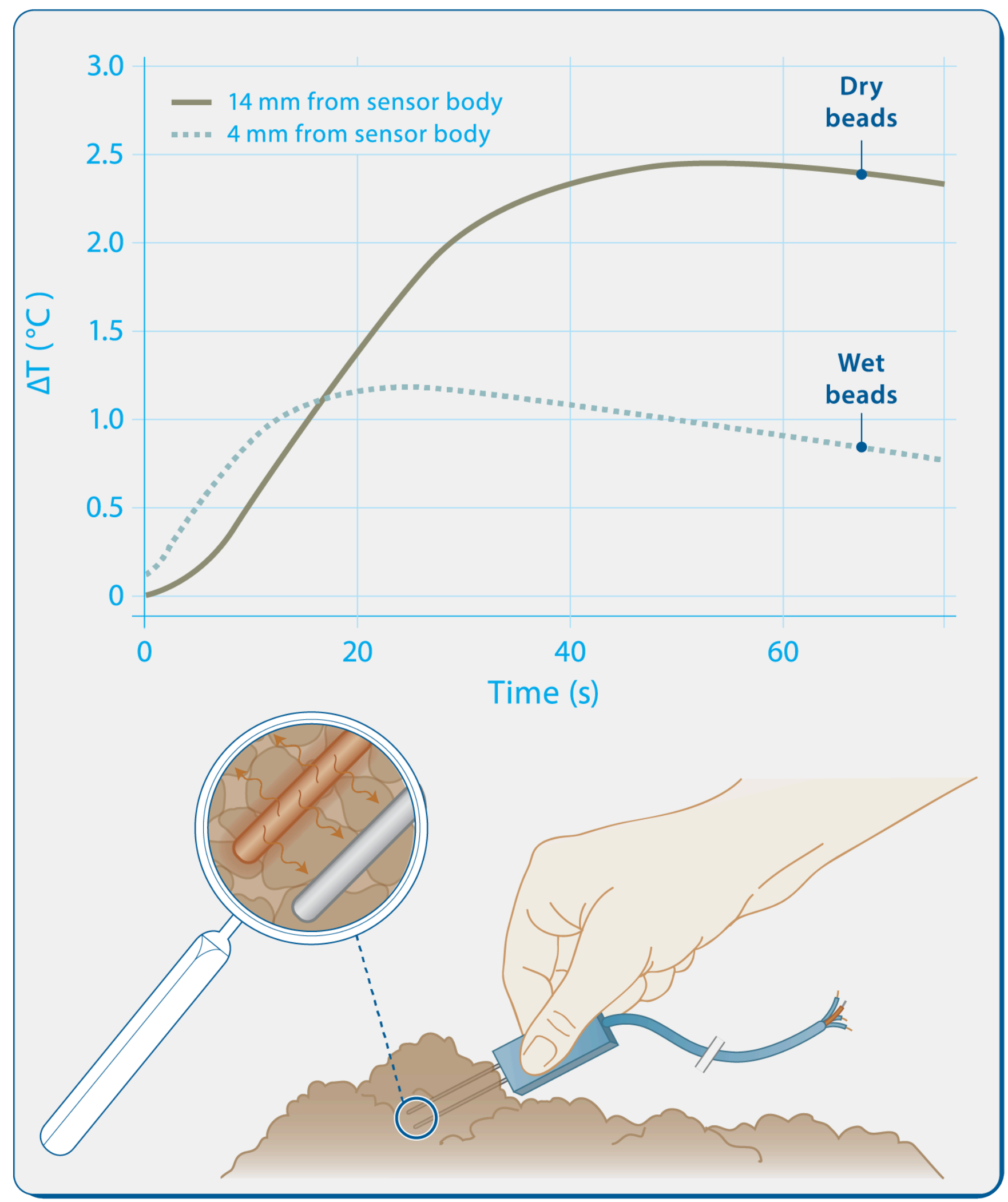

Figure 5.10 The dual probe heat probe method allows both the measurement of the response within the needle which provides the heat, as well as a sensing needle which is separated by a known distance. Combining the two measurements constrains thermal properties much more than does the single probe approach. 
Heat is generated for a short time by application of a constant electric current along the needle, while measuring its temperature rise with respect to time using a thermocouple collocated with the heater. The radial flow of heat from a linear heat source may be described using a variation of Eq.168) for a radial coordinate system. An approximate solution for a constant linear heat source of strength $\mathrm{q}\left(\mathrm{W} \mathrm{m}^{-1}\right)$ is given in terms of temperature rise with time from its initial value $\left(\mathrm{T}_{0}\right)$ by:

$$
T-T_{0}=\frac{q}{4 \pi \lambda} \ln \left(t+t_{0}\right)+d, \quad \frac{r^{2}}{4 D t}<<1
$$

where $\mathrm{d}$ is a constant, and $\mathrm{t}_{0}$ is a time correction which may be ignored for probes of $0.1 \mathrm{~cm}$ diameter or less, and for $\mathrm{t}>60 \mathrm{~s}$. A linear regression of $\mathrm{T}$ vs. $\ln (\mathrm{t})$ yields a line from which the thermal conductivity may be estimated as:

$$
\lambda=\frac{q}{4 \pi \cdot \text { slope of the line }}
$$

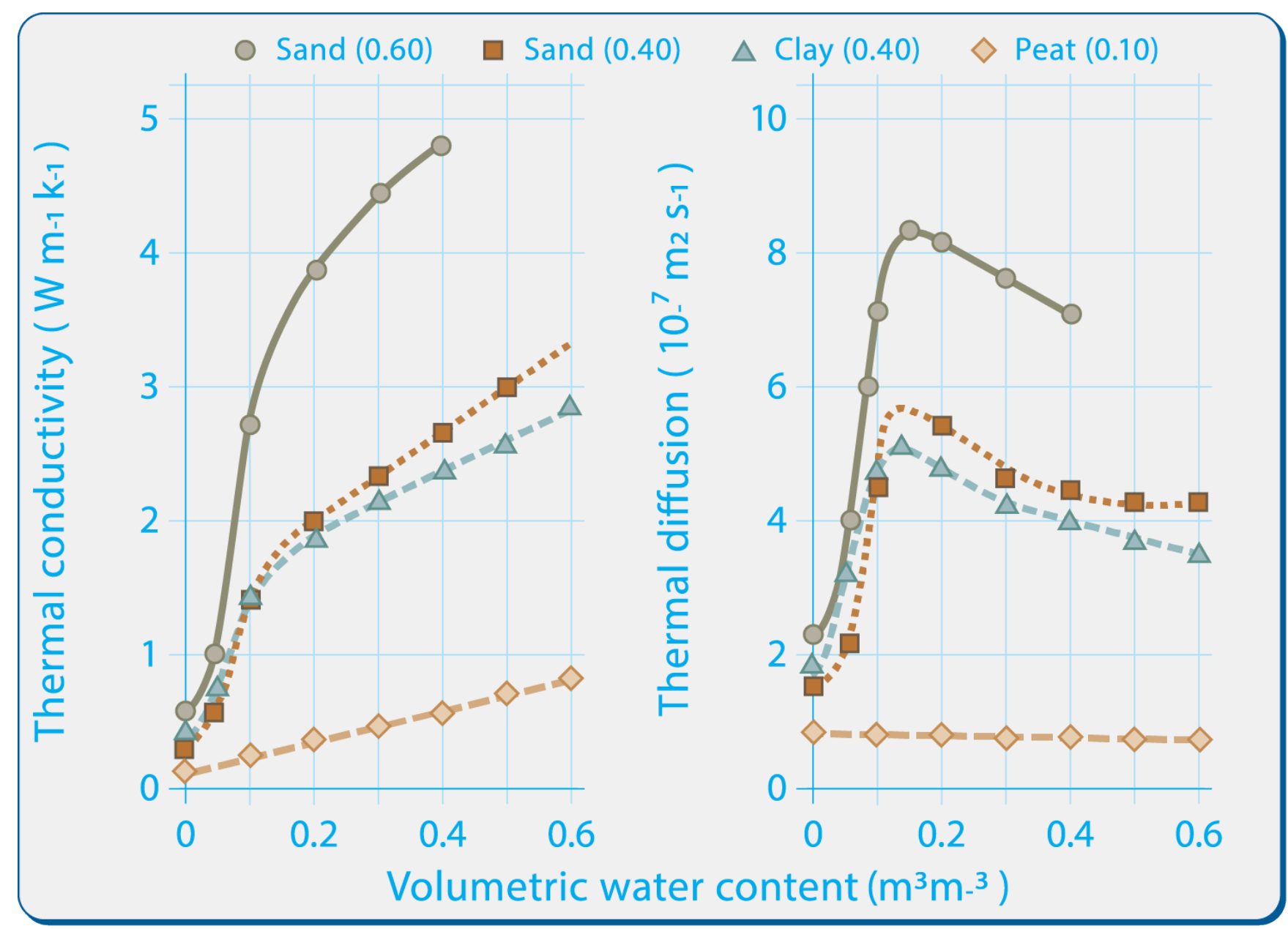

Figure 5.11 Soil thermal conductivity (a) and thermal diffusivity (b) as a function of water content for various soil types [Source: Jury et al. 1991; reprinted with permission of John Wiley \& Sons, Inc.].

\section{Thermal Diffusivity}

Thermal diffusivity is the ratio of the thermal conductivity to the volumetric heat capacity: $D_{H}=\lambda / c_{v}$. It may be estimated from measurements of $\lambda$ and $c_{v}$, or it may be measured directly. Thermal diffusivity at first increases rapidly 
with increasing water content, then decreases at a slower rate (Figure 5.11). This behavior results from the fact that while heat capacity $\left(c_{v}\right)$ increases linearly with water content, $\lambda$ increases most rapidly at low water contents (Figure 5.11). It is important to note that $\mathrm{D}_{\mathrm{H}}$ is not monotonic for many soils - so that the same value may be found for two different water contents. This is a key feature that must be kept in mind if one seeks to determine water content from soil thermal properties: any expression dependent primarily on $\mathrm{D}_{\mathrm{H}}$ will not be useful to estimate water content.

One method for measuring $\mathrm{D}_{\mathrm{H}}$ directly is based on placing a heat source having constant temperature $\left(\mathrm{T}_{\mathrm{S}}\right)$ in contact with the surface of a soil column having constant cross sectional area and insulated sides, initially at ambient temperature $\left(\mathrm{T}_{0}\right)$. A solution of Eq.168) relating temperature (T) along a plane at distance $\mathrm{x}$ from the source to time is given by (Carslaw and Jaeger, 1959):

$$
\frac{T-T_{0}}{T_{s}-T_{0}}=\operatorname{erfc}\left[\frac{x}{\sqrt{4 D_{H} t}}\right]
$$

where $\operatorname{erfc}(\mathrm{u})$ is the complementary error function, equal to $2 \pi^{-1 / 2} \int \exp \left(-\mathrm{u}^{2}\right) \mathrm{du}$. The complementary error function looks like a smoothing step function. Tabulated values of erfc(u) may be found in mathematical handbooks, similar to finding values of log, sin, cos, etc., or calculated using approximate solutions. Many recent versions of computer spreadsheets also feature erf and/or erfc as built-in functions. The solution is applicable only if the soil column is sufficiently long that the temperature at the other face of the column remains unchanged.

A procedure for finding explicit solutions to Eq.172) from observations of temperature (T) vs. time (t) is:

1. Calculate $\mathrm{p}=\left\{-\ln \left[0.5 *\left(\mathrm{~T}-\mathrm{T}_{0}\right) /\left(\mathrm{T}_{\mathrm{s}}-0\right)\right]\right\}^{1 / 2}$ for a given $\mathrm{t}$.

2. The argument in $\operatorname{erfc}(\mathrm{u})$ where $\mathrm{u}=\mathrm{x} /\left(4 \mathrm{D}_{\mathrm{H}} \mathrm{t}\right)^{1 / 2}$ may be approximated by (van Genuchten and Wierenga, 1986):

$$
\mathrm{u}=\mathrm{p}-\frac{1.881796+0.9425908 \mathrm{p}+0.0546028 \mathrm{p}^{3}}{1+2.356868 \mathrm{p}+0.3087091 \mathrm{p}^{2}+0.0937563 \mathrm{p}^{3}+0.0219104 \mathrm{p}^{4}}
$$

$D_{H}$ may then be calculated from:

$$
\mathrm{D}_{\mathrm{H}}=\frac{\mathrm{x}^{2}}{\mathrm{u}^{2} 4 \mathrm{t}}
$$

\section{Example 4-1: Thermal Diffusivity and Heat Capacity}

Given: A soil with $\theta_{\mathrm{v}}=0.3 \mathrm{~m}^{3} \mathrm{~m}^{-3}, \rho_{\mathrm{b}}=1350 \mathrm{~kg} \mathrm{~m}^{-3}$ and thermal conductivity $\lambda=2 \mathrm{~J} \mathrm{~m}^{-1} \mathrm{~s}^{-1}{ }^{\circ} \mathrm{C}^{-1}$. Find the thermal diffusivity $\mathrm{D}_{\mathrm{H}}$.

Solution: Recalling that $D_{H}=\lambda / c_{v}$, the first step is to compute $c_{v}$ using Eq.167). Neglecting organic matter we use $\mathrm{n}=1-\rho_{\mathrm{b}} / \rho_{\mathrm{s}}$, to find:

$$
\mathrm{c}_{\mathrm{v}}=1.94\left(1-\left[1-\frac{1350}{2650}\right]\right)+4.19 \cdot 0.3=2.245 \mathrm{MJm}^{-3-1}
$$

The thermal diffusivity is thus: 


$$
\mathrm{D}_{\mathrm{H}}=\frac{\lambda}{\mathrm{c}_{\mathrm{v}}}=\frac{2 \mathrm{~J} \mathrm{~m}^{-1} \mathrm{~s}^{-1}{ }^{-1}}{2.245 \cdot 10^{6} \mathrm{~J} \mathrm{~m}^{-1}{ }^{\circ} \mathrm{C}^{-1}}=8.907 \cdot 10^{-7} \mathrm{~m}^{2} \mathrm{~s}^{-1}=0.077 \mathrm{~m}^{2} \mathrm{~d}^{-1}
$$

\section{Diurnal and Seasonal Variations in Soil Temperature}

In the field, soil thermal regime is characterized by periodic changes in response to the natural periodicity in atmospheric conditions controlling energy inputs to the soil surface. There is a diurnal (daily) cycle (Figure 5.12), as well as a superimposed seasonal cycle (Fig.5.14). These diurnal and seasonal cycles are perturbed by irregular meteorological events including cloudiness, warm and cold fronts, precipitation, etc.

We may identify three fundamental characteristics of diurnal and annual soil thermal regimes. First, because these energy inputs are cyclic, we observe diurnal and annual temperature cycles in response to these fluctuating inputs. Secondly, because much of the heat traveling down through a soil profile is utilized to change the local temperature of the soil, there is a decrease in energy flux at depth. Thus we observe the phenomenon of amplitude damping, or a reduction in the magnitude of these temperature cycles with increasing depth. Finally, because it takes time for heat to travel into and out of the soil, there is a delay in the time at which any specific location on the temperature cycle reaches a given point in the soil, and this time lag becomes more pronounced with increasing distance. Each of these characteristics may be observed in field data plotted as Figure 5.12?. Note also the gradual increase in temperature at $1 \mathrm{~m}$ depth, which represents springtime warming associated with the annual temperature cycle at that depth. This phenomenon is not as readily apparent from data at the shallower depths in Figure 5.12? due to the higher frequency and larger amplitude daily cycles superimposed upon the annual temperature fluctuations. 


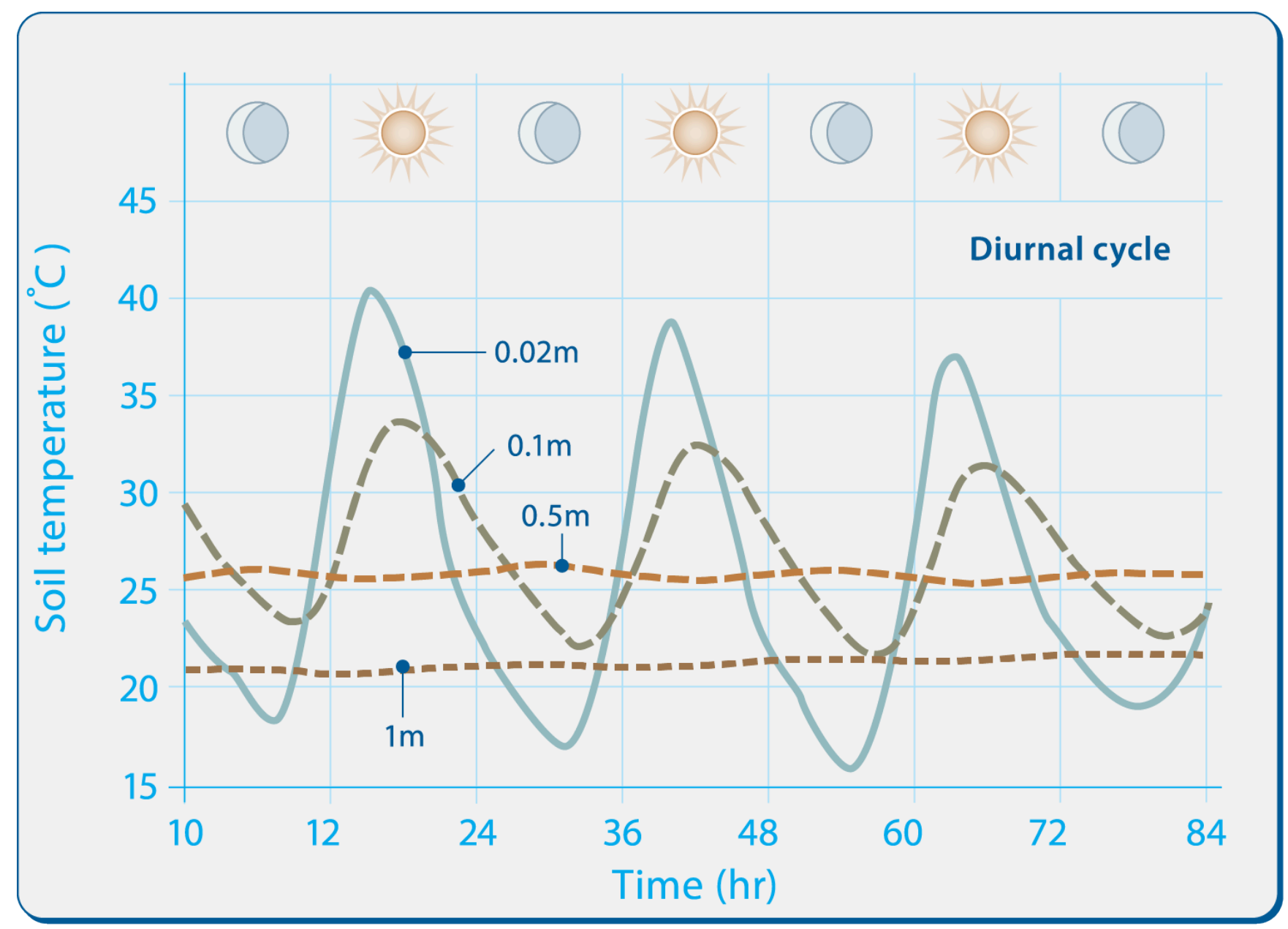

Figure 5.12 Soil temperature measured at four depths under corn near Logan, UT during May 27 to 30, 1988 [Wraith and Hanks, 1992]. 


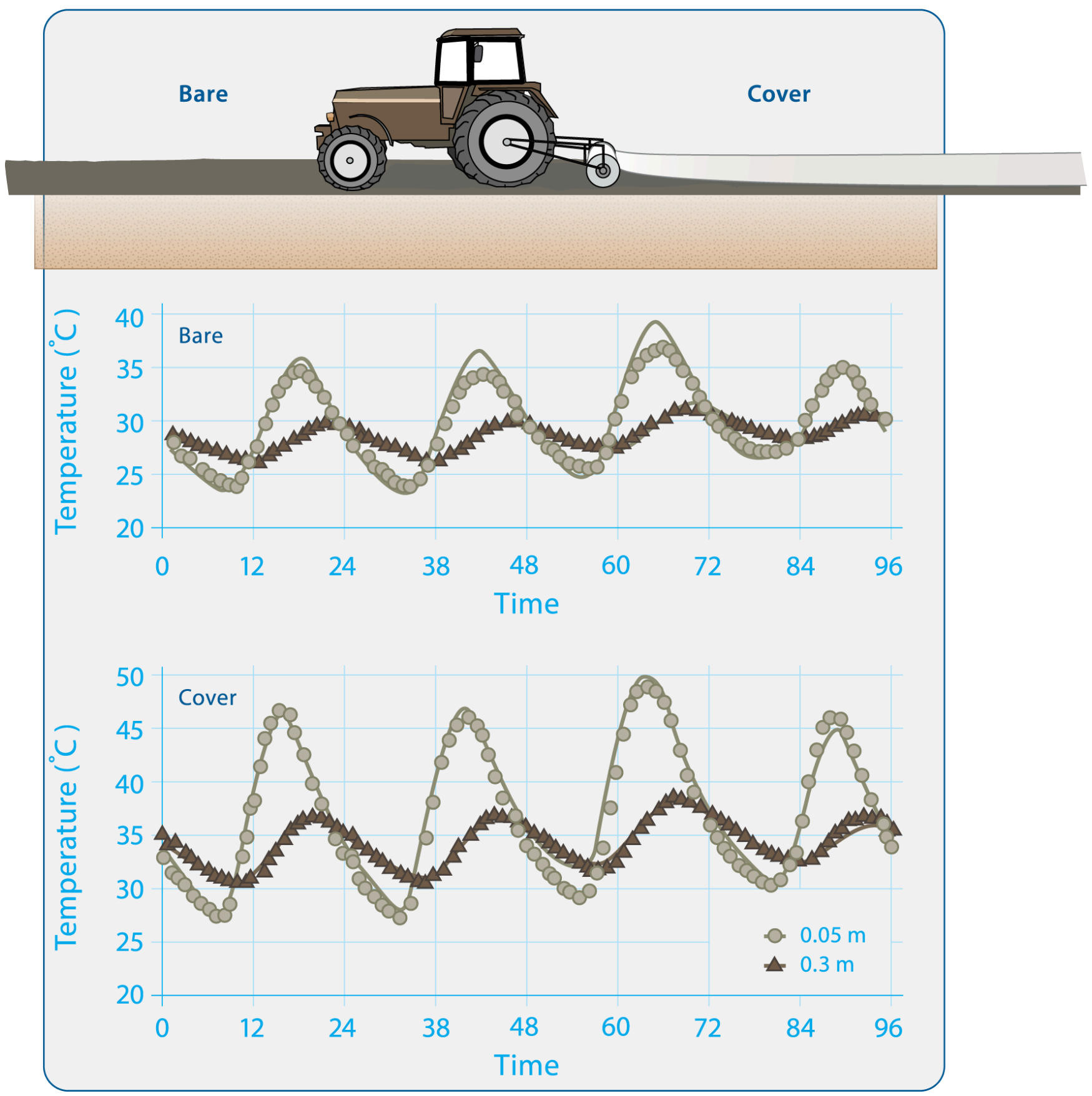

Figure 5.13 Inclusion of a cover on a field has many implications for water and thermal behaviors. Here we show data from a 4 day comparison of a bare and covered field. The covered field shows $10^{\circ} \mathrm{C}$ higher daytime surface temperatures, and $5^{\circ} \mathrm{C}$ average temperature. Data from XXX. These effects are well captured by numerical modeling (solid line). 


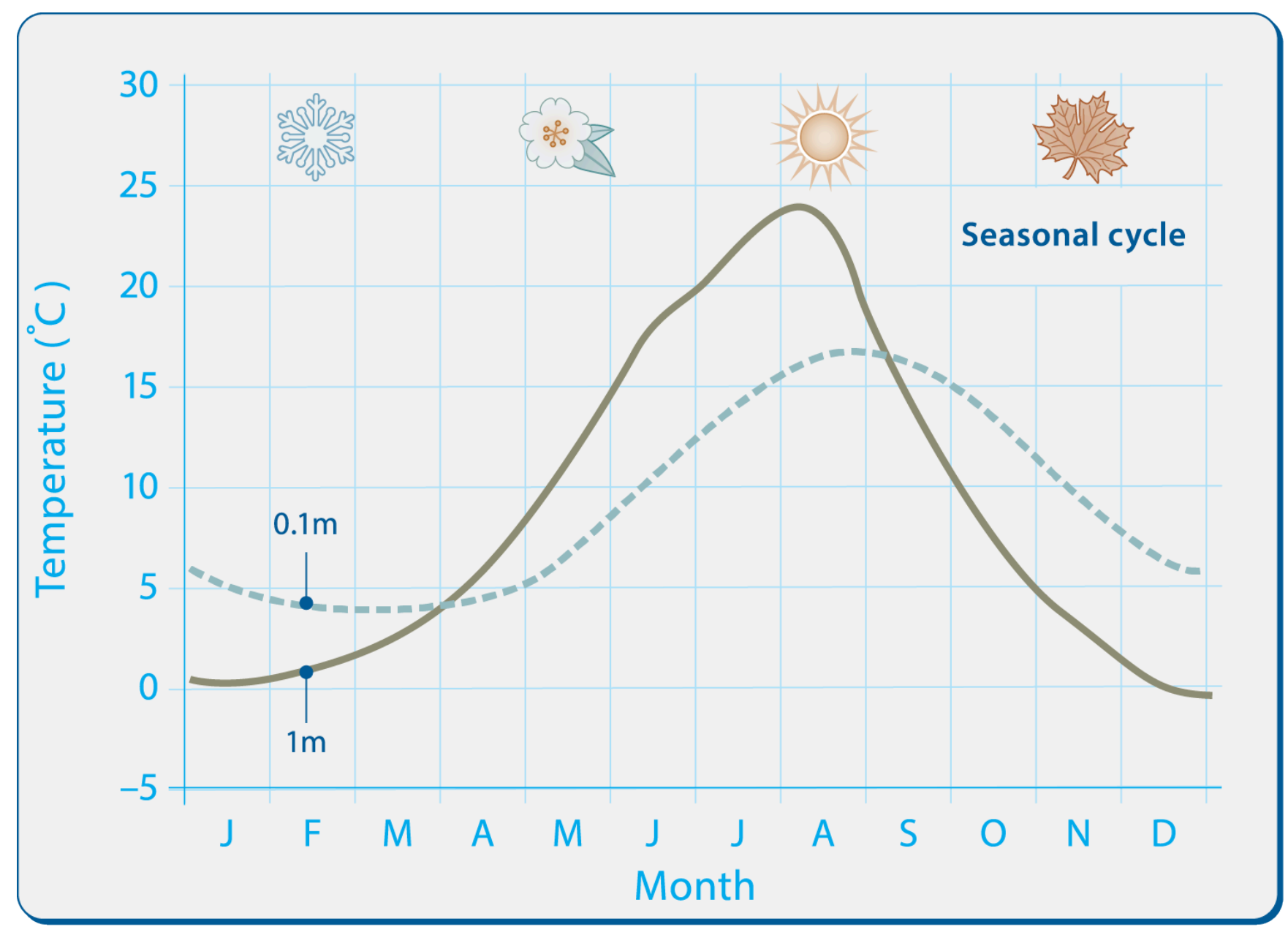

Figure 5.14 Mean monthly soil temperatures measured at two depths in Logan UT [Source: Hanks, 1992].

In order to obtain a mathematical description for a soil's thermal regime, it is common to assume that soil temperature oscillates as a pure harmonic (sinusoidal) function of time about some mean value. For example, we may represent the surface temperature $(\mathrm{z}=0)$ as a function of time, $\mathrm{T}(0, \mathrm{t})$, by:

(5.19) $T(0, t)=T+A_{0} \sin \omega t$

where $\mathrm{T}$ is the mean temperature at the soil surface (as well as of the soil profile), $\mathrm{A} 0$ is the amplitude of temperature fluctuation $\left[\mathrm{A}_{0}=\right.$ (maximum - minimum) $\left./ 2\right]$ at the soil surface, $\omega=2 \pi / \mathrm{P}$ is the angular frequency and $\mathrm{P}$ is the period of oscillation. For diurnal variations, $\mathrm{P}=24 \mathrm{~h}$ or $86400 \mathrm{sec}$; and for yearly variations, $\mathrm{P}=12$ months or 365 days. If we assume that Eq.175) applies at the soil surface, and at a very large depth the temperature is constant (T), we may obtain a solution to Eq. 168) for temperature variations as a function of time and depth:

$$
T(z, t)=T+A_{0} \exp ^{d} \sin \left[\omega(t-8)-\frac{z}{d}\right] \quad 0 \leq z<\infty
$$

where $\mathrm{z}$ is depth below the surface expressed as positive numbers, the 8 in ' $(\mathrm{t}-8)$ ' is an offset in hours to the sine function to obtain maximum soil surface temperature at 2 p.m. consistent with many field measurements, and $d$ is the characteristic damping depth:

$$
d=\sqrt{2 D_{H} / \omega}=\sqrt{P D_{H} / \pi}
$$

or depth at which the amplitude $A_{z}$ decreases to the fraction $1 /$ e $(1 / 2.718=0.37)$ of the surface amplitude $\left(A_{0}\right)$. The offset value may be modified somewhat consistent with measured temperatures, to obtain optimal agreement. 
From the solution (Eq.176)), one can see that: (1) the amplitude at each depth is reduced to $A_{z}=A_{0} e^{z / d} ;(2)$ there is an increase in phase (time) lag with depth equal to $\mathrm{z} / \mathrm{d}$; (3) the average temperature and the period are the same for all depths.

In-situ measurements of soil temperature with time and depth, coupled with the above solution (Eq.176)) may be used to infer soil thermal properties. For example, one can use the phase (time) lag, or the amplitude damping, to infer the soil thermal diffusivity $\mathrm{D}_{\mathrm{H}}$. Using the ratio of two amplitudes $\left(\mathrm{A}_{1}=\mathrm{A}_{0} \mathrm{e}^{\mathrm{z} 1 / \mathrm{d}} ; \mathrm{A}_{2}=\mathrm{A}_{0} \mathrm{e}^{\mathrm{z} 2 / \mathrm{d}}\right)$ measured at different depths $(\mathrm{z} 1$ and $\mathrm{z}_{2}$ ) yields:

$$
\mathrm{D}_{\mathrm{H}}=\frac{\mathrm{P}\left(\mathrm{z}_{1}-\mathrm{z}_{2}\right)^{2}}{4 \pi\left(\mathrm{t}_{1}-\mathrm{t}_{2}\right)^{2}}
$$

Similarly, one may use the difference between two time lags (e.g., the argument in the sine function at the two depths for the attainment of maximal temperature should equal $\pi / 2$ ), thus:

$$
\mathrm{D}_{\mathrm{H}}=\frac{\pi\left(\mathrm{z}_{1}-\mathrm{z}_{2}\right)^{2}}{\mathrm{P}\left(\ln \left[\frac{\mathrm{A}_{1}}{\mathrm{~A}_{2}}\right]\right)^{2}}
$$

Values of $\mathrm{D}_{\mathrm{H}}$ resulting from these two estimation methods may differ due to violation of assumptions made in the derivation of the solution, e.g. the soil may be nonuniform, the measured temperature wave may not be well represented by a sine function, etc.

\section{Example 5-2: Diurnal Variations in Soil Temperature}

Given: The thermal diffusivity $\mathrm{D}_{\mathrm{H}}$ is $0.003 \mathrm{~m}^{2} \mathrm{hr}^{-1}$, the average temperature of the soil is $20^{\circ} \mathrm{C}$, and the daily amplitude at the soil surface is $20^{\circ} \mathrm{C}$.

Find: The temperature at a depth of $\mathrm{z}=0.2 \mathrm{~m}$ and at $\mathrm{t}=8 \mathrm{hr}$.

Solution: To use Eq.176) we first compute the characteristic damping depth:

$$
\mathrm{d}=\left(\frac{24 \mathrm{hr} \cdot 0.003}{3.14}\right)^{1 / 2}=0.151 \mathrm{~m}
$$

Then we plug in the appropriate values (remember to use radians for the computation of the sine function) and obtain:

$$
\mathrm{T}(-0.2,8)=20+20 \mathrm{e}^{(-0.2 / 0.151)} \sin \left[\frac{2 \pi(8-8)}{24}-\frac{0.2}{0.151}\right]=14.8 \mathrm{C}
$$




\section{Example 4-3: Estimation of Thermal Diffusivity (DH)}

Given: The data in Figure 5.12? and the following information for the first day: the maximum and minimum temperatures at 20 and $100 \mathrm{~mm}$ were $(40.0,18.7)$ and $(33.6,23.4)^{\circ} \mathrm{C}$, respectively. Their respective occurrence times were 1500 and $1800 \mathrm{hrs}$ for the maximum temperature, 0700 and $0900 \mathrm{hrs}$ for the minimum.

Find: The thermal diffusivity $\mathrm{D}_{\mathrm{H}}$ for the soil using the time lag and the amplitude damping methods.

Solution: For the time lag method we observe that the time difference between the maximum temperatures at $-0.02 \mathrm{~m}$ and $-0.10 \mathrm{~m}$ was $\left(\mathrm{t}_{1}-\mathrm{t}_{2}\right)=3.0 \mathrm{hrs}$. Using Eq.179) with $\mathrm{P}=24 \mathrm{hrs}$, we find:

$$
\mathrm{D}_{\mathrm{H}}=\frac{24(-0.02+0.10)^{2}}{4 \pi \cdot 3^{2}}=0.00136 \mathrm{~m}^{2} \mathrm{~h}^{-1}=0.03269 \mathrm{~m}^{2} \mathrm{~d}^{-1}
$$

For the amplitude damping method we compute the amplitude at $-0.02 \mathrm{~m}$ as:

$$
\mathrm{A}_{1}=\frac{40.0-18.7}{2}=10.65
$$

And at $\mathrm{z}=-0.10 \mathrm{~m}$ :

$$
\mathrm{A}_{2}=\frac{33.6-23.4}{2}=5.10
$$

Now we can calculate thermal diffusivity as:

$$
\mathrm{D}_{\mathrm{H}}=\frac{\pi(-0.02+0.10)^{2}}{24 \cdot\left(\ln \left[\frac{10.65}{5.10}\right]\right)^{2}=0.00154 \mathrm{~m}^{2} \mathrm{~h}^{-1}=0.0371 \mathrm{~m}^{2} \mathrm{~d}^{-1}}
$$

The two values of $\mathrm{D}_{\mathrm{H}}$ are in reasonable agreement.

\section{SUMMARY - Chapter 5}

Heat transport in soils is a result of the interaction of energy transfer mechanisms, primarily near the soil surface, and of dynamic thermal properties of the soil body. Fundamental soil thermal properties include the specific and volumetric heat capacities, the thermal conductivity, and the ratio of thermal conductivity to volumetric heat capacity or thermal diffusivity. Each of these properties arises as a result of the relative proportions and specific relationships between the three soil phases as discussed in Section 1. The interaction of the surface energy balance with soil thermal properties results in diurnal and annual temperature cycling in soil profiles, each of which exhibit characteristic properties of time lag and amplitude damping. While often considered to be of secondary concern relative to the status of water or chemicals in soils, the soil thermal regime may often have a significant impact on these processes. Kinetics of physical, chemical, and biological processes are greatly influenced by ambient temperature, with resultant consequences for nearly any aspect of soil science or soil behavior we might consider. 


\section{REFERENCES - Chapter 5}

Alam, M.R., M.F. Zain, A.B. Kaish, A. Jamil. 2015. Underground Soil and Thermal Conductivity Materials Based Heat Reduction for Energy Efficient Building in Tropical Environment. Indoor and Built Environment 24(2):185-200. DOI: 10.1177/1420326X13507591.

Assouline, S., K. Narkis, K, S.W. Tyler, I. Lunati, M.B. Parlange, and J.S. Selker. 2010. On the Diurnal Soil Water Content Dynamics during Evaporation using Dielectric Methods Vadose Zone J., 9 (3): 709-718.

Assouline, S., S.W. Tyler, J.S. Selker, I. Lunati, C.W. Higgins, M.B. Parlange. 2013. Evaporation from a shallow water table: Diurnal dynamics of water and heat at the surface of drying sand. Water Resour. Res 49(7):1944-7973. DOI 10.1002/ wrcr.20293.

Brutsaert, W. 1982. Evaporation into the Atmosphere. P. Reidel Publishing Company. Dordrecht, Holland.

Carslaw, H.S., and J.C. Jaeger. 1959. The Conduction of Heat in Solids. Oxford University Press. London.

Hanks, R.J., 1992. Applied Soil Physics. 2nd ed., Springer Verlag. New York. NY.

Hillel, D. 1980. Fundamentals of Soil Physics. Academic Press, San Diego, CA.

Hiraiwa, Y. and T. Kasubuchi. 2000. Temperature dependence of thermal conductivity of soil over a wide range of temperature $\left(5-75^{\circ} \mathrm{C}\right)$. European Journal of Soil Science. vol. 51(2): 211-218.

Jury, W.A., W.R., Gardner, and W.H. Gardner. 1991. Soil Physics. John Wiley and Sons, New York, NY.

Wraith, J.M., and R.J. Hanks. 1992. Soil thermal regime influence on water use and yield under variable irrigation. Agron. J. 84:529-536.

\section{Problems - Section 4}

1. How much heat $\left(\mathrm{W} / \mathrm{m}^{2}\right)$ will be needed to change the temperature of $35 \mathrm{~kg}$ of Silt-Loam soil from 20 to $28^{\circ} \mathrm{C}$ if water content is $0.25 \mathrm{~m}^{3} \mathrm{~m}^{-3}$, and the volumetric fraction of organic matter is $0.03 \mathrm{~m}^{3} \mathrm{~m}^{-3}$ ?

2. What is the heat flux density in peat soil with $2_{\mathrm{v}}=0.4 \mathrm{~m}^{3} \mathrm{~m}^{-3}$, if temperature at the surface is $35^{\circ} \mathrm{C}$, and temperature at a depth of $0.3 \mathrm{~m}$ is $25^{\circ} \mathrm{C}$ ?

3. A soil sample at uniform initial temperature $\mathrm{T} 0=24^{\circ} \mathrm{C}$ was brought into contact with a heat source of constant temperature $\mathrm{TS}=40^{\circ}$ At a distance of $\mathrm{x}=30 \mathrm{~mm}$ from the "hot" surface the temperature was $\mathrm{T}=28^{\circ} \mathrm{C}$ after $360 \mathrm{~s}$; (i) find the soil's thermal diffusivity $\mathrm{D}_{\mathrm{H}}$, and (ii) predict the amount of time needed to raise the temperature at $\mathrm{x}=100 \mathrm{~mm}$ by $2^{\circ} \mathrm{C}$ (Hint: use Eq.174).

4. Use the information in the figure below to estimate the soil thermal diffusivity by the time-lag and amplitude damping methods. 


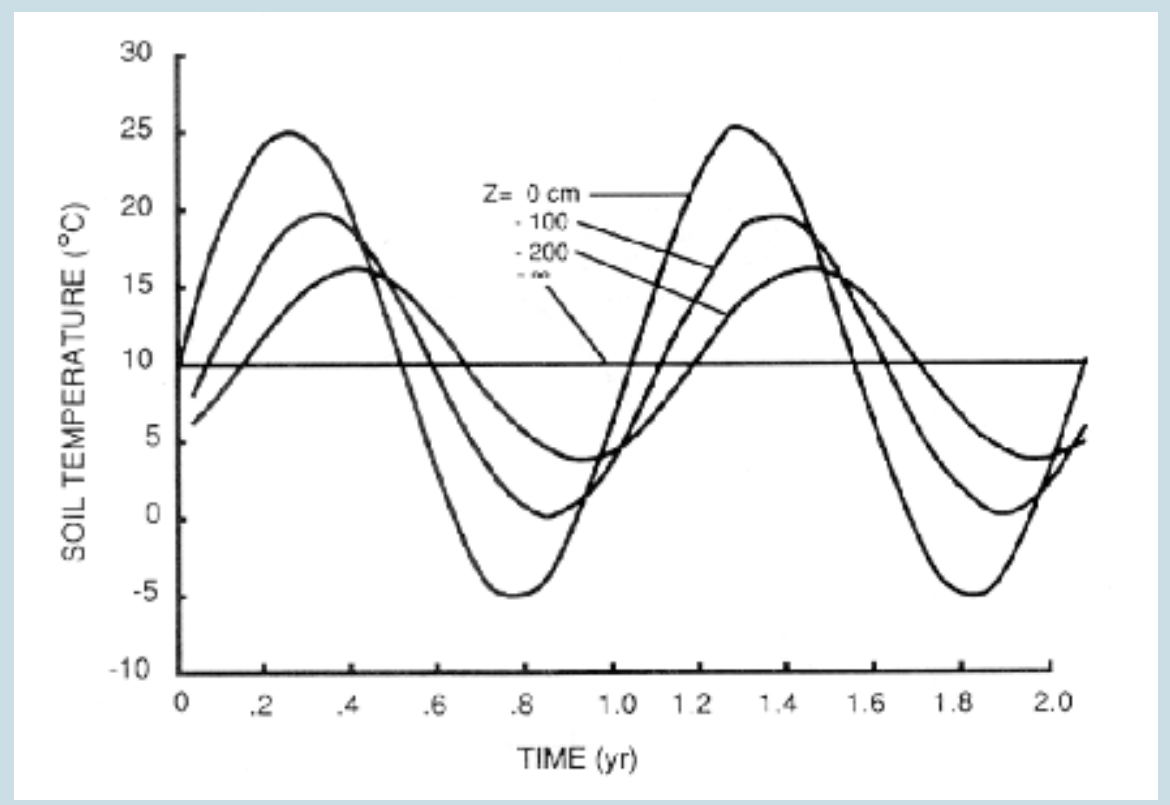

5. Two thermocouples buried at $\mathrm{z}_{1}=-0.75 \mathrm{~m}$ and $\mathrm{z}_{2}=-1.5 \mathrm{~m}$ recorded an annual $\mathrm{T}_{\max }$ of 20.75 and $17.7^{\circ} \mathrm{C}$, and $\mathrm{T}_{\min }$ of -0.75 and $2.3^{\circ} \mathrm{C}$ for $\mathrm{z}_{1}$ and $\mathrm{z}_{2}$, respectively. Calculate: (i) the soil thermal diffusivity; (ii) the annual average temperature; and (iii) the amplitude of the temperature at the soil surface.

6. Given $\mathrm{D}_{\mathrm{H}}=430 \mathrm{~cm}^{2} \mathrm{~d}^{-1}, \mathrm{~T}_{\text {average }}=7^{\circ} \mathrm{C}$, and $\mathrm{A}_{\mathrm{O}}=17^{\circ} \mathrm{C}$ Find: (i) the depth at which the minimum temperature is above freezing (so one may safely bury water pipes); and (ii) the depth at which the time lag is 6 months (so a basement will be pleasantly warm in the Winter and cool in the Summer). 


\section{Solute Transport in Soils and Vadose Materials}

Much of the recent interest in vadose zone processes has come out of the need to predict the movement of contaminants to aquifers. Contaminants have diverse sources, chemistries, and time-evolutions of deposition in soils, all of which effect their fate. Spills can be rapid or slow, from points of entry, or spread across a field, injected at or below the surface, and may be made up of substances that are nearly insoluble in water (diesel oil), or essentially completely soluble (e.g., nitrate) (Figure 6.1 below). Investigation of solute transport through the vadose zone has consequently received great attention, with a number of distinct approaches having been developed to match the diversity of situation that arise. We first review the basic formulations of sorption and degradation, then review the approaches to solute modeling with a focus on the approach which we believe is of greatest utility, the convection-dispersion equation. Some

of the more recently introduced formulations for modeling transport are briefly discussed, including use of transfer functions, stream tube models, and mobile-immobile approaches. 


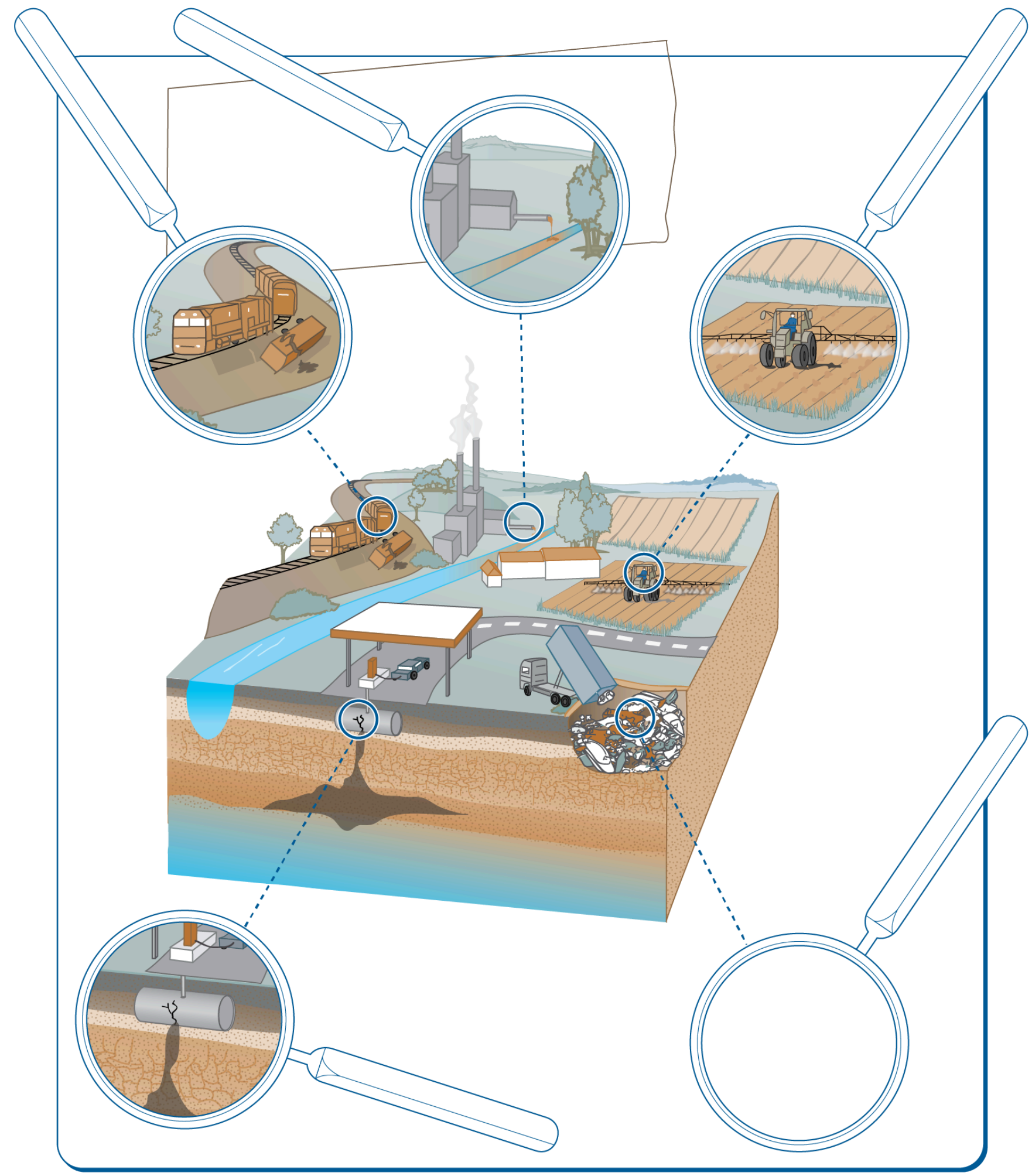

Figure 6.1

The description of solute movement is fundamentally dependent upon the accuracy of the understanding of the solvent (typically water for vadose problems) movement. Any errors in the solvent description will be propagated and likely 
amplified in the solute model. So step one in your thinking should always be to understand any uncertainties you have in your description of water flow, ad address these to the greatest degree possible.

There are three basic mechanisms by which solutes may move: advection, diffusion, and dispersion. Advection, also known as convection, refers to movement of the solute with the bulk water in a macroscopic sense (Figure 6.2). Advective transport ignores the microscopic processes, but simply follows the bulk Darcian flow vectors. Imagine that you were in a great tightly packed crowd, say at a political rally with your brothers and sisters by your side. You would be advected if the speaker told everyone to move three steps to the east, and the whole pack moved without changing the local positions between you and your siblings. Generally when we speak of advection we ignore all processes that lead to solute spreading, referring to advective flow as "piston" flow or "plug" flow, evoking the sense that the solutes march in lock-step with the water. With pure-advection (no adsorption, degradation, or dispersion), if a column of soil contains $25 \%$ water content, then if we apply a pulse of solutes, then $25 \mathrm{~cm}$ of water, the solute will all end up at exactly one meter depth. We would then define the "pore volume" of this system to be $25 \mathrm{~cm} / \mathrm{m}$. In Figure 6.2 we illustrate a pore volume of 10 balls, so if a solute ball is applied at the inlet, which will emerge after 10 more balls follow this solute. Notice that the "concentration" of solute has not changed from that which was put in to that which came out. By contrast, if we had five parallel tubes, which held eight, nine, ten, eleven and twelve balls, then after putting eight balls in each tube, we would get one "solute" ball, and four clean balls. So the concentration would be $1 / 5$ of the applied solute concentration. this would continue to be the outcome until the 13th ball, at which point the outflow would be free of solutes. While in each of the five tubes there was plug flow, taken together they resulted in smearing the solutes.

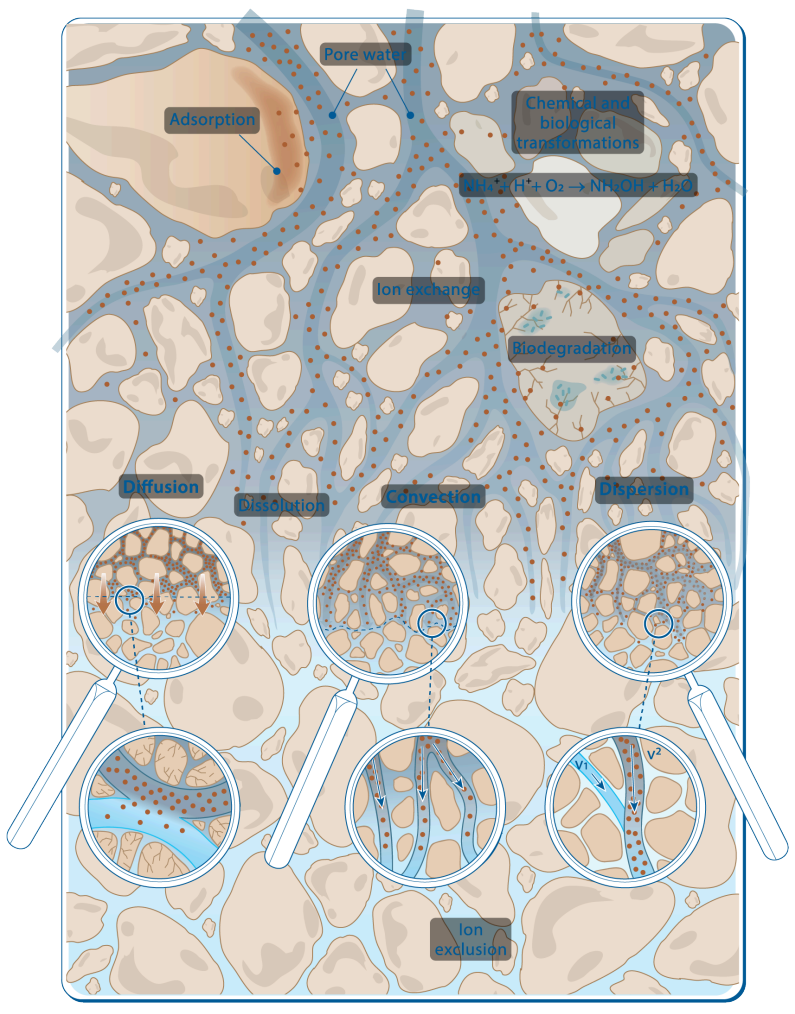

Figure 6.2. Illustration of "plug flow" as balls in a pipe. 


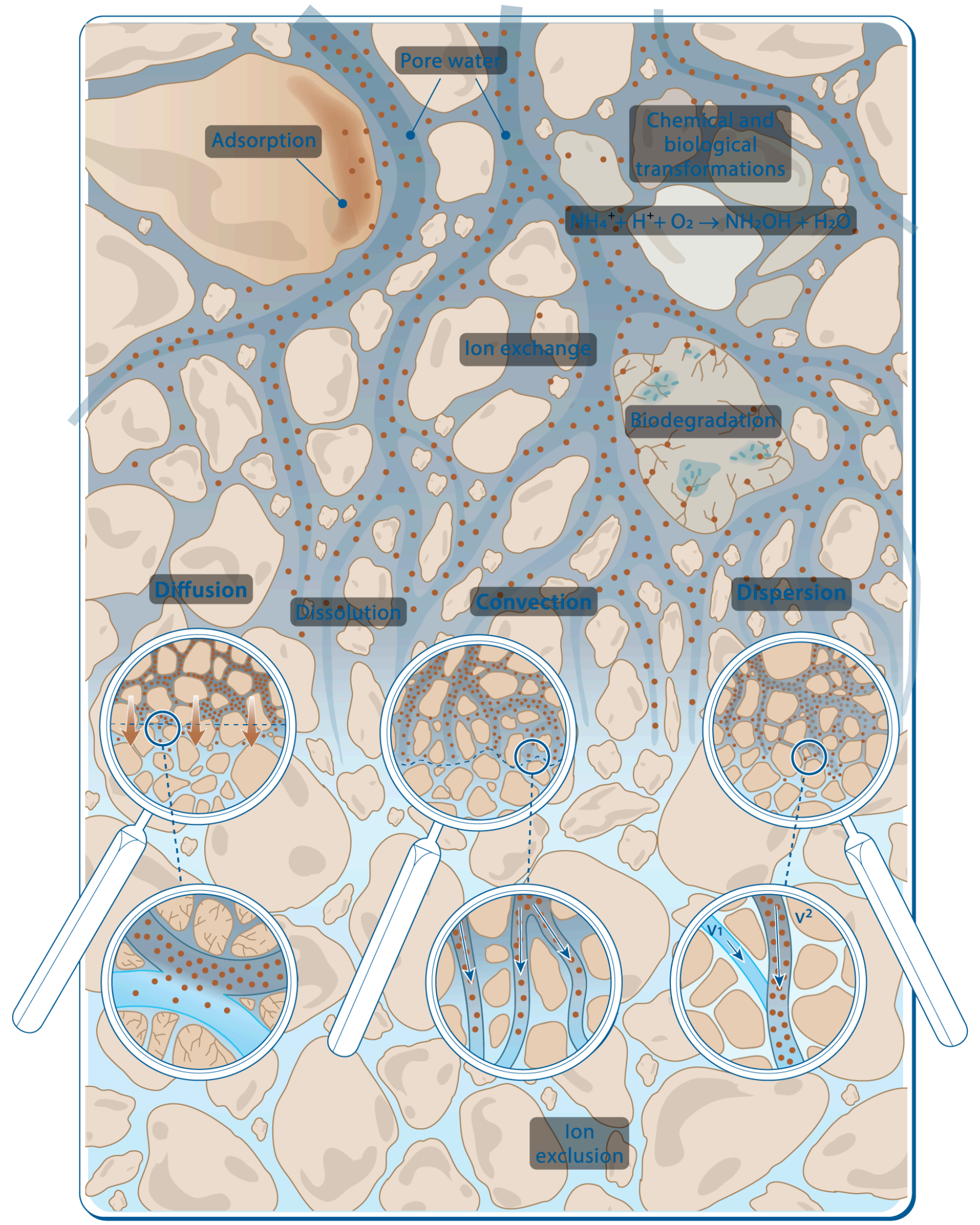

Figure 6.3 
Diffusion refers to the spreading of a compound through the effects of molecular motion. Governed by Fick's law, molecular movement tends to mix areas of high concentration with areas of lower concentration. Diffusion from a point source of solutes, in absence of advection or physical obstacles to movement, will lead to a Gaussian distribution of the solute, with the width of the plume growing with the square root of time. Since the process is governed by molecular movement, the rapidity of diffusive spreading is linked to the typical velocities of molecular movement as well as the typical path length of movement between molecular collisions. For a given temperature, any given molecule has a particular energy, and thus velocity. Since kinetic energy is related to the square of velocity, diffusion rates changes with the square-root of temperature (as measured in degrees $\mathrm{K}$ ), and thus vary little over typical groundwater temperature ranges. The diffusion rates, as summarized by the diffusion coefficients, are on the order of $0.2 \mathrm{~cm}^{2} / \mathrm{s}$ in gases and $0.00002 \mathrm{~cm}^{2} / \mathrm{s}$ in liquids; a factor of 10,000 higher in gases due to the lower rate of molecular collisions. (Note: the spreading caused by diffusion goes with the square root of the diffusion coefficient, so liquid phase diffusion spreads stuff about 100 times slower than gas phase diffusion). This should make sense if you think that a mole of gas fills about $20,000 \mathrm{~cm}^{3}$ while the same mole of liquid (say water) would fill about $20 \mathrm{~cm}^{3}$. This alone would suggest that typical path lengths between collisions should differ by a factor of $1000^{1 / 3}=10$. This discrepancy is further amplified by the fact that much of the volume of liquids is taken up by the molecules themselves, leaving little room for free motion without collision. In the political rally, diffusion would correspond to the movement that happens when they put the dance music on as darkness falls. Now people start bouncing around, and slowly you and your siblings will spread out in the crowd, making your mother very anxious about how you will all ever be brought together again. She is right to worry in that she is working in direct opposition to the aggressive force of entropy, a tough foe.

Dispersion refers to mixing which occurs due to differences in velocities of neighboring parcels of fluid. Dispersion is a process which occurs at many scales, while diffusion is strictly a molecular-scale process. Harking back to the political rally, imagine that they have turned the music off, and your mother has reassembled the family. Now it is time to leave the pavilion. Unfortunately there are many pillars holding up the roof, and as the crowd exits, some of the family members get stripped off from the group by the crowd moving past these obstructions. Dispersive processes in ground water flow start at the scale of the intergranular channels which the fluid moves through. In these channels the fluid velocity is proportional to the square of the distance from the local surfaces (see derivation of Hagen-Poiseuille law, chapter 2), leading to separation of particles across these areas, as shown in Figure 6.4 in elements A and B. The tortuousity of the intergranular space also smears solutes as they pass through porous media, as shown in elements $\mathrm{C}$ and D in Figure 6.4. At a larger scale (say the $1 \mathrm{~m}$ scale), there is typically heterogeneity between materials of differing permeability, which will again lead to areas of higher and lower flow velocity, which leads to further dispersion of materials distributed across such regions: the magnitude of dispersion increases with increasing scale as each new dispersive process is added to those which occur at all of the lower scales.

Figure 6.4 Sketch of intergranular-scale dispersive processes. Here the porous media is saturated with fluid which is moving from left to right. A straight-line of molecules in position A are distorted to the parabolic line of molecules in $\mathrm{B}$ due to the laminar flow velocity profile between particles. The region of solutes in position $\mathrm{C}$ is distorted around a particle to shape D due to the tortuousity of the inter-granular channels.

\section{Flux and Resident Concentration}

Before launching into a description of solute movement, we must understand what is meat when specifying a certain concentration at a point. In the volume about a given point, the resident aqueous concentration, $\mathrm{C}_{1}^{\mathrm{r}}$, may be defined as the mass of solute per unit mass of liquid

$$
\mathrm{C}_{\mathrm{l}}^{\mathrm{r}}=\frac{\mathrm{M}_{\mathrm{sw}}}{\mathrm{M}_{\mathrm{w}}}
$$


where $\mathrm{M}_{\mathrm{SW}}$ is the mass of solute found in a mass of water $\mathrm{M}_{\mathrm{w}}$. We may define the resident concentration of a sorbed species, $\mathrm{C}_{\mathrm{S}}^{\mathrm{r}}$, as the mass sorbed per mass of solid

$$
\mathrm{C}_{\mathrm{s}}^{\mathrm{r}}=\frac{\mathrm{M}_{\mathrm{ss}}}{\mathrm{M}_{\mathrm{s}}}
$$

where $\mathrm{M}_{\mathrm{SS}}$ is the mass of solute found sorbed to a mass of solid $\mathrm{M}_{\mathrm{S}}$. Since the water content of the vadose zone is variable in time, it is most convenient to define the total resident concentration, $\mathrm{C}^{\mathrm{r}}$, on a volumetric basis, in which case the concentration is the total species mass per volume of vadose material $\mathrm{V}$

$$
\mathrm{C}^{\mathrm{r}}=\frac{\mathrm{M}_{\mathrm{sw}}+\mathrm{M}_{\mathrm{ss}}}{\mathrm{V}}
$$

You might think that that is the end of the concentration story, but there is one more wrinkle that we must address. Suppose two people are assigned the task of monitoring solute concentration in time at a given subsurface location. To further simplify the problem, their task has been defined as only being responsible to report aqueous concentrations, with some other team looking at sorbed material. There certainly should be no ambiguity in this task, right? Well, suppose that one of the field workers uses a porous cup suction sampler which operates by quickly extracting the solution in the vicinity of the cup. For the sake of argument, we will suppose that the cup device instantaneously extracts all of the water residing in the soil immediately surrounding the point of interest. The concentration observed in this sample corresponds to the resident aqueous concentration defined above, $\mathrm{C}_{1}^{\mathrm{r}}$. Now suppose that the second field worker has a small intercepting pan device that is inserted at the depth of interest and provides a continuous set of samples of the water passing the point of interest without disturbing the flow regime. An example of such an instrument is the Passive Capillary Sampler (PCAPS) employed recently by a number of researchers (e.g., Brandi-Dorhn et al., 1997). The concentrations of samples collected in this manner will be called aqueous flux concentrations, $\mathrm{C}_{1}^{\mathrm{f}}$.

Is our team bound for strife or harmony? Is $\mathrm{C}_{1}^{\mathrm{r}}=\mathrm{C}_{1}^{\mathrm{f}_{2}}$ ? In general the answers are strife and no, respectively. To intuitively understand this, consider an extreme case of a unit of damp soil penetrated by a single worm hole which carries half of the percolating water. $\mathrm{C}_{1}^{\mathrm{r}}$ about the worm hole will be dominated by the state of water in the damp soil volume, while $\mathrm{C}_{1}^{\mathrm{f}}$ will be made up in equal parts of water from the bulk soil and the worm hole. Assuming that the worm hole is fed from the soil surface, the solute concentration in each of these samples would clearly be different. Perhaps less obvious is the fact that even had the worm hole not been there, the concentrations would have differed, as shown by Parker and van Genuchten (1984), and discussed in section 5.3.2.5. Generally in this text we will be using the resident concentration since our formulations generally are built on a control volume approach, which dictates that we keep track of the mass resident in particular volumes of material. 


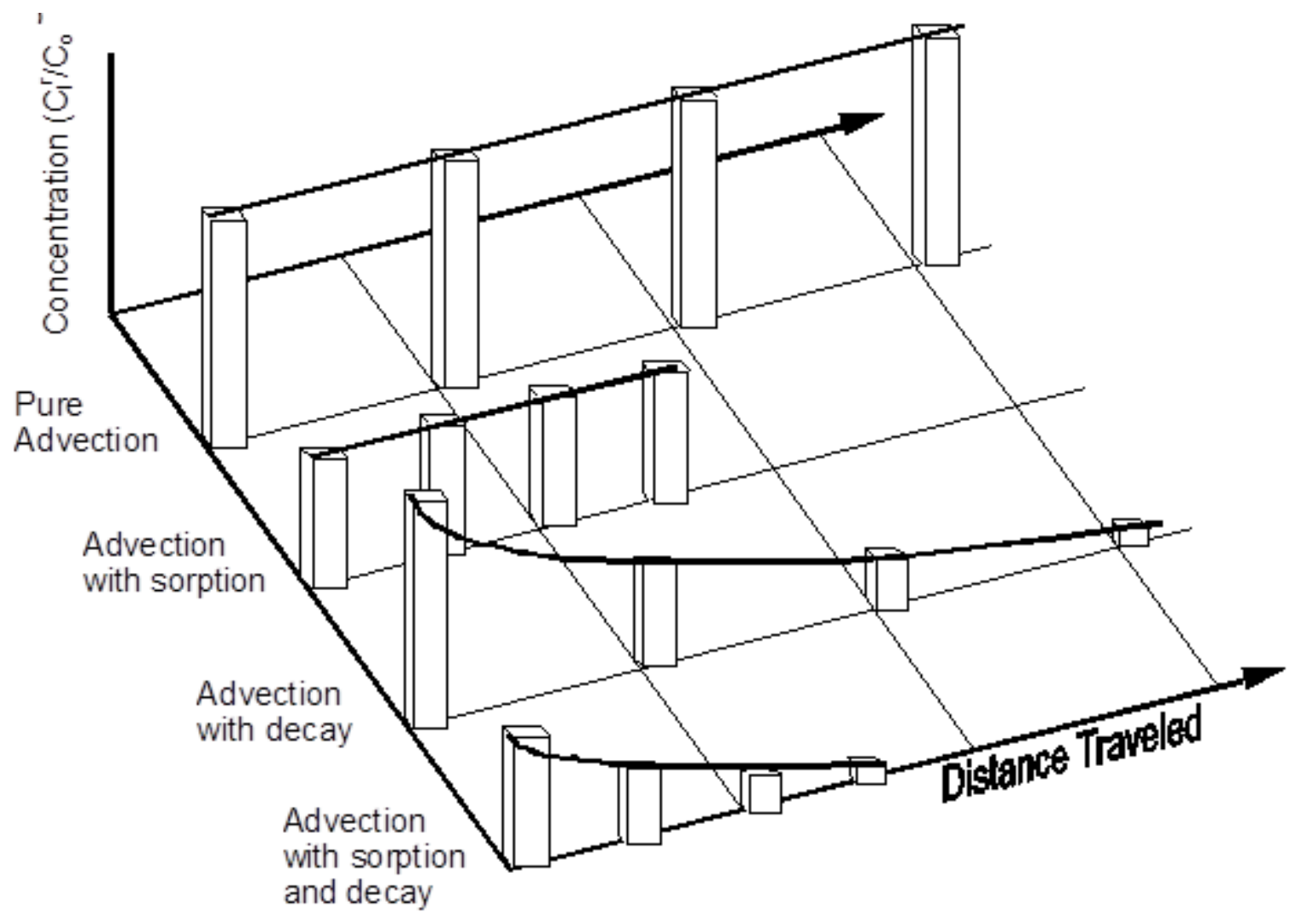

Figure 6.5 Schematic representation of various implementations of plug flow models.

\section{Plug Flow Models}

The most basic approach to modeling transport is application of a "plug-flow" or "piston flow" model. In this case solute movement is purely advective, moving through the soil without dispersion or diffusion of the dissolved components. While it is clear that such a simplification does not do justice to the true complexity of solute movement, there are several reasons why such a simplified approach is adequate and appropriate for particular cases. First, we often do not have data on the magnitude of the dispersion which might occur in a given media. Determination of the magnitude of dispersion is difficult in the best of cases, so one can argue that it is better to be explicit with our lack of knowledge by not including this term rather than making a wild guess which makes it look like we know more about the situation that we actually do.

Furthermore, dispersion is only a major factor in transport if the solute concentration varies significantly in the region of interest. Many times this is not the case. Take the example of nutrient modeling under agricultural production. On sites where practices have been quite constant in time, the shape of the nutrient profile with depth will be most strongly controlled by (1) the rate of nutrient application; (2) the degree and vertical distribution of root uptake; (3) spatial variability in applied water and soil water retention properties, and (4) any sorption/transformation processes which might occur for the compound of interest. Although many authors have spent considerable effort including dispersive processes in nutrient transport models, we suggest that this effort obfuscates the true nature of the problem, diverting effort and attention from these central issues.

In many vadose transport calculations one is primarily interested in how much of a surface applied mass makes it to the water table. While it is true that in cases of moderate adsorption and very high dispersion and degradation the mass loading can be significantly effected by dispersion, for a great number of cases dispersion has little effect on the 
mass of compound that arrives to the water table. In summary, the simplicity of plug flow models allow more explicit computation (typically easily carried out with pencil and paper) which is highly desirable, and highly appropriate for many problems.

Processes of adsorption and degradation can be incorporated in plug flow models (Figure 6.5). If the model is implemented numerically, it is possible to employ any sorption isotherm of choice, simply by computing the remaining dissolved portion and advancing the aqueous phase with the water at each time step. The same is true of the degradation model selected. In the case of sorption, if a first order sorption model is selected the plume does not spread due to sorption. This compliments the simplicity of the plug flow approach, making the computations readily carried out with pencil and paper with explicit dependence upon the physical parameters. Resident and flux concentrations $\left(\mathrm{C}^{\mathrm{r}}\right.$ and $\mathrm{C}^{\mathrm{f}}$ ) are identical in the plug flow set-up.

\section{Example}

Plug Flow Transport (Following example III-12 of Mills et al., 1985)

Consider a spill of 50,000 $\mathrm{g} / \mathrm{ha}$ of naphthalene which is leaching from an abandoned waste disposal site in a sandy loam soil with bulk density of $1.5 \mathrm{~g} / \mathrm{cm}^{3}$, field capacity $\theta_{\mathrm{f}}=0.22 \mathrm{~cm}^{3} / \mathrm{cm}^{3}$, water table at 1.5 meters, and mean annual percolation of $40 \mathrm{~cm}$. Using first order sorption and degradation, we find a partition coefficient of 11 , and a half life of 1,700 days ( $4.66 \mathrm{y}$, or equivalently, a decay rate, $\left.\mathrm{k}_{\mathrm{s}}=0.149 \mathrm{yr}^{-1}\right)$ for the site. It is desired to calculate the quantity of naphthalene that will reach the aquifer.

The annually averaged naphthalene plug flow velocity may be calculated as

$$
\begin{aligned}
\mathrm{X} & =\frac{\mathrm{Q}}{\theta_{\mathrm{f}}+\rho \mathrm{K}_{\mathrm{D}}} \\
& =\frac{40}{0.22+1.5(11)} \\
& =2.3 \mathrm{~cm} / \mathrm{y}
\end{aligned}
$$

(6.4)

thus, the predicted time to reach the water table is 65 years. The final mass of contamination entering the groundwater, $\mathrm{M}(\mathrm{T})$, is calculated based on first order degradation 


$$
\begin{aligned}
\mathrm{M}(\mathrm{T}) & =\mathrm{M}(0) \exp \left(-\mathrm{k}_{\mathrm{s}} \mathrm{t}\right) \\
& =50,000 \exp [-0.149(65)] \\
(6.5) \quad & =3.1 \mathrm{~g} / \mathrm{ha}
\end{aligned}
$$

It would be straightforward to bracket this value based on uncertainty in any of the parameters keeping the entire calculation completely explicit.

\section{Advective-Dispersion Equation}

The Advective Dispersion Equation (ADE), also called the Convection-Dispersion Equation (CDE) is used as the basis to describe solute transport in porous media. The equation is derived by imposing the conservation of mass upon transport which includes convection, diffusion, and dispersion. Scale dependent dispersion is achieved through selection of appropriate local dispersion coefficients to describe the processes at the scale of the REV, then generating further dispersion by assigning various permeability and sorption characteristics at larger scales. In general, the ADE requires numerical methods for its solution. There are, however, a number of very useful analytical solutions to the ADE for special cases which give insight into many real world problems. We will derive the ADE for the resident concentration and provide the predicted relationship between resident and flux concentrations.

\section{Scope of Application}

The ADE is used to describe contaminant transport in contexts as varied as riverine discharges, industrial smoke-stack emissions, and groundwater transport. In the context of the vadose zone, the equation may be used to describe the movement of most solutes. It is important to note that the ADE assumes the solutes are hydrodynamically inactive. i.e. concentrations are so small that density induced flow is ignored (which is a minor concern to vadose problems, but can be very significant in saturated systems where the potential gradients are much smaller), as well as effects on viscosity and surface tension (which can have profound effects on vadose transport). As mentioned above, prior to solving the $\mathrm{ADE}$, the flow field must be known. The ADE requires average pore water velocities obtained by dividing specific flow rates by the effective porosity or volumetric water content. Therefore any error in the flow modeling will cause errors in the solute modeling exercise: the $\mathrm{ADE}$ is less predictive than the flow equation.

\section{${ }_{5}$ Derivation of the Advection-Dispersion Equation}

The derivation that follows is not unique to this text, with similar derivations found in Bear (1972) who uses tensor 
notation to derive a general equation in three dimensions. A basic road map of our approach is that we will (1) use a mass balance on a representative elemental volume (REV) to obtain a solute mass conservation equation in three dimensions; (2) look at the flux term at a microscopic and macroscopic level to identify transport processes and add these processes into the solute conservation equation; and finally (3) add in chemical reactions (decay and absorption) to obtain the ADE in three dimensions.

Solute mass conservation equation.

A mass balance requires that the total flux into the REV is equal to the change of stored mass in the REV. That is to say that the sum of flux leaving the volume through the surface (the dot product of the three dimensional flux vector, $\mathbf{J}$, and the unit normal vector, $\mathbf{n}$, over the boundary surface, S) and sources and sinks within the volume, $\sigma$, equals the time rate of change in storage of mass inside the REV (Figure 6.6). $\mathrm{C}^{\mathrm{r}}$ is the total mass of solute per unit volume in the volume and $\partial \mathrm{C}^{\mathrm{r}} / \partial t$ is the time rate of change of solute mass. This mass balance is shown in [5.4].

$$
-\oint_{S}(\mathbf{J} \cdot \mathbf{n}) \mathrm{d} S+\int_{V} \sigma \mathrm{dV}=\int_{\mathrm{V}} \frac{\partial \mathrm{C}^{\mathrm{r}}}{\partial \mathrm{t}} \mathrm{dV}
$$

The first term in [5.4] is the mass entering into the volume as a flux through the surface, with a flux across the surface into the volume is defined as being negative, as drawn in Figure 6.6. The second term is the source/sink term or the mass of solute generated or decayed per unit time in the volume. The third term on the right hand side of [5.4] is the time rate of change of mass in the volume. To simplify [5.4] it is handy to transform the first surface integral into a volume integral to correspond with the latter volume integrals by means of the Gauss Divergence Theorem, [5.5].

$$
-\oint_{\mathrm{S}}(\mathrm{k} \cdot \mathrm{n}) \mathrm{dS}=\int_{\mathrm{V}}(\nabla \cdot \mathrm{k}) \mathrm{dV}
$$

Applying this to the surface integral we find,

$$
-\oint_{\mathrm{S}}(\mathbf{J} \cdot \mathrm{n}) \mathrm{dS}=\int_{\mathrm{V}}(\nabla \cdot \mathbf{J}) \mathrm{dV}
$$

Substituting [5.6] into [5.4] we obtain three volume integrals over the same patch of space $\mathrm{V}$

$$
-\int_{\mathrm{V}}(\nabla \cdot \mathbf{J}) \mathrm{dV}+\int_{\mathrm{V}} \sigma \mathrm{dV}=\int_{\mathrm{V}} \frac{\partial \mathrm{C}^{\mathrm{r}}}{\partial \mathrm{t}} \mathrm{dV}
$$

which can gathered to one side of the equality as

$$
\int_{\mathrm{V}}\left[(\nabla \cdot \mathbf{J})+\frac{\partial \mathrm{C}^{\mathrm{r}}}{\partial \mathrm{t}}-\sigma\right] \mathrm{dV}=0
$$

Since the volume $\mathrm{V}$ is completely arbitrary, we could choose this to be any given point. 


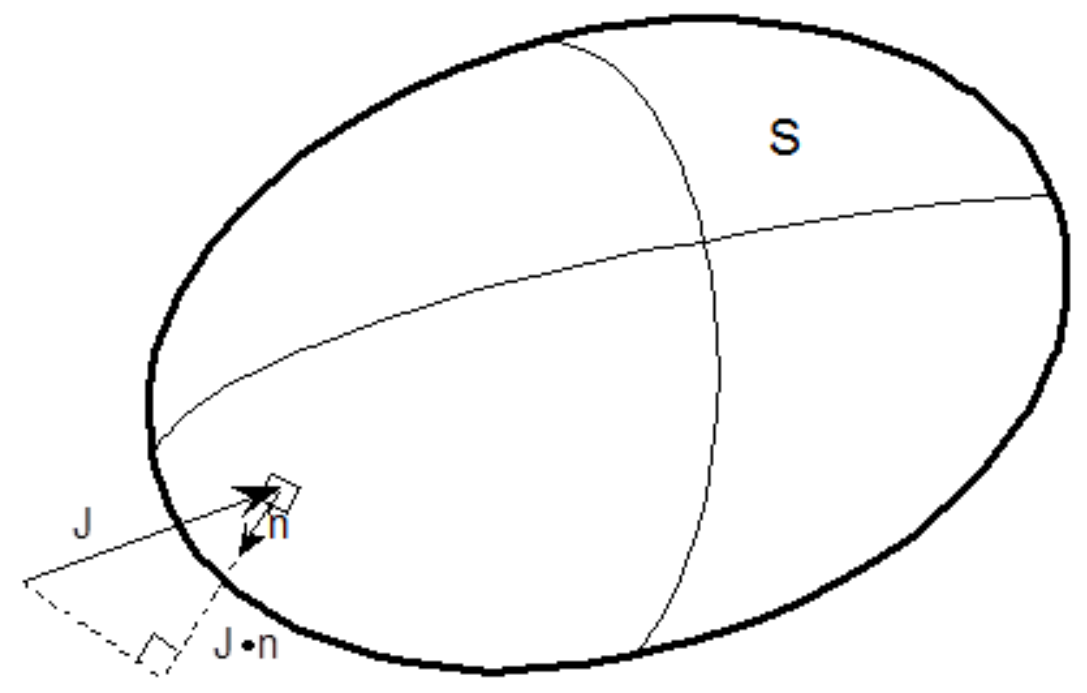

Figure 6.6 Mass balance on a representative elemental volume (REV). System parameters include $\mathbf{J}$ and $\mathbf{n}$, the flux and surface normal vectors respectively, $\mathrm{S}$ the surface of the volume $\mathrm{V}$.

So we see that the integrand under the volume integral is zero everywhere. So we have

$$
(\nabla \cdot \mathbf{J})+\frac{\partial \mathrm{C}^{\mathrm{r}}}{\partial \mathrm{t}}-\sigma=0
$$

or

$$
\frac{\partial \mathrm{C}^{\mathrm{r}}}{\partial \mathrm{t}}=-(\nabla \cdot \mathrm{J})+\sigma
$$

which can be summarized as saying

(6.13) Change in storage with time $=$ Fluxes into $(-)$ and out + sources and sinks which is a clear statement of the conservation of mass

Flux term, J.

We now must consider the flux term, $\mathbf{J}$, at a microscopic and macroscopic level to identify and parameterize the transport processes of advection, diffusion and dispersion and show how these transport processes fit into the solute conservation of mass equation.

\section{Convective Transport}

The rate of convective mass flow through the area, $d \mathrm{~A}$, at point $\mathbf{x}$ is the dot product of the three dimensional fluid velocity vector, $\mathbf{u}$ and the unit normal vector, $\mathbf{n}$ to the area, $d \mathrm{~A}$, all multiplied by the concentration of solute in the fluid, $\mathrm{C}^{\mathrm{r}}$. The solute concentration has units of mass of solute per unit volume of fluid, 


$$
\text { convective mass transport though } \mathrm{dA}=(\mathbf{u} \cdot \mathbf{n}) \mathrm{C}^{\mathrm{r}} \mathrm{dA}=\left(\mathbf{J}_{\text {conv }} \cdot \mathrm{n}\right) \mathrm{dA}\left[\frac{\text { mass }}{\text { time }}\right]
$$

where the convective mass flux, $\mathbf{J}_{\text {conv }}$, is defined as.

$$
\mathbf{J}_{\text {conv }}=u C^{r}\left[\frac{\text { mass }}{\text { time } \times \text { area }}\right]
$$

Diffusive Transport

Fickís law states that the net rate of diffusive mass transport is proportional, through the diffusion coefficient $\mathrm{D}$, to the negative gradient of concentration normal to the area, $\mathrm{dA}$. This may be written

$$
\begin{gathered}
\text { diffusive mass transport through } \mathrm{dA}=-\mathrm{D} \frac{\partial \mathrm{c}}{\partial \mathrm{n}} \mathrm{dA}=-\mathrm{D}\left(\nabla \mathrm{C}^{\mathrm{r}} \cdot \mathrm{n}\right) \mathrm{dA}\left[\frac{\text { mass }}{\text { time }}\right] \\
-\mathrm{D}\left(\nabla \mathrm{C}^{\mathrm{r}} \cdot \mathrm{n}\right) \mathrm{dA}=\left(\mathbf{J}_{\text {diff }} \cdot \mathrm{n}\right) \mathrm{dA}\left[\frac{\text { mass }}{\text { time }}\right]
\end{gathered}
$$

where the diffusive mass flux, $\mathbf{J}_{\text {diff, is defined }}$

$$
\mathbf{J}_{\text {diff }}=-\mathrm{D} \nabla \mathrm{C}^{\mathrm{r}}\left[\frac{\text { mass }}{\text { time } \times \text { area }}\right]
$$

Notice that the fluxes are vectors, while $D$ is a scalar, and $\nabla C^{r}$ is a vector quantity.

The total microscopic scale flux, $\mathbf{J}$, is the sum of both the convective, $\mathbf{J}_{\text {conv }}$, and diffusive, $\mathbf{J}_{\text {diff, }}$ mass fluxes.

(6.18) $\mathbf{J}=\mathbf{J}_{\text {conv }}+\mathbf{J}_{\text {diff }}=\mathrm{uC}^{\mathrm{r}}-\mathrm{D} \nabla \mathrm{C}^{\mathrm{r}}$

The three-dimensional vector, $\mathbf{J}$, represents the mass flux of solute at a point in space.

Macroscopic Phenomena

On a macroscopic scale, the total solute flux, $\mathbf{J}$, is the sum the convective flux, $\mathbf{J}_{\text {conv }}$, the flux due to molecular diffusion,

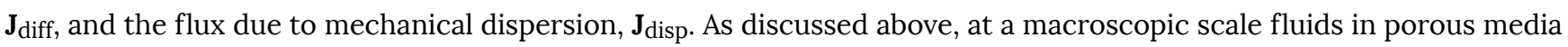
move at different velocities depending upon where they are relative to particle surfaces or other obstructions. Thus, solute particles carried by the fluid will travel with a velocity either faster or slower than the average fluid velocity depending on the particular flow path followed. To introduce dispersion into the ADE we consider how local velocities deviate from the average fluid velocity. Ultimately we want to identify how the degree of variability in velocity and solute concentration relate to the degree of dispersion.

For a given point in space we may consider the local velocity $\mathbf{u}$ to be composed of a sum of the average local velocity $\overline{\mathbf{u}}$ with a deviation term $\delta \mathbf{u}$ accounting for the departure of the local velocity from the average

(6.19) $\mathbf{u}=\overline{\mathbf{u}}+\delta \mathbf{u}$

We may apply the same notation to the solute concentration on a point wise basis

(6.20) $\mathrm{C}^{\mathrm{r}}=\overline{\mathrm{C}^{\mathrm{r}}}+\delta \mathrm{C}^{\mathrm{r}}$

Now substituting [5.18] and [5.19] into the flux equation derived on a microscopic scale, [5.17] we can write 


$$
\mathbf{J}=(\overline{\mathbf{u}}+\delta \mathbf{u})\left(\overline{\mathrm{C}^{\mathrm{r}}}+\delta \mathrm{C}^{\mathrm{r}}\right)-\mathrm{D} \nabla\left(\overline{\mathrm{C}^{\mathrm{r}}}+\delta \mathrm{C}^{\mathrm{r}}\right.
$$

$$
\mathbf{J}=\overline{\mathbf{u}} \mathrm{C}^{\mathrm{r}}+\overline{\mathbf{u}} \delta \mathrm{C}^{\mathrm{r}} \delta \mathbf{u} \overline{\mathrm{C}^{\mathrm{r}}}+\delta \mathbf{u} \delta \mathrm{C}^{\mathrm{r}}-\mathrm{D} \nabla \overline{\mathrm{C}^{\mathrm{r}}}-\mathrm{D} \nabla \delta \mathrm{C}^{\mathrm{r}}
$$

To obtain a volume averaged flux we multiply the right side of [5.21] by the fraction of the volume taking part in the flow which is the volumetric water content, $\theta$ and take averages of all terms.

$$
\mathbf{J}=\theta\left(\overline{\overline{\mathbf{u} C^{r}}}+\overline{\overline{\mathbf{u}} \delta \mathrm{C}^{r}}+\overline{\delta \mathbf{u} \overline{\mathrm{C}^{r}}}+\overline{\delta \mathbf{u} \delta \mathrm{C}^{\mathrm{r}}}-\overline{\mathrm{D} \nabla \overline{\mathrm{C}^{r}}}-\overline{\mathrm{D} \nabla \delta \mathrm{C}^{r}}\right)
$$

To simplify [5.22] we note that the average of a deviation is zero, so any constant time the average of a deviation is also zero. Thus we see that

(6.23) $\overline{\overline{\mathbf{u}} \delta \mathrm{C}^{\mathrm{r}}}=\overline{\delta \mathbf{u} \overline{\mathrm{C}^{\mathrm{r}}}}=\overline{\mathrm{D} \nabla \delta \mathrm{C}^{\mathrm{r}}}=0$

With these results, equation [5.18] becomes

$$
\overline{\mathbf{J}}=\theta\left(\overline{\overline{\mathbf{u} \mathrm{C}^{\mathrm{r}}}}+\overline{\delta \mathbf{u} \delta \mathrm{C}^{\mathrm{r}}}-\overline{\mathrm{D} \nabla \overline{\mathrm{C}^{\mathrm{r}}}}\right)
$$

The total flux is then expressed as the sum of three separate fluxes.

$$
\text { connective flux }=\mathbf{J}_{\text {conv }}=\theta \overline{\overline{\mathbf{u C}^{\mathrm{r}}}}
$$

$$
\text { diffusive flux }=\mathbf{J}_{\text {diff }}=-\theta \overline{\mathrm{D} \nabla \overline{\mathrm{C}^{\mathrm{r}}}}
$$

$$
\text { dispersive flux }=\mathbf{J}_{\text {disp }}=\overline{\theta \delta \mathbf{u} \delta \mathrm{C}^{\mathrm{r}}}
$$

So we see that dispersion is due to the correlation between variations in solute concentrations and fluid velocities (Figure 6.7). Physically, the small-scale dispersive flux is due to variable pore size, the velocity profile in a pore, and tortuous flow channels (see Figure 6.4). Now this dispersive flux depends on the area over which you take your averages. This really boils down to the same difficulty we discussed way back in chapter 1 regarding the REV, but with a new twist. In the case of the REV, any given parameter would not have a well defined tendency to grow or shrink based on the REV selection. In the case of the dispersive flux, the larger the volume over which this is calculated, the larger the magnitude of the dispersive flux! This arises due to heterogeneity. In the extreme case, imagine that a site has mixed areas of pure sand and pure clay. Any solute in a sandy area would zip along, while material stuck in the clay regions would be nearly stagnant. This would smear the plume out, increasing the spreading of the plume due to dispersive processes. The proper approach is to select a value of dispersion coefficient that matches the size of the plume that you are modeling with attention to the heterogeneity of your site. 


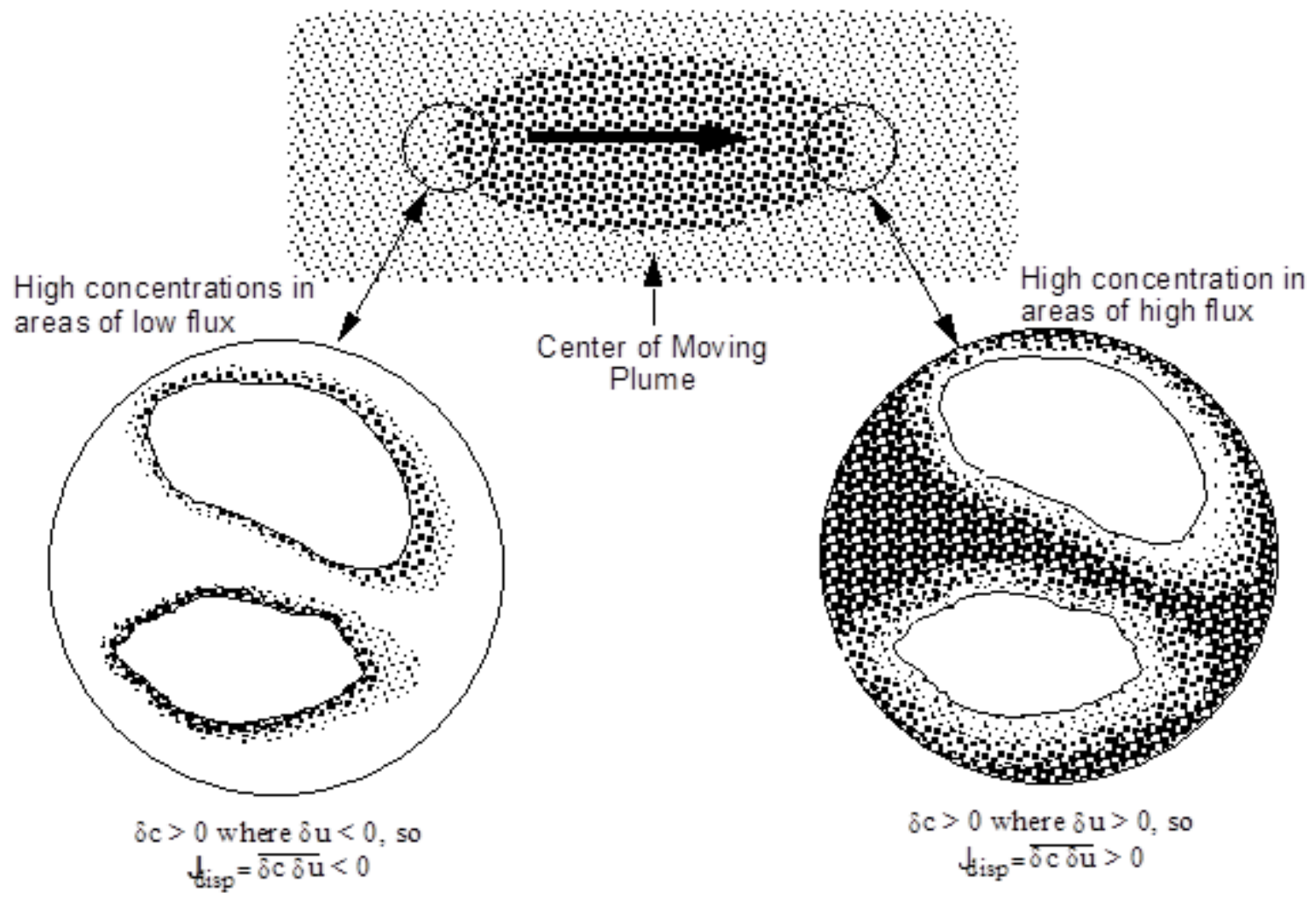

Figure 6.7 Illustration of the processes of pore-scale dispersion at the leading and trailing edges of a plume. Where concentration is high in regions of high velocity (positive deviation in velocity), dispersion generates a positive contribution to solute velocity. In the trailing edge the contribution is negative, where the areas of high concentration are in stagnant regions (where the deviation velocity is negative).

It is not practical to measure fluid velocities and solute concentrations on a macroscopic scale to determine the velocity variations and concentration variations. In practice, the effects of dispersion give rise to mixing that looks much like a diffusion process, so it is often mathematically modeled as a random Fickian process analogous to diffusion.

$$
\mathbf{J}_{\text {disp }}=\theta \overline{\delta \mathbf{u} \delta \mathrm{C}^{r}}=-\theta \mathbf{D} \nabla \mathrm{C}^{\mathrm{r}}
$$

This is justified if the velocity variations of a particle will experience the whole range of possible velocities. $\mathbf{D}$ is the dispersion coefficient, and is a second rank tensor. Watch out: $\mathbf{D}$ is always anisotropic even if flow is isotropic. Dispersion in the longitudinal direction (in the direction of flow) is always much greater than in the transverse direction. It is usually possible to align the coordinate system (and thereby $\mathbf{D}$ ) in the direction of flow, so that $\mathbf{D}$ is symmetric with no off diagonal components.

Total flux may now be written using [5.24] and [5.28] where all concentrations, $C^{r}$, and velocities, $\mathbf{u}$, now represent averages throughout the REV to remove overbars.

$$
\mathbf{J}=\theta \mathbf{u} \mathrm{C}^{\mathrm{r}}-\theta(\mathrm{D}+\mathbf{D}) \nabla \mathrm{C}^{\mathrm{r}}
$$

Now we may recall the solute mass conservation equation, [5.10], and substitute for the total flux given by [5.29].

$$
\frac{\partial \mathrm{C}^{\mathrm{r}}}{\partial \mathrm{t}}=-\nabla \cdot\left(\theta \mathbf{u} \mathrm{C}^{\mathrm{r}}-\theta(\mathrm{D}+\mathrm{D}) \nabla \mathrm{C}^{\mathrm{r}}\right)+\sigma
$$

or,

$$
\frac{\partial \mathrm{C}^{\mathrm{r}}}{\partial \mathrm{t}}+\nabla \cdot\left(\theta \mathbf{u} \mathrm{C}^{\mathrm{r}}\right)-\nabla \cdot\left(\boldsymbol{\theta}(\mathrm{D}+\mathrm{D}) \nabla \mathrm{C}^{\mathrm{r}}\right)-\sigma=0
$$

Equation [5.31] is the ADE solute transport equation for the REV defined in Figure 6.6. It includes terms for the time 
rate of change of solute, transport by advection, molecular diffusion, mechanical dispersion and internal sources or sinks of solute in the REV with dimensional units expressed in rates per unit volume.

Dispersion coefficients

In this section we will look more closely at dispersion coefficients. The dispersion tensor in three dimensions can be written out

$$
\mathbf{D}=\left[\begin{array}{ccc}
\mathrm{D}_{\mathrm{xx}} & \mathrm{D}_{\mathrm{yx}} & \mathrm{D}_{\mathrm{zx}} \\
\mathrm{D}_{\mathrm{xy}} & \mathrm{D}_{\mathrm{yy}} & \mathrm{D}_{\mathrm{zy}} \\
\mathrm{D}_{\mathrm{xz}} & \mathrm{D}_{\mathrm{yz}} & \mathrm{D}_{\mathrm{zz}}
\end{array}\right]
$$

If $\mathbf{D}$ is aligned with the velocity, as is usually the case, then the off diagonal terms go to zero

$$
\mathbf{D}=\left[\begin{array}{lll}
\mathrm{D}_{\mathrm{x}} & 0 & 0 \\
0 & \mathrm{D}_{\mathrm{y}} & 0 \\
0 & 0 & \mathrm{D}_{\mathrm{z}}
\end{array}\right]
$$

It is often the case that the dispersion in the two axes perpendicular to the direction of flow (transverse) can be taken as equal. When the transverse dispersion is equal, the dispersion tensor may be represented as a $2 \times 2$ tensor

$$
\mathbf{D}=\left[\begin{array}{cc}
\mathrm{D}_{\mathrm{L}} & 0 \\
0 & \mathrm{D}_{\mathrm{T}}
\end{array}\right]
$$

where $\mathrm{D}_{\mathrm{L}}$ is the longitudinal dispersion in the direction of the flow field and $\mathrm{D}_{\mathrm{T}}$ is the transverse dispersion perpendicular to the flow field with units of length ${ }^{2}$ per unit time. $\mathrm{D}_{\mathrm{L}}$ is typically on the order of ten times the value of $\mathrm{D}_{\mathrm{T}}$.

Now it is rather awkward that these dispersion coefficients depend on the fluid velocity: Since flow in natural porous media is almost always laminar, the dependency of the dispersion coefficients on velocity is linear. To simplify this situation, we define the longitudinal dispersivity, $\alpha_{\mathrm{L}}$, and transverse dispersivity, $\alpha_{\mathrm{T}}$, with units of length, in relation to the average pore water velocity in the longitudinal direction, $u$.

$$
\begin{aligned}
& \mathrm{D}_{\mathrm{L}}=\alpha_{\mathrm{L}}|\mathrm{u}| \\
& \mathrm{D}_{\mathrm{T}}=\alpha_{\mathrm{T}}|\mathrm{u}|
\end{aligned}
$$

dispersivities, which are a function of the media alone, are rarely known a-priori, but the following rules of thumb are commonly used in solute transport problems to estimate dispersivity at the small-scale are

$$
\begin{aligned}
& \frac{\alpha_{\mathrm{L}}}{\sqrt{\mathrm{k}}} \approx 25 \operatorname{to} 50 \\
& \frac{\alpha_{\mathrm{T}}}{\alpha_{\mathrm{L}}} \approx 0.1
\end{aligned}
$$

where $\mathrm{k}$ is the intrinsic permeability of the medium. As we have mentioned often, dispersivity is scale dependent and the random Fickian model does not fully describe the dispersion process because dispersion is not a molecular level process. Values of longitudinal dispersivity measured in laboratory column experiments are usually on the order of one centimeter, but in the field using tracer experiments they are found to be much larger than laboratory measurements 
(Figure 6.8). Scaling of dispersivity is due to increased heterogeneity and small scale variations in permeability found in the field.

Figure 6.8 Scale dependence of longitudinal dispersivity (after Beims (1982), found in Kinzelbach (1986), Fig. 6.9, p. 201.

In [5.29] - [5.31] we saw that the coefficients of dispersion and diffusion appear together. It is convenient to combine these terms into a single coefficient of hydrodynamic dispersion, $\mathbf{D}^{\prime}=\mathrm{D}+\mathbf{D}$. To assign vales to $\mathbf{D}^{\prime}$ we need some way to assess the relative importance of diffusion and dispersion. The relationship between molecular diffusion, dispersion and pore water velocity is expressed in terms of the dimensionless Peclet number. The Peclet number, Pe, is calculated as the ratio between the average pore water velocity, $u$, times a characteristic length expressed as the mean grain size, $d$, to the molecular diffusion coefficient, D as in [5.38].

$$
\mathrm{Pe}=\frac{u d}{\mathrm{D}}
$$

As is demonstrated in Figure 6.9, the relationship between $\mathbf{D}$ and $\mathbf{D}$ is a well described function of Pe. The curve shown can be divided into several zones. In Zone I, molecular diffusion dominates, $\mathrm{Pe}<0.4$. In this zone the velocity is such that the time of travel through a pore is equal to or greater than the time required for diffusion of the solute. In Zone II, molecular diffusion is of the same order of magnitude as dispersion, $0.4<\mathrm{Pe}<5$, and diffusional processes are as important as dispersion processes. In Zone III, $5<\mathrm{Pe}<10$ the main spreading is due to mechanical dispersion combined with transverse molecular diffusion, and transverse diffusion reduces longitudinal dispersion. In Zone IV, Pe $\leq 10$, mechanical dispersion dominates and diffusion is negligible. In Zone V, mechanical dispersion is important, but because of the high pore water velocity, the effects of inertia and turbulence may no longer be neglected.

Figure 6.9 Relationship between molecular diffusion and convective dispersion (after Pfannkuch (1963) and Saffman (1960) as found in Bear (1972), Fig. 10.4.1, p 607.

Molecular diffusion coefficients for solutes in pure water are readily found in handbooks, but in porous media these diffusion coefficients are reduced due to tortuousity, porosity, media structure and water content. To account for these effects in porous media, an effective diffusion coefficient, $\mathrm{D}_{\mathrm{e}}$ is used where $\mathrm{D}_{\mathrm{e}}$ for typical ions range from $10^{-9}$ to $10^{-14}$ $\mathrm{m}^{2} / \mathrm{s}$. For dispersion to dominate we need Pe $>5$. For a sandy soil, with a mean grain diameter, $d=1 \times 10^{-3} \mathrm{~m}$ and $\mathrm{D}_{\mathrm{e}}$ $=10^{-11} \mathrm{~m}^{2} / \mathrm{s}$ using [5.38] one finds a pore water velocity needs to be greater than about $5 \times 10^{-8} \mathrm{~m} / \mathrm{s}(1.6 \mathrm{~m} /$ year) to neglect the effects of diffusion on the longitudinal spreading of the solute. Had we considered a finer texture of soil this velocity would increase inversely. We see that in the vadose zone we are typically in the tough regions II and III. For instance in a typical site in western Oregon, USA, Brandi-Dorhn et al. (1996) found that mean vadose velocity was $1.1 \mathrm{~m} /$ y. It is, therefore, critical to correctly identify the values of $d$ and $u$ that apply to your problem to determine the relative importance of diffusion and dispersion.

Chemical reactions, solute decay and adsorption

As was made clear in Chapter 4, many solutes can undergo chemical reactions in porous media. Terms that account for solute decay and reversible adsorption of solutes onto the solid matrix of the porous media can be added into the transport equation. Although one may use arbitrary expressions in the differential equation and solve for concentration numerically, here we will employ the simplest first-order decay and linear, reversible adsorption due to the availability of useful analytical solutions for these cases. Many important features of the true influence of sorption and decay processes are not well described by these simplifications, as discussed by Brusseau (1995). The effects of these features are discussed qualitatively at the end of this section.

A first order decay reaction is where solute gain or loss is proportional to its concentration as described by [5.39],

$$
\frac{\mathrm{d} \mathrm{C}^{\mathrm{r}}}{\mathrm{dt}}=-\lambda \mathrm{C}^{\mathrm{r}}=\sigma=\text { source or sink }
$$


where $\lambda$ is the proportionality constant, $\mathrm{C}^{\mathrm{r}}$ is the total resident solute concentration and all other variables are previously defined.

The simplest case of reversible adsorption of solute onto the solid matrix of the porous media can be accounted for using a linear adsorption isotherm where we consider solute concentrations in both the liquid and solid phase. The total resident solute concentration in the porous matrix, $\mathrm{C}^{\mathrm{r}}$ is the sum of the concentrations in the liquid and the solid phase with units [mass total solute per total volume]. For the linear case,

$$
\mathrm{C}^{\mathrm{r}}=\rho_{\mathrm{b}} \mathrm{C}_{\mathrm{s}}+\theta \mathrm{C}_{1}^{\mathrm{r}}
$$

where $\rho_{b}$ is the bulk density of the porous media with units [mass dry media per total volume], $C_{s}$ is the solute concentration adsorbed on the porous media with units [mass of solute adsorbed per mass of dry media], $\theta$ is the volumetric water content with units [volume of water per total volume], $\mathrm{C}_{1}^{\mathrm{r}}$ is the resident solute concentration in the liquid phase with units [mass of solute in liquid phase per volume of water]. For the case of a linear isotherm,

(6.39) $\mathrm{C}_{\mathrm{s}}=\mathrm{k}_{\mathrm{d}} \mathrm{C}_{\mathrm{l}}^{\mathrm{r}}$

where $k_{d}$ is the proportionality constant relating the adsorbed solute to the solute concentration in the media. Substituting [5.41] into [5.40] to express the total concentration in terms of the liquid solute concentration.

$$
\begin{gathered}
\mathrm{C}^{\mathrm{r}}=\rho_{\mathrm{b}} \mathrm{k}_{\mathrm{d}} \mathrm{C}_{\mathrm{l}}^{\mathrm{r}}+\theta \mathrm{C}_{\mathrm{r}}^{\mathrm{l}} \\
\mathrm{C}^{\mathrm{r}}=\left(\rho_{\mathrm{b}} \mathrm{k}_{\mathrm{d}}+\theta\right) \mathrm{C}_{\mathrm{l}}^{\mathrm{r}} \\
\mathrm{C}^{\mathrm{r}}=\left(1+\frac{\rho_{\mathrm{b}} \mathrm{k}_{\mathrm{d}}}{\theta}\right) \mathrm{C}_{\mathrm{l}}^{\mathrm{r} \theta} \\
\mathrm{C}^{\mathrm{r}}=\mathrm{RC}_{\mathrm{l}}^{\mathrm{r}} \theta
\end{gathered}
$$

where $\mathrm{R}$ is the retardation factor defined as

$$
\mathrm{R}=1+\frac{\rho_{\mathrm{b}} \mathrm{k}_{\mathrm{d}}}{\theta}
$$

First order decay and solute adsorption can now be added into the solute transport equation defined in [5.31].

$$
\frac{\partial\left(\mathrm{RC}_{\mathrm{l}}^{\mathrm{r}} \theta\right)}{\partial \mathrm{t}}+\nabla \cdot\left(\theta \mathbf{u} \mathrm{C}_{\mathrm{l}}^{\mathrm{r}}\right)-\nabla \cdot\left(\theta \mathrm{D}^{\prime} \nabla \mathrm{C}_{\mathrm{l}}^{\mathrm{r}}\right)-\lambda \mathrm{R} \theta \mathrm{C}_{\mathrm{l}}^{\mathrm{r}}=0
$$

Equation [5.47] is the advection dispersion equation for linear, reversible adsorption and first order decay expressed in three dimensions. We have a few major problems left before we can hope to solve this equation analytically. The big problem is that this is a non-linear equation, with $\mathbf{D}^{\text {‘ }}$ and $\theta$ varying in space, multiplied by $\mathrm{C}_{1}^{\mathrm{r}}$, which also varies in space. To make progress, we need to eliminate both of these "problems" by assuming them away. [Watch Out! While these assumptions are mighty handy, that doesn't make them physically correct!] When applying the solutions that we will come up with, keep in mind all of the simplifications that we have had to introduce, and that the real world doesn't abide by most of them.

We start by assuming that $\theta$ is constant in space and time. Dividing [5.47] by R $\theta$ we obtain 


$$
\frac{\partial \mathrm{C}_{\mathrm{l}}^{\mathrm{r}}}{\partial \mathrm{t}}+\nabla \cdot\left(\frac{\mathbf{u}}{\mathrm{R}} \mathrm{C}_{\mathrm{l}}^{\mathrm{r}}\right)-\nabla \cdot\left(\frac{\mathbf{D}^{\prime}}{\mathrm{R}} \nabla \mathrm{C}_{\mathrm{l}}^{\mathrm{r}}\right)-\lambda \mathrm{C}_{\mathrm{l}}^{\mathrm{r}}=0
$$

With the further assumptions that the fluid velocities, retardation factor and the dispersivity are constant in space and time, we may pull them outside of the derivatives. We then note that since $\mathrm{C}$ is a scalar, $\nabla \cdot \mathrm{C}=\nabla \mathrm{C}$, and $\nabla \cdot \nabla \mathrm{C}=$ $\nabla^{2} \mathrm{C}$. With all of this, we obtain

$$
\frac{\partial \mathrm{C}_{\mathrm{l}}^{\mathrm{r}}}{\partial \mathrm{t}}+\frac{\mathbf{u}}{\mathrm{R}} \nabla \mathrm{C}_{\mathrm{l}}^{\mathrm{r}}-\frac{\mathrm{D}^{\prime}}{\mathrm{R}} \nabla^{2} \mathrm{C}_{\mathrm{l}}^{\mathrm{r}}-\lambda \mathrm{C}_{\mathrm{l}}^{\mathrm{r}}=0
$$

Notice that we now have a linear equation with $\mathrm{C}_{1}^{\mathrm{r}}$ as the only variable, which gives us some hope of being able to solve the equation for concentration in space given fixed values of hydrodynamic dispersion and moisture content. The advection dispersion equation is often expressed in one dimension, where it looks like

$$
\frac{\partial \mathrm{C}_{1}^{\mathrm{r}}}{\partial \mathrm{t}}+\frac{\mathrm{u}}{\mathrm{R}} \frac{\partial \mathrm{C}_{1}^{\mathrm{r}}}{\partial \mathrm{x}}-\frac{\mathrm{D}_{\mathrm{L}}}{\mathrm{R}} \frac{\partial^{2} \mathrm{C}_{1}^{\mathrm{r}}}{\partial \mathrm{x}^{2}}-\lambda \mathrm{C}_{1}^{\mathrm{r}}=0
$$

If the fluid velocity is moving in the $\mathrm{x}$-direction we can look at solute spreading in two dimensions by examining the effects of transverse dispersion using [5.51].

$$
\frac{\partial \mathrm{C}_{\mathrm{l}}^{\mathrm{r}}}{\partial \mathrm{t}}+\frac{\mathrm{u}}{\mathrm{R}} \frac{\partial \mathrm{C}_{\mathrm{l}}^{\mathrm{r}}}{\partial \mathrm{x}}-\frac{\mathrm{D}_{\mathrm{L}}}{\mathrm{R}} \frac{\partial^{2} \mathrm{C}_{\mathrm{l}}^{\mathrm{r}}}{\partial \mathrm{x}^{2}}-\frac{\mathrm{D}_{\mathrm{T}}}{\mathrm{R}} \frac{\partial^{2} \mathrm{C}_{\mathrm{l}}^{\mathrm{r}}}{\partial \mathrm{y}^{2}}-\lambda \mathrm{C}_{\mathrm{l}}^{\mathrm{r}}=0
$$

Let's take a little closer look at this retardation factor. Multiplying both sides of [5.50] by $\mathrm{R}$ we obtain

$$
\frac{\partial \mathrm{C}_{1}^{\mathrm{r}}}{\partial \mathrm{t} / \mathrm{R}}+\mathrm{u} \frac{\partial \mathrm{C}_{\mathrm{l}}^{\mathrm{r}}}{\partial \mathrm{x}}-\mathrm{D}_{\mathrm{L}} \frac{\partial^{2} \mathrm{C}_{\mathrm{l}}^{\mathrm{r}}}{\partial \mathrm{X}^{2}}-\mathrm{R} \lambda \mathrm{C}_{\mathrm{l}}^{\mathrm{r}}=0
$$

Defining $t^{*}=t / R$, and taking the decay rate to be zero, [5.52] reduces to the ADE without adsorption

$$
\frac{\partial \mathrm{C}_{1}^{\mathrm{r}}}{\partial \mathrm{t}^{*}}=\mathrm{D}_{\mathrm{L}} \frac{\partial^{2} \mathrm{C}_{\mathrm{l}}^{\mathrm{r}}}{\partial \mathrm{x}^{2}}-\mathrm{u} \frac{\partial \mathrm{C}_{\mathrm{l}}^{\mathrm{r}}}{\partial \mathrm{x}}
$$

Now here's the punch line: the spatial distribution of solutes are the same in the case of non-adsorbed vs. adsorbed compounds! For a given boundary condition and time $t^{*}$, the solution is unique and independent of $R$. The key difference between the retarded and non-retarded pulses is how fast the apparent time runs: the bigger $\mathrm{R}$ is, the slower things happen, while if $\mathrm{R}<1$, events are accelerated (useful, for instance, in the case of ion exclusion). Of course, $\mathrm{C}_{1}^{\mathrm{r}}$ is lower than $\mathrm{C}^{\mathrm{r}}$ (the total resident concentration) since a portion of the material is sorbed to the solids. This retardation feature is evident in the solutions provided below.

The relationship between resident and flux concentrationsfor the ADE

You may recall way back at the beginning of this chapter (in section 5.1, to be exact) that we had brought up the possibility that the concentration at a point was not as easily defined as one might suppose on first examination. In particular, we showed that in a soil with macroscopic structure, such as worm holes, that the aqueous concentration about a point in space $\left(\mathrm{C}^{\mathrm{r}}\right)$ would be different than the concentration flowing by that point $\left(\mathrm{C}^{\mathrm{f}}\right)$. We used the example of effects of macropore flow to illustrate this situation because it is relatively easy to see how the two concentrations might be dramatically different under this situation. Having now painstakingly developed the ADE, it is worthwhile to identify the relationship between $C^{f}$ and $C^{r}$ for in the case of the ADE description of movement. Although not at all obvious from the outset, even in an idealized continuous media in which we ignore almost all of the "real-world" issues in solute transport (i.e., the $\mathrm{ADE}$ ), the concentration at a point depends upon how you define it, as illustrated by the fact 
that $C^{f}$ and $C^{r}$ are not equal. This conundrum was brought to the attention of the groundwater community by Parker and van Genuchten (1984) who drew on the results developed in the context of chemical engineering by Kreft and Zuber (1978).

In the development of the $\mathrm{ADE}$ we considered the mass balance about a point, with all concentrations representing $\mathrm{C}^{\mathrm{r}}$. The resident concentration was that which if integrated over a volume provided a measure of the mass in that region. Now suppose we go to the field to measure the concentration in recharge to an aquifer. A natural tool for this purpose would be an intercepting device which reports the volume of water and concentration of solute passing through some plane in the vadose zone. Since this device would impose no gradients in concentration (it is passive with respect to solute concentration), the only driving mechanism for flux into the device would be the movement of water (i.e., there would be no concentration gradient at the soil-sampler interface which would cause diffusion or dispersion driven transport). So if the local aqueous flux perpendicular to the sampler is given by $\theta \mathrm{u}_{\mathrm{p}}$, and the solute flux into the device is given by $\mathbf{J}$, they will be related by the flux concentration

$$
\theta u_{\mathrm{p}} \mathrm{C}^{\mathrm{f}}=\mathbf{J}
$$

We can now obtain the relationship between resident and flux concentrations by equating the flux to that computed in [5.29] to obtain

$$
\mathrm{C}^{\mathrm{f}}=\mathrm{C}^{\mathrm{r}}-\frac{(\mathrm{D}+\mathrm{D})}{\mathrm{u}_{\mathrm{p}}} \nabla \mathrm{C}^{\mathrm{r}}
$$

So we see clearly that the two concentrations are not equal even within the ADE framework. What is the source of the discrepancy here? The flux concentration does not account for the contribution of local hydrodynamic dispersion in that it creates a boundary where there is no gradient in concentration. In the undisturbed media, on the other hand, the flux would be a function of the local gradients in concentration. The flux concentration attributes all mass movement due to advection into the sampler, which is indeed what the world looks like to the sampler, but is not how the world looks to a solute particle which feels the effects of the neighboring solute particles via Ficks Law etc. The point here is not to suggest that you compute both concentrations for each measurement of concentration that you take, but rather to be aware of what you are measuring. As pointed out by Brandi-Dorhn et al. (1996), different devices will provide measurements which may disagree by up to $100 \%$ due to their capturing of either $\mathrm{C}^{\mathrm{f}}$ or $\mathrm{C}^{\mathrm{r}}$.

Solutions of the ADE

While the assumptions that went into the derivation of the ADE (as shown in [5.49]) imposed significant constraints on the properties of solutes, the ability to actually write down solutions makes it an extremely important starting point for almost any solute transport analysis. We present a number of the most commonly used solutions in the following sections, with many more available in the literature (e.g., Carslaw and Jaeger, 1948). In any real site almost all of the assumptions (except conservation of mass) will be violated to some degree. On the other hand, the cost of "fully" characterizing a site with the myriad of parameters required to capture the effects of all of these "non-idealities" is prohibitive. Table 6.1 provides a brief summary of some of the effects you should expect due to each of the possible violations of assumptions.

Table 6.1 Summary of effects of non-ideal features of real setting compared to the solutions of the ADE as given in [5.49]. 


\begin{tabular}{ll}
\hline Assumption & Effects if violated \\
\hline$\theta$ Constant in space & 1. Retardation coefficient lower where $\theta$ is higher. \\
& $\begin{array}{l}\text { 2. If flux is constant, areas of higher and lower } \theta \text { will give lower and higher, } \\
\text { respectively, fluid velocity. }\end{array}$ \\
\hline D constant in space & $\begin{array}{l}\text { If D varies strongly in space, the media is highly heterogeneous and the macro-scale } \\
\text { dispersion will be much greater than would be suggested by the value of D at any } \\
\text { given location . Concentrations likely to vary in irregular manner (plume will have } \\
\text { "tattered" edges). }\end{array}$ \\
\hline D independent of scale & $\begin{array}{l}\text { The plume will grow more slowly that predicted at first, then more broadly than } \\
\text { predicted . Plume will be less regular than predicted with shape that imitates the } \\
\text { causes of the scale dependent D (e.g., following layering, crack structure etc.) }\end{array}$ \\
\hline Reversible sorption & $\begin{array}{l}\text { Irreversible sorption will deposit solute alone the entire flow path, greatly spreading } \\
\text { the plume, and reducing solution concentration. }\end{array}$ \\
\hline Equilibrium sorption & $\begin{array}{l}\text { Causes "tailing" at both the front and back of the plume, with the leading edge of the } \\
\text { plume faster, and the trailing edge slower than predicted. }\end{array}$ \\
\hline Linear sorption & $\begin{array}{l}\text { Nonlinearity arises from sorption sites being filled. Results in higher than expected } \\
\text { solution concentration at peak, lower retardation, more tailing. }\end{array}$ \\
\hline Linear decay & $\begin{array}{l}\text { Typically violated by decay rates being time dependent: slower at first if local } \\
\text { bacterial can degrade, or slower in time if a critical nutrient is fully consumed. Peak } \\
\text { degradation rate dictated by environmental conditions rather than local solute } \\
\text { concentration. }\end{array}$ \\
\hline $\begin{array}{l}\text { In sedimentary materials the conductivity and dispersion coefficients are greater } \\
\text { along beds than across beds (anisotropic). Causes stretching/smearing along } \\
\text { bedding. }\end{array}$ \\
\hline \begin{tabular}{l} 
Heterogeneity can cause all of the effects discussed above. \\
\hline
\end{tabular}
\end{tabular}

Instantaneous Point Injection in I-D infinite column

Here we take the governing equation to be [5.50]. We will predict the distribution of solute arising from an instantaneous point injection of total mass $\mathrm{M}$ put in at $\mathrm{x}=0$ in a 1-D column of infinite length both ahead of and behind the injection point with a cross-sectional area of A. The water is moving at a steady, constant velocity $\mathrm{u}$. The boundary and initial conditions are taken as

$$
\mathrm{C}(\mathrm{x}, 0)=0 \text { except at } \mathrm{x}=0
$$

$$
\mathrm{M}(0,0)=\mathrm{M}
$$

The solution is given by

$$
c_{L}(x, t)=\frac{M}{2 \theta A R \sqrt{\pi \mathrm{Dt} / \mathrm{R}}} \exp \left(-\frac{(\mathrm{x}-\mathrm{ut} / \mathrm{R})^{2}}{4 \mathrm{Dt} / \mathrm{R}}\right) \exp (-\lambda \mathrm{t})
$$

Of course you don't need to take our word for it that this really a solution: you can prove it by simply plugging it in to [5.50] and checking that the equality is satisfied as are the boundary conditions. Since the governing equation is linear, the solution is unique. To start to get a feel for the properties of this solution, lets consider the special case of no adsorption $(R=1)$ and no decay $(\lambda=0)$. If we write the mass per unit area of injection as $m=M / A$, the solution reduces to the important case of a conservative non-reactive tracer 


$$
c_{L}(x, t)=\frac{m}{\theta \sqrt{\pi 4 \mathrm{Dt}}} \exp \left(-\frac{(\mathrm{x}-\mathrm{ut})^{2}}{4 \mathrm{Dt}}\right)
$$

This is the standard form of the Gaussian distribution (the "bell curve") with a standard deviation of $\sqrt{4 \mathrm{Dt}}$, an area under the curve of $\mathrm{m} / \theta$, and its peak (and center of mass) at $\mathrm{x}$-ut. So we see that the pulse spreads out in direct proportion to the square root of $\mathrm{Dt}$, it moves along at a speed of ut, and has concentration directly proportional to the mass applied.

(a)

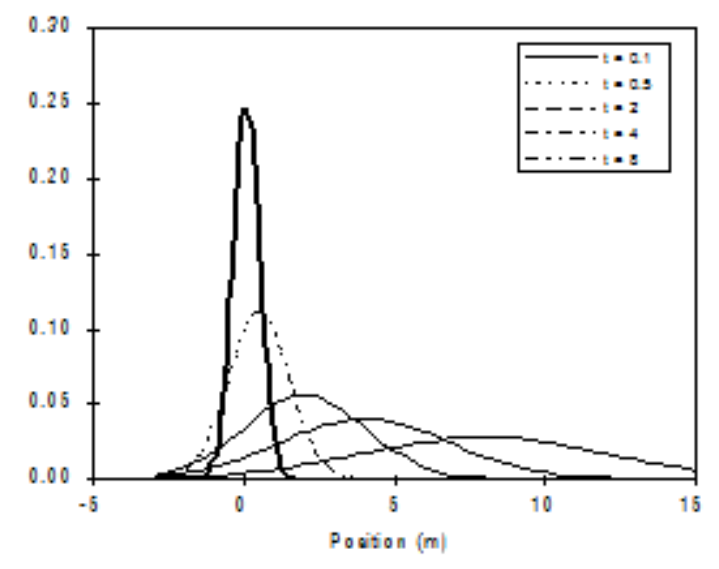

(b)

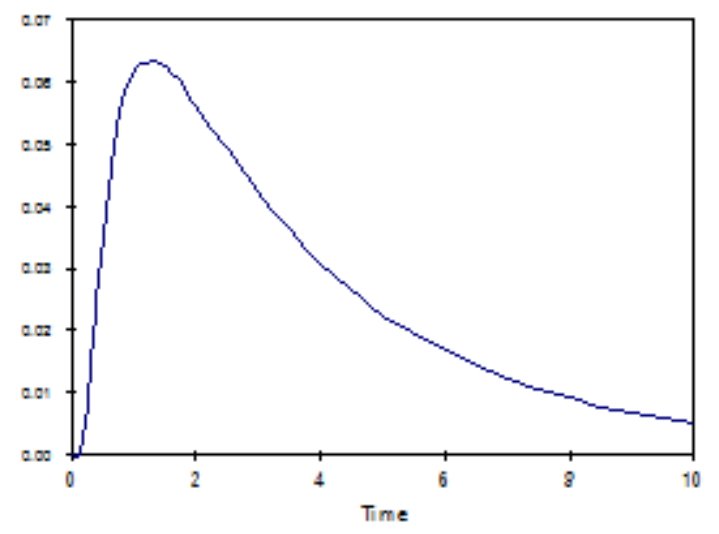

Figure 6.10 Plot of concentration of instantaneous injection at $x=0$ in an infinite 1-dimensional column with $u=1 \mathrm{~m} /$ day, $\mathrm{D}=1 \mathrm{~m}^{2} /$ day, $m=\theta$. In (a) concentration is plotted against position for $t=0.1,0.5$, 2,4 , and 8 days, while in (b) the concentration is plotted in time as measured $2 \mathrm{~m}$ from the point of injection. Note that the concentration is symmetric in position, but not in time. Further note that the peak in (b) occurs at $1.235 \mathrm{hr}$, well before the center of mass arrives at that point after $2 \mathrm{hr}$.

It is well known that the Gaussian curve is symmetric in space, and the shape of the plume is indeed symmetric at any given time in that the concentration is equal for distances equally far from the peak (Figure 6.10). Though the solution is symmetric in space, the solution IS NOT symmetric in time! That is, if you are sampling at a given location and watching the plume go by, the concentration profile of the increasing concentration side will be different than the falling limb. Moreover, the time at which you observe the peak concentration WILL NOT correspond to the center of mass of the plume, nor will it be seen at $\mathrm{t}=\mathrm{x} / \mathrm{u}$ ! To prove this to yourself, calculate $\partial \mathrm{c} / \partial \mathrm{t}$ and put in $\mathrm{t}=\mathrm{x} / \mathrm{u}$, and you will find that it is 
not 0 , so you are not at a local maximum. What to do? Fit your well observation data to the solution using a non-linear least squares procedure to obtain the best estimates of $u$ and $D$ (Toride et al., 1995).

Now getting back to our full solution, what about the effects of decay? You see that the entire solution is multiplied by an exponential decay term, which is the only place that the loss term shows up. So first order decay uniformly diminishes the concentration without altering the geometry or velocity of the plume.

The effects of adsorption are threefold. First, the aqueous concentration is reduced by a factor of $1 / \mathrm{R}$ at all points due to the removal from the liquid of mass that is stuck to the solid phase. Second, the time evolution of the plume is slowed by a factor of 1/R. Without decay, the solution is identical for the non-sorbed and sorbed plumes at times of $t$ and Rt respectively up to the factor of $1 / R$ in concentration. Finally, decay goes on regardless of retardation, but would also have slowed down by $1 / \mathrm{R}$ if we had insisted that only the aqueous phase was subject to decay, as is quite commonly employed for biologically driven processes.

In the solution provided in [5.59] the plume has positive values of concentration both in the -x and positive $x$ direction. The most common application of a one-dimensional solution is in the description of a plume growing from a uniform application to a surface where it is not acceptable to be accounting for mass occurring in the atmosphere above the soil. To handle this situation we make use of the linearity of the ADE. Remember that by the uniqueness property of solution to linear differential equations, once we have a solution for the equation that satisfies the boundary conditions our job is done. For the semi-infinite one-dimensional set-up (i.e. $x$ goes from 0 to $\infty$ ), we simply add two solutions to the ADE which in sum satisfy the boundary conditions. In this case the boundary and initial conditions are

$$
\mathrm{C}^{\mathrm{r}}(\mathrm{x}, 0)=0 \text { except at } \mathrm{x}=0 \quad \text { (initially uncontaminated) }
$$

$$
\frac{\partial \mathrm{C}^{\mathrm{r}}(0, \mathrm{t})}{\partial \mathrm{x}}=0 \quad(\text { no flux at the soil surface })
$$

$$
\mathrm{M}(0,0)=\mathrm{M}
$$

The need for [5.61] comes out of the need to maintain no flux out of the soil which would be driven by Fickian diffusion if there were a gradient in concentration at the boundary. We could have just as well replaced [5.61] with a no-flux boundary condition.

To satisfy [5.61] we will use the method of superposition. Specifically, we will superimpose two Gaussian solutions, one heading in the positive $\mathrm{x}$-direction and on in the negative $\mathrm{x}$-direction. At $\mathrm{x}=0$ [5.61] will be satisfied by means of the symmetry of the solution, while [5.61] is satisfied as before. What about the predicted concentration above the ground surface? Since this region is outside of the bounds of the problem, we simply ignore such values as being irrelevant to our area of concern. The solution is given by

$$
\mathrm{c}_{\mathrm{L}}(\mathrm{x}, \mathrm{t})=
$$

$$
\frac{\mathrm{M}}{2 \theta \mathrm{AR} \sqrt{\pi \mathrm{Dt} / \mathrm{R}}}\left[\exp \left(-\frac{(\mathrm{x}-\mathrm{ut} / \mathrm{R})^{2}}{4 \mathrm{Dt} / \mathrm{R}}\right)+\exp \left(-\frac{(\mathrm{x}+\mathrm{ut} / \mathrm{R})^{2}}{4 \mathrm{Dt} / \mathrm{R}}\right)\right] \exp (-\lambda \mathrm{t})
$$

The method of superposition employed in the semi-infinite column solution can be employed to solve a wide range of problems. In fact, the Gaussian solution is the Green's function for the ADE with linear sorption and first order decay (for a detailed discussion of Green's functions, see Hildebrand, 1952, p388-401). The way you can think of this is that the 
Gaussian solution provides the plume that would arise from a point source. To determine the plume that would arise from a source of arbitrary shape and concentration, simply divide the source into a collection of point sources, each of which will have a Gaussian plume, and sum up all the plumes. The semi-infinite column is one use of this method, superimposing the solutions in space, while the solution given for a continuous injection also make use of this method, this time summing the plumes over time rather than space.

Continuous Point injection in I-D

Now consider an infinite initially uncontaminated column to which contaminants are continuously injected at $\mathrm{x}=0$. The rate of mass injection will be taken to be $\mathrm{m}$. The direct solution of [5.50] for continuous injection can be derived analytically using a La Place transform method to be

$$
\begin{gathered}
\mathrm{C}_{\mathrm{l}}^{\mathrm{r}}(\mathrm{x}, \mathrm{t})= \\
\frac{\mathrm{m}}{2 \mathrm{~A} \theta \mathrm{u}} \exp \left(\frac{\mathrm{x}}{2 \alpha}\right)\left[\exp \left(\frac{-\mathrm{x} \gamma}{2 \alpha}\right) \operatorname{erfc}\left(\frac{\mathrm{x}-\mathrm{ut} \gamma / \mathrm{R}}{2 \sqrt{\alpha \mathrm{utR}}}\right)-\exp \left(\frac{\mathrm{x} \gamma}{2 \alpha}\right) \operatorname{erfc}\left(\frac{\mathrm{x}+\mathrm{ut} \gamma / \mathrm{R}}{2 \sqrt{\alpha u t R}}\right)\right]
\end{gathered}
$$

where erfc is the complementary error function (Figure 6.11), $\gamma=\sqrt{1+4 \lambda \alpha \mathrm{R} / \mathrm{u}}, \alpha$ is the longitudinal dispersivity, and $\mathrm{m}=\mathrm{C}_{\mathrm{O}} \mathrm{A} \theta \mathrm{u}$ is the rate of mass injection per unit area with $\mathrm{C}_{\mathrm{o}}$ being the effective concentration of the injected material at the source. To get a feel for how this solution looks for a simple case, consider a plume with no sorption or degradation. In this case [5.64] reduces to

$$
\mathrm{C}_{\mathrm{l}}^{\mathrm{r}}(\mathrm{x}, \mathrm{t})=\frac{\mathrm{m}}{2 \mathrm{~A} \theta \mathrm{u}}\left[\operatorname{erfc}\left(\frac{\mathrm{x}-\mathrm{ut}}{2 \sqrt{\alpha \mathrm{ut}}}\right)-\exp \left(\frac{\mathrm{x}}{\alpha}\right) \operatorname{erfc}\left(\frac{\mathrm{x}+\mathrm{ut}}{2 \sqrt{\alpha u t}}\right)\right]
$$

This is the first time that we have come across the complementary error function, erfc, which we plot below (Figure 6.11). Think of it just like you think of the sin, cos, exp, or any other function that you can't just compute the value of without a table or specially programmed calculator. As it turns out, the Gaussian and the complementary error function are closely related. If you think about what we said about the Gaussian being the Green's function for the ADE, the solution for a continuous injection should act just like a rapid sequence of individual injections. Now if you made many such repeated injections such that the total rate of mass injection was $\mathrm{m}$, the solution had better look a lot like the one quoted in [5.65]. As it turns out, the complementary error function is simply the shape you get by adding infinitely many Gaussians in exactly this manner, and therefore may be computed as 


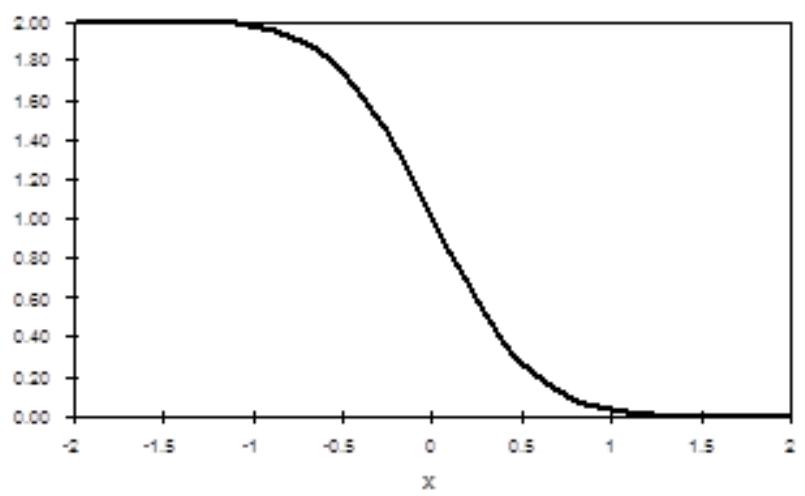

Figure 6.11 Plot of the complimentary error function erfc(x).

$$
\operatorname{erfc}(x)=1-\frac{2}{\sqrt{\mu}} \int_{0}^{x} e^{-y^{2}} d y
$$

Now we have only mentioned the complementary error function, but it is typically computed using its relative, the error function, $\operatorname{erf}(\mathrm{x})$

$$
\operatorname{erfc}(\mathrm{x})=1-\operatorname{erf}(\mathrm{x})
$$

and we also note the handy identity

(6.60) $\operatorname{erf}(-\mathrm{x})=-\operatorname{erf}(\mathrm{x})$

To compute the complementary error function you may use the approximation (Hastings, 1955)

$$
\operatorname{erfc}(\mathrm{x})=\left(\mathrm{at}+\mathrm{bt} \mathrm{t}^{2}+c \mathrm{t}^{3}+d \mathrm{t}^{4}+\mathrm{gt} \mathrm{t}^{5}\right) \exp \left(-\mathrm{x}^{2}\right)+\epsilon(\mathrm{x})
$$

for values $0 \leq \mathrm{x}<\infty$. For negative values of $\mathrm{x}$, just use [5.67] and [5.68], which tell us that $\operatorname{erfc}(-\mathrm{x})=2-\operatorname{erfc}(\mathrm{x})$. Notice that in [5.64] and [5.65] the erfc function multiplies other exponential terms in $x$. Since the erfc has a negative exponential of $\mathrm{x}$ squared, it effectively squelches the exponential terms in the two equations which can quickly blow up. In computations it is best to carry out the multiplication of the exponentials algebraically before attempting to compute the values numerically. The parameter $t$ in [5.69] is defined as

$$
\text { (6.62) } t=\frac{1}{1+p x}
$$

and the error, $\varepsilon(\mathrm{x})$, is always bounded by

(6.63) $|\epsilon(\mathrm{x})| \leq 1.5 \times 10^{-7}$

The values of the constants are: $\mathrm{p}=0.3275911 ; \mathrm{a}=0.25482 ; \mathrm{b}=-0.284496736 ; \mathrm{c}=1.421413741 ; \mathrm{d}=-1.453152027$; and $\mathrm{g}=$ 1.0614054 .

Point Sources in 2 and 3-Dimensions

The one-dimensional solutions that we have presented thus far are appropriate for column studies, and field situations where solutes are distributed uniformly in the lateral directions. We now present solutions for an infinite line source (two-dimensional solution) and a point source in three dimensions. It must be emphasized that there are many other very useful solutions for finite plane sources, finite line sources, etc. which are not presenting here. 


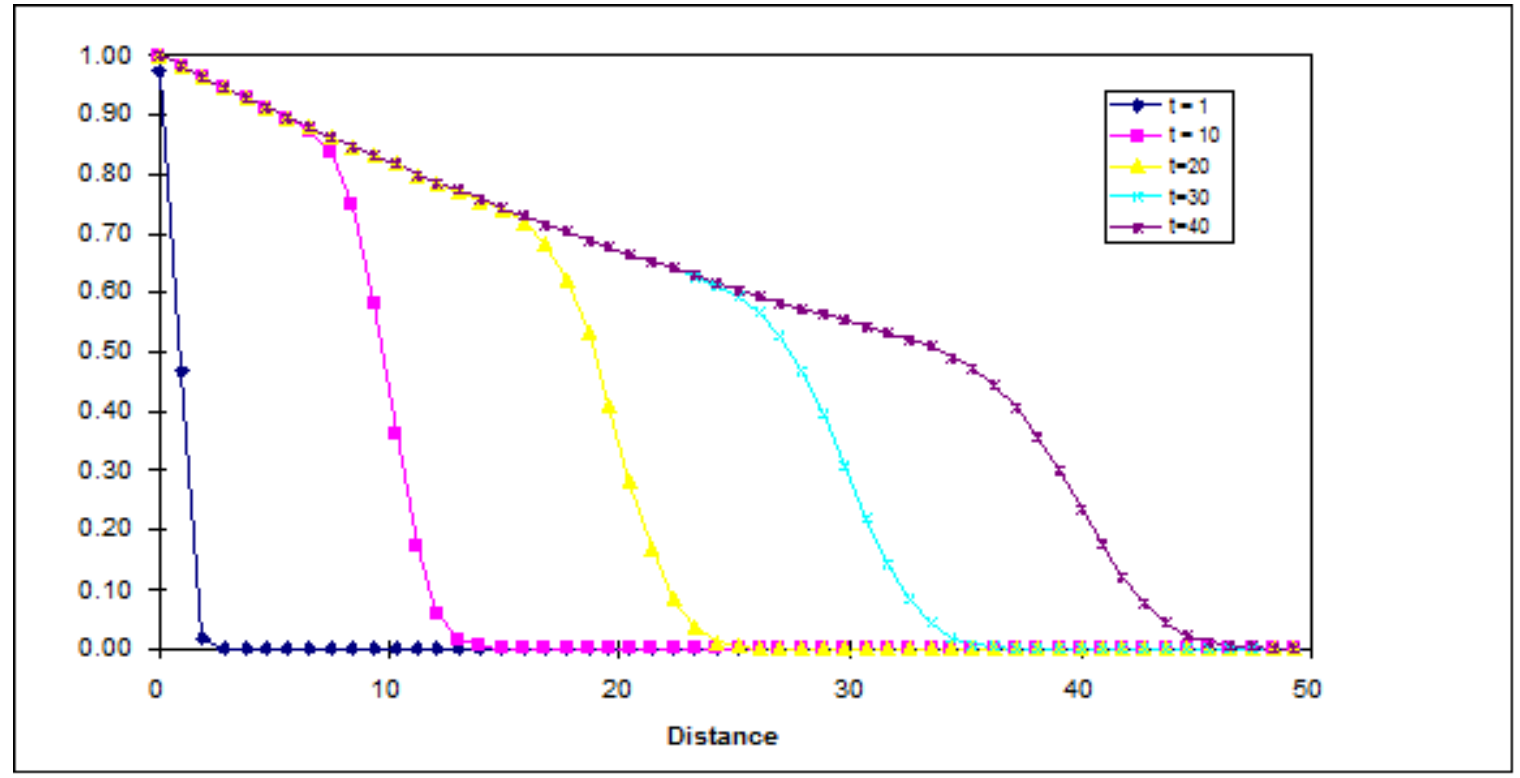

Figure 6.12 Illustration of a typical solution of the concentration arising downstream of a continuous injection with first order decay (equation [5.65]). Parameter values are $\alpha=0.1, R=1, \lambda=0.02, u=1.0$, and $\mathrm{m}=1$.

The instantaneous line source problem presents the evolution of concentration in the plane perpendicular to a line source. The direction of flow is taken to be in the $\mathrm{x}$ direction, while the line course is oriented along the $\mathrm{y}$-axis (note that since gravity effects are not being considered, there is no special significance of the $\mathrm{z}$ axis). We assume that the media is infinite in all directions. The boundary and initial conditions for an instantaneous infinite line source in an infinite domain are

$$
\mathrm{C}^{\mathrm{r}}(\mathrm{x}, \mathrm{y}, \mathrm{z}, 0)=0
$$

$$
\mathrm{M}(0, \mathrm{y}, 0,0)=\mathrm{M}[\mathrm{mass} / \text { length }]
$$

which has solution

$$
\mathrm{C}_{\mathrm{l}}^{\mathrm{r}}(\mathrm{x}, \mathrm{t})=\frac{\mathrm{M}}{4 \pi \mathrm{t} \theta \sqrt{\mathrm{D}_{1} \mathrm{D}_{\mathrm{t}} \mathrm{t}}} \exp \left(-\frac{(\mathrm{x}-\mathrm{ut} / \mathrm{R})^{2}}{4 \mathrm{D}_{1} \mathrm{t} / \mathrm{R}}-\frac{\mathrm{y}^{2}}{4 \mathrm{D}_{\mathrm{t}} \mathrm{t} / \mathrm{R}}\right) \exp (-\lambda \mathrm{t})
$$

One quickly observes that the two-dimensional solution is Gaussian in both axis.The three dimensional instantaneous point source solution

$$
\mathrm{C}_{\mathrm{l}}^{\mathrm{r}}(\mathrm{x}, \mathrm{y}, \mathrm{z}, \mathrm{t})=\frac{\mathrm{M} \exp (-\lambda \mathrm{t})}{4 \pi \theta\left(\mathrm{D}_{1} \mathrm{D}_{\mathrm{y}} \mathrm{D}_{\mathrm{z}}\right)^{3 / 2}} \exp \left(-\frac{(\mathrm{x}-\mathrm{ut} / \mathrm{R})^{2}}{4 \mathrm{D}_{1} \mathrm{t} / \mathrm{R}}-\frac{\mathrm{y}^{2}}{4 \mathrm{D}_{\mathrm{y}} \mathrm{t} / \mathrm{R}}-\frac{\mathrm{z}^{2}}{4 \mathrm{D}_{\mathrm{z}} \mathrm{t} / \mathrm{R}}\right)
$$

Which again is Gaussian, but now in three-dimensions. 


\section{Sources and models for non-ideal transport}

The ADE provides a flexible model with the great advantage of having a rich set of solutions which can be added, and can incorporate effects of linear sorption and degradation naturally. Yet most sorption and degradation processes are not linear, and porous media has regions of very fast flow and essentially stagnant fluid, which are not well described in the ADE, as reviewed in Table 6.1. Since at least the 1950's scientists have developed more complete models of solute transport which explicitly include these non-ideal features. In this section we briefly review the literature in this area. Right up front we apologize to all the authors who we will slight in this review, as there are literally hundreds of publications covering these topics, of which we will only have space to mention but a very few.

\section{The various non-ideal flow processes}

Departures from the assumptions of the ADE occur at many physical scales, with the non-ideal processes being closely aligned to particular ranges of scale. We shall consider these processes in order of declining scale.

At the field scale the key concern should be heterogeneity. As demonstrated by the ponded infiltration study of Nielson et al. (1973), heterogeneity in the vadose zone is not only ubiquitous, but extreme, typically following a lognormal distribution. These observations have been supported by many field studies which have followed, with recent pan-sampling tracer transport studies supporting the log-normal velocity observations (Boll et al., 1998). To capture some of the behavior of these effects one consider a simplified model which identifies effective surface area of soil in terms of the typical rate of percolation. If we are concerned about the field average of material which arrives to the aquifer, then we don't have to worry about where these areas are, but just what fraction of the field falls into each velocity group. We could then model each velocity group separately, and add all the solutions up to obtain a reasonable estimate of what the aquifer will see. This is referred to as a stream tube modeling approach, where the site is divided into a number of "tubes", each of which is characterized by particular velocity, and dispersion coefficient. The CXTFIT model will fit such models to field sampling data (Toride et al., 1995). Clearly one would need many sampling locations to characterize such a spatially variable process. Brandi-Dorhn et al. (1997) observed in their field experiment that spatial variability dictated that to obtain an estimate of the mean concentration of a frequently applied solute such as nitrate to obtain a $95 \%$ confidence interval which was plus or minus $30 \%$ of the true mean concentration required 8 of the $0.35 \mathrm{~m}^{2}$ passive capillary samplers (PCAPS), while sampling a pulse applied tracer required 37 suction cup samplers or 25 PCAPS.

\section{Retardation revisited: incorporating geochemistry}

In the past ten years, many studies of how to improve the characterization of reactions given the simplifying assumptions underlying use of $\mathrm{R}$ in [5.47]. This area of "reactive transport modeling" takes ADE as point of departure and generally couples a transport module for concentrations only (after [5.31]) with a code module(s) which perform mass-transfer (geochemical) calculations. Typically the transport module advances, the resulting concentrations are modified by the mass-transfer calculations and returned to the transfer module for the next time step. This procedure permits inclusion of multiple species - including solid phases - in the calculation of activities and concentrations (section 4.2.5). Suarez and Simunek (1996) provide a recent review focused on vadose environments. LEACHMS (Wagenet and Hutson, 1987) is an example of a well-known model developed for soils. In some cases reactive transport models for groundwater systems (e.g. PHREEQEM, Appelo et al. 1990; HYDROGEOCHEM, Yeh and Tripathi, 1991) have been modified for application to vadose zones (e.g. Hansen and Postma 1995).

The most ambitious current work couples heat and water flow, mass transport, and mass transfer (e.g. Simunek 
and Suarez, 1994, 1996). Richards' equations (flow module) are solved (chapter 3) using the K model of van Genuchten (chapter 2), resulting velocities are substituted into equations for each species taking form of [5.31] (transport module). The computed concentrations are used as inputs to an aqueous speciation model (sec. 4.2.5.3) which is expanded to include selected minerals sorbents, and $\mathrm{CO} 2$ in the gas phase (mass transfer module). The latter two modules are then solved iteratively to convergence at each time-step. Precipitation of minerals or expansion of clays causes changes in hydraulic properties which are accounted for in the flow module for the next time-step.

Modeling transport with explicit geochemistry in a coupled geochemical code module is going to be a bigger job than the "normal" way of handling reactions using R embedded in the ADE (eq. [5.46] and following): we will not be able to use analytical solutions; the numerical solutions will be complicated and not very user-friendly as of this writing; and we will need geochemical input parameters in addition to the transport parameters, which as we have seen are difficult enough to estimate. Thus we are often justifiably (or at least understandably) discouraged from tackling the reactive-transport problem.

Now, having confronted reality, let's be explicit about what we can legitimately handle in the "normal" fashion, i.e. using $\mathrm{R}$ in the ADE. The most mathematically restrictive (but attractive!) situation is use of analytical solutions to equations like [5.50] in which $\mathrm{R}$ (not to mention $\mathbf{D}, \mathrm{u}$, and $\theta$ !) is assumed invariant in time and space. This necessarily implies not only that the solid-phase concentration is a simple function of the solution concentration (eq. [4.102] and [5.41]), but also, as noted in section 4.5.7, that the distribution coefficient $\mathrm{k}_{\mathrm{d}}$ in that function is constant. Physically, this limits the geochemical processes we can describe to linear, instantaneous, nonhysteretic exchange (section 4.5.4) or sorption (section 4.5.5). Mercifully, the reactive behaviors of contaminant metals and organics (section 4.5.6) actually conform to this description in certain situations provided contaminant concentrations are low enough to render equations like 4.101 valid. This is why so much batch- $k_{d}$ work (Figure 4.16 ?) has been done in the subsurfacecontamination-remediation business in recent decades.

Now, let's relax the constant-R restriction, corresponding to use of equations like [5.47] and [5.48]. Even without a coupled geochemical module, the non-linearities in these equations require numerical solutions. In exchange for this considerable inconvenience we can, in principle, model sorption using Langmuir or Freundlich isotherms ([4.103] and [4.105]). Mathematically, [5.42] takes a more complex form which propagates into the coefficients in the storage and decay terms of [5.47]. Geochemically, this allows us to model sorption where "sites" are limited, e.g. over larger concentration ranges than would be possible otherwise (section 4.5.5.1).

As a final thought on this topic, let's explicitly consider what we cannot handle using R in the ADE. A key insight here is that all of the proportionality expressions underlying R concern the solute of interest only. In the "real world" there are always other chemical species in the vadose mix which may exert strong control on the solute of interest; and the point at which that control becomes important often depends on the geochemistry of those other species. Two important cases are variable ionic strength (variation in the concentrations of major ions) affecting the activity of a contaminant metal (section 4.2.5), and solubility control by a mineral phase on a contaminant metal. A simple example of the latter is provided by $\mathrm{CdCO}_{3(\mathrm{~s})}$ : threshold conditions for dissolution or precipitation of cadmium, in analogy with calcium or magnesium ([4.72]) will always depend on the carbonate ion concentration, which depends in turn on the whole $\mathrm{pH}^{-}$ dependent carbonate system. Another key point to remember is that where reactions are slow relative to transport rates, reaction kinetics may be important. There is no single reference with an elegant summary of these effects for the universe of dissolved species of interest; Stumm and Morgan (1996), Langmuir (1998), and Drever (1997) all provide useful information, and the first of these is particularly encyclopedic. For inorganics, the short summaries provided by Hem (1985) are often an excellent basis for getting started.

Frequently some good geochemical thinking will define geochemical limits within which an R-ADE transport treatment is defensible, thereby strengthening the reliability of a transport prediction. 


\section{The Mobile-immobile conceptual model}

The process of dispersion which was presented above attributes spreading of solute to the joint variation in velocity and solute concentration in a porous media. In naturally occurring porous media not only is there variation in flow velocity due to differences in distance from no-slip solid surfaces, but there are entire volumes of liquid which are isolated from the general flow field. Such isolated and dead-end pores can exchange solutes with the moving fluid by means of diffusion. Models which account for such active and passive pools of liquid are referred to as mobile-immobile models. They have been discussed in the fields of chemical engineering and chromatography for decades (e.g., Turner, 1958), and were introduced in the literature of solute movement through natural media by Skopp and Warrick (1974). Skopp and Warrick (1974) presented an analytical solution for the system shown in Figure 6.13, where dispersion was not included. In this model, Skopp and Warrick considered the transport within the mobile phase to be non-dispersive plug flow, and the mobile phase to be entirely stagnant. All plume spreading came from the exchange between these phases. Mechanistically, there still would be expected to be dispersion within the actively moving pore-space, and mobileimmobile models have includes such features (e.g., Gerke and van Genuchten, 1993), although the resulting systems of equations appear to be soluble only through numerical means. In addition, the number of parameters included in such models, for instance exceeding 20 in the work of Gerke and van Genuchten (1993), renders this approach beyond the complexity reasonable for other than research applications. It should be noted, however, that numerical inversion of tracer experiment breakthrough curves to obtain parameters for a 19-parameter non-equilibrium mobile-immobile model is presented by Toride et al. (1995) which greatly increases the feasibility of application of these tools. Great attention should be paid to the uniqueness of any parameter set obtained for such a complex model since predictions could be very sensitive to highly uncertain parameters. That is to say, the fact that a complex model fits experimental data sets does not imply either that the model is accurately describing the processes nor that it is accurate in predicting experimental outcomes under differing boundary and initial conditions.

\section{Transfer Function Modeling}

The modeling approaches that we have described thus far are mechanist, in that the transport process is built up of various mechanisms to ever greater levels of detail. At various points in this text we have suggested that we might either throw up our hands and give up, or plod ahead and try to simplify the situation sufficiently to make it manageable. Guess what: we can both throw our hands up in the air and plod ahead! To be more specific, what if we accept the fact that the transport processes through the vadose zone are too complex to model mechanistically, yet we still want to describe the movement of a compound as observed experimentally. One option is to run a single experiment and attempt to extrapolate from that experiment to determine what might happen under differing circumstances. This is the essence of transfer function modeling: it is an organized way to extrapolate from experience. It follows in the distinguished tradition of "black-box" hydrologic models such as the instantaneous unit hydrograph for stream flow, and is quite analogous in its underpinnings. An excellent reference text on this subject is "Transfer Functions and Solute Movement Through Soil" (Jury and Roth, 1990) where the full, elegant, mathematical basis of the technique is developed.

The basic notion is that we need a Green's function (impulse response) for the vadose transport process, as the Gausian solution was for an instantaneous application for the ADE. Let's say that we were interested in the contaminant transport below the root zone of a non-reactive tracer that had been applied to the site over time, and the only information that we had for the site was a breakthrough curve for a bromide pulse that had been applied to the site some years before. Perhaps we applied the bromide at the soil surface, and we observed the breakthrough using a set of lysimeters located at a depth of $2 \mathrm{~m}$. The idea of transfer function modeling is that the bromide pulse represents the basic pattern of non-reactive solute movement, and that any other similar solute may be modeled as a summation of the output of the bromide curve weighted for the relative amounts of water and solute applied in time. So if we wanted 
to model, say, the expected breakthrough of two successive bromide applications, we simply would superimpose two copies of the original breakthrough with the second curve shifted by the interval which separated the two bromide applications, keeping track of all movement in terms of the volume of water passing through the level of observation. Each of the two breakthrough curves would be multiplied by the ratio of the mass applied in the experiment to that applied to obtain the original bromide breakthrough curve.

Figure 6.13 Illustration of mobile, immobile, and solid regions as thought of in the mobile-immobile model of transport. Figure (a) depicts a actual porous medium and (b) the parameterization of the system in the model (after Skopp and Warrick, 1974).

As mentioned above, in this procedure it is important to put all measurements in terms of the amount of water which has past through the system rather than identifying the breakthrough curves against time, for instance. The most logical way to keep track of this is in terms of "pore volumes" of water that have passed the point of interest. A pore volume is simply the equivalent depth of water between the point of application and the point of observation of the breakthrough. For our example, if the average moisture content of the soil above the lysimeters was $22 \%$ by volume, then one pore volume would be $0.44 \mathrm{~m}$ of water. Note that the pore volume accounting system suggests that any sequence of experiments which involved the same total volume of water applied would generate exactly the same solute breakthrough result regardless, for instance, of the total time employed to apply the water.

We see that the transfer function method allows us to extrapolate from observations at a given location to the outcome of a different experiment at the same position in the soil profile. By supposing that the soil is homogeneous, one could ask how could we extrapolate to other positions within the soil profile. Jury and Roth (1990) introduce the hypothesis that if a particle required $\mathrm{n}_{1}$ pore volumes of water (where $\mathrm{n}$ may any positive real number) to arrive at depth $\mathrm{z}_{1}$, then to arrive at depth $\mathrm{z}_{2}$ will require $\mathrm{n}_{2}=\mathrm{n}_{1} \mathrm{z}_{2} / \mathrm{z}_{1}$ pore volumes of water. This amounts to assuming that the moment of particles is statistically linear in space with equal distances traveled with equal applications of water. Using this hypothesis, the breakthrough curve obtained at depth $\mathrm{z}_{1}$, if plotted against pore volumes, would maintain its shape with depth, with the duration increasing linearly with depth, and the concentrations decreasing linearly with depth.

The linear transport hypothesis has intuitive appeal, and is fundamental to the transfer function approach. It is problematic for processes where microscopic diffusion plays an appreciable role (i.e., situations with Peclet numbers of the order of 5 or less). To see this, note that if there were no water movement, the transfer function approach predicts no solute spreading or displacement. But we know that diffusion inexorably spreads solutes regardless of bulk water flow. The transfer function approach ascribes all solute transport to dispersive processes. It is therefore reasonable to apply the transfer function approach to describe processes with common effective dispersivity, which implies appropriate use for any set of experiments in which dispersion dominates (Pe $>10)$, or in comparing with identical Peclet number if $\mathrm{Pe}<$ 10. Many of the problems of interest to vadose transport have $\mathrm{Pe}<10$, in which case the underlying assumptions of the transfer function approach limit the range of extrapolation to solute profiles expected at varying depths under identical water flow boundary conditions.

\section{Lab and Field Methods to Measure Transport Parameters}

In the preceding sections we have developed a simplified conceptual model for solute transport through natural media. To make use of this description we must, at a minimum, obtain parametric values describing the soil including the dispersion coefficient, diffusion coefficient, and solute partition coefficient. Perhaps we could look up the diffusion coefficient for the solute in pure water and adjust for an effective tortuosity, and perhaps we could carry out an equilibrium batch experiment to obtain the partition coefficient, but how could we determine the hydrodynamic dispersion coefficient? Clearly this depends upon the structure of the material, the rate of flow, and the degree of saturation of the media. The only realistic method to obtain this parameter is to carry out an experiment, either in a small laboratory column, or in a field experiment. Presumably we would obtain a breakthrough curve similar to those shown in Figure 6.10 and Figure 6.11.

Solute Transport in Soils and Vadose Materials | 295 
Our problem then is to "back out" the parameters in the ADE (or what ever other transport model we select) from the observed breakthrough curve. What this amounts to is finding a reliable procedure to select the set of parameters which when put in the model most closely reproduce the observed experimental results. Fortunately, we do not need to start from scratch on this problem, as carefully crafted numerical solution packages have been developed to automatically complete this task (e.g., CXTFIT presented by Toride et al., 1995). Typically the fitting procedure is driven by minimizing the sum of square difference between the experimental data and the model generated values.

We must be cautious in getting excited when we see the lovely overlay of the fitted model to our data. The ADE, transfer functions, and more complex models are all quite flexible in there ability to fit a smoothly changing solute concentrations. On the other hand, a good fit by no means implies that the model in fact provides either a good description of the physical processes involved or that it will be predicted under differing conditions or at differing scales of time of space. Of course, we may have no choice but to proceed in using the calibrated model for predictive purposes for lack of a better alternative, but this should be done with great humility, understanding that significant errors will likely be encountered.

\section{Nonaqueous Fluids in the Vadose}

Much of the study of vadose processes is directed toward the understanding of contaminant transport. One set of contaminates requires special treatment; those that are not miscible in water. These are referred to as Non-Aqueous Phase Liquids, or NAPLs, and are characterized by their low solubility in water. NAPLs are non-polar compounds which if introduced into a natural environment will remain as a separate liquid phase (as opposed to going into solution as alcohol or latex do). This overall grouping is subdivided into those which have density lower than that of water (LNAPLs - Light; e.g., gasoline, diesel, kerosene) and those which are denser than water (DNAPL - Dense, e.g., TCE, carbon tetrachloride)

The sources of LNAPL are, unfortunately, very numerous in today's industrial society. The most ubiquitous consist of leaking underground storage tanks (LUST's). The most widely regulated subset of these are at gas stations where approximately $10 \%$ of single walled steel tanks leaked, with plumbing leaks in approximately $30 \%$ of these installations (lesson: don't assume that the plume will be under the tank since most arise from delivery system failure) (Selker, 1991). Most of these tanks have been removed in the United States due to tightened regulation. Another major source of LNAPLs are household heating oil tanks. Although long overlooked, it is clear that there are a vast number of leaking buried oil tanks, most likely in the same or higher proportions as old gas station tanks. These problems are rarely noticed until there are catastrophic failures, since unlike commercial tanks, there are no records of consumption. The lower volatility of heating oil also limits the observation of leaks through vapor transport into basements etc.

DNAPLs in the environment typically arise from disposal of cleaning compounds. Whereas LNAPLs are most commonly observed at points of delivery, DNAPLs are found at points of delivery, use, and disposal. "Dry wells" and other ad hoc disposal sites represent a major portion of plume generators, often near the point of use, or at waste disposal sites. Spills are typically of smaller volume than LNAPLs, but more serious due to higher toxicity and bulk penetration of aquifers, as discussed below.

\section{The Anatomy of a NAPL Spill}

The prediction of NAPL movement is complicated by a wide range of physical and chemical processes making quantitative prediction generally impossible for field spills (Osborne and Sykes, 1986; Cary et al., 1989b; Essaid et al., 1993). From this perspective, it is most productive to understand the qualitative characteristics of typical site movement, rather than spend inordinate energy on quantitative prediction of NAPL disposition. In this section we address the typical features of a NAPL plume.

A key point in understanding the fate of NAPL is to note that residual saturation can account for a large fraction of a spill. By residual, we refer to blobs of NAPL that are isolated to the extent that their movement is not governed by local gradients in fluid potential. Since most natural sites will be composed of media which is coated with water, NAPLs 
tend to form small droplets (a.k.a. ganglia) in the unsaturated zone. On the order of $5 \%$ of the volume of the region which experienced NAPL transport will remain NAPL filled with residual product (Cary et al., 1989c). This is of interest for planning in soil clean up, as well as understanding how much of the product may have reached the upper aquifer.

\section{Example}

A spill of 10,000 1 of product $10 \mathrm{~m}$ above an unconfined aquifer. Assuming, for convenience, that the NAPL wetted area of $4 \mathrm{~m}$ by $4 \mathrm{~m}$, that there was no lateral spreading, and the residual saturation was $5 \%$, how much of this original spill makes it to the water table in liquid form?

Solution:

The residual volume in the vadose zone is:

$$
10 \mathrm{~m} \times 4 \mathrm{~m} \times 4 \mathrm{~m} \times 5 \%=8 \mathrm{~m}^{3}=8.000 \mathrm{l}
$$

therefore about 2,000 liters (20\%) makes it to the water table. Given our very uncertain values of residual, and highly restrictive assumptions, we should probably conclude that there is a high likelihood of NAPLs reaching the water table, and NOT quote a specific expected amount that WILL enter the aquifer.

The second key point is that the geologic configuration of the site is very influential in the final disposition of the NAPL. In the case of LNAPLs, the vadose zone is of primary importance, since the bulk liquid does not penetrate the saturated zone, while in the case of DNAPLs, the structure in both saturated and unsaturated regions will have a major impact on disposition. The main issue here is layers between media of different texture. In particular, horizontal bedding features will cause the plume to spread laterally with a dominant down-dip movement (Schroth et al., 1997).

The rate at which the NAPL was introduced is also highly influential in its ultimate disposition (Poulsen and Kueper, 1992). Rapid spills require broader areas to carry the flow, and thus leave larger residual saturation in the unsaturated zone, and less free product on aquifers. They are also less susceptible to extreme lateral flow due to textural interfaces. Slow leaks are more susceptible to lateral diversion along textural interfaces in the vadose zone, and will likely follow more isolated paths of flow. In this way the slow leaks tend to contaminate a larger area, while still delivering a greater fraction of the product to the aquifer.

In the vadose zone DNAPLs and LNAPLs behave quite similarly so long as regions of saturation are not encountered. This should make sense, as the only distinction we have made between these is their relative density in comparison to water. As long as the NAPL has not encountered a region of saturation, there will be no buoyancy effects, and therefore the physics of flow is essentially the same. Once saturated regions have been broached, the nature of free product migration differs dramatically for LNAPLs and DNAPLs. Here LNAPLs travel in direction of the slope of the top of the water table, while for DNAPLs, the gradient in lower boundary of aquifer dictates the movement of free product. Furthermore, prior to reaching lower boundary of the aquifer, DNAPLs typically move through aquifers in web like networks of pores rather than moving in a unified front (e.g., Held and Illangasekare, 1995). Since in this pattern many of the pores are not wetted with the DNAPL, this reduces residual saturation, thus increasing the free product available to spread through the aquifer. 


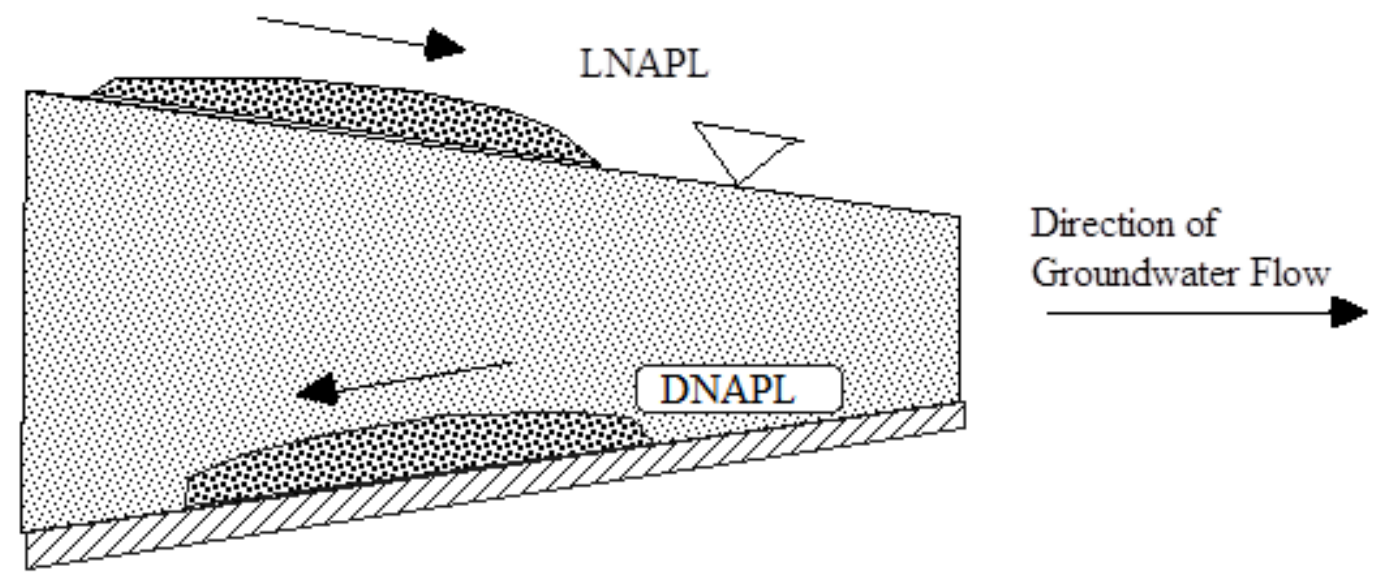

Figure 6.13 Contrasting migration of LNAPLs and DNAPLs in the vicinity of regions of saturation.

\section{Observing LNAPLs in Wells}

Often the first indication of NAPL contamination is the observation of the product in a well. The extent of a plume at a site is often then delineated by installing additional wells on the site. The extent of contamination is then delineated by obtaining core sample sand observing the depth of "free product" in the wells. BE CAREFUL: The depth observed in wells is not the free product depth on the aquifer (see Abdul et al., 1989). It is useful to carry out a few calculations to show how deceptive such in-well measurements can be.

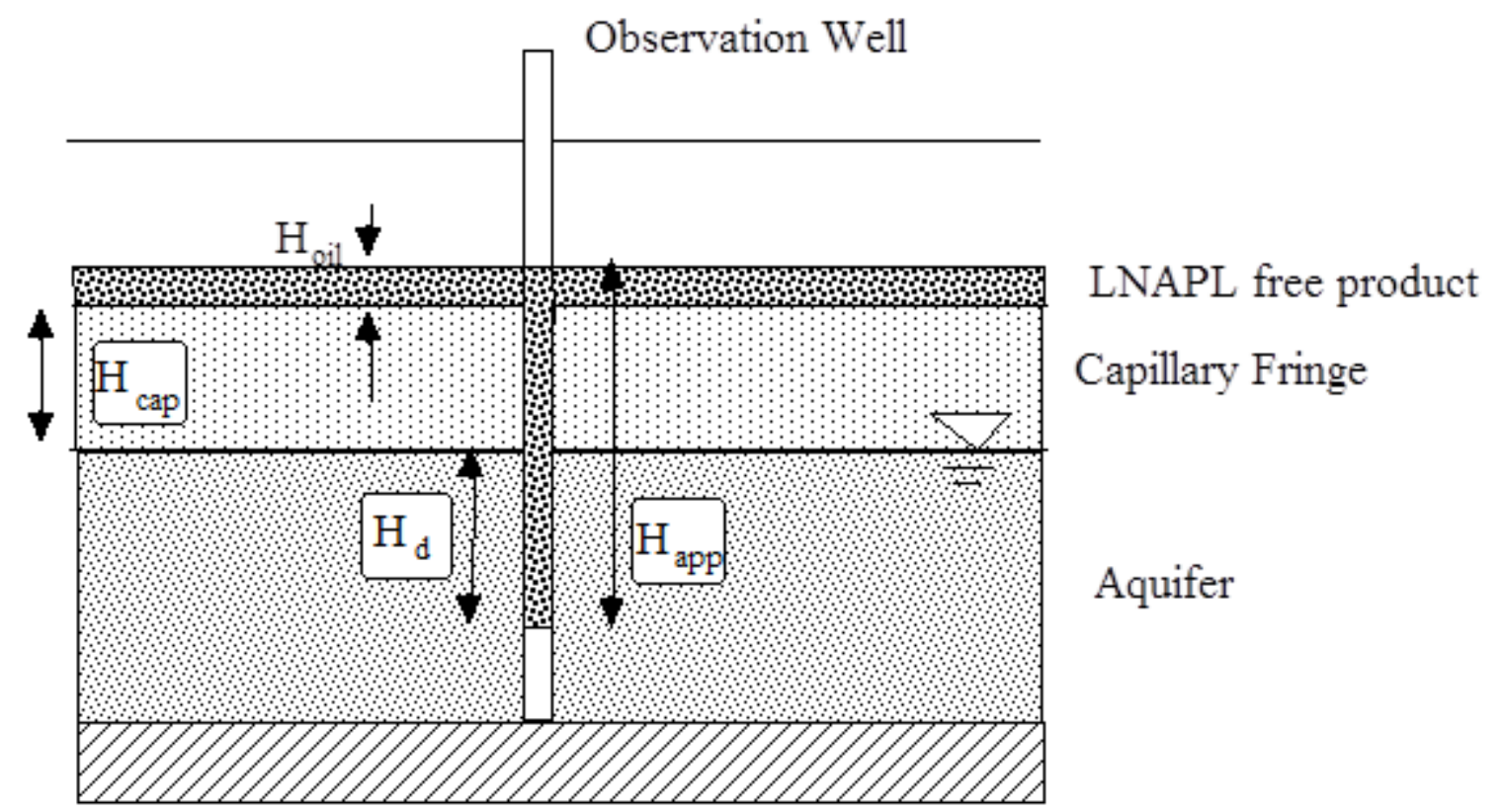

Figure 6.14 Typical observation well at an LNAPL spill site where Hoil is the "True" depth of free product, Hcap is the thickness of the capillary fringe, Happ is the "apparent" depth of free product, and Hd - the depression of the water surface in the well (meters) 
Consider the configuration shown in Figure 6.14. At the oil-water interface in the well, the total head is

$$
\mathrm{H}_{\mathrm{O}-\mathrm{w}}=\gamma_{\mathrm{oil}} \mathrm{H}_{\mathrm{app}}
$$

where goil is the specific gravity of the oil, and we take the total head at the water table to be 0 (datum).

We also know that the total head at all points in the aquifer is constant (assuming that we are not pumping from the well), so head at the interface is also given by

$$
\text { (6.69) } \gamma_{\mathrm{o}-\mathrm{w}}=\gamma_{\mathrm{w}} \mathrm{H}_{\mathrm{d}}
$$

Equating $(\underline{6.68})$ and $(\underline{6.69})$ we have

$$
\text { (6.70) } \gamma_{\mathrm{w}} \mathrm{H}_{\mathrm{d}}=\gamma_{\mathrm{oil}} \mathrm{H}_{\mathrm{app}}
$$

From the geometry of the set-up we see that

$$
\text { (6.71) } \mathrm{H}_{\mathrm{app}}-\mathrm{H}_{\mathrm{d}}=\mathrm{H}_{\mathrm{cap}}+\mathrm{H}_{\mathrm{oil}}
$$

solving for $\mathrm{H}_{\mathrm{d}}$

(6.72) $\mathrm{H}_{\mathrm{d}}=\mathrm{H}_{\mathrm{app}}-\mathrm{H}_{\mathrm{cap}}-\mathrm{H}_{\text {oil }}$

We may rewrite (6.71) using (6.70) as

$$
\gamma_{\mathrm{oil}} \mathrm{H}_{\mathrm{app}}=\gamma_{\mathrm{w}}\left(\mathrm{H}_{\mathrm{app}}-\mathrm{H}_{\mathrm{cap}}-\mathrm{H}_{\mathrm{oil}}\right)
$$

Solving for $\mathrm{H}_{\mathrm{oil}}$ we find:

$$
\mathrm{H}_{\mathrm{oil}}=\mathrm{H}_{\mathrm{app}}\left[1-\frac{\gamma_{\mathrm{oil}}}{\gamma_{\mathrm{w}}}\right]-\mathrm{H}_{\mathrm{cap}}
$$

or

$$
\mathrm{H}_{\mathrm{app}}=\frac{\mathrm{H}_{\mathrm{oil}}+\mathrm{H}_{\mathrm{cap}}}{\left[1-\mathrm{d} \frac{\gamma_{\mathrm{oil}}}{\gamma_{\mathrm{w}}}\right]}
$$

Since the densities of the NAPL and water are quite similar, the denominator of $(\underline{6.75})$ can be quite small, making $\mathrm{H}_{\mathrm{app}}$ much larger than either $\mathrm{H}_{\mathrm{oil}}$ or $\mathrm{H}_{\text {cap }}$.

\section{Example}

For typical NAPLs $\left(\gamma_{o i l} / \gamma_{w}\right)$ is about 0.8 . Taking $H_{\text {cap }}$ to be $50 \mathrm{~cm}$ (typical for a silt loam texture), and assuming the true depth of free product to be $2 \mathrm{~cm}$, we can use $(\underline{6.75})$ to calculate the "apparent depth" of NAPL in the well

$$
\mathrm{H}_{\mathrm{app}}=\frac{2 \mathrm{~cm}+50 \mathrm{~cm}}{1-0.8}=\frac{52}{0.2}=260 \mathrm{~cm}
$$

almost $3 \mathrm{~m}$ of "free product" in the well! If you don't mind being off by a factor of 100 then you can ignore this. Note that this result is very sensitive to: (a) the height of the capillary fringe, and (b) the density contrast of the liquids. Typically we can be fairly sure of the density contrast, but the height of the effective capillary fringe is difficult to measure. In fact, the height of the capillary fringe depends on the infiltration history as well as the surface tension between the NAPL and aquifer water, which is a function of the changing degree of contamination of each of these liquids.

In the case of DNAPLs, wells present a more serious threat. If a well screen crosses an aquitard, the well itself can 
become a pathway for transport, with a DNAPL draining off the aquitard, into the well, and out the well in the lower aquifer. On the other hand for LNAPLs, by creating a cone of depression about a well you may facilitate removal of the contaminant which will then flow to the well.

\section{Movement and Retention of NAPLs}

We will now briefly discuss the underlying physics of the movement of NAPLs in porous media to bring out the key differences between two phase flow (e.g., air/water) and three phase flow (e.g., air/water/NAPL). In the case of NAPL transport, there are three primary pathways. First we have the bulk flow of the NAPL. Here the movement will follow Darcy's law but with conductivity a function of $\theta_{\mathrm{w}}$ and $\theta_{\mathrm{oil}}$. The second important transport process is in the vapor phase, which can be responsible for a large fraction of transport in the case of volatile compounds. Finally, although largely immiscible, NAPLs are slowly carried throughout an aquifer in solution. Solubility is typically between 1 and 500 ppb. Dissolved phase transport is mitigated by partitioning out of solution through adsorption to mineral and organic constituents, creating a retardation between bulk water movement and that of the dissolved contaminant.

Predicting NAPL movement is much more complex than for soluble compounds. One of the thorniest issues is that of the surface tension effects between the medium, water and NAPL. From our discussion in section 2.3 we know that surface tension is responsible for many of the features of vadose zone transport. In the case of NAPL movement, we have not two interfaces to think about (i.e., the air/water and air solid surfaces), but six (air/solid, air/water, air/NAPL, solid/water, solid/NAPL, and water/NAPL). Moreover, each of the four phases (gaseous, solid, water and NAPL) can now be contaminated with the either water or NAPL to varying degrees (Schroth et al., 1995), which has a huge effect on the interfacial surface tension. As if this wasn't bad enough, as the NAPL phase ages, its characteristics will change (McCaffery and Mungan, 1970). This again leads us to limit our expectations of being able to predict NAPL movement in a quantitative sense. Still it is useful to consider the underlying physics to be able to understand the characteristics of the transport process.

Just as shown in Figure 2.11? for the air/water/solid combination, each of the possible junctions between three different surfaces will be characterized by a contact angle. A critical issue in the realm of surface tension in the context of NAPL movement is the issue of spreading vs. non-spreading NAPLs, which depends upon the contact angle of the air/ water/NAPL triplet. Spreading NAPLsare those which have a contact angle of zero with water in air, and therefore will spontaneously spread over a water/air interface yielding a layered water in contact with only NAPL and air in contact only with NAPL. Non-spreading NAPLs are those with non-zero NAPL/water contact angle, and will remain in isolated blobs on water surfaces. Examples of these phenomena abound in the world around us, for example oil floating in droplets on soup (non spreading), and the rainbow sheen of a drop of oil spread in a micron thick layer on a puddle of water (spreading). These differences are crucial in terms of NAPL movement in porous media. A spreading oil will move quickly over the entire surface of the water in a wetted soil, while the non-spreading oil will quickly go into a state of residual saturation as isolated blobs of oil in the soil, leaving a much higher residual fluid content. A word of caution is in order here; as shown by Schroth et al. (1995) and McBride et al. (1992), NAPLs that are initially non-spreading can become spreading even if the NAPL is, to all appearances, immiscible in water and nonvolatile.

Wetability refers to the strength of the physical attraction of a fluid to a solid. Highly wetting fluids (e.g., water) are at lowest energy when coating solid surfaces while nonwetting fluids (e.g., mercury) are at lowest energy when in isolated droplets. In porous media, the wetting fluid will fill the smallest pores, while the non-wetting fluid will fill the largest pores. In the case of the familiar air/water system, air is the non-wetting fluid.

To estimate the potential for bulk transport we need to consider how Darcy's law will apply to multiphase systems. Recall that the intrinsic permeability is defined as

$$
\text { (6.77) } K=K_{i} \frac{v_{i}}{g}
$$


where $K_{\mathrm{i}}$ is the hydraulic conductivity of the medium to fluid $\mathrm{i}$, and $v_{\mathrm{i}}$ is the kinematic viscosity of the fluid. To obtain $\mathrm{K}$, one must measure $\mathrm{K}_{\mathrm{i}}$ for some particular liquid. Darcy's Law can then be rewritten as

$$
\text { (6.78) } \mathrm{q}=-\kappa \frac{\mathrm{v}_{\mathrm{i}}}{\mathrm{g}} \nabla \mathrm{H}
$$

for the general category of Newtonian fluids (e.g., a material in which viscosity is well defined; not tooth paste). In situations where there is only one wetting liquid in the presence of a gaseous phase, this result will be applicable to unsaturated conditions as discussed in section 2.4. If, on the other hand, two liquid phases are present, the situation becomes far more complex. Typically water will be the most wetting phase, with the NAPL phase then layered on the water. Now the permeability of the system to the oil phase will be dependent not only on the degree of oil saturation, but also upon the degree of water saturation, as the water will "lift" the oil into larger pore spaces which tend to offer less resistance to flow. The theory of multi-liquid pressure/saturation/permeability relationships has been studied extensively, and some very useful results have been obtained, but go far beyond the scope of this text (see Parker et al, 1987; Parker and Lenhard, 1987; and Lenhard and Parker, 1987).

Figure 2.38? Relative permeability of water and NAPL phases in porous media (the NAPL phase saturation is simply 1- $\theta$ where $\theta$ is the water phase saturation). Note that for a given degree of fluid content, the NAPL phase has higher permeability than the water phase due to the water occupying the smaller pores (from Land, 1968).

\section{Review Questions}

1. What is the relationship between accuracy in prediction in the movement of water and that of solutes?

2. What are typical magnitudes of the coefficient of molecular diffusion for gas and liquid phases? Why the big difference?

3. Would there be dispersion in a cylindrical capillary tube under pure laminar flow? Why/why not?

4. Under what conditions is use of a plug flow model justified?

5. Explain two advantages of plug flow modeling.

6. From a mathematical perspective, dispersion could be taken as either a scalar or a tensor. Under what conditions, if any, would it be appropriate to take the dispersion coefficient as a scalar?

7. Under what conditions may the dispersion tensor be diagonalized? Taken as a $2 \times 2$ rather than $3 \times 3$ tensor?

8. What is the relationship between dispersivities and dispersion coefficients? Which is a property of the media alone, and which is a function of the flow velocity.

9. What is the range of relationships between longitudinal and transverse dispersivities? How could you explain a site in which these parameters are of equal magnitude?

10. Explain in words the Peclet number. Explain the five regions of Peclet number in terms of the relationship between both longitudinal and transverse dispersivity in contrast to the diffusion coefficient.

11. In absences of degradation, and using linear sorption, how different will the spatial distribution be for two solutes which have different sorption, but started with equal total mass, and when looked at when the center of mass is an equal distance from the point of injection.

12. For an instantaneous injection with linear sorption, will the plume which develops be symmetric spatially? Temporally?

13. Why is it correct to use superposition in solving the ADE? What assumptions must be made to make this work?

14. Explain the relationship between the Gaussian curve and the error function. 
15. What is meant by a black-box model, as the Transfer Function approach is described? Are physical processes considered?

16. Why might calculating the distribution of aqueous species be important for predicting transport of a heavy metal, whereas it might not be very important for predicting transport of a halogenated organic? (ANS: for ions, $\mathrm{mi}=\mathrm{f}(\mathrm{mi}, \mathrm{j})$; also mineral dissolution/precipitation could be important)

17. Consider the problem of trying to predict vadose transport of $\mathrm{Cd}$ (mentioned briefly in section 5.X). We know that otavite (CdCO3) could exert solubility control on transport, i.e. precipitation could drastically reduce its mobility in solution. (a) Is this likelier to occur in a carbonate terrain or in a silicate terrain? Why? (b) How could you evaluate whether otavite should control Cd mobility in a given setting?

\section{Problems}

1. Show that [5.58] satisfies [5.50] by computing the derivatives and obtaining the equality shown in [5.50].

2. A compound with a half-life of $2 \mathrm{y}$ is applied to a soil with a uniform and constant moisture content of 0.30 $\mathrm{cm}^{3} / \mathrm{cm}^{3}$. The percolation is $0.5 \mathrm{~m} / \mathrm{y}$, and there is no sorption. Using a plug flow approach, what fraction of the compound makes it to the water table at a depth of $5 / 0.3 \mathrm{~m}$ ? Had the retardation factor been 2 , what fraction would have made it to the water table?

3. For a situation where the coefficient of molecular diffusion in $0.00002 \mathrm{~cm}^{2} / \mathrm{s}$, the velocity is $0.02 \mathrm{~cm} / \mathrm{d}$, the mean soil grain sizes is $0.002 \mathrm{~cm}$ and the dispersivity is $20 \mathrm{~cm}$ :

a. what is the Peclet number?

b. for each longitudinal and transverse spreading of the plume, comment on

whether the spreading is dominated by diffusion or dispersion.

4. Reynolds et al. (1982) studied sorption of strontium $\left(\mathrm{Sr}^{2+}\right)$ to Chalk River sand. Batch tests (Fig. 4.16) yielded $\mathrm{k}_{\mathrm{d}}=15+/-0.6 \mathrm{~mL} \mathrm{~g}^{-1}$ over the concentration range of concern. The bulk density of the sand is approximately $1.75 \mathrm{~g} \mathrm{~cm}^{-3}$. Assuming volumetric water content $\theta=0.1$, estimate (a) $\mathrm{R}$, and (b) the vertical migration rate through a vadose zone at a recharge rate of $30 \mathrm{~cm} / \mathrm{y}$. What physical and geochemical issues are we forced to neglect given the limitations of our information?

\section{Index}

A

ADE

adsorption

advection

advective dispersion equation

anisotropy

asymmetry 
black-box model

breakthrough curve

C

CDE

chemical reactions

complementary error function

conservation of mass

conservative

continuous injection

convection

convection-dispersion equation

CXTFIT

D

dead-end pores

decay

degradation

diagonalized

diffusion

diffusion coefficients

dispersion

dispersion coefficient

dispersivity

dispertivity

E

effective diffusion coefficient

equilibrium sorption

error function

F

Fick's law

Fickian model

Fickian process

first order decay

fitting procedure

flux concentration

Freundlich isotherm

G

Gauss Divergence Theorem

Gaussian distribution

Gaussian Distribution

geochemistry

Green's function

H

heterogeneity

hydrodynamic dispersion coefficient

hydrodynamically inactive

hydrograph

I 
infinite line source

instantaneous injection

intercepting pan sampler

intrinsic permeability

irreversible sorption

isotropic media

L

laminar

laminar flow

Langmuir isotherm

linear adsorption

linear decay

linear isotherm

linear sorption

longitudinal

longitudinal dispersion

longitudinal dispersivity

longitudinal dispersivity, scale dependence

M

macroscopic processes

mass balance

measuring transport parameters

mechanistic modeling

mobile-immobile model

molecular diffusion coefficient

molecular diffusion coefficinets

$\mathbf{N}$

non-ideal transport

non-idealities

non-reactive

nutrient transport

O

one-dimensional solution

$\mathbf{P}$

passive capillary sampler

PCAPS

Peclet number

plug flow model

pore volume

R

reactive transport modeling

representative elementary volume

resident concentration

retardation coefficient

retardation factor

REV

reversable sorption

reversible adsorption 
S

scale dependence

sorption

sorption sites

stream tube model

suction cup sampler

superposition

surface tension

symmetry

T

tailing

temperature

three dimensional point source

tortuosity

tracer

transfer function model

transfer function modeling

transfer functions

transverse

transverse dispersion

transverse dispersivity

V

viscosity

W

worm hole 


\section{The Gas Phase in Soils}

- 1 Introduction

- 2 Composition of the Soil Gaseous Phase

- 3 Mechanisms for Gas Transport in Soil

- 4 Basic issues of time (daily vs seasonal); space (micro-site; profile, lateral); and sources/sinks (vegetation and microbial actors)

- 4.1 Convective Transport of Soil Air

- 4.2 The Klinkenberg Effect

- 4.3 Diffusive Transport (JD)

- 5 Modeling Gas Transport in Soils

- 5.1 Steady State Gas Diffusion

- 5.2 O2 Diffusion to Plant Roots (Radial Flow)

- 5.3 Water Vapor Flow

- 5.3.1 Fick's law

- 5.3.2 Condensation and evaporation in soils

- 5.3.3 Philip and deVries enhancement effect

- 5.4 Measurements of Soil Gaseous Phase (Taylor and Ashcroft, 1972)

- 5.5 Soil Air Content

- 5.6 In-Situ Measurement of Gas Concentration and flux. Licor chambers, CO tracer gas

- 5.7 Mass Air Flow

- 6 SUMMARY

- 7 REVIEW QUESTIONS

- 8 PROBLEMS

- 9 REFERENCES

- 10 APPENDICES 


\section{Bio-Physics of the Vadose Zone}

\section{Introduction}

Most of our discussion so far has concerned the occurrence and movement of liquid water in vadose zones. In this chapter we turn our attention to the chemistry of these environments. Our objectives are to understand and develop predictive relations for the chemical composition of vadose pore water and its evolution in space and time. This can provide useful insights into the controls on shallow subsurface water quality and factors governing the subsurface transport and fate of specific chemicals. Ironically perhaps, vadose chemistry also offers some of the most promising means of addressing what is usually thought of as a physical problem: quantifying rates of water movement beneath soils.

We (following many others before us) have noted that hydrologically, the subsurface is a physical continuum from ground surface through soils, subsoils, and saturated zones. While properties and dynamics vary greatly in space and time, water occurrence and movement are physically connected and follow the same general "rules" throughout subsurface systems. It is less often stated, but equally true, that the subsurface is a chemical continuum, or, more specifically, a biological and geochemical continuum. This understanding guides our biogeochemical approach in this chapter, which is to explore biological and geochemical "space" in vadose zones, and to understand the links between the biotic and abiotic processes which yield the chemistry we observe.

In a number of important ways, vadose zones may be considered as vertically-extended soils. The "driving force", biologically, is usually the respiration and decay of plant roots which generate acids and sustain microbial communities. Abiotically, acids are consumed by dissolution of mineral material, the solutes are partitioned among water, gas, and solid (including biological) phases, and mass transport occurs in the fluid phases. The links between biotic and abiotic processes lie at the conceptual foundation of pedology: climate and character of the geologic material determine flow, fluid-rock interaction, and transport regimes within which plant and associated microbial communities develop; and this development modifies the original regimes over time. Some features of this linkage and its consequences for vadose hydrochemistry are summarized in Figure 8.1. 


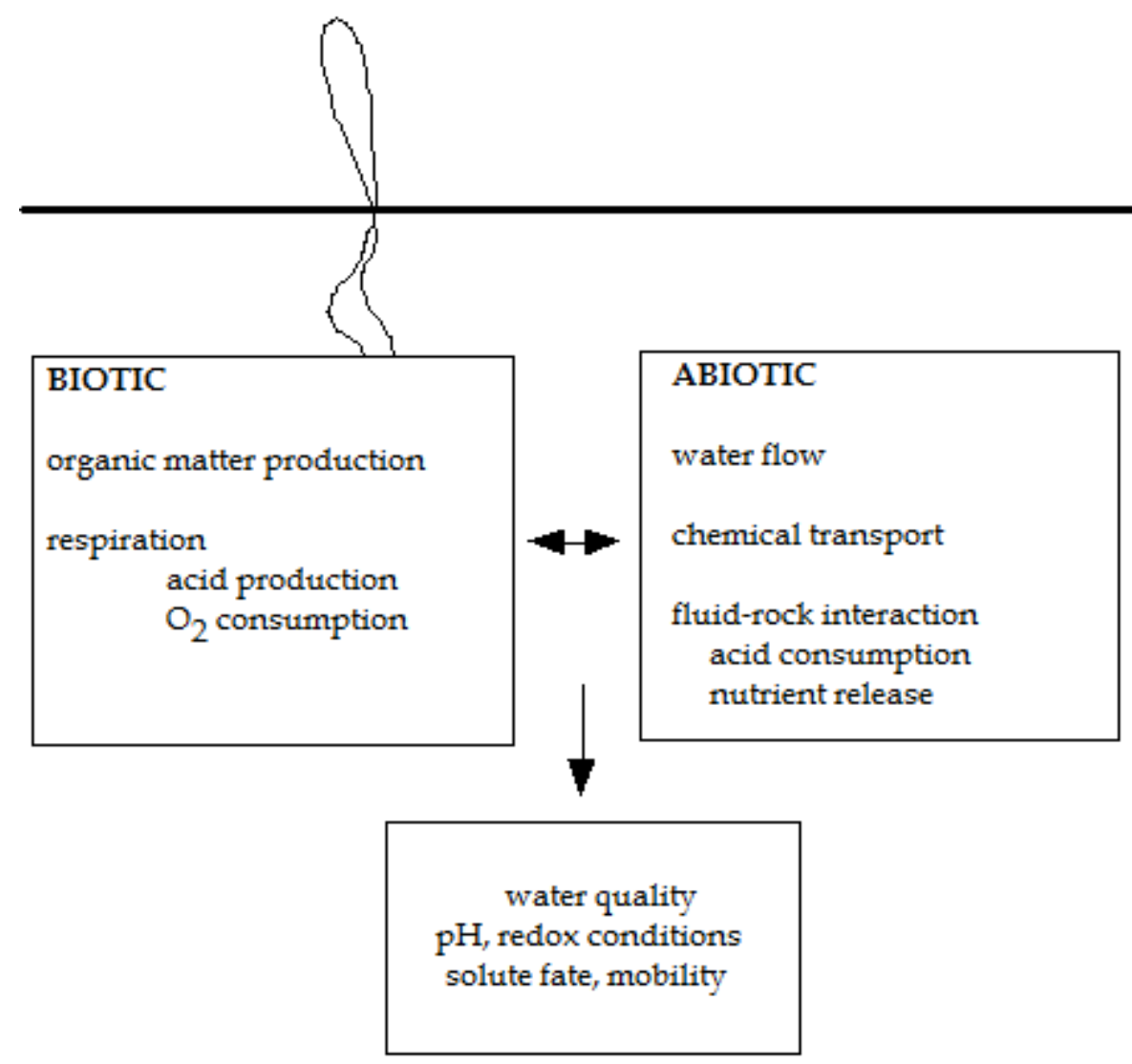

Figure 8.1 Interaction of biotic and abiotic processes to yield vadose hydrochemistry.

But large depth intervals of vadose zones are, in some important respects, biogeochemically more like groundwater zones than like soils. These intervals contain no living plant roots; any plant-derived organic matter present must be physically transported from above, during which time that organic matter ages and loses its capacity to serve as fuel for microbial activity. In these settings, geologic sources of organic matter may become relatively important as biological substrates and solute sorbents. Environmental fluctuations - in water flow, moisture content, and temperature are rapidly attenuated with depth, such that conditions are physically stable beneath depths of a few meters. Water residence times in vadose zones are frequently years to many thousands of years. In these environments the notion of biotic-abiotic linkage is still useful, as we will see; however, the commonality with groundwater environments means that the perspectives of groundwater geochemistry can also be useful.

In this chapter we will thus navigate a terrain that straddles soil chemistry and biochemistry, groundwater geochemistry, and the physical processes discussed in previous chapters. While frequent reference will be made to the soils and groundwater literatures, we make no attempt to fully present their perspectives and contributions; other excellent texts are available for this, and we will try to refer to them as appropriate. We will focus on concepts and research that apply to entire vadose zones, and thus we will omit coverage of large bodies of work in soil science. Also, as in the rest of this book, we will focus on clear illustration of key ideas at the expense of complete review. Our premise is that mastery of fundamentals positions the reader to tackle a wide range of practical problems. This results in a treatment that addresses several current issues (e.g. physical chemistry of non-aqueous phase liquids; transformation of synthetic organic compounds) only briefly.

Section 4.2 is a brief discussion of some fundamentals of physical chemistry as applied to the subsurface. Readers with minimal backgrounds in chemistry may want to read Appendix I in conjunction with this material, while other readers may skip it entirely. Then we will turn our attention to selected aspects of Figure 8.1. Section 4.3 will focus 
on biotic processes: the occurrence of organic matter, the geomicrobiology of vadose zones, the generation of acids and consumption of oxygen by microbial metabolism. Section 4.4 will focus on the abiotic processes of fluid-rock interaction which moderate the acidity generated by the biologic processes and give rise to the bulk hydrochemistries we observe in vadose porewaters and groundwaters. Section 4.5 concerns sorptive processes, geochemically important in pristine vadose zones and particularly crucial to understanding and predicting solute transport (chapter 5). Section 4.6 attempts,in first-order fashion, to apply the biogeochemical insights developed in this chapter to the vadose occurrence and fate of major categories of solutes of interest. Section 4.7 is a very brief review of how biogeochemical modeling is facilitated by widely-available computer codes. Section 4.8 discusses natural geochemical indicators of vadose flow and transport processes; widely-used approaches are compared with relatively new techniques utilizing simple measurements on vadose solids.

\section{Geochemical Fundamentals}

\section{Kinetics.}

Consider a schematic reaction:

(8.1) $\mathrm{aA}=\mathrm{bB}$

The symbols mean "a units of substance A react to form b units of substance B, and vice-versa". The unit coefficients $\mathrm{a}$ and $\mathrm{b}$, which indicate the relative proportions of the substances consumed and produced, are collectively referred to as the stoichiometry of the reaction. The substances A and B may be any entity or "species" with a definable chemical composition, including minerals, natural and artificial organic substances, and dissolved chemicals of all kinds.

Kinetics addresses the fundamental question "how fast does a reaction proceed, and what are the controls on this rate?" Sposito (1989, chap. 4) gives a cogent discussion which we will follow here. The observed rate of forward (left-to$\backslash$ right) reaction may be written with reference to [4.1] as:

$$
-\left(\frac{\mathrm{dA}}{\mathrm{dt}}\right)=\mathrm{r}_{\mathrm{f}}-\mathrm{r}_{\mathrm{r}}
$$

where $r_{f}$ and $r_{r}$ are forward and reverse reaction rates and $t$ is time. Typically, experiment shows that these rates are functions of the concentrations of the species participating in the forward and reverse reactions:

$$
-\left(\frac{\mathrm{d}(\mathrm{A})}{\mathrm{dt}}\right)=\mathrm{k}_{\mathrm{f}}(\mathrm{A})^{\alpha}+\mathrm{k}_{\mathrm{r}}(\mathrm{B})^{\beta}
$$

where the $\mathrm{k}$ parameters are rate constants $\left[\mathrm{t}^{-1}\right]$, the parentheses denote species concentrations, and $\alpha$ and $\beta$ are the orders of the reaction with respect to the associated species. The sum of these orders is referred to as the order of the reaction. If we consider reaction [4.1] under conditions in which the reverse reaction is negligible, we can separate variables and rewrite 4.3 as:

$$
\int\left(\frac{\mathrm{d}(\mathrm{A})}{(\mathrm{A})^{\mathrm{a}}}\right)=-\mathrm{kt}
$$

Given this framework, we can estimate kinetic parameters by analysing concentration vs. time data. Taking a graphical approach, for example, we can see by integrating the left side of [4.4] for various cases that a reaction that is zeroth order with respect to (A) exhibits linearity between (A) and $t$ with slope - $k$; first order with respect to (A) exhibits linearity between $\backslash \ln (\mathrm{A})$ and $t$ with slope $-\mathrm{k}$; second order with respect to (A) exhibits linearity between $1 /(\mathrm{A})$ and $t$ with slope $k$; 
and so on. Sposito (1989) presents a tabular summary of these relations and their respective half-lives, along with kinetic parameters for selected reactions and references to primary data sources.

The rates of many biologically-mediated reactions are proportional to substrate concentration up to some threshold, beyond which resources are sufficiently abundant that substrate concentration has no rate effect. These relations may be described by a hyperbolic "Michaelis-Menten" equation, in which the dependent (rate) variable reaches an asymptote:

$$
r=r_{\max }\left(\frac{(A)}{k_{m}+(A)}\right)
$$

Dimensional analysis shows that $\mathrm{k}_{\mathrm{m}}$, the Michaelis constant, has units of concentration; that concentration is defined as (A) for the condition $r=1 / 2 r_{\max }$. When (A) is much smaller than $k_{m}, r$ is directly proportional to (A), i.e. kinetics are first-order. Conversely when (A) is much larger than $k_{m}$, then $r=r_{\max }$ (the asymptote) regardless of (A), i.e. kinetics are zeroth-order. Paul and Clark (1985) note that [4.5] is called the Monod equation when used to describe microbial metabolism, and that experiments have shown that microbial decomposition of plant residues in soils is first-order. This implies that microbial growth in soils responds quickly to resource availability. We will see that this is not necessarily true for the vadose zone as a whole.

Geochemical reaction rates are generally sensitive to temperature according to the relation of Arrhenius (1889):

$$
\frac{\mathrm{dlnk}}{\mathrm{dT}}=\frac{\mathrm{E}_{\mathrm{a}}}{\mathrm{RT}^{2}}
$$

where $\mathrm{T}$ is absolute temperature (Kelvins), $\mathrm{E}_{\mathrm{a}}$ is the activation energy, and $\mathrm{R}$ is the gas constant. Now, [4.6] may be integrated and written as:

$$
\ln \mathrm{k}=\left(\frac{-\mathrm{E}_{\mathrm{a}}}{\mathrm{R}}\right)\left(\frac{1}{\mathrm{~T}}\right)+\mathrm{C}
$$

where $\mathrm{C}$ is a constant of integration. Experimental rate constants $\mathrm{k}$, obtained at different temperatures $\mathrm{T}$, may be plotted as $\backslash \mathrm{ln} k$ vs $1 / \mathrm{T}$ to obtain a straight line with slope $-E_{a} / R$. The activation energy thus obtained reflects the mechanism of the reaction. (Note that use of eq. (4.7) assumes a constant activation energy, which will only be true in practice over a limited temperature range within which the reaction mechanism does not vary.) Activation energies vary widely in geochemistry, probably less so within the "environmental" temperature range characteristic of vadose zones. Lasaga (1984) reports activation energies, for various common minerals dissolving abiotically in water, ranging from 30-80 $\mathrm{kJ} \mathrm{mol}^{-1}$. Paul and Clark (1985) report activation energies of this magnitude for microbial processes, but note that these values may depend strongly on other factors such as $\mathrm{pH}$. Unfortunately for our purposes, relatively little is known about the situation of most interest to us: minerals dissolving and precipitating in the context of, and mediated by, biological processes in soils and subsoils (e.g. Drever, 1994).

\section{Equilibrium}

Returning to [4.1], let us now consider the special case in which rates of forward and reverse reactions are equal. This case, called equilibrium, is of great geochemical importance for at least two reasons. First, many geochemical processes attain equilibrium quickly, relative to the timescale of geologic or anthropogenic perturbations, such that it is useful to be able to predict the equilibrium state. Second, even when equilibrium is attained slowly, it is useful to know what that state is because the reaction of interest will proceed (net conversion of reactants to products) in that direction. We begin our search for tools to predict equilibrium states by noting that the condition is dynamic at the molecular level but steady-state at the macroscopic level. This means that the time derivative in [4.2] is zero and we obtain, from [4.3]:

(8.8) $\mathrm{k}_{\mathrm{f}}(\mathrm{A})^{\alpha}=\mathrm{k}_{\mathrm{r}}(\mathrm{B})^{\beta}$ 
which we can write as:

$$
\frac{\mathrm{k}_{\mathrm{f}}}{\mathrm{k}_{\mathrm{r}}}=\frac{(\mathrm{B})^{\beta}}{(\mathrm{A})^{\alpha}}=\mathrm{k}_{\mathrm{cond}}
$$

where "cond" means conditional. This relation indicates that there is a number $\mathrm{K}_{\text {cond }}$ which represents (and can in principle be calculated as) a ratio of rate constants or, alternatively, a ratio of concentrations of reactants and products at equilibrium. Equations like 4.9 are of limited utility for predicting equilibrium states because the kinetic parameters, which are difficult to obtain in any case, are conditioned on the composition of the system - particularly, in our case, the amounts of all the species dissolved in a gas or aqueous solution.

Coefficients like $\mathrm{K}_{\text {cond }}$ - but which do not depend on solution composition, and therefore are much more generally useful predictors of equilibrium - can be obtained thermodynamically. We will illustrate the basis and use of these coefficients, called equilibrium constants, later in this section. First we recall two key thermodynamic relations for geochemical applications, and apply them to a simple but important problem. The relations, obtained in appendix I as [I.27] and [I.28], are:

$$
\begin{aligned}
& \Delta \mathrm{G}(\mathrm{P}, \mathrm{T})<0 \quad \text { (spontaneous process) } \\
& \Delta \mathrm{G}(\mathrm{P}, \mathrm{T})=0 \\
& \text { (equilibrium process) }
\end{aligned}
$$

These mean, in a geochemical context, that equilibrium is a condition in which the free energies $\mathrm{G}$ of reacting species are the same, such that transformation of one to another involves no free energy change, i.e. the free energy of the system is minimized. Conversely when a free energy difference exists, a reaction proceeds ("spontaneously") such that free energy is minimized. This definition holds and can be used when the total pressure on the system $\mathrm{P}$, and temperature of the system T, are constant at specified values. G, P, and T are variables of state or state functions whose values are independent of how a given state was achieved (the "path" to that state). The theoretical basis of these statements is developed in appendix. I.

Equilibrium between pure substances; standard states

Let us apply [4.10] and [4.11] to the problem of predicting conditions of equilibrium between liquid water and water vapor, a condition typical of vadose zones which was addressed in chap. 2. We can write:

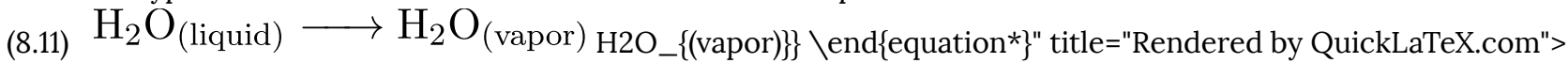
or, schematically after [4.1], as:

(8.12) $\mathrm{aA} \longrightarrow \mathrm{bB}$ bB $\} \backslash$ end $\{$ equation*\}" title="Rendered by QuickLaTeX.com"> or, schematically in Figure 8.2 as:

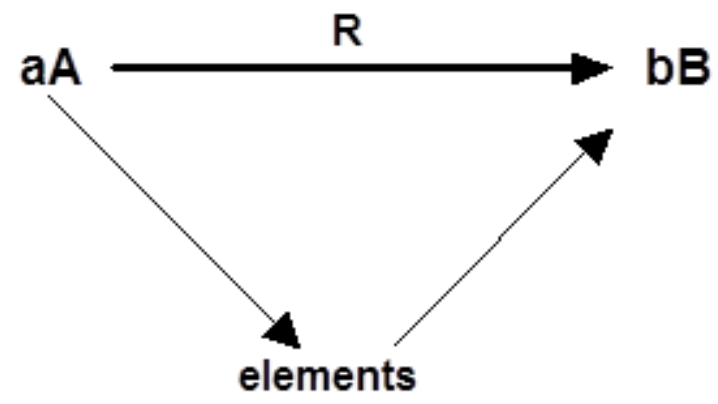

Figure 8.2 
To figure out whether [4.13] proceeds as written ([4.10] holds) or, alternatively, is at equilibrium ([4.11] holds), we must determine the free energy change $\Delta G_{R}$ associated with the reaction $R$. There are so many possible processes of geochemical interest that there is no way to catalog, let alone measure, $\Delta \mathrm{G}_{\mathrm{R}}$ for all these processes and so we must be clever. The Figure shows one alternate path from A to B: decomposition of species A (liquid water in our example) to the elements, followed by reconstitution of those elements to form species B (water vapor). Because $\mathrm{G}$ is a state function, its value depends only on initial and final states (not the path between those states) and we can write:

$\Delta \mathrm{G}_{\mathrm{R}}=\mathrm{b} \Delta \mathrm{G}_{\mathrm{fB}}-\mathrm{a} \Delta \mathrm{G}_{\mathrm{fA}}$

which may be generalized as:

$$
\Delta \mathrm{G}_{\mathrm{R}}=\sum \operatorname{products}_{\mathrm{i}} \Delta \mathrm{G}_{\mathrm{fi}}-\sum \operatorname{reactants} v_{\mathrm{i}} \Delta \mathrm{G}_{\mathrm{fi}}
$$

where " $\mathrm{f}$ " denotes a reaction of formation from constitutive elements - one of the diagonal paths in Figure I-2 - and $v_{i}$ are stoichiometric coefficients. The first term on the right-hand side is the $\mathrm{G}$ change associated with formation of the product; the second term is the $\mathrm{G}$ change for the decomposition of the reactant (algebraically, minus the formation of the reactant). The stoichiometric coefficients appear because $\mathrm{G}$ is extensive, i.e. its value is mass-dependent. The superscript "o" denotes geochemical "datum" or standard state, so the left-hand-side term is the standard free energy of reaction and the free energies on the right are standard free energies of formation. Standard states, like any other point of reference, are chosen for convenience. We will adopt a common standard state for environmental applications: total pressure $P_{\text {tot }}=1$ bar (very close to atmospheric pressure at Earth surface), $\mathrm{T}=298 \mathrm{~K}(25 \mathrm{C})$, solids are pure substances, gases are pure at $\mathrm{P}=1$ bar.

To see how this works, we will compute $\Delta \mathrm{G}^{\circ} \mathrm{R}$ for [4.12]:

$$
\Delta \mathrm{G}_{\mathrm{R}}=1 \Delta \mathrm{G}_{\mathrm{f} \text { (vapor) }}-1 \Delta \mathrm{G}_{\mathrm{f}(\text { liquid })}
$$

Nordstrom and Munoz (1994) present a concise complilation of $\Delta \mathrm{G}^{\circ}$ f data for many important species, employing the standard state we have chosen. (They also provide reference to additional, more extensive, data compilations.) From that list, we obtain for substitution in [4.15]:

$$
\Delta \mathrm{G}_{\mathrm{R}}=-228.58 \mathrm{kJmol}_{(\mathrm{vap})}^{-1}-\left(-237.14 \mathrm{kJmol}^{-1}\right)_{(\mathrm{liq})}
$$

or $\Delta \mathrm{G}_{\mathrm{R}}^{\circ}=8.56 \mathrm{~kJ} \mathrm{~mol}^{-1}$. Our result is non-zero, which means, with reference to [4.10] and [4.11], that liquid water does not coexist in equilibrium with pure water vapor at 1 bar pressure, at total pressure of 1 bar and $25 \mathrm{C}$. In fact, the positive sign of the result - reflecting the smaller $\Delta \mathrm{G}^{\circ} \mathrm{f}$ of the liquid, in this case -indicates that the reaction, as written, would proceed spontaneously in reverse: pure water vapor at 1 bar, having the greater free energy, would condense to form liquid water, thus minimizing the free energy. This is consistent with everyday experience, which also tells us that if we redid the calculations with free energy data for $\mathrm{T}=100 \mathrm{C}(398 \mathrm{~K})$ and other conditions the same, i.e. the "boiling point" of water, we should obtain $\Delta \mathrm{G}^{\circ} \mathrm{R}=0$.

So: can equilibrium exist in the vadose zone between water vapor and liquid water? We assumed equilibrium in section 2.3, to demonstrate how pressure differences across gas-water interfaces are related to interfacial curvature. In fact, the conditions in microscopic pore space do roughly correspond to the macroscopic experience of evaporation, high humidity, and condensation in places like closed greenhouses and indoor swimming pools. Here we have liquid-vapor equilibria at temperatures much less than boiling. How can this be, in light of the calculations just completed in [4.16]? The answer lies in the fact that in the vadose zone, greenhouse, and indoor swimming pool, the water vapor is one gas in a mixture of gases, each of which has a partial pressure which is only a fraction of the 1 bar total pressure. Thus, to describe this equilibrium, we must be able to quantify the free energy of a substance (water vapor, in this case) whose concentration varies from standard-state concentration (pure gas at $1 \mathrm{bar}$, in this case) in mixtures.

The equilibrium constant.

Our approach to this problem is to employ the chemical potential, which was introduced in Appendix I as a tool for handling the energy state of phases of variable composition. With respect to our example, the variable-composition 
phase is the vadose-zone gas phase or "atmosphere", and it is the chemical potential of water (vapor) in that phase which is of immediate interest. We begin by writing down the free energy of water vapor in differential form ([I.22]):

(8.16) $\mathrm{dG}=\mathrm{VdP}-\mathrm{nSdT}$

where $\mathrm{S}$ is the entropy ([1.7]). If $\mathrm{T}$ does not change during the reaction then:

(8.17) $\mathrm{dG}=\mathrm{VdP}$

where $\mathrm{P}$ is the partial pressure of the vapor. We introduce the partial molal volume $\mathrm{v}$ (OVERBAR) and reintroduce the partial molal free energy G (OVERBAR) or chemical potential $\mu$ :

$$
\overline{\mathrm{v}} \mathrm{i}=\left(\frac{\partial \mathrm{V}}{\partial \mathrm{n}_{\mathrm{i}}}\right)_{\mathrm{n}_{\mathrm{i}}, \mathrm{P}, \mathrm{T}} \overline{\mathrm{G}} \mathrm{i}=\mu_{\mathrm{i}}=\left(\frac{\partial \mathrm{G}}{\partial \mathrm{n}_{\mathrm{i}}}\right)_{\mathrm{n}_{\mathrm{i}}, \mathrm{P}, \mathrm{T}}
$$

where $\mathrm{n}$ is the mass, usually taken as number of moles, of the component $\mathrm{i}$. Now we can rewrite [4.18] for water vapor in terms of partial molal (per mole) quantities:

(8.19) $\mathrm{d} \mu=\mathrm{vdP}$

Note that [4.19] is precisely the same as [2.43], which was obtained in chap. 2 from a different form of [4.17] (see Appendix I for the relations between [4.19] and the antecedents to [2.43]). The ideal gas equation of state, PV $=\mathrm{nRT}$, expressed on a per mole basis and rearranged, is:

$$
\mathrm{v}=\frac{\mathrm{RT}}{\mathrm{P}}
$$

which we substitute into [4.19] to obtain

$$
\mathrm{d} \mu=\frac{\mathrm{RT}}{\mathrm{P}} \mathrm{dP}
$$

Integrating over the difference between the partial pressure of the vapor and some (arbitrary) standard-state pressure $\mathrm{P}^{\circ}$ we obtain:

$$
\mu=\mu+R T \ln \left(\frac{\mathrm{P}}{\mathrm{P}}\right)
$$

The pressure ratio is the activity of an ideal gas. All substances have activities which are likewise dimensionless, and whose numerical values likewise depend on the standard state in use. We see from this example that the activity is closely related to concentration (measured as pressure, in this case); it is useful to think of activity as the thermodynamically effective concentration. A more general expression is:

(8.23) $\mu_{\mathrm{i}}=\mu_{\mathrm{i}}+\mathrm{RT} \ln \mathrm{a}_{\mathrm{i}}$

where a is the activity of the ith component. Now [4.14] may be rewritten for states other than standard states (to accommodate variable composition), using the chemical potential, as:

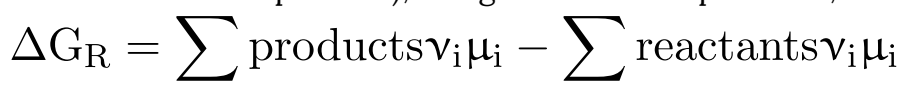

We can now rewrite the free energy change for reaction 4.13 by substituting [4.20] into [4.21]:

$$
\Delta \mathrm{G}_{\mathrm{R}}=\mathrm{b}\left(\mu_{\mathrm{B}}+\mathrm{RT} \ln \mathrm{a}_{\mathrm{B}}\right)-\mathrm{a}\left(\mu_{\mathrm{A}}+\mathrm{RT} \ln \mathrm{a}_{\mathrm{A}}\right)
$$

Rearranging:

$$
\Delta \mathrm{G}_{\mathrm{R}}=\mathrm{b} \mu_{\mathrm{B}}-\mathrm{a} \mu_{\mathrm{A}}+\mathrm{RT} \ln \left(\frac{\mathrm{a}_{\mathrm{B}}^{\mathrm{b}}}{\mathrm{a}_{\mathrm{A}}^{\mathrm{a}}}\right)
$$

The $\mu^{\circ}$ are equivalent to $\Delta \mathrm{G}^{\circ}$, so the first two terms on the right-hand side are the standard free energy of reaction $\Delta \mathrm{G}^{\circ} \mathrm{R}$ (see [4.14]), and we can write: 


$$
\Delta \mathrm{G}_{\mathrm{R}}=\Delta \mathrm{G}_{\mathrm{R}}+\mathrm{RT} \ln \left(\frac{\mathrm{a}_{\mathrm{B}}^{\mathrm{b}}}{\mathrm{a}_{\mathrm{A}}^{\mathrm{a}}}\right)
$$

which may be generalized (for all reactions and their stoichiometries) as:

$$
\Delta \mathrm{G}_{\mathrm{R}}=\Delta \mathrm{G}_{\mathrm{R}}+\mathrm{RT} \ln \Pi \mathrm{a}_{\mathrm{i}}^{v_{\mathrm{i}}}
$$

With this expression we can compute the free energy change, or free energy difference between products and reactants, at any stage of a reaction, not just for standard-state conditions (compare with [4.14]); a positive result indicates the reaction proceeds spontaneously in reverse as written (e.g. the liquid-vapor calculation in [4.16]) whereas a negative result indicates spontaneity in the forward direction. Problem 4 following appendix I provides an example. For the special case of equilibrium, substituting [4.11] into [4.23] gives:

\section{(8.29) $\Delta \mathrm{G}_{\mathrm{R}}=-\mathrm{RT} \ln \Pi \mathrm{a}_{\mathrm{i}}^{\nu_{\mathrm{i}}} \quad$ (equilibrium)}

The path-independence of free energies (of the standard-state reactants and products, in this case) means that the left-hand side has only one value for a given reaction. This means that:

$$
\Pi \mathrm{a}_{\mathrm{i}}^{v_{\mathrm{i}}}=\operatorname{constant}_{(\mathrm{P}, \mathrm{T})}=\mathrm{K}_{(\mathrm{P}, \mathrm{T})} \quad \text { (equilibrium) }
$$

where $\mathrm{K}_{(\mathrm{P}, \mathrm{T})}$ is the equilibrium constant. The subscripts $(\mathrm{P}, \mathrm{T})$ remind us of a crucial point, developed in appendix I and raised in [4.11], which is that our working definition of equilibrium requires specification of constant total pressure and temperature. Because these are variables of state, each different combination thereof represents a different energy state for the system of interest and therefore the values of free energies, standard free energies of reaction, and equilibrium constants vary accordingly.

Two consequences of [4.24] and [4.25] deserve special emphasis:

- at equilibrium the activity product ratio has a unique value at a given P,T. However the individual activities may have any of an infinite number of values. We can say that it is the overall balance between the activities of reactants and products which determines the equilibrium.

- at equilibrium the activity product ratio is uniquely valued (at a given $\mathrm{P}, \mathrm{T}$ ) irrespective of other species present in the system. This follows, as noted above, directly from the path-independence of the state function $\mathrm{G}$. The equilibrium constant is thus not conditioned on system composition as are the $\mathrm{K}_{\text {cond }}$ defined in [4.9].

These features of [4.24] and [4.25] mean that we can use tabulated standard-state thermodynamic data $\left(\Delta \mathrm{G}_{\mathrm{f}}^{\circ}\right)$ to compute standard free energies of reactions $\left(\Delta \mathrm{G}^{\circ}\right.$, e.g. [4.14]) and, thence, by rearranging [4.24], activity product ratios which must pertain at equilibrium:

$$
\exp \left(\frac{-\Delta \mathrm{G}_{\mathrm{R}}}{\mathrm{RT}}\right)=\Pi \mathrm{a}_{\mathrm{i}}^{v_{\mathrm{i}}}=\mathrm{K}_{(\mathrm{P}, \mathrm{T})} \quad \text { (equilibrium) }
$$

\section{Activity conventions.}

Before using [4.26] we must write down rules for quantifying activity in terms of the more generally measurable quantity concentration. Examination of [4.24] and [4.25] shows that these conventions depend, via $\Delta \mathrm{G}^{\circ} \mathrm{R}$, on our standard-state convention. (Wood and Fraser (1978) and Nordstrom and Munoz (1994, pp. 135-137) provide useful illustrations.) Given the choice we made above, appropriate activity conventions are:

- for water and minerals: $\mathrm{a}_{\mathrm{i}}=\mathrm{X}_{\mathrm{i}}$ where $\mathrm{X}_{\mathrm{i}}$ is the ratio of moles of $\mathrm{i}$ to total number of moles of the substance, or mole fraction of $\mathrm{i}$. When the water or mineral is pure or nearly so, $\mathrm{X}_{\mathrm{i}}$ may be taken as 1 . 
- for gases: $\mathrm{a}_{\mathrm{i}}=\mathrm{P} / \mathrm{P}^{\circ}$ where $\mathrm{P}$ is the partial pressure in bars and $\mathrm{P}^{\circ}$, the standard-state pressure, is 1 bar. Thus $\mathrm{a}_{\mathrm{i}}=\mathrm{P}$ numerically. This assumes ideal-gas behavior, which is reasonable for pressures less than 10 bars, i.e. for most environmental purposes.

- for aqueous solutes: $a_{i} \rightarrow m_{i}$ as $\Sigma m_{i} \rightarrow 0$ where $m$ is the molality of species $i$, defined as:

$$
\mathrm{m}_{\mathrm{i}}=\frac{\text { moles } \mathrm{i}}{\mathrm{kg} \mathrm{H}_{2} \mathrm{O}}
$$

This is referred to as the infinite dilution convention; it implies that activity is less directly proportional to concentration (molality), i.e. less ideal, as the bulk concentration of the solution increases. We will deal quantitatively with this nonideality later.

Henry's Law.

We now apply the fruits of our labors, [4.26], to the problem of equilibrium between water liquid and vapor in a vadose zone. The reaction is (from [4.12]):

$$
\mathrm{H}_{2} \mathrm{O}_{(\text {liquid })}=\mathrm{H}_{2} \mathrm{O}_{(\text {vapor })}
$$

For simplicity we choose total $\mathrm{P}$ and $\mathrm{T}$ equal to our standard state $(1 \mathrm{bar}, 25 \mathrm{C})$. We have already computed the standard free energy of reaction in [4.16]: $\Delta \mathrm{G}^{\circ} \mathrm{R}=8.56 \mathrm{~kJ} \mathrm{~mol}^{-1}$. With $\mathrm{R}=8.3143 \mathrm{~J} \mathrm{~mol}^{-1} \mathrm{~K}^{-1}$ and $\mathrm{T}=298 \mathrm{~K}$, we obtain from [4.26]:

$$
K_{(\mathrm{P}, \mathrm{T})}=\exp \left(\frac{-8,560 \mathrm{Jmol}^{-1}}{8.314 \mathrm{Jmol}^{-1} \mathrm{~K}^{-1}(298 \mathrm{~K})}\right)=\frac{\mathrm{a}_{\text {vapor }}}{\mathrm{a}_{\text {liquid }}}
$$

Doing the arithmetic we obtain:

$$
\mathrm{K}_{(\mathrm{P}, \mathrm{T})}=0.0316=\frac{\mathrm{a}_{\text {vapor }}}{\mathrm{a}_{\text {liquid }}}
$$

If the liquid is pure or nearly so, then $\mathrm{a}_{\text {liquid }}=1$ and $\mathrm{a}_{\mathrm{vapor}}=\mathrm{P}_{\mathrm{vapor}}=0.0316$ bar. This equilibrium partial pressure may be termed "saturation" of the atmosphere with respect to water vapor, in that liquid water precipitates out of the atmosphere (at the same rate as water vapor evaporates from the liquid).

Equation 4.27 is an example of Henry's law, which is simply the name given to the proportionality, at equilibrium, between gas- and aqueous-phase activities of a solute of interest. Henry's law appears in the scientific literature in many different forms, and often the distinction between activity and concentration is neglected, e.g.

(8.36) $\mathrm{P}_{\mathrm{i}}=\mathrm{H}_{\mathrm{i}} \mathrm{C}_{\mathrm{i}}$

where $\mathrm{P}$ is the partial pressure in the gas phase, $\mathrm{C}$ is the concentration in the aqueous phase, and $\mathrm{H}$ is the Henry's law constant for the phase partitioning of $\mathrm{i}$, corresponding to $\mathrm{K}$ in [4.27]. In the geochemical literature, Henry's-law expressions are often inverted with respect to [4.27], e.g.:

$$
K_{(P, T)}=\frac{a_{\text {liquid }}}{a_{\text {vapor }}}
$$

which corresponds to writing reaction 4.12 in reverse.

\section{Temperature and pressure dependence of equilibria.}

Equilibria like [4.27] are generally temperature-dependent. Returning to [4.17] we see that: 


$$
\left(\frac{\partial \mathrm{G}}{\partial \mathrm{T}}\right)_{\mathrm{P}}=-\mathrm{S}
$$

and, if we assume no change in total pressure on the system, we can write for a reaction

$$
\left(\frac{\mathrm{d} \Delta \mathrm{G}_{\mathrm{R}}}{\mathrm{dT}}\right)-\Delta \mathrm{S}_{\mathrm{R}}
$$

The free energy change for a reaction may be expressed as ([I.18]):

(8.40) $\Delta \mathrm{G}_{\mathrm{R}}=\Delta \mathrm{H}_{\mathrm{R}}-\mathrm{T} \Delta \mathrm{S}_{\mathrm{R}}$

where $\Delta \mathrm{H}_{\mathrm{R}}$ is the enthalpy change of the reaction ([I.15]). Substituting this into [4.29] and specifying equilibrium via [ 4.24] and [4.25] (e.g. Garrels and Christ, 1967, pp. 348-349), we obtain the van't Hoff equation which gives the variation of the equilibrium constant with temperature as a function of the standard enthalpy change of reaction:

$$
\frac{\mathrm{d} \operatorname{lnK}}{\mathrm{dT}}=\frac{\Delta \mathrm{H}_{\mathrm{R}}}{\mathrm{RT}^{2}}
$$

Separating variables, integrating with respect to temperature over an interval $\mathrm{T}^{-\mathrm{T}^{\circ}}$, and converting to common logarithms gives:

$$
\log \mathrm{K}_{\mathrm{T}}-\log \mathrm{K}_{\mathrm{T}}=\frac{-\Delta \mathrm{H}_{\mathrm{R}}}{2.303 \mathrm{R}}\left(\frac{1}{\mathrm{~T}}-\frac{1}{\mathrm{~T}}\right)
$$

The standard enthalpy change was assumed constant with respect to $\mathrm{T}$ in the integration of [4.31]. Enthalpies, like free energies and for the same reason, actually vary with $\mathrm{T}$; however for many reactions the variation of $\Delta \mathrm{H}^{\circ} \mathrm{R}$ within environmental $\mathrm{T}$ intervals is small enough that [4.32] may be used reliably to adjust $\mathrm{K}$ values from their values at standard-state $\left(25 \mathrm{C}\right.$ ) temperatures. (The variation of $\mathrm{H}$ with $\mathrm{T}$ is quantified by the state function heat capacity $\mathrm{C}_{\mathrm{P}}$, which may be used when necessary to include $\Delta \mathrm{H}^{\circ} \mathrm{R}$ in the integration of [4.32]; see Nordstrom and Munoz, 1994, sec. 3.10.) Use of [4.32] requires only $\Delta \mathrm{H}^{\circ} \mathrm{R}$ values, which are calculated from standard enthalpies of formation using an expression entirely analogous to [4.14] and obtained by virtue of path-independence in exactly the same way:

$$
\Delta \mathrm{H}_{\mathrm{R}}=\sum \operatorname{products}_{\mathrm{i}} \Delta \mathrm{H}_{\mathrm{fi}}=\sum \operatorname{reactants}_{\mathrm{i}} \Delta \mathrm{H}_{\mathrm{fi}}
$$

A problem at the end of this chapter explores the variation of $\mathrm{K}$ with $\mathrm{T}$ for the water liquid-vapor equilibrium.

Pressure dependence. To obtain [4.27] we assumed that the total pressure, i.e. the sum of the partial pressures of the gases comprising the gas phase as well as the pressure on the liquid water, was 1 bar. The attentive reader will have recalled that in the vadose zone, the pressure of liquid water is generally less than the total pressure of the coexisting gas phase due to surface tension acting on curved fluid interfaces (chap. 2, section 2.3.2). The vadose atmosphere is generally hydraulically well connected to the above-ground atmosphere, so the 1-bar total P assumption is reasonable for the gas phase and invalid, at least in principle, for the liquid phase. Let's now look at the practical effects of this for purposes of geochemical calculations.

Following the analysis of varying partial pressure in the gas phase, we can use [4.17] to describe varying pressure on the liquid phase, and we can rewrite [4.19] for the liquid:

(8.44) $\mathrm{d} \mu=\operatorname{vdP}$

We can integrate over some finite pressure interval - say standard-state Po to some smaller pressure $\mathrm{P}$ - to obtain:

$$
\Delta \mu=\mathrm{v}(\mathrm{P}-\mathrm{P})=\mu+\mathrm{RT} \ln \mathrm{a}_{\mathrm{P}}-\mu-\mathrm{RT} \ln \mathrm{a}_{\mathrm{P}} \textrm{ }
$$

where the outer equality holds by virtue of [4.20]. This can be rewritten as:

$$
\mathrm{v} \Delta \mathrm{P}=\operatorname{RT} \ln \left(\frac{\mathrm{a}_{\mathrm{P}}}{\mathrm{a}_{\mathrm{p}}}\right)
$$

or 


$$
\exp \left(\frac{v \Delta P}{R T}\right)=\frac{a_{P}}{a_{P}}
$$

(the reader should compare [4.40] with [2.52]!). Evaluating this for a few values of $\Delta \mathrm{P}$ pertinent to vadose conditions, with $\mathrm{T}=298 \mathrm{~K}, \mathrm{v}=0.018 \mathrm{~L} \mathrm{~mol}^{-1}$ (Nordstrom and Munoz, 1994, p. 126), $\mathrm{R}=0.0820597 \mathrm{~L} \mathrm{~atm} \mathrm{~mol}^{-1} \mathrm{~K}^{-1}, 1 \mathrm{bar}=0.9869 \mathrm{~atm}$ $=10.20 \mathrm{~m} \mathrm{H}_{2} \mathrm{O}$ (Table 2.2?), and noting that the standard-state activity is 1 , we obtain:

Table 8.1

\begin{tabular}{|c|c|c|c|}
\hline$\Delta \mathrm{P}$ (bar) & $\Delta \mathrm{P}\left(\mathrm{m} \mathrm{H}_{2} \mathrm{O}\right)$ & $\underline{a}_{p}$ & typical vadose condition \\
\hline-0.01 & -0.102 & 0.99999 & capillary fringe \\
\hline-0.1 & -1.02 & 0.99993 & near water table or humid climate \\
\hline-1.0 & -10.2 & 0.99927 & garden-variety conditions \\
\hline-10 & -102 & 0.99276 & permanent wilting point for plants \\
\hline-100 & -1020 & 0.92995 & extremely dry soils \\
\hline
\end{tabular}

We see from this analysis that the over the entire spectrum of moisture potentials in vadose zones, the variability of activity of liquid water due to pressure variation is less than $10 \%$; the variation is little more than $1 \%$ for most conditions of interest. (This observation can reliably be extended to all "condensed" phases, i.e. solids and liquids.) Therefore the assumption of 1-bar total pressure in the preceeding problem was wrong in principle, but in practice it caused negligible error.

\section{Mineral equilibria and speciation.}

Vadose pore water dissolves mineral material; this is the primary source of the solutes in subsurface and surface waters. In many cases, which we will address specifically in section 4.4 below, pore-water residence times are large relative to the time required for water-mineral equilibrium, and so an equilibrium approach is useful. Let's take as an example the dissolution of gypsum (hydrous calcium sulfate):

(8.48) $\mathrm{CaSO}_{4} \bullet{ }_{2} \mathrm{H}_{2} \mathrm{O}=\mathrm{Ca}^{2+}+\mathrm{SO}_{4}{ }^{2-}+2 \mathrm{H}_{2} \mathrm{O}$

This reaction says that when 1 mole of gypsum dissolves it releases 1 mole of the cation calcium with ionic charge of +2 , one mole of the anion sulfate with ionic charge -2 , and 2 moles of water from the crystal structure. If dissolution proceeds to equilibrium - which generally occurs, at environmental temperatures, in a matter of hours - then [4.26] pertains, and we can compute the equlibrium constant $\mathrm{K}_{\text {gyp }}$ from the standard free energy of reaction. Going to data tables - for this example those in Nordstrom and Munoz (1994, their appendix D), we obtain: 


$\begin{array}{ll}\Delta \mathrm{Go}_{\mathrm{f}(\mathrm{gyp})} & =-1797.36 \mathrm{~kJ} \mathrm{~mol}^{-1}(\mathrm{P}=1 \mathrm{bar}, \mathrm{T}=25 \mathrm{C}) \\ \Delta \mathrm{Go}_{\mathrm{f}\left(\mathrm{Ca}^{2+}\right)} & =-552.8 \\ \Delta \mathrm{Go}_{\left(\mathrm{SO}_{4}{ }^{2-}\right)} & =-744.0 \\ \Delta \mathrm{Go}_{\mathrm{f}\left(\mathrm{H}_{2} \mathrm{O}\right)} & =-237.14\end{array}$

Note that the data are reported with different numbers of decimal places, reflecting varying uncertainties. Nordstrom and Munoz (1994, chaps. 11-13) present excellent discussions of the effects of these uncertainties on equilibrium calculations, as well as how thermodynamic data are measured and reported in the first place. This material is highly recommended for readers who perform their own thermodynamic calculations. We simply note that some data are routinely obtained by difference from others (e.g. entropies can be computed from enthalpies and free energies, using [I.17]) such that experimental errors tend to be compounded, leading to disagreements among published sources which can be significant with respect to a given problem.

We can use the data in [4.41] to compute the standard free energy of reaction via [4.14], and, carefully observing the stoichiometric coefficients, we obtain $\Delta \mathrm{G}^{\circ} \mathrm{R}=26280 \mathrm{~J} \mathrm{~mol}^{-1}$. Then from [4.26] we compute Kgyp:

$$
\exp \left(\frac{-\Delta \mathrm{G}_{\mathrm{R}}}{\mathrm{RT}}\right)=10^{-4.61}=\mathrm{a}_{\mathrm{Ca}^{2+} \mathrm{a}_{\mathrm{SO}^{2-}}}=\mathrm{K}_{\mathrm{gyp}} \quad(1 \mathrm{bar}, 25 \mathrm{C})
$$

where we have assumed unity actitivies, i.e. purity, for water and gypsum so that they do not appear explicitly in the activity product ratio. The base-10 notation for the value of $\mathrm{K}_{\mathrm{gyp}}$ is conventional because of the convenience of manipulating quantities in the form of common logs, e.g.:

$$
\log \mathrm{K}_{\text {gyp }}=\log \mathrm{a}_{\mathrm{Ca}^{2+}}+\log \mathrm{a}_{\mathrm{SO}_{4}^{2-}}=-4.61
$$

This result tells us that a vadose water which is in equilibrium with gypsum - or a shallow groundwater which has equilibrated with gypsum in the overlying vadose zone - should exhibit an activity product of $10^{-4.61}$ (provided the temperature is not too different from $25 \mathrm{C}$ !) To put some numbers to this, we can assume the very simple system of water and gypsum only, such that $\mathrm{Ca}^{2+}$ and $\mathrm{SO}_{4}{ }^{2-}$ are equimolal in solution. Recalling our activity convention for aqueous solutes and assuming for the moment that $\mathrm{a}_{\mathrm{i}}=\mathrm{m}_{\mathrm{i}}$ (the solution is infinitely dilute), we get:

$$
\log \mathrm{m}_{\mathrm{Ca}^{2+}}=\log \mathrm{m}_{\mathrm{SO}_{4}^{2-}} \log \mathrm{a}_{\mathrm{Ca}^{2+}}=\log \mathrm{a}_{\mathrm{SO}_{4}^{2-}}=0.5(-4.61)=-2.31
$$

or $\mathrm{m}_{\mathrm{Ca}^{2+}}{ }^{2+} \mathrm{m}_{\mathrm{SO}}{ }^{2-}=0.0050$, which is $199 \mathrm{mg} \mathrm{L}^{-1}$ for calcium and $476 \mathrm{mg} \mathrm{L}^{-1}$ for sulfate ${ }^{1}$.

1. the relationship between molality and $\mathrm{mg} \mathrm{L}-1$ is:

$$
\mathrm{m}_{\mathrm{i}}=\frac{\mathrm{mg}}{\mathrm{L}}\left(\frac{\mathrm{g}}{1000 \mathrm{mg}}\right)\left(\frac{1}{\rho_{\mathrm{s}}}\right)\left(\frac{1}{\mathrm{GFW}}\right)
$$

where $\rho$ s is density of solution in $\mathrm{kg} \mathrm{H} 2 \mathrm{O}$ L-1, and GFW is grams solute per mole solute. Densities of 
We have just produced a prediction - albeit a crude one - of what we should see in vadose pore waters where gypsum is present in the geologic material. Let's now compare our prediction to observation. Following is the major-element chemistry of a water sample collected at the water table, just following its percolation through approximately $7 \mathrm{~m}$ of gypsiferous clayey till (Keller et al., 1991):

6.85

Table 8.2

\begin{tabular}{lrr}
\hline \multicolumn{1}{c}{ ion } & concentration $\left(\mathrm{mg} \mathrm{L}^{-1}\right)$ & concentration $(\mathrm{m})$ \\
$\mathrm{Ca} 2+$ & 501 & 0.0125 \\
$\mathrm{Mg} 2+$ & 382 & 0.0157 \\
$\mathrm{Na}+$ & 382 & 0.0166 \\
$\mathrm{HCO}-$ & 866 & 0.0142 \\
$\mathrm{SO} 42-$ & 2800 & 0.0292 \\
$\mathrm{pH}$ & & \\
$\mathrm{T}\left({ }^{\circ} \mathrm{C}\right)$ & & \\
\hline
\end{tabular}

Clearly $\mathrm{Ca}^{2+}$ and $\mathrm{SO}_{4}{ }^{2-}$ are not equimolal in solution; sulfate molality is more than twice that of calcium. This is inconsistent with our simple model, but it can be explained by the presence of other minerals in the till which also affect calcium and sulfate concentrations. Even so, because the the path-independence of state functions as embodied in [4.25], water in equilibrium with gypsum should exhibit an activity product equal to the equilibrium constant Kgyp. We can check this by again assuming $\mathrm{a}_{\mathrm{i}}=\mathrm{m}_{\mathrm{i}}$ and computing:

$$
\log \mathrm{m}_{\mathrm{Ca}^{2+}}+\log \mathrm{m}_{\mathrm{SO}_{4}^{2-}}=-1.90-1.53=-3.43
$$

This result is more than an order of magnitude greater than the equilibrium value ([4.44]). The temperature of the water sample is $20^{\circ} \mathrm{C}$ less than our standard state, but correction of mineral solubility products using [4.32] over such temperature ranges only yields differences up to $50 \%$ or so - not a factor of ten. To adequately explain the discrepancy between [4.44] and [4.45], and the inadequacy of our simple model, we must take three brief but important detours.

Activity coefficients. As noted in passing above, the activity of an ion in solution is directly proportional to its concentration only in the limit of its "infinite dilution". Departure from the proportionality is due to the solutesolute and solute-water interactions which increasingly affect the free energies of solute molecules at increasing bulk concentrations. This departure is quantified by the ionic activity coefficient $\gamma_{\mathrm{i}}$, where $\mathrm{a}_{\mathrm{i}}=\mathrm{m}_{\mathrm{i}} \gamma_{\mathrm{i}}$, and it follows from the infinite dilution convention that $\gamma_{i} \rightarrow 1$ as $\Sigma \mathrm{m}_{\mathrm{i}} \rightarrow 0$. A great deal of theoretical and experimental work has gone into formulating the ionic activity coefficient in terms of solution properties. Here we mention two of the most commonly used relations:

aqueous solutions differ negligibly from $1 \mathrm{~kg} \mathrm{H} 2 \mathrm{O}$ L-1 for total dissolved solutes (TDS) concentrations up to about 7,000 $\mathrm{mg} \mathrm{L-1}$, such that for lesser TDS , the approximation $\mathrm{mg} \mathrm{L-1}=$ ppm is acceptable:

$$
\frac{\mathrm{mg}_{\text {solute }}}{\mathrm{L}_{\text {water }}}\left(\frac{\mathrm{L}}{10^{6} \mathrm{mg}}\right)=\frac{\mathrm{mg}_{\text {solute }}}{10^{6} \mathrm{mg}_{\text {water }}}=\mathrm{ppm}
$$




$$
\log \gamma_{i}=\frac{-A z_{i}^{2} \sqrt{I}}{1+B a_{\circ} \sqrt{I}}
$$

$\log \gamma_{i}=\frac{-A z_{i}^{2} \sqrt{I}}{1+B a_{\circ} \sqrt{I}}+b I$

where $\mathrm{A}$ and $\mathrm{B}$ are constants dependent on $\mathrm{P}$ and $\mathrm{T}, \mathrm{b}$ is a constant whose value depends on the solute species of interest, $\mathrm{z}_{\mathrm{i}}$ is the ionic charge and $\mathrm{a}_{\mathrm{o}}$ is the hydrated radius of that species, and $\mathrm{I}$ is the ionic strength defined as:

$$
\mathrm{I}=\frac{1}{2} \sum \mathrm{m}_{\mathrm{i}} \mathrm{z}_{\mathrm{i}}^{2}
$$

Equations [4.46] and [4.47] are called "Debye-Huckel" and "extended Debye-Huckel" respectively (Truesdell and Jones, 1974) after the authors of the electrostatic theory on which they are based. Both expressions satisfy the infinite dilution convention: if I $=0, \gamma_{\mathrm{i}}=1$. In [4.46], $\gamma_{\mathrm{i}}$ declines from 1 as I increases; this behavior reflects the decline of the activity of an individual ion associated with increasing electrostatic interactions with its neighbors. The same behavior holds for [4.47] except at high concentrations, for which the positive value of the second term becomes relatively large. DebyeHuckel is considered to accurately quantify activity coefficients for these ions with charges of magnitude one or two, at ionic strengths up to about 0.1, whereas extended Debye-Huckel is adequate for many purposes for these ions to ionic strengths at least 7 times larger (e.g. seawater). This range in ionic strength probably includes most vadose waters, but the data basis for this statement is very small. Some pore waters in arid environments will undoubtedly exceed ionic strengths of 1.0; for these and greater bulk concentrations, other theory is available which better accounts for the complexity of solute-solute interactions. Also, neither Debye-Huckel nor extended Debye-Huckel can be used for trivalent ions for ionic strengths greater than approximately 0.01. Drever (1997, chap. 2) provides a brief but useful discussion.

We can now see that non-zero ionic strength depresses activities below their molalities; this should bring the activity product for gypsum, for the water in Table 8.2, more in line with its equilibrium value. Let's see: the ionic strength, after [4.48], is:

$$
\begin{gathered}
\mathrm{I}=0.5\left[0.0125(2)^{2}+0.0157(2)^{2}+0.0166(1)^{2}+0.0142(-1)^{2}+0.0292(-2)^{2}\right] \\
=0.130
\end{gathered}
$$

At this ionic strength, we should use [4.47]. Values of A and B are temperature dependent; these and the remaining required parameters are given in Table 8.3 .

Table 8.3 (after Drever, 1997, Table 2-1)

\begin{tabular}{llllll}
\hline$\underline{\text { Ion }}$ & $\underline{\text { ao }}$ & $\underline{\mathrm{b}}$ & $\underline{\mathrm{T}}(\mathrm{C})$ & $\underline{\mathrm{A}}$ & $\underline{\mathrm{B}}$ \\
$\mathrm{Ca}^{2+}$ & 5.0 & 0.165 & 5 & 0.4883 & 0.3241 \\
$\mathrm{Mg}^{2+}$ & 5.5 & 0.20 & 15 & 0.5000 & 0.3262 \\
$\mathrm{Na}^{+}$ & 4.0 & 0.075 & 25 & 0.5085 & 0.3281 \\
$\mathrm{~K}^{+}, \mathrm{Cl}^{-}$ & 3.5 & 0.015 & & & \\
$\mathrm{HCO}_{3}^{-}$ & 5.4 & 0.0 & & & \\
$\mathrm{SO}_{2}-$ & 5.0 & -0.04 & & & \\
$\mathrm{H}^{+}$ & 9.0 & & & & \\
\hline
\end{tabular}


The activity coefficients for calcium and sulfate are then (from [4.47]) $\gamma \mathrm{Ca2+}=0.375$ and $\gamma \mathrm{SO} 42^{-}=0.353$. The activities $\mathrm{a}_{\mathrm{i}}=$ $\mathrm{m}_{\mathrm{i}} \gamma_{\mathrm{i}}$ are then 0.00469 and 0.0103 respectively - depressed to less than $40 \%$ of the concentrations! - and the recomputed $\log$ ion activity product is:

$$
\log \mathrm{a}_{\mathrm{Ca}^{2+}}+\log \mathrm{a}_{\mathrm{SO}_{4}^{2-}}=-2.33-1.99=-4.32
$$

This result is a big improvement over [4.45], but it is still larger than it should be if the water is indeed in equilibrium with gypsum ([4.44]).

Complexes.

Another fact of life in solution chemistry is that ionic solutes associate with one another, in solution, to form complexes. This means that not all of a given element in solution (known from a water analysis like that reported in Table 8.2) exists in its "free" form, e.g. as $\mathrm{Ca}^{2+}$; some of the calcium ion will be "tied up" in complexes with other ions called ligands. Complexes may be thought of as either ion pairs or coordination compounds. The former are also called outer-sphere complexes because the water molecules electrostatically associated with the ion of interest, called its hydration sheath, are retained. The latter are called inner-sphere complexes because the ionligand bonding is stronger and water of hydration is moved aside. Complexes involving ligands with more than one site available for binding to the same metal ion are called chelates. Drever (1997, chap. 2) provides a brief discussion with examples; Stumm and Morgan (1996, chap. 6) give examples using somewhat different terminology. For our purposes, the differences among complex-formation processes result in a wide range of stabilities, which can be expressed as a stability constant:

$$
\mathrm{M}^{+}+\mathrm{L}^{-} \leftrightarrow \mathrm{ML} \quad \text { where } \quad \mathrm{K}_{\text {stab }}=\frac{\mathrm{a}_{\mathrm{ML}}}{\mathrm{a}_{\mathrm{M}^{+}} \mathrm{a}_{\mathrm{L}^{-}}}
$$

where $\mathrm{M}^{+}$is a cation and $\mathrm{L}^{-}$is a ligand, and the charges on all three species may vary. The use of an equilibrium relation to describe the existence of ionic complexes is valid because the kinetics of their formation are generally rapid relative to other processes of interest. Values of stability constants may be calculated from $\Delta \mathrm{G}_{\mathrm{f}}^{\circ}$ data via [4.26]; they are commonly reported directly in data tabulations as $\log \mathrm{K}_{\text {stab. }}$.

We can now see that the actual activities of $\mathrm{Ca}^{2+}$ and $\mathrm{SO}_{4}{ }^{2-}$ will be depressed even further, relative to our calculation in [4.49], to the extent that complexes involving these two ions form. Perusal of Nordstrom and Munoz (1994, app. D) shows that standard-state $\log \mathrm{Kstab}$ values for the complexes $\mathrm{CaSO}_{4}{ }^{\circ}, \mathrm{CaCO}^{\circ}, \mathrm{CaHCO}_{3-}$, and $\mathrm{MgSO}_{4}{ }^{\circ}$ are $2.30,3.22,1.18$, and 2.37 respectively; the large activity product ratios for these equilibria mean that substantial ionic mass is indeed "tied up" in these complexes. Can we quantify this effect? We could, using the activities of $\mathrm{Ca}^{2+}$ and $\mathrm{SO}_{4}{ }^{2-}$ appearing in [4.49], compute the activity of each complex from its stability constant [4.50]; and then compute the molality of each complex

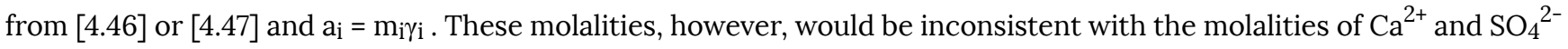
used in the calculations of their activities (which assumed all of each ion was "free"). We can see from this example that determining the distribution of species in a water with multiple ionic solutes requires simultaneous consideration of the total mass of each ion and all the equilibria affecting its distribution among various complexes.

\section{Computing distribution of species ("speciation”)}

The speciation problem is solved as a system of simultaneous equations. For each element (e.g. Ca) we can write a mass balance:

$$
\mathrm{m}_{\mathrm{Ca} \text { total }}=\mathrm{m}_{\mathrm{Ca}^{2+}}+\mathrm{m}_{\mathrm{Ca} 1}+\mathrm{m}_{\mathrm{Ca} 2}+\ldots \mathrm{m}_{\mathrm{CaN}}
$$

where $\mathrm{Ca} 1, \mathrm{Ca} 2, \ldots \mathrm{CaN}$ are complexes of $\mathrm{Ca}^{2+}$. Then for each of those complexes we can write an equilibrium: 


$$
\begin{aligned}
\mathrm{K}_{\mathrm{stab}_{\mathrm{Ca} 1}} & =\frac{\mathrm{m}_{\mathrm{Ca} 1} \gamma_{\mathrm{Ca} 1}}{\mathrm{~m}_{\mathrm{Ca}^{2}}+\gamma_{\mathrm{Ca}^{2}+\mathrm{m}_{1^{2}} \gamma_{1^{2-}}}} \\
\mathrm{K}_{\mathrm{stab}_{\mathrm{C} 2}} & =\frac{\mathrm{m}_{\mathrm{Ca} 2} \gamma_{\mathrm{Ca} 2}}{\mathrm{~m}_{\mathrm{Ca}^{2}+} \gamma_{\mathrm{Ca}^{2}} \mathrm{~m}_{2^{2-}} \gamma_{2^{2-}}}
\end{aligned} \ldots
$$

Now [4.52] represents $\mathrm{N}$ equations (for $\mathrm{N}$ complexes) which, when added to [4.51], gives $\mathrm{N}+1$ equations in total. Considering the unknowns: $m_{\text {Catotal }}$ is known from the water analysis (e.g. Table 8.2) and the stability constants can be computed or looked up. If we assume for the moment that activities equal molalities, i.e. $\gamma_{\mathrm{i}}=1$, then we have $\mathrm{N}+1$ unknowns (the molalities of all the terms on the right-hand sides of [4.51] and [4.52]). As thus constituted the system can then be solved simultaneously. There exists such a system for each element considered, such that the full system for simultaneous solution is large but determinate in principle.

We know that the assumption $\mathrm{a}_{\mathrm{i}}=\mathrm{m}_{\mathrm{i}}$ does not hold for many waters, certainly not for the water represented in Table 8.2 where $\gamma$ for $\mathrm{Ca}^{2+}$ and $\mathrm{SO}_{4}{ }^{2-}$ appear to be on the order of 0.4 . We get around this difficulty using an iterative scheme:

step 1: compute I ([4.48]) using $\mathrm{m}_{\mathrm{i}}{ }^{\circ}$ directly from a laboratory analysis (e.g. Table 8.2 ), where "o" denotes initial value

step 2: compute $\gamma_{\mathrm{i}}{ }^{\circ}$ for all free ions ([4.46] or [4.47])

step 3: write the systems of equations for each element ([4.51] and 4.52) including the non-unity $\gamma_{\mathrm{i}}{ }^{\circ}$ computed in step 2

step 4: solve the full system for $\mathrm{m}_{\mathrm{i}}^{1}$

step 5: compare $\mid \mathrm{m}_{\mathrm{i}} 1-\mathrm{m}_{\mathrm{i}}$ 粮 to a convergence criterion; STOP or

step 6: recompute I using $\mathrm{m}_{\mathrm{i}}^{1}$ and iterate to step 2.

After the step 4 of the first cycle, nonzero $m_{i}$ will exist for all free ions and complexes of interest, such that step 2 is performed for every species, including complexes, upon iteration. The classic paper describing and documenting this scheme (and its implementation in the computer code WATEQ) was published by Truesdell and Jones (1974). Numerous extensions of the original data base, i.e. the list of species and their complexes along with stability constants (e.g. WATEQ4F, Ball and Nordstrom, 1991) constitute the WATEQ "family" of speciation programs. All data bases include $\Delta \mathrm{H}^{\circ} \mathrm{R}$ data for complex formation reactions so that the stability constants may be adjusted for non-standard-state water sample temperatures ([4.32]) from 0-30 ${ }^{\circ} \mathrm{C}$. Many other computer codes with similar capabilities exist; see Nordstrom et al. $(1979,1990)$ for reviews.

Speciation thus simultaneously accounts for the effects of complex formation and solution nonideality in the determination of species activity coefficients and activities. Speciating the water of Table 8.2 using WATEQ4F (Ball and Nordstrom, 1991) yields the following:

$$
\text { Species } \mathrm{m} \quad \gamma \quad \mathrm{a}=\mathrm{m} \gamma
$$

Taking the ion activity product for gypsum, we obtain:

$$
\mathrm{a}_{\mathrm{Ca}^{2+}} \mathrm{a}_{\mathrm{SO}_{4}^{2-}}=10^{-4.61}
$$

or

$$
\log \mathrm{a}_{\mathrm{Ca}^{2+}}+\log \mathrm{a}_{\mathrm{SO}_{4}^{2-}}=-4.61
$$

which is very close to the equilibrium value ([4.43] and [4.44]), as expected given the confirmed presence of gypsum in the vadose zone this water comes from. We characterize this condition as saturation, defined and quantified in terms of the saturation index SI:

$$
\mathrm{SI}=\frac{\mathrm{IAP}}{\mathrm{K}_{\mathrm{sp}}}
$$

or 
Species $\quad \begin{aligned} & \gamma \\ & \mathrm{a}\end{aligned}=\mathrm{m} \gamma$

where IAP is the ion activity product and $\mathrm{K}_{\mathrm{sp}}$ is the dissolution equilibrium constant or solubility product for the mineral of interest. (We note here that IAP and $\mathrm{K}_{\mathrm{sp}}$ always have an identical form - in our present example, the former is [4.53] while the latter is [4.43] - but that IAP is computed for a water of interest, while $\mathrm{K}_{\mathrm{sp}}$ is a path- and solutionindependent value describing equilibrium at given $\mathrm{P}$ and T.) In our case we find from [4.55] and [4.43], and [4.56] and [4.44], that $\operatorname{SI}_{\text {gyp }}=1.0$ and $\log \operatorname{SI}_{\text {gyp }}=0.0$. These are quantitative expressions of saturation with respect to gypsum. As a rough generality, we consider the ranges $0.7<\mathrm{SI}<1.3$ and $-0.1<\operatorname{logSI}<0.1$ to indicate saturation; the ranges allow for unavoidable errors introduced by sampling procedures, sample handling, and laboratory analysis, all of which affect IAP; and (last but not least!) errors in the thermodynamic data affecting $\mathrm{K}_{\mathrm{sp}}$. Speciation programs such as the WATEQ family contain $\mathrm{K}_{\mathrm{sp}}$ in their data bases; they automatically compute and report logSI for all such minerals for which a logIAP can be calculated.

If we did not have detailed lithologic descriptions of geologic material available to us the way Keller et al. (1991) did, and we didn't have field confirmation of the presence of gypsum, we could reasonably infer from the the speciation of the Table 8.2 water that this water had "seen" gypsum along its flow path. Such gypsum could, in principle, be primary i.e. deposited with the geologic material, or secondary, i.e. formed in situ in one among the linked vadose processes of biogenic $\mathrm{CO}_{2}$ production, oxidation, and/or evaporation. If, for example, geologic insight tells us that gypsum cannot be primary in a certain deposit, then the gypsum-saturation result of the speciation exercise alone provides the beginning of a conceptual model of biogeochemical processes in that vadose zone. This is not to suggest that there is any substitute for direct field observation, particularly lithologic description. Furthermore, this kind of reasoning must be applied with caution because minerals vary widely in the rates at which they attain saturation in vadose waters, such that observed undersaturation may simply mean that vadose pore water residence time is inadequate for attainment of equilibrium. These issues are explored in more detail below.

\section{Biological Processes}

\section{Overview.}

Biologically, the vadose zone is fundamentally like the rest of the terrestrial environment: all of its aspects depend on primary production by plants which fix carbon from the atmosphere via photosynthesis:

(8.64) $\left.\mathrm{CO}_{2}+\mathrm{H}_{2} \mathrm{O} \longrightarrow \mathrm{CH}_{2} \mathrm{O}+\mathrm{O}_{2} \mathrm{CH} 2 \mathrm{O}+\mathrm{O} 2\right\} \backslash$ end $\{$ equation*\}" title="Rendered by QuickLaTeX.com">

The term $\mathrm{CH}_{2} \mathrm{O}$, the simplest carbohydrate, is useful geochemical shorthand for generic plant tissue; actual plant materials contain substantial nitrogen as well as a large number of minor and trace elements. Photosynthesis fixes solar energy in the chemical bonds of the plant tissue, i.e. $\Delta \mathrm{G}^{\circ} \mathrm{R}$ of reaction 4.57 is positive. The terrestrial carbon cycle vadose and otherwise - is completed by the multitude of life processes which extract the chemical energy in the plant materials for their own metabolic needs via respiration:

(8.65) $\left.\mathrm{CH}_{2} \mathrm{O}+\mathrm{O}_{2} \longrightarrow \mathrm{CO}_{2}+\mathrm{H}_{2} \mathrm{O} \mathrm{CO} 2+\mathrm{H} 2 \mathrm{O}\right\} \backslash$ end $\{$ equation*\}" title="Rendered by QuickLaTeX.com">

Application of equation 4.14 shows that, as we would intuitively expect, $\Delta \mathrm{G}^{\circ}{ }_{\mathrm{R}}$ for reaction 4.58 is equal in magnitude and opposite in sign to reaction 4.57. If respiration balances photosynthesis, the reservoirs of organic matter, oxygen, carbon dioxide, water, and solar energy in chemical bonds remain unchanged by the combined processes, and an environmental steady state exists. In the grand scheme of things, the geologic record shows that this steady state has existed on a global scale for at least 2 billion years; our ancestors could not have arisen and evolved in an oceanatmosphere system which did not have approximately the same bulk chemistry as today's (Holland, 1978). Of course, a vast body of work in the life and earth sciences shows that spatially and temporally local fluctuations about this steady state are the norm.

In vadose zones, reaction 4.57 proceeds essentially at the upper boundary. From that boundary, plant material is 
translocated (by processes such as plant rooting, animal burrowing, and transport in solution) into a lightless domain where reaction 4.58 takes over. In this domain, reaction 4.58 is actually the sum of sub-reactions which accomplish parts of the overall transformation: plant tissue is oxidized to $\mathrm{CO}_{2}$ in a series of steps, in which reaction products become the reactants for the next step, by linked communities of microorganisms each of which extracts part of the overall free energy and carbon needed for growth. (Such organisms, like ourselves, are termed chemoheterotrophs; phototrophs obtain energy from the sun, while lithotrophs obtain carbon from an inorganic source, so green plants are photolithotrophs.) Some of the individual steps may be fermentative rather than respirative, e.g.:

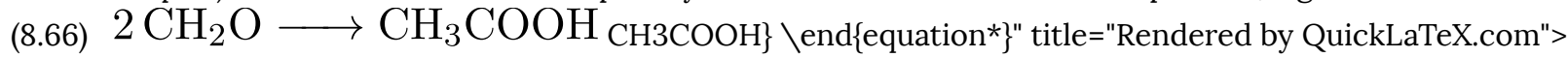

in which case no oxygen or other inorganic oxidant is involved; however the fermentation products are available for subsequent oxidation, e.g.:

(8.67) $\left.\mathrm{CH}_{3} \mathrm{COOH}+2 \mathrm{O}_{2} \longrightarrow 2 \mathrm{CO}_{2}+2 \mathrm{H}_{2} \mathrm{O} 2 \mathrm{CO} 2+2 \mathrm{H} 2 \mathrm{O}\right\} \backslash$ end\{equation*\}" title="Rendered by QuickLaTeX.com">

such that the overall result, summing [4.59] and[ 4.60], is respiration.

In the solum, this sequence can proceed very rapidly: plant material is transformed to $\mathrm{CO}_{2}$ within weeks to months as the most labile and soluble or "juiciest" plant tissue compounds (e.g. carbohydrates and low-molecular-weight organic acids) are "skimmed" by fast-acting microbes. At the same time, though, more refractory - less labile and less soluble - tissues (e.g. lignins) tend to accumulate, as do certain reaction intermediates (e.g. humic substances). From the agronomic perspective, this is the accumulation of soil organic matter essential to fertility. The essential point for us, considering the vadose system as a whole, is that as downward transport of organic matter proceeds, the proportion of labile to refractory compounds decreases with time and depth. Thus the solum is characterized by high levels biological activity and reaction-intermediate production, fueled by labile goodies, while the subsolum is characterized by activity levels which fall off with depth, fueled by refractory table scraps from the overlying solum (Figure 8.3).

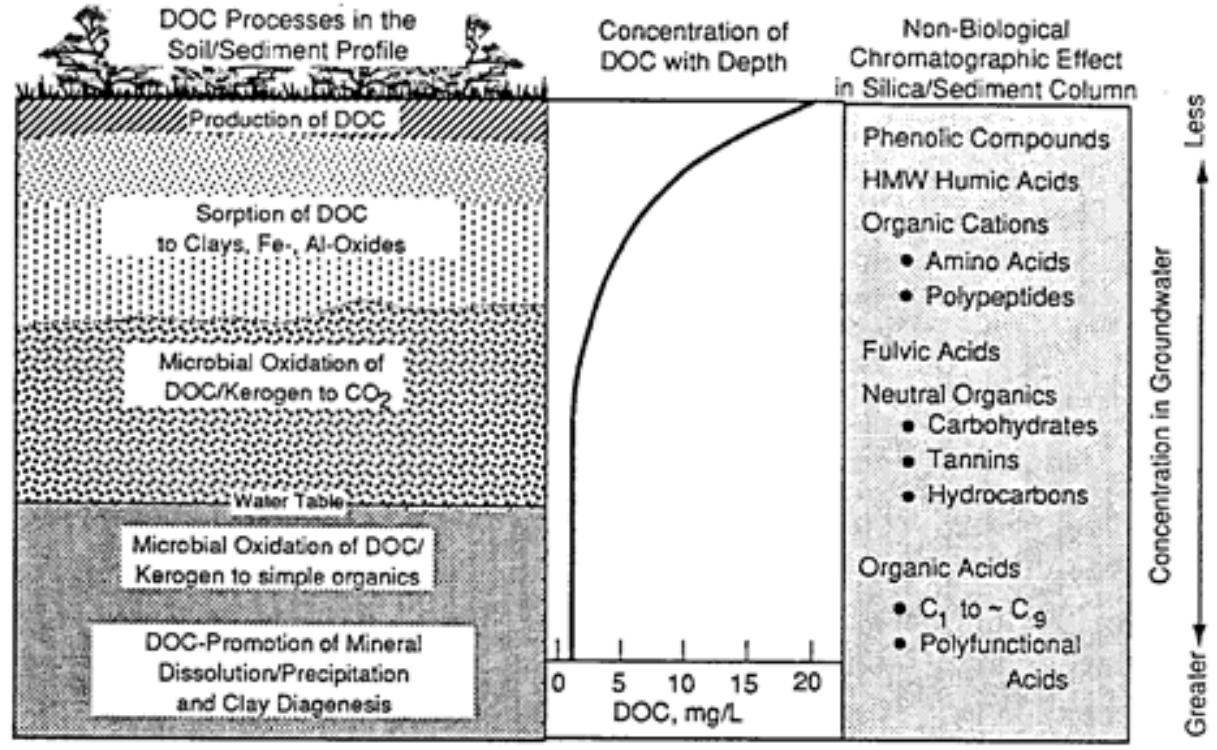

Figure 8.3 Processes affecting concentration and lability of dissolved organic carbon (DOC) with increasing depth and transport distance from ground surface. (After Murphy and Aiken, in prep; permission to be requested)

\section{Vadose geomicrobiology.}

The approach and techniques for study of life in the vadose zone have been borrowed largely from environmental and soil microbiology (e.g. Paul and Clark, 1989). In many decades of soil-microbiologic studies, workers have shown by 
direct microscopic observation and culture/incubation techniques that sola are populated primarily by (from mostto least-numerous) bacteria, fungi, and protozoa. The bacteria are single-celled prokaryotes, while the fungi are eukaryotic; the protozoa are single-celled animals. The species in each of these groups are believed to be dominantly chemoheterotropic, feeding in many cases on each other as well as on plant tissue and reaction intermediates. The qualifier "believed to be" is necessary because laboratory cultures inevitably select for and thus bias observations toward certain kinds of organisms at the expense of others.

Application of soil-microbiologic techniques to saturated-zone materials reveals largely bacteria whose numbers are orders of magnitude smaller than in the solum. In saturated zones the "higher" life forms, fungi and protozoa, recede into apparent numerical insignificance (Chapelle, 1993). This may be due to the relatively greater propensity of bacteria to be transported via porous media flow, the greater capability of bacteria to survive under conditions of nearstarvation, or some combination of both. Applying this line of reasoning to the vadose zone, we would expect to find transitional conditions. Indeed, bacterial populations appear to decline rapidly beneath the solum such that saturatedzone population levels are achieved within a few meters of ground surface (Figure 8.4; Balkwill and Ghiorse, 1985). 
(a)

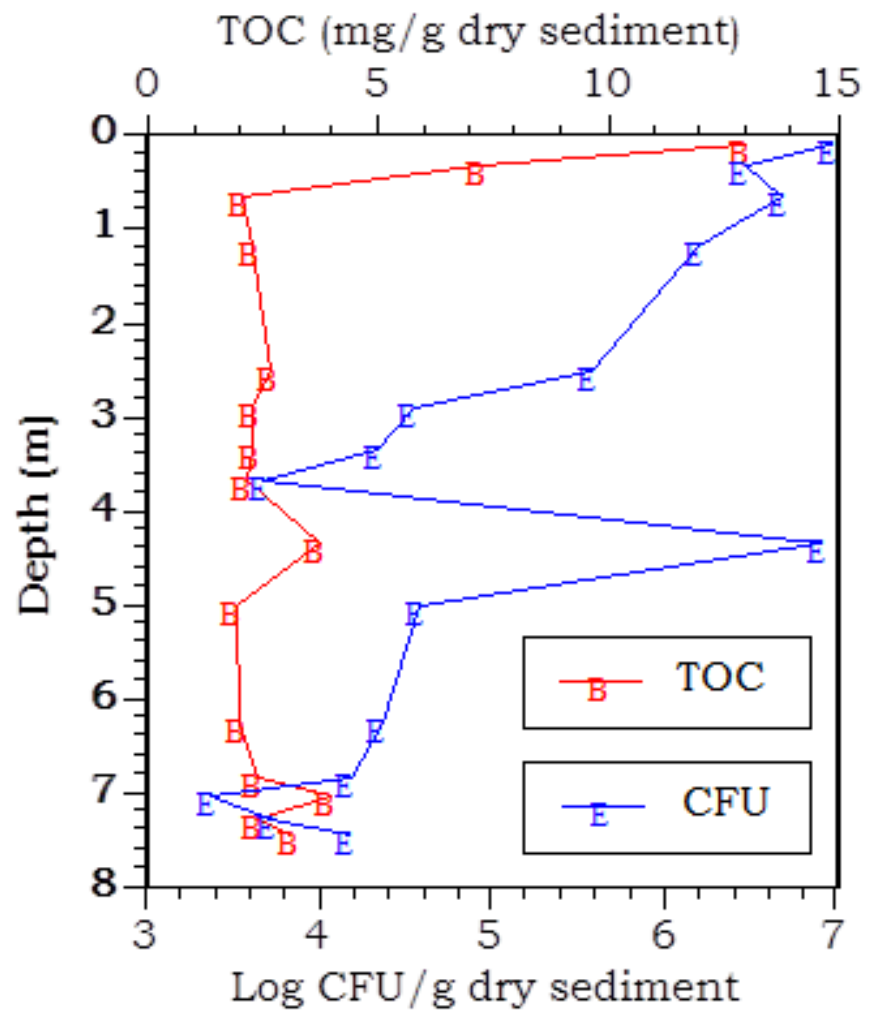

(b)

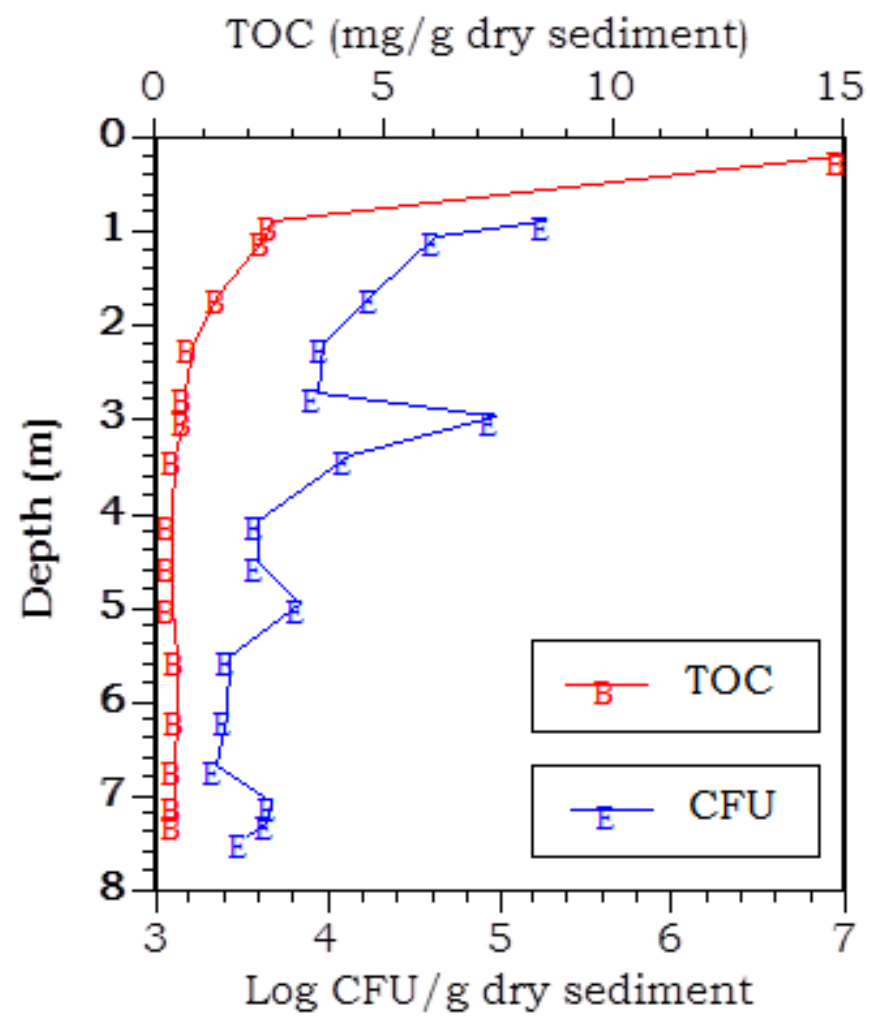

Figure 8.4 Depth profiles of total organic carbon (TOC) and culturable 
microorganisms (colony-forming units, CFU) at two sites (Severson et al., 1991). (a)

Dalmeny site, southern Saskatchewan; (b) WSU site, southeastern Washington state.

In deep vadose zones Brockman et al. (1992) found increased numbers and diversity in microbial communities associated with buried paleosols; they suggested that organic matter in these horizons was responsible for the contrast with other sediments. Severson et al. (1991) found greater diversity in microbial populations occurring in sediment containing kerogen (ancient, refractory organic matter) than in sediments at similar depths without kerogen; they also suggested that greater numbers of bacteria existed near fractures which might function as nutrient conduits. Taken together, these observations suggest that in general, bacterial numbers decline and bacterial predominance increases rapidly beneath soils, but that stratigraphic and hydraulic heterogeneities may introduce complexities.

\section{Vadose bacterial activity and $\mathrm{CO}_{2}$ generation.}

According to our purposes, we will define bacterial activity as the rate of $\mathrm{CO}_{2}$ generation by microorganisms per unit volume of vadose zone. This emphasizes our interest in the abundance of $\mathrm{CO}_{2}$, which is a key geochemical factor in vadose zones, and our potential interest in the microbial degradation of organic matter such as a pollutant hydrocarbon. We might expect activity to be proportional to numbers of viable bacteria. There are two problems with this logic: (1) it is known that living communities can exist in the subsurface in virtual stasis, waiting (perhaps thousands of years) for their next "meal" (e.g. Amy et al., 1993) ; (2) enumeration of viable bacteria is a disruptive, biasing process as noted above; organisms which do not respond to laboratory culturing may in fact be key players in subsurface respiration.

4.3.3.1 Laboratory measurements. Subsurface microbial-activity measurements have been made in two ways. The first, using established soil-microbiological techniques, is to monitor increasing $\mathrm{CO}_{2}$ concentration in a closed vessel containing a sample of vadose material. Such samples are generally amended with a labile carbon source (such as glucose or acetate) to raise the production rate to a detectable level. These measurements are obviously affected by sampling and measurement disturbances such that they do not represent actual in-situ activities. They may be used, however, to look for trends and controls on activity. For example, Kieft et al. (1992) assessed the effect of water content on activity in dry-climate, subsoil vadose zones by treating water content as an amendment variable. Because water potentials in these enviornments (not less than approximately $-500 \mathrm{kPa}$; refer to Table 8.1 ) were not small enough to directly cause dessication stress, their positive findings suggested that ambient water contents were small enough in some cases to limit the rate of nutrient diffusion through water films to the bacterial cells.

4.3.3.2 Field measurements. The second approach to activity measurement is to monitor $\mathrm{CO}_{2}$ concentrations in the vadose zone, rather than in a lab vessel (Amundson and Davidson, 1990, provide a review). In this case the system is not closed and so transport of $\mathrm{CO}_{2}$ must be accounted for in a statement of mass balance:

$$
\nabla \bullet \mathrm{J}=-\frac{\partial \mathrm{C}}{\partial \mathrm{t}}+\alpha
$$

where $\mathrm{J}$ is the flux, $\mathrm{C}$ is the concentration, $\mathrm{t}$ is time, and $\alpha$ is the production rate. This expression says that the change in the flux across a control volume (the divergence of the flux, left-hand side) is associated with a concentration change within that volume over time, plus some rate of production. In the special case of transport by gas-phase diffusion only in the vertical direction - a reasonable simplification for many vadose zones (Massman and Farrier, 1992) - [4.61] becomes:

$$
\frac{\partial}{\partial \mathrm{z}}\left(\mathrm{D} \frac{\partial \mathrm{C}}{\partial \mathrm{z}}\right)+\alpha=\frac{\partial \mathrm{C}}{\partial \mathrm{t}}
$$

where $\mathrm{D}$ is the effective diffusion coefficient for $\mathrm{CO}_{2}$ through gas-filled pore space, and the diffusive flux described 
by Fick's first law is shown in the parenthesis. We can see by looking at [4.62] that if the concentration changes with time and depth can be measured in a vadose profile, and if D can be reasonably estimated, we can solve directly for $\alpha$. Wood et al. (1993) took essentially this approach to determining production rates in two vadose zones beneath temperate grasslands; however their version of [4.62] included a dissolved-flux term which was calculated by estimating the vertical specific discharge (chapter 3 ) and assuming equilibrium between gas- and aqueous-phase $\mathrm{CO}_{2}$ (discussed below). Davidson and Trumbore (1995) used a slightly simplified form of [4.62] obtained by assuming steady state ( $\partial \mathrm{C} / \partial \mathrm{t}$ $=0$ ) in vadose profiles beneath forest and pasture land in the Brazilian Amazon.

Alternatively, a production distribution with depth can be assumed and, with D known and boundary and initial conditions specified, [4.62] may be solved for $\mathrm{C}=\mathrm{C}(\mathrm{z}, \mathrm{t})$. These results are then compared to observation as a test of the reasonableness of the production distribution which is then adjusted to obtain a best "fit". Bacon (1997) used this method in a transient, heterogeneous numerical study of the sites studied previously by Wood et al. (1993); Solomon and Cerling (1987) used essentially the same approach with a much simpler model in their study of the annual cycle of $\mathrm{CO}_{2}$ in a montane soil. If the production distribution can be described in simple mathematical form, and it is assumed that the concentration profile is steady $(\partial \mathrm{C} / \partial \mathrm{t}=0)$ and $\mathrm{D}$ does not vary, then analytical solutions are feasible. Taking this approach, Wood and Petraitis (1984) published solutions for several cases, including the case for production decreasing exponentially with depth:

$$
\alpha=\alpha_{0} \exp (-\mathrm{kz})
$$

where the subscript "o" indicates ground surface and $\mathrm{k}$ is a "rate" constant $\left[\mathrm{L}^{-1}\right]$ quantifying the steepness of production decline with depth. Wood and Petraitis (1984) present the solution of [4.62] and [4.63] for $\mathrm{a}_{\mathrm{o}}(\mathrm{C}, \mathrm{z}, \mathrm{k})$ and a curve-matching procedure to evaluate $\mathrm{k}$, in their study of production in two vadose zones beneath the High Plains of west Texas. 


\section{$\mathrm{CO}_{2}$ Production, mmol nin hri ${ }^{-1}$}

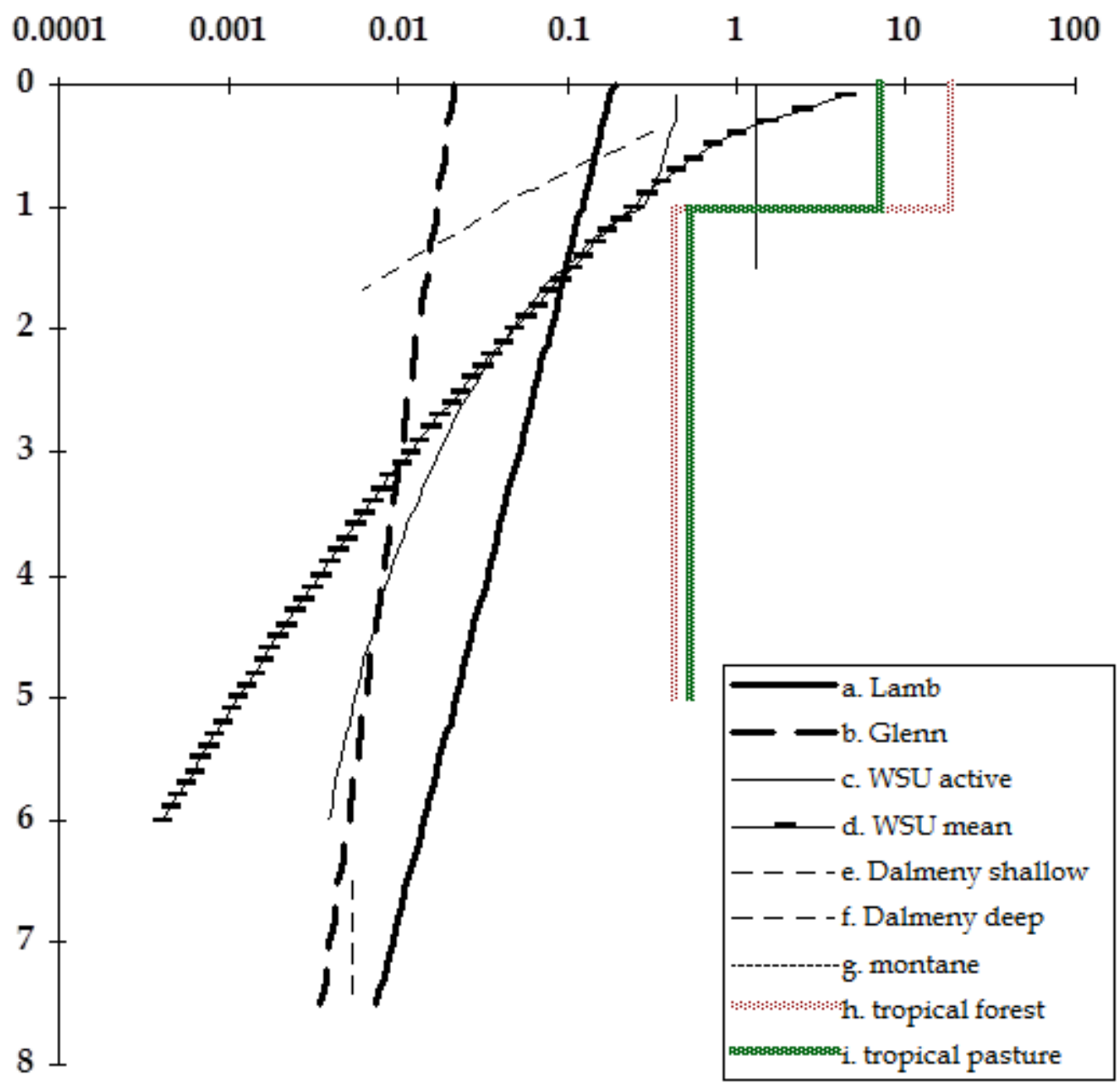

Figure 8.5 Carbon dioxide production vs. depth as determined by field measurements. (a) and (b): west Texas, calculated from Wood and Petraitis (1984); (c): Washington active season, calculated from Wood et al. (1993); (d) Washington mean annual, Bacon (1997); (e), (f): Saskatchewan mean annual, Bacon (1997) and Keller and Bacon (1998); (g): Utah, Solomon and Cerling (1987); (h), (i): Brazil, calculated from Davidson and Trumbore (1995). Production between 2 and $6.5 \mathrm{~m}$ depths at Dalmeny is below detection.

4.3.3.3 $\mathrm{CO}_{2}$ production distributions. Carbon dioxide production distributions determined by the five studies mentioned above are shown in Figure 8.5. The shapes of the plots vary according to mathematical treatment used. We must first note that the results span a very broad range. Part of this range is certainly due to difference in modeling approach and the seasonality of observation, which varies from study to study (note the differences between profiles c and d obtained for the same site). Total production, expressed as the flux from the ground to the atmosphere (conventionally termed soil respiration, reflecting conceptual neglect of subsoil production), is similarly variable (Table 8.4), averaging on an annual basis from $0.09 \mathrm{mmol} \mathrm{m}^{-2} \mathrm{hr}^{-1}$ (west Texas Glenn site) to $22 \mathrm{mmol} \mathrm{m}^{-2} \mathrm{hr}^{-1}$ (Brazilian forest site). The Brazilian vadose zones are pervaded by abundant tree roots to depths of at least $8 \mathrm{~m}$. These roots directly inject sloughed root hairs and exudates throughout this interval, and probably deeper. Perhaps the great contrast in labile substrate availability explains most of the production difference between this setting and the high, dry, low-primary-production ecosystems studied by Wood and Petraitis (1984). Cold-season "shut down" of shallow respiration in the temperate 
climates is probably also important. It should be noted that Solomon and Cerling (1987) and Davidson and Trumbore (1995) constrained their production estimates with measured values of "soil" respiration, using $\mathrm{CO}_{2}$ traps at the ground surface, while Wood and Petraitis (1984), Wood et al. (1993), and Bacon (1997) did not. This may partially explain why the latter production and "soil"-respiration rates tend to be low relative to the other studies and to widely-accepted estimates of net primary production for their ecosystem types (e.g. Schlesinger, 1991).

(\%)

Table 8.4

\begin{tabular}{|c|c|c|c|c|c|}
\hline Study & $\begin{array}{l}\text { monitored depth } \\
\text { (m) }\end{array}$ & $\begin{array}{c}\text { "soil" respiration" * } \\
(\mathrm{mmol} \mathrm{m-2} \mathrm{hr-1)} \\
\text { TOTAL z>1m** }\end{array}$ & $\%>1 m^{* *}$ & & CO2 max. \\
\hline \multicolumn{6}{|c|}{ Wood and Petraitis (1984) } \\
\hline Lamb & 28 & 0.43 & 0.28 & 65 & 2.62 \\
\hline Glenn & 50 & 0.09 & 0.07 & 78 & 0.88 \\
\hline \multicolumn{6}{|c|}{ Solomon and Cerling (1987) } \\
\hline montane & 0.9 & 1.3 & 0.4 & 31 & $0.6-1.4$ \\
\hline \multicolumn{6}{|c|}{ Wood et al. (1993) } \\
\hline WSU active & 6 & 0.5 & 0.18 & 35 & 1.5 \\
\hline \multicolumn{6}{|c|}{ Bacon (1997), Keller and } \\
\hline \multicolumn{6}{|l|}{ Bacon (1998) } \\
\hline WSU mean & 6 & 1.3 & 0.14 & 11 & 2 \\
\hline Dalmeny & 7.5 & 0.3 & 0.02 & 7 & 4 \\
\hline \multicolumn{6}{|c|}{ Davis and Trumbore (1995) } \\
\hline forest & 8 & 22 & 3.8 & 17 & 7 \\
\hline pasture & 8 & 10 & 2.5 & 25 & 6 \\
\hline & $\begin{array}{l}* \text { calculated fror } \\
* * \text { respiration } \mathrm{p}\end{array}$ & $\begin{array}{l}\text { m reported data. } \\
\text { roduced at depth grea }\end{array}$ & ter than $1 \mathrm{r}$ & & \\
\hline
\end{tabular}

Noting the logarithmic x-axis in Figure 8.5, the hypothesis that $\mathrm{CO}_{2}$ production generally declines exponentially with depth seems reasonable. Rapid, if not exponential, declines with depth are consistent with our conception of production as controlled strongly by availability of labile substrates. Table 8.4 shows the respiration flux from that portion of the vadose zone deeper than $1 \mathrm{~m}$ and its percentage of the total "soil" respiration. 


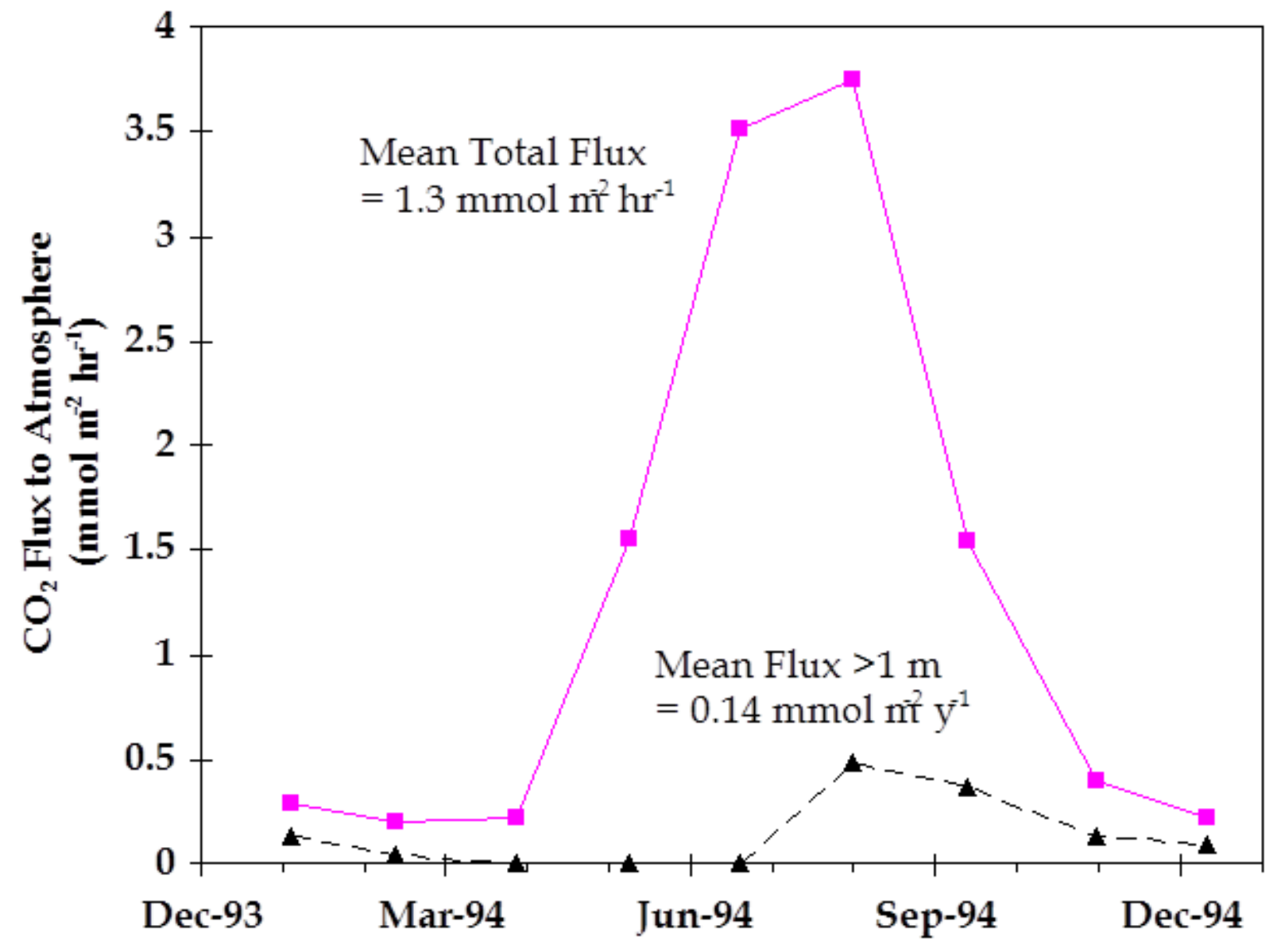

Figure 8.6 shows how the total and deep respiration flux components vary over a seasonal cycle at the WSU site (Bacon, 1997). These and the foregoing data suggest that a substantial fraction of ecosystem respiration in fact occurs beneath soils, particularly when plants are inactive, and that the turnover dynamics of subsoil carbon stocks must be considered in attempts to understand ecosystem-atmosphere carbon exchanges. The term "suggest" in the previous sentence is important, because the available data are few; furthermore, neither Wood and Petraitis (1984) nor Solomon and Cerling (1987) designed their studies to address this issue. And there is an additional twist: the isotopic signatures of carbon in vadose $\mathrm{CO}_{2}$ and organic matter indicate that the substrate for the deep respiration at the Dalmeny site (Figure 8.5, Table 8.4) is mostly the Cretaceous kerogen component of the glacial till there (Keller and Bacon, 1998). This, then, is "geo"respiration, i.e. carbon being cycled between lithologic and ocean-atmosphere reservoirs on a timescale of tens to hundreds of millions of years (Holland, 1978; Berner and Canfield, 1989). Others (e.g. Parkin and Simpkins, 1995; Wassenaar et al., 1990) have observed that Pleistocene plant material entrained in glacial tills can support subsoil microbial production of $\mathrm{CO}_{2}$ and methane. It is unknown whether the turnover times of such vadose carbon stocks are perturbed by anthropogenic disturbances - such as cultivation and deforestation - which perturb soil-derived carbon stocks.

Regardless of the specific magnitude and distribution of vadose $\mathrm{CO}_{2}$ production, $\mathrm{CO}_{2}$ concentrations can be expected to generally increase with depth. We can see why, in a solution of the steady-state form of [4.62] for $\mathrm{C}(\mathrm{z})$. For the boundary conditions:

$$
\mathrm{C}_{\mathrm{O}}=0 \quad \text { atz }=0 \text { (ground surface) } \quad \frac{\partial \mathrm{C}}{\partial \mathrm{z}}=0 \quad \text { atz }=\mathrm{L}(\text { top of saturated zone })
$$

the solution is, for constant production a throughout the depth $\mathrm{L}$ of the vadose zone, 


$$
\mathrm{C}_{\mathrm{z}}-\mathrm{C}_{\mathrm{O}}=\frac{\alpha}{\mathrm{D}}\left(\mathrm{LZ}-\frac{\mathrm{z}^{2}}{2}\right)
$$

You can show yourself that as $\mathrm{z}$ increases to approach $\mathrm{L}$, the value of the parenthesis term (and therefore $\mathrm{C}_{\mathrm{z}}$ ) increases. The same can be shown to be true for other production distributions like those discussed above (Wood and Petraitis, 1984). The key to this result is the zero-flux condition at the bottom boundary: $\mathrm{CO}_{2}$ produced at depth can only leave the domain upwards, and to do this by diffusion it must attain a concentration greater than concentrations above. In fact, diffusion of $\mathrm{CO}_{2}$ through water-filled pore space in the saturated zone is more than $10^{4}$ times slower than through gas-filled pore space, so the bottom boundary condition is a reasonable approximation. Furthermore, removal of $\mathrm{CO}_{2}$ by dissolution into downward-flowing water (e.g. Wood et al., 1993) reduces the concentrations but does not change the downward-increasing trend. 


\section{a. WSU site, southeastern Washington}

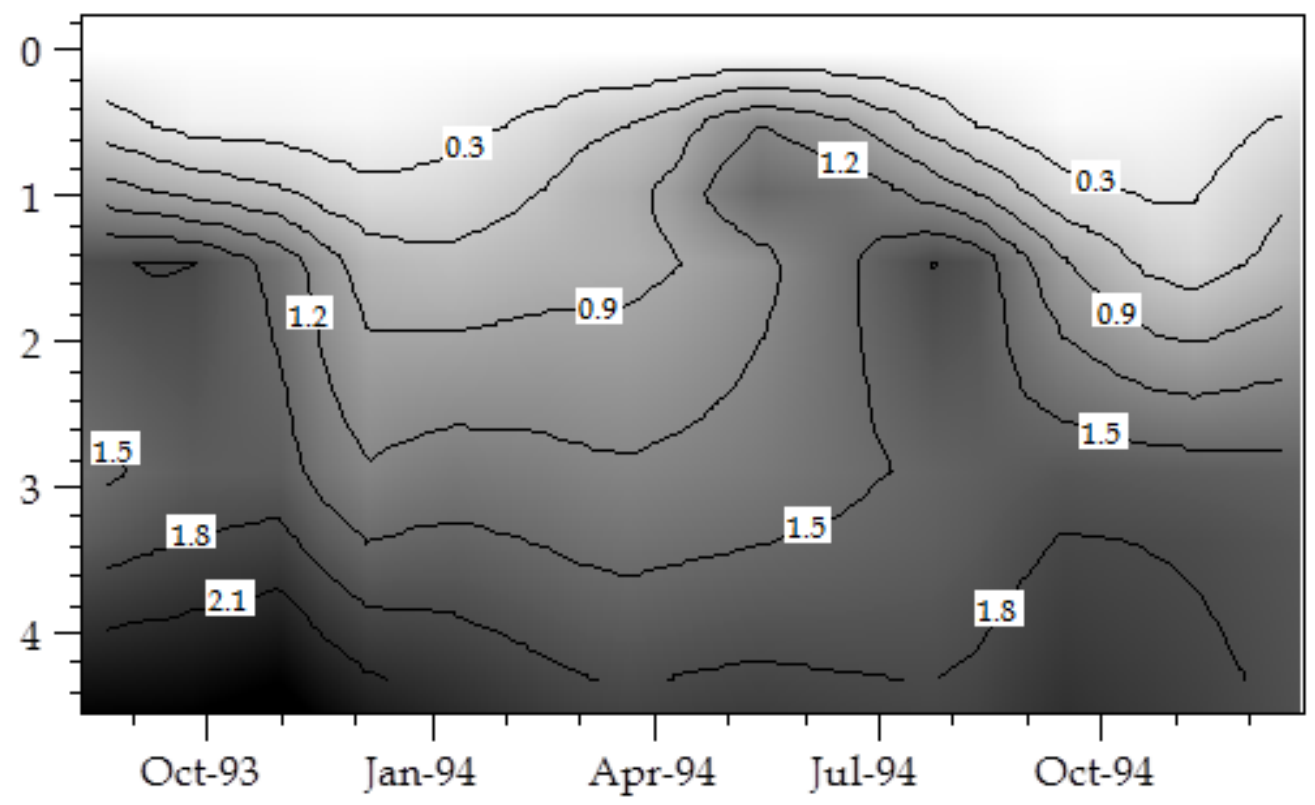

b. Dalmeny site, southern Saskatchewan

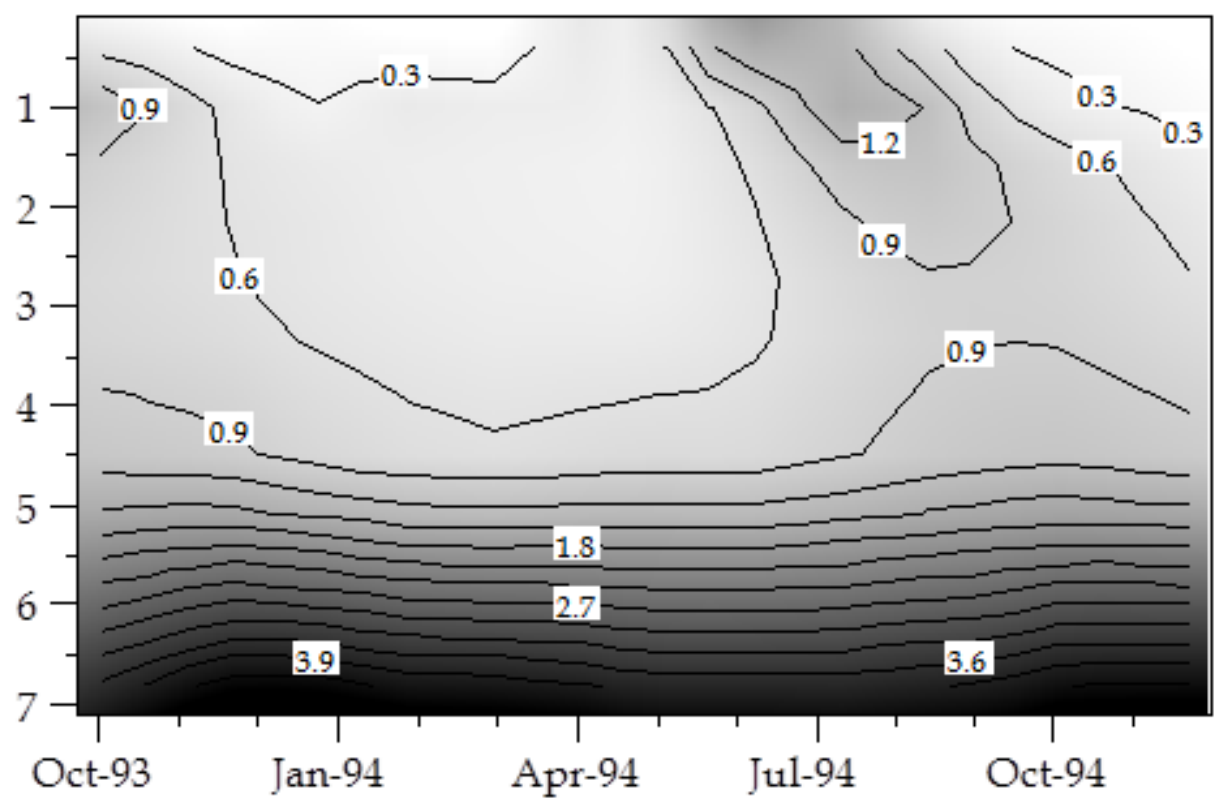

Figure $8.7 \mathrm{CO}_{2}$ concentrations vs. depth and time at two field sites (from Bacon, 1997, permission to be asked). Concentration gradients are dominantly downard and diffusive fluxes upward at both sites, due to finite subsoil production. See also Wood et al. (1993), Keller and Bacon (1998), and Bacon and Keller (1998). 
Theory aside, what do field observations tell us? Data reported in all the aforementioned studies show increasing $\mathrm{CO}_{2}$ concentrations with depth (Figure 8.7), and while (again) the data base is small, it appears that this is indeed the general pattern. If this is true, a piece of the conventional wisdom of hydrogeology is misleading: groundwater does not attain its initial "charge" of dissolved $\mathrm{CO}_{2}$ so much in the soil zone (e.g. Freeze and Cherry, 1979, chapter 7) as at depth in the vadose zone beneath, where the highest concentrations are attained. This is important because it suggests that the concentration of dissolved $\mathrm{CO}_{2}$ in groundwater at the water table cannot reliably be predicted from measurement of soil $\mathrm{CO}_{2}$. It is true that high $\mathrm{CO}_{2}$ concentrations which decline with depth are attained during growing seasons in temperate-zone soils, but this is a transient effect on a diannual timescale (Figure 8.7). Fluctuations of vadose $\mathrm{CO}_{2}$ concentrations have important implications for carbonate chemistry, as we will see later.

The region immediately above the saturated zone is also the locus of the imprinting of the dissolved $\mathrm{CO}_{2}$ with its isotopic signature - attained by equilibrium with the gas phase - before it moves into the saturated zone. That isotopic signature may differ considerably from the soil zone; in particular, it may contain less ${ }^{14} \mathrm{C}$ due to the age of the organic substrate (Keller, 1991). Subsequent studies (Trumbore et al., 1995; Keller and Bacon, 1998) illustrate this effect, which may be dramatic when the substrate, or a fraction thereof, is very old. These observations have at least two practical implications: (1) vadose $\mathrm{CO}_{2}$ with an isotopic signature different from that of the soil is not necessarily a tracer of the degradation of a contaminant such as hydrocarbon (Suchomel et al., 1990); and (2) the dissolved $\mathrm{CO}_{2}$ in recharging groundwater may not necessarily be "modern", as conventionally assumed in radiocarbon-dating studies of groundwater age (e.g. Fontes and Garnier, 1979). Figure 8.8 shows how assuming modern $(100 \mathrm{pmc}) \mathrm{CO}_{2}$ at point of recharge at the Dalmeny site leads to large overestimates of shallow groundwater age using well-known age-dating models.

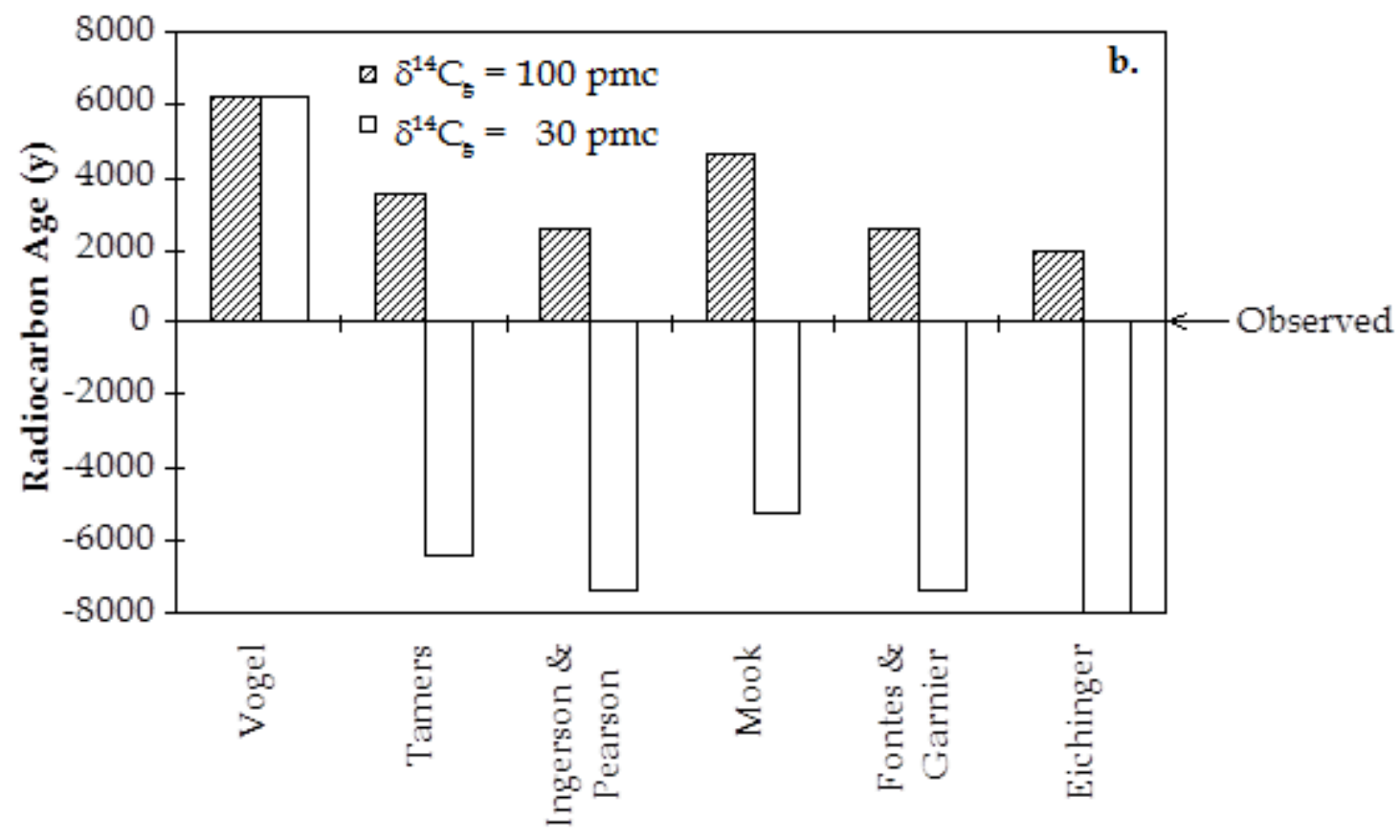

Figure 8.8 Erroneous radiocarbon age estimates, generated by various published models, based on ${ }^{14} \mathrm{C}$ and ${ }^{13} \mathrm{C}$ content of dissolved $\mathrm{CO}_{2}$ in modern shallow groundwater at the Dalmeny site (Bacon and Keller, 1998). $\mathrm{Cg}$ is assigned soil-zone gas-phase $\mathrm{CO}_{2}$, pmc is percent modern carbon.

This is due to the fact that modern recharge is in contact and equilibrium with ancient kerogenic $\mathrm{CO}_{2}$ deep in the Dalmeny vadose zone. The Figure also shows that simply adjusting the $14 \mathrm{C}$ content of point-of-recharge $\mathrm{CO}_{2}$ does not produce any better results; this is because the age-dating models assume closed-system dissolution of carbonate minerals, while the dissolution process at Dalmeny appears to occur in the vadose zone under open-system conditions 
(see section 4.4.1.1 below). Bacon and Keller (1998) suggest that these findings at Dalmeny have implications for other settings where vadose zones contain "geologic" (non-solum) organic carbon.

Table 8.4 shows the maximum $\mathrm{CO}_{2}$ concentrations attained at the deepest monitoring points reported in the four studies we have compared. Solomon and Cerling (1987) report diannual fluctuation due to shallow depth, while the other studies report nearly constant concentrations. The data span less than an order of magnitude, which, in the face of a paucity of data, we believe represents most vadose zones. This is a much smaller range than exhibited by many hydrologic variables. The reason for this somewhat surprising uniformity from place to place, despite great variation in geologic setting and ecosystem type, is unknown. We can, though, do some informed speculation. First, root respiration, which may constitute half the total (microbial plus root) respiration in soils (Trumbore et al., 1995), is highly variable in root zones but its contribution - and associated variability - usually declines in importance with increasing depth. (This may not be true at the Brazilian sites of Davidson and Trumbore (1995), which also accounts for the large $\mathrm{CO}_{2}$ concentrations observed at depth there (Table 8.4). Second, vadose zones are shallow where precipitation is high and deep where precipitation is low (chapter 3). All else equal, $\mathrm{CO}_{2}$ levels would be larger in the deep vadose zones where the diffusion path to ground surface is longer. Countervailing, however, is the fact that primary (and labile substrate) production is generally larger in the high-precip, shallow-vadose-zone settings. There may be other balancing mechanisms by which the biogeochemical environments of vadose zones have remained stable through Earth history, stabilizing chemical weathering rates and therefore atmospheric $\mathrm{CO}_{2}$ levels, thus conferring a kind of homeostasis over geologic time (Keller and Wood, 1993).

\subsection{4 $\mathrm{CO}_{2}$, acid, and $\mathrm{pH}$.}

Carbon dioxide dissolves into water, quickly attaining equilibrium in the intimate confines of vadose pore space:

$$
\mathrm{CO}_{2(\mathrm{~g})}=\mathrm{CO}_{2}
$$

where the superscript denotes the charge of the species dissolved in water. Dissolved $\mathrm{CO}_{2}$ exists predominantly as a loosely hydrated species $\mathrm{CO}_{2}{ }^{\mathrm{O}}$ but a small proportion forms carbonic acid:

(8.73) $\mathrm{CO}_{2}+\mathrm{H}_{2} \mathrm{O}=\mathrm{H}_{2} \mathrm{CO}_{3}$

Conventionally these two equations are added together (right side plus right side, left side plus left side) to obtain the single equilibrium:

$$
\mathrm{CO}_{2(\mathrm{~g})}+\mathrm{H}_{2} \mathrm{O}=\mathrm{H}_{2} \mathrm{CO}_{3} \quad \mathrm{~K}_{\mathrm{CO}_{2}}=\frac{\mathrm{a}_{\mathrm{H}_{2} \mathrm{CO}_{3}}}{\mathrm{P}_{\mathrm{CO}_{2(\mathrm{~g})}}}
$$

where we have taken the activity of the gas as equal to its partial pressure and assumed that the water is pure ( $a=1)$ in writing the Henry's law equilibrium constant (recall [4.27a]). Now carbonic acid takes its name from its propensity to dissociate, releasing a proton or hydrogen ion $\mathrm{H}+$ and thus becoming bicarbonate ion:

$$
\mathrm{H}_{2} \mathrm{CO}_{3}=\mathrm{H}^{+}+\mathrm{HCO}_{3}^{-} \quad \mathrm{K}_{1}=\frac{\mathrm{a}_{\mathrm{HCO}_{3}^{-}} \mathrm{a}_{\mathrm{H}^{+}}}{\mathrm{a}_{\mathrm{H}_{2} \mathrm{CO}_{3}}}
$$

Looking back quickly, we see that the proton actually comes from the hydrated water molecule associated with the $\mathrm{CO}_{2}{ }^{\mathrm{O}}$. This process is called hydrolysis. Bicarbonate is also an acid, dissociating in a second hydrolysis step to yield another proton and carbonate ion:

$$
\mathrm{HCO}_{3}^{-}=\mathrm{H}^{+}+\mathrm{CO}_{3}^{2-} \quad \mathrm{K}_{2}=\frac{\mathrm{a}_{\mathrm{CO}_{3}^{2-}} \mathrm{a}_{\mathrm{H}^{+}}}{\mathrm{a}_{\mathrm{HCO}_{3}^{-}}}
$$

We see from [4.65-4.67] that carbonic acid, bicarbonate, and carbonate coexist at equilibrium in solution, and that 
their activities are related to each other and, via hydrolysis, to the activity of the hydrogen ion. The three species are collectively referred to as the carbonate species (which includes carbonate ion), or as disssolved inorganic carbon (DIC).

The upshot of [4.65-4.67] is that when $\mathrm{CO}_{2}$ is present in a gas phase, an accompanying water phase will be "charged" with DIC and acid, or, more specifically, hydrogen ion. Furthermore, we can see that the greater the $\mathrm{CO}_{2}$ level (or $\mathrm{P}_{\mathrm{CO} 2}$ in 4.65), the more carbonic acid, bicarbonate, carbonate, and $\mathrm{H}^{+}$will coexist at equilibrium. We can now specify a typical vadose $\mathrm{CO}_{2}$ concentration as a partial pressure (e.g. from Table 8.4), calculate the values of the equilibrium constants from thermodynamic data using [4.26] or get the values from a table (Table 8.5), and solve the three equations for the various activities. We'll illustrate a rough but useful solution here, deferring a more rigorous approach to later. Suppose we want to know the $\mathrm{H}^{+}$activity of water in equilibrium with the atmosphere (e.g. rain). From 4.65 we get

$$
\mathrm{a}_{2} \mathrm{CO}_{3}=\mathrm{P}_{\mathrm{CO}_{2}} \mathrm{~K}_{\mathrm{CO}_{2}}
$$

The $\mathrm{PCO}_{2}$ of the atmosphere is $0.03 \%=0.0003$ bar. For a temperature of $25 \mathrm{C}$ (Table 8.5), $\mathrm{K}_{\mathrm{CO} 2}=10^{-1.47}$ and the carbonic acid activity is then $1.0 \times 10^{-5}$ or $10^{-4.99}$. We could solve 4.66 for $\mathrm{H}^{+}$in terms of this activity and $\mathrm{K}_{1}$, but aHCO3in that expression is unknown and depends in turn on aco32- in 4.67, which is also unknown. The way out is to note that $\mathrm{K}_{2}=10^{-10.33}$ at this temperature, and to recall from experience that rainwater is acid, i.e. its $p H$ is something less than a "neutral" 7. Using the definition

(8.78) $\mathrm{pH}=-\log \mathrm{a}_{\mathrm{H}}+$

and assuming an upper-limit $\mathrm{pH}=7$, we obtain $\log \mathrm{H}^{+}=-7$ and, from [4.67], that the ratio of carbonate to bicarbonate activities would be

$$
\frac{\mathrm{a}_{\mathrm{CO}_{3}^{2-}}}{\mathrm{a}_{\mathrm{HCO}_{3}^{-}}}=\frac{\mathrm{K}_{2}}{\mathrm{a}_{\mathrm{H}^{+}}}=\frac{10^{-10.33}}{10^{-7}}=10^{-3.33}
$$

i.e. so small that for a first approximation we can neglect the presence of carbonate ion at neutral $\mathrm{pH}$ and lower. This makes the problem much easier, because we see that in 4.66 one mole of $\mathrm{H}^{+}$is generated for each mole of $\mathrm{HCO}_{3}$-, i.e. their concentrations will be equimolal. If we neglect the difference between activities and concentrations then their activities will also be equal, and we can rewrite 4.66 as

$$
\mathrm{K}_{1}=\frac{\mathrm{a}_{\mathrm{H}^{+}}^{2}}{\mathrm{a}_{\mathrm{H}_{2} \mathrm{CO}_{3}}}
$$

or

$$
\mathrm{a}_{\mathrm{H}^{+}}=\left(\mathrm{K}_{1} \mathrm{a}_{\mathrm{H}_{2} \mathrm{CO}_{3}}\right)^{1 / 2}
$$

which gives $\mathrm{aH}_{+}=2.1 \times 10^{-6}$ or $\mathrm{pH}=5.7$. This is indeed the approximate $\mathrm{pH}$ of pristine rainwater (which, we see, is actually "acid" in that its $\mathrm{pH}$ is substantially less than 7!). Were we skeptical of our result, we could re-examine the validity of our simplifying assumption of equality of activities and concentrations. Clinging to that idea for one more moment, we can approximate the ionic strength from [4.48] and then the ionic activity coefficient gi from [4.46] . This would give us a good idea, from $a_{i}=m_{i} \gamma_{i}$, of the magnitude of the deviation of activity and concentration, and the associated magnitude of the error in our result.

So much for rainwater. Considering the vadose zone - where $\mathrm{PCO}_{2} \mathrm{~S}$ are higher - we see that more carbonic acid and hence more $\mathrm{H}^{+}$are generated (4.65 and 4.66 respectively), i.e. computed $\mathrm{pH}$ values are even lower. As we will see, though, these reaction simulations do not match observed vadose or groundwater pHs very well. In soils, the organic acids secreted by roots and generated as reaction intermediates exert aditional pH control (e.g. Cronan and Aiken, 1985). And in both soils and subsoils, organic and carbonic acids are consumed (buffered) by reactions with solids. The "big picture", hydrogeochemically, is that the acidity generated by vadose life processes interacts with the inorganic, mineral realm to yield water qualities which sustain us all, plant and animal. We now turn to these processes. 


\subsection{Acid Consumption and Fluid-Rock Interaction}

We may expand the pertinent parts of Figure 8.1 as follows (for convenience, assuming mineralization of respiration reaction intermediates) :

$$
\mathrm{H}_{2} \mathrm{O}+\mathrm{H}_{2} \mathrm{CO}_{3}{ }^{+} \text {minerals }=\mathrm{H}_{2} \mathrm{O}+\mathrm{DIC}+\text { inorganic solutes }
$$

We have just seen how DIC is generated as carbonic acid dissociates. The inorganic solutes - the elements and their complexes which go into solution when mineral crystals are attacked by water molecules - are the predominantly ionic species which are called "nutrients" by agronomists and ecologists, essential building blocks of plant and animal tissue in combination with volatile elements $(\mathrm{C}, \mathrm{N}, \mathrm{O}, \mathrm{H})$ from the atmosphere. The same solutes are perceived by geologists as the "chemical weathering" flux carried in terrestrial runoff to the oceans.

There are some important details embedded in [4.69], which actually represents a large number of reactions in a real vadose zone. The extent to which the reactions proceed will depend on the amount of acid available and on the solubilities of the specific minerals. The mineral assemblage also determines the number and concentrations of the inorganic solutes, and whether the reactions may be treated as kinetic or equilibrium processes. Reaction [4.69] does not represent all fluid-rock interactions in vadose zones; in particular, dissolution of soluble salts like gypsum does not consume acid because no hydrolysis (e.g. [4.66] and [4.67]) is involved. Nonetheless, [4.69] is ubiquitous and it characterizes water-quality controlling reactions in non-arid regions. We will now discuss two important "genera" of these reactions in the context of the geologic settings that determine their applicability.

\subsection{Carbonate minerals and terranes.}

Calcite $\left(\mathrm{CaCO}_{3}\right)$ and dolomite $\left(\mathrm{CaMg}\left(\mathrm{CO}_{3}\right)_{2}\right)$ are formed in many geologic environments. They comprise substantial fractions of the mineral mass of many marine and some terrestrial sedimentary rocks. These rocks and their "reworked" (eroded and redeposited) products, such as glacial tills, comprise the surficial deposits in many parts of the world, where they are referred to as carbonate terranes. These terranes occur across much of the North American continent (Back et al., 1988), with extensive coverage in the central Mississippi River basin, Texas and the High Plains, the Great Basin, the Yucatan and Florida, and the Saint Lawrence Lowlands-Great Lakes-Interior Plains megaregion (as glacial till). Less extensive occurrences are widespread. For our purposes, the important point is that in all these regions, rock fragments containing carbonate minerals are incorporated into soil and subsoil vadose zones where they interact with flowing pore water.

4.4.1.1 The carbonate system. Calcite is usually present where dolomite is; it equilibrates with water more quickly than dolomite and its chemical formula is simpler, so we will follow the time-honored tradition (at least since Garrels and Christ, 1965) of using calcite in our illustrations. A pertinent form of [4.69] is

$$
\mathrm{H}_{2} \mathrm{CO}_{3}+\mathrm{CaCO}_{3}=2 \mathrm{HCO}_{3}^{-}+\mathrm{Ca}^{2+}
$$

where we have dropped the water - cancelling it from both sides - for simplicity. Before we employ this in attempts to predict water chemistry, we note that [4.70] is the sum of two reactions

$$
\begin{aligned}
\mathrm{H}_{2} \mathrm{CO}_{3} & =\mathrm{HCO}_{3}^{-}+\mathrm{H}^{+} \\
\mathrm{H}^{+}+\mathrm{CaCO}_{3} & =\mathrm{Ca}^{2+}+\mathrm{HCO}_{3}^{-}
\end{aligned}
$$

the first of which is [4.65]. This explicitly shows the consumption of $\mathrm{H}^{+}$which makes [4.70] so important in the buffering of atmospheric and biologic acids.

Now: how much buffering occurs? How far does reaction 4.70 proceed in vadose zones? We have the great advantage here of using an equilibrium analysis: calcite reaches aqueous equilibrium at environmental temperatures relatively quickly, within days to weeks. We can straightaway write the equilibrium constant for [ 4.70]: 


$$
\mathrm{K}=\frac{\mathrm{a}_{\mathrm{HCO}_{3}^{-}}^{2} \mathrm{a}_{\mathrm{Ca}^{2+}}}{\mathrm{a}_{\mathrm{H}_{2} \mathrm{CO}_{3}}}
$$

but all three terms in the reaction quotient are unknown. Mathematically, we need additional constraints (equations). Reviewing our development, we have a constraint on $\mathrm{aH}_{2 \mathrm{CO}} \mathrm{Co}$ in [4.65] and two constraints on aHCO3- in [4.66] and [4.67]. Translated into physical terms, this reminds us that aqueous DIC is in equilibrium contact with $\mathrm{CO}_{2}$ in the gas phase ([4.65]) and that all three carbonate species coexist in mutual equilibrium with $\mathrm{H}^{+}([4.66]$ and [4.67]) as well as with calcite ([4.70]). Therefore we must solve all these equations simultaneously.

The physical setup, a three-phase equilibrium, is shown schematically in Figure 8.9.

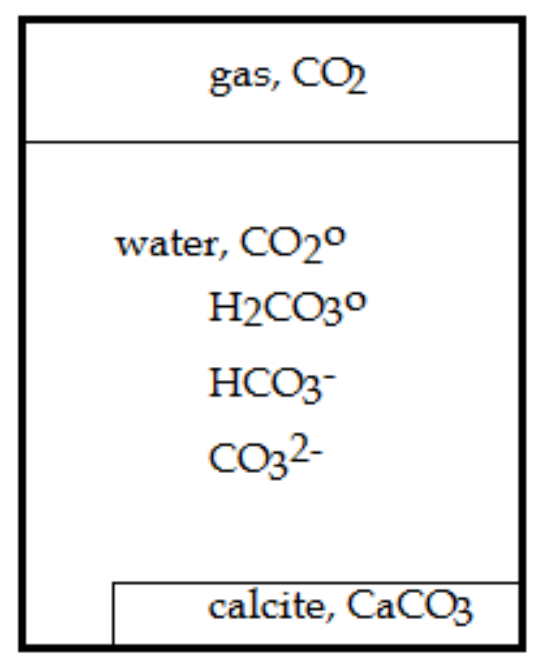

Figure 8.9 The carbonate system.

It is convenient arithmetically to subtract two $\mathrm{H}+$ from each side of [4.70] to obtain

$$
\mathrm{CaCO}_{3}=\mathrm{Ca}^{2+}+\mathrm{CO}_{3}^{2-} \quad \mathrm{K}_{\mathrm{cal}}=\mathrm{a}_{\mathrm{Ca}^{2+}} \mathrm{a}_{\mathrm{CO}_{3}^{2-}}
$$

With [4.65 - 4.67] and [4.72], all species and phases in Figure 8.9 are represented. Now it should be possible to pose and solve the problem: given a certain $\mathrm{P}_{\mathrm{CO} 2}$, as a consequence of vadose biological activity and $\mathrm{CO}_{2}$ transport, what are the equlibrium activities of the aqueous species and how much calcite is dissolved or precipitated?

The equilibrium constants are well known: their values are tabulated (Table 8.5) or may be computed from tabulated free-energy data via [4.26], and these values may be adjusted for temperature deviations from standard state via [4.39] and [4.32].

Table 8.5 Equilibrium constants for the carbonate system. Values reported as - $\log _{10} \mathrm{~K}$. (from Drever, 1997, Table 3-1 and Freeze and Cherry, 1979, Table 3.4) 


\begin{tabular}{llllll}
\hline $\mathrm{T}\left({ }^{\circ} \mathrm{C}\right)$ & $\mathrm{pKCO} 2$ & $\mathrm{pK1}$ & $\mathrm{pK2}$ & $\mathrm{pKcal}$ & $\mathrm{pKw}$ \\
5 & 1.19 & 6.52 & 10.55 & 8.39 & 14.73 \\
10 & 1.27 & 6.46 & 10.49 & 8.41 & 14.53 \\
15 & 1.34 & 6.42 & 10.43 & 8.43 & 14.35 \\
20 & 1.41 & 6.38 & 10.38 & 8.45 & 14.17 \\
25 & 1.47 & 6.35 & 10.33 & 8.48 & 14.00 \\
30 & 1.52 & 6.33 & 10.29 & 8.51 & 13.83 \\
\hline
\end{tabular}

As a system of equations, [4.65-4.67] and [4.72] isindeterminate: taking the equilibrium constants as known, we see that if we specify $\mathrm{P}_{\mathrm{CO} 2}$ (take $\mathrm{P}_{\mathrm{CO} 2}$ as the independent variable), there remain 5 unknown variables - the activities of carbonic acid, bicarbonate, carbonate, hydrogen, and calcium ion. We need another independent equation:

$$
\mathrm{H}_{2} \mathrm{O}=\mathrm{H}^{+}+\mathrm{OH}^{-} \quad \mathrm{K}_{\mathrm{w}}=\mathrm{a}_{\mathrm{H}^{+}} \mathrm{a}_{\mathrm{OH}^{-}}
$$

which describes the equilibrium dissociation (hydrolysis) of the water molecule, where $\mathrm{OH}^{-}$is the hydroxide ion. (Here we see that water itself, by dissociating to yield a hydrogen ion, may be considered to be an acid.) This equation amplifies our description of the system but does not gain us any ground mathematically because we have introduced another variable, the activity of hydroxide. However, if we invoke the condition of charge balance as applied to all the ions, we can write an independent equation without introducing any new species:

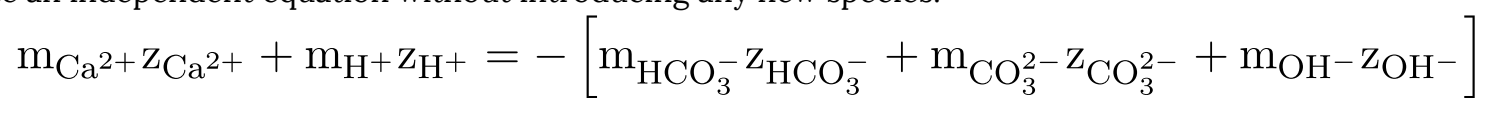

In this expression we know the respective ionic charges but now molalities have been introduced. The way out of this quandary is again to make, at least provisionally, the assumption of ideality $-\mathrm{a}_{\mathrm{i}}=\mathrm{m}_{\mathrm{i}}$. This permits direct substitution of molalities for all the activity terms in [4.65-4.67]and [4.72-4.74] to yield a determinate system of 6 equations in 6 unknowns. Garrels and Christ (1967, chap. 3, case 2) present the classic outline of this open (to an equilibrium gas phase) system problem and its manual solution, which involves expression of all the variables in [4.74] in terms of hydrogen ion and equilibrium constants, then solving the resulting 4th-order polynomial. They obtained, for the condition of atmospheric $\mathrm{P}_{\mathrm{CO} 2}=0.0003 \mathrm{bar}=10^{-3.5}$ bar and $\mathrm{T}=25^{\circ} \mathrm{C}$, the result $\mathrm{m}_{\mathrm{H}^{+}}=10^{-8.4}$ or $\mathrm{pH}=8.4$. For the rest of the species activities, Garrels and Christ (1967) obtained the following molalities: $\mathrm{HCO}_{3}{ }^{-}=10^{-3.0}, \mathrm{CO}_{3}{ }^{2-}=10^{-4.9}, \mathrm{H}_{2} \mathrm{CO}_{3}{ }^{\circ}=10^{-5.0}, \mathrm{Ca}^{2+}$ $=10^{-3.4}$, and $\mathrm{OH}^{-}=10^{-5.6}$. You should check these results by evaluating the carbonate equilibria and $\mathrm{K}_{\mathrm{w}}$, [4.65-4.73], at $\mathrm{pH}=8.4$ using the appropriate equilibrium constant values in Table 8.5. Be prepared to find some inconsistency: the 1967 calculations employed different equilibrium-constant values!

Let us compare, now, the results of this new calculation - representing precipitation in contact with atmosphere and calcite - with our earlier results, for the case of no calcite present. The clearest change is the increase of the $\mathrm{pH}$ from 5.7 to 8.4. The DIC has also increased dramatically. In the no-calcite case, the only species appreciably present were $\mathrm{H}_{2} \mathrm{CO}_{3}{ }^{\circ}$ and $\mathrm{HCO}_{3}{ }^{-}$at $\mathrm{m}=10^{-4.99}$ and $10^{-5.7}$ respectively, for a total of $\mathrm{m}_{\mathrm{DIC}}=10^{-4.91}$. In the calcite case, these two species' concentrations are $10^{-5.0}$ and $10^{-3.0}$ respectively; then there is $\mathrm{CO}_{3}{ }^{2-}$ also present at $\mathrm{m}=10^{-4.9}$, for a total of mDIC $=$ $10^{-3.0}$, nearly two orders of magnitude greater. The change in $\mathrm{HCO}_{3}{ }^{-}+\mathrm{CO}_{3}{ }^{2-}$, which comprises the carbonate alkalinity and quantifies the buffering capacity with respect to acid addition (producing $\mathrm{H}_{2} \mathrm{CO}_{3}{ }^{\circ}$ and $\mathrm{HCO}_{3}{ }^{-}$by driving reactions 4.65 and 4.66 backwards, instead of driving down the $\mathrm{pH}$ ) is even larger, from $10^{-5.7}$ to $10^{-3.0}$. Finally, $\mathrm{Ca}^{2+}$, an essential nutrient, is present only in the calcite case.

Of course, the $\mathrm{PCO}_{2}$ distributions characteristic of vadose zones mean that calcite equilibria result in different $\mathrm{pH}$ and ion concentrations; you can explore this with a simple spreadsheet, but we can note qualitatively that as the $\mathrm{PCO}_{2}$ increases relative to the atmosphere, the $\mathrm{pH}$ decreases and $\mathrm{HCO}_{3}{ }^{-}, \mathrm{CO}_{3}{ }^{2-}$, and $\mathrm{Ca}^{2+}$ increase. Reardon et al. (1979, 1980) showed this to be the case in a carbonate terrane in subhumid southern Ontario. That seminal work showed unequivocally the establishment of calcite equilibrium, and the associated gross water-chemistry features of shallow groundwater, in the vadose zone. Others before and since (e.g. Deines et al., 1974; Keller, 1991) have speculated that 
the vadose zone is "where the action is" in the establishment of carbonate groundwater chemistry; Reardon et al. (1979) demonstrated it.

The dissolution of calcite into downward-flowing pore water eventually - on a timescale of hundreds to thousands of years - leads to observable depletion of the mineral. We can see that given a certain calcite content, the rate of that depletion would be proportional to the $\mathrm{PCO}_{2}$ and to the net water flux, i.e. the volume of recharging water per unit area subject to calcite equilibration within a given time. Thus it is that calcite is leached from soils (and, sometimes, subsoils) in settings where appreciable groundwater recharge occurs. The leaching boundary, above which calcite has been removed and below which it remains, is often quite sharp - consistent with rapid attainment of equilibrium (Reardon et al., 1979; Keller et al., 1991). This means that in a favorable geologic setting, calcite leaching can be quantified and related through Garrels and Christ's (1967) model to the long-term groundwater recharge rate. This problem is discussed further in section 4.6.4.

In vadose zones of arid regions, ionic concentrations may achieve high levels in part due to evaporative enrichment of shallow pore waters and/or the presence of highly-soluble salts of sulfate (e.g. gypsum) or chloride. The effects of such conditions on the carbonate system may be modeled by appropriate modification of [4.74]. For instance, to simulate the effect of high chloride in pore water equilibrating with calcite, a specified molality of $\mathrm{Cl}$ - is added to the charge balance. Drever (1997 chap. 3) gives clear examples of how simplifying assumptions yield easy, approximate manual solutions.

The attentive, experienced reader may be thinking - correctly - that calcite sometimes occurs in highly localized fashion, at the base of the soil zone, in geologic settings where carbonate minerals are otherwise absent. This "caliche" or "hardpan" does in fact reflect pore-water equilibrium with calcite, but to understand its occurrence we must discuss the hydrogeochemistry of silicate terranes.

\subsubsection{Silicate minerals and terranes.}

Silicate minerals, chemically dominated by silicon and oxygen, comprise the bulk of Earth's crust in the form of igneous, metamorphic, and sedimentary rocks. Thus silicate minerals are ubiquitous and occur in the vadose zones of carbonate terranes where they are mixed by depositional and soil-forming processes with carbonate minerals. Why, then, aren't all vadose zones considered silicate terranes - why do carbonate minerals exert such a dominant hydrochemical influence when they are present? The answer to this lies in the nature of silicate-water interaction; dissolution is gradual and limited by the formation of clay minerals, such that the products of equlibrium with carbonate minerals predominate in solution. Thus silicate terranes - hydrologically - are found only where carbonate minerals are present in negligible concentrations.

4.4.2.1 Silicate weathering. Recall that we are still, here, talking about mineral-water reactions which consume acid and yield solutes driving life and other geochemical cycles (Figure 8.1). Thus [4.69] applies, and it can be written as:

(8.89) $\mathrm{H}_{2} \mathrm{CO}_{3}{ }^{+}$silicates $=\mathrm{DIC}+\mathrm{H}_{4} \mathrm{SiO}_{4}{ }^{+}$inorganic solutes + clays

where, again, we have dropped the water on both sides for brevity. This form has two terms we have not seen before: $\mathrm{H}_{4} \mathrm{SiO}_{4}{ }^{\circ}$ or silicic acid (because it can generate a proton by hydrolysis, just like carbonic acid in [4.66], under the right pH conditions), and clays. So in this case, unlike the carbonate case, one (primary silicate) solid dissolves while another (secondary clay) forms, in a dissolution process that is termed incongruent: the stoichiometry of the products yielded into solution differs from the stoichiometry of the dissolving phase (by the stoichiometry of the secondary phase). These processes underlie the geochemical transformation of Earth's crust by weathering, and pedogenesis - which is characterized by formation and translocation of clays - in particular. An important note: here we are talking about clay minerals rather than the particle size fraction $(<2 \mu \mathrm{m})$ referred to as "clay" when we discuss hydraulic properties (chapter 2). As a matter of fact, clay minerals frequently are clay-sized, but the clay size fraction usually contains both clay and non-clay minerals, so the distinction is important.

Let's further explore the importance of silicate weathering by examining a particular reaction, the weathering of one of the most common of the primary silicates, plagioclase, to the clay, kaolinite: 


$$
\begin{array}{r}
2 \mathrm{Na}_{0.5} \mathrm{Ca}_{0.5} \mathrm{Al}_{1.5} \mathrm{Si}_{2.5} \mathrm{O}_{\mathrm{S}(\mathrm{plag})}+3 \mathrm{H}_{2} \mathrm{CO}_{3}+\frac{5}{2} \mathrm{H}_{2} \mathrm{O}= \\
3 \mathrm{HCO}_{3}^{-}+2 \mathrm{H}_{4} \mathrm{SiO}_{4}+\mathrm{Na}^{+}+\mathrm{Ca}^{2+}+\frac{3}{2} \mathrm{Al}_{2} \mathrm{Si}_{2} \mathrm{O}_{5}(\mathrm{OH})_{4(\mathrm{kaol})}
\end{array}
$$

This is a much "nastier" (stoichiometrically) reaction than the analogue for calcite ([4.70]), partly because of the additional solid term and partly because the formulae of plagioclase and kaolinite are more complicated. Plagioclase, in particular, contains decimal stoichiometric subscripts because this is the conventional way of writing the formulae for minerals which exhibit solid-solution behavior. (In this case, albite, $\mathrm{NaAlSi}_{3} \mathrm{O}_{8}$, may be considered to be dissolved in anorthite, $\mathrm{CaAl}_{2} \mathrm{Si}_{2} \mathrm{O}_{8}$ - or vice versa. There is nothing particularly significant about the 50/50 solution in 4.75; real plagioclases span the full range of solid-solution composition from pure albite to pure anorthite, depending on the rock type within which the plagioclase originally crystallized.)

Now we can look at the incongruency of silicate weathering more closely. The key is that all of the aluminum, $\mathrm{Al}$, in the reactant plagioclase goes into the product kaolinite. Al, in fact, generally "hates" to be in aqueous solution, to the point that in waters in the $\mathrm{pH}$ range 5-9, it may be assumed to be conserved in solid phases as in [4.75]. In other words, if $\mathrm{Al}$ is being released by dissolution, something else (kaolinite in this case) will precipitate to remove it from solution ${ }^{2}$. (A similar point can be made about vadose iron: when released from a primary mineral structure and oxidized to $\mathrm{Fe}^{3+}$, it is rapidly and almost completely precipitated into an oxide or hydroxide.) We can understand this on a thermodynamic basis by writing a reaction for the dissolution of kaolinite:

$$
\mathrm{Al}_{2} \mathrm{Si}_{2} \mathrm{O}_{5}(\mathrm{OH})_{4(\mathrm{kaol})}+6 \mathrm{H}^{+}=2 \mathrm{Al}^{3+}+2 \mathrm{H}_{4} \mathrm{SiO}_{4}+\mathrm{H}_{2} \mathrm{O}
$$

for which the equilibrium constant, using [4.25] and assuming pure water and kaolinite, is

$$
\mathrm{K}_{\mathrm{kaol}}=\frac{\mathrm{a}_{\mathrm{Al}^{3}+\mathrm{a}_{\mathrm{H}_{4} \mathrm{SiO}_{4}}^{2}}}{\mathrm{a}_{\mathrm{H}^{+}}^{6}}
$$

While thermodynamic data for clay minerals are generally poorly known, values suitable for rough calculations are given in Table 8.6, which also gives values for pertinent species in solution. Using [4.14] we obtain:

$$
\Delta \mathrm{G}_{\mathrm{R}}=\sum \operatorname{products}_{\mathrm{i}} \Delta \mathrm{G}_{\mathrm{fi}}-\sum \operatorname{reactants}_{\mathrm{i}} \Delta \mathrm{G}_{\mathrm{fi}}=-40,330 \mathrm{Jmol}^{-1}
$$

and, evaluating [4.26], we get:

$$
\mathrm{K}_{\mathrm{kaol}}=\frac{\mathrm{a}_{\mathrm{Al}^{3}+\mathrm{a}_{\mathrm{H}_{4} \mathrm{SiO}_{4}}^{2}}}{\mathrm{a}_{\mathrm{H}^{+}}^{6}}=10^{7.1}
$$

\section{Table 8.6*}

2. this is the incongruency mechanism called "via solution". Another mechanism is called "topotactic solution"; in this case the dissolving mineral is selectively leached, leaving behind the secondary phase. There is a continuum between the two mechanisms. 


\begin{tabular}{|c|c|c|}
\hline Species & $\underline{\text { formula }}$ & $\Delta \mathrm{G}_{\mathrm{f}}^{\circ}\left(\mathrm{kJ} \mathrm{mol}^{-1}, 298 \mathrm{~K}, 1 \mathrm{bar}\right)$ \\
\hline gibbsite & $\mathrm{Al}(\mathrm{OH})_{3}$ & -1151 \\
\hline kaolinite & $\mathrm{Al}_{2} \mathrm{Si}_{2} \mathrm{O}_{5}(\mathrm{OH})_{4}$ & -3800 \\
\hline Ca beidellite & $\mathrm{Ca}_{0.167} \mathrm{Al}_{2.33} \mathrm{Si}_{3.67} \mathrm{O}_{10}(\mathrm{OH})_{2}$ & -5388 \\
\hline Na beidellite & $\mathrm{Na}_{0.33} \mathrm{Al}_{2.33} \mathrm{Si}_{3.67} \mathrm{O}_{10}(\mathrm{OH})_{2}$ & -5382 \\
\hline liquid water & $\mathrm{H}_{2} \mathrm{O}$ & -237.13 \\
\hline aluminum ion & $\mathrm{Al}^{3+}$ & -485 \\
\hline calcium ion & $\mathrm{Ca}^{2+}$ & -553.58 \\
\hline sodium ion & $\mathrm{Na}^{+}$ & -261.91 \\
\hline silicic acid & $\mathrm{H}_{4} \mathrm{SiO}_{4}^{\circ}$ & -1316.6 \\
\hline \multicolumn{3}{|c|}{ *after Drever (1997) chap. 10 and their Appendix II. } \\
\hline
\end{tabular}

Now: how much plagioclase needs to dissolve before kaolinite equilibrium or saturation is reached? According to the stoichiometry of our plagioclase in [4.75], when one $\mathrm{mmol} \mathrm{L}^{-1}$ dissolves, $1.5 \mu \mathrm{mol} \mathrm{Al}$ and $2.5 \mu$ mol Si are released from the crystal structure. If these elements went into solution as $\mathrm{Al}^{3+}$ and $\mathrm{H}_{4} \mathrm{SiO}_{40}$ at $\mathrm{pH}=5.5$, the ion activity product (section 4.2.5) would be:

$$
\mathrm{IAP}_{\text {kaol }}=\frac{\left[1.5 * 10^{-6}\right]^{2}\left[2.5 * 10^{-6}\right]^{2}}{\left[10^{-5.5}\right]^{6}}=10^{9.6}
$$

and the saturation index would be (using [4.56]):

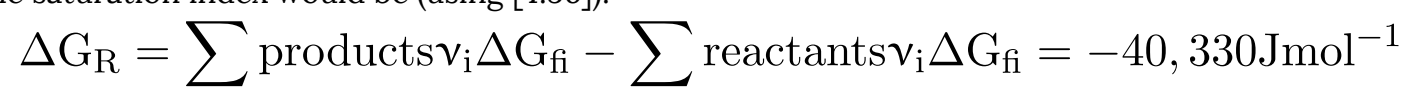

Recalling that saturation corresponds to $\log \mathrm{SI}=0$, we see that this solution would be supersaturated with respect to kaolinite, i.e. not even this very small micromolar concentration of $\mathrm{Al}^{3+}$ should remain in solution at this $\mathrm{pH}$, but instead should be scavenged from solution by precipitation. In soils where organic matter and dissolved organic carbon concentrations are large (section 4.3), $\mathrm{Al}^{3+}$ is complexed in solution by organic acids and attains concentrations of 0.1 millimolar (e.g. Zabowski and Ugolini, 1992) - $10^{2}$ times higher than our analysis neglecting organic acids would indicate. As far as we know, these effects are generally restricted to the solum. 


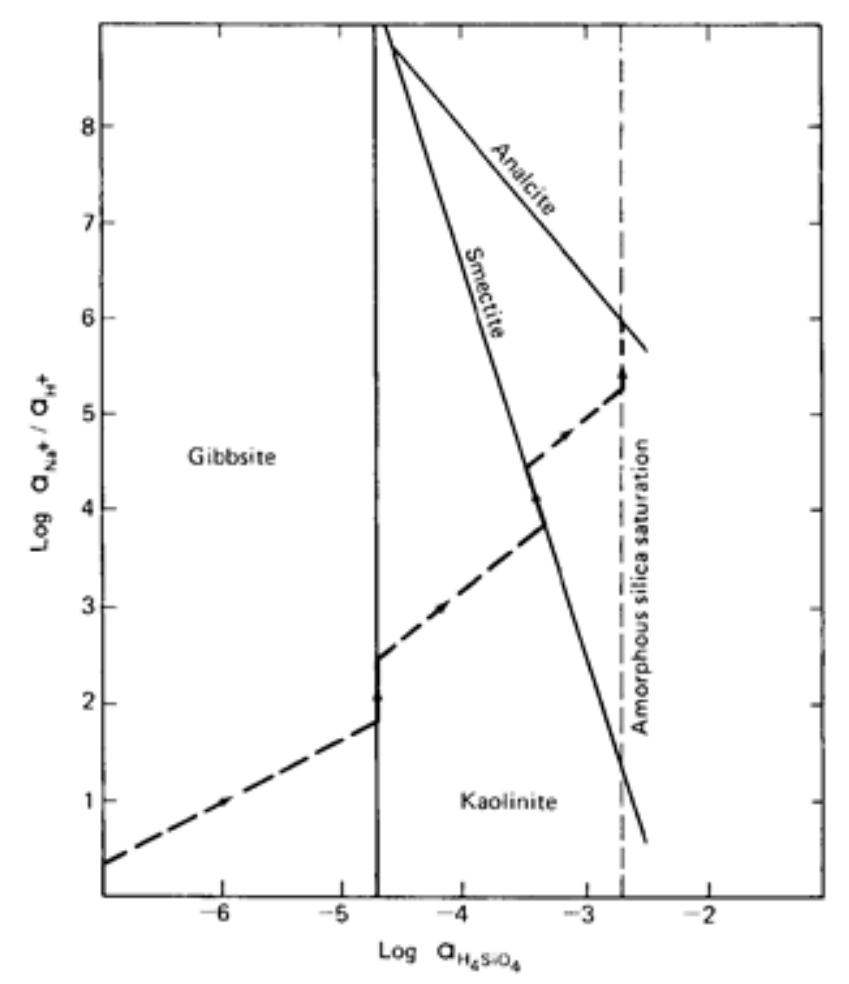

Figure 8.10 Incongruent dissolution of Na-feldspar. Precipitating secondary mineral changes as dissolution procresses. "Smectite" is a category encompassing montmorillonite and beidellites. From Drever (1997), permission to be asked.

It is important to pause here and explicitly recognize that our treatment of silicate-water interaction differs fundamentally from that of the other mineral families carbonates (represented by calcite) and evaporites (represented by gypsum). In the latter cases, we represented the minerals as dissolving to equilibrium (saturation) in solution, a thermodynamically predictable state; here, by contrast, we represent a silicate as dissolving gradually ("kinetically") while a secondary clay mineral precipitates in equilibrium (saturation) with the solution. As the silicate dissolves, $\mathrm{Al}$ and some of the $\mathrm{Si}$ are sequestered in the clay mineral; the rest of the $\mathrm{Si}$ (as silicic acid) and the cations $\mathrm{Na}^{+}, \mathrm{Ca}^{2+}$, build up in solution. This process is represented for pure albite (Na) feldspar in Figure 8.10, which also illustrates that the precipitating secondary mineral actually varies in a predictable sequence: very early in the reaction progress, when there is very little $\mathrm{H}_{4} \mathrm{SiO}_{4}{ }^{\circ}$ present, kaolinite is undersaturated ([4.76]) and gibbsite precipitates. $\mathrm{As}_{4} \mathrm{H}_{4} \mathrm{SiO}_{4}{ }^{\circ}$ increases, kaolinite reaches saturation and precipitates in preference to gibbsite. Eventually, when the cations and $\mathrm{H}_{4} \mathrm{SiO}_{4}{ }^{\circ}$ reach sufficient concentrations, smectite precipitates in preference to kaolinite and gibbsite. And all the while carbonic acid is being consumed (as in [4.75]) so the $\mathrm{pH}\left(\mathrm{a}_{\mathrm{H}^{+}}\right.$in Figure 8.10) tends to rise. For a given solution composition along the reaction path, we can predict which of the clays should precipitate by writing reactions between the pairs gibbsitekaolinite and kaolinite-beidellite, e.g.:

$$
\mathrm{Al}(\mathrm{OH})_{3(\text { gibb })}+\mathrm{H}_{4} \mathrm{SiO}_{4}=0.5 \mathrm{Al}_{2} \mathrm{Si}_{2} \mathrm{O}_{5}(\mathrm{OH})_{4(\mathrm{kaol})}+2.5 \mathrm{H}_{2} \mathrm{O}
$$

The equilibrium constant, assuming pure solids and water, and evaluated using data from Table 8.6 as above, is:

$$
\mathrm{K}_{(\text {gibb-kaol })}=\frac{1}{\mathrm{a}_{4} \mathrm{SiO}_{4}}=10^{4.4}
$$

This means that kaolinite does not become the preferred precipitate until silicate dissolution has generated more than 
$10^{-4.4} \mathrm{~m}$ of silicic acid; for smaller concentrations gibbsite should precipitate. (The alert reader will notice the conflict with our earlier finding on $\mathrm{p}$. _- that kaolinite would precipitate at solute concentrations of $10^{-6} \mathrm{~m}$. That conclusion was, very specifically, that kaolinite would be supersaturated (stable) at such concentrations, and that conclusion is correct. What we neglected to consider was that some other mineral, i.e. gibbsite, might be more stable than kaolinite and "outcompete" it for precipitating aluminum. This illustrates an important point about the application of geochemical principles, i.e. the utility of our findings depends on the adequacy of our geological model.) The same procedure applied to the kaolinite-beidellite pair yields a more complicated equilibrium constant containing activities of $\mathrm{Ca}^{2+} / \mathrm{Na}^{+}$and $\mathrm{H}^{+}$ as well as $\mathrm{H}_{4} \mathrm{SiO}_{4}{ }^{\circ}$. Drever (1997) gives detailed examples, and shows how the solution compositions corresponding to the various minerals' stability ranges may be presented in graphical form.

4.4.2.2 Weathering progress concepts. Now: in real vadose systems in silicate terranes, how fast and how far does this weathering process go? Primary silicate dissolution is a kinetic process, so a theoretician might say: "Simple: select the appropriate form of the rate law ([4.4]), and evaluate." Such rate expressions have indeed been experimentally developed, but it turns out that they predict weathering rates $10^{2}-10^{4}$ times greater than what hydrologists and geochemists observe in the field (Swoboda-Colberg and Drever, 1993). The many variables controlling natural subsurface rates, including occlusion of fresh silicate surfaces by precipitating clays, extent of contact with silicate surfaces during macroporous flows, and effects of plant exudates in the rhizosphere, are very difficult to reproduce in the laboratory. Thus although this is an active field of research (Lasaga et al., 1994) driven by a variety of fundamental questions (e.g. how do plant communities obtain their nutrients? How does the rate of reactions like 4.75 , which remove $\mathrm{CO}_{2}$ from the atmosphere in the conversion of carbonic acid to bicarbonate, vary with ecologic and geologic conditions?), we have no theoretically satisfactory means of predicting silicate weathering rates in the field.

Garrels and MacKenzie (1967) showed that the chemistries of ephemeral spring waters in the Sierra Nevada were consistent with silicate-to-kaolinite weathering, while perennial spring chemistries in the same area could be derived by weathering of the same primary minerals to a combination of kaolinite and Ca-beidellite. This interpretation, which underscored the correspondence of increased residence time to increased reaction progress (Figure 8.11), has been been applied with considerable success by hydrologists and geochemists to a wide variety of surface and groundwaters in silicate terranes in the decades since up to the present (e.g. Hyman et al., 1998). Waters precipitating kaolinite and/or beidellite are common; reaction progress to the point of saturation with respect to dissolving primary silicate minerals is rarely, if ever, reported. Rather, the characteristics of "evolved" waters are circumneutral to slightly alkaline $\mathrm{pH}$, moderate total dissolved solids (tens to hundreds of $\mathrm{mg} / \mathrm{L}$ ), with bicarbonate as the dominant anion ([4.75]). While these surface and groundwaters are the geochemical descendents of vadose pore waters, little attention has been paid to the extent to which vadose processes contribute to the observed results (Keller and Wood, 1993). 


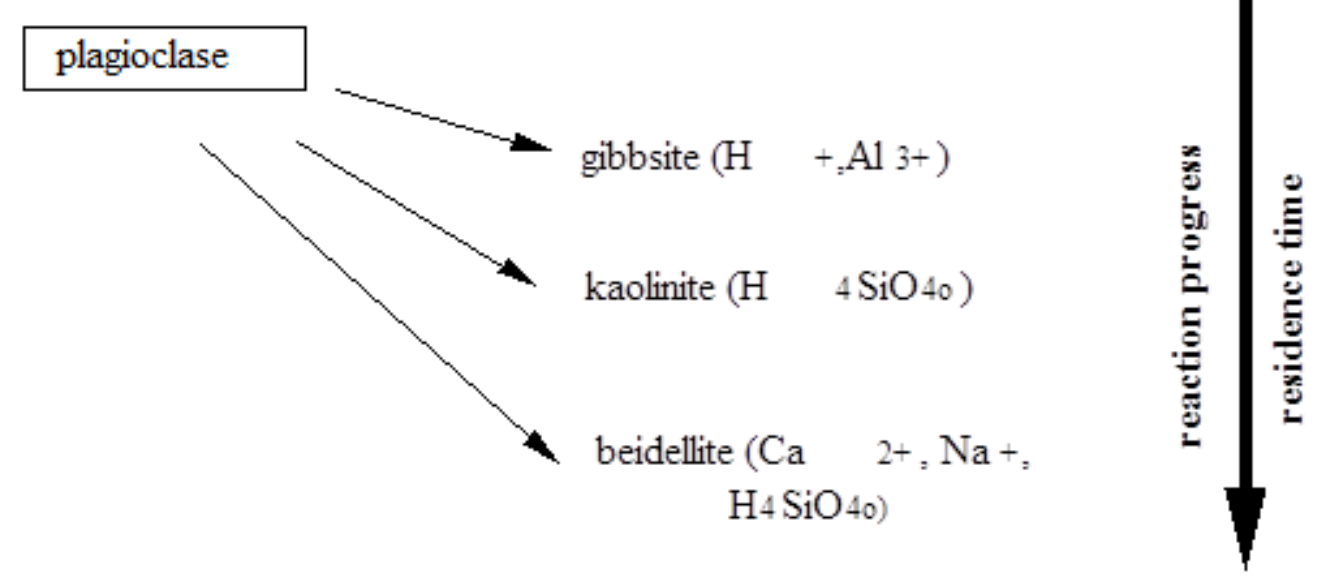

Figure 8.11 Geochemical evolution of subsurface waters in silicate terranes, according to the reaction-progress model used by geochemists and hydrologists. Arrows correspond to the reaction-progress trajectory shown in Figure 8.10. Plagioclase is example of common primary weathering mineral. Parenthesis indicates dominant cations in the water corresponding to precipitating clay mineral (formulae in Table 8.6).

In the soils community, the perspective is quite different (Jenny, 1980). The Jackson-Sherman weathering stages (Jackson and Sherman, 1953; e.g. Sposito, 1989) emphasize the ultimate availability of elements at a given moment in the development of a soil, as the primary control on clay mineralogy and water chemistry. For example (Figure 8.12), when weatherable plagioclase is present, beidellites and related clays form; later in pedogenesis, when the plagioclase (and the primary reservoir for $\mathrm{Ca}^{2+}$ and $\mathrm{Na}^{+}$) is exhausted, beidellite dissolves and kaolinite is the dominant clay mineral; and in advanced development, all silicon-bearing minerals (including kaolinite) have also been dissolved and leached away, such that gibbsite is dominant. This sequence leads ultimately to aluminous soils (e.g. bauxite) and low-TDS, acidic pore waters - a consequence of lack of soluble mineral material to consume acids (e.g. [4.75]). This weathering-stage model is a time-tested tenet of soil science; its empirical basis is much stronger than is the hydrogeochemical evolution model in application to waters. 


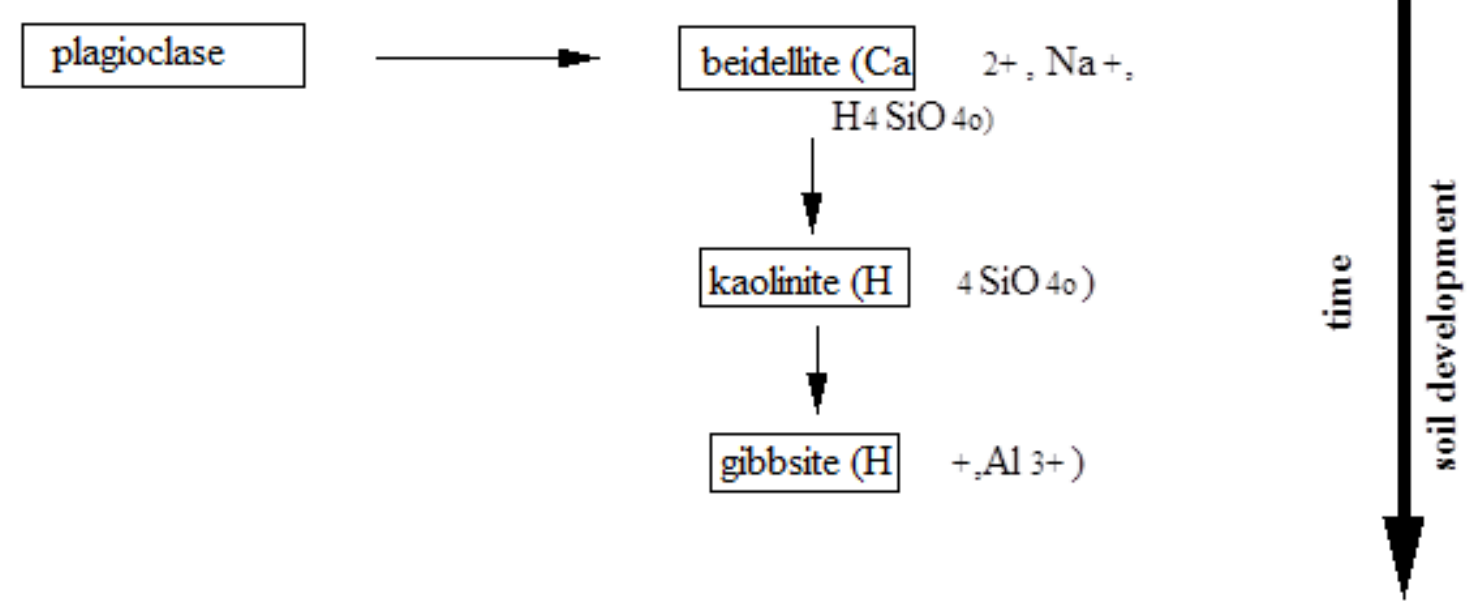

Figure 8.12 Silicate mineralogy and pore water chemical evolution in soils, according to concept of weathering stages. Plagioclase is an example of a common primary weathering mineral. Parenthesis indicates dominant cations in the water corresponding to dominant clay mineral (formulae in Table 8.6).

It is worth noting that the time sequences of clay-mineral occurrence and solution characteristics in Figures 8.11 and 8.12 are reversed. One key reason for this apparent opposition is difference in reaction rates associated with the nutrient needs amd physiology of plants: abundance of organic acids, physical structures, and intense freezethaw and wetting-drying cycles undoubtedly accelerate silicate weathering in soils relative to deeper regions of the subsurface. Plagioclase-to-beidellite reaction progress may occur annually in temperate-zone soils, while the same reaction progress may require tens to thousands of years in groundwater systems. Another clear difference between the two sequences is that plagioclase (like all primary silicates) in the solum is exhaustible on the timescale of soil development, whereas it is present in effectively infinite amounts - as a geochemical reservoir - along most subsoil flow paths.

4.4.2.3 Caliche. We do know that in silicate terranes, calcite precipitation often occurs at some point along a subsurface flow path. How can this occur: calcite out of thin air? There is no arguing with the facts, and [4.75] reveals the mechanism. As silicate dissolution progresses, three things happen: (a) $\mathrm{Ca}^{2+}$ increases, (b) $\mathrm{HCO}_{3}{ }^{-}$increases, and (c) $\mathrm{H}^{+}$is consumed, i.e. the $\mathrm{pH}$ increases. The latter two processes result in progressive increase in $\mathrm{CO}_{3}{ }^{2-}$ ([4.67]), which, together with the first process, eventually leads to calcite saturation ([4.72]). Both evaporation and loss of $\mathrm{CO}_{2}$ by degassing can be the "trigger" for calcite precipitation under these circumstances, the former by concentrating solutes and the latter by elevating the $\mathrm{pH}([4.65-4.67])$. Both of these processes can occur in soils on a seasonal basis with wetting/drying and the waxing/waning of respiration ([4.58]). The resulting caliche (also known colloquially in the North American west as "hardpan") is common in the soils of semi-arid and arid regions, where calcite saturation is attained in pore waters immediately beneath the solum. Caliche formation is extremely important: the secondary calcite in these horizons is fine-grained, reducing gas and water permeability; plant growth and contaminant-transport variables (pore-water $\mathrm{pH}, \mathrm{Ca}^{2+}$, and carbonate species) are strongly affected; and, more recently, geologists have realized that the calcite in caliche contains carbon- and oxygen-isotopic information about the atmosphere at the time of formation (Kelley et al; McFadden et al.). Conversely, humid conditions favor deep percolation before calcite saturation. Also, we know that increase in humidity favors greater biological productivity, hence greater acid production; this should elevate the rate at which soil development proceeds and primary silicates are exhausted ([4.75] and using Figure 8.12), in which case $\mathrm{Ca}^{2+}$ production and $\mathrm{pH}$ increase do not occur.

Our statement that primary silicate availability is prerequisite to caliche formation is correct but actually somewhat incomplete. Primary silicate mineral assemblages vary chemically, as shown in Figure 8.13. A syenite (or its volcanic equivalent trachyte) contains very little $\mathrm{Ca}$, while the plagioclases and pyroxenes in gabbroic/basaltic rocks contain 
abundant $\mathrm{Ca}$. The mineralogies of real soils and vadose zones are often complicated mixtures which depend on transport and depositional as well as petrologic processes, but the Figure shows that other factors being equal, caliche formation (and $\mathrm{Ca}^{2+}$-rich pore waters) will be favored given the latter mineral assemblage. However, mafic (Ca-Fe-Mg rich) mineral assemblages also weather more quickly than felsic assemblages, due to their lower stability under Earth surface conditions, and this means that caliche generation in mafic terranes is enhanced early in soil development relative to later stages. Here we see a particular illustration of a general rule, which is that soils derived from different parent assemblages converge geochemically (in this case, to absence of calcite) as soil development advances.

\begin{tabular}{|c|c|c|c|c|c|}
\hline $\begin{array}{l}\text { IGNEOUS } \\
\text { ROCK }\end{array}$ & $\begin{array}{c}\text { syenite } \\
\text { (trachyte) }\end{array}$ & $\begin{array}{c}\text { granite } \\
\text { (rhyolite) }\end{array}$ & $\begin{array}{c}\text { diorite } \\
\text { (andesite) }\end{array}$ & $\begin{array}{l}\text { gabbro } \\
\text { (basalt) }\end{array}$ & peridotite \\
\hline
\end{tabular}

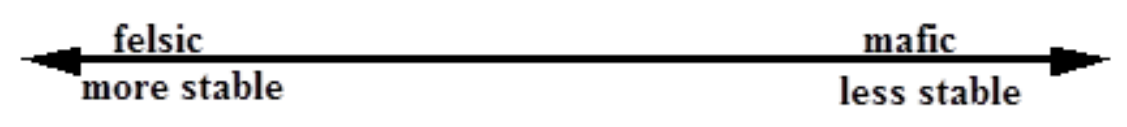

\section{DOMINANT MINERALS}

\section{LEACHABLE CATIONS*}

\author{
orthoclase
}

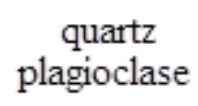
plagioclase pyroxene

pyroxene olivine

Figure 8.13 Some aspects of the mineralogy and chemistry of igneous rocks. Volcanic equivalents of plutonic rocks are given in parentheses. See e.g. Brownlow (1996) for more details. Mixtures of igneous, metamorphic, and sedimentary rocks can also be located on these continuua.

*excludes $\mathrm{Si}$, which is abundant in all minerals shown.

Percolating pore waters may encounter a reservoir of weatherable silicates beneath soils which have been leached of these same minerals. Under these conditions, saturation with respect to calcite may be attained more slowly along subsurface flow paths. This process has been reported in groundwater systems (Freeze and Cherry, 1979) and it likely occurs in vadose zones as well.

\subsubsection{Evaporites and evaporation.}

Evaporites are the minerals which form by precipitation out of surface waters, including seawater, by evaporative concentration. They are highly soluble, i.e. their solubility products are large, such that subsurface waters encountering these minerals become rapidly and highly mineralized. The "flip side" of this observation is that high solubilities lead to rapid leaching of these minerals from soil and vadose environments, such that they are not usually encountered in recharge (net downward flow) settings; their occurrence is reknowned in much deeper environments such as petroleum reservoirs where flow and thus leaching rates are very small. We can reason, however, that we should sometimes find vadose evaporites (1) whose solubilities are relatively small, where (2) they are abundant in the mineral fraction, where (3) recharge rates are small, and where (4) the shallow subsurface is geologically youthful, i.e. has been exposed to weathering/leaching for a short time.

4.4.3.1 Gypsum. The four criteria lead to two regional, shallow-subsurface occurrences of gypsum $\left(\mathrm{CaSO}_{4} \bullet 2 \mathrm{H}_{2} \mathrm{O}\right.$; 
section 4.2.5) in the U.S. and Canada. One is in west Texas and southeastern New Mexico, where high-level nuclear waste disposal is being implemented as of this writing. The second, most geographically widespread case is in the surficial clayey tills of the Interior Glaciated Plains (Back et al., 1988). This gypsum is actually a secondary mineral in that it has formed in till vadose zones by oxidation of sulfides (species containing reduced sulfur), derived from the marine shales incorporated into the tills, to sulfate (Hendry et al., 1986):

$$
\mathrm{FeS}+\frac{9}{4} \mathrm{O}_{2}+\frac{5}{2} \mathrm{H}_{2} \mathrm{O} \longrightarrow \mathrm{Fe}(\mathrm{OH})_{3}+\mathrm{SO}_{4}^{2-}+2 \mathrm{H}^{+}
$$$$
\mathrm{Fe}(\mathrm{OH}) 3+\mathrm{SO}^{\wedge}\{2-\} 4+
$$

$\backslash$ end\{equation*\}" title="Rendered by QuickLaTeX.com">

followed by combination with calcium (derived from carbonate minerals also abundant in the shales):

$$
\mathrm{CaSO}_{4} \bullet 2 \mathrm{H}_{2} \mathrm{O}=\mathrm{Ca}^{2+}+\mathrm{SO}_{4}{ }^{2-}+2 \mathrm{H}_{2} \mathrm{O}
$$

The unidirectional arrow in [4.77] is used to indicate that the oxidation is strongly spontaneous (doesn't reach equilibrium) in vadose zones where atmospheric $\mathrm{O}_{2}$ is present. These reactions have generated a reservoir of gypsum in the vadose zones of clayey tills which is being slowly depleted where recharge rates are small.

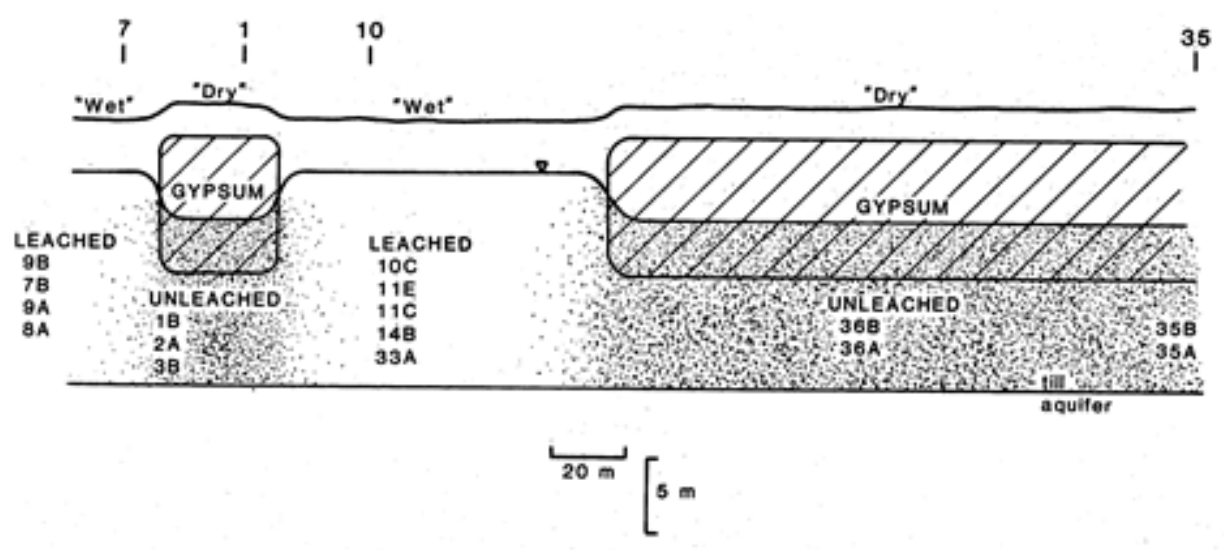

Figure 8.14 Cross section showing lateral variation in leaching of gypsum from vadose till, due to microtopographic focussing of recharge (Keller et al., 1991). Topographic variation is exaggerated with respect to overall vertical scale by 2 times. Alphanumeric entries indicate lateral positions of wells; waters from wells in unleached zones are saturated with respect to gypsum and have sulfate concentrations about 10x greater than wells in leached zones.

Beneath depressions in prairie landscapes, where springtime recharge is focussed, vadose (oxidizing) zones are thin and the gypsum never formed at all: in these environments sulfate generation is outpaced by leaching, in analogy with lack of caliche formation in humid climates. The result (Figure 8.14) is a laterally-variegated vadose geochemistry, wherein groundwater in underlying aquifers is undersaturated with respect to gypsum - and potable - because most of it has percolated through gypsum-free vadose zones beneath recharge foci (Keller et al., 1991; Fortin et al., 1991).

4.4.3.2 Evaporative concentration. There is another important vadose process - evaporation - which can yield highly mineralized pore waters and (sometimes) secondary evaporite mineral formation. There are two genera of important evaporation scenarios (Figure 8.15): (1) concentration of solutes in recharging pore waters as they move through the active zone; and (2) concentration of solutes in discharging pore waters. 


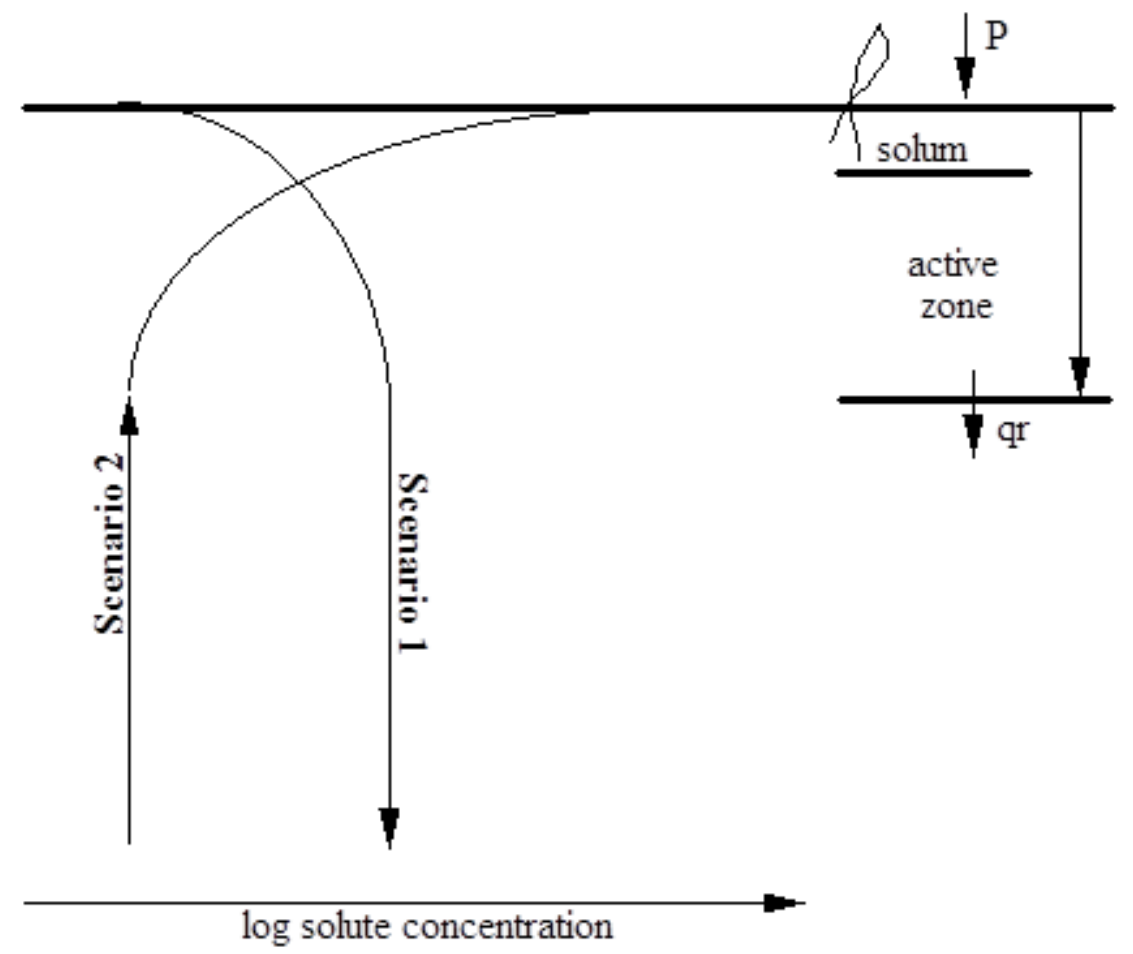

Figure 8.15 Two scenarios by which evaporation concentrates solutes in vadose pore waters. Flow is downward in scenario 1, which has been documented in research on chloride mass balance (P and $q_{\mathrm{r}}$ refer to [4.79]). Upward flow (scenario 2) is a common cause of natural soil salinization.

Scenario (1) has received much attention recently in application to estimating recharge rates by chloride $\left(\mathrm{Cl}^{-}\right)$mass balance. This approach relates the $\mathrm{Cl}^{-}$concentration achieved in recharge at the base of the active zone, $\mathrm{C}_{\mathrm{r}}$, to the $\mathrm{Cl}^{-}$ concentration in precipitation, $\mathrm{C}_{\mathrm{p}}$, via the evaporation ratio $\mathrm{P} / \mathrm{qr}$ :

$$
\frac{\mathrm{C}_{\mathrm{r}}}{\mathrm{C}_{\mathrm{p}}}=\frac{\mathrm{P}}{\mathrm{q}_{\mathrm{r}}}
$$

where $\mathrm{P}$ is the precipitation flux $\left(\mathrm{LT}^{-1}\right)$ and $\mathrm{qr}_{\mathrm{r}}$ is the recharge flux as in [4.78]. The mass-balance nature of [4.79] is readily apparent if we cross-multiply the equation by the two denominator terms; this yields an equality of solute fluxes. We explore the practical application of this relationship below (section 4.6). In Scenario 2 (Figure 8.15), because the flow path is upward, evaporation and concentration of solutes also increase in the upward direction. This situation can result in very large pore-water concentrations and precipitation of evaporites more soluble than gypsum in soils, due to intensity of evaporation near the ground surface. Which evaporites form, and how the pore-water chemistry evolves, depends on the initial solution composition and follows two rules: less-soluble minerals reach saturation and precipitate first; and ions concentrated in solution are those with larger initial concentrations than their precipitating co-ions. These rules, known as the Hardie - Eugster model (Hardie and Eugster, 1970), explain why saline solutions obtained by evaporation are characterized by a small number of "surviving" ionic solutes usually including $\mathrm{Na}^{+}$and $\mathrm{Cl}^{-}$(because salts of sodium and chloride are so soluble). Drever (1997) presents clear examples and case studies. These processes have implications for understanding the occurrence and effects of soil salinization in connection with irrigated agriculture, discussed briefly below in section 4.5.4.2. 


\subsubsection{Vadose hydrochemical evolution.}

In this section we will attempt a brief summary of natural vadose hydrogeochemistry in the form of a semiquantitative portrayal of bulk pore-water concentration as it evolves along a downward vadose flow path (Figure 8.16). The Figure "works" as follows: choose a starting point at ground surface; follow the shaded envelope of silicate concentrations downward through leached zones until an additional mineral assemblage is encountered; move laterally to the shaded envelope representing that assemblage before resuming downward progress. Where such assemblages are present, i.e. in non-silicate terranes, the depths of leached zones could have almost any value depending on rock relations, vadose hydrology, and landscape maturity. (This is why no depth scale is shown.) Conversely where such assemblages are not present, i.e. in a silicate terrane, no lateral moves are made. Figure 8.16 represents a combination of observation and prediction: it attempts to honor published data, which usually pertain to only a part of the profile; and it attempts to interpolate and extrapolate using the predictive tools - gas-water-solid equilibrium models, kinetic weatheringprogress models, geologic and pedologic observations - which we have discussed earlier in this section. We have no doubt that interesting and perhaps important considerations have been omitted in this brief treatment, and we ask readers to view our work here as stimulus for further thought and discussion. We defer discussion of the nuts-and-bolts of modeling calculations to section 4.7 below.

Let's begin with the silicate concentration envelope which represents most soils - due to the occurrence of at least some leaching, even in dry-climate settings - and many subsoils. Dominant cations are shown in Figures 8.11 and 8.12, and the dominant anion is HCO3-. The TDS range shown in Figure 8.16 is fairly well defined by reported soil porewater chemistries (e.g. Zabowski and Ugolini, 1992), and shallow groundwater chemistries (Freeze and Cherry, 1979) respectively; and they are consistent with the few vadose-hydrochemical studies which encompass soils and subsoils in these terranes (e.g. Antweiler and Drever, 1983; White et al., 1998). The two perspectives on silicate weathering represented by Figures 8.13 and 8.14 have contrasting implications for hydrochemical evolution as shown in Figure 8.16. Following Figure 8.11 the hydrogeochemical ("HG") model implies a gradual increase with depth to the water table, below which reaction progress may also continue. 


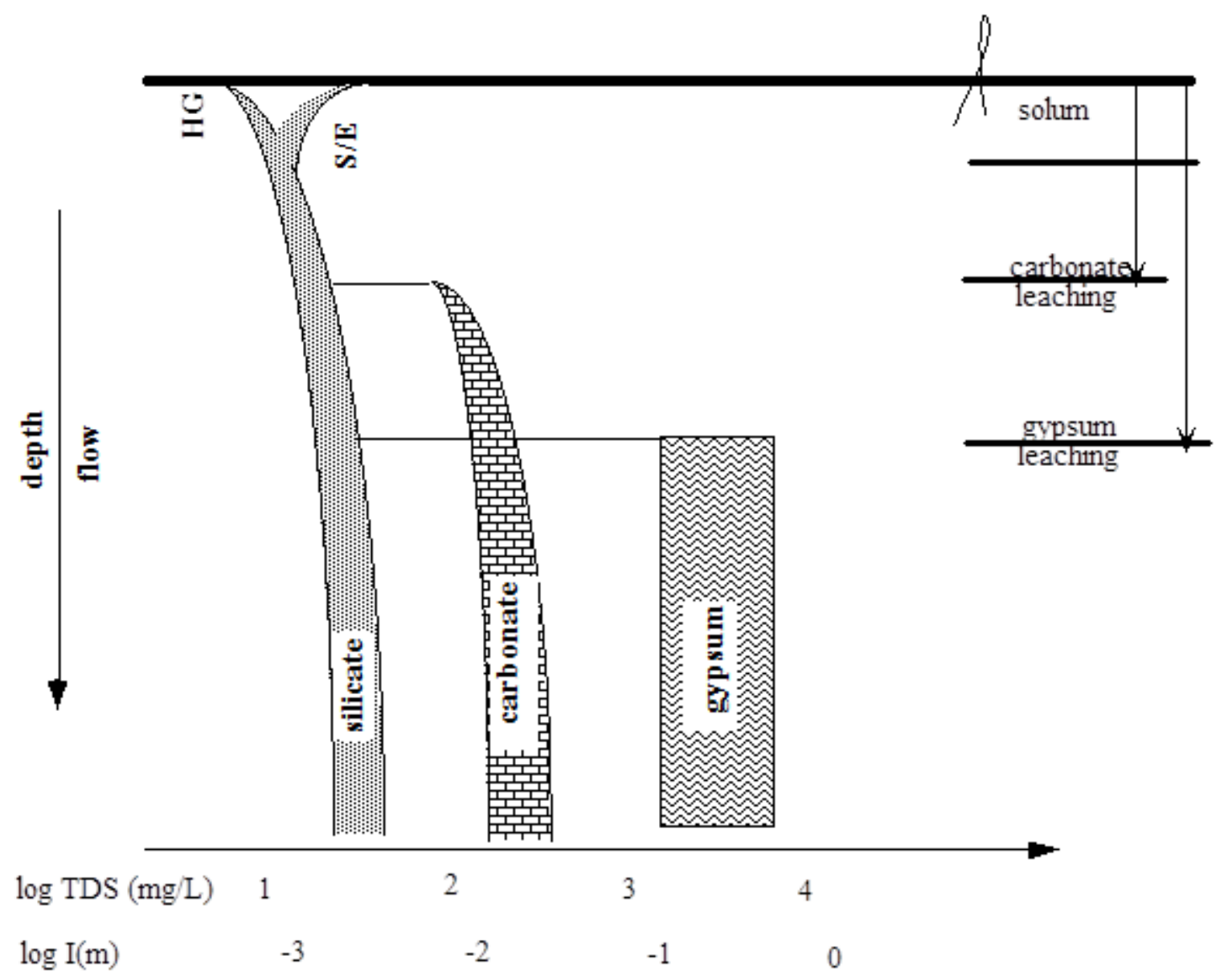

Figure 8.16 Geochemical evolution trajectories along a downward flow path through the vadose zone. Solution concentration axis is approximate; patterned areas suggest naturally-occurring ranges of pore-water mineralization.

The soils/ecosystems ("S/E") model, also represented in Figure 8.16, implies that pore-water mineralization attains relatively high levels in the biophysically dynamic soil zone. Following Figure 8.12, we might logically think that these occurrences should be limited to settings from which weatherable primary silicates have not been exhausted. But this is not the case; even in heavily leached tropical forest soils, pore waters exhibit substantial concentrations of the major elements (e.g. White et al. 1998). Also, the profile for the S/E model in Figure 8.16 suggests concentration decreases along downward flowpaths through the solum. These apparently puzzling features of the S/E model are explained by ecosystem ecologists' understanding of how nutrient cycling in soils is controlled by plant-community activity (Schlesinger, 1997; Likens and Bormann, 1995). Throughfall leaches solutes from above-ground plant surfaces; at the ground surface a litter layer rapidly decomposes and further releases solutes in large quantities to infiltrating water. These nutrients are then subject to root uptake, satisfying plant-metabolic needs, as water moves through the solum. If a plant community is highly efficient at recycling its nutrients in this manner, it may subsist at extremely low levels of net mineral-weathering input (the tropical scenario). In this way of thinking, the solute load in water moving below the solum is "leakage" of nutrients which are lost to the immediate ecosystem.

Few studies have observed major element pore-water chemistry from the litter layer downward through soil-vadose continuua; however the weight of available evidence (e.g. Zabowski and Ugolini, 1990; Arthur and Fahey, 1993; White et al., 1998; summary by Schlesinger, 1997 (chapter 6)) probably supports the soils/ecosystem model at least for humid climates. We must re-emphasize the speculative nature of both the shapes of the profiles as well as how they might vary with soil development and geologic setting. It's also worth saying explicitly that primary silicate mineral weathering 
generates the clay minerals present in vadose and all other environmental systems; and clay minerals themselves exert strong controls on water quality and solute transport via their crystallographic and sorptive properties, as discussed in section 4.5 and chapter 5 . The clays we find in a given vadose location may, of course, have been generated in situ, and/ or transported and deposited there.

Another scenario consistent with both hydrogeochemists' and soils/ecosystems scientists' observations in silicate terranes is that deep percolation (recharge) substantially bypasses the solum via macropore flow, injecting fresh water directly into the region of relatively slow reaction progress. This may, in fact, be common in many winter-precipitation climates.

Now we turn to the envelopes of pore-water chemistry profiles for carbonate terranes (Figure 8.16) which we encounter below the carbonate-leached zone. In this case the vadose data base is somewhat better, partly because gas-phase monitoring yields water-chemistry insight via the $\mathrm{PCO}_{2}$ (sections 4.3 and 4.4.1). Total dissolved solids are greater than in silicate terranes because of the relative rapidity with which calcite - a fairly soluble mineral - dissolves to equilibrium. Dominant cations are $\mathrm{Ca}^{2+}$ (and $\mathrm{Mg}^{2+}$ where dolomite is present) and the dominant anion is $\mathrm{HCO}_{3}{ }^{-}$. Note that as we trace a downward flow path, TDS remains low at the silicate-terrane level until we traverse the depth to which carbonate minerals have been leached, which depends on initial mineral mass fraction, recharge rate, time, and $\mathrm{PCO}_{2}$ - i.e. the factors represented in [4.78]. This leached depth may vary from negligible in young soils to many meters in hydrologically mature settings. Note, too, that the TDS is shown to increase throughout the zone of carbonate occurrence. This is not analogous to the silicate case - kinetic dissolution of the primary mineral - but is due, rather, to increase of $\mathrm{PCO}_{2}$ with depth (section 4.3.3) coupled with equilibrium dissolution of carbonate, yielding increasing solution concentrations with depth, in response (sections 4.3.3.3 and 4.4.1). While the $\mathrm{PCO}_{2}$ is represented as the "driving force" in this scenario, note that the $\mathrm{PCO}_{2}$ of the solum gas phase does not determine water quality deeper in the vadose zone, much less in groundwater at the bottom (Figure 8.16).

When gypsum is present, it dissolves rapidly to equilibrium and pore-water concentrations are large. Given its large solubility, we might expect a greater leaching depth than for carbonates (Figure 8.16), although of course this depends on the relative quantities of gypsum and carbonates initially present and any ongoing depositional inputs. Recalling that a given equilibrium has a unique constant at a given pressure and temperature ([4.25] and [4.26), we might be tempted to try to predict pore water composition using [4.32], [4.54], and speciation calculations (section 4.4.1.3). However, vadose gypsum is often accompanied by other soluble minerals which complicate the situation and give rise to a range of pore-water concentrations as suggested in the Figure. The most common and important of these accompaniments is carbonate minerals. Dissolution of gypsum in a water already saturated with respect to calcite produces $\mathrm{Ca}^{2+}([4.41])$ and thus immediately causes supersaturation and precipitation of calcite ([4.72]); this is, incidentally, called the common ion effect in solution chemistry. As $\mathrm{Ca}^{2+}$ is consumed by this precipitation and its activity is reduced, more gypsum dissolves and $\mathrm{SO}_{4}{ }^{2-}$ builds up in solution until the ion activity product equals the solubility product ([4.43]). The result is a high- $\mathrm{SO}_{4}{ }^{2-}$ water - often thousands of $\mathrm{mg} / \mathrm{L}$ of sulfate - saturated with respect to both gypsum and calcite. The $\mathrm{Ca}^{2+}$ concentration depends on the $\mathrm{PCO}_{2}$ via the carbonate-system equilibria and also, typically, on ion-exchange processes associated with clays (section 4.5.4) which may yield $\mathrm{Na}^{+}$into solution. The water of Table 8.2 is a typical example of the result. Geochemical modeling of these coupled processes is usually handled with a computer code (section 4.7 below).

Figure 8.16 illustrates the results of water-mineral interactions, which explain most available data collected in humidto semi-arid climates. In arid settings, studies of chloride profiles suggest that evaporation may be the most important of the processes controlling pore-water chemical evolution. The concentration axis in Figure 8.15 is not scaled, because available hydrochemical data in these settings is largely restricted to single solutes. However, these data indicate that arid-climate pore waters attain TDS levels in the $10^{4} \mathrm{mg} / \mathrm{L}$ range in scenario 1 (recharge settings), and even larger concentrations in scenario 2 (discharge settings).

We've mentioned cation exchange only in passing here. Our justification is that this process affects pore-water chemical evolution transiently, i.e. when pore-water chemistry is changing in time due to reasons other than exchange. Clearly these situations may be very interesting and important, and we discuss the issues further below in section 4.5.4. 


\subsection{Sorptive Processes.}

Equilibrium expressions are often used to quantify the effects of sorptive processes on pore water chemistry. In order to discuss these expressions in light of their limitations, we must investigate some aspects of underlying mechanisms. First, as to terminology: sorption is a general, catch-all term which takes in a wide variety of processes by which solutes bind to solids. Adsorption refers to the subset of such processes which occur at the solution-solid interface; a bsorption refers to processes by which solute is partitioned into the solid. The latter process appears to be particularly important for organic solutes, and we will defer further discussion until later.

\subsection{Adsorption.}

In section 2.3.2.2.1, the equation of state for a surface phase was derived from the fundamental equation ([I.33]) by subtracting the internal energy components of the two phases separated by the surface, from the total system internal energy. That equation of state ([2.30]) may be written as:

(8.102) $\mathrm{d} \sigma \Sigma=\mathrm{S}_{\mathrm{a}} \mathrm{dT}$

where $\sigma$ is an intensive state function called the surface tension or energy per unit area required to create the interface (section 2.3.2), the greek sigma $\Sigma$ is the interfacial area, and $\mathrm{S}_{\mathrm{a}}$ is the entropy of the interface. Recalling [4.17], we see that for constant-pressure conditions:

(8.103) $\mathrm{S}_{\mathrm{a}} \mathrm{dT}=-\mathrm{dG}$

which, substituted into [4.80] gives:

(8.104) $\mathrm{d} \sigma \Sigma=-\mathrm{d} \mathrm{G}_{\mathrm{a}}$

or

$$
-\frac{\mathrm{d} \sigma}{\mathrm{d} G_{\mathrm{a}}}=\frac{1}{\Sigma}
$$

This expression is generalized for component $i$ of the surface phase by multiplying both sides by the number of moles of substance i, which transforms $\mathrm{G}_{\mathrm{a}}$ to $\mu_{\mathrm{i}}$ and gives (e.g. Stumm and Morgan,1981, Table 10.3):

$$
-\left(\frac{\partial \sigma}{\partial \mu_{i}}\right)_{P, T, u_{j}}=\text { Gamma }_{i}
$$

where $\Gamma_{\mathrm{i}}$ is moles of surface component i per unit interfacial area, $\mathrm{n}_{\mathrm{i}} \Sigma^{-1}$. Relation [4.81] is known as the Gibbs equation and it shows how adsorption (the right-hand side) is related to variation in surface tension. Specifically, adsorption will increase to the extent that the derivative becomes more negative, i.e. to the extent that surface tension decreases with increase in chemical potential (or activity, via [4.20]). Qualitatively, this means that solutes which decrease the surface tension of the solution phase will tend to be increasingly adsorbed as their concentration (activity) in solution increases, whereas solutes which increase the surface tension of the solution will tend not to be. We can understand this as the tendency of surface-tension-lowering solutes to migrate to the interface because less energy per unit area is thereby required to establish it. Thus, as Stumm and Morgan point out, solutes which break up the strong, hydrogen-bonded structure of water - nonionic solutes, particularly organic substances - are adsorbed at solid surfaces; and conversely, many ions, which increase the strength of the water-molecular structure, cannot be adsorbed unless there exists some countervailing binding force between the ion and the solid.

The ubiquity of the adsorption of ionic solutes, particularly cationic ones, means that such binding forces must also be ubiquitous. Adsorptive binding of solutes to surfaces may occur via electrostatic forces or, alternatively, by stronger bonds of a chemical nature. A huge literature exists discussing these binding variations and their implications for environmental problems; Weber et al. (1991) provide a recent review within a subsurface-hydrologic context. For our 
purposes, the categorization suggested by Sposito (1989) is useful in that it refers to distinctions already adopted for complexes: adsorbed species may exist as (1) inner-sphere surface complexes, where water of hydration is eliminated from the immediate bonding region; as (2) outer-sphere surface complexes, where hydration sheaths remain more or less intact and interposed between the species and the surface; or as (3) diffuse species, associated with the surface in only a delocalized sense. The former two are associated with surface functional groups (section 1.5.3) as illustrated in Figure 8.17 .

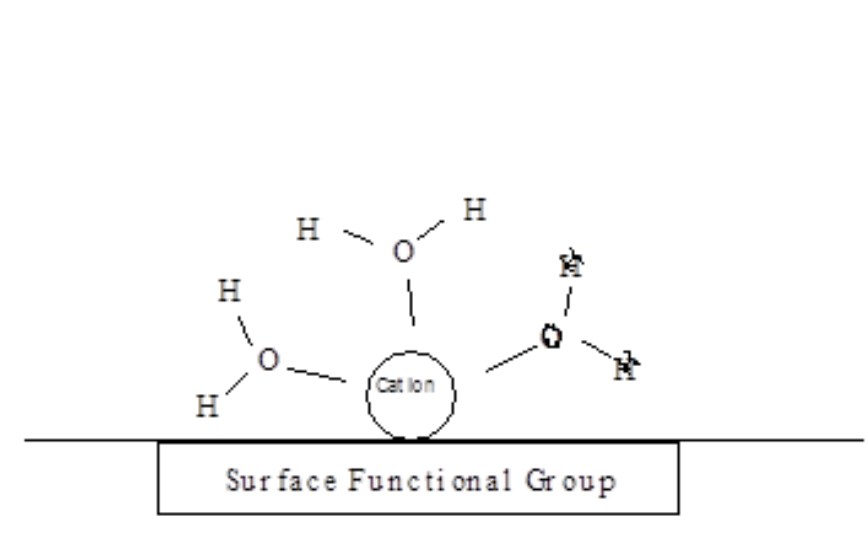

(a) Inner sphere complex

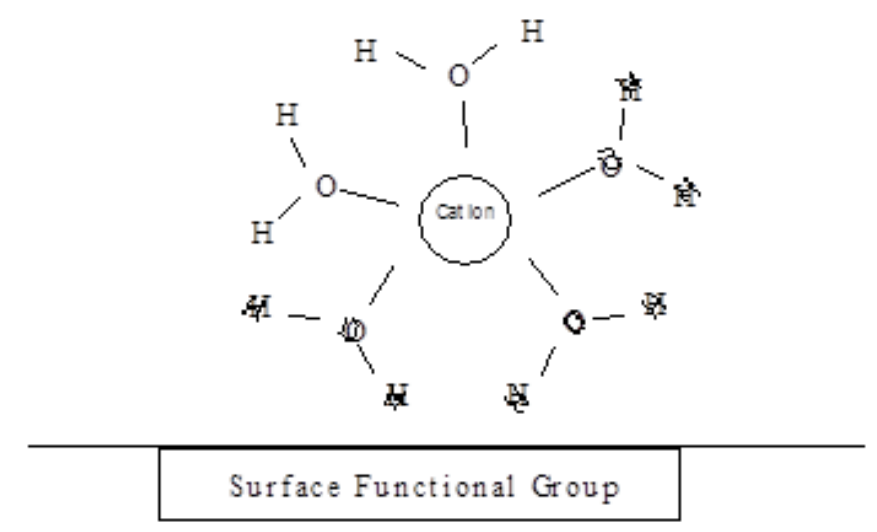

(b) Out er spher e complex

Figure 8.17 Two kinds of ionic binding to mineral surfaces. Surface fuctional groups are discussed in section 1.5.3. (a) Inner-sphere complexes are specifically adsorbed, while (b) outer-sphere complexes are non-specifically adsorbed. The latter, along with diffuse species, are exchangeable.

The latter two kinds of binding are relatively weak and reversible, and are also commonly called nonspecific (as contrasted with type (1), or specific) adsorption. Nonspecific adsorption is thus relatively amenable to equilibrium treatment, whereas specific adsorption tends to be relatively irreversible.

\subsubsection{The diffuse double layer.}

We saw in section 1.5.3 that both nonspecific and specific adsorption can occur (a) in interlayers of clay minerals or layer silicates, and (b) at $\mathrm{H}^{+}$-exchanging surfaces of a variety of solid materials. Now we will attempt to reach some generalizations about the application of these concepts in vadose environments where water contents are variable, such that sorbing surfaces are "bathed" in varying amounts of water. To do this we must make an excursion into the theory of aqueous ion distribution near a sorbing surface.

4.5.3.1 Diffuse Layer Theory. The charge associated with a solid surface is neutralized by an accumulation of counterions in the solution close to the interface. van Olphen's (1977, pp. 29-30) characterization is apt:

"The counter-ions are electrostatically attracted by the opppositely charged surface. At the same time, however, these ions have a tendency to diffuse away from the surface towards the bulk of the solution, where their concentration is lower. This situation is analogous to that in the earth's atmosphere, in which the gas molecules are subject to the competition between gravitation and diffusion. The action of the two competitive tendencies results in an equilibrium distribution of gas molecules in which their concentration gradually decreases with increasing distance from the earth's surface...The same type of distribution is obtained in the double layer...The counter-ion atmosphere is often referred to as the diffuse or Gouy layer." 
There is also, simultaneously, a deficiency of co-ions in the diffuse layer due to electrostatic repulsion which is counterbalanced to some extent by diffusion from the bulk solution toward the repulsive surface. This deficiency is not equal in magnitude to the counter-ion excess; if it were, the diffuse layer would not serve to neutralize the surface charge. The situation is shown schematically in Figure 8.18. This schematic does not show any ions selectively adsorbed at the surface in what is known as a Stern layer (Sposito, 1989; Bohn et al., 1979; Stumm and Morgan, 1981, 1996) which can be important under certain circumstances.

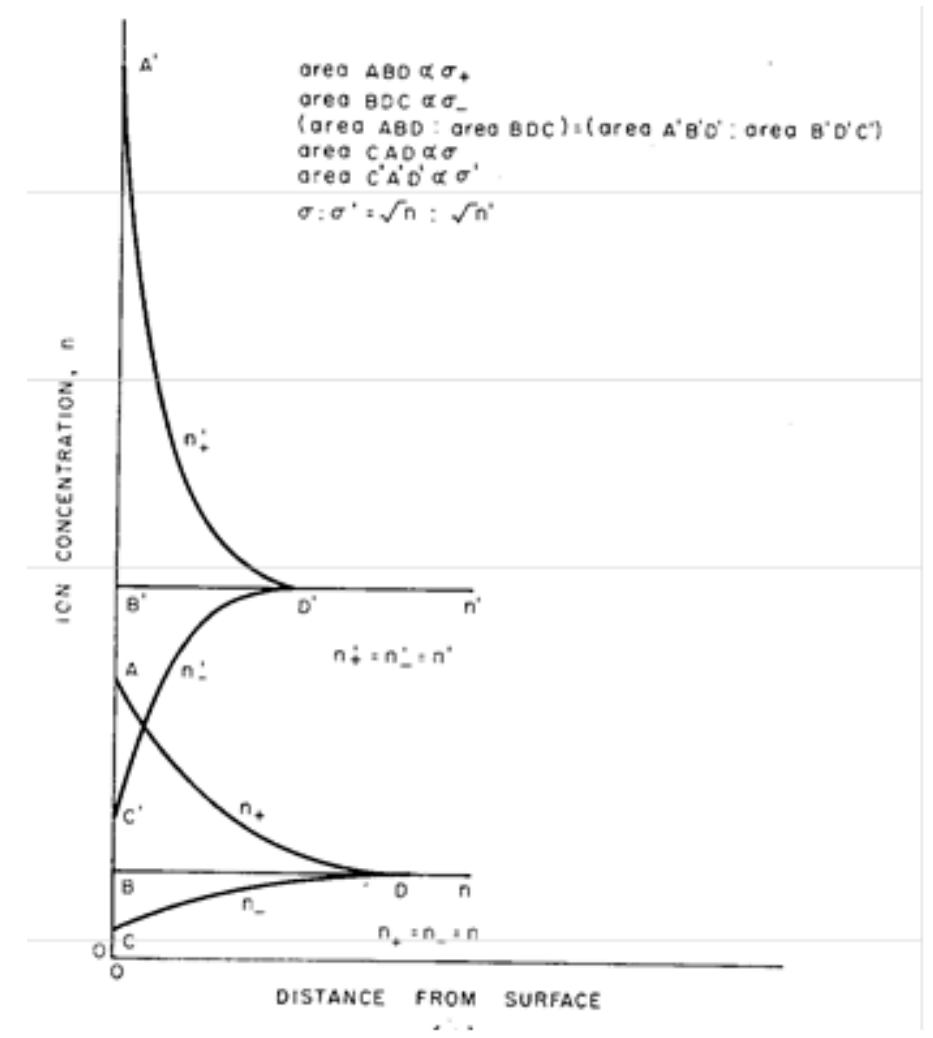

Figure 8.18 Charge distribution in the diffuse layer as a function of distance from a surface with constant charge (van Olphen, 1977, Fig. 10, permission to be asked). Symbols $n+$ and $n$ - are local volumetric ion concentrations. Distributions are shown for 2 different ionic strengths. Note proportionalities of areas representing charges $\sigma$.

Qualitatively we can see that the magnitude and sign of the surface charge determine whether cations or anions will be in excess in the diffuse layer, and how many of these ions are required to neutralize the surface charge. Gouy-Chapman theory, which neglects a Stern layer and treats diffuse-layer ions as point charges with coulombic interactions, is often used to quantify the charge distribution, and we will summarize the theory briefly here following Stumm and Morgan (1981). First, we define electrochemical potential, $\mu_{\mathrm{e}}$, as an extension of the chemical potential we are now familiar with:

$$
\text { (8.107) } \mu_{\mathrm{e}}=\mu+\mathrm{zF} \Psi
$$

where F is Faraday's constant, $\Psi$ is electrical potential, and $\mathrm{z}$ and $\mu$ are as defined previously. We can understand the second right-hand-side term, by analogy with [4.20], as an electrical "activity" term. The Gouy-Chapman concept is that at equilibrium, all ions in the diffuse layer have the same electrochemical potential, and in particular:

$$
\mu_{\mathrm{e}(\mathrm{x})}^{+}=\mu_{\mathrm{e}(\mathrm{x} \rightarrow \infty)}^{+}
$$$$
\mu_{\mathrm{e}(x)}^{-}=\mu_{\mathrm{e}(x \rightarrow \infty)}^{-}
$$

where $\mathrm{x}$ is distance from the surface. This leads to an expression relating the local potential (at $\mathrm{x}$ ) relative to the bulk solution $($ at $x=\infty)$, to the local ion concentration relative to the bulk solution: 


$$
\mathrm{zF}\left(\Psi_{(x)}-\Psi_{(x \rightarrow \infty)}\right)=-\mathrm{RT} \ln \left(\frac{\mathrm{n}_{(\mathrm{x})}^{+}}{\mathrm{n}_{(\mathrm{x} \rightarrow \infty)}^{+}}\right)=-\mathrm{RT} \ln \left(\frac{\mathrm{n}_{(\mathrm{x})}^{-}}{\mathrm{n}_{(\mathrm{x} \rightarrow \infty)}^{-}}\right)
$$

where the $\mathrm{n}$ are volumetric ion concentrations. Here we see that electrical activity is expressible as a dimensionless ratio of charge concentrations, in precise analogy with [4.20]. Application of Poisson's equation to the $\Psi-n$ relationship gives, after substituting [4.83] for $n$ :

$$
\frac{\mathrm{d}^{2} \Psi}{\mathrm{dx}^{2}}=\frac{\mathrm{\kappa}^{2} \sinh (\mathrm{zF} \Psi / \mathrm{RT})}{(2 \mathrm{~F} / \mathrm{RT})}
$$

where

$$
\kappa=\left(\frac{\mathrm{e}^{2} \sum \mathrm{in}_{\mathrm{i}} \mathrm{z}_{\mathrm{i}}^{2}}{\epsilon \epsilon_{\mathrm{o}} \mathrm{kT}}\right)^{1 / 2}
$$

where $\mathrm{e}$ is the elementary charge of the electron, the product of $\varepsilon$ and $\varepsilon_{0}$ is the permittivity of water, and $\mathrm{k}$ is the Boltzmann constant. Readers are referred to Stumm and Morgan (1981, Table 10.4) or van Olphen (1977, his Appendix III) for the details of the derivation. For our purposes it is enough to note that $\mathrm{k}$ has dimensions of length ${ }^{-1}$ and is the distance within which half of the surface charge is neutralized, or the double-layer thickness. In [4.84] all the terms are constants except for $\mathrm{T}$ and the summation, which has the general form of ionic strength I ([4.48]). We can therefore express the double-layer thickness as a function of $\mathrm{T}$ and $\mathrm{I}$ :

$$
\kappa^{-1}=\frac{1.64 \times 10^{-2}}{\sqrt{ }} \sqrt{\mathrm{I}} \quad(\mathrm{nm})
$$

It is important to remember that details of the charge-balancing characteristics of the solution are not represented by Gouy-Chapman theory. A solution of divalent ions (e.g. $\mathrm{Ca}^{2+}-\mathrm{SO}_{4}{ }^{2-}$, from dissolution of gypsum) neutralizes charge more "effectively" than, and has double-layer thicknesses about one-half of, a solution of monovalent ions (e.g. $\mathrm{Na}^{+}-\mathrm{Cl}^{-}$) of the same ionic strength. This distinction is not reflected by [4.85], but is important with respect to development and behavior of saline soils (section 4.5.4.3).

4.5.3.2 Ion exclusion. [4.85] gives a thickness of solution from solid surfaces in which counter-ions are preferred and co-ions are excluded. In typical vadose zones as in soils, surface charge is dominantly negative so counter-ions are cations and co-ions are anions. (There are important exceptions to this characterization: as discussed in section 1.5.3, certain kinds of surface charge are $\mathrm{pH}$-dependent such that anion exchange capacity is developed at $\mathrm{pH}$ less than the zero point of charge. This tends to be a concern in forest and other relatively acid soils.) Let us now consider two pore waters: fresh pore water which has been minimal evaporated and has "seen" no minerals more soluble than calcite, with an ionic strength of $0.001 \mathrm{~m}$; and a strongly brackish pore water which has been highly evaporated, or has "seen" soluble salts such as sulfates and halides, or both, with an ionic strength of $1.0 \mathrm{~m}$ (slightly greater than seawater). [4.85] gives:

$$
\begin{array}{ll}
\text { for } I=0.001 \mathrm{~m}, & \kappa^{-1}=9 \mathrm{~nm} \\
\text { for } \mathrm{I}=1.0 \mathrm{~m}, & \kappa^{1}=0.3 \mathrm{~nm}
\end{array}
$$

These are rough estimates (subject to all the limitations of Gouy-Chapman theory) of anion-exclusion distances from negatively-charged surfaces. Let us now compare these distances with some important water-layer-thickness ranges:

Table 8.7 


\begin{tabular}{ll}
\hline region & water thickness (nm) \\
mica interlayers & $<0.1$ \\
smectite interlayers & $0.1-2$ or greater \\
"wet" surfaces & $10^{2}-10^{5}$ \\
"dry" surfaces & $0.1-1$ \\
\hline
\end{tabular}

"Wet" and "dry" denote the extremes of pores nearly filled with water, and surfaces wet by only a few molecular layers of water, respectively. We see from this that for fresh pore waters, double-layer thicknesses are greater than water layer thicknesses except in relatively wet pore space. So except for the latter pore-space environment, double-layer theory (which is developed assuming infinite available distance from a charged surface) does not strictly hold, the solution does not achieve charge balance, and anions are excluded. On the other hand the double-layer thickness for the brackish case is on the order of the hydrated radius of an anion, which may be smaller than smectite interlayers and even the thickness of water layers on relatively "dry" surfaces such that anions are only excluded from mica interlayers. This analysis is semiquantitative at best, but its implications for vadose-zone transport and other processes should not be overlooked (chapter 5).

Bohn (1979, app. 6.1) and Sposito (1989, sec. 8.4) present relations with which anion exclusion volumes and distances can be computed from results of adsorption experiments.

\subsubsection{Cation exchange reactions.}

Nonspecifically-adsorbed cations - in electric double-layers and in layer-silicate interlayers - may switch places with cations in bulk solution in a process called cation exchange. Exchangeability, mentioned in section 1.5.3, is operationally defined in the context of measurements designed to quantify the adsorbed reservoirs of cations readily available to plants for their nutritive needs. Soil is suspended in water to which a large concentration of a displacer cation is added; due to its large chemical potential in bulk solution, the displacer cation diffuses into and displaces cations from doublelayers and interlayers; then the gain of the displaced cations into bulk solution is determined. The process can be represented as:

(8.114) $\mathrm{H}_{2} \mathrm{CO}_{3}{ }^{+}$silicates $=\mathrm{DIC}+\mathrm{H}_{4} \mathrm{SiO}_{4}{ }^{+}$inorganic solutes + clays

where $\mathrm{A}^{+}$is the displacer cation and the subscript indicates the sorbent or exchanger phase. Usually the displacer cation is itself subsequently re-displaced by another cation and also determined in bulk solution (important details of cation-exchange measurements are discussed in chapter 9). The cation exchange capacity or CEC is then reported as the magnitude of this re-displacement in units of equivalents of cationic charge per mass of dry soil (e.g. eq $\mathrm{g}^{-1}$ ). The cations displaced in the first step by the initial displacer are reported on the same basis as exchangeable cations. The timescale of the entire procedure is hours; therefore cation exchange is operationally defined as both rapid and reversible.

The CEC is an important index of soil fertility, and exchangeable cations denote a soil's nutritive status for purposes of growing particular crops, making specific soil amendments, and so on. Because the exchange process obviously affects the chemical composition of percolating waters, it would also be useful to employ CEC and exchangeable-cations data predictively in water-quality modeling. An equilibrium approach is justified by the rapid-reversible nature of the process, and if we define equilibrium for [4.87] as equality of chemical potential for each species in bulk solution and exchanger phases (after [I.38]), we can apply [4.25] and write:

$$
\mathrm{K}_{(\mathrm{P}, \mathrm{T})}=\operatorname{constant}_{(\mathrm{P}, \mathrm{T})}=\Pi \mathrm{a}_{\mathrm{i}}^{v_{\mathrm{i}}}=\frac{\mathrm{a}_{\mathrm{Ax}_{\mathrm{ex}}} \mathrm{a}_{\mathrm{B}}}{\mathrm{a}_{\mathrm{A}}+\mathrm{a}_{\mathrm{B}}}
$$

The potential value of this exchange constant is immediately apparent: if, for example, we know the ratio of the 
exchanger-phase activities then we can predict the ratio of the aqueous activities at equilibrium. There is, however, a major roadblock: we cannot quantify the activities of ions in the exchanger phase the way we can for ions in the bulk aqueous phase (e.g. ai $=\mathrm{m}_{\mathrm{i}} \gamma_{\mathrm{i}}$ via [4.46] and [4.47]). On a moment's reflection, this is no surprise: the exchanger phase of geologic material is different for every vadose zone, even within a given vadose zone, because it is a particular, composition- and $\mathrm{pH}$ - dependent aggregate of nonselective adsorption sites. The crucial ramification of this is that no aex means, practically speaking, no $\mu_{\mathrm{ex}}$ nor $\mu^{\mathrm{o}}{ }_{\mathrm{ex}}$ ([4.20]), which means no $\Delta \mathrm{G}_{\mathrm{R}}{ }^{\mathrm{o}}$ for the exchange reaction (4.22] and [4.23]) and therefore no path-independent value for $\mathrm{K}_{(\mathrm{P}, \mathrm{T})}([4.26])$. This is the meaning of the cryptic characterization in soils texts of cation-exchange equilibria as "not thermodynamically well defined".

4.5.4.1 Selectivity coefficient. Not all is lost, however, if we face these facts and seek only to characterize the exchange equilibrium for a particular material as follows:

$$
K_{\mathrm{B}}^{\mathrm{A}}=\frac{\left(\mathrm{X}_{\mathrm{A}}\right) \mathrm{a}_{\mathrm{B}^{+}}}{\mathrm{a}_{\mathrm{A}}+\left(\mathrm{X}_{\mathrm{B}}\right)}
$$

where the left-hand side is the exchange coefficient, or selectivity coefficient for A over B on the exchanger, and the parentheses denote exchanger-phase concentration in equivalent fraction on the exchange sites, where $\Sigma \mathrm{X}_{\mathrm{i}}=1$ (caution: several conventions are in use for exchanger-phase concentrations; each is valid provided it is applied consistently). In substituting exchanger-phase concentrations for activities we resign ourselves to an "apparent equliibrium constant" or conditional equilibrium coefficient in the sense of [4.9]. These coefficients can still be very useful hydrologically in several ways, as long as we remember that their actual values depend on both the solid material and solution composition. First, selectivity coefficients can be used to make semi-quantitative predictions of effects of exchanger phases on vadose water quality. For example, given the exchange reaction:

$$
\mathrm{Ca}^{2+}+\mathrm{Mg}_{\mathrm{ex}}=\mathrm{Mg}^{2+}+\mathrm{Ca}_{\mathrm{ex}}
$$

the selectivity coefficient, applying [4.25] conditioned on a particular material, is:

$$
\mathrm{K}_{\mathrm{Mg}}^{\mathrm{Ca}}=\frac{\left(\mathrm{X}_{\mathrm{Ca}}\right) \mathrm{a}_{\mathrm{Mg}^{2+}}}{\mathrm{a}_{\mathrm{Ca}^{2+}}\left(\mathrm{X}_{\mathrm{Mg}}\right)}
$$

If we assume that pore water and the exchanger are in equilibrium, we can measure exchangeable $\mathrm{Ca}$ and $\mathrm{Mg}$, determine the ion chemistry of the pore water and speciate it to obtain the aqueous activities, and evaluate the righthand side. Exchangeable-cations measurements on 14 samples of Borden sand (Reardon et al., 1983), summarized in Table 8.8, may be used for an example.

mean concentration,

Table 8.8 Chemical characteristics of Borden sand (from Reardon et al., 1983)

\begin{tabular}{llll}
\hline \multicolumn{5}{c}{ mean concentration, } \\
$\underline{\text { cation }}$ & exchanger $\left(\mathrm{meq} \mathrm{g}^{-1}\right)$ & $\underline{\mathrm{X}_{\text {cation }}}$ & pore water $\left(\mathrm{mm} \mathrm{L}^{-1}\right)$ \\
$\mathrm{Ca}^{2+}$ & 0.00497 & 0.92 & 1.27 \\
$\mathrm{Mg}^{2+}$ & 0.000265 & 0.049 & 0.084 \\
$\mathrm{Na}^{+}$ & 0.0000412 & 0.0076 & 0.296 \\
$\mathrm{~K}^{+}$ & 0.000129 & 0.024 & 0.033 \\
\hline
\end{tabular}

Taking aqueous molalities as equal to activities (dilute solution, $\left.\gamma_{\mathrm{i}}=1\right)$ [4.91] becomes:

$$
\mathrm{K}_{\mathrm{Mg}}^{\mathrm{Ca}}=\frac{(0.92) 8.4 \times 10^{-5}}{0.00127(0.049)}=1.24
$$

The slight elevation of this value above unity indicates that the $\mathrm{Ca} / \mathrm{Mg}$ ratio on the exchanger is slightly larger than 
the same ratio in solution, i.e. this sand exhibits a slight selectivity or affinity for Ca over $\mathrm{Mg}$ on the exchanger phase. Combining and rearranging [4.91] and [4.92], we get:

$$
\mathrm{K}_{\mathrm{Mg}}^{\mathrm{Ca}} \frac{\left(\mathrm{X}_{\mathrm{Mg}}\right)}{\left(\mathrm{X}_{\mathrm{Ca}}\right)}=1.24 \frac{(0.049)}{(0.92)}=0.066=\frac{\mathrm{a}_{\mathrm{Mg}^{2+}}}{\mathrm{a}_{\mathrm{Ca}^{2+}}}
$$

The important implication of this representation is that if the exchanger reservoirs are large relative to the equlibrating mass of cations in pore water such that they are not substantially changed during exchange, then constancy of the left-hand side may be assumed, thereby fixing the activity ratio. This is how vadose materials with appreciable exchange capacity modify the chemistry of waters flowing through them.

4.5.4.2 Exchanger evolution. We can assess the reasonableness of the constant-exchanger-composition assumption by comparing the cationic mass on the Borden exchanger phase, which has a CEC at the low end of surficial geologic materials, with cationic mass in coexisting pore water. The porosity of Borden sand is about 0.35 , so $1 \mathrm{~cm}^{3}$ of formation at vadose volumetric water content of 0.1 contains $0.035 \mathrm{~g}$ of water. From Table 8.8 we obtain a total of $3.04 \mathrm{meq} \mathrm{L}^{-1}$ in

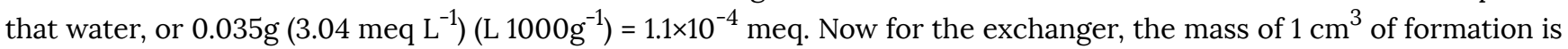
(the bulk density of) $1.8 \mathrm{~g}$ and the CEC is (totaling the exchangeable cations, Table 8.6) $0.0054 \mathrm{meq} \mathrm{g}^{-1}$, so we obtain 1.8 $\mathrm{g}\left(0.0054 \mathrm{meq} \mathrm{g}^{-1}\right)=9.7 \times 10^{-3}$ meq. So in this case the ratio of cation equivalents on the exchanger to cation equivalents in pore water is about 100; even at full saturation the ratio would be about 10. For trace cationic contaminants subject to exchange (such as radionuclides), the ratios tend to be orders of magnitude larger still; and where the aqueous concentration of the dominant exchanging cation is large enough that exchange affects it negligibly, the concentration of the trace cation is effectively fixed (see [4.93]). This means that the exchanger phase has a large and relatively predictable capacity to attenuate the migration of exchangeable contaminant cations. Over the course of many pore volumes, the exchanger composition evolves; the co-evolution of water and exchanger composition can be represented by simple mixing-cell models (e.g. Dance and Reardon, 1983). We deal with this below in section 4.8.

The calcium-sodium exchange reaction is:

$$
\mathrm{Ca}^{2+}+2 \mathrm{Na}_{\mathrm{ex}}=2 \mathrm{Na}^{+}+\mathrm{Ca}_{\mathrm{ex}}
$$

and the selectivity coefficient for Borden sand, based on the averages reported in Table 8.6, is (assuming $\mathrm{a}_{\mathrm{i}}=\mathrm{m}_{\mathrm{i}}$ ):

$$
\mathrm{K}_{\mathrm{Na}}^{\mathrm{Ca}}=\frac{\left(\mathrm{X}_{\mathrm{Ca}}\right) \mathrm{a}_{\mathrm{Na}^{+}}^{2}}{\mathrm{a}_{\mathrm{Ca}^{2+}}\left(\mathrm{X}_{\mathrm{Na}}\right)^{2}}=\frac{(0.92)\left(2.96 \times 10^{-4}\right)^{2}}{1.27 \times 10^{-3}(0.0076)^{2}}=1.10
$$

Monovalent-divalent exchange introduces squared terms in the activity product ratio of [4.95] with interesting implications for the dependence of exchangeable cations populations on their natural pore water environment. The exchanger-phase equivalent ratio, from [4.95], is:

$$
\frac{\left(\mathrm{X}_{\mathrm{Ca}}\right)}{\left(\mathrm{X}_{\mathrm{Na}}\right)^{2}}=\mathrm{K}_{\mathrm{Na}}^{\mathrm{Ca}} \frac{\mathrm{a}_{\mathrm{Ca}^{2+}}}{\mathrm{a}_{\mathrm{Na}^{+}}^{2}}
$$

Now consider a hypothetical pore water 100 times more concentrated than the water in Table 8.6 - about half the ionic strength of seawater. How would the Borden sand exchanger change, if it evolved over time to equilibrate with this new water? Concentrating both aqueous activities in [4.95] by 100 times and evaluating [4.96] gives:

$$
\frac{\left(0.9376-\mathrm{X}_{\mathrm{Na}}\right)}{\left(\mathrm{X}_{\mathrm{Na}}\right)^{2}}=159.4
$$

where the total equivalent fraction occupied by $\mathrm{Ca}$ and $\mathrm{Na}$ is assumed to be constant at 0.9376 (Table 8.6), so that the numerator represents $\mathrm{X}_{\mathrm{Ca}}$. Solving the $\backslash$ quadratic gives $\mathrm{X}_{\mathrm{Na}}=0.076$ and $\mathrm{X}_{\mathrm{Ca}}=0.86$. The cation equivalent ratio on the exchanger is now $\mathrm{X}_{\mathrm{Na}} / \mathrm{X}_{\mathrm{Ca}}=0.089$, more than ten times larger than the dilute case (Table 8.6) where the ratio is 0.0083. What we see is that divalent cations dominate the exchanger phase in equilibrium with dilute waters, whereas monovalent cations are increasingly favored on the exchanger with increasing ionic strength. This has important implications for water quality in vadose zones. First, the provenance (geologic source) of the sediments in vadose zones 
is generally important. Marine clays, equilibrated with seawater high in $\mathrm{Na}^{+}$, have exchanger-phase cation populations dominated by Na which is released by exchange with $\mathrm{Ca}^{2+}$ when exposed to relatively fresh vadose percolates. Such clays appear to control the exchanger-phase properties of some tills in the Glaciated Interior Plains; in one documented case (Keller et al., 1991), $\mathrm{K}^{\mathrm{Ca}}$ Na for vadose clayey till is approximately 260 and exchange along percolation paths now produces high-sodium groundwaters more than $10^{4} \mathrm{yr}$ after the tills were deposited. A second point, following from the first, is that the provenance of the exchanger becomes less important and local hydrogeochemical conditions become more important with the passage of time. These points help us understand the development of many naturally saline soils (section 4.5.4.3 below). We will also use these facts to estimate vadose flow rates in section 4.6.

For calcium-potassium exchange on Borden sand, the reaction is just like [4.94] with $\mathrm{K}$ substituted for Na; for the selectivity coefficient we obtain (using data in Table 8.6):

$$
\mathrm{K}_{\mathrm{K}}^{\mathrm{Ca}}=\frac{\left(\mathrm{X}_{\mathrm{Ca}}\right) \mathrm{a}_{\mathrm{K}^{+}}^{2}}{\mathrm{a}_{\mathrm{Ca}}^{2+}\left(\mathrm{X}_{\mathrm{K}}\right)^{2}}=\frac{(0.92)\left(3.3 \times 10^{-5}\right)^{2}}{1.27 \times 10^{-3}(0.0239)^{2}}=0.00138
$$

Comparing [4.95] and [4.98] we see that the exchanger selectivity for Ca over $\mathrm{K}$ is much smaller than selectivity for Ca over Na, i.e. $\mathrm{K}$ has much greater affinity for the exchanger phase than Na. This is an instance of marked selectivity. The cause is probably the excellent "fit" of the potassium ion into mica interlayers, resulting in relatively specific adsorption for that species where micas are abundant in the layer-silicate mineral fraction - which is true at Borden.

Aside from instances of marked selectivity, which are entirely medium-specific, we can draw a rough generalization about cationic affinity for exchangers: the greater the ratio of charge to hydrated radius, the greater the affinity. This gives the normal affinity sequence:

$$
\mathrm{Th}^{4+} \mathrm{Th}^{4+}
$$

$$
\mathrm{Rb}^{+} \mathrm{Rb}^{+} \mathrm{Al}^{\wedge}\{3+\}
$$

$\backslash$ approx $\left.\mathrm{La}^{\wedge}\{3+\}>\mathrm{Ba}^{\wedge}\{2+\} \backslash \operatorname{approx} \mathrm{Sr}^{\wedge}\{2+\}>\mathrm{Ca}^{\wedge}\{2+\}>\operatorname{Mg}^{\wedge}\{2+\} \backslash \operatorname{approx} \mathrm{Cs}^{\wedge}\{2+\}>\right\} \backslash \backslash \backslash \backslash \backslash \operatorname{ce}\left\{\mathrm{Rb}^{\wedge}\{+\}>\mathrm{NH}_{-}\{4\}^{\wedge}\{+\}\right.$

$\backslash$ approx $\mathrm{K}^{\wedge}\{+\}>\mathrm{Na}^{\wedge}\{+\} \backslash$ approx $\left.\mathrm{Li}^{\wedge}\{+\}\right\} \backslash$ end $\{$ multline* $\}$ " title="Rendered by QuickLaTeX.com">

which provides a crude, qualitative tool for estimating relative cationic masses and retention on exchange sites as a function of pore water chemistry. Written in reverse order, the affinity sequence becomes the lyotropic series of relative ion replaceability on an exchanger (Bohn et al., 1979).

4.5.4.3 Saline soils. This topic is covered in virtually all soil-chemistry and many soil-physics texts. Sposito (1989) devotes a chapter of his book to a thorough and practical treatment. We will discuss it briefly here as a consequence, in many cases, of natural vadose geochemical processes. To illustrate, consider a typical hydrogeochemical scenario in the Glaciated Interior Plains, discussed just previously on p. _-_- and earlier in section 4.4.3.1: vadose dissolution of calcite and gypsum under high $\mathrm{PCO}_{2}$ generates high- $\mathrm{Ca}^{2+}$ pore waters which displaces $\mathrm{Na}^{+}$from marine-clay exchange sites. This yields shallow groundwaters with large concentrations of both cations. At a groundwater discharge point (which may be either nearby or distant, seasonal or perennial) solutes become evaporatively concentrated as discussed in section 4.4.3.2 (van der Kamp and van Stempvoort, 1992). If, as in the Interior Plains, the groundwaters are already highly mineralized, relatively modest evaporation associated with semi-arid climate is sufficient to raise ionic strength in soil pore water to levels causing salinity hazard to crops. In this scenario, diffuse-layer thicknesses are compressed 
such that van der Waals attractive forces are sufficient, when clay particles approach each other, to cause clay-particle clumping or flocculation - leaving permeable void space in the soil. However if, in addition, the $\mathrm{Na}^{+}$concentration is elevated according to the Hardie-Eugster rules (p. _- ) so that it eventually displaces exchangeable divalent cations on the clays, the diffuse layer thickness may rise to the point that closest approach distances are too large to be overcome by van der Waals forces, and clay dispersion occurs. This can cause dramatic reduction of the size of interconnected pore space, permeabilility, and the water- and nutrient-exchange capacities of plants rooted in these soils. Soils subject to this problem are called sodic. In general we can see that sodicity will tend to be offset by sufficiently high salinity (Figure 8.18) - but this, of course, tends to cause salinity hazard.

In irrigated agriculture water is applied to soil from above instead of discharged from below, but the same kind of evaporative concentration occurs due to crop transpiration and prevailing aridity of climate in irrigated settings. In this case pore-water quality is a function of both water applied and the weathering status and mineralogy of the soil (section 4.4.2.). Irrigated agriculture has provided many decades of practical experience in how these factors influence the development and management of salinity and sodicity hazards (e.g. Sposito 1989, chap. 12).

\section{Sorption isotherms.}

Equation [4.89] may be rearranged as:

$$
\frac{\left(\mathrm{X}_{\mathrm{A}}\right)}{\mathrm{a}_{\mathrm{A}^{+}}}=\mathrm{K}_{\mathrm{B}}^{\mathrm{A}} \frac{\left(\mathrm{X}_{\mathrm{B}}\right)}{\mathrm{a}_{\mathrm{B}^{+}}}
$$

If $\mathrm{A}$ is a trace species (e.g. a contaminant metal) such that exchange does not appreciably change the aqueous- and exchanger-phase activities of $\mathrm{B}$, then the right-hand-side can be taken as constant:

$$
\frac{\left(\mathrm{X}_{\mathrm{A}}\right)}{\mathrm{a}_{\mathrm{A}^{+}}}=\mathrm{const}
$$

or, substituting molality for activity $\left(\gamma_{i}=1\right)$,

$$
\text { (8.129) }\left(\mathrm{X}_{\mathrm{A}}\right)=\operatorname{const}\left(\mathrm{m}_{\mathrm{A}^{+}}\right)
$$

or, more generally:

(8.130) $\mathrm{C}_{\text {ads }}=\mathrm{K}_{\mathrm{d}} \mathrm{C}_{\text {soln }}$

where $K_{d}$ is the distribution coefficient for A between solution and exchanger, and C is concentration of A in any practical units. The simple linear relation between solution and adsorbed concentrations described by [4.102] is the linear isotherm, so named because the relation pertains for a particular temperature (and pressure). Isotherms are generated experimentally by reacting a series of solutions, with known initial concentrations of the species of interest, with aliquots of sediment of known mass. Following equilibration, measurement of final solution concentration reveals the loss of mass onto the sorbent, which can be expressed as a concentration. A plot of the results of these batch experiments - adsorbed concentration vs. mean solution concentration - yields points on the isotherm which, if it is linear, has slope $K_{d}$ (Figure 8.19). 


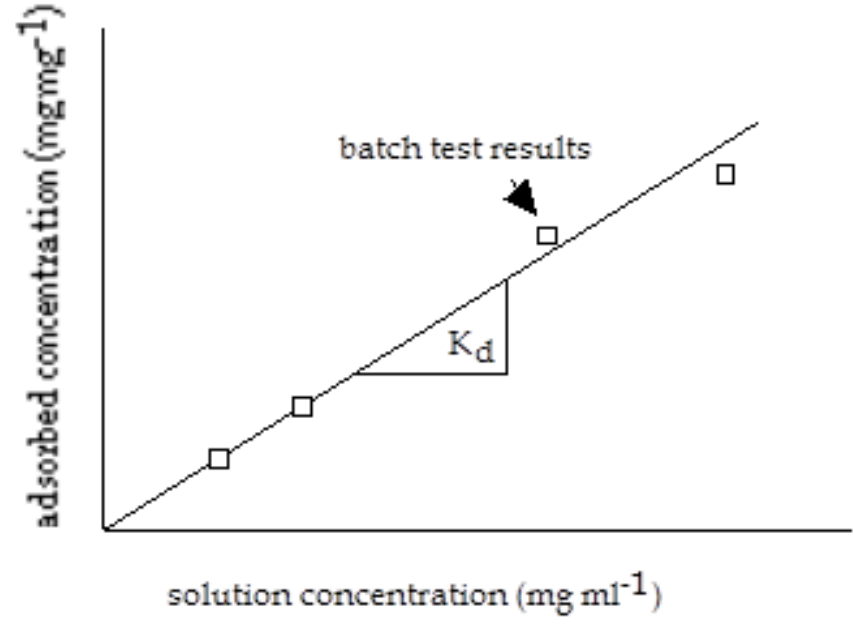

Figure 8.19

The focus of [4.102] is on the (single) species of interest, rather than on the overall exchange reaction from which it was derived; this suggests that isotherms may be generated for any adsorption reaction:

(8.131) $\mathrm{A}_{\mathrm{soln}}=\mathrm{A}_{\mathrm{ads}}$

where the subscripts refer to the solution and sorbent phases, regardless of whether or not the process can be characterized as exchange.

4.5.5.1 Adsorption models. It is absolutely crucial to note that [4.102] describes an isotherm only if it is linear which, in turn, depends on the assumption made going from [4.100] to [4.101]: that the properties of the exchanger, or, more generally, adsorbent, do not vary - at least, within the parameters of the investigation. The physical concept is of a sorbent with an effectively unlimited number of identical adsorption "sites". This concept often applies for trace species over limited concentration ranges, but it will not always be appropriate. Where the number of sites is effectively finite, a simple equilibrium model can be applied (following Jury et al., 1991, p. 26). If the rates of adsorption $\mathrm{r}_{\text {ads }}$ and desorption $\mathrm{r}_{\text {des }}$ are first-order, then:

$$
\text { (8.132) } \mathrm{r}_{\mathrm{des}}=\mathrm{k}_{\mathrm{des}} \mathrm{C}_{\mathrm{ads}} \quad \mathrm{r}_{\mathrm{ads}}=\mathrm{k}_{\mathrm{ads}}\left(\mathrm{C}_{\mathrm{t}}-\mathrm{C}_{\mathrm{ads}}\right) \mathrm{C}_{\mathrm{soln}}
$$

where the $\mathrm{k}$ are rate constants and $\mathrm{C}_{\mathrm{t}}$ is total concentration of available sites. The rate of desorption is proportional to the adsorbed concentration, while the rate of adsorption depends on both the solution concentration and the concentration of unfilled available sites. At equilibrium, the two rates must be equal; solving the equality for $\mathrm{C}_{\mathrm{ads}}$ gives:

$$
\mathrm{C}_{\mathrm{ads}}=\frac{\mathrm{K}_{1} \mathrm{C}_{\mathrm{t}} \mathrm{C}_{\mathrm{soln}}}{1+\mathrm{K}_{1} \mathrm{C}_{\mathrm{soln}}}
$$

where $K_{1}=k_{a d s} / k_{\text {des }}$. This is referred to as the Langmuir isotherm. From the functional form we can see that at low concentrations the $\mathrm{C}_{\mathrm{ads}}$ increases approximately linearly with $\mathrm{C}_{\text {soln }}$; the magnitude of this initial slope is $\mathrm{K}_{l} \mathrm{C}_{\mathrm{t}}$. As concentrations increase, $\mathrm{C}_{\mathrm{ads}}$ is asymptotic to $\mathrm{C}_{\mathrm{t}}$. (This functional behavior is analogous to Michaelis-Menten rate dependence on concentration substrate, [4.5].) In the more general case in which the adsorption-site population is both finite and comprised of sub-populations with different characteristics, Sposito (1981, chap. 4) shows that generalizing [4.103] over a continuum of such sub-populations yields:

$$
\mathrm{C}_{\mathrm{ads}}=\frac{\mathrm{K}_{\mathrm{f}} \mathrm{C}_{\mathrm{soln}}^{\beta}}{1+\left(\mathrm{K}_{\mathrm{f}} / \mathrm{K}_{1}\right) \mathrm{C}_{\mathrm{soln}}^{\beta}}
$$


where $K_{f}$ and $\beta$ are positive, and $0<\beta<1 . \beta$ quantifies the variance of the Langmuir $K_{l}$ parameters across the subpopulation continuum; for $\beta=1$, corresponding to zero variance, [4.104] reduces to the form of [4.103]. For sufficiently small $\mathrm{C}_{\text {soln }},[4.104]$ reduces to

$$
\text { (8.135) } \mathrm{C}_{\mathrm{ads}}=\mathrm{K}_{\mathrm{f}} \mathrm{C}_{\text {soln }}^{\beta}
$$

which is the usual form of the van Bemmelen-Freundlich or, more commonly, the Freundlich isotherm. For $\beta=1$, [4.105] becomes [4.102]. Sposito (1981) explains how [4.103-4.105] can be parameterized from experimental data. Kinniburgh (1986) and Travis and Etnier (1981) also provide good general discussions of isotherm formulations and applications.

The assumption of linear-isotherm adsorption behavior ([4.102]) in transport modeling has been ubiquitous; in fact, it is surprising (and fortunate) how frequently that assumption appears to be fulfilled in practice. However, careful work should generally proceed from the initial conservative assumption that adsorption is nonlinear and in this sense "nonideal" (e.g. Brusseau, 1994) such that a more sophisticated formulation is required ([4.103] or [4.105]). Fortunately, one longstanding rationale (excuse?) for assuming linear adsorption behavior - computational tractability - is rapidly fading with the expansion of computational power.

Adsorptive processes which are rate-limited, i.e. slow compared to fluid and solute residence times such that solutesorbent equilibrium may not be achieved, present a more serious conceptual and practical problem. Rate-limitations give rise to isotherms which vary as a function of timescale; there is relatively little experience, yet, with application of such time-dependent information to a field-scale problem. The great bulk of recent work aimed at understanding the underlying processes has been done in studies of sorption of organic compounds, to which we now turn.

\subsubsection{Sorption of organics.}

This field has exploded in recent years with rising concerns about the fate of contaminant organic compounds which have been released to the surface and shallow subsurface. Theory and experience are firmly rooted in studies of sorption of nonionic or hydrophobic contaminant organics to soils and bottom sediments of rivers and lakes, in which natural organic matter is relatively abundant. An up-to-date general reference on the topic is the encyclopedic work of Schwartzenbach et al. (1993).

We have seen, as a consequence of the thermodynamics of interfaces ([4.80] and [4.81]), that nonionic aqueous solutes tend to concentrate at the interface with another phase. The same considerations dictate that the nonionic solute would migrate across the interface and into solution in a phase for which that solute increases the surface tension. Such a phase would be chemically "like" the solute; for nonionic organic solutes, a useful reference phase is the organic solvent octanol, which is only slightly soluble in water and thus immiscible with water. The reaction representing this process, referred to as partitioning of an organic solute A from aqueous solution to an immiscible organic phase can be written as:

(8.136) $\mathrm{A}_{\mathrm{W}} \rightarrow \mathrm{A}_{\mathrm{O}}$

and described as (ab)sorption, for which the equilibrium constant would be

$$
\mathrm{K}_{\mathrm{ow}}=\frac{\mathrm{q}_{\mathrm{O}}}{\mathrm{a}_{\mathrm{A}_{\mathrm{W}}}}=\frac{\gamma_{\mathrm{A}_{\mathrm{O}}} \mathrm{C}_{\mathrm{A}_{\mathrm{O}}}}{\gamma_{\mathrm{A}_{\mathrm{W}}} \mathrm{C}_{\mathrm{A}_{\mathrm{W}}}}
$$

where $\mathrm{K}_{\mathrm{ow}}$ is the octanol-water partition coefficient. In the case of ideality - analogous to the case of infinite dilution for ionic solutes, i.e. $\gamma_{\mathrm{i}}=1-$ this expression can be rewritten as:

$$
\log \mathrm{K}_{\mathrm{ow}}=\log \mathrm{C}_{\mathrm{A}_{\circ}}-\log \mathrm{C}_{\mathrm{A}_{\mathrm{w}}}
$$

where the superscript indicates ideality. Representing $\left(\backslash \operatorname{mathrm}\left\{\mathrm{C}_{-}\left\{\mathrm{A}_{-}\{\backslash\right.\right.\right.$ circ $\left.\left.\left.\}\right\}\right\}\right)$ as inversely proportional to the solute molar volume $\mathrm{v}$ and $\left(\backslash \operatorname{mathrm}\left\{\mathrm{C}_{-}\left\{\mathrm{A}_{-}\{\mathrm{w}\}\right\}\right\}\right)$ as the aqueous solubility of the solute $\mathrm{s}_{\mathrm{w}}$, Chiou et al. (1983) reformulated this expression for soil organic matter as the organic phase:

$$
\log _{\mathrm{om}}=-\log \left(\mathrm{s}_{\mathrm{w}} \mathrm{v}\right)-0.622
$$


where the equilibrium constant is now the soil organic matter partition coefficient. The constant term lumps quantities arising from theoretical treatment of the organic phase, SOM, as an organic polymer. Choiu et al. (1983) then generated batch isotherms for twelve common nonionic organic contaminant solutes on a silt loam soil. The isotherms were linear over large concentration ranges, and batches containing two solutes produced the same isotherms as for each solute individually. This noncompetitive sorption was taken as evidence for partition into the organic phase, as opposed to an adsorptive process which would be expected to exhibit Langmuir or Freundlich isotherms. The isotherm slopes yielded $\mathrm{K}_{\mathrm{d}}$ values and, from these, $\mathrm{K}_{\mathrm{om}}$ for each solute:

$$
\mathrm{K}_{\mathrm{om}}=\frac{\mathrm{K}_{\mathrm{d}}}{\mathrm{f}_{\mathrm{om}}}
$$

where $\mathrm{f}_{\mathrm{om}}$ is the mass fraction of organic matter in the soil. The $\mathrm{K}_{\mathrm{om}}$ results for all the solutes fit the regression

(8.141) $\log \mathrm{K}_{\mathrm{om}}=-0.813 \log \left(\mathrm{s}_{\mathrm{w}} \mathrm{v}\right)-0.993$

with $\mathrm{r}^{2}=0.995$, where $\mathrm{s}_{\mathrm{w}}$ is $\mathrm{mol} \mathrm{L}^{-1}$ and $\mathrm{v}$ is $\mathrm{L}^{-1} \mathrm{~mol}$. The deviation of [4.86] from [4.107] was attributed to the inferiority of natural SOM as an organic solvent, relative to the organic polymer for which the theory was developed.

In more common practice the direct proportionality between $\log \left(\mathrm{s}_{\mathrm{w}} \mathrm{v}\right)$ and $\log \mathrm{K}_{\mathrm{ow}}$ (compare [4.106] and [4.107]) has been exploited to generate equations functionally equivalent to [4.109] of the form:

$$
\log \mathrm{K}_{\mathrm{OC}}=\mathrm{a} \log \mathrm{K}_{\mathrm{ow}}+\mathrm{b}
$$

where

$$
K_{\mathrm{OC}}=\frac{K_{\mathrm{d}}}{\mathrm{f}_{\mathrm{OC}}}
$$

and "oc" refers to organic carbon (instead of organic matter, [4.108]), and a and b are constants. Taking logs of [4.111] and substituting [4.110] yields:

(8.144) $\log \mathrm{K}_{\mathrm{d}}=\mathrm{a} \log \mathrm{K}_{\mathrm{ow}}+\log \mathrm{f}_{\mathrm{oc}}+\mathrm{b}$

Schwartzenbach and Westall (1981) showed in an influential study that this expression, with $\mathrm{a}=0.72$ and $\mathrm{b}=0.49$, described sorption of many common nonionic organic solutes to a surprising variety of sorbents.

The great importance and practical appeal of [4.112] is that $K_{d}$ values for many dissolved organics may be predicted from easily-obtainable information. Numerous investigations since that of Schwartzenbach and Westall (1981) have more or less agreed with their results. However for our purposes we must note an important qualification which has arisen over the past decade or so: the sorptive quality of natural organic matter varies substantially. To put it another way, $\mathrm{K}_{\mathrm{oc}}$ ([4.111]) for a given contaminant varies greatly, depending upon organic-matter provenance. It appears, for example, that old, "hard" kerogenic organic matter has $K_{\mathrm{oc}}$ values for hydrophobic organic solutes which are up to $10^{2}-10^{3}$ times greater than would be predicted by [4.112] for young, "soft" organic matter (Allen-King et al., 1997; Weber et al., 1992). Furthermore, the extent to which such organic matter has been weathered may exert additional strong control (Binger et al., 1998 ). As we have seen, "hard", weathered, and unweathered organic matter is predominant in some vadose zones like that of the Dalmeny till (sections 4.3 and 4.4 above).

A second important qualification with respect to our use of [4.112] arises in connection with the "soft" carbon Schwartzenbach and Westall (1981) studied. Recent studies have found substantial variation in the values of a and b in [4.112] when a large fraction of the sorbing organic matter exists as coatings on mineral grains, due to variability in the composition and configuration of the coatings (Murphy et al. 1990; Xing et al. 1994). This, too, is relatively liable to be important in subsoil parts of vadose zones where organic matter levels are low, and it probably partly explains Schwartzenbach and Westall's caution that their relation is unreliable for $\mathrm{f}_{\mathrm{oc}}<0.001$. Others have examined this problem from a more general perspective in which partitioning theory breaks down, due to insufficient organic matter to function thermodynamically as solvent for organic solutes, such that prediction of sorption involves superposition of noncompetitive (partitioning) and potentially competitive (adsorptive) effects. This issue is a topic of current interest with respect to sorption of organics in low- $f_{\text {oc }}$ aquifers (e.g. Werth and Reinhard, 1997; Ball and Roberts, 1991a; Brusseau, 1991). Luthy et al. (1997) provide a recent perspective on these and related issues, as well as an extensive bibliography.

4.5.6.1 Nonequilibrium sorption. Another general problem in prediction of organic sorption is recognized to be rate- 
limitation or nonequlibrium. Pignatello (1989) gives a particularly clear discussion of issues involved in this problem, in which he points out that nonequilibrium is probably a leading cause of observed hysteresis in sorption-desorption experiments, as evidenced by the desorption isotherm falling above the sorption isotherm (refer to Figure 8.19). Investigators have developed two kinds of mathematical descriptions of the process (Pignatello, 1989). Nondiffusion kinetics retains explicit time derivatives in Langmuir-type adsorption and desorption expressions (which we set equal to obtain [4.103] above), sometimes for multiple site populations each with different rate characteristics. Diffusion kinetics treats the process as diffusive transport of the solute into and out of solid grains which are taken to be porous aggregates of mineral material and organic matter. In this latter approach, which is conceptually more consistent with sorption by partitioning, sorption to the interiors of micropores is assumed instantaneous and the kinetic effect is associated with the time required for intra-grain diffusion according to Fick's second law (chapter 5). In a Fickian process, the time scale $t$ is related to length scale $L$ and effective diffusion coefficient $D_{\text {eff }}$ by:

$$
\mathrm{t}=\frac{\mathrm{L}^{2}}{\mathrm{D}_{\mathrm{eff}}}
$$

Quick evaluation of this expression shows that for L typical of mineral grains in soils and subsoils (mm to mm), $\mathrm{D}_{\text {eff }}$ must be very small to yield times of the order of observed equilibration times in batch experiments, i.e. hours to weeks or more. Wu and Gschwend (1986) derived an expression for Deff :

$$
\mathrm{D}_{\mathrm{eff}}=\frac{\mathrm{D} \theta \tau}{\left(1-\theta_{\mathrm{s}}\right) \rho_{\mathrm{s}} \mathrm{K}_{\mathrm{d}}+\theta}
$$

where $\mathrm{D}^{\circ}$ is the diffusion coefficient in water and $t$ is the tortuosity of the diffusion path, and the water content and density terms apply to the grains themselves. This expression shows how $D_{\text {eff }}$ can become very small if $K_{d}-$ which quantifies the propensity of solute molecules to reside in the solid, thereby slowing their migration - is sufficiently large. Wood et al. (1990), Brusseau et al., (1991) and Ball and Roberts (1991b) explore application of these ideas to aquifer sediments. Luthy et al. (1997) also discuss this issue in their overview.

\subsubsection{Retardation.}

All the quantities other than $\mathrm{D}^{\circ}$ and $\tau$ on the right-hand side of [4.114] may be collected and expressed as the retardation coefficient R:

$$
\mathrm{R}=\frac{\left(1-\theta_{\mathrm{s}}\right) \rho_{\mathrm{s}} \mathrm{K}_{\mathrm{d}}+\theta}{\theta}
$$

or, because $\rho_{b}=\rho_{S}(1-n)$, for the special case of pore water saturation $\left(\theta=\theta_{S}\right)$,

$$
\mathrm{R}=1+\frac{\rho_{\mathrm{b}} K_{\mathrm{d}}}{\mathrm{n}}
$$

which is the form more familiar to hydrogeologists. Substituting [4.115] into [4.114], we obtain for diffusive transport:

$$
\mathrm{D}_{\mathrm{eff}}=\frac{\mathrm{D} \tau}{\mathrm{R}}
$$

and we can write by analogy, for advective transport:

$$
\overline{\mathrm{v}}_{\text {solute }}=\frac{\overline{\mathrm{v}}_{\text {water }}}{\mathrm{R}}
$$

Equation [4.115] is derived in the context of the transport equation in section 5.3. [4.115-4.117] are commonly employed in transport modeling formulations because of their computational tractability (chap. 5). Note that the partition 
coefficient underlying these formulations, $\mathrm{K}_{\mathrm{d}}$, reflects linear, instantaneous, non-hysteretic sorptive behavior. These, then, along with invariant $\theta$, are all necessary conditions for $\mathrm{R}$ to be treated as constant in the transport problem (section 5.3).

\subsection{Summary: controls on vadose solute occurrence}

Up to now we have discussed or mentioned a wide variety of solutes of potential interest in the vadose zone. We have also covered a broad array of biogeochemical processes which influence or control the occurrence of these species in vadose pore water. It seems useful to try to summarize these insights in a condensed form (Table 8.8). In doing this we have made numerous simplifications and ignored some complexities; this is strictly a first-order attempt.

There are many ways such a Table could be organized. Here we list solutes with predominantly natural (lithologic, atmospheric) sources first followed by solutes with important anthropogenic sources. Characterization of sources is a bit tricky: for example, bicarbonate naturally comes from the atmosphere and, if carbonates are present, rock sources (section 4.4.1); sulfate and nitrate have substantial anthropogenic, atmopheric sources where acid rain is important (Johnson et al., 1981; Likens and Bormann, 1995). All of these sources are immediate; one can easily make the case that every solute of interest passes through or ultimately comes from a lithologic reservoir in its biogeochemical cycle. For example, sodium and chloride are both derived from Earth's crust but most vadose chloride - and, occasionally, sodium too (e.g. Hansen and Postma, 1995) - has been transported in atmospheric precipitation from the oceans (section 4.8.1 below).

Table 8.8

\begin{tabular}{|c|c|c|c|}
\hline Solute & Principal source(s)* & $\begin{array}{l}\text { Principal biogeochemical } \\
\text { control(s)** }\end{array}$ & Prediction of occurrence \\
\hline $\mathrm{Ca}^{2+}, \mathrm{Mg}^{2+}, \mathrm{K}^{+}$ & $\mathrm{L}$ & W-R-G, B & biogeochemical \\
\hline $\mathrm{Na}^{+}, \mathrm{Fe}^{2+}, \mathrm{Mn}^{2+}, \mathrm{Al}^{3+}, \mathrm{SiO}_{2}{ }^{\mathrm{O}}$ & $\mathrm{L}$ & W-R-G & biogeochemical \\
\hline $\mathrm{HCO}_{3}^{-}, \mathrm{SO}_{4}^{2-}$ & $\mathrm{L}, \mathrm{A}, \mathrm{An}$ & W-R-G, B & biogeochemical \\
\hline $\mathrm{Cl}^{-}$ & A, An & -- & transport \\
\hline $\mathrm{NO}_{3}^{-}$ & A, An & W-R-G, B & biogeochemical, transport \\
\hline heavy metals & $\mathrm{L}, \mathrm{An}$ & W-R-G & biogeochemical, transport \\
\hline synthetic organics & An & W-R-G, B & biogeochemical, transport \\
\hline $\begin{array}{l}* \mathrm{~L}=\text { lithologic }, \\
\quad * * \mathrm{~W}-\mathrm{R}-\mathrm{G}=\mathrm{W}\end{array}$ & $\begin{array}{l}\text { tmospheric, } \mathrm{An}=\text { antl } \\
\text { rock-gas, } \mathrm{B}=\text { biologi }\end{array}$ & $\begin{array}{l}\text { genic } \\
\text { ake, release, transformation }\end{array}$ & \\
\hline
\end{tabular}

It makes intuitive sense that the group of solutes derived principally from natural sources contains all the major elements (dominating the total dissolved solids) of vadose pore waters and groundwaters. These solutes $-\mathrm{Ca}^{2+}, \mathrm{Mg}^{2+}$ $, \mathrm{K}^{+}, \mathrm{Na}^{+}, \mathrm{HCO}_{3}{ }^{-}, \mathrm{SO}_{4}{ }^{2-}, \mathrm{Cl}^{-}, \mathrm{SiO}_{2}{ }^{\mathrm{O}}-$ are predominantly ionic. A subset of the natural solutes $-\mathrm{Fe}^{2+}, \mathrm{Mn}^{2+}$, and $\mathrm{Al}^{3+}$ - occurs primarily in soils (rarely in subsoils) where the combination of acid generation and complexation with dissolved organic matter is most intense. All of these solutes are strongly controlled by water-rock-gas interactions precipitation/dissolution, speciation in solution, exchange, sorption - with the result that predicting their occurrence 
is mostly a biogeochemical problem relying on methods outlined in this chapter. Additionally, however, occurrence in the solum is directly affected by biological cycling for several of these species which plant scientists call "nutrients".

Chloride deserves its own line in Table 8.8 because of its relative lack of reactivity. This has made chloride a very important tracer of vadose flow and transport processes in pristine (section 4.8 .1 below) as well as contaminated settings. Other halides like bromide and iodide behave similarly and salts of these anions are commonly dissolved in the water used in artificial vadose transport experiments (chapter 6). Chloride's reputation as "conservative" in solution is not altogether deserved: it is actually subject to some plant uptake, as is bromide (Bowman et al., 1997), and it can be affected by anion exchange and anion exclusion. These latter effects are often small relative to hydrologic uncertainties in prediction exercises, and plant uptake is not an issue when timescales are long enough that vegetation may be considered at steady state.

Nitrate gets its own line in Table 8.8 because it is so widespread as a dissolved contaminant in the shallow subsurface. Although the great bulk of the nitrogen in the environment has been fixed from the atmosphere by plants, that $\mathrm{N}$ is cycled "tightly" in unperturbed ecosystems such that very little $\mathrm{N}$ in any form moves beneath the solum. Nitrogen fertilizers, however, are commonly nitrified to nitrate in soils. Where agricultural nitrate inputs are greater than the nutritive needs of the plants, which is common, excess nitrate is available for transport into subsoils. There, nitrate is relatively unaffected by plant uptake and (as an anion) sorption/exchange processes such that transport processes become paramount in predicting occurrence (chapter 5). However under reducing conditions, which can occur seasonally in the solum, biogeochemical processes can transform and attenuate nitrate (Paul and Clark, 1989).

In their cationic forms heavy metals are strongly controlled and attenuated by exchange and sorption (sections 4.5.4, 4.5.5). However, they may not occur as cations in pore water due to $\mathrm{pH}$-dependent speciation (e.g. section 4.4.1). Many heavy metals are also controlled by precipitation/dissolution due to the solubility of a mineral - cadmium, for example, by $\mathrm{CdCO}_{3}$. Hem (1985) provides brief overviews, and Lindsay (1979) gives authoritative treatments from a soils perspective. Where their primary source is contaminant rather than lithologic (e.g. an industrial discharge), such that the metal is not ubiquitously distributed through the vadose zone, prediction of occurrence may rely strongly on understanding transport processes (chapter 5).

"Synthetic organics" in Table 8.8 refers to the many thousands of compounds, ranging from pesticides to pharmaceuticals, which underlie "better living through chemistry" and which have been found dissolved in water around the world. These compounds are thermodynamically unstable in the environment and tend to degrade by biologically-mediated oxidation like all other organic matter to $\mathrm{CO}_{2}$ and other simple inorganic compounds (section 4.3 above). However the environmentally-effective rates of these transformations vary over many orders of magnitude, and reaction intermediates may be toxic. In fact, the environmental fate of most of these chemicals is not well known. Their importance in vadose studies over the long haul lies not so much in their aggregate dissolved mass (very small relative to inorganic species; usually parts per billion to parts per \quadrillion) as in their relatively unknown epidemiological effects. In a very real sense, we are all participants in ongoing global-scale experiments on the long-term health effects of chronic exposure to multiple low-level synthetic organics.

Certain industrial and dry-cleaning chemicals - mostly halognenated organic solvents such as PCE and TCE - occur locally at high and clearly dangerous concentrations in vadose zones across the industrialized world. These pointsource occurrences, along with spills of petroleum hydrocarbons, have been a major force behind the recent explosive growth of the "bioremediation" and "intrinsic remediation" industries (e.g. Watts, 1998). Petroleum components (not actually synthetic) and halogenated solvents are generally nonionic in aqueous solution, so that their tendency to partition into or onto solid organic matter (sections 4.5.1, 4.5.6) is important to consider in attempts to predict vadose occurrence. Schwartzenbach (1993) provides encyclopedic coverage of these characteristics. Transport processes (chap. 5) are likewise important to consider for all but the most strongly sorbed and quickly transformed of these compounds. 


\subsection{Reaction Simulations}

At several points in this chapter we have used conceptual models, realized in the form of a few equations, to make predictions of vadose porewater chemistry. The systems of equations have been kept simple to make the examples instructive. There is this virtue in simple models, which we emphasize throughout this book: if you understand them you can try them out readily, using pencil and paper or a microcomputer spreadsheet, to test basic approaches to understanding data.

Frequently in practice there is reason to refine a prediction. In most such cases, as exemplified in section 4.2.5, refinement is achieved by keeping track of interacting species in addition to the species of interest. This is usually done by expanding a simple system of equations representing a chemical equilibrium, and using a computer code to store and solve the equations. When implemented to compute the distribution of dissolved species (section 4.2.5.3), this is called an "aqueous model" ("model" because it is a prediction based on equilibrium assumptions and thermodynamic data) which forms the core of most hydrochemical modeling programs. The approach may be generalized: chemical reactions are written among species of interest, equilibrium constants are specified, and material balance(s) are introduced to constrain the system for solution (Plummer et al., 1983; Bethke, 1996 provides a comprehensive discussion).

In reaction simulations of vadose processes of interest, available codes allow addition of a species to an existing solution (e.g. spill of a contaminant), addition of mineral phases (e.g. movement of pore water into a deeper horizon), and addition of sorbents (e.g. cation exchange). The calcite equilibrium problem in section 4.4.1 provides an example of addition of a mineral to an aqueous equilibrium. This works mathematically as long as each addition, which adds an equilibrium-constant equation like $4.72\left(\mathrm{~K}_{\mathrm{cal}}\right)$, also adds a dependent variable $\left(\mathrm{Ca}^{2+}\right)$ so that the system does not become overconstrained. The same thing happens with a sorbent: we add an equilibrium constant $\mathrm{K}_{\mathrm{d}}$ and a dependent variable $\mathrm{C}_{\mathrm{ads}}$ in [4.102] to the problem of predicting $\mathrm{C}_{\text {soln }}$. Problems 2 and 3 following appendix I explore this matter in the context of the phase rule.

As we have characterized them thus far, reaction simulations can be called "forward" models: we start with an initial condition (porewater chemistry) and predict the result of changes. Widely-used forward codes include PHREEQE (Parkhurst et al. 1980) and its descendents and MINTEQA2 (Allison et al., 1991). These codes can be readily adapted to simulate kinetic processes by incremental addition of species. In the past decade or so an "inverse" approach has been used widely. This method takes initial and final water chemistries as given and "backs out" the mass-transfer reactions responsible for the change, based on mass balance. It is fundamentally different from the forward method in that no assumptions about equilibrium are needed, basically because we already know the initial and final states. It is satisfying to say that this method, too, is nothing really new, but rather an elaboration of the approach taken in the classic study of silicate weathering by Garrels and MacKenzie (1967) mentioned in section 4.4.2.2. Computerization allows rapid identification of all possible combinations of reactions as constrained by user-specified phases (Plummer et al. 1991).

There are many excellent, in-depth treatments of geochemical reaction simulation. Among the most recent are the books by Bethke (1996) and several sections of the text by Langmuir (1997). Suarez and Simunek (1996) give a review of application of reaction simulation codes to vadose environments in which they address the problem of coupling reaction simulation to transport simulation (chapter 5).

\subsection{Geochemical indicators of vadose flow processes}

The occurrence of selected chemical species (e.g. oxidized and reduced iron, carbonate minerals) has been used by soil scientists for generations to discern qualitative differences among soil flow regimes. Over the past 3 decades a mix of soil scientists, hydrologists, and geologists has begun quantitative application of this approach to the vadose zone. Gee and Hillel (1988) contended that these geochemical (including isotopic) methods are more promising than water-balance 
and hydrodynamic methods for estimating groundwater recharge, wherever the relative errors in these methods are large relative to the estimated recharge flux, i.e. in dry climates. They also noted that the geochemical indicators could reveal and account for certain kinds of spatial and temporal variability that other methods could not.

The thrust of most of this work has been to somehow sample vadose pore water and determine vertical pattern of occurrence of some "environmental tracer" in that water, then relate the subsurface tracer pattern to a known or inferred historic pattern of atmospheric deposition. Usually the simplifying assumption is made that the tracer does not volatilize or react with vadose solids; usually an estimate of groundwater recharge is the goal. However, there is now increasing interest in what environmental tracers can tell us about processes affecting vadose transport such as preferential flow and extreme hydrologic events. Also a few investigators have looked at chemical species that do react, and that are even produced entirely by reactions in the subsurface. These kinds of studies rely on insights developed in the rest of this chapter.

\subsection{Mass balances on conservative solutes.}

Let's begin with the simplest case: all of the mass of a solute in precipitation is transmitted, via deposition and infiltration, into the vadose zone. Where surface runoff is negligible this will be approximately true for chloride $(\mathrm{Cl})$, which is not evaporated and for which limited plant uptake may often be assumed to be recycled back into soils. We will use $\mathrm{Cl}$ as the characteristic "conservative solute" for this discussion because so many studies have taken this approach. Figure 8.20 shows generic, schematic profiles of $\mathrm{Cl}$ concentration in vadose pore water vs. depth. Such profiles are usually obtained by extracting sediment samples from known depths with distilled water, measuring the $\mathrm{Cl}$ concentration of the resulting extract solution, and adjusting that concentration to the pore-water concentration by multiplying by the ratio of the extract and in-situ water contents (e.g. Murphy et al., 1996). 


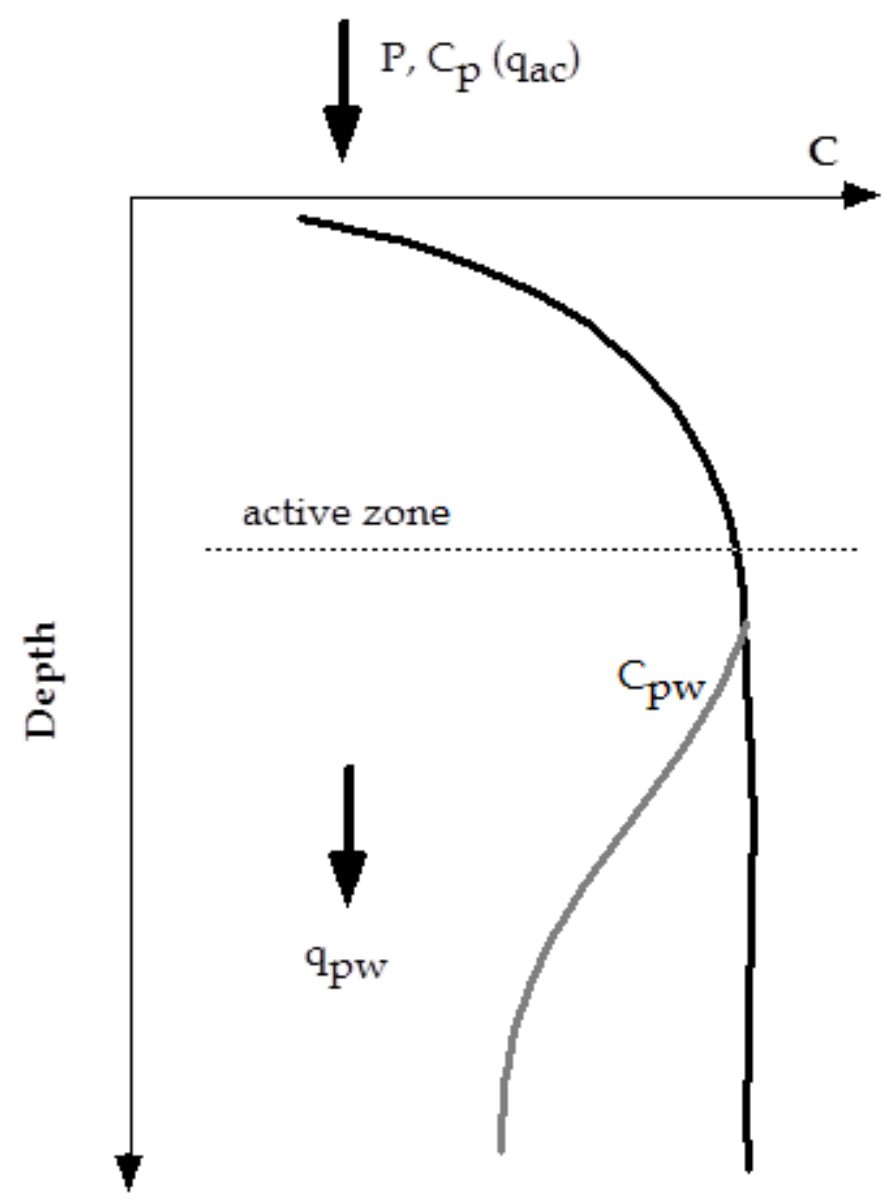

Figure 8.20 Schematic profiles of $\mathrm{Cl}$ concentration in vadose pore water. Symbols are defined in text.

If we assume piston flow then in this simple case, the mass of $\mathrm{Cl}$ in the profile down to a given depth - which we will call the "inventory" - represents all the $\mathrm{Cl}$ deposited by precipitation over some time period:

(8.151) inventory $=$ deposition $*$ time

Using the symbols in Figure 8.20, [4.118] becomes more precisely:

(8.152) $\int_{0}^{\mathrm{z}} \mathrm{C}_{\mathrm{pw}} \theta \mathrm{dz}=\int_{0}^{\mathrm{t}} \mathrm{C}_{\mathrm{p}} \mathrm{Pdt}$

where $C_{p w}$ is concentration in pore water $\left[\mathrm{ML}^{-3}\right], \theta$ is volumetric water content $\left[\mathrm{L}^{3} \mathrm{~L}^{-3}\right], \mathrm{C}_{\mathrm{p}}$ is concentration in precipitation $\left[\mathrm{ML}^{-3}\right]$, and $\mathrm{P}$ is precipitation $\left[\mathrm{LT}^{-1}\right]$. Sometimes an important portion of a solute is deposited dry, as in dust; in this case the product $\mathrm{C}_{\mathrm{p}} \mathrm{P}$ is replaced by the total accumulation flux $\mathrm{q}_{\mathrm{ac}}\left[\mathrm{ML}^{-2} \mathrm{~T}^{-1}\right]$ :

(8.153) $\int_{0}^{\mathrm{z}} \mathrm{C}_{\mathrm{pw}} \theta \mathrm{dz}=\int_{0}^{\mathrm{t}} \mathrm{q}_{\mathrm{ac}} \mathrm{dt}$

If the deposition is assumed constant over a period of interest $t$, we get

$$
\int_{0}^{\mathrm{z}} \mathrm{C}_{\mathrm{pw}} \theta \mathrm{dz}=\mathrm{q}_{\mathrm{ac}} \mathrm{t}
$$

or 


$$
\mathrm{t}=\frac{\int_{0}^{\mathrm{z}} \mathrm{C}_{\mathrm{pw}} \theta \mathrm{dz}}{\mathrm{q}_{\mathrm{ac}}}
$$

Let's work an example: estimate the mean residence time of water at 8-m depth in top-slope and mid-slope vadose profiles in Figure 8.21. The data are given in the first five columns of Table 8.9. Assume that $\mathrm{q}_{\mathrm{ac}}$ is $120 \mathrm{mg} \mathrm{Cl} \mathrm{m}^{-2} \mathrm{yr}^{-1}$.

Lege
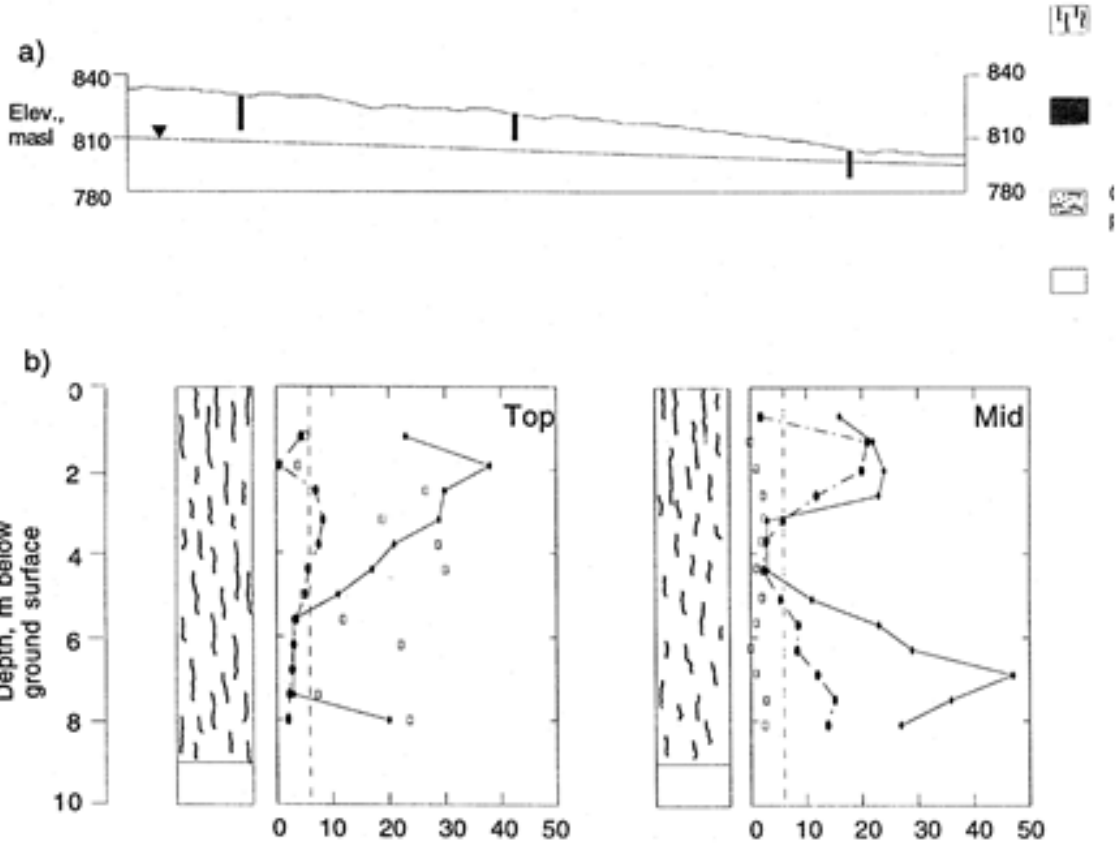

$\bullet 3_{\mathrm{H}}$ Concentration, TU $\bullet-\mathrm{NO}_{3}$ Concentration, $\mathrm{mg} /$

口 $\mathrm{Cl}$ Concentration, mg/

Figure 8.21 (O’Brien et al. 1996)

Table 8.9 


\begin{tabular}{|c|c|c|c|c|c|c|c|}
\hline \multirow[t]{8}{*}{ top-slope } & $\begin{array}{l}\text { depth } \\
\text { (m) }\end{array}$ & $\begin{array}{l}\mathrm{dz} \\
(\mathrm{m})\end{array}$ & $\begin{array}{c}\mathrm{q} \\
\left(\mathrm{m}^{3} \mathrm{~m}^{-3}\right)\end{array}$ & $\begin{array}{c}\mathrm{Cl} \\
\left(\mathrm{mgL}^{-1}\right)\end{array}$ & $\begin{array}{l}\text { inventory in } \mathrm{dz} \\
\qquad\left(\mathrm{mg} \mathrm{m}^{-2}\right)\end{array}$ & $\begin{array}{l}\text { cum. inventory } \\
\qquad\left(\mathrm{mg} \mathrm{m}^{-2}\right)\end{array}$ & $\begin{array}{l}\text { MRT } \\
(\mathrm{yr})\end{array}$ \\
\hline & 1.2 & 1.55 & 0.24 & 5.41 & 2013 & 2013 & 17 \\
\hline & 1.9 & 0.65 & 0.19 & 3.9 & 482 & 2494 & 21 \\
\hline & 2.5 & 0.65 & 0.26 & 26.69 & 4511 & 7005 & 58 \\
\hline & 4.4 & 0.6 & 0.36 & 30.02 & 6484 & 21868 & 182 \\
\hline & 5 & 0.6 & 0.33 & 4.78 & 946 & 22814 & 190 \\
\hline & 5.6 & 0.6 & 0.39 & 11.88 & 2780 & 25594 & 213 \\
\hline & 8 & 0.4 & 0.4 & 23.66 & 3786 & 37003 & 308 \\
\hline \multirow[t]{8}{*}{ mid-slope } & 0.9 & 1.25 & 0.32 & 1.85 & 740 & 740 & 6 \\
\hline & 1.6 & 0.65 & 0.38 & 0 & 0 & 740 & 6 \\
\hline & 2.2 & 0.6 & 0.39 & 1.04 & 243 & 983 & 8 \\
\hline & 2.8 & 0.6 & 0.42 & 2.27 & 572 & 1555 & 13 \\
\hline & 3.4 & 0.6 & 0.4 & 2.48 & 595 & 2151 & 18 \\
\hline & 4 & 0.6 & 0.36 & 2.07 & 447 & 2598 & 22 \\
\hline & 7.6 & 0.65 & 0.46 & 2.74 & 819 & 4415 & 37 \\
\hline & 8.3 & 0.35 & 0.37 & 2.49 & 322 & 4737 & 39 \\
\hline
\end{tabular}

Depth intervals "dz" in Table 8.9 are center-weighted about the reported depth. We calculate the inventory for each $\mathrm{dz}$, then these inventories are summed downward. Each "cumulative inventory" entry is the total mass of $\mathrm{Cl}$ per square meter in the profile above and including the depth of that entry. The MRT is then the number of years it took to accumulate total mass to that depth. Figure 8.22 shows how these estimates of pore-water age increase with depth. According to the $\mathrm{Cl}$ mass balance ("CMB"), pore water is about ten times as old beneath the top-slope as beneath the mid-slope. 


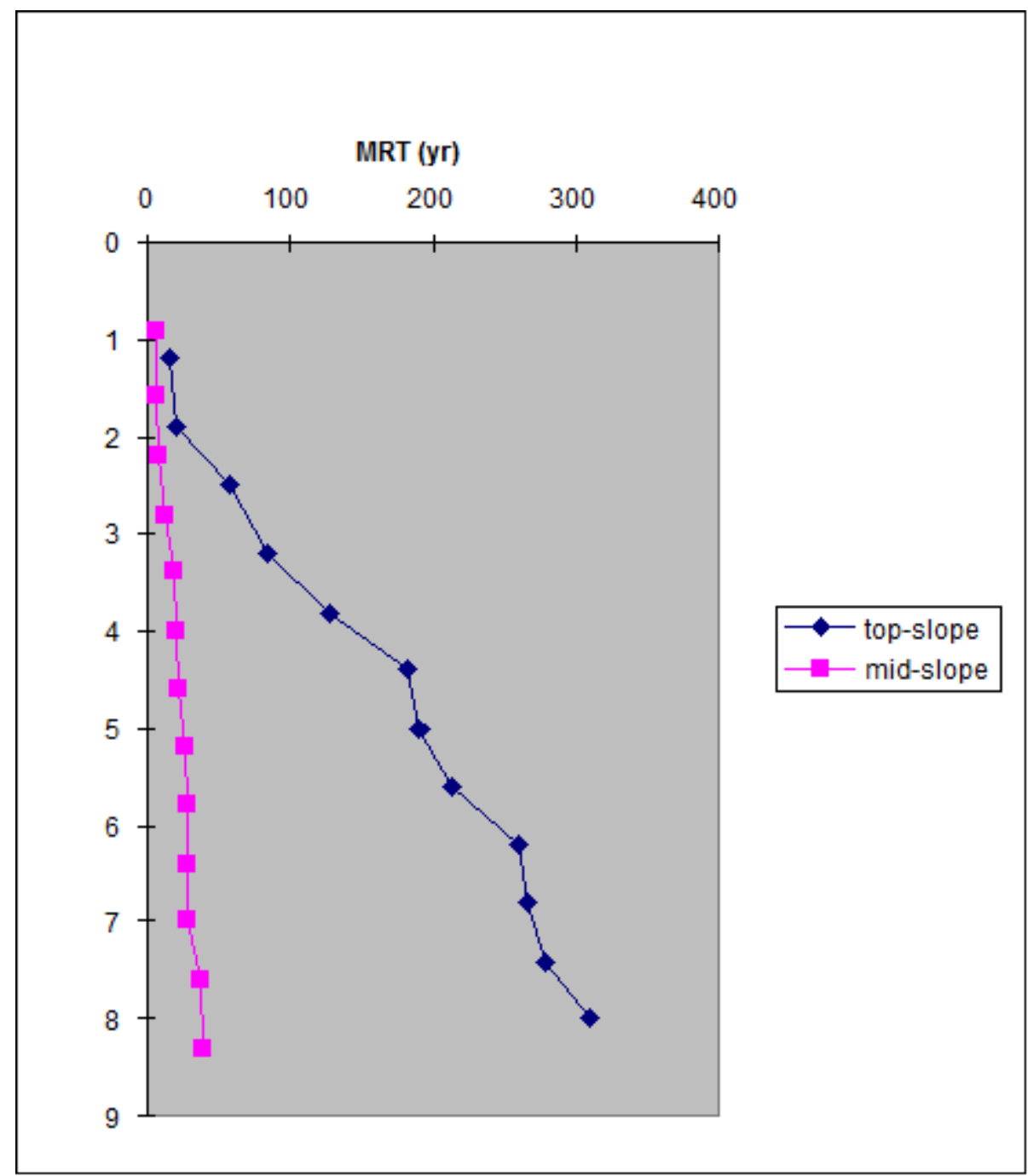

Figure 8.22 Age of water calculated by chloride mass balance for profiles in Figure 8.21 using data of Table 8.9 and [4.121].

With these MRT results we can easily calculate recharge rates because we know the time elapsed, at each depth, for accumulation of the water in an overlying depth interval:

$$
\mathrm{R}_{1-2}=\frac{\int_{\mathrm{z}_{1}}^{\mathrm{z}_{2}} \theta \mathrm{dz} \text { (155) }}{\mathrm{t}_{2}-\mathrm{t}_{1}}
$$

This is the quotient of the "water inventory" by time of accumulation. Let's do this for depth intervals beneath the active zone of infiltration/exfiltration, i.e. beneath about $2 \mathrm{~m}$ in the study of O'Brien et al. (1996). If we always take $\mathrm{z}_{1}$ and $t_{1}$ to refer to the $1.6 \mathrm{~m}$ and $1.9 \mathrm{~m}$ depths at the base of the active zone, we can generate the profiles in Figure 8.23. Each point represents the result of [4.122] applied from its plotted depth up to $z_{1}$, over the period of its MRT minus $t_{1}$. Each successively deeper point thus represents more water and a greater time interval. The deepest results, i.e. the longestterm average recharge rates for the profiles, are about $7 \mathrm{~mm} \mathrm{yr}^{-1}$ for the top-slope and $70 \mathrm{~mm} \mathrm{yr}^{-1}$ for the mid-slope. 


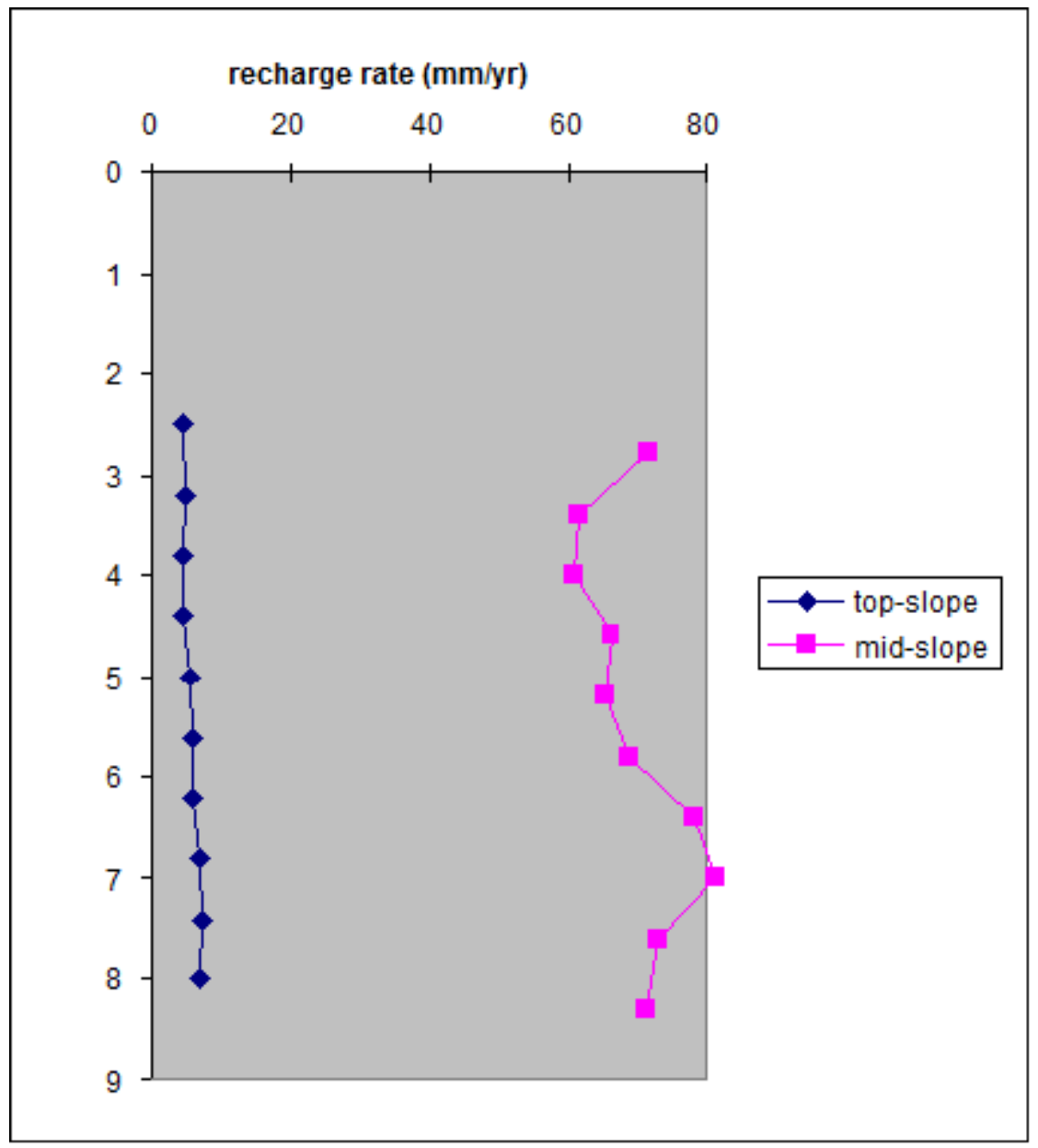

Figure 8.23

We can get an alternative to equation 4.123 by equating the accumulation flux with the vadose flux:

(8.156) $\mathrm{q}_{\mathrm{ac}}=\mathrm{RC}_{\mathrm{pw}}$

This equation says explicitly that at each time of interest, the $\mathrm{Cl}$ load in deposition is equal to the $\mathrm{Cl}$ flux out the bottom of the active zone. Plants take up water, progressively enriching the pore water in $\mathrm{Cl}$ as it moves through the active zone, so the smaller is $\mathrm{R}$ in [4.123] the larger is $\mathrm{C}_{\mathrm{pw}}$ for a given accumulation rate. This concept is implicit in [4.118]. Rearranging [4.123] we get

(8.157) $\mathrm{R}=\mathrm{q}_{\mathrm{ac}} / \mathrm{C}_{\mathrm{pw}}$

The very simplest application of this relationship is to best-estimate Cpw over some depth interval beneath the active zone, assume an accumulation rate, and perform the division. Doing this for the data below $2 \mathrm{~m}$ in Table 8.9 yields $\mathrm{C}_{\mathrm{pw}}$ $=17.7 \mathrm{mg} \mathrm{L}^{-1}$ for the top slope and $1.55 \mathrm{mg} \mathrm{L}^{-1}$ for the mid slope (O'Brien et al. 1996). Again assuming qac is $120 \mathrm{mg} \mathrm{Cl} \mathrm{m}^{-2}$ $\mathrm{yr}^{-1}$, we get:

$$
\begin{aligned}
& \mathrm{R}_{\text {topslope }}=6.8 \mathrm{mmyr}-1 \\
& \mathrm{R}_{\text {midslope }}=77 \mathrm{mmyr}-1
\end{aligned}
$$

These results are very similar to those in Figure 8.23. A slightly more elaborate and interesting way of using [4.124] is to plot the water inventory (the numerator in [4.122]) as a function of $\mathrm{Cl}$ inventory (Figure 8.24). 


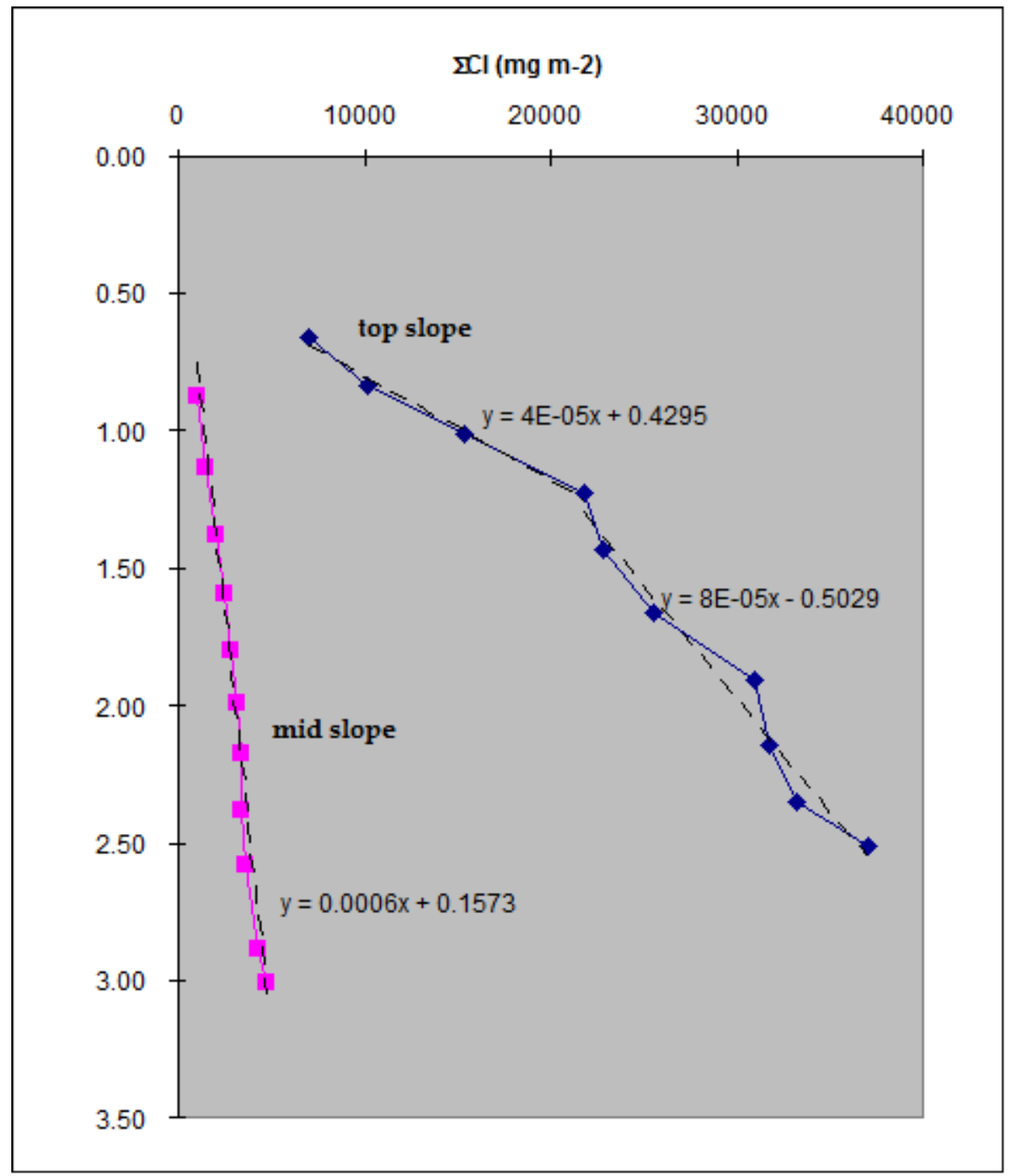

Figure 8.24

The slope of any segment on these plots is the reciprocal of the $\mathrm{Cl}$ concentration for the depth interval represented by that segment (check the units!). If we use a little imagination to divide the top-slope profile into two segments, the $\mathrm{Cl}$ concentrations from best-fit slopes (Figure 8.24) come out to $25 \mathrm{mg} \mathrm{L}^{-1}$ for the shallow segment and $13 \mathrm{mg} \mathrm{L}^{-1}$ for the deeper segment, yielding recharge rates from [4.124] of 4.8 and $9.6 \mathrm{~mm} \mathrm{yr}^{-1}$ respectively. There is a hint here that there was a slight decrease in the recharge rate at this point on the landscape around $200 \mathrm{yr}$ ago (Figure 8.22). Phillips (1994) and Murphy et al. (1996) show much more dramatic changes in best-fit concentrations over time intervals of thousands of years which suggest changes in climatic regime. Even using our imaginations, it would be hard to detect any deflection in the mid-slope profile in Figure 8.24, which yields a best-fit concentration of $1.7 \mathrm{mg} \mathrm{L}^{-1}$ and recharge rate of $72 \mathrm{~mm} \mathrm{yr}^{-1}$. This is essentially indistinguishable from the results using the simple average ([4.125]) and the water inventory ([ 4.122], Figure 8.23).

Let's return for a moment to the statements of material balance ([4.118] and [4.123]) which underlie this whole development. They contains an important assumption: all $\mathrm{Cl}$ comes from atmospheric deposition, and no $\mathrm{Cl}$ is lost or gained from soils or subsoils intervals by runoff, preferential flow, reactions, sources, or sinks. The runoff issue is negligible in many topographically flat settings; O'Brien et al. (1996) considered that problem and the possibility of 
preferential flow at their hilly site. The source-sink assumption is probably reasonable in many environments where chloride has not been applied to soils in fertilizers or other forms. We must state, however, a geochemical caution: common mineral assemblages contain chloride (Kuroda and Sandell, 1953), and some of the chloride-rich phases, such as the glasses which occur in volcanic rocks like basalt, dissolve relatively rapidly (Gislason et al., 1987) - that is, they could dissolve appreciably on the timescales of vadose pore water residence times in certain settings. This would be a source of "stealth" $\mathrm{Cl}$ which would upwardly bias $\mathrm{C}_{\mathrm{pw}}$ and cause overprediction of MRT in [4.121] or underprediction of recharge in [4.124]. The magnitude of the error depends on the extent to which the rock $\mathrm{Cl}$ is released and concentrated in the active zone (Figure 8.25).

(a)

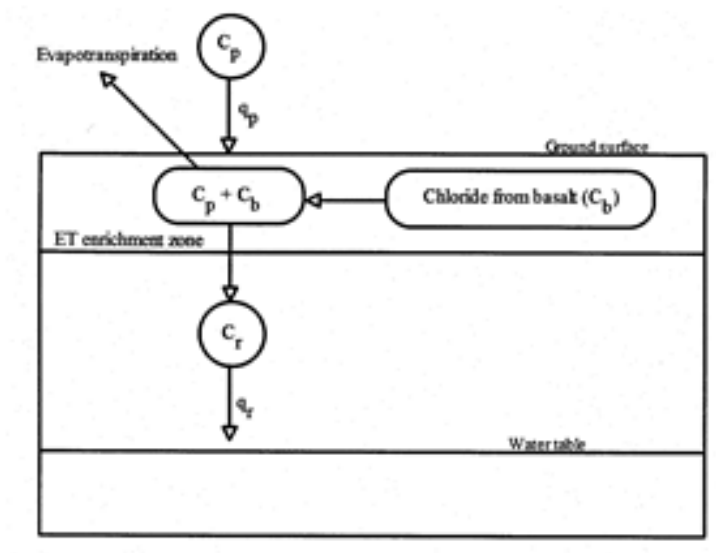

(b)

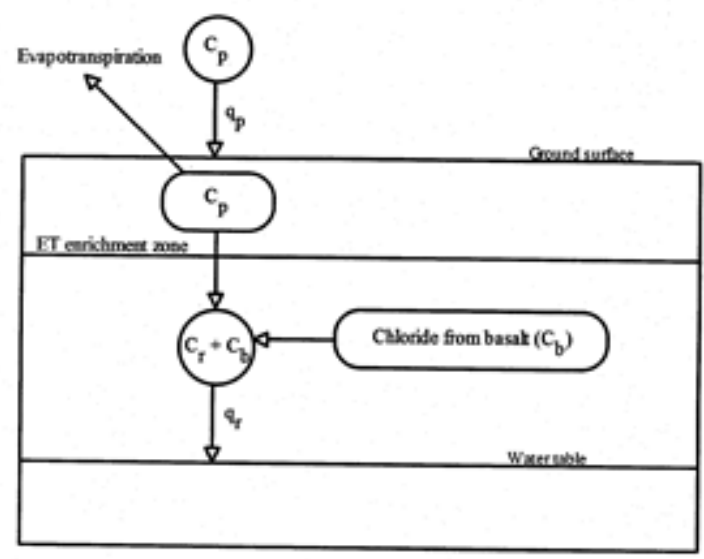

Figure 8.25 Two end-member cases of the effect on the $\mathrm{Cl}$ mass balance of a source of vadose rock chloride (from Roe, 1995, permission to be asked). (a) A source which is evaporatively enriched (along with the $\mathrm{Cl}^{-}$ from precipitation) causes larger recharge underprediction errors than (b) a source beneath the active zone, here termed "ET enrichment zone".

In many dry climates, particularly arid regions, rock chloride sources are probably small relative to evaporative enrichment such that [4.118] and [4.123] may be used with confidence (e.g. Murphy et al., 1996). Other cautions and issues relative to interpreting vadose chloride mass balances are discussed by Allison et al. (1994) and Tyler and Walker (1994). 


\subsubsection{Mass balances on evaporating species.}

Some of the most useful tracers of water are actually isotopes of hydrogen and oxygen in the water molecule itself. Tritiated water, ${ }^{3} \mathrm{HHO}$ (usually just called "tritium"), is an interesting example as it was produced in above-ground bomb testing at levels up to $10^{4}$ times the limit of detection in precipitation during the 1950s and 1960s. In recent decades tritium concentrations in meteoric water have declined due to both "rainout" and radioactive decay (tritium's half-life is $12.4 \mathrm{yr}$ ), back to near the pre-bomb levels of about 100 times the detection limit. Thus there is a favorably large signal/ noise ratio in water that was precipitated within a fairly short time; this particular property of environmental tritium has been exploited without recourse to mass balance (section 4.8.3). Right now it is important to see that tritium will be evapotranspired like other water molecules, so that deposition is not all retained in the vadose inventory as [4.118] says - much of deposition will instead be returned to the atmosphere. What we can say, then, is:

$$
\frac{\text { inventory }}{\text { deposition } * \text { time }}=\frac{\overline{\mathrm{R}}}{\overline{\mathrm{P}}}
$$

i.e. the ratio of the mean recharge rate to the mean precipitation rate should be the same as the ratio of vadose tritium to total tritium deposited. Both ratios are less than one to the extent that precipitation and tritium are removed from the profile by evapotranspiration. We can express the inventory and deposition*time terms exactly as we did for $\mathrm{Cl}$ in [4.119]; doing this and solving for $\mathrm{R}$ we get

$$
\overline{\mathrm{R}}=\frac{\int_{0}^{\mathrm{z}} \mathrm{C}_{\mathrm{pw}} \theta \mathrm{dz}}{\overline{\mathrm{P}}^{-1} \int_{0}^{\mathrm{t}} \mathrm{C}_{\mathrm{p}} \mathrm{Pdt}}
$$

We also need to account for the fact that precipitated tritium has decayed over the time between deposition and inventory:

$$
\bar{R}=\frac{\int_{0}^{z} C_{p w} \theta d z}{\bar{P}^{-1} \int_{0}^{t} C_{p} P \exp (-t \lambda) d t}
$$

where $\lambda$ is the radioactive decay constant $\left[\mathrm{T}^{-1}\right]$. Using yearly precip. data, we get:

$$
\overline{\mathrm{R}}=\frac{\int_{0}^{\mathrm{z}} \mathrm{C}_{\mathrm{pw}} \theta \mathrm{dz}}{\overline{\mathrm{P}}^{-1} \sum_{\mathrm{i}=1}^{\mathrm{t}} \mathrm{C}_{\mathrm{pi}} \mathrm{P}_{\mathrm{i}} \exp (-\mathrm{t} \lambda) 1 \mathrm{yr}}
$$

We wrote mean precipitation in the denominator to show that each year's deposition is weighted with respect to that quantity. Equation [4.127] is equivalent to [5] of Allison et al. (1994). As they point out, it is quite general in that preferential flow is not a problem as long as all the tritium in a profile is captured in the inventory. The reason this formulation has not been used more may be the difficulty of assigning the right $\mathrm{C}_{\mathrm{pi}}$ to each $\mathrm{P}_{\mathrm{i}}$ in each yearly term of the deposition: for example, in many settings only winter precipitation becomes recharge, but the tritium signature of that winter precipitation may differ substantially from the reported or estimated yearly mean.

In principle [4.127] could be applied to other evaporating species such as ${ }^{2} \mathrm{HHO}$ or $\mathrm{H}_{2}{ }^{18} \mathrm{O}$. However in practice trends in these concentrations have been used to count seasonal cycles where rainfall is large (e.g. Bath et al., 1982) or to inversely relate evaporative isotope fractionation to recharge (Allison et al., 1984) in dry settings. 


\subsection{3 "Bomb pulse" methods.}

We noted in the previous section that tritium deposition in precipitation reached very large concentrations during the time of atmospheric thermonuclear testing. This property of the tritium "history" of precipitation may be exploited in a very simple manner using very simple logic: water samples with substantial tritium concentrations must contain at least a component of water precipitated since testing began in 1952. "Substantial" means less than one TU: pre-testing levels in precipitation may have been as high as $10 \mathrm{TU}$ which, when decayed the four half-lives (at time of this writing) since then, corresponds to about 0.6 TU today. Current "low-level" analytical technique with a detection limit of $0.1 \mathrm{TU}$ is, therefore, good enough to detect even small fractions of post-bomb water in environmental samples. Unfortunately for us, vadose water samples for tritium analysis must generally be distilled or squeezed from core samples of sediment; this typically yields volumes on the order of a few $\mathrm{mL}$ which are too small for low-level analysis and must instead be counted by a standard technique with a detection limit of 5-15 TU. Even with this great loss of resolution it is possible to identify post-bomb water in vadose profiles, where concentrations commonly range from a few tens to hundreds of TU.

Shapes of vertical vadose tritium profiles may be relatively smooth, as in Figure 8.21, or they may exhibit great irregularity (e.g. Hendry, 1983). When we see a smooth peak like that beneath the top-slope position of the study site of O'Brien et al. (1996) (Figure 8.21) it is tempting to identify that peak with the peak in global atmospheric tritium deposition in 1963. Were this the case, pore water above the peak could be considered the increment to the subsurface system since 1963, i.e. a measure of the local rate of recharge with a sampling time of decades. The allure of such a recharge estimate is great, but - be careful! The underlying assumption of plug flow (chapter 3) may not be met, leading to large errors. O'Brien et al. (1996) show, in fact, how meters-deep bomb tritium peaks in semi-arid vadose profiles could occur due to preferential flow where there is no recharge beneath the soil zone. van der Kamp (1992) gives related illustrations for other semi-arid settings. Our experience, reflected more broadly in the literature, is that patterns of tritium occurrence in vadose zones are most useful in comparison with other environmental tracers, whereby they illustrate flow processes as affected by heterogeneity, and where they can help corroborate recharge estimates made by other means such as water balance and chloride mass balance (e.g. Wood et al., 1997; O'Brien et al., 1996; Scanlon, 1992; Phillips et al., 1988).

Chlorine-36 is another environmental tracer which, like tritium, has gained attention since mid-20th-century thermonuclear bomb testing. A very small fraction of atmospheric chloride deposition occurs naturally as ${ }^{36} \mathrm{Cl}$, a radioisotope with a half-life of 301,000 yr generated in the upper atmosphere by cosmic ray bombardment. These concentrations are typically reported as the fraction of total chloride, ${ }^{36} \mathrm{Cl} / \mathrm{Cl}$, with present-day natural background values in precipitation (probably similar to Holocene and late-Pleistocene time) around $500 \times 10^{-15}$ to $1000 \times 10^{-15}$ in North America. The great advantage of chlorine-36 relative to tritium is its large half-life which, in combination with its low detection limit, permits the radiometric dating of very old waters (e.g. Bentley et al., 1986). This property can in principle be exploited to estimate the mean residence times and recharge rates of very old vadose pore water in arid regions (e.g. Tyler et al., 1996). On the down side is the expense and difficulty of chlorine-36 analyses (by specialized accelerator mass spectrometry) and the difficulty of estimating chlorine-36 and chloride deposition rates tens to hundreds of thousands of years in the past. These practical problems have focussed most applications on the bomb pulse of chlorine-36 which was generated by thermonuclear test explosions on Pacific atolls in the 1950s. This bomb pulse was "washed out" of the atmosphere by precipitation which thereby attained ${ }^{36} \mathrm{Cl} / \mathrm{Cl}$ ratios several hundreds of times greater than natural background, ending in the early 1960s.

Vadose profiles of chlorine-36 concentrations present similar seductions and opportunities as do tritium profiles. As with tritium, their great utility (if the cost obstacle can be surmounted!) is in comparison with other tracers to elucidate flow processes. A good example is the recent recognition that vadose bomb pulses of chlorine-36 and tritium will move at different rates to the extent that the vapor-phase contribution to the total water flux is important: as a solute, chlorine-36 does not partition into the vapor phase and cannot move across "vapor gaps", while tritium does (Phillips et al., 1988; Scanlon, 1992). Observation of bomb-pulse chlorine-36 levels deep within desert vadose zones is being used to bolster arguments that rapid preferential flow can occur in these settings (Fabryka-Martin et al., 1996). 


\subsubsection{Mass balances on dissolving solids.}

In some geologic settings the distribution of a chemical species in the sediment - rather than in the pore water - is a useful indicator of the distribution of flow rates. This approach has been used semiquantitatively, in fact, by pedologists for a long time. Illustrate, we return to Figure 8.14 which showed localized removal of sulfate-containing gypsum from till.

Assuming that sulfate is removed in recharging groundwater, the total mass depletion per unit area, $\mathrm{M}_{\mathrm{d}}$, would be:

$$
\mathrm{M}_{\mathrm{d}}=\int_{\mathrm{t}_{0}}^{\mathrm{t}_{1}} \mathrm{q}_{\mathrm{r}} \mathrm{C}_{\mathrm{r}} \mathrm{dt}
$$

where $\mathrm{qr}_{\mathrm{r}}$ is the recharge flux $\left(\mathrm{LT}^{-1}\right), \mathrm{C}_{\mathrm{r}}$ is the sulfate concentration $\left(\mathrm{ML}^{-3}\right)$, and $\mathrm{t}$ is time. If conditions have been sufficiently steady through the time interval of interest, then the integration yields (Keller et al., 1991):

(8.164) $\mathrm{M}_{\mathrm{d}}=\mathrm{q}_{\mathrm{r}} \mathrm{C}_{\mathrm{r}} \Delta \mathrm{t}$

Knowledge of any three of the variables in [4.78] permits solution for the fourth. The most potentially widespread application of this expression is in exploring time/recharge hypotheses, i.e. $\Delta \mathrm{t} / \mathrm{qr}$ pairs in [4.128], against observed mass-depletion $\left(\mathrm{M}_{\mathrm{d}}\right)$ and concentration $\left(\mathrm{C}_{\mathrm{r}}\right)$ values.

\section{Example}

At the Dalmeny site Darcy's Law was applied to piezometric data in the saturated zone to estimate the mean annual rate of recharge in the leached area at piezometer nest 10 (Figure 8.14). The result was approximately $\mathrm{qr}_{\mathrm{r}}$ $=3.5 \mathrm{~cm} \mathrm{yr}^{-1}$ (Keller et al., 1988). Because of the notorious difficulty of accurately estimating the vertical hydraulic conductivity of sediments in the field, we want to test the consistency of this result with observed vadose depletion of major elements in the till through which recharge occurs. Keller et al. (1991) estimated the mean concentration of DIC at this location as $190 \mathrm{mg} \mathrm{L}^{-1}$. Because this DIC is the result of dissolution of carbonate minerals by carbonic acid, [4.70] shows that half - the half we are interested in here - is attributable to the minerals (the other half coming from biogenic $\mathrm{CO}_{2}$ ); thus $\mathrm{C}_{\mathrm{r}}=95 \mathrm{mg} \mathrm{L}^{-1}$. If we assume that leaching has been going on at this location since the end of the last glaciation, we take $\mathrm{Dt}=11,500 \mathrm{yr}$. Equation [4.128] then yields $\mathrm{M}_{\mathrm{d}}=3.8 \times 10^{4} \mathrm{gC} \mathrm{m}^{-2}$, that is, about $40 \mathrm{~kg}$ of inorganic carbon (mostly in the form of $\mathrm{HCO}_{3}^{-}$) has been dissolved from calcite and dolomite per square meter of till beneath this topographically depressed area. 


\section{LEACHED (34)}

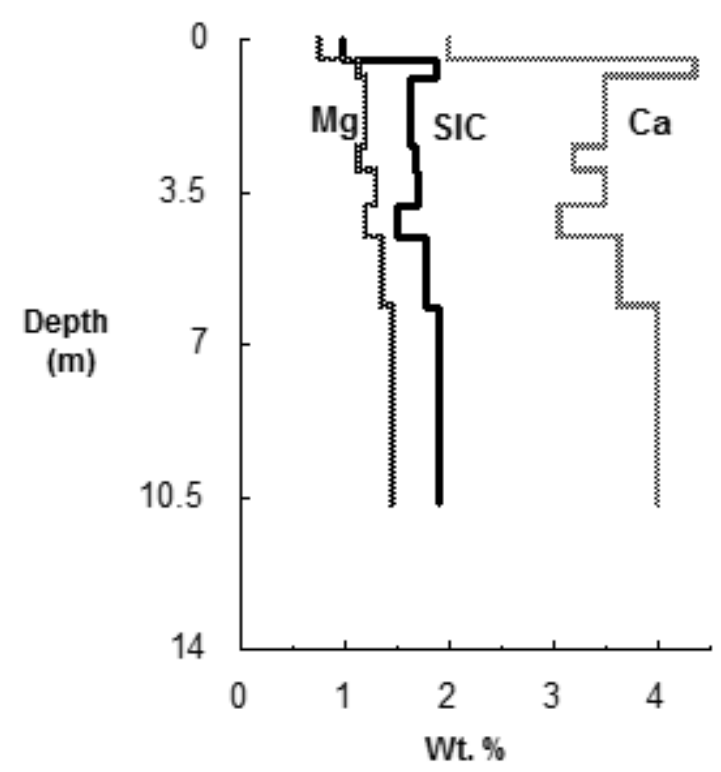

Figure 8.26 Concentrations of calcium, magnesium, and solid inorganic carbon in carbonate minerals in till at location 34 (near piezometer 10, Figure 8.14) at the Dalmeny site. Profiles show elemental depletion in the upper $6 m$ due to dissolution of carbonate minerals and removal in downward flow at this recharge focus.

Now: how does this hypothesis (of recharge rate and its constancy through the Holocene) square with observation? Figure 8.26 shows a solid inorganic carbon concentration (SIC) profile for testhole 34, drilled and sampled near piezometer 10. We can qualitatively see depletion of inorganic $\mathrm{C}$ in the vadose zone as reduction of SIC to levels less than the deep-till, unweathered value of $1.9 \%$ by weight. Quantitatively the depletion is the sum of the depletions for each of the sampled intervals, times their respective thicknesses:

$$
\mathrm{M}_{\mathrm{c}(\mathrm{obs})}=\sum\left(\mathrm{C}_{\mathrm{i}}-\mathrm{C}_{\mathrm{un}}\right) \rho_{\mathrm{i}}\left(1-\mathrm{n}_{\mathrm{i}}\right) \mathrm{z}_{\mathrm{i}}
$$

where $\mathrm{C}$ is solid-phase concentration $\left[\mathrm{g} \mathrm{g}^{-1}\right] \mathrm{C}_{\mathrm{un}}$ is that concentration in unweathered till, $\rho$ is density of solid particles $\left(2.7 \times 10^{6} \mathrm{~g} \mathrm{~m}^{-3}\right), \mathrm{n}$ is porosity (about 0.26 for this till), and $\mathrm{z}$ is depth. Because the physical properties are effectively constant in this case, we can replace [4.129] with

$$
\mathrm{M}_{\mathrm{c}(\mathrm{obs})}=\left(\mathrm{C}_{\text {ave }}-\mathrm{C}_{\mathrm{un}}\right) \rho(1-\mathrm{n}) \mathrm{z}
$$

where $\mathrm{C}_{\mathrm{ave}}$ is the average depletion for the whole weathered and leached interval. Eyeballing Figure 8.26, we can guesstimate $\mathrm{C}_{\mathrm{ave}}$ as about $1.6 \%$ or $0.016 \mathrm{~g} \mathrm{~g} \mathrm{~g}^{-1}$, which yields $\mathrm{M}_{\mathrm{d}(\mathrm{obs})}=3.6 \times 10^{4} \mathrm{gC} \mathrm{m}^{-2}$. Keller et al. (1991) used the actual SIC concentration data and [4.129] to obtain $\mathrm{M}_{\mathrm{d}(\mathrm{obs})}=3.3 \times 10^{4} \mathrm{gC} \mathrm{m}^{-2}$. Both of these results are within 20\% of $\mathrm{M}_{\mathrm{d}}$ as estimated above by [4.128]. In this case of good agreement between predicted and observed depletion, we gain confidence in our Darcian recharge-rate estimation and, particularly, the value of that elusive vertical hydraulic conductivity term. We also find support for other features of our conceptual model of this site: that water quality is controlled by the carbonate system (section 4.4.1) and, most practically important, that time-averaged hydraulic and geochemical conditions over the past 10,000 years or so have been much like today's.

This exercise hammers home the fact that observable leaching of major elements is not limited to soils and may extend, in fact, all the way through vadose zones (the mean water table depth at testhole 34 is about 3-4 m). Valuable, 
time-integrated hydrologic information would be lost if geochemical monitoring were not performed all the way from ground surface into the saturated zone. As a practical matter, we see that success of this exercise depends upon accurate knowledge of the geochemical initial condition $\left(\mathrm{C}_{\mathrm{un}}\right)$ and depletions which are larger than measurement uncertainties. Keller et al. (1991) noted that this analysis can in principle be extended to any element which is predictably soluble and leachable on the timescale of interest, and is not subject to depletion via gas-phase transport (e.g. $\mathrm{C}$ as $\mathrm{CO}_{2}$, section 4.3.3).

\subsubsection{Mass balances on exchanging solids.}

We noted in section 4.5 that cation exchangers change composition with time unless they are in equilibrium with pore water. Sometimes, as when the exchanger is imprinted with a particular (say marine) composition and is exposed to altered pore-water chemistry at some point in the past, this fact can be used to estimate the "characteristic time" of cation-exchanger control on water quality. Our approach is to divide the exchangeable cation reservoir by the cation flux through that reservoir. This is a steady-state mass balance that gives essentially the mean residence time of a cation in the exchangeble reservoir:

$$
\frac{\mathrm{CEC}\left(\mathrm{meq} \bullet \mathrm{m}^{-3}\right) \mathrm{L}(\mathrm{m})}{\mathrm{q}\left(\mathrm{m} \bullet \mathrm{yr}^{-1}\right) \text { cationconc }\left(\mathrm{meq} \bullet \mathrm{m}^{-3}\right)}
$$

where $\mathrm{L}$ is flow path length, and CEC (numerator) and aqueous cation concentration (denominator) are both per unit volume of formation.

\section{Example}

The till at the Dalmeny site (section 4.8.5 above) contains a marine-derived, initially Na-dominated cation exchanger. Let's estimate how long this exchanger should maintain elevated Na levels in subsurface pore water. The till has CEC $=0.1 \mathrm{meq} \mathrm{g}^{-1}$, bulk density $\mathrm{r}_{\mathrm{b}}$ is $2 \times 10^{6} \mathrm{~g} \mathrm{~m}^{-3}$, and porosity averages 0.26 . The previous discussion shows that we actually have two calculations to make, one for leached recharge foci and one for unleached low-recharge areas. If we consider a vertical flowpath, which is the shortest distance L through the till, its uniform thickness (Figure 8.14) and physical characteristics mean the numerator of [4.130] is the same for both cases: the CEC times $\rho \mathrm{b}$ times L yields $5.2 \times 10^{6} \mathrm{meq}^{-2}$.

\section{Table 8.10}

\begin{tabular}{ccc} 
setting & leached & unleached \\
$\mathbf{q}\left(\mathbf{m ~ y r}^{-\mathbf{1}}\right)$ & 0.035 & $<0.001$ \\
cation concentration $\left(\mathbf{m e q ~}^{-\mathbf{1}}\right)$ & 12 & 70 \\
$\mathbf{q}\left(\mathbf{m}^{\mathbf{3}} \mathbf{~ m}^{-\mathbf{3}}\right)$ & 0.26 & 0.26 \\
cation flux $\left(\mathbf{m e q ~}^{-\mathbf{2}} \mathbf{y r}^{-\mathbf{1}}\right)$ & 110 & 18 \\
$\operatorname{time~}(\mathbf{y r})$ & $5 \times 10^{4}$ & $3 \times 10^{5}$ \\
\hline
\end{tabular}


Table 8.10 shows typical vertical flow rates and aqueous cation concentrations needed to calculate the cation flux (denominator) for each setting. The volumetric water content $\theta$ is needed to convert the aqueous concentrations to a formation basis. The resulting times indicate that if the exchanger began to re-equilibrate to present-day conditions about 10,000 yr ago (which the previous example suggests is reasonable), the leached settings at Dalmeny are just getting a good start in their evolution to producing less-sodic water. In fact we made this observation in section 4.5. The times in Table 8.10 are also consistent with the fact that $\mathrm{Na} /(\mathrm{Na}+\mathrm{Ca})$ milliequivalent ratios in groundwaters are only somewhat smaller in leached than in unleached settings (Keller et al., 1991).

In principle this solid-phase mean residence time approach could be extended to other phases, like (for example) primary carbonate minerals in vadose zones. Reardon et al. (1979) recognized this possibility when they observed clearlydefined zones of calcite depletion in a humid-climate sand vadose zone. Hydrogeologists have predicted attenuation of acid mine drainage (e.g. Morin et al., 1982) this way.

4.9 Review Questions

1. Why does an equilibrium constant have a unique value (at given $\mathrm{P}, \mathrm{T}$ ) regardless of the complexity of a geochemical system?

2. What is chemical activity? Why is the distinction between activity and concentration necessary?

3. Give an example of how we might use Henry's Law in a practical vadose zone problem.

4. Demonstrate and discuss the similarity of [4.40] to [2.52].

5. Describe how a pore water is speciated and how the results can be used interpretively.

6. Why is vadose biological activity chemotrophic? Why does this activity decline rapidly with increasing depth in natural vadose systems?

7. Speculate on what conditions might lead to (a) high $\mathrm{CO}_{2}$ concentrations, (b) high $\mathrm{CO}_{2}$ production rates deep within vadose zones.

8. Could you use the $\mathrm{PCO}_{2}$, measured on a vadose gas sample, to estimate the $\mathrm{pH}$ of pore water at that point? What else would you need to know?

9. What is the biological significance of vadose acid (including $\mathrm{CO}_{2}$ ) generation?

10. Silicate minerals are omnipresent in vadose zones, but they play "second fiddle" in the hydrochemical orchestra when carbonate minerals are present. Why?

11. Other things equal, would vadose porewater $\mathrm{pH}$ values be higher in carbonate or in silicate terranes? Why?

12. Briefly outline what happens to dissolving primary silicate minerals in vadose zones. Which constituents are retained, which are transported, and why?

13. Define caliche and describe how it forms.

14. Define a mafic mineral assemblage.

15. There are two basic pathways leading to highly mineralized (high ionic strength) vadose pore waters. What are they? Briefly discuss the geographic occurrence of these pathways.

16. If you wanted to characterize the quality of water in a vadose zone, how would knowledge of the local stratigraphy (stacking of rock/sediment layers) help you plan your sampling?

17. According to the soils/ecosystems model of hydrochemical evolution in a silicate terrane (no carbonates nor evaporites), does soil porewater quality adequately represent the quality of groundwater?

18. How does the surface tension of the solution phase (water) affect sorption of solutes to solids?

19. How does double-layer thickness vary with ionic strength?

20. Briefly explain why selectivity coefficients (for cation exchange) are conditional rather than 
thermodynamic constants.

21. How can a selectivity coefficient be measured?

22. How can a selectivity coefficient be used to predict pore-water quality? What about a distribution coefficient $\left(\mathrm{K}_{\mathrm{d}}\right)$ ?

23. List some important limitations of [4.112] for predicting organic sorption in vadose environments.

24. Argue that predicting the gross distribution of nitrate in vadose zones is primarily a biogeochemical problem.

25. Argue that the best species for indicators of vadose flow rates do not interact with solids (are "conservative").

26. Argue that the best species for indicators of vadose flow rates are subject to equilibrium dissolution/ precipitation/exchange.

\subsection{Problems}

1. Derive [4.40] from [4.26].

2. A simple pore water has the following composition at $25 \mathrm{C}$ :

\begin{tabular}{ll}
$\mathrm{Ca}^{2+}$ & $608 \mathrm{mg} / \mathrm{L}$ \\
$\mathrm{SO}_{4}{ }^{2-}$ & $1456 \mathrm{mg} / \mathrm{L}$ \\
\hline
\end{tabular}

A. Write a spreadsheet to "speciate" the solution, i.e. to calculate $\mathrm{m}_{\mathrm{i}}, \gamma_{\mathrm{i}}$, and $\mathrm{a}_{\mathrm{i}}$ for these two species and their neutral complex $\mathrm{CaSO}_{4}{ }^{\circ}$. Take $\mathrm{K}_{\mathrm{stab}}=10^{+2.3}$ at $25 \mathrm{C}$ for the complex. Assume these are the only species in solution and that the density of the solution differs negligibly from that of liquid water. Use the Debye-Huckel relation to calculate $\mathrm{g}_{\mathrm{i}}$; obtain the necessary solvent parameters and effective diameters from Table 8.3. Use a simple iterative technique, following section 4.2.5.3. Use a convergence criterion mi $=0.00005 .4$.

B. From your results, compute the IAP for gypsum, $\mathrm{CaSO}_{4} \bullet 2 \mathrm{H}_{2} \mathrm{O}$. Assume $\mathrm{a}_{\text {water }}=1$.

C. Assume that the solution is in fact saturated w.r.t. gypsum. If $\Delta \mathrm{G}^{\mathrm{o}}$ f(gypsum) is $-1797197 \mathrm{~J} \mathrm{~mol}^{-1}$ and $\Delta \mathrm{G}_{\mathrm{f}}^{\mathrm{O}}\left(\mathrm{Ca}^{2+}\right)$ is $-553540 \mathrm{~J} \mathrm{~mol}^{-1}$ (from Robie et al., 1978), what is $\Delta \mathrm{G}^{\mathrm{O}}\left(\mathrm{SO}_{4}{ }^{2-}\right)$ ?

3. Consider the following laboratory analysis of a water-table groundwater sample: 


\begin{tabular}{lrl}
\hline $\mathrm{Na}$ & 51 & $\mathrm{mg} / \mathrm{L}$ \\
$\mathrm{K}$ & 0.9 & \\
$\mathrm{Ca}^{2+}$ & 40 & \\
$\mathrm{Mg}^{2+}$ & 11 & \\
$\mathrm{SO}^{2-}$ & 57 & \\
$\mathrm{Cl}$ & 2.5 & \\
$\mathrm{HCO}_{3}-$ & 240 & \\
$\mathrm{pH}$ & 7.4 & units \\
$\mathrm{T}$ & $15 \mathrm{C}$ & \\
\hline
\end{tabular}

Compute the following:

a. TDS

b. meq/L for each of the solutes

c. millimoles $/ \mathrm{kg}(\mathrm{m})$ for each of the solutes (assume density of solution $=1 \mathrm{E} 3 \mathrm{~kg} \mathrm{~m}^{-3}$ )

d. I

e. activity coefficient $(\gamma)$ for each of the solutes (do not bother with an interative scheme for parts e. and f.)

f. activity for each of the solutes.

Suggestion: these calculations quickly get tedious and boring, and are thus subject to error when done by hand. This is an excellent application for a microcomputer spreadsheet with columns mg/L, gfw, m, z, etc.

g. is there evidence of gypsum in the vadose zone? (estimate logSIgyp)

4. Reconsider the water analysis in problem XX.

a. estimate the $\mathrm{PCO}_{2}$ at the bottom of the vadose zone.

b. do you think calcite is present in the vadose zone?

5. In section 4.3.4 a simple reaction simulation was performed in which atmospheric precipitation was equilibrated with atmospheric $\mathrm{CO}_{2}$. Estimate the ionic strength of the simulated water, and the magnitude of the error in results due to the assumption $\mathrm{a}_{\mathrm{i}}=\mathrm{m}_{\mathrm{i}}$.

6.

A. Consider calcite in water, with $\mathrm{pH}$ and $\mathrm{PCO}_{2}$ arbitrarily variable. Caclulate and plot as a function of $\mathrm{pH}$

a. activities of $\mathrm{HCO}^{-}$, and

b. activity of $\mathrm{Ca}^{2+}$,

for $\mathrm{PCO}_{2}=10^{-1.5}, 10^{-2.0}, 10^{-2.5}$, and $10^{-3.5}$ bar , at $\mathrm{T}=25 \mathrm{C}$. Use the equilbrium constants in Table 8.5 and the "open system" assumptions in section 4.4.1. (A spreadsheet is recommended, especially for trial-anderror solution of the 4 th-order polynomial.)

B. Now consider a natural system (Figure 8.11). Suppose, as is usually the case, that highly soluble (sulfate, chloride) salts and carbonate minerals have been flushed from the solum by percolating 
groundwater. Estimate the $\mathrm{pH}$ in the solum during the growing season $\left(\mathrm{PCO} 2=10^{-2}\right.$ bar), neglecting any silicate mineral dissolution. Should this estimate be an upper limit or a lower limit for a real soil?

C. Now assume that porewater from part B moves beneath the solum into a zone containing fine grains of calcite. If the PCO2 remains about the same, how does the pH change?

D. Now assume that the $\mathrm{PCO}_{2}$ declines, following the growing season, to $10^{-3.5}$ bar. How much $\mathrm{Ca}^{2+}$ leaves the porewater solution? If a distinct horizon forms in the vadose zone by this process, what do we call it?

E. Suppose our porewater percolates further downward into a zone containing gypsum. Use the system of in part A above and mass-action reasoning to qualitatively explain the changes in the chemistry of the water.

7. Draw part of Figure 8.10 for yourself: compute and plot the stability boundaries for gibbsite-kaolinite and kaolinite - Na-beidellite. Use free-energy data in Table 8.6.

A. Neglecting differences between activities and molalities, plot the following two water compositions (from Garrels and MacKenzie, 1967, Table I) on your Figure.

$\begin{array}{lll} & \text { molality, } & \text { molality, } \\ \text { species } \quad \text { ephemeral springs } & \text { perennial springs }\end{array}$

springs

$\begin{array}{lll}\mathrm{Ca} 2+ & 0.78 \mathrm{E}-4 & 2.6 \mathrm{E}-4 \\ \mathrm{Na}+ & 1.34 \mathrm{E}-4 & 2.59 \mathrm{E}-4 \\ \mathrm{HCO}- & 3.28 \mathrm{E}-4 & 8.95 \mathrm{E}-5 \\ \mathrm{SiO} 2 & 2.73 \mathrm{E}-4 & 4.1 \mathrm{E}-4 \\ \mathrm{pH} & 6.2 & 6.8\end{array}$

B. Do you see evidence for evolution of water chemistry along flow paths? Is there any evidence for difference in subsurface residence time?

C. This problem demonstrates a classical hydrogeochemical analysis of a silicate terrane. Do crude calculations to indicate that these waters did not evolve in vadose zones containing carbonate minerals.

8. The following are chemical properties of Chalk River sand (Reynolds et al. 1982, Table 1): 


\begin{tabular}{lll}
\hline & mean concentration, & mean concentration, \\
cation & exchanger (meq g-1) & pore water $(\mathrm{mg} \mathrm{L}-1)$ \\
$\mathrm{Ca}^{2+}$ & 0.0437 & 82.7 \\
$\mathrm{Mg}^{2+}$ & 0.0024 & 4.63 \\
$\mathrm{~K}^{+}$ & 0.0106 & 4.74 \\
\hline
\end{tabular}

A. Compute exchangeable cations, ratios of exchanger : solution cation equivalents, and the cation exchange capacity of the sand.

B. Batch-derived $\mathrm{K}_{\mathrm{d}}$ for dissolved strontium (Sr) on the sand is $15 \mathrm{~mL} \mathrm{~g}^{-}$. Also sand bulk density = $1.75 \mathrm{~g}$ $\mathrm{cm}^{-3}$, and saturated volumetric water content $\mathrm{q}_{\mathrm{s}}=0.34^{1}$ (Reynolds et al., 1982). Assuming vadose volumetric water content $\mathrm{q}=0.1$, estimate the $\mathrm{Sr}$ retardation coefficient. If mean downward porewater velocity in the vadose zone is $0.3 \mathrm{~m} \mathrm{yr}^{-1}$, what is the mean downward velocity of dissolved $\mathrm{Sr}$ ?

9. At the location of testhole 34 at the Dalmeny site (Figure 8.26), mean calcium concentration in groundwater is $\mathrm{C}_{\mathrm{r}}=198 \mathrm{mg} \mathrm{L}^{-1}$. Assuming that downward specific discharge $\mathrm{qr}_{\mathrm{r}}=3.5 \mathrm{~cm} \mathrm{yr}^{-1} \mathrm{through}$ the Holocene, and taking till physical properties reported in section 4.8.4, decide whether predicted and observed calcium mass depletions support the idea that hydrogeologic conditions have been constant over this period.

10. The Borden site in southern Ontario is characterized by sandy glaciofluvial sediment and a more humid climate than at Dalmeny (section 4.8.5). Estimate the characteristic time of exchanger evolution at Borden, assuming a typical flow-path length of $100 \mathrm{~m}, \mathrm{r}_{\mathrm{b}}=1.5 \times 10^{6} \mathrm{~g} \mathrm{~m}^{-3}$, and $\mathrm{q}=0.1-0.3 \mathrm{~m} \mathrm{yr}^{-1}$. Get CEC and cation concentration data from Table 8.8; recall that the CEC is the sum of exchangeable cations. Is the exchanger in equilibrium with present-day environmental conditions?

\section{References for Chapter 4}

Allen-King, R.M., McKay, L. D., Trudell, M. R., 1997. Organic carbon dominated trichloroethene sorption in a clay-rich glacial deposit. Ground Water 35: 124-130.

Allison, G.B., G.W. Gee, and S.W. Tyler, 1994. Vadose-zone techniques for estimating groundwater recharge in arid and semiarid regions. Soil Sci. Soc. Am. J. 58: 6-14.(all)

Allison, G.B., C.J. Barnes, M.W. Hughes, and F.W.J. Leaney, 1984. Effect of climate and vegetation on oxygen-18 and deuterium profiles in soils. In: Isotope Hydrology, Proc. Int. Symp. Isot. Hydrol Water Resour. Dev., Vienne, 12-16 Sept. 1983, IAEA.

Allison, J.D., D.S. Brown, and K.J. Novo-Gradac, 1991. MINTEQA2/PRODEFA2, a geochemical assessment model for environmental systems, version 3.0 user's manual. U.S. Environmental Protection Agency Report EPA/600/3-91/021. Amundson, R.G., and E.A. Davidson, 1990. Carbon dioxide and nitrogenous gases in the soil atmosphere. J. Geochem. Explor. 38: 13-41. 
Amy, P.S., C. Durham, D. Hall, and D.L. Haldeman, 1993. Starvation-survival of deep subsurface isolates. $\underline{\text { Current }}$ Microbiol. 26: 345-352.

Antweiler, R.C., and J.I. Drever, 1983. The weathering of a late Tertiary volcanic ash: Importance of organic solutes. Geochim. Cosmochim. Acta 47: 623-239.

Arthur, M.A., and T.J. Fahey, 1993. Controls on soil solution chemistry in a subalpine forest in north-central Colorado. Soil Sci. Soc. Am. J. 57: 1122-1130.

Back, W., J.S. Rosenshein, and P.R. Seaber, eds., 1988. Hydrogeology: The Geology of North America, Volume O-2, Geol. Society of America, $510 \mathrm{pp}$.

Bacon, D.H. 1997. Residence time of labile carbon in the vdose zone. PhD dissertation, Washington State University, 256 pp.

Bacon, D.H., and C.K. Keller, in press. Carbon dioxide respiration in the deep vadose zone: Implications for groundwater age dating. Water Resources Research.

Ball, J.W., and D.K. Nordstrom, 1991. User's manual for WATEQ4F, with revised thermodynamic data base and test cases for calculating speciation of major, trace, and redox elements in natural waters. U.S. Geological Survey Open-File Report 91-183, 61 pp.

Balkwill, D.L., and W.C. Ghiorse, 1985. Characterization of subsurface bacteria associated with two shallow aquifers in Oklahoma. Appl. Environ. Microbiol. 50: 580-588.

Bath, A.H., W.G. Darling, and A.P. Brunsden, 1982. The stable isotopic composition of infiltration moisture in the unsaturated zone of English Chalk. In: H.L. Schmidt (ed.), Stable isotopes, Elsevier, pp. 161-166.

Bentley, H.W., F.M. Phillips, S.N. Davis, M.A. Habermehl, P.L. Airey, G.E. Claf, D. Elmore, H.E. Gove, and T. Torgersen, 1986. Chlorine 36 age dating of very old ground waters, 1., The Great Artesian Basin, Australia. Water Resour. Res. 22: 1991-2002.

Berner, R.A., and D.E. Canfield, 1989. A new model for atmospheric oxygen over Phanerozoic time. Am. J. Sci. 289: 333-361.

Binger, C.A., Martin, J. P., Allen-King, R. M., Fowler, M., in prep. Variability of chlorinated solvent sorption associated with oxidative weathering of kerogen. Submitted, Environ. Sci. Technol.

Bohn, H.L., B.L McNeal, and G.A. O'Connor, 1979. Soil Chemistry. Wiley, 329 pp.

Bowman, R.S., J. Schroeder, R. Bulusu, M, Remmenga, and R. Heightman, 1997. Plant toxicity and plant uptake of fluorobenzoate and bromide water tracers. J. Environ. Qual. 26: 1292-1299.

Bowman, R.S., G.W. Gross, R.L Liddle, and J.B. Kool, 1990. Study of hydrologic poperties to include the use of tracers. Report no. 1 to the U.S. Geological Survey, contract no. 14-08-0001-22637, 554 pp.

Brockman, F.J., T.L. Kieft, J.K. Fredrickson, B.N. Bjornstad, Shu-mei W. Li, W. Spangenburg, and P.E. Long, 1992. Microbiology of vadose zone paleosols in south-Central Washington State. Microb. Ecol. 23: 279-301.

Brownlow, A.H., 1996. Geochemistry. 2nd. ed. Prentice-Hall, 580 pp.

Brusseau, M.L., 1994. Transport of reactive contaminants in heterogeneous porous media. Rev. of Geophys. 32:285-313.

Chapelle, F.H., 1993. Ground-water microbiology and geochemistry. Wiley, 424 pp.

Cronan, C.S., and G.R. Aiken, 1985. Chemistry and transport of soluble humic substances in forested watersheds of the Adirondack Park, New York. Geochim cosmochim. Acta 49: 1697-1705.

Dance, J.T., and E.J. Reardon, 1983. Migration of contaminants in groundwater at a landfill: a case study. 5. Cation migration in the dispersion test. J. Hydrol. 63: 109-130.

Davidson, E.A., and S.E. Trumbore, 1995. Gas diffusivity and production of $\mathrm{CO}_{2}$ in deep soils of the eastern Amazon. Tellus 47B: 550-565.

Deines, P., D. Langmuir, and R.S. Harmon, 1974. Stable carbon isotope ratios and the existence of a gas phase in the evolution of carbonate ground waters. Geochim Cosmochim. Acta 38: 1147-1164.

Drever, J.I., 1994. The effect of land plants on weathering rates of silicate minerals. Geochim. Cosmochim. Acta 58(10): 2325-2332.

Drever, J.I, 1997. The geochemistry of natural waters: Surface and groundwater environments. 3rd. ed. Prentice-Hall, 436 pp. 
Johnson, N.M., C.T. Driscoll, J.S. Eaton, G.E. Likens, and W.H. McDowell, 1981. Acid rain, dissolved aluminum and chemical weathering at the Hubbard Brook Experimental Forest, New Hampshire. Geochim. Cosmochim. Acta 45(9): 1421-1437.

Fabryka-Martin, J.T., et al., 1996. Summary report of chlorine-36 studies: Sampling, Analysis, and simulation of Cl-36 in the Exporatory Studies Facility (Yucca Mountain). U.S. Dept. of Energy, Milestone 3783M, draft August 1996.

Fortin, G., G. van der Kamp, and J.A. Cherry, 1991. Hydrogeology and hydrogeochemistry of an aquifer-aquitard system within the glacial drift, Saskatchewan, Canada. J. Hydrol. 126: 265-292.

Freeze, R.A. and J.A. Cherry, 1979. Groundwater. Prentice-Hall, 604 pp.

Garrels, R.M., and C.L. Christ, 1965. Solutions, minerals, and equilibria. Freeman, 450 pp.

Garrels, R.M., and F.T. MacKenzie, 1967. Origin of the chemical compositions of some springs and lakes. In: Equlibrium concepts in natural water systems, W. Stumm, ed., American Chemical Society, Advances in chemistry series \# 67, pp. 222-232.

Gislason, S.R., and H.P. Eugster, 1987. Meteroic water-basalt interactions, II. A field study in northeastern Iceland. Geochim. Cosmochim. Acta 51: 2841-2855.

Grim, R.E., 1962. Applied clay mineralogy. McGraw Book Co.

Hansen, B.K., and D. Postma, 1995. Acidification, buffering, and salt effects in the unsaturated zone of a sandy aquifer, Klosterhede, Denmark. Water Resour. Res. 31(11): 2795-2809.

Hardie, L.A., and H.P. Eugster, 1970. The evolution of closed-basin brines. Mineral. Soc. Am. Spec. Publ. 3: 273-290.

Hem, J.D., 1985. Study and interpretaiton of the chemical characteristics of natural water, 3rd. ed. U.S. Geological Survey Water-Supply Paper 2254, 263 pp.

Hendry, M.J., J.A. Cherry, and E.I. Wallick, 1986. Origin and distribution of sulfate in a fractured till in southern Alberta, Canada. Water Resour. Res. 22(10), 45-61.

Hendry, M.J., 1983. Ground water recharge through a heavy-textured soil. J. Hydrol. 62(314): 201-209.

Holland, H.D., 1978. The chemistry of the atmoshpere and oceans. Wiley-Interscience, $351 \mathrm{pp}$.

Hyman, M.E., C.E. Johnson, S.W. Bailey, R.H. April, and J.W. Hornbeck, 1998. Chemical weathering and cation loss in a base-poor watershed. Geol. Soc. Am. Bull. 110: 85-95.

Jenny, H., 1980. The soil resource. Ecological Studies, v. 37. Springer-Verlag, 377 pp.

Jury, W.A., W.R. Gardner, and W.H. Gardner, 1991. Soil physics, 5th Ed. Wiley, 328 pp.

Kieft, T.L., P.S. Amy, F.J. Brockman, J.K. Fredrickson, B.N. Bjornstad, and L.L. Rosacker, 1993. Microbial abundance and activities in relation to water potential in the vadose zones of arid and semiarid sites. Microb. Ecol. 26: 59-78.

Keller, C.K., and G. van der Kamp, 1988. Hydrogeology of two Saskatchewan tills, II. Occurrence of sulfate and implications for soil salinity. J. Hydrol. 101: 123-144.

Keller, C.K., J.A. Cherry, and G. van der Kamp, 1991. Hydrogeochemistry of a clayey till 1. Spatial variability. Water Resour. Res. 27(10): 2543-2554.

Keller, C.K., and B.D. Wood, 1993. Possibility of chemical weathering before the advent of vascular land plants. Nature 364: 223-225.

Keller, C.K., and D.H. Bacon, 1998. Soil respiration and georespiration distinguished by transport analyses of vadose $\mathrm{CO}_{2}$, ${ }^{13} \mathrm{CO}_{2}$, and ${ }^{14} \mathrm{CO}_{2}$. Global Biogeochemical Cycles 12: 361-372.

Kuroda, P.K., and E.B. Sandell, 1953. Chlorine in igneous rocks. Geol. Soc. Am. Bull. 64: 879-896.

Lasaga, A.C., J.M. Soler, J. Ganor, T.E. Burch, and K.L. Nagy, 1994. Chemical weathering rate laws and global geochemical cycles. Geochim. Cosmochim. Acta 58: 2361-2386.

Langmuir, D., 1997. Aqueous environmental geochemistry. Prentice-Hall, 600 pp.

Likens, G.E., and F.H. Bormann, 1995. Biogeochemistry of a forested ecosystem, 2nd ed. Springer-verlag, 159 pp.

Lindsay, W.L., 1979. Chemical equilibria in soils. Wiley, 449 pp.

Luthy, R.G., G.R. Aiken, M.L. Brusseau, S.D. Cunningham, P.M. Gschwend, J.J. Pignatello, M. Reinhard, S.J. Traina, W.J. Weber Jr., and J.C. Westall, 1997. Sequestration of hydrophobic organic contaminants by geosorbents. Environ. Sci. Technol. 31(12): 3341-3347.

Massman, J., and D.F. Farrier, 1992. Effects of atmoshperic pressures on gass transport in the vadose zone. Water Resour. Res. 28: 771-791. 
Morin, K.A., J.A. Cherry, T.P. Lim, and A.J. Vivyurka, 1982. Contaminant migration in a sand aquifer near an inactive uranium tailings impoundment, Elliot Lake, Ontario. Can. Geotech. J. 19: 49-62.

Murphy, E.M., and G.R. Aiken, in prep. Natural organic carbon in groundwater. In: The Chemistry of Groundwater, ed. by C.D. Palmer. chap. 7.

Murphy, E.M., T.R. Ginn, and J.L Phillips, 1996. Geochemical estimates of paleorecharge in the Pasco Basin: Evaluation of the chloride mass balance technique. Water Resour. Res. 32(9): 2853-2868.

Murphy, E.M., Zachara, J. M., Smith, S. C., 1990. Influence of mineral-bound humic substances on the sorption of hydrophobic organic compounds. Environ. Sci. Technol. 24: 1507-1516.

Nordstrom, D.K., and J.L Munoz, 1994. Geochemical thermodynamics 2nd ed. Blackwell Scientific Publications, 493 pp.

Nordstrom, D.K., L.N. Plummer, and T.M.L. Wigley, 1979. A comparison of computerized chemical models for equilibrium calculations in aqueous systems. In: E.A. Jenne, ed., Chemical modeling in aqueous systems. American Chemical Society Symposium Series 93: 857-892.

Nordstrom, D.K., L.N. Plummer, D. Langmuir, E. Busenberg, H.M. May, B.F. Jones, and D.L. Parkhurst, 1990. Revised chemical equilibrium data for major water-mineral reactions and their limitations, In: D.C. Melchior and R.L. Bassett, eds., Chemical modeling in aqueous systems II. American Chemical Society Symposium Series 416: 398-413.

Parkhurst, D.L., D.C. Thorstenson, and L.N. Plummer, 1980. PHREEQE: a computer program for geochemical calculations. U.S. Geol Surv. Water Resour. Invest. 80-86, 210 pp.

Parkhurst, D.L., 1995. User's guide to PHREEQEC, a computer model for speciation, reaction-path, advective transport and inverse geochemical calculations. U.S. Geological Survey Water Resour. Invest. 95-4227, 143 pp.

Parkin, TB., and W.W. Simpkins, 1995. Contemporary groundwater methane production from Pleistocene carbon. J. Environ. Qual. 24: 367-372.

Paul, E.A., and F.E. Clark, 1989. Soil microbiology and biochemistry. Academic Press, 273 pp.

Phillips, F.M., 1994. Environmental tracers for water movement in desert soils of the American Southwest. Soil Sci. Soc. Am. J. 58: 15-24.

Phillips, F.M., J.L Mattick, T.A. Duval, D. Elmore, and P.W. Kubik, 1988. Chlorine-36 and tritium from nuclear weapons fallout as tracers for long-term liquid and vapor movement in desert soils. Water Resour. Res. 24(11): 1877-1891.

Plummer, L.N., D.L. Parkhurst, and D.C. Thorstenson, 1983. Development of reaaction models for ground-water systems. Geochim. Cosmochim. Acta 47: 665-686.

Plummer, L.N., E.C. Prestemon, and D.L. Parkhurst, 1994. In interactive code (NETPATH) for modeling net geochemical reactions along a flow path, version 2.0. U.S. Geol. Survey Water Resour. Invest. 94-4169.

Pignatello, J.J., 1989. Sorption dynamics of organic compounds in soils. In: B.L. Sawhney and K. Brown, eds, Reactions and movement of organic chemicals in soils. Soil Sci. Soc. Am. Spec. Publ. No. 22, pp. 45-80.

Reardon, E.J., G.B. Allison, and P. Fritz, 1979. Seasonal chemical and isotopic variations of soil CO2 at Trout Creek, Ontario. J. Hydrol. 43: 355-371.

Reardon, E.J., A.A. Mozeto, and P. Fritz, 1980. Recharge in northern clime clacareous sandy soils: Soil water chemical and carbon-14 evolution. Geochim. Cosmochim. Acta 44: 1723-1735.

Reardon, E.J., J.T. Dance, and J.L. Lolcama, 1983. Field determination of cation exchange properties for calcareous sands. Ground Water 21: 421-428.

Roe, R.B., 1995. Release of chloride from basalt: implications for the chloride mass-balance approach to estimating groundwater recharge. M.S. thesis, Washington State University.

Scanlon, B.R., 1992. Evaluation of liquid and vapor water flow in desert soils based on chlorine-36 and tritium tracers and noniosthermal flow simulations. Water Resour. Res. 28(1): 285-297.

Severson, K.J., D.L. Johnstone, C.K. Keller, and B.D. Wood, 1992. Hydrogeologic parameters affecting vadose-zone microbial distributions. Geomicrobiol. J. 9: 197-216.

Schlesinger, W.H., 1991. Biogeochemistry: An analysis of global change. Academic Press, 423 pp.

Schwartzenbach,R.P., and J. Westall, 1981. Environ. Sci. Technol. 15: 1360-1367.

Schwartzenbach, R.P., P.M. Gschwend, and D.M. Imboden, 1993. Environmental Organic Chemistry. Wiley.

Bio-Physics of the Vadose Zone | 389 
Simunek, J., and D.L. Suarez, 1994. Two-dimensional transport model for variably saturated porous media with major ion chemistry. Water Resour. Res. 30(4): 1115-1133.

Suarez, D.L., and J. Simunek, 1996. Solute transport modeling under variably saturated water flow conditions. IN: P.C. Lichtner, C.I Steefel, and E.H. Oelkers, eds., Reactive transport in porous media. Reviews in Mineralogy 34: 229-268.

Solomon, D.K., and T.E. Cerling, 1987. The annual carbon dioxide cycle in a montane soil: observations, modeling and implications for weathering. Water Resour. Res. 23:2257-2265.

Sposito, G., 1989. The chemistry of soils. Oxford University Press, 277 pp.

Stephens, D.B., 1994. A perspective on diffuse natural recharge mechanisms in areas of low precipitation. Soil Sci. Soc. Am. J. 58: 40-48. (all)

Stumm, W., and J.J. Morgan, 1981 and 1996. Aquatic chemistry, 2nd and 3rd eds. Wiley-Interscience.

Swoboda-Colberg,N.G., and J.I. Drever, 1993. Mineral dissolution rates in plot-scale field and laboratory experiments. Chem. Geol. 105: 51-69.

Truesdell, A.H., and B.F. Jones, 1974. WATEQ, a computer program for calculating chemical equilibria of natural waters. U.S. Geological Survey Journal of Research, vol. 2 pp. 233-274.

Trumbore, S.E., E.A. Davidson, P.B. deCamarga, D.C. Nepstad, and L.A. Martinelli, 1995. Below-ground cycling of carbon in forests and pastures of Eastern Amazonia. Global Biogeochemical Cycles 9: 515-528.

Tyler, S.W., J.B. Chapman, S.H. Conrad, D.P. Hammermeister, D. Blout, J. Miller, and J.M. Ginanni, 1996. Soil-water flux in the southern Great Basin, United States: Temporal and saptial variations over the last 120,000 years. Water Resour. Res. 32: 1481-1499.

Tyler, S.W., and G.R. Walker, 1994. Root zone effects on tracer migration in arid zones. Soil Sci. Soc. Am. J. 58: 25-31. (all) van der Kamp, G., 1992. The continuity between groundwater and soil water in the prairie setting. Proceedings, National Hydrology Research Centre Workshop on Soil Moisture Modelling, 9-10 March, 1992, Saskatoon, SK.

van der Kamp, G., and D.Van Stempvoort, 1992. Role of groundwater flow in soil salinization: some advances in the past ten years. In: H. Steppuhn and D. Curtin, eds., Salinity and Sustainable Agriculture, Workshop on Soil Salinization, Swift Current, Saskatchewan, March 19-20, 1992, Prairie Salinity Publication no. 1, pp. 36-43.

van Olphen, H., 1977. An introduction to clay colloid chemistry. Wiley, 318 pp.

Wassenaar, L.I., M.J. Hendry, R. Aravena, and P. Fritz, 1990. Organic carbon isotope geochemistry of clayey deposits and associated pore waters, southern Alberta. J. Hydrol. 12: 251-270.

Watts, R.J., 1998. Hazardous wastes: Sources, pathways, receptors. Wiley, 764 pp.

Weber, W. J., McGinley, P. M., Katz, L. E., 1992. A distributed reactivity model for sorption by soils and sediments 1. Conceptual basis and equilibrium assessments. Environ. Sci. Technol. 26: 1955-1962.

Weber, W.J., P.M McGinley, and L.E. Katz, 1991. Sorption phenomena in subsurface systems: Concepts, models and effects on contaminant fate and transport. Wat. Res. 25(5): 499-528.

Werth, C.J., Reinhard, M., 1997. Effects of temperature ontrichloroethylene desorption from silica gel and natural sediments 1. Isotherms. Environ. Sci. Technol. 31: 689-696.

White, A.F., A.E. Blum, M.S. Schulz, D.V. Vivit, D.A. Stonestrom, M. Larsen, S.F. Murphy, ad D. Eberl, 1998. Chemical weathering in a tropical watershed, Luquillo Mountains, Puerto Rico: I. Long-term versus short-term weathering fluxes. Geochim. Cosmochim. Acta 62(2): 209-226.

Wood, B.D., C.K. Keller, and D.L. Johnstone, 1993. Carbon dioxide as a measure of microbial activity in the unsaturated zone. Water Resour. Res. 29: 647-659.

Wood, B.J., and D.G. Fraser, 1977. Elementary thermodynamics for geologists. Oxford Science Publications, 303 pp.

Wood, W.W., K.A. Rainwater, and D.B. Thompson, 1997. Quantifying macropore recharge: Examples from a semi-arid area. Ground Water 35(6): 1097-1106.

Wood, W.W., and M.J. Petraitis, 1984. Origin and distribution of carbon dioxide in the unsaturated zone of the southern high plains of Texas. Water Resour. Res. 20: 1193-1208.

Xing, B., McGill, W. B., Dudas, M. J., 1994. Cross-correlation of polarity curves to predict partition coefficients of nonionic organic contaminants. Environ. Sci. Technol. 28: 1929-1933. 
Zabowski, D., and F.C. Ugolini, 1990. Lysimeter and centrifuge soil solutions: Seasonal differences between methods. $\underline{\text { Soil }}$ Sci. Soc. Am. J. 54: 1130-1135.

Zabowski, D., and F.C. Ugolini, 1992. Seasonality in the mineral stability of a subalpine spodosol. Soil Sci. 154(6): 497-507 . 


\section{Mechanics of Soil}

- 1 Goals and major applications: roads, buildings, ecosystem changes, landslides

- 2 Attending to cross-disciplinary jargon: parameterization and symbols

- 3 Stress, Strain, and Strength

- 4 Pores, voids and effective stress

- 5 Soil compression and failure modes

- 6 Measurement Methods: uniaxial, triaxial; penetrometer, elastic properties

- 7 Applications:

- 7.1 Worms and roots and their contributions to tensile strength

- 7.2 Landslides

- 7.3 Alteration in conductivity and retention

- 8 REVIEW QUESTIONS

- 9 PROBLEMS

REFERENCES 


\section{Io. Selected Applications Across Scales}

- 1 Challenges and strategies in applying unsaturated zone concepts at large scales

- 2 "Deep" flow and Unit gradients

- 3 Lateral flow

- 3.1.1 Scale limitations of Richards Equation for lateral flow

- 3.1.2 Capillary Barriers

- 3.1.3 Hydraulic theory and the Boussinesq equation: Short and Long time solutions and fundamentals of Recession analysis

- 4 Data assimilation combining models and remote sensing

- 5 Patterns of oxygen content in soils from grain to landscape scales

- 6 Water tracing and determination of age

- 6.1 Isotopic methods

- 6.2 Natural tracers

- 6.3 Anthropogenic tracers

- 7 Drought methods across scale: soil, hillslope, stream, river, and reservoir droughts

- 7.1 Water balance, climate extremes, agronomic water (soil), hydrologic water (streams); meteorological water (precipitation and ETP), Thornwaite; precipitation recycling at the continental scale

- 8 REVIEW QUESTIONS

- 9 PROBLEMS

REFERENCES 



\section{Appendix I}

\section{Thermodynamic Fundamentals}

Thermodynamics is the study of macroscopic energy states and changes in these states as process driving forces. The range of disciplinary thought across which its generalizations apply is unmatched by any other coherent body of scientific material. This book illustrates the preceeding statement; we use thermodynamic tools to develop predictive formulations for a variety of vadose-zone processes. For these reasons we present a brief overview of thermodynamic principles in the following pages. Our endeavor is constrained by space and the backgrounds of our readers, which are assumed to be minimal. We will proceed by means of illustration rather than proof. We refer readers desiring greater rigor and depth to any of a large number of texts on the subject (classics include those by Lewis and Randall, 1923; Denbigh, 1957; Callen, 1960; Reiss, 1965; Badger, 1967; and Klotz and Rosenberg, 1972).

We begin with definitions:

- A system is a region of the Universe.

- Variables of state, also called state functions, are path-independent properties of a system.

- The values of extensive variables of state depend on the "extent" of material in a system (e.g. mass, volume); values of intensive variables of state (e.g. temperature, pressure) do not.

We illustrate the path-independence of state functions by a simple thought experiment, represented in Figure I-1.

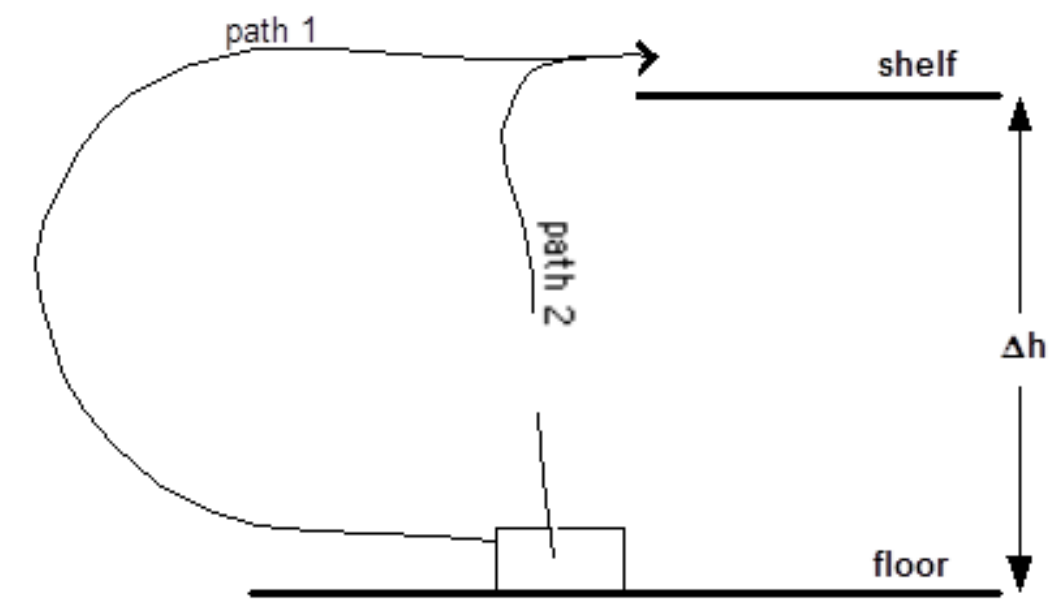

Figure I-1.

When the box is raised from the floor to the shelf, its gravitational potential energy is increased by an amount $\Delta \mathrm{E}=$ $\mathrm{mg} \Delta \mathrm{h}$. This is true whether the box follows path 1 or path 2 or, in fact, any other path during the operation. Furthermore, if the box is lifted and then returned to the floor, $\Delta \mathrm{E}=\mathrm{mg} \Delta \mathrm{h}=0$, again regardless of path. The value of $\Delta \mathrm{E}$ depends

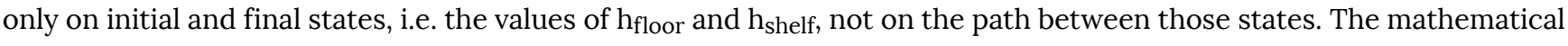
statement encapsulating all of these observations is:

$$
\oint \mathrm{dE}=0
$$


This is true of any variable of state, and is thus a definition: the change in its value, integrated over any closed path, is zero.

To continue:

- First Law: the internal energy $U$ is a state function defined as

$$
\mathrm{d} U=\mathrm{dq}+\mathrm{dw}
$$

where $q$ is heat absorbed by a system and $w$ is work done on a system. The internal energy of the Universe is always conserved.

The classic illustrations of the First Law are Joule's experiments in which water in an insulated drum was heated by various means - stirring, resistance heating, rubbing iron blocks together, and so on. Joule found that a given expenditure of mechanical work raised the temperature of the water the same amount, regardless of the particular means by which work was transformed into heat. Other studies have shown that pressure and composition, as well as temperature, determine the internal energy of a substance. In 150 years of experimentation since Joule, no one has refuted the First Law.

Writing $\mathrm{q}$ and $\mathrm{w}$ as lower-case letters (eq. (I.2) indicates that unlike $\mathrm{U}$, heat and work are not state functions. To illustrate, we consider the isothermal expansion of gas against a piston in a cylinder (the analogy of the setup to the "guts" of a steam engine is no accident: much of thermodynamics was developed in connection with steam power design). This time-tested illustration is somewhat abstract but useful in its simplicity: the system is closed (no mass transfer in or out) and constant in composition. The gas has volume $\mathrm{V}$ and pressure $\mathrm{P}$, both of which change over the course of the expansion, in a manner we can plot:

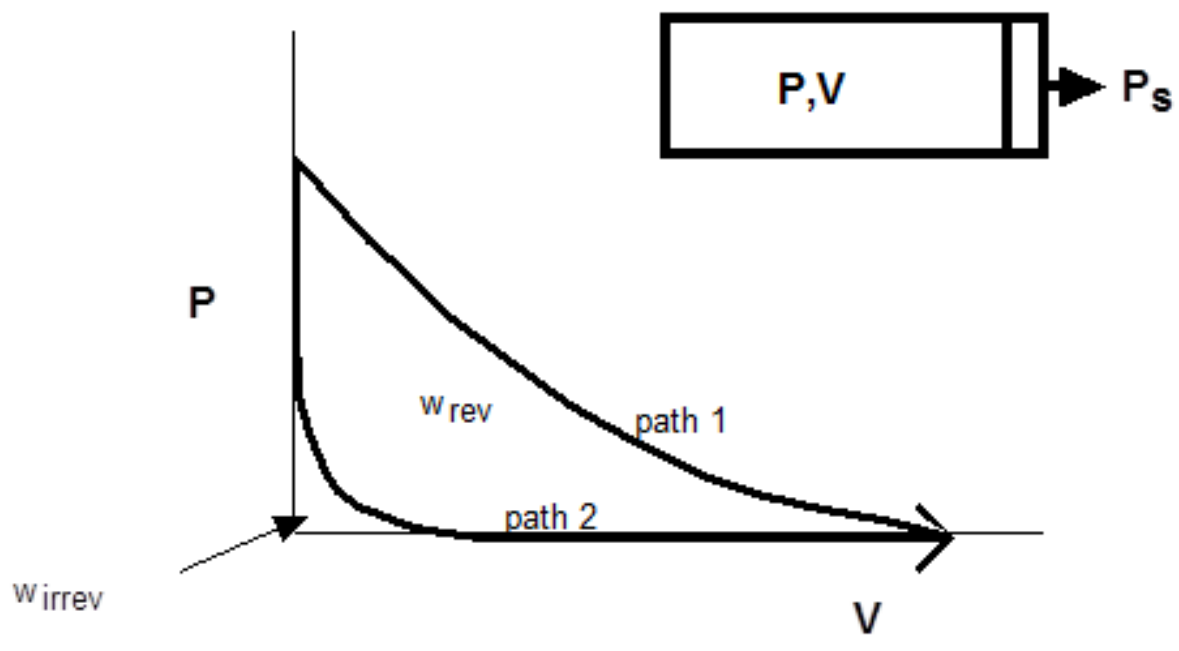

Figure I-2.

The plot shows two possible paths by which the expansion might take place. Path 1 represents infinitesimally slow reduction of the pressure $P_{S}$ exerted by the surroundings on the piston. Such a process, whose direction would be changed by an infinitesimal change in the surroundings, is defined as reversible. Path 2 represents a rapid reduction of $\mathrm{P}_{\mathrm{s}}$, such that the system gas expands spontaneously or irreversibly. The work done by the system on the environment is the area under the curve in each case; it is the sum of the infinitesimals -PdV:

(I.3) $\mathrm{dw}=-\mathrm{PdV}$

The negative sign is required because work energy is transferred from the system to the surroundings (note the definition of work in the First Law), and both $\mathrm{P}$ and $\mathrm{dV}$ are positive. We thus see that the areas under the P-V curves are different, i.e. the work varies depending on the path, and in particular:

(I.4) Wirrev $>\mathrm{W}_{\text {rev w_ }}\{$ rev $\} \backslash$ end $\{$ equation*\}" title="Rendered by QuickLaTeX.com"> 
(the sense of the inequality is explained by the fact that $\mathrm{w}_{\text {rev }}$ is more negative than $\mathrm{w}_{\text {irrev }}$, i.e. the system does more work on the surroundings in the reversible case). Furthermore, because the initial and final conditions (P,T,V) are the same for both paths and therefore $\Delta \mathrm{U}$ must be the same, the First Law dictates (compare eqs. I.4 and I.2):

(I.5) qirrev $_{\text {irev }}$

Thus work and heat are processes by which energy transfers occur, but not generally state functions.

If we rewrite the First Law for the reversible path, using eq. I.3, as:

(I.6) $\mathrm{dU}=\mathrm{dq} \mathrm{q}_{\mathrm{rev}}+\mathrm{dw}_{\mathrm{rev}}=\mathrm{dq}_{\mathrm{rev}}-\mathrm{PdV}$

we see that $d w_{\text {rev }}=-P d V$. In words, we may say that $w_{r e v}$ is the energy transfer associated with the driving force $P$ exerted over a displacement dV. Furthermore, we note that $\mathrm{dw}_{\mathrm{rev}}$ is (for the special case of reversibility only) a state function: try thought experiments to obtain different $d w_{\text {rev }}$ values for the same initial and final P,V states shown in Figure $\mathrm{I}-2$, and you will fail! Therefore $\mathrm{dq}_{\mathrm{rev}}$ is also an energy-transfer state function, for which there is a driving force (T) and a thermal displacement analogous to $\mathrm{dV}$ for (mechanical) work. The measure of thermal displacement is entropy, $\mathrm{S}$, which is the matter of the

- Second Law: the state function entropy $\mathrm{S}$ is defined as

$$
\mathrm{dS}=\frac{\mathrm{dq} \mathrm{rev}}{\mathrm{T}}
$$

and the entropy of the Universe always increases for any spontaneous process.

Eq. I.7 gives us a way to quantify the entropy change for a process such as that represented in Figure I-2: divide each infinitesimal of heat absorbed by the absolute temperature, over a reversible path. The Second Law then says that this quantity is always positive - heat is always absorbed, or some energy is always lost for the purpose of doing work - as an inevitable part of any spontaneous process for the Universe as a whole. (This is a thermodynamically complete statement of the Second Law; the tools of statistical mechanics can be employed to show that increase in entropy corresponds to increase in positional freedom or "randomness" at the molecular level.) Because nature is an ensemble of spontaneous processes - from sediment moving to the ocean, to the birth of a child - the Second Law says that the entropy of the Universe is always increasing. Conversion of inorganic substances to the complex of organic chemicals comprising a child involves an entropy decrease, which could in principle be calculated. However, the fact that the child is a lowerentropy entity than the "dust" of which she is constituted should not confuse us; the child is thermodynamically one system among many, and other systems have experienced entropy increases during conception and gestation which more than counterbalance the entropy decrease embodied in the child. The generation of a baby involves large flows of heat! The distinction between the system and the Universe, relative to application of the Second Law, has been lost on some advocates of creation "science". Anderson and Crerar (1993, section 6.6) discuss related issues. As with the First Law, the foundation of the Second Law is experience, and many years of experimentation have failed to disprove it. Denbigh (1957) provides several clear illustrations.

The flip side of the Second Law is that the entropy of the Universe does not increase for a reversible process. We can demonstrate this by computing the entropy changes, for both system and surroundings, associated with the reversible expansion of a gas (Fig. 2): using eq. I.7, we see that each infinitesimal dS is positive for the system (heat is absorbed) and negative for the surroundings (heat is released), but equal in magnitude. This insight is of great importance to us because one of our main goals in applying thermodynamics is to describe and predict conditions of reversibility, also called equilibrium states.

So we now seek the mathematical expression of equilibrium in terms of constant-entropy conditions. We start by combining the First and Second Laws, written for a reversible process (substitute the outer equality of I.6 into eq. I.7):

$$
\mathrm{dS}=\frac{\mathrm{dU}+\mathrm{PdV}}{\mathrm{T}}
$$

We see from eq. I.8 that $\mathrm{dS}=0$, i.e. entropy is constant, for reversible processes that occur under conditions of constant $\mathrm{U}$ and $\mathrm{V}$, and formally we write: 


\section{(I.9) $\mathrm{dS}(\mathrm{U}, \mathrm{V})=0 \quad$ (equilibrium)}

which is also a mathematical statement that $\mathrm{S}$ achieves a maximum for given $\mathrm{U}, \mathrm{V}$ (Anderson and Crerar, 1993, chap. 5; Anderson, 1996). This is fine except that constant- $\mathrm{U}, \mathrm{V}$ conditions characterize either a totally isolated system, or the Universe as a whole - neither of which is experimentally convenient or descriptive of the natural systems we study! So this is a valid but not very useful definition of equilibrium.

A rephrasing of eq. I.9 is that $\mathrm{S}$ is the characteristic function for the independent variables of state $\mathrm{U}$ and V; what we want is the characteristic function for a more appropriate pair of independent variables (Nordstrom and Munoz, 1994). For our purposes, this pair is pressure and temperature: it is usually reasonable to (a) specify the $\mathrm{P}$ and $\mathrm{T}$ at which a process occurs, and (b) assume that $\mathrm{P}$ and $\mathrm{T}$ do not vary appreciably during attainment of equilibrium. We seek the characteristic function for $\mathrm{P}$ and $\mathrm{T}$ by rewriting eq. I.8:

(I.10) $\mathrm{dU}=\mathrm{TdS}-\mathrm{PdV}$

This relation has been called the fundamental equation of thermodynamics because so many other useful relations may be readily derived from it. It follows from eq. I.10 that partial derivatives of $U$ are:

$$
\begin{aligned}
& \left(\frac{\partial \mathrm{U}}{\partial \mathrm{S}}\right)_{\mathrm{v}}=\mathrm{T} \\
& \left(\frac{\partial \mathrm{U}}{\partial \mathrm{V}}\right)_{\mathrm{s}}=-\mathrm{P}
\end{aligned}
$$

Also, using functional notation we may rewrite eq. I.10 as:

$$
\mathrm{U}=\mathrm{U}(\mathrm{S}, \mathrm{V})
$$

or, equally well, $\mathrm{dU}(\mathrm{S}, \mathrm{V})=0$ (after eq. I.9 above). All of these eqs. I.10 - I.13 show that $\mathrm{U}$ is the characteristic function for $\mathrm{S}$ and $\mathrm{V}$.

We can obtain additional characteristic functions by application of the Legendre Transform, which is simply a way to write a new function in terms of the original function ( $U$ in this case) and one or more of its partial derivatives (eqs. I.11 and I.12 in this case), without losing any information (e.g. Anderson and Crerar, 1993, chap. 5). Application to eq. I.13 yields:

$$
\mathrm{A}=\mathrm{U}-\mathrm{S}\left(\frac{\partial \mathrm{U}}{\partial \mathrm{S}}\right)_{\mathrm{v}}=\mathrm{U}-\mathrm{TS}
$$

$$
\mathrm{H}=\mathrm{U}-\mathrm{V}\left(\frac{\partial \mathrm{U}}{\partial \mathrm{V}}\right)_{\mathrm{s}}=\mathrm{U}+\mathrm{PV}
$$

$$
\mathrm{G}=\mathrm{U}-\mathrm{S}\left(\frac{\partial \mathrm{U}}{\partial \mathrm{S}}\right)_{\mathrm{v}}-\mathrm{V}\left(\frac{\partial \mathrm{U}}{\partial \mathrm{V}}\right)_{\mathrm{s}}=\mathrm{U}-\mathrm{TS}+\mathrm{PV}
$$

In each of these expressions the second equality was obtained by substitution of eq. I.11 and/or I.12 for the partial derivative(s). The new state functions obtained are the Helmholtz Free Energy A, the Enthalpy H, and the Gibbs Free Energy (often just the "free energy") G. Enthalpy can be shown to be the heat released or absorbed for a process which does $\mathrm{P}-\mathrm{V}$ (expansion-contraction) work at constant pressure: $\mathrm{H}=\mathrm{qp}_{\mathrm{p}}$. This is the "heat content" of a chemical reaction carried out in a calorimeter. Substituting eq. I.15 into I.16 shows that $\mathrm{G}$ may also be defined as:

(I.17) $\mathrm{G}=\mathrm{H}-\mathrm{TS}$

where we understand TS as heat or "bound" energy unavailable for work, subtracted from the enthalpy in this case. The function G could thus be called the residual or "free" enthalpy; by similar reasoning, looking at eq. I.14, A could be called the "free" energy (Nordstrom and Munoz, 1994). We will, however, stick to the common practice of using "free energy" to mean G. Commonly used forms of eq. I.17 are:

(I.18) $\mathrm{dG}=\mathrm{dH}-\mathrm{TdS}$ 
(I.19)

$$
\mathrm{dG}=\mathrm{dq}_{\mathrm{p}}-\mathrm{TdS}
$$

where conditions of constant $\mathrm{T}$ and constant $\mathrm{P}, \mathrm{T}$ are assumed, respectively.

Recalling our search for the characteristic function for $\mathrm{P}$ and $\mathrm{T}$, we take the total differentials of the outer equalities of eqs. I.14-I.16 and, substituting eq. I.10 for dU, we get:

(I.20) $\mathrm{dA}=\mathrm{dU}-\mathrm{TdS}-\mathrm{SdT}=-\mathrm{PdV}-\mathrm{SdT}$

(I.21) $\mathrm{dH}=\mathrm{dU}+\mathrm{PdV}+\mathrm{VdP}=\mathrm{TdS}+\mathrm{VdP}$

(I.22) $\mathrm{dG}=\mathrm{dU}-\mathrm{TdS}-\mathrm{SdT}+\mathrm{PdV}+\mathrm{VdP}=\mathrm{VdP}-\mathrm{SdT}$

By looking at these outer equalities we have, by analogy with eqs. I.9, I.10, and I.13:

$$
\begin{array}{ll}
\text { (I.23) } \mathrm{A}=\mathrm{A}(\mathrm{V}, \mathrm{T}) \text { or, at equilibrium, } \mathrm{dA}(\mathrm{V}, \mathrm{T})=0 \\
\text { (I.24) } \mathrm{H}=\mathrm{H}(\mathrm{S}, \mathrm{P}) \text { or, at equilibrium, } \mathrm{dH}(\mathrm{S}, \mathrm{P})=0 \\
\text { (I.25) } \mathrm{G}=\mathrm{G}(\mathrm{P}, \mathrm{T}) \text { or, at equilibrium, } \mathrm{dG}(\mathrm{P}, \mathrm{T})=0
\end{array}
$$

From this we see that $\mathrm{G}$ is the characteristic function for $\mathrm{P}$ and $\mathrm{T}$, and is therefore the state function we seek for description of equilibrium. To make this practical, we return to the Second Law:

$$
\text { (I.17) } \mathrm{dS}=\frac{\mathrm{dq} \text { rev }}{\mathrm{T}}
$$

and recall that, for a nonequilibrium process, i.e. occurring spontaneously or irreversibly, the heat absorbed is less than for the same process by the reversible path (eq. I.5) such that

$$
\left.\mathrm{dS}>\frac{\mathrm{dq}}{\mathrm{T}} \quad(\text { spontaneous }) \backslash \text { frac }\{\mathrm{dq}\}\{\mathrm{T}\}\right\} \backslash \text { qquad }(\backslash \operatorname{text}\{\text { spontaneous }\}) \backslash \text { end }\{\text { equation* } * \text { " title="Rendered }
$$

by QuickLaTeX.com">

or

$$
\text { (I.26) } \mathrm{dq}-\mathrm{TdS}<0 \quad \text { (spontaneous) }
$$

Substituting eq. I.18 into the right-hand side, we get:

$$
\mathbf{d G}_{(\mathbf{P}, \mathbf{T})}<\mathbf{0} \text { (spontaneous process) }
$$

and, for completeness, we rewrite eq. I.25:

$$
\mathbf{d} \mathbf{G}_{(\mathbf{P}, \mathrm{T})}=\mathbf{0} \text { (equilibrium process) }
$$

where the subscripts remind us of the conditions for which eq. I.25 was derived. Eqs. I.27 and I.28 are often written in finite-difference forms $\Delta \mathrm{G}<0$ and $\Delta \mathrm{G}=0$ respectively.

We can summarize our achievements to this point: we have combined the First and Second Laws (eqs. I.2 and I.7) to obtain the fundamental equation (eq. I.10) which we then transformed to obtain G, the characteristic function for P and $\mathrm{T}$ (eq. I.22) whose differential is zero under conditions of constant $\mathrm{P}$ and T (eq. I.25). We then re-applied the Second Law (eq. I.26) to find that spontaneous processes occur so as to decrease dG (eq. I.27) toward zero, which is the equilibrium state (eqs. I.25 and I.28). We can thus think of equilibration as the natural, spontaneous "minimization of free energy" which corresponds conceptually to a rolling ball reaching stability in a potential-energy "trough".

We will see that eqs. I.27 and I.28 work well for processes involving "pure" substances - like pure water and minerals - for which free energies $\mathrm{G}$ are tabulated in thermodynamic data compilations. Most of the processes of interest to us, however, involve at least one mixture of substances none of which is "pure". Examples: vadose "water" is actually a mixture of $\mathrm{H}_{2} \mathrm{O}$ and dissolved salts and organic substances; the atmosphere of the vadose zone is actually a mixture of $\mathrm{N}_{2}, \mathrm{O}_{2}$, and $\mathrm{CO}_{2}$ with traces of other gases. These mixtures - called solutions in this case - are phases: macroscopic substances whose substituent components cannot be separated by physical means. The "pure" substances mentioned above are one-component phases.

To determine the spontaneity or equilibrium of processes involving multi-component phases like solutions, we need to generalize the foregoing results to accomodate systems of variable composition. Specifically, we mean by this that 
the numbers of moles of the components (a mole is defined as the mass of the component in grams, divided by its gram formula weight) may vary. For the simple case of a 2-component phase we rewrite eq. I.25 as:

$$
\mathrm{G}=\mathrm{G}\left(\mathrm{P}, \mathrm{T}, \mathrm{n}_{1}, \mathrm{n}_{2}\right)
$$

where $\mathrm{n} 1$ and $\mathrm{n} 2$ are the mole numbers of components 1 and 2 . The total differential is

$$
\mathrm{dG}=\left(\frac{\partial \mathrm{G}}{\partial \mathrm{P}}\right)_{\mathrm{V}, \mathrm{n}_{1}, \mathrm{n}_{2}} \mathrm{dP}+\left(\frac{\partial \mathrm{G}}{\partial \mathrm{T}}\right)_{\mathrm{P}, \mathrm{n}_{1}, \mathrm{n}_{2}} \mathrm{dT}+\left(\frac{\partial \mathrm{G}}{\partial \mathrm{n}_{1}}\right)_{\mathrm{P}, \mathrm{V}, \mathrm{n}_{2}} \mathrm{dn}_{1}+\left(\frac{\partial \mathrm{G}}{\partial \mathrm{n}_{2}}\right)_{\mathrm{P}, \mathrm{V}, \mathrm{n}_{1}} \mathrm{dn}_{2}
$$

The first two terms in eq. I.29 correspond to the constant-composition case, i.e. the outer equality in eq. I.22. Comparing these two equations, the first two partial derivatives in eq. I.29 equal V and -S respectively, and we can rewrite I.29 as:

$$
\mathrm{dG}=\mathrm{VdP}-\mathrm{SdT}+\left(\frac{\partial \mathrm{G}}{\partial \mathrm{n}_{1}}\right)_{\mathrm{P}, \mathrm{V}, \mathrm{n}_{2}} \mathrm{dn} \mathrm{n}_{1}+\left(\frac{\partial \mathrm{G}}{\partial \mathrm{n}_{2}}\right)_{\mathrm{P}, \mathrm{V}, \mathrm{n}_{1}} \mathrm{dn}_{2}
$$

The partial derivatives in eq. I.30 are the partial molal free energies or chemical potentials of the two components. We can understand the chemical potential of component $i$ as the effect upon the energy of the phase of varying the amount of $i$, all other components and conditions being held constant, and we denote it by the symbol:

$$
\mu_{\mathrm{i}}=\left(\frac{\partial \mathrm{G}}{\partial \mathrm{n}_{\mathrm{i}}}\right)_{\mathrm{n}_{\mathrm{j}}, \mathrm{P}, \mathrm{T}}
$$

Eq. I.30 may then be rewritten in general form as:

$$
\mathrm{dG}=\mathrm{VdP}-\mathrm{SdT}+\sum \mathrm{i} \mu_{\mathrm{i}} \mathrm{dn} \mathrm{n}_{\mathrm{i}}
$$

Parallel consideration of the effect of variable composition (mole numbers) for the state functions $\mathrm{U}, \mathrm{A}$, and $\mathrm{H}$, using equations I.13 and X10, I.23 and I.20, and I.24 and I.21 respectively, yields:

$$
\mathrm{dE}=\mathrm{TdS}-\mathrm{PdV}+\sum \mathrm{i} \mu_{\mathrm{i}} \mathrm{dn}_{\mathrm{i}}
$$

$$
\begin{aligned}
& \mathrm{dA}=-\mathrm{SdT}-\mathrm{PdV}+\sum \mathrm{i} \mu_{\mathrm{i}} \mathrm{dn}_{\mathrm{i}} \\
& \mathrm{dH}=\mathrm{TdS}+\mathrm{VdP}+\sum \mathrm{i} \mu_{\mathrm{i}} \mathrm{dn}_{\mathrm{i}}
\end{aligned}
$$

where the $\mu_{\mathrm{i}}$ are partial derivatives of $\mathrm{U}, \mathrm{A}$, and $\mathrm{H}$ with respect to $\mathrm{n}_{\mathrm{i}}$. Denbigh $(1957, \mathrm{sec} .2 .7)$ underlines the importance of eqs. I.32-I.35 by saying that they constitute the basis of chemical thermodynamics, and shows that the chemical potentials $\mu_{\mathrm{i}}$ in all four equations are in fact equivalent.

What are the working definitions of equilibrium in this case of variable composition? This is most easily grasped by assuming constancy of the variables for which G, U, A, and H are characteristic in eqs. I.32-I.35. Doing this for G (eq. I.32; constant $\mathrm{P}$ and $\mathrm{T}$ ) we get:

$$
\mathrm{dG}=\sum \mathrm{i} \mu_{\mathrm{i}} \mathrm{d} \mathrm{n}_{\mathrm{i}}
$$

From eq. I.28 we obtain, for equilibrium:

$$
\sum_{\mathrm{i}} \mu_{\mathrm{i}} \mathrm{dn} n_{\mathrm{i}}=0
$$

In, for example, a very simple system with 1 component and two phases (e.g. water liquid and water vapor in a closed container) eq. I.37 says that at equilibrium: 


$$
\mu_{\mathrm{H}_{2} \mathrm{O}}^{\text {vapor }} \mathrm{dn}{ }^{\text {vapor }}=-\mu_{\mathrm{H}_{2} \mathrm{O}}^{\text {liquid }} \mathrm{dn} \text { liquid }
$$

In this simple system it must be true that $\mathrm{dn}^{\mathrm{vapor}}=-\mathrm{dn}^{\text {liquid }}$, and this means:

$$
\mu_{\mathrm{H}_{2} \mathrm{O}}^{\text {vapor }}=\mu_{\mathrm{H}_{2} \mathrm{O}}^{\text {liquid }}
$$

This illustrates an important working definition of equilibrium: the chemical potential of each component is the same in every phase in which that component is present. Note that we could have obtained these results by starting with I.33, I.34, or I.35 and assuming constancy of different pairs of variables of state.

The tendency toward vapor-liquid equilibrium is a key consideration in the vadose zone, where both phases usually coexist. Problem 4 (following this chapter) illustrates this tendency explicitly. We will refer to these ideas throughout this book.

\section{Problems}

1. Equation I.10 was derived by considering a reversible process. Argue that this equation and its derivatives (e.g. eqs. I.20-I.22) apply to spontaneous processes as well.

2. In a closed system of fixed composition, the First Law says that there are 2 ways of changing the energy of a system: work and heat. Duhem's principle says that as a consequence, assigning values to $a$ maximum of 2 variables of state (e.g. P and T; or $\mathrm{T}$ and $\mathrm{V}$; or $\mathrm{U}$ and $\mathrm{S}$ ) determines the values of all remaining variables of state at equilibrium. Suggest a modification of Duhem's principle to characterize equilibrium in systems which may vary in composition.

3. Duhem's principle, introduced in problem 2 above, refers to a "maximum" of 2 variables of state. This qualification is needed to accommodate cases of coexisting phases at equilibrium, as in the example of water liquid and water vapor given at the end of the chapter. Manipulate this example by simple thought experiment to discover a further modification of Duhem's principle, which characterizes equilibrium in systems with multiple coexisting phases as well as variable composition.

4. Working from the water liquid-vapor equlilibrium example given at the end of the chapter, show that inequality in the chemical potential of water between phases causes spontaneous transformation of the high-potential phase to the low-potential phase.

\section{Answers to Problems}

1. Eq. I.10 holds for spontaneous as well as reversible processes, because all the variables in it are variables of state whose values are path-independent. (What varies with path is dw and dq, as shown in the example illustrated by Figure I-2.)

2. Compositional variation provides additional means by which system energy may vary. Therefore, for each compositional variable, an additional variable of state must be specified to determine all the other variables at equilibrium. For example, pure liquid water - one phase, all by itself, in a closed container exists stably at various combinations of $\mathrm{P}$ and $\mathrm{T}$, i.e. within certain limits we may choose or assign equilibrium $\mathrm{P}$ and $\mathrm{T}$, which in turn determines all other aspects of the state of the water (its internal 
energy, entropy, and so on). If we introduce a solute like $\mathrm{NaCl}$, this compositional variable affects system energy by altering molecular behavior, and specifying two variables no longer fully determines the state; one more specification (e.g. the amount of $\mathrm{NaCl}$ dissolved in the water) is needed. So the modification would read "...assigning values to a maximum of $\mathrm{c}+2$ variables of state..." where $\mathrm{c}$ is number of independent system compositional variables. This " $\mathrm{c}$ " is also correctly called the number of components. In the example of $\mathrm{NaCl}$ dissolved in water, $\mathrm{c}=2$ (for water and salt).

3. In the water liquid-vapor system, experiments show that only 1 variable of state can be independently specified if both phases are to coexist at equilibrium. For example, if we specify a high $\mathrm{T}$ and atmospheric $\mathrm{P}$, all the water exists stably as vapor, i.e. the two-phase equilibrium is not possible; but if we specify only that same $\mathrm{T}$, there exists some sufficiently high $\mathrm{P}$ - which is thus a dependent variable in this example at which the two-phase equilibrium is possible. This illustrates the principle that each additional phase at equilibrium reduces by 1 the number of variables of state which are specified to determine equilibrium. The final modification would then read "...assigning values to $c-p+2$ variables of state..." where $p$ is number of coexisting phases. This is the Phase Rule.

4. Suppose the spontaneous process is:

liquid $\rightarrow$ vapor

Then we know, by combining eqs. I.36 and I.27, that:

$$
\begin{aligned}
& \mu_{\mathrm{H}_{2} \mathrm{O}}^{\text {vapor }} \mathrm{dn} \\
& \quad \text { vapor }+\mu_{\mathrm{H}_{2} \mathrm{O}}^{\text {liquid }} \mathrm{dn} n^{\text {liquid }}<0 \\
& \quad \text { and, because } \mathrm{dn}^{\text {vapor }}=-\mathrm{dn}^{\text {liquid: }} \\
& \mu_{\mathrm{H}_{2} \mathrm{O}}^{\text {vapor }}-\mu_{\mathrm{H}_{2} \mathrm{O}}^{\text {liquid }}<0
\end{aligned}
$$

or

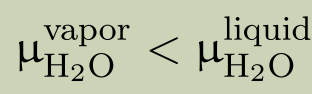

i.e. the chemical potential is lower in the phase to which water spontaneously moves. The spontaneous transfer of mass from high-potential to low-potential phases means that chemical potential may be thought of as a chemical "pressure".

\section{References}

Anderson, G.M., 1996. Thermodynamics of natural systems. Wiley, $382 \mathrm{pp}$.

Anderson, G.M., and D.A.Crerar, 1993. Thermodynamics in geochemistry: the equilibrium model. Oxford University Press, 588 pp.

Badger, P.H., 1967. Equilibrium thermodynamics. Allyn and Bacon, 626 pp.

Callen, H.B., 1960. Thermodynamics. Wiley and Sons, 376 pp.

Denbigh, K., 1957. The principles of chemical equilibrium. University Press (Cambridge), 491 pp.

Klotz, I.M., and R.M. Rosenberg, 1972. Chemical thermodynamics, 3rd Ed. W.A. Benjamin, 444 pp.

Nordstrom, D.K., and J.L. Munoz, 1994. Geochemical thermodynamics, 2nd Ed. Blackwell Science, 493 pp.

Lewis, G.N., and M. Randall, 1923. Thermodynamics, McGraw-Hill.

Reiss, M.H., 1965. Methods of thermodynamics. Blaisdell, 217 pp. 


\section{References}

Abdul, S.A., S.F. Kia and T.L. Gibson. 1989. Limitations of monitoring wells for the detection and quantification of petroleum products in soils and aquifers. Ground Water Monitoring Review. 9:90-99.

Ankeny, M.D., T.C. Kaspar and R. Horton. 1988. Design for an Automated Tension Infiltrometer. Soil Sci. Soc. Am. J. 52:893-896.

Ankeny, M.D., M. Ahmed, T.C. Kaspar, and R. Horton. 1992. Simple method for determining unsaturated hydraulic conductivity. Soil Sci. Soc. Am. J. 55:467-470.

Appelo, C.A.J., A. Willemsen, H.E. Beekman, and J. Griffioen, 1990. Geochemical calculations and observations on salt water intrusions, 2. Validation of a geochemical model with column experiments. J. Hydrol. Amsterdam 120: $225-250$.

Arcone, S. 1986. Conductivity Limitations in Single-Reflection Time-Domain Reflectometry. J. Phys. E.: Sci. Instrum. 19:1067-1069.

Arya, L.M. and J.F. Paris. 1981. A Physicoemperical Model to Predict the Soil Moisture Characteristic from Particle Size Distribution and Bulk Density Data. Soil Sci. Soc. Am. J. 45:1023-1030.

ASTM, 1990. Standard test method for field measurement of infiltration rate using a double ring infiltrometer with a sealed-inner ring. Annual Book of ASTM Standards, Vol. 04.08. D 5093-90.

Bartell, F.E. and A.D. Wooley. 1933. Solid-liquid-air contact angles and their dependence upon the surface condition of the solid. J. Amer. Chem. Soc.. 55:5318-3527.

Baumgartner, N., G.W. Parkin and D.E. Elrick. 1994. Soil Water Content and Potential Measured by Hollow Time Domain Reflectometry Probe. Soil Sci. Soc. Am. J. 58:315-318.

Bear, J. 1972. Dynamics of Fluids in Porous Media. Elsevier. Mineola, N.Y.

Beven, K. and P. Germann. 1981. Water flow in soil macropores II. A combined flow model J. Soil Sci. 32:15-29.

Biggar, J.W. and D.R. Nielsen. 1976. Spatial Variability of the Leaching characterisitics of a Field Soil. Water Resour. Res. 12:78-84.

Boll, J., J.S. Selker, G. Shalit, and T.S. Steenhuis. Frequency Distribution of water and solute transport properties derived from pan sampler data. Water Resour. Res. 33: 2655-2664. 1997.

Bousinnesq, J. 1868. Mémoire sur L'influence des Frottements dans les Mouvements Réguliers des Fluides. Liouville, Jour. Math., t. 13, p395.

Bousinnesq, J. 1904. Recherches Theoriques sur l'écoulement des nappes d'eau infiltrées dans le sol. J. Math. Pures Appl. 10:363-394.

Bouwer, H. 1966. Rapid Field Measurement of air entry value and hydraulic conductivity of soil as significant parameters in flow system analysis. Water Resour. Res. 2:729:738.

Brandi-Dorhn, F.M., R.P. Dick, M. Hess, and J.S. Selker. 1996a. Suction cup bias in leashing characterization of an undisturbed field soil. Water Resour. Res. 32:1173-1182.

Brandi-Dohrn, F.M., R.P. Dick, M. Hess, and J.S. Selker. 1996b. Field Evaluation of Passive Capillary Samplers. Soil Sci. Soc. Am. J. 60:1705-1713.

Broadbridge, P. .1988. Integrable forms of the one-dimensional flow equation for unsaturated heterogeneous porous media. J. Math. Phys. 29:622-627.

Brooks, R.H., and A.T. Corey. 1964. Hydraulic properties of porous media. Hydrology Papers, Colorado State University, Ft. Collins, CO.

Bruce, R.R. and A. Klute. 1956. The measurement of Soil Water Diffusivity. Soil Sci. Soc. Am. Proc. 20:458-462.

Brusseau. M.L. 1995. Transport of Reactive Contaminants in Heterogeneous Porous Media. Rev. Geophysics. 32:285-313.

Brutsaert, W. 1966. Probability laws for pore size distributions. Soil Sci. 101:85-92.

Brutsaert, W. 1968. The adaptability of an exact solution to horizontal infiltration. Wat. Resourc. Res. 4:785-789.

Brutsaert, W. 1977. Vertical infiltration in dry soil. Wat. Resour. Res. 13:363-368.

Buckingham, E. 1904. Contribution to our knowledge of the aeration of soils. Bull 25, USDA Bur. of Soils.

References | 403 
Buckingham, E. 1907. Studies in the Movement of Soil Moisture. U.S. Dept. Agr. Bur. Soils Bull. 38. Washington, D.C. $29-61$. Burdine, N.T. 1953. Relative Permeability Calculations from Pore Size Distribution Data. Pet. Trans. AIME. 198:71-78.

Campbell. 1990. Dielectric properties and influence of conductivity in soils at one to fifty megahertz. Soil Sci. Soc. Am. J. 54:332-341.

Carslaw, H.S. and J.C. Jaeger. 1948. Conduction of heat in solids. Claredon Press. Oxford, England.

Carter, M.R. 1993. Soil Sampling and Methods of Analysis. Lewis Publishers, NY.

Cary, J.W., J.F. McBride and C.S. Simmons. 1989a. Electro-Optical Detection of Liquid in Translucent Porous Material. Soil Sci. Soc. Am. J. 53(5):1591-1595.

Cary, J.W., J.F. McBride and C.S. Simmons. 1989b. Observation of water and oil infiltration into soil: Some simulation challenges. Water Resour. Res. 25:73-80.

Cary, J.W., J.F. Mc Bride, and C.S. Simmons. 1989c. Trichlorethylene residuals in the capillary fringe as affected by airentry pressures. J. Environ. Qual. 18:72-77.

Childs, E.C., N. Collis-George. 1950. The Permeability of Porous Materials. Roy. Soc. Lon. Proc. A. 201:392-405.

Clothier, B.E. and I. White. 1981. Measurement of sorptivity and soil water diffusivity in the field. Soil Sci. Soc. Am J. 45:241-245.

Cook, F.J. and A. Broeren. 1994. Six methods for determining sorptivity and hydraulic conductivity with disc permeameters. Soil Sci. 157:2-11.

Corey, A.T. Air Permeability. Chap. 48 in Methods of Soil Analysis, Part 1. Physical and Mineralogical Methods. Agronomy Monograph no. 9. American Society of Agronomy, Madison, WI.

Cuenca, R.C., M. Ek and L. Mahrt. 1996. Impact of Soil Water Property Parameterization on Atmospheric Boundary-layer Simulation. J. Geophys. Res. 101:7269-7277.

Dagan, G. 1986. Statistical theory of groundwater flow and transport: Pore to laboratory, laboratory to domain, and domain to regional scale. Wat. Resour. Res. 22:120S-135S.

Dagan, G. 1994. The significance of heterogeneity of evolving scales to transport in porous media. Wat. Resour. Res. 30:3327-3336.

Dalton, F.N., W.N. Herkelrath, D.S. Rawlins and J.D. Rhoades. 1984. Time-Domain Reflectometry: Simultaneous Measurement of Soil Water Content and Electrical Conductivity with a Single Probe. Science. 224:989-990.

Darcy, H. 1856. Les Fountaines Publique de la Ville de Dijon. Dalmont, Paris.

Das and Kluitenberg. 1996. WRR.

Elrick, D.E. and W.D. Reynolds. 1992a. Methods for analyzing constant-head well permeameter data. Soil Sci. Soc. Am. J. 56:320-323.

Elrick, D.E. and W.D. Reynolds. 1992b. Infiltration from constant-head well permeameters and infiltrometers. Chapter 1 in Advances in Measurement of Soil Physical Properties: Bringing Theory into Practice. Soil Sci. Soc. Am. Special Publication No. 30, Madison, WI.

Elrick, D.E., and W.D. Reynolds. 1993. Reply to "Comments on 'Methods for anaylzing constant-head well permeameter data."' Soil Sci. Soc. Am. J. 57:560-563.

Enderby, J.A. 1955. The domain model of hysteresis. 1. Independent domains. Trans. Faraday Soc 51:835-848.

Essaid, H.I., W.N. Herkelrath and K.M. Hess. 1993. Simulation and fluid distributions observed at a crude oil spill site incorporating hysteresis, oil entrapment, and spatial variability of hydraulic properties. Water Resour. Res. 29:1753-1770.

Essert. S.J. and J.W. Hopmans. 1994. Development and testing of a combined tension-solution probe. Agronomy Abstracts. American Society of Agronomy. Seattle, WA, November 13-18.

Everett, D.H. 1955. A general approach to hysteresis, An alternative formulation of the domain model. Trans. Faraday Soc. 51:1551-1557.

Fellner-Feldegg, H. 1969. The Measurement of Dielectric in the Time Domain. J. Physical. Chem. 73:616-623.

Fuentes, C., R. Haverkamp and J.-Y Parlange. 1992. Parameter constraints on closed-form soilwater relationships. J. of Hydro. 134:117-142. 
Fujita, H. 1952. The exact pattern of a concentration-dependent diffusion in a semi-infinite medium, Part II. Text Res. J. 22:823-827.

Gardner, W. H. 1986. Water Content. Chapter 21 in Methods of Soil Analysis, Part 1. Physical and Minerological Methods. Agronomy Monograph no. 9. American Society of Agronomy, Madison, WI.

Gardner, W.H., G.S. Campbell and C. Calissendorff. 1972. Systematic and random errors in dual gamma energy soil bulk density and water content measurements. Soil Sci. Soc. Am. Proc. 36:393-401.

Gardner, W.R. 1958. Some steady state solutions of the unsaturated moisture flow equation with application to evaporation from a water table. Soil Sci. 85:228-232.

Gee, .W., M.D. Campbell, J.H. Campbell and G.S. Campbell. 1992. Rapid measurement of low soil water potentials using a water activity meter. Soil Sci. Soc. Am. 56:1068-1070.

Germann, P.F., and K. Beven. 1986. A distribution function approach to water flow in soil macropores based on kinematic wave theory. J. Hydro. 83:173-183.

Gish, T.J. and A. Shirmohammadi, editors. 1991. Preferential Flow. Proceedings of the National Symposium held 16-17 December, 1991, Chicago, Illinois. Am. Soc. Ag. Eng., St. Joseph, MI.

Glass, R.J, T.S. Steenhuis, and J.-Y. Parlange. 1989. Mechanism for Finger Persistence in Homogeneous, Unsaturated, Porous Media: Theory and Verification. Soil Sci. 148(1):60-70.

Glass, R.J, and L. Yarrington. 1996. Simulation of gravity fingering in porous media using a modified invasion percolation model. Geoderma 70:231-252.

Green, W.H. and G.A. Ampt. 1911. Studies on Soil Physics: I. Flow of Air and Water Through Soils. J. Agric. Sci. 4:1-24.

Gouy, G. 1910. J. Phys. (4)9:457

Gouy, G. 1917. Ann. Phys. (9)7:129.

Gupta, R.K., R.P. Rudra, W.T. Dickinson, N.K. Patni, and G.J. Wall. 1993. Comparison of saturated hydraulic conductivity measured by various field methods. Trans. ASAE. 36:51-55.

Guymon, G.L. 1994. Unsaturated Zone Hydrology. Prentice Hall, Cliffwood, NJ.

Haines, B.W. 1930. Studies in Physical Properties of Soil: 5. The Hysteresis Effect in Capillary Properties, and the Modes of Moisture Distribution Associated Therewith. J. Agr. Sci. 20:97-116.

Hastings, C. 1955. Approximations for digital computers. Princeton, Univ. Press, Princeton, N.J.

Haverkamp, R, P.J. Ross, K.R.J. Smettem and J.Y. Parlange. 1994. Three-dimensional analysis of infiltration from the disc infiltrometer 2. Physically based infiltration equation. Water Resourc. Res. 30:2931-2935.

Held, R.J. and T.H. Illangaskare. 1995. Fingering of dense nonaqueous phase liquids in porous media 1. experimental investigations. Water Resourc. Res. 31:1213-1222.

Hildebrand, F.B. 1952. Methods of Applied Mathematics. Prentice-Hall, Englewood Cliffs, N.J.

Hill, D.E. and J.-Y. Parlange. 1972 Wetting Front Instability in Layered Soils. Soil Sci. Soc. Am. Proc. 36:697-702.

Hillel, D. 1980. Fundamentals of Soil Physics. Academic Press, N.Y., N.Y.

Hillel, D. 1980. Applications of Soil Physics. Academic Press, N.Y., N.Y.

Hillel, D. 1982. Introduction to Soil Physics. Academic Press, N.Y., N.Y.

Ho, C.K. and S.W. Webb. 1998. Capillary barrier performance in heterogeneous porous media. Wat. Resour. Res. 34:603-609.

Hoa, M.T. 1981. A New Method Allowing the Measurement of Rapid Variations in Water Content in Sandy Porous Media. Water Resourc. Res. 17:41-48.

Hoekstra, P. and A. Delaney. 1974. Dielectric Properties of Soils at UHF and Microwave Frequencies. J. Geophys. Res. 79:1699-1708.

Hoogmoed, W.B. and J. Bouma. 1980. A simulation model for predicting infiltration into cracked clay soil. Soil Sci. Soc. Am. J. 44:458-461.

Hook, W.R. Livingston, N.J. Sun, Z.J. Hook, P.B. 1992. Remote diode shorting improves measurement of soil water by time domain reflectometry. Soil Sci. Soc. Am. J. 56:1384-1391.

Hutson, J.L. and R.J. Wagenet. 1995. A multiregion model for describing water flow and solute transport in heterogeneous soils. Soil Sci. Soc. Am. J. 59:743-751. 
Jury, W.A. and K. Roth. 1990. Transfer Functions and Solute Movement through Soil. Birkhäuser, Basel. 226 pp.

Jury, W.A., W.R. Gardner and W.H. Gardner. 1991. Soil Physics, fifth edition. John Wiley and Sons, N.Y., N.Y.

Kelly, S.F., J.S. Selker and J.L. Green. 1995. Using short soil moisture probes with high-bandwidth time domain reflectometry instruments. Soil Sci. Soc. Am. J. 59:97102.

Kirkham, D and W.L. Powers. 1972. Advanced Soil Physics. R.E. Krieger Publishing, Malabar, FL.

Klinkenberg, L.J. 1941. The permeability of porous media to liquids and gases. Drilling and Production Practice. 200-213. Am. Petro. Inst., NY.

Klute. A. 1986. Methods of Soil Analysis Part 1. Physical and Mineralogical Methods. Second Edition. Soil Sci. Soc. Am., Madison, WI.

Knight, J.H. 1992. Sensitivity of Time Domain Reflectometry Measurements to Lateral Variations in Soil Water Content. Water Resour. Res. 28:2345-2352.

Knox, R.C., D.A. Sabatini, and L.W. Canter. 1993. Subsurface Transport and Fate Processes. Lewis Press, Boca Raton, FL.

Kosugi, K. 1994. Three-parameter lognormal distribution model for soil water retention. Water Resour. Res. 30:891-901.

Kozeny, I. 1927. Über Kapillarleitfähigkeit des Wassers im Boden. Sitzungsber. Akad. Wiss. Wein 136:271-303.

Kreft, A. and A. Zuber. 1978. On the physical meaning of the dispersion equation and its solutions for different initial and boundary conditions. Chem Eng. Sci. 33:1471-1480.

Kunze, R.J. and W.H. Shayya. 1993. Assessing the Constancy of the Potential Term in the Green and Ampt Infiltration Equation. Trans. Am. Soc. Ag. Eng. 36:1093-1098.

Land, C.S. 1968. Calculation of imbibition relative permeability for two- and three-phase flow from rock properties. Soc. Pet. Eng. J. 243:149-156.

Lenhard, R.J. and J.C. Parker. 1987. A model for hysteretic constitutive relations governing multiphase flow 2. Permeability-saturation relations. Water Resour. Res. 23:2197-2206.

Lenhard, R. J.; Parker, J. C.; Kaluarachchi, J. J. 1991. Comparing simulated and experimental hysteretic two-phase transient fluid flow phenomena. Wat. Resour. Res. 27:2113-2124.

Leverett, M.C. 1938. Flow of Oil-water Mixtures through Unconsolidated Sands. Trans. AIME 132:149-171.

Leverett, M.C. 1941 Capillary Behavior in Porous Media. Trans. AIME 142:341-358.

Li, J. and D. Helm. 1998. Viscous drag, driving forces, and their reduction to Darcy's Law. Wat. Resour. Res. 34:1675-1684.

Lui, Y., B.R. Bierck, J.S. Selker, T.S. Steenhuis, and J-Y. Parlange. 1993. High Intensity X-Ray and Tensiometer Measurements in Rapidly Changing Preferential Flow Fields. Soil Sci. Soc. Am. J. 57:1188-1192.

Logsdon, S.D. and D.B. Jaynes. 1993. Methodology for determining hydraulic conductivity with tension infiltrometers. Soil Sci. Soc. Am. J. 57:1426-1431.

Luxmoore, R.J. 1981. Micro, meso-, and macroporosity of soil. Soil Sci. Soc. Am. J. 45:671-672.

Maheshwarla, S.V., R. Venkatasubramanian and R.F. Boehm. 1995. Comparison of Time Domain Reflectometry Performance Factors for Several Dielectric Geometry's: Theory and Experiments. Water Resourc. Res. 31:1927-1933.

Maidment, D.R. 1992. Handbook of Hydrology. McGraw Hill, NY.

Mallants, D. D. Jaques, M. Vanclooster, J. Diels and J. Feyen. 1996. A stochastic approach to simulate water flow in a macroporous soil. Geoderma 70:299-324.

Marshall, T.J. 1958. A Relation Between Permeability and Size Distribution of Pores. J. Soil Sci. 9:1-8.

Merabet, M. and T.K. Bose. 1988. Dielectric Measurements of Water in the Radio and Microwave Frequencies by Time Domain Reflectometry. J. Phys. Chem. 92:6149-6150.

McBride, J.F., C.S. Simmons and J.W. Cary. 1992. Interfacial spreading effects on one-dimensional organic liquid imbibition in water-wetted porous media. J. Contam. Hydro. 11:1-25.

McCaffery, F.G. and N. Mungan. 1970. Contact angle and interfacial tension studies of some hydrocarbon-water-solid systems. J. Can. Pet. Tech. 9:185-196.

Miller, E.E. and R.D. Miller. 1956 Physical Theory for Capillary Flow Phenomena. J. Appl. Phys. 27:324-332.

Miller, E.E., and A. Salehzadeh. 1993. Stripper for bubble-free tensiometry. Soil Sci. Soc. Am. J. 57:1470-1473.

Millington, R.J. and J.P. Quirk. 1959. Permeability of Porous Media. Nature. 183:387-388.

Millington, R.J. and J.P. Quirk. 1961. The Permeability of Porous Solids. Trans. Faraday Soc. 57:1200-1207. 
Mohanty, B.P., R.S. Bowman, J.M.H. Hendrickx, and M.T. van Genuchten. 1997. New piecewise-continuous hydraulic functions for modeling preferential flow in an intermittent-flood-irrigated field. Water Resour. Res. 33:2049-2063.

Moore, H.M. and K. Attenborough. 1992. Acoustic determination of air-filled porosity and relative air permeability. J. Soil Sci.. 43:211-228.

Morrison and Szecsody. 1987. A Tensiometer and Pore Water Sampler for Vadose Monitoring. Soil Sci.. 144(5):367-372.

Mualem, Y. 1976. A New Model for Predicting the Hydraulic Conductivity of Unsaturated Porous Media. Wat. Resour. Res. 12:513-522.

Mualem, Y. 1973. Modified Approach to Capillary Hysteresis Based on a Similarity Hypothesis. Water Resour. Res. 9:1324-1331.

Mualem, Y. 1974. Modified approach to capillary hysteresis based on a similarity hypothesis. Wat. Resour. Res., 9:1324-1331.

Nielson, D.R., J.W. Biggar and K.T. Erh. 1973. Spatial Variability of Field Measured Soil-Water Properties. Higardia 42:215-259.

Ogden, C.B., R.J. Wagenet, H.M. van Es, and J.L. Hutson. 1992 Quantification and modeling of macropore drainage. Geoderma 55:17-35.

Osborne, M and J. Sykes. 1986. Numerical modeling of immiscible organic transport at the Hyde Park landfill. Wat. Resour. Res. 22:25-33.

Parker, J.C., and M.Th. van Genuchten. 1984. Flux-averaged and volume-averaged concentrations in continuum approaches to solute transport. Water Resour. Res. 20:866-872.

Parlange, Y-.J. Capillary hysteresis and the relationship between drying and wetting curves. Wat. Resour. Res., 12(2):224-228. 1976.

Parlange, J.-Y. and D.E. Hill. 1976. Theoretical Analysis of Wetting Front Instability in Soils. Soil Sci. 122:236-239.

Parlange, J.Y. Vauclin, M. Haverkamp, R. Lisle, I. 1985. The relation between desorptivity and soil-water diffusivity. Soil Sci. 139:458-461

Parlange, J.-Y, R.J. Glass and T.S. Steenhuis. 1990. Application of Scaling to the Analysis of Unstable Flow Phenomena. In Scaling in Soil Physics, Soil Sci. Soc. Am. Special Publication No. 25, Madison, WI.

Parker, J.C., R.J. Lenhard and T. Kuppusamy. 1987. A parametric model for constitutive properties governing multiphase flow in porous media. Water Resour. Res. 23:618-624.

Parker, J.C. and R.J. Lenhard. 1987. A model for hysteretic constitutive relations governing multiphase flow 1. Saturation-0pressure relations. Water Resour. Res. 23:2187-2196.

Perroux, K.M. and I. White. 1988. Designs for Disc Permeameters. Soil Sci. Soc. Am. J. 52:1205-1215.

Philip, J.R. 1960a. General method of exact solution of the concentration-dependent diffusion equation. Australian J. Physics. 13:1-12.

Philip, J.R. 1960b. A very general class of exact solutions in concentration-dependent diffusion. Nature 185:233.

Philip, J.R. 1993. Variable-Head Ponded Infiltration Under Constant of Variable Rainfall. Wat. Resour. Res. 29:2155-2165.

Pierzynski, G.M., J.T. Sims and G.F. Vance. 1994. Soils and Environmental Quality. Lewis Press, Boca Raton, FL.

Pivetz, B.E. and T.S. Steenhuis. 1995. Soil matrix and macropore biodegradation of 2,4-D. J. Env. Qual. 24:564-570.

Polubarinova-Kochina. 1962. Theory of Ground Water Movement. Princeton Univ. Press, Princeton, NJ.

Poulovassilis, A. 1962. Hysteresis of Pore Water, an Application of the Concept of Independent Domains. Soil Sci. 93:405-412.

Poulsen, M.M. and B.H. Kueper. 1992. A field experiment to study the behavior of tetrachloroethylene in unsaturated porous media. Environ. Sci. Technol. 26:889-895.

Rawlins, S.L., and G.S. Campbell. 1986. Water Potential: Thermocouple Psychrometry. Chapter 24 in Methods of Soil Analysis Part 1. Physical and Mineralogical Methods. ASA, Madison, WI.

Rawls, W.J. and D.L. Brakensiek. 1983. A procedure to predict Green and Ampt infiltration parameters. Adv. Infiltration, Am. Soc. Agric. Eng, pp 102-112.

Reeve, R.C. 1953. A method of determining stability of soil structure based on air and water permeability measurements. Soil Sci. Soc. Am. Proc. 17:324-329. 
Reynolds, W.D., and D.E. Elrick. 1985. In situ measurement of field-saturated hydraulic conductiviy, sorptivity, and the a-parameter using the Guelph permeameter. Soil Sci. 140:292-302.

Reynolds, W.D., R.W. Gillham, and J.A. Cherry. 1992. Evaluation of distribution coefficients for the prediction of strontium and cesium migration in a uniform sand. Can. Geotech. J. 19: 91-203.

Richards, L.A. 1931. Capillary Conduction of Liquids Through Porous Media. Physics. 1:318-333.

Rieu, M. and G. Sposito. 1991 Fractal Fragmentation, Soil Porosity, and Soil Water Properties: 1. Theory. Soil. Sci. Soc. Am. J. 55:1231-1238.

Rieu, M. and G. Sposito. 1991 Fractal Fragmentation, Soil Porosity, and Soil Water Properties: II. Applications. Soil. Sci. Soc. Am. J. 55:1239-1244.

Ritgema, P.E. 1965. An analysis of actual evapotranspiration. Rep. 659, 107 pp. Center for Agri. Publ. and Doc., Wageningen, Netherlands.

Roth, K, R. Schulin, H. Fluhler, and W. Attinger. 1990. Calibration of time domain reflectometry for water content measurement using a composite dielectric approach. Water Resour. Res. 26:2267-2273.

Salvucci, G.D. and D. Entekhabi. 1994. Explicit Expressions for Green-Ampt (Delta Function Diffusivity) Infiltration Rate and Cumulative Storage. Wat. Resourc. Res. 30:2661-2663.

Schiegg, H.O., with translation editors J.F. Mc Bride and D.N. Graham. Laboratory setup and results of experiments on two-dimensional multiphase flow in porous media. Pacific Northwest Laboratory report PNL-7453. Pacific Northwest Laboratory, Richland Washington, 99352. 1990.

Schroth, M.H., J.D. Istok, S.J. Ahearn and J.S. Selker. 1995. Geometry and position of light nonaqueous-phase liquid lenses in water-wetted porous media. J. Contam. Hydro. 19:269-287.

Schroth, M.H., S.J. Ahearn, J.S. Selker and J.D. Istok. 1996. Characterization of Miller-Similar Silica Sands for Laboratory Hydrologic Studies. Soil Sci. Am. J. 60:1331-1339.

Scotter, D.R. 1978. Preferential solute movement through larger soil voids. I. Some computations using simple theory. Aust. J. Soil Res. 16:257-67.

Selker, J.S., P. Leclerq, J.Y. Parlange and T.S. Steenhuis. 1991. Fingered flow in two dimensions 1. Measurement of matic potential. Water Resour. Res. 28:2513-2521.

Selker, J.S., T.S. Steenhuis and Y.-J. Parlange. 1992. Fingered Flow in Unlayered Media under Uniform Irrigation. Soil Sci. Soc. Am. J. 56:1346-1350.

Selker, J.S. 1994. Comment on "Comparison of three methods for assessing soil hydraulic properties" by G.B. Paige and Hillel. Soil Sci. 157:120-121.

Selker, J.S., L. Graff and T. Steenhuis. 1993. Noninvasive time domain reflectometry moisture probe. Soil Sci. Soc. Amer. J. 57:934-936.

Selker, J.S. and M.H. Schroth. 1998. Evaluation of Hydrodynamic scaling in porous media using finger dimensions. Water Resour. Res. 34:1875-80

Simunek, J., M.Th. van Genuchten and J.W. Hopmans. 1995. Parameter estimation of soil hydraulic properties from the tension disc infiltrometer experiment. Abstracts. European Geophysical Union. The Hague. May 6-10.

Sisson, J.B. and T.K. Honeycutt. 1994. Tension Bore Hole Permeameter Annual Report. Idaho National Engineering Laboratory report INEL-94/0107. October 1994.

Skopp, J. 1981 Comment on "Micro, meso-, and macroporosity of soil." Soil Sci. Soc. Am. J. 45:1246.

Smettem, K.R.J., J.-Y. Parlange, P.J. Rose and R. Haverkamp. 1994. Three-Dimensional Analysis of Infiltration from the Disc Infiltrometer. I. A Capillary-based Theory. Water Resourc. Res. 30:2925-2929.

Smettem, K.R.J., P.J. Ross, R. Haverkamp and J.Y. Parlange. 1995. Three-dimensional analysis of infiltration from the disk infiltrometer. 3. Parameter estimation using a double-disk tension infiltrometer. Water Resourc. Res. 31:2491-2495.

Slichter, C.S. 1899. Theoretical Investigation of the Motion of Ground Waters. 19th annual report of the U.S. Geological Survey 1897-98. Washington, D.C.

Soil Survey Staff. 1994. Keys to Soil Taxonomy, Sixth Edition. USDA

Soil Conservation Service, Washington, D.C.

Spanner, D.C. 1951. The Peltier effect and its use in the measurement of suction pressure. J. Exp. Bot. 11:145-168. 
Sposito, G. 1989. The Chemistry of Soils. Oxford Press. N.Y., N.Y.

Stephens, D.B. 1995. Vadose Zone Hydrology. Lewis Press. Boca Raton, FL.

Stolte, J, J.I. Freijer, W. Bouten, C. Dirksen, J.M. Halbertsma, J.C. Van Dam, J.A. Van den Berg, G.J. Veerman, and J.H.M. Wösten. 1994. Comparison of six methods to determine unsaturated soil hydraulic conductivity. Soil Sci. Soc. Am. J. 58:1596-1603.

Suarez, D.L., and J. Simunek, 1996. Solute transport modeling under variably saturated water flow conditions. IN: P.C. Lichtner, C.I Steefel, and E.H. Oelkers, eds., Reactive transport in porous media. Reviews in Mineralogy 34: 229-268.

Tamari, S., J.-C. Gaudu and T. Simonneau. 1993 Tensiometric Measurement and Metastable State of Water Under Tension. Soil Sci. 156:149-155.

Tokunaga, T. 1992. The Pressure Response of the Soil Water Sampler and Possibilities for Simultaneous Soil Solution Sampling and Tensiometry. Soil Science, 1992.

Topp, G.C., J.L. Davis and A.P. Annan. 1980. Electromagnetic determination of soil water content: measurement in coaxial transmission lines. Water Resour. Res. 16:574-582.

Toride, N., F.J. Leij and M.Th. van Genuchten. 1995. The CXTFIT code for estimating transport parameters from laboratory or field tracer experiments. Research Report 137, U.S. Salinity Laboratory, Riverside, CA.

van Genuchten, M. T. 1980. A Closed Form Equation for Predicting the Hydraulic Conductivity of Unsaturated Soils. Soil Sci. Soc. Am. J. 44:892-898.

van Genuchten, M.T., F.J. Leij and S.R. Yates. 1991 The RETC code for quantifying the hydraulic functions of unsaturated soils. U.S. EPA report 600/2-91/065. Washington D.C.

Vepraskas, M.J. 1992. Redoximorphic Features for Identifying Aquic Conditions. Tech. Bul. 301, North Carolina State University, Raleigh, N.C.

Wagenet, R.J. and J.L Hutson. 1987. LEACHM: A finite-difference model for simulating water, salt, and pesticide movement in the plant root zone. Continuum 2, New York State Resources Institute, Cornell Univ., Ithaca, NY.

Warrick, A. 1990. Application of scaling to the characterization of spatial variability of soils. p39-52 in Scaling in Soil Physics: Principles and Applications. Soil Sci. Soc. Am. Special Publication \# 25. Soil Sci. Soc. Am., Madison, WI.

Warrick, A.W., P.J. Wierenga and L. Pan. 1997. Downward water flow through sloping layers in the vadose zone: Analytical solutions for diversions. J. Hydrol 192:321-337.

Weast, R.C., Editor of the CRC Handbook of Chemistry and Physics, 68th edition. CRC Press, Boca Raton, FL. 1987.

Webb, E.K. and M.P. Anderson. 1996. Simulation of preferential flow in three-dimensional heterogeneous conductivity fields with realistic internal structure. Water Resour. Res. 32:533-545.

Webb, S.W. 1997. Generalization of Ross' tilted capillary barrier diversion formula for different two-phase characteristic curves. Wat. Resour. Res. 33:1855-1859.

Weeks, E. 1979. Barometric fluctuations in wells tapping deep unconfined aquifers. Water Resourc. Res. 15:1167-1176.

Whalley, W.R. 1993. Considerations on the Use of Time-Domain Reflectometry (TDR) for Measuring Soil Water Content. J. Soil Sci. 44:1-9.

White, M. D.; Oostrom, M.; Lenhard, R. J. 1995. Modeling fluid flow and transport in variably saturated porous media with the STOMP simulator; I, Nonvolatile three-phase model description. Adv. Wat. Resour. 18:353-364.

White, R.E., G.W. Thomas and M.S. Smith. 1984. Modeling water flow through undisturbed soil cores using a transfer function model derived from $3 \mathrm{HOH}$ and $\mathrm{Cl}$ transport. J. of Soil Sci. 35:159-168.

Wooding, R.A. 1968. Steady infiltration from a shallow circular pond. Water Resour. Res. 4:1259-1273.

Yeh, G.T., and V.S. Tripathi. 1991. A model for simulating transport of reactive multispecies components: model development and demonstration. Water Resour. Res. 27: 3075-3094.

Zangar, C.N. 1953. Theory and problems of water percolation. Eng. Monogr. no. 8. Bur of Reclamation, U.S. Dep. of Interior, Washington D.C.

Zaradny, H. 1993. Groundwater Flow in Saturated and Unsaturated Soil. A.A. Balkema, Rotterdam.

Zheng, Q, D.J. Durben, G.H Wolf, and C.A. Angell. 1991. Liquids at Large Negative Pressures: Water at the Homogenous Nucleation Limit. Science. 254:829-832. 


\section{Symbols used}

Symbol, Dimensions, and International System (SI) of Units for Soil Water Terms 


\section{Term}

Bowen ratio

Chemical potential

Cumulative depth of infiltration

Degree of saturation

Depth ratio of soil water

Discharge rate

Dispersivity

Distribution coefficient

Dry bulk density

Equivalent depth of soil water (Soil water storage)

Gravitational head

Gravitational potentiala

Heat capacity

Hydraulic head

Hydraulic potentiala

Hydrodynamic dispersion coefficient

Infiltration rate

Latent heat flux

\section{Symbol Dimensions SI Units}

$\beta \quad--$

(sensible heat flux)/(latent heat flux)

$\mu$

L2t-2

$\mathrm{J} / \mathrm{kg}$

I

$\mathrm{L}$

m

S - -

(volume water)/(volume fluids)

$\theta \mathrm{v}$

(depth water)/(unit depth soil)

Q L $3 \mathrm{t}-1 \quad \mathrm{~m} 3 / \mathrm{s}$

$\lambda \quad L$

$\mathrm{m}$

$\mathrm{kd} \quad--\quad$ (adsorbed concentration)/ (solute concentration)

ob $\quad$ ML-3 $\quad \mathrm{Mg} / \mathrm{m} 3, \mathrm{~g} / \mathrm{cm} 3$

De L

$\mathrm{m}$

Z L

$\mathrm{m}$

$\psi \mathrm{Z} \quad \mathrm{ML}-1 \mathrm{t}-2 \quad \mathrm{~N} / \mathrm{m} 2, \mathrm{~Pa}$

c $\quad$ ML2t-2K-1 J/K

$\begin{array}{lll}\mathrm{H} & \mathrm{L} & \mathrm{m}\end{array}$

$\psi$ h $\quad$ ML-1t-2 N/m2, Pa

Dh L2t-1 m2/s

i $\quad$ Lt-1 $\mathrm{m} / \mathrm{s}$

LEt Mt-3 W/m2 


\section{Term}

Liquid dynamic viscosity

Mass water content

Matric potentiala

Mean particle density

(Density of solids)

Net radiation

Permeability

Pore water velocity

Porosity

Pressure potentiala

Retardation factor

Saturated hydraulic conductivity

Saturated water content

Sensible heat flux

Soil thermal conductivity

Soil water head (matric head)

Soil bulk dielectric constant

Soil heat flux

Soil water diffusivity

412 | Symbols used

\section{Symbol Dimensions SI Units}

$\eta \quad$ ML-1t-1 Pa s

$\theta \mathrm{m} \quad--\quad$ (mass water)/(mass dry soil)

$\psi \mathrm{m} \quad \mathrm{ML}-1 \mathrm{t}-2 \quad \mathrm{~N} / \mathrm{m} 2, \mathrm{~Pa}$

os $\quad$ ML-3 $\quad \mathrm{Mg} / \mathrm{m} 3, \mathrm{~g} / \mathrm{cm} 3$

RN $\quad$ Mt-3 W/m2

k $\quad$ L2 $\quad$ m2

v Lt-1 $\mathrm{m} / \mathrm{s}$

n $\quad--\quad$ (volume fluids)/(total volume)

$\psi \mathrm{p} \quad \mathrm{ML}-1 \mathrm{t}-2 \quad \mathrm{~N} / \mathrm{m} 2, \mathrm{~Pa}$

$\mathrm{R} \quad--\quad$ (change in concentration)/ (change in time)

Ks Lt-1 $\quad \mathrm{m} / \mathrm{s}$

$\theta \mathrm{s} \quad--\quad$ (volume water at saturation)/ (total volume)

$\mathrm{H} \quad \mathrm{Mt}-3 \quad \mathrm{~W} / \mathrm{m} 2$

$\lambda \quad$ Mt-3K-1 J/m s K

h L $\quad \mathrm{m}$

eb $\quad$-- $\quad$ [(propagation velocity in vacuum)/(propagation velocity in medium) $] 2$

G $\quad$ Mt-3 $\quad \mathrm{W} / \mathrm{m} 2$

D Lt-1 $\quad \mathrm{m} / \mathrm{s}$ 
Term

\begin{tabular}{|c|c|c|c|}
\hline Solute (osmotic) potentiala & $\psi \mathrm{s}$ & ML-1t-2 & $\mathrm{N} / \mathrm{m} 2, \mathrm{~Pa}$ \\
\hline Sorptivity & S & $\mathrm{Lt}-1 / 2$ & $\mathrm{~m} / \mathrm{s} 1 / 2$ \\
\hline Total potentiala & $\psi \mathrm{T}$ & ML-1t-2 & $\mathrm{N} / \mathrm{m} 2, \mathrm{~Pa}$ \\
\hline Void ratio & $\mathrm{e}$ & -- & (volume fluids)/(volume solids) \\
\hline Volumetric water content & $\theta \mathrm{v}$ & -- & (volume water)/(total volume) \\
\hline Water density & $\rho \mathrm{w}$ & ML-3 & $\mathrm{g} / \mathrm{cm} 3$ \\
\hline Water vapor pressure & $e$ & ML-1t-2 & $\mathrm{Pa}, \mathrm{N} / \mathrm{m} 2$ \\
\hline Water flux density & $\mathrm{JW}$ & Lt-1 & $\mathrm{m} / \mathrm{s}$ \\
\hline Water vapor potentiala & $\psi \mathrm{w}$ & ML-1t-2 & $\mathrm{N} / \mathrm{m} 2, \mathrm{~Pa}$ \\
\hline
\end{tabular}

a Dimensions and units given for volumetric potential (energy per unit volume).

\section{Symbol Dimensions SI Units}




\section{Physical units and conversions}

\section{Conversion Factors}

Length (L):

Système International (SI) base unit: meters (m)

Dimensions: L

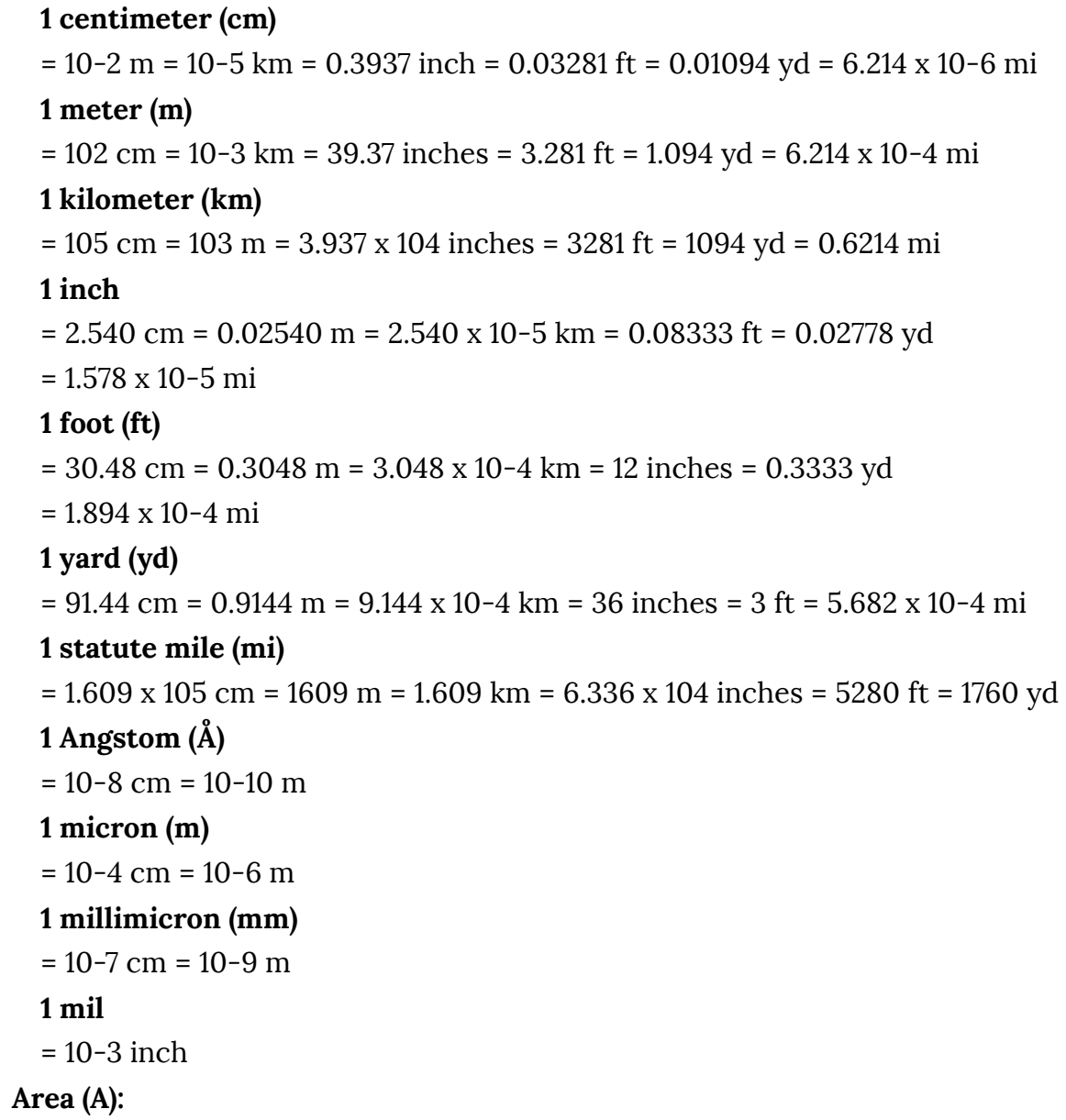

Système International (SI) base unit: square meters (m2)

Dimensions: L2

\section{1 square centimeter $(\mathrm{cm} 2)$}

$=10-4 \mathrm{~m} 2=10-10 \mathrm{~km} 2=10-8$ ha $=0.1550$ inch $2=1.076 \times 10-3 \mathrm{ft} 2$

$=1.196 \times 10-4 \mathrm{yd} 2=3.861 \times 10-11 \mathrm{mi} 2=2.471 \times 10-8$ acre

1 square meter $(\mathrm{m} 2)$

$=104 \mathrm{~cm} 2=10-6 \mathrm{~km} 2=10-4$ ha $=1550$ inches $2=10.76 \mathrm{ft} 2$

$=1.196 \mathrm{yd} 2=3.861 \times 10-7 \mathrm{mi} 2=2.471 \times 10-4$ acre 


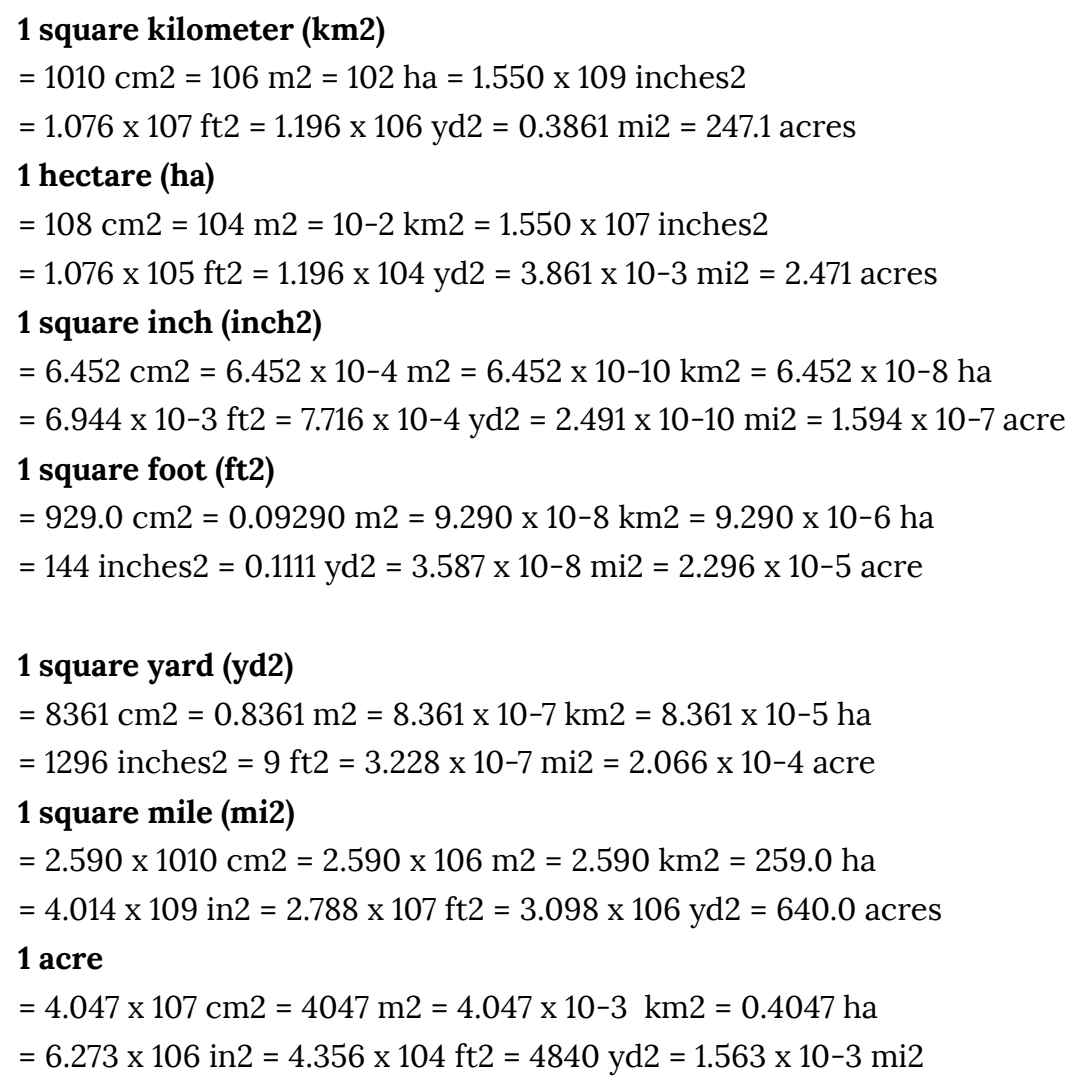

\section{1 square yard (yd2)}

$=8361 \mathrm{~cm} 2=0.8361 \mathrm{~m} 2=8.361 \times 10-7 \mathrm{~km} 2=8.361 \times 10-5$ ha

$=1296$ inches $2=9 \mathrm{ft} 2=3.228 \times 10-7 \mathrm{mi} 2=2.066 \times 10-4$ acre

\section{1 square mile (mi2)}

$=2.590 \times 1010 \mathrm{~cm} 2=2.590 \times 106 \mathrm{~m} 2=2.590 \mathrm{~km} 2=259.0$ ha

$=4.014 \times 109$ in $2=2.788 \times 107 \mathrm{ft} 2=3.098 \times 106 \mathrm{yd} 2=640.0$ acres

1 acre

$=4.047 \times 107 \mathrm{~cm} 2=4047 \mathrm{~m} 2=4.047 \times 10-3 \mathrm{~km} 2=0.4047$ ha

$=6.273 \times 106$ in $2=4.356 \times 104 \mathrm{ft} 2=4840 \mathrm{yd} 2=1.563 \times 10-3 \mathrm{mi} 2$

\section{Volume (V):}

Système International (SI) base unit: cubic meters (m3)

Dimensions: L3

\section{1 cubic centimeter $(\mathrm{cm} 3)$}

$=10-6 \mathrm{~m} 3=10-3$ liter $=0.06102$ inch $3=3.531 \times 10-5 \mathrm{ft} 3$

$=0.03381 \mathrm{fl} \mathrm{oz}=2.642 \times 10-4 \mathrm{gal}$

\section{1 cubic meter $(\mathrm{m} 3)$}

$=106 \mathrm{~cm} 3=103$ liters $=6.102 \times 104$ inches $3=35.31 \mathrm{ft} 3$

$=3.381 \times 104 \mathrm{fl} \mathrm{oz}=264.2 \mathrm{gal}=9.729 \times 10-3$ acre-inch

\section{1 liter}

$=103 \mathrm{~cm} 3=10-3 \mathrm{~m} 3=61.03$ inches $3=0.03532 \mathrm{ft} 3=33.81 \mathrm{fl} \mathrm{oz}=0.2642 \mathrm{gal}$

$=0.2270$ U.S. dry gallon $=1.057$ U.S. fluid quarts $=0.9081$ U.S. dry quart

$=0.02838 \mathrm{bu}$

\section{1 cubic inch (inch3)}

$=16.39 \mathrm{~cm} 3=1.639 \times 10-5 \mathrm{~m} 3=0.01639$ liter $=5.787 \times 10-4 \mathrm{ft} 3$

$=0.5541 \mathrm{fl} \mathrm{oz}=4.329 \times 10-3 \mathrm{gal}$

\section{1 cubic foot (ft3)}

$=2.832 \times 104 \mathrm{~cm} 3=0.02832 \mathrm{~m} 3=28.32$ liters $=1728$ inches 3

$=957.5 \mathrm{fl} \mathrm{oz}=7.481 \mathrm{gal}$ 


\section{U.S. fluid oz (fl oz)}

$=29.57 \mathrm{~cm} 3=2.957 \times 10-5 \mathrm{~m} 3=0.02957$ liter $=1.805$ inches 3

$=1.044 \times 10-3 \mathrm{ft} 3=7.813 \times 10-3 \mathrm{gal}=2.755 \times 10-4$ acre- inch

1 U.S. fluid gallon (gal)

$=3785 \mathrm{~cm} 3=3.785 \times 10-3 \mathrm{~m} 3=3.785$ liters $=231$ inches 3

$=0.1337 \mathrm{ft} 3=128 \mathrm{fl} \mathrm{oz}=8$ U.S. fluid pints $=4$ U.S. fluid quarts

$=3.683 \times 10-5$ acre inch

1 U.S. bushel (bu)

$=35.24$ liters $=0.3524$ hectoliter $=64$ U.S. dry pints

$=32$ U.S. dry quarts $=9.309$ gal. $=8$ U.S. dry gallons $=4$ U.S. dry pecks

1 acre-inch

$=102.8 \mathrm{~m} 3=2.715 \times 104 \mathrm{gal}=3630 \mathrm{ft} 3$

Mass (m):

Système International (SI) base unit: kilogram (kg)

Dimensions: $\mathrm{M}$

\section{1 gram (g)}

$=10-3 \mathrm{~kg}=10-5 \mathrm{q}=10-6$ metric ton $=6.852 \times 10-5 \mathrm{slug}$

$=0.03527 \mathrm{oz}=2.205 \times 10-3 \mathrm{lb}=2.205 \times 10-5 \mathrm{cwt}=1.102 \times 10-6$ U.S. short ton

1 kilogram (kg)

$=103 \mathrm{~g}=10-2 \mathrm{q}=10-3$ metric ton $=0.06852 \mathrm{slug}$

$=35.27 \mathrm{oz}=2.205 \mathrm{lb}=0.02205 \mathrm{cwt}=1.102 \times 10-3$ U.S. short ton

\section{1 metric ton}

$=106 \mathrm{~g}=103 \mathrm{~kg}=10 \mathrm{q}=68.52$ slugs $=3.527 \times 104 \mathrm{oz}$

$=2205 \mathrm{lb}=22.05 \mathrm{cwt}=1.102$ U.S. short tons

\section{1 slug}

$=1.459 \times 104 \mathrm{~g}=14.59 \mathrm{~kg}=0.1459 \mathrm{q}=0.01459$ metric ton

$=514.8 \mathrm{oz}=32.17 \mathrm{lb}=0.3217 \mathrm{cwt}=0.01609$ U.S. short ton

1 avoirdupois ounce (oz)

$=28.35 \mathrm{~g}=0.02835 \mathrm{~kg}=2.835 \times 10-4 \mathrm{q}=2.835 \times 10-5$ metric ton

$=1.94 \times 10-3 \mathrm{slug}=0.0625 \mathrm{lb}=6.25 \times 10-4 \mathrm{cwt}=3.125 \times 10-5$ U.S. short ton

1 avoirdupois pound (lb)

$=453.6 \mathrm{~g}=0.4536 \mathrm{~kg}=4.536 \times 10-3 \mathrm{q}=4.536 \times 10-4$ metric ton

$=0.03108 \mathrm{slug}=16 \mathrm{oz}=10-2 \mathrm{cwt}=5 \times 10-4$ U.S. short ton

1 short hundredwight (cwt)

$=4.536 \times 104 \mathrm{~g}=45.36 \mathrm{~kg}=0.4536 \mathrm{q}=0.04536$ metric ton

$=3.108$ slugs $=1600 \mathrm{oz}=102 \mathrm{lb}=0.05$ U.S. short ton

1 U.S. short ton

$=9.072 \times 105 \mathrm{~g}=907.2 \mathrm{~kg}=9.072 \mathrm{q}=0.9072$ metric ton $=$

$=62.16$ slugs $=3.2 \times 104 \mathrm{oz}=2000 \mathrm{lb}=20 \mathrm{cwt}$

\section{Density $(\rho)$ :}

Système International (SI) base unit: mass/volume $(\mathrm{kg} / \mathrm{m} 3)$

Dimensions: ML-3

416 | Physical units and conversions 
1 gram per cubic centimeter $(\mathrm{g} / \mathrm{cm} 3)$

$=103 \mathrm{~kg} / \mathrm{m} 3=1.940$ slugs $/ \mathrm{ft} 3=0.03613 \mathrm{lb} /$ inch $3=62.43 \mathrm{lb} / \mathrm{ft} 3$

1 kilogram per cubic meter $(\mathrm{kg} / \mathrm{m} 3)$

$=10-3 \mathrm{~g} / \mathrm{cm} 3=1.940 \times 10-3 \mathrm{slug} / \mathrm{ft} 3=3.613 \times 10-5 \mathrm{lb} /$ inch $3=0.06243 \mathrm{lb} / \mathrm{ft} 3$

1 slug per cubic foot (slug/ft3)

$=0.5154 \mathrm{~g} / \mathrm{cm} 3=515.4 \mathrm{~kg} / \mathrm{m} 3=0.01862 \mathrm{lb} / \mathrm{inch} 3=32.17 \mathrm{lb} / \mathrm{ft} 3$

1 pound per cubic inch $(\mathrm{lb} / \mathrm{inch} 3)$

$=27.68 \mathrm{~g} / \mathrm{cm} 3=2.768 \times 104 \mathrm{~kg} / \mathrm{m} 3=53.71 \mathrm{slugs} / \mathrm{ft} 3=1728 \mathrm{lb} / \mathrm{ft} 3$

1 pound per cubic foot $(\mathrm{lb} / \mathrm{ft} 3)$

$=0.01602 \mathrm{~g} / \mathrm{cm} 3=16.02 \mathrm{~kg} / \mathrm{m} 3=0.03108 \mathrm{slug} / \mathrm{ft} 3=5.787 \times 10-4 \mathrm{lb} /$ inch 3

\section{Velocity (V):}

Système International (SI) base unit: meter/second (m/s)

Dimensions: Lt-1

\section{1 centimeter per second $(\mathrm{cm} / \mathrm{sec})$}

$=10-2 \mathrm{~m} / \mathrm{sec}=0.036 \mathrm{~km} / \mathrm{hr}=0.03281 \mathrm{ft} / \mathrm{sec}=0.02237 \mathrm{mi} / \mathrm{hr}=0.01944 \mathrm{knot}$ 1 meter per second $(\mathrm{m} / \mathrm{sec})$

$=102 \mathrm{~cm} / \mathrm{sec}=3.6 \mathrm{~km} / \mathrm{hr}=3.281 \mathrm{ft} / \mathrm{sec}=2.237 \mathrm{mi} / \mathrm{hr}=1.944 \mathrm{knots}$

1 kilometer per hour $(\mathbf{k m} / \mathbf{h r})$

$=27.78 \mathrm{~cm} / \mathrm{sec}=0.2778 \mathrm{~m} / \mathrm{sec}=0.9113 \mathrm{ft} / \mathrm{sec}=0.6214 \mathrm{mi} / \mathrm{hr}=0.5400 \mathrm{knot}$

1 foot per second (ft/sec)

$=30.48 \mathrm{~cm} / \mathrm{sec}=0.3048 \mathrm{~m} / \mathrm{sec}=1.097 \mathrm{~km} / \mathrm{hr}=0.6818 \mathrm{mi} / \mathrm{hr}=0.5925 \mathrm{knot}$ 1 mile per hour ( $\mathrm{mi} / \mathrm{hr}$ or $\mathrm{mph}$ )

$=44.70 \mathrm{~cm} / \mathrm{sec}=0.4470 \mathrm{~m} / \mathrm{sec}=1.609 \mathrm{~km} / \mathrm{hr}=1.467 \mathrm{ft} / \mathrm{sec}=0.8690 \mathrm{knot}$

1 nautical mile/hr (knot)

$=51.44 \mathrm{~cm} / \mathrm{sec}=0.5144 \mathrm{~m} / \mathrm{sec}=1.852 \mathrm{~km} / \mathrm{hr}=1.688 \mathrm{ft} / \mathrm{sec}=1.151 \mathrm{mi} / \mathrm{hr}$

Force (F):

Système International (SI) base unit: newton (nt or N)

Dimensions: MLt-2

\section{1 dyne}

$=10-5 \mathrm{nt}=2.248 \times 10-6 \mathrm{lb}$

\section{1 newton (nt)}

$=105$ dynes $=0.2248 \mathrm{lb}$

1 pound (lb)

$=4.448 \times 105$ dynes $=4.448 \mathrm{nt}$

Pressure (p):

Système International (SI) base unit: newton/area (nt/m2)

Dimensions: ML-1t-2

1 dyne per square centimeter [barye] (dyne/cm2)

$=10-1 \mathrm{nt} / \mathrm{m} 2=1.450 \times 10-5 \mathrm{lb} /$ inch $2=2.089 \times 10-3 \mathrm{lb} / \mathrm{ft} 2=9.869 \times 10-7 \mathrm{~atm}$

$=10-6 \mathrm{bar}=10-3 \mathrm{mb}=4.015 \times 10-4$ inch $\mathrm{H} 2 \mathrm{O}=7.501 \times 10-5 \mathrm{~cm} \mathrm{Hg}$

1 newton per square meter [pascal] (nt/m2) 
$=10$ dynes $/ \mathrm{cm} 2=1.450 \times 10-4 \mathrm{lb} /$ inch $2=0.02089 \mathrm{lb} / \mathrm{ft} 2=9.869 \times 10-6 \mathrm{~atm}$

$=10-5 \mathrm{bar}=10-2 \mathrm{mb}=4.015 \times 10-3$ inch $\mathrm{H} 2 \mathrm{O}=7.501 \times 10-4 \mathrm{~cm} \mathrm{Hg}$

1 pound per square inch $(\mathrm{lb} /$ inch 2 or $\mathrm{psi})$

$=6.895 \times 104$ dynes $/ \mathrm{cm} 2=6.895 \times 103 \mathrm{nt} / \mathrm{m} 2=144 \mathrm{lb} / \mathrm{ft} 2$

$=0.06805 \mathrm{~atm}=0.06895 \mathrm{bar}=68.95 \mathrm{mb}=27.68$ inches $\mathrm{H} 2 \mathrm{O}=5.171 \mathrm{~cm} \mathrm{Hg}$

1 pound per square foot $(\mathrm{lb} / \mathrm{ft} 2)$

$=478.8$ dynes $/ \mathrm{cm} 2=47.88 \mathrm{nt} / \mathrm{m} 2=6.944 \times 10-3 \mathrm{lb} / \mathrm{inch} 2=4.725 \times 10-4 \mathrm{~atm}$

$=4.788 \times 10-4 \mathrm{bar}=0.4788 \mathrm{mb}=0.1922$ inch $\mathrm{H} 2 \mathrm{O}=0.03591 \mathrm{~cm} \mathrm{Hg}$

1 atmosphere (atm)

$=1.013 \times 106$ dynes $/ \mathrm{cm} 2=1.013 \times 105 \mathrm{nt} / \mathrm{m} 2=14.70 \mathrm{lb} /$ inch 2

$=2116 \mathrm{lb} / \mathrm{ft} 2=1.013 \mathrm{bars}=1013 \mathrm{mb}=406.8$ inches $\mathrm{H} 2 \mathrm{O}=76 \mathrm{~cm} \mathrm{Hg}$

1 bar

$=106$ dynes $/ \mathrm{cm} 2=105 \mathrm{nt} / \mathrm{m} 2=14.50 \mathrm{lb} / \mathrm{in} 2=2089 \mathrm{lb} / \mathrm{ft} 2=0.9869 \mathrm{~atm}=103 \mathrm{mb}$

$=401.5$ inches $\mathrm{H} 2 \mathrm{O}=75.01 \mathrm{~cm} \mathrm{Hg}$

1 millibar (mb)

$=103$ dynes $/ \mathrm{cm} 2=102 \mathrm{nt} / \mathrm{m} 2=0.01450 \mathrm{lb} /$ inch $2=2.089 \mathrm{lb} / \mathrm{ft} 2$

$=9.869 \times 10-4 \mathrm{~atm}=10-3 \mathrm{bar}=0.4015$ inch $\mathrm{H} 2 \mathrm{O}=0.07501 \mathrm{~cm} \mathrm{Hg}$

1 inch of water at $4^{\circ} \mathrm{C}$ (inch $\mathrm{H} 2 \mathrm{O}$ )

$=2491$ dynes $/ \mathrm{cm} 2=249.1 \mathrm{nt} / \mathrm{m} 2=0.03613 \mathrm{lb} / \mathrm{inch} 2=5.202 \mathrm{lb} / \mathrm{ft} 2$

$=2.458 \times 10-3 \mathrm{~atm}=2.491 \times 10-3 \mathrm{bar}=2.491 \mathrm{mb}=0.1868 \mathrm{~cm} \mathrm{Hg}$

1 centimeter of mercury at $0^{\circ} \mathrm{C}(\mathrm{cm} \mathrm{Hg})$

$=1.333 \times 104$ dynes $/ \mathrm{cm} 2=1333 \mathrm{nt} / \mathrm{m} 2=0.1934 \mathrm{lb} /$ inch $2=27.85 \mathrm{lb} / \mathrm{ft} 2$

$=0.01316 \mathrm{~atm}=0.01333 \mathrm{bar}=13.33 \mathrm{mb}=5.353$ inches $\mathrm{H} 2 \mathrm{O}$

\section{Energy (E):}

Système International (SI) base unit: joule $(\mathrm{J}=\mathrm{nt} \mathrm{m})$

Dimensions: ML2t-2

\section{$1 \mathrm{erg}$}

$=10-7$ joule $=2.778 \times 10-14 \mathrm{kw} \mathrm{hr}=7.376 \times 10-8 \mathrm{ft} \mathrm{lb}$

$=3.725 \times 10-14 \mathrm{hp} \mathrm{hr}=2.389 \times 10-8 \mathrm{cal}=9.485 \times 10-11 \mathrm{Btu}$

1 joule

$=107 \mathrm{ergs}=2.778 \times 10-7 \mathrm{kw} \mathrm{hr}=0.7376 \mathrm{ft} \mathrm{lb}$

$=3.725 \times 10-7 \mathrm{hp} \mathrm{hr}=0.2389 \mathrm{cal}=9.485 \times 10-4 \mathrm{Btu}$

1 kilowatt hour (kw hr)

$=3.6 \times 1013 \mathrm{ergs}=3.6 \times 106$ joules $=2.655 \times 106 \mathrm{ft} \mathrm{lb}$

$=1.341 \mathrm{hp} \mathrm{hr}=8.601 \times 105 \mathrm{cal}=3415 \mathrm{Btu}$

1 foot pound ( $\mathbf{f t} \mathbf{~ l b}$ )

$=1.356 \times 107 \mathrm{ergs}=1.356$ joules $=3.766 \times 10-7 \mathrm{kw} \mathrm{hr}$

$=5.051 \times 10-7 \mathrm{hp} \mathrm{hr}=0.3239 \mathrm{cal}=1.286 \times 10-3 \mathrm{Btu}$

1 horsepower hour (hp hr)

$=2.685 \times 1013 \mathrm{ergs}=2.685 \times 106$ joules $=0.7457 \mathrm{kw} \mathrm{hr}$

$=1.98 \times 106 \mathrm{ft} \mathrm{lb}=6.413 \times 105 \mathrm{cal}=2546 \mathrm{Btu}$

1 calorie at $15^{\circ} \mathrm{C}$ (cal)

$=4.186 \times 107 \mathrm{ergs}=4.186$ joules $=1.163 \times 10-6 \mathrm{kw} \mathrm{hr}=3.087 \mathrm{ft} \mathrm{lb}$

418 | Physical units and conversions 
$=1.559 \times 10-6 \mathrm{hp} \mathrm{hr}=3.970 \times 10-3 \mathrm{Btu}$

1 British thermal unit (Btu)

$=1.054 \times 1010$ ergs $=1054$ joules $=2.929 \times 10-4 \mathrm{kw} \mathrm{hr}$

$=777.6 \mathrm{ft} \mathrm{lb}=3.928 \times 10-4 \mathrm{hp} \mathrm{hr}=251.9 \mathrm{cal}$

Power (P):

Système International (SI) base unit: watt (J/s)

Dimensions: ML2t-3

1 watt [1 joule per second]

$=10-3 \mathrm{kw}=0.7376 \mathrm{ft} \mathrm{lb} / \mathrm{sec}=44.25 \mathrm{ft} \mathrm{lb} / \mathrm{min}$

$=1.341 \times 10-3 \mathrm{hp}=0.2389 \mathrm{cal} / \mathrm{sec}=3.414 \mathrm{Btu} / \mathrm{hr}$

\section{1 kilowatt (kw)}

$=103$ watts $=737.6 \mathrm{ft} \mathrm{lb} / \mathrm{sec}=4.425 \times 104 \mathrm{ft} \mathrm{lb} / \mathrm{min}$

$=1.341 \mathrm{hp}=238.9 \mathrm{cal} / \mathrm{sec}=3414 \mathrm{Btu} / \mathrm{hr}$

1 foot pound per second ( $\mathrm{ft} \mathrm{lb} / \mathrm{sec}$ )

$=1.356$ watts $=1.356 \times 10-3 \mathrm{kw}=60 \mathrm{ft} \mathrm{lb} / \mathrm{min}$

$=1.818 \times 10-3 \mathrm{hp}=0.3239 \mathrm{cal} / \mathrm{sec}=4.629 \mathrm{Btu} / \mathrm{hr}$

1 foot pound per minute (ft $\mathrm{lb} / \mathrm{min}$ )

$=0.02260$ watt $=2.260 \times 10-5 \mathrm{kw}=0.01667 \mathrm{ft} \mathrm{lb} / \mathrm{sec}=3.030 \times 10-5 \mathrm{hp}$

$=5.398 \times 10-3 \mathrm{cal} / \mathrm{sec}=0.07716 \mathrm{Btu} / \mathrm{hr}$

1 horsepower (hp)

$=745.7$ watts $=0.7457 \mathrm{kw}=550 \mathrm{ft} \mathrm{lb} / \mathrm{sec}$

$=3.300 \times 104 \mathrm{ft} \mathrm{lb} / \mathrm{min}=178.1 \mathrm{cal} / \mathrm{sec}=2546 \mathrm{Btu} / \mathrm{hr}$

1 calorie per second (cal/sec)

$=4.186$ watts $=4.186 \times 10-3 \mathrm{kw}=3.087 \mathrm{ft} \mathrm{lb} / \mathrm{sec}$

$=185.2 \mathrm{ft} \mathrm{lb} / \mathrm{min}=5.613 \times 10-3 \mathrm{hp}=14.29 \mathrm{Btu} / \mathrm{hr}$

1 British thermal unit per hour (Btu/hr)

$=0.2929$ watt $=2.929 \times 10-4 \mathrm{kw}=0.2160 \mathrm{ft} \mathrm{lb} / \mathrm{sec}=12.96 \mathrm{ft} \mathrm{lb} / \mathrm{min}$

$=3.928 \times 10-4 \mathrm{hp}=0.06997 \mathrm{cal} / \mathrm{sec}$

\section{Temperature (T):}

Système International (SI) base unit: Kelvin (K)

Dimensions: $\mathrm{T}$

Degrees Celcius $\left({ }^{\circ} \mathrm{C}\right)$

$=\mathrm{K}-273=(5 / 9)(\mathbf{o F}-32)$

Kelvin (K)

$={ }^{\circ} \mathrm{C}+273=(5 / 9)(\mathrm{oF}-32)+273$

Degrees Fahrenheit (oF)

$=(9 / 5)^{\circ} \mathrm{C}+32=(9 / 5)(\mathrm{K}-273)+32$

Yield:

1 kilogram per hectare (kg/ha)

$=10-3$ metric ton $/$ ha $=0.8922 \mathrm{lb} /$ acre $=8.922 \times 10-3 \mathrm{cwt} /$ acre

$=4.461 \times 10-4$ short ton/acre

1 metric ton per hectare (metric ton/ha) 
$=103 \mathrm{~kg} / \mathrm{ha}=892.2 \mathrm{lb} / \mathrm{acre}=8.922 \mathrm{cwt} / \mathrm{acre}=0.4461$ short ton $/$ acre

1 pound per acre (lb/acre)

$=1.121 \mathrm{~kg} / \mathrm{ha}=1.121 \times 10-3$ metric ton $/ \mathrm{ha}=10-2 \mathrm{cwt} /$ acre

$=5 \times 10-4$ short ton/acre

1 hundredweight per acre (cwt/acre)

$=112.1 \mathrm{~kg} / \mathrm{ha}=0.1121$ metric ton $/ \mathrm{ha}=100 \mathrm{lb} /$ acre $=0.05$ short ton $/$ acre

1 short ton per acre (short ton/acre)

$=2242 \mathrm{~kg} / \mathrm{ha}=2.242$ metric ton $/ \mathrm{ha}=2000 \mathrm{lb} / \mathrm{acre}=20 \mathrm{cwt} / \mathrm{acre}$

1 liter per hectare (liter/ha)

$=10-2 \mathrm{hl} / \mathrm{ha}=0.01148 \mathrm{bu} / \mathrm{acre}$

1 hectoliter per hectare (hg/ha)

$=102$ liters $/$ ha $=1.148 \mathrm{bu} /$ acre

1 U.S. bushel per acre (bu/acre)

$=87.08$ liters $/$ ha $=0.8708 \mathrm{hl} / \mathrm{ha}$

\section{Flow (Q) :}

Système International (SI) base unit: meter3/sec (m3/s)

Dimensions: L3t-1

1 cubic centimeter per second $(\mathrm{cm} 3 / \mathrm{sec})$

$=10-3$ liter $/ \mathrm{sec}=0.06$ liter $/ \mathrm{min}=3.531 \times 10-5 \mathrm{ft} 3 / \mathrm{sec}=2.119 \times 10-3 \mathrm{ft} 3 / \mathrm{min}$

$=2.642 \times 10-4 \mathrm{gal} / \mathrm{sec}=0.01585 \mathrm{gal} / \mathrm{min}$

1 liter per second (liter/sec)

$=103 \mathrm{~cm} 3 / \mathrm{sec}=60$ liters $/ \mathrm{min}=0.03532 \mathrm{ft} 3 / \mathrm{sec}$

$=2.119 \mathrm{ft} 3 / \mathrm{min}=0.2642 \mathrm{gal} / \mathrm{sec}=15.85 \mathrm{gal} / \mathrm{min}$

1 liter per minute (liter/min)

$=16.67 \mathrm{~cm} 3 / \mathrm{sec}=0.01667$ liter $/ \mathrm{sec}=5.886 \times 10-4 \mathrm{ft} 3 / \mathrm{sec}=0.03532 \mathrm{ft} 3 / \mathrm{min}$

$=4.403 \times 10-3 \mathrm{gal} / \mathrm{sec}=0.2642 \mathrm{gal} / \mathrm{min}$

1 cubic foot per second ( $\mathrm{ft} 3 / \mathrm{sec})$

$=2.832 \times 104 \mathrm{~cm} 3 / \mathrm{sec}=28.32$ liters $/ \mathrm{sec}=1699$ liters $/ \mathrm{min}=60 \mathrm{ft} 3 / \mathrm{min}$

$=7.481 \mathrm{gal} / \mathrm{sec}=448.8 \mathrm{gal} / \mathrm{min}=0.9917 \mathrm{acre}-\mathrm{inch} / \mathrm{hr}$

1 cubic foot per minute (ft3/min)

$=471.9 \mathrm{~cm} 3 / \mathrm{sec}=0.4719$ liter $/ \mathrm{sec}=28.32$ liters $/ \mathrm{min}=0.01667 \mathrm{ft} 3 / \mathrm{sec}$

$=0.1247 \mathrm{gal} / \mathrm{sec}=7.481 \mathrm{gal} / \mathrm{min}=0.01653 \mathrm{acre}-\mathrm{inch} / \mathrm{hr}$

1 U.S. fluid gallon per second (gal/sec)

$=3785 \mathrm{~cm} 3 / \mathrm{sec}=3.785$ liters $/ \mathrm{sec}=227.1$ liter $/ \mathrm{min}=0.1337 \mathrm{ft} 3 / \mathrm{sec}$

$=8.021 \mathrm{ft} 3 / \mathrm{min}=60 \mathrm{gal} / \mathrm{min}$

1 U.S. fluid gallon per minute (gal/min)

$=63.09 \mathrm{~cm} 3 / \mathrm{sec}=0.06309$ liter $/ \mathrm{sec}=3.785$ liter $/ \mathrm{min}=2.228 \times 10-3 \mathrm{ft} 3 / \mathrm{sec}$

$=0.1337 \mathrm{ft} 3 / \mathrm{min}=0.01667 \mathrm{gal} / \mathrm{sec}$

Dynamic (absolute) Viscosity $(\mu)$ :

Système International (SI) base unit: (nt s /m2)

Dimensions: ML-1t-1

1 slug $/ \mathrm{ft} \mathbf{s}=1 \mathrm{lb} \mathrm{s} / \mathrm{ft} 2=47.88 \mathrm{~kg} / \mathrm{m} \mathrm{s}=47.88 \mathrm{~N} \mathrm{~s} / \mathrm{m} 2$

420 | Physical units and conversions 
1 poise $=1$ dynes s $/ \mathrm{cm} 2=0.1 \mathrm{~N} \mathrm{~s} / \mathrm{m} 2$

\section{Kinematic Viscosity (v):}

Système International (SI) base unit: meter $2 / \mathrm{sec}(\mathrm{m} 2 / \mathrm{s})$

Dimensions: $\mathrm{L} 2 \mathrm{t}-1$

$1 \mathrm{ft} 2 / \mathrm{s}=0.0929 \mathrm{~m} 2 / \mathrm{s}$

1 stoke $=1 \mathrm{~cm} 2 / \mathrm{s}=0.00108 \mathrm{ft} 2 / \mathrm{s}$

$1 \mathrm{~m} 2 / \mathrm{s}=10.764 \mathrm{ft} 2 / \mathrm{s}$ 


\section{Selected physical constants}

\section{Selected Physical Constants}

cpa Specific heat capacity of dry air $=1.003 \times 107 \mathrm{ergs} / \mathrm{g}^{\circ} \mathrm{C}=1003$ joules $/ \mathrm{kg}^{\circ} \mathrm{C}$

$=0.2396 \mathrm{cal} / \mathrm{g}^{\circ} \mathrm{C}$

e $\quad$ Base of the natural logarithms $=2.71828$

F $\quad$ Faraday constant $=96,500$ coulombs per gram equivalent

$g$ Acceleration of gravity: normal acceleration $=980.665 \mathrm{~cm} / \mathrm{sec} 2=9.80665 \mathrm{~m} / \mathrm{sec} 2=$

$32.1740 \mathrm{ft} /$

$\sec 2$

$k \quad$ von Karman constant $=$ about 0.41 (unitless)

$\mathrm{L} \quad$ Latent heat of vaporization of water:

at $0^{\circ} \mathrm{C}=2.494 \times 1010 \mathrm{ergs} / \mathrm{g}=2.494 \times 106$ joules $/ \mathrm{kg}=595.9 \mathrm{cal} / \mathrm{g}$

at $20^{\circ} \mathrm{C}=2.448 \times 1010 \mathrm{ergs} / \mathrm{g}=2.448 \times 106 \mathrm{joules} / \mathrm{kg}=584.9 \mathrm{cal} / \mathrm{g}$

at $100^{\circ} \mathrm{C}=2.259 \times 1010 \mathrm{ergs} / \mathrm{g}=2.259 \times 106$ joules $/ \mathrm{kg}=539.55 \mathrm{cal} / \mathrm{g}$

NA Avogadro $=\mathrm{s}$ number $=6.023 \times 1023$ molecules $/ \mathrm{mole}$

$\mathrm{Pa}$ Pressure of the atmosphere: standard atmosphere $=1.01325 \times 106$ dynes $/ \mathrm{cm} 2=1.01325 \times 105 \mathrm{nt} / \mathrm{m} 2=$ $14.69 \mathrm{lb} /$ inch $2=2117 \mathrm{lb} / \mathrm{ft} 2$

$\mathrm{R}^{*} \quad$ Universal gas constant (gas constant per mole) $=8.314 \times 107 \mathrm{ergs} / \mathrm{K}$ mole $=$ 8.314 joules $/ \mathrm{K}$ mole $=1.986 \mathrm{cal} / \mathrm{K}$ mole

$\begin{array}{lll}\text { Rs } & \text { Solar constant }=1.35 \times 106 \mathrm{ergs} / \mathrm{cm} 2 \mathrm{sec}=1.35 \times 103 \text { watts } / \mathrm{m} 2=1.94 \mathrm{cal} / \mathrm{cm} 2 \mathrm{~min} \quad \text { (langleys } / \mathrm{min}) \\ \gamma & \text { Psychrometric constant at } 1 \mathrm{standard} \text { atmosphere and } 20^{\circ} \mathrm{C}=0.667 \mathrm{mb} / \mathrm{K}=\end{array}$ $0.278 \mathrm{~mm} \mathrm{Hg} / \mathrm{oF}$

$\varepsilon \quad$ Ratio of the molecular weight of water to that of air $=0.622$ (unitless)

pa Density of dry air: at 1 standard atmosphere and $0^{\circ} \mathrm{C}=1.293 \times 10-3 \mathrm{~g} / \mathrm{cm} 3=$

$1.293 \mathrm{~kg} / \mathrm{m} 3=2.508 \times 10-3 \mathrm{slug} / \mathrm{ft} 3$

○w Density of water: maximum density at 1 standard atmoshphere and $3.98^{\circ} \mathrm{C}=$

$0.99997 \mathrm{~g} / \mathrm{cm} 3=1.00000 \mathrm{~g} / \mathrm{ml}$

$\rho \mathrm{Hg} \quad$ Density of mercury: at 1 standard atmosphere and $0^{\circ} \mathrm{C}=13.595 \mathrm{~g} / \mathrm{cm} 3=$

$13,595 \mathrm{~kg} / \mathrm{m} 3=26.38$ slugs $/ \mathrm{ft} 3$

$\pi \quad=3.14159$

$\sigma \quad$ Stefan-Boltzmann constant $=5.67 \times 10-5$ ergs $/ \mathrm{K} 4 \mathrm{~cm} 2 \mathrm{sec}=5.67 \times 10-8$ watts $/ \mathrm{K} 4 \mathrm{~m} 2=$

$8.127 \times 10-11$ $\mathrm{cal} / \mathrm{K} 4 \mathrm{~cm} 2 \mathrm{~min}$ (langley/K4 min)

Boltzmann constant $=1.381 \times 10-16$ ergs $/ \mathrm{K}=1.381 \times 10-23$ joules $/ \mathrm{K}=$

$3.298 \times 10-24 \mathrm{cal} / \mathrm{K}$

Ice point $=273.16 \mathrm{~K}$ 


\section{Properties of liquid water}

\section{Properties of Liquid Water at typical environmental temperatures}

\begin{tabular}{|c|c|c|c|c|c|c|c|}
\hline Temperature & Density & $\begin{array}{l}\text { Specific Heat } \\
\text { Capacity }\end{array}$ & $\begin{array}{l}\text { Vapor } \\
\text { Pressure }\end{array}$ & Viscosity & $\begin{array}{l}\text { Thermal } \\
\text { Conduc- } \\
\text { tivity }\end{array}$ & $\begin{array}{l}\text { Dielectric } \\
\text { Constant }\end{array}$ & $\begin{array}{l}\text { Surface } \\
\text { Tension }\end{array}$ \\
\hline $\mathrm{oC}$ & $\mathrm{g} / \mathrm{cm} 3$ & $\mathrm{~J} / \mathrm{g} \mathrm{K}$ & $\mathbf{k P a}$ & $\mathrm{mPa} s$ & $\mathrm{~mW} / \mathrm{K} \mathrm{m}$ & & $\mathrm{mN} / \mathrm{m}$ \\
\hline 0 & 0.99984 & 4.2176 & 0.6113 & 1793 & 561.0 & 87.90 & 75.64 \\
\hline 10 & 0.99970 & 4.1921 & 1.2281 & 1307 & 580.0 & 83.96 & 74.23 \\
\hline 20 & 0.99821 & 4.1818 & 2.3388 & 1002 & 598.4 & 80.20 & 72.75 \\
\hline 30 & 0.99565 & 4.1784 & 4.2455 & 797.7 & 615.4 & 76.60 & 71.20 \\
\hline 40 & 0.99222 & 4.1785 & 7.3814 & 653.2 & 630.5 & 73.17 & 69.60 \\
\hline 50 & 0.98803 & 4.1806 & 12.344 & 547.0 & 643.5 & 69.88 & 67.94 \\
\hline 60 & 0.98320 & 4.1843 & 19.932 & 466.5 & 654.3 & 66.73 & 66.24 \\
\hline 70 & 0.97778 & 4.1895 & 31.176 & 404.0 & 663.1 & 63.73 & 64.47 \\
\hline 80 & 0.97182 & 4.1963 & 47.373 & 354.4 & 670.0 & 60.86 & 62.67 \\
\hline 90 & 0.96535 & 4.2050 & 70.117 & 314.5 & 675.3 & 58.12 & 60.82 \\
\hline 100 & 0.95840 & 4.2159 & 101.325 & 281.8 & 679.1 & 55.51 & 58.91 \\
\hline
\end{tabular}

a All values (except vapor pressure) given for $100 \mathrm{kPa}$ (1bar) pressure.

b Specific heat capacity at constant pressure $(\mathrm{Cp})$. 


\section{Creative Commons License}

This work is licensed by John Selker \& Dani Or under a

Creative Commons Attribution-NonCommerical-Share Alike 4.0 International License (CC BY-NC-SA)

You are free to:

Share - copy and redistribute the material in any medium or format

Adapt - remix, transform, and build upon the material

The licensor cannot revoke these freedoms as long as you follow the license terms.

\section{Under the following terms:}

Attribution - You must give appropriate credit, provide a link to the license, and indicate if changes were made. You may do so in any reasonable manner, but not in any way that suggests the licensor endorses you or your use.

NonCommercial - You may not use the material for commercial purposes.

ShareAlike - If you remix, transform, or build upon the material, you must distribute your contributions under the same license as the original.

No additional restrictions - You may not apply legal terms or technological measures that legally restrict others from doing anything the license permits. 


\section{Recommended Citations}

APA outline:

Source from website:

- (Full last name, first initial of first name). (Date of publication). Title of source. Retrieved from https://www.someaddress.com/full/url/

Source from print:

- (Full last name, first initial of first name). (Date of publication). Title of source. Title of container (larger whole that the source is in, i.e. a chapter in a book), volume number, page numbers.

Examples

If retrieving from a webpage:

- Berndt, T. J. (2002). Friendship quality and social development. Retrieved from insert link.

If retrieving from a book:

- Berndt, T. J. (2002). Friendship quality and social development. Current Directions in Psychological Science, 11, 7-10.

MLA outline:

Author (last, first name). Title of source. Title of container (larger whole that the source is in, i.e. a chapter in a book), Other contributors, Version, Number, Publisher, Publication Date, Location (page numbers).

Examples

- Bagchi, Alaknanda. "Conflicting Nationalisms: The Voice of the Subaltern in Mahasweta Devi's Bashai Tudu." Tulsa Studies in Women's Literature, vol. 15, no. 1, 1996, pp. 41-50.

- $\quad$ Said, Edward W. Culture and Imperialism. Knopf, 1994.

\section{Chicago outline:}

Source from website:

- Lastname, Firstname. "Title of Web Page." Name of Website. Publishing organization, publication or revision date if available. Access date if no other date is available. URL . 
Source from print:

- Last name, First name. Title of Book. Place of publication: Publisher, Year of publication.

Examples

- $\quad$ Davidson, Donald, Essays on Actions and Events. Oxford: Clarendon, 2001. https://bibliotecamathom.files.wordpress.com/2012/10/essays-on-actions-and-events.pdf.

- $\quad$ Kerouac, Jack. The Dharma Bums. New York: Viking Press, 1958. 


\section{Versioning}

This page provides a record of changes made to this guide. Each set of edits is acknowledged with a 0.01 increase in the version number. The exported files for this toolkit reflect the most recent version.

If you find an error in this text, please fill out the form at bit.ly/33cz3Q1

\begin{tabular}{|l|l|l|l|}
\hline Version & Date & Change Made & Location in text \\
\hline 0.1 & MM/DD/YYYY & & \\
\hline & & & \\
\hline & & & \\
\hline
\end{tabular}

